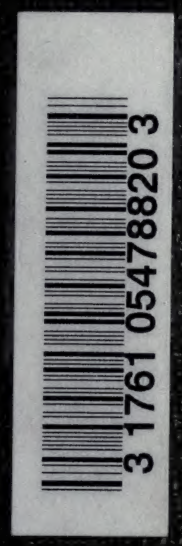



Digitized by the Internet Archive in 2007 with funding from Microsoft Corporation 





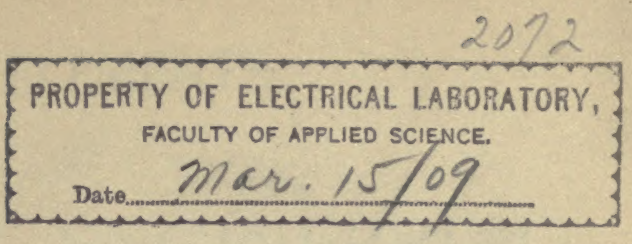

\title{
THE ELEMENTS OF
}

\section{ELECTRICAL ENGINEERING}

\author{
VOLUME I.
}

DIRECT CURRENT MACHINES

ELECTRIC DISTRIBUTION AND LIGHTING 
Thestixo. 


\section{THE ELEMENTS}

OF

\section{ELECTRICAL ENGINEERING}

A TEXT BOOK FOR

TECHNICAL SCHOOLS AND COLLEGES

BY

WILLIAM SUDDARDS FRANKLIN

PROFESSOR OF PHYSICS IN LEHIGH UNIVERSITY

AND

WILLIAM ESTY

PROFHSSOR OF ELECTRICAL ENGINEERING IN LEHIGH UNIVERSITY

VOLUME I.

DIRECT CURRENT MACHINES

ELECTRIC DISTRIBUTION AND LIGHTING

THIRD EDITION, EIGHTH THOUSAND

那elo 思ork

THE MACMILLAN COMPANY

LONDON: MACMILLAN \& CO., LTD.

1907

All rights reserved 


\section{COPYRIGHT, Ig06}

\section{By W. S. FRANKLIN AND WILLIAM ESTY}

Advance Edition, January, I9o6.

First Regular Edition, September x, rgo6.

Second Edition, October, 1906.

Third Edition, August, 1907 .

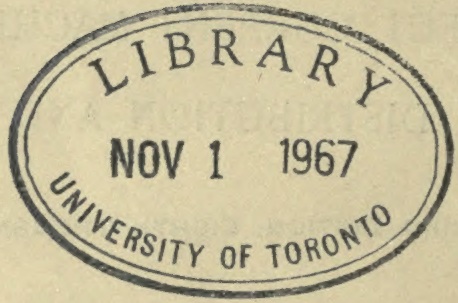




\section{PREFACE.}

THis treatise on the elements of electrical engineering represents the combined experience of the authors in teaching the subject for thirteen years. The aim has been to give a clear and concise treatment of the elements of the subject illustrated by numerous practical examples and problems.

In almost every branch of engineering a simple working knowledge of the electrical problems involved in the generation, distribution, and utilization of power is becoming imperative. Students pursuing a course in engineering, other than electrical, are limited as to the time to be devoted to electrotechnology, while students taking a course in electrical engineering are not so restricted.

The problem which the authors undertook to solve in the preparation of this treatise was to so select and arrange the subject matter that the book might be advantageously used as an introductory course, not only for electrical engineering students, but also for students specializing in other branches of engineering.

This somewhat difficult problem has been solved by treating the more essential parts of the subject consecutively in a series of chapters, and by placing the more elaborate developments in in a series of appendices. This arrangement makes it possible for a student to easily cover the fundamental portions of the text in one semester, by omitting the more highly specialized matter that is given partly in fine print and partly in Appendices A, B and $\mathrm{C}$.

An important feature of the book is an extended list of carefully chosen problems given as a final appendix. These problems are arranged in an order following closely the development of the subject matter of the text. They have been designed not merely to illustrate principles, but to supplement the information given in the text. The answers to these problems have been checked with extreme care. 
The book as a whole seems to be comparatively free from inaccuracies and errors which the authors hope to eliminate entirely. Lists of errata from those who use the book will be thankfully received by

\section{The Authors.}

\section{South Bethlehem,}

July, 1906. 


\section{TABLE OF CONTENTS.}

PAGES.

Classification and Notation . . . . . . . IX-XIII

CHAPTER I.

INTRODUCTION.

Elementary Electricity and Magnetism . . . . . . . . I- 34

CHAPTER II.

The Dynamo ................. 35- 73

CHAPTER III.

The Operation of the Dynamo as a Generator . . . . . . 74- 94

CHAPTER IV.

The Operation of the Dynamo as a Motor . . . . . 95-126

CHAPTER V.

Power Losses in Generators and Motors. Efficiency . . . . 127-144

CHAPTER VI.

RATINGS AND GUARANTEES. ............... I45-171

CHAPTER VII.

The Practical Operation of Dynamos. Station Equipment . . . 172-237

CHAPTER VIII.

Storage Batteries. . . . . . . . . . . . . 238-265

CHAPTER IX.

Electric Distribution AND Wiring . . . . . . . . . . . 266-307

CHAPTER $\mathrm{X}$.

Photometry and Electric Lighting. . . . . . . . . . 308-350

APPENDIX A.

Electromagnets. Magnetism of Iron. . . . . . . 351-382 
viii

\section{TABLE OF CONTENTS}

APPENDIX B.

Characteristic Curves. ................. 383-417

APPENDIX C.

ARMATURE Windings. . . . . . . . . . . . 418-447

APPENDIX D.

Problems . . . . . . . . . . . . . . . 448-509

INDEX ........... 5111-517 


\section{CLASSIFICATION AND NOTATION.}

\section{CLASSIFICATION OF MACHINES.*}

A direct current is a unidirectional current.

A continuous current is a steady, or non-pulsating, direct current.

An alternating current is a current of equal half-waves in successively opposite directions.

An oscillating current is a current alternating in direction, and of decreasing amplitude.

Electrical Apparatus will be treated under the following heads :

I. Commutating Machines, which comprise a constant magnetic field, a closed coil armature, and a multi-segmental commutator connected thereto.

Under this head may be classed the following: Continuouscurrent generators ; continuous-current motors ; continuous-current boosters; motor-generators; dynamotors; converters and closed-coil arc machines.

A booster is a machine inserted in series in a circuit to change its voltage, and may be driven either by an electric motor, or otherwise. In the former case it is a motor-booster.

A motor-generator is a transforming device consisting of two machines; a motor and a generator, mechanically connected together.

A dynamotor is a transforming device combining both motor and generator action in one magnetic field, with two armatures, or with an armature having two separate windings.

For converters, see III.

* This classification constitutes a part of the Report of the Committee on Standard. ization, of the American Institute of Electrical Engineers. 
II. Synchronous Machines, which comprise a constant magnetic field, and an armature receiving or delivering alternating currents in synchronism with the motion of the machine; i. $\ell$., having a frequency equal to the product of the number of pairs of poles and the speed of the machine in revolutions per second.

III. Synchronous Commutating Machines:- These include: (I) Synchronous converters, commonly called "converters"; $i$. e., converters from alternating to direct, or from direct to alternating current, and (2) Double-current generators; $i$. $\ell$., generators producing both direct and alternating currents.

A converter is a machine employing mechanical momentum in changing electric energy from one form into another.

A converter may be either :

a. A direct-current converter, converting from a direct current to a direct current, or

b. A synchronous converter, formerly called a rotary converter, converting from an alternating to a direct current or vice versa.

Phase converters are converters from an alternating-current system to an alternating-current system of the same frequency, but in different phase.

Frequency converters are converters from an alternating-current system of one frequency to an alternating-current system of another frequency, with or without change in the number of phases.

IV. Rectifying Machines, or Pulsating-Current GeneraTORS, which produce a unidirectional current of periodically varying strength.

V. Stationary Induction Apparatus, $i$. e., stationary apparatus changing electric energy to electric energy through the medium of magnetic energy. These comprise :

a. Transformers, or stationary induction apparatus in which the primary and secondary windings are electrically insulated from each other. 
$b$. Auto-transformers, also called compensators: $i$. $e$., stationary induction apparatus in which part of the primary winding is used as a secondary winding; or conversely.

c. Potential regulators, or stationary induction apparatus having a coil in shunt, and a coil in series with the circuit, so arranged that the ratio of transformation between them is variable at will.

These may be divided into the following types, or combinations thereof:

I. Compensator potential regulators, in which the number of turns of one of the coils is changed.

2. Induction potential-regulators, in which the relative positions of primary and secondary coils is changed.

3. Magneto potential-regulators, in which only the direction of the magnetic flux with respect to the coils is changed.

d. Reactors, or reactance coils, formerly called choking coils : $i$. e., stationary induction apparatus used to produce impedance or phase displacement.

VI. Rotary Induction Apparatus, which consist of primary and secondary windings rotating with respect to each other. They comprise :

a. Induction motors.

b. Induction generators.

c. Frequency converters.

d. Rotary phase converters.

\section{NOTATION.*}

The following table shows the more important symbols used in this text and explains their meaning. A few of these symbols are used at different times to represent different quantities. Thus

* Non-standard symbols which are used but once or twice in this treatise are not given in this table. The significance of such symbols is fully explained whenever and wherever they are used. 
$t$ in some places represents temperature and at another place it may stand for an interval of time. The meanings of these symbols are explained every time an equation is discussed in the text, and therefore symbols that are used only once or twice in the text are not given in this table.

$a, \beta, \phi, \theta$ are used for angle; $\beta$ is also used for temperature coefficient of resistance, and for coefficient of linear expansion.

$\mathscr{B}$ magnetic flux density.

$C$ capacity.

$E, e$ electromotive force or voltage.

$E_{a}=$ electromotive force induced in an armature. $E_{x}=$ electromotive force between the terminals of a dynamo.

$f$ frequency of an alternating electromotive force or current in cycles per second. The letter $f$ is also used for electric field intensity.

$F$ force; sometimes used for field loss of a dynamo.

F magnetomotive force.

$H$ quantity of heat expressed in ergs or joules.

$h$ conical intensity of a beam of light in hefners or in candles.

$\mathscr{H}$ magnetic field intensity.

$I, i$ current.

$I_{a}=$ armature current, $I_{s}=$ shunt field current.

$I_{c}=$ series field current, $I_{x}=$ current in external - circuit.

The letter $I$ is also used for the sectional intensity of a beam of light, or for intensity of illumination.

$\mathfrak{g}$ intensity of magnetization.

$k$ inductivity of a dielectric.

$L$ inductance.

$l$ length.

$m$ magnetic pole strength.

$\mu$ magnetic permeability. 
$n$ speed in revolutions per minute or in revolutions per second. This letter is frequently used where any number of things, $n$, are to be represented in a formula.

$P$ power.

$p$ number of field magnet poles of a dynamo.

The letter $p$ is also used for power factor.

$p^{\prime}$ number of current paths in an armature.

$\Phi, \phi$ magnetic flux.

$Q, q$ quantity of electric charge.

$R, r$ resistance.

$R_{a}=$ armature resistance, $R_{s}=$ shunt field resistance, $R_{c}=$ series field resistance.

$\mathscr{R}$ magnetic reluctance.

$\rho$ specific resistance.

$s$ area of a surface, sectional area.

$S$ stray power loss in a dynamo.

$t$ time; also temperature.

$T$ torque.

$v$ linear velocity.

$\omega$ angular velocity.

$W$ work.

$X, x$ reactance. The letter $x$ is also frequently used as a coördinate.

$Z, z$ impedance. The letter $Z$ is also used for the number of conductors on the surface of an armature, and for the number of turns of wire in a coil. The letter $z$ is also used for the number of turns of wire per unit length of a coil.

$Z^{\prime}=p Z \mid p^{\prime} 10^{8}$ where $Z$ is the number of conductors on the surface of an armature. 



\section{ELEMENTS OF}

\section{ELECTRICAL ENGINEERING.}

\section{CHAPTER I.}

\section{INTRODUCTION.}

\section{ELEMENTARY ELECTRICITY AND MAGNETISM.}

1. The magnet. - The name magnet was originally applied to the lodestone, a mineral composed of iron oxide which, in its native state, possesses the power of attracting iron. The lodestone imparts its magnetic property to pieces of iron or steel which are brought near to it. Such pieces of iron or steel are said to be magnetized and they are called magnets. The methods at present employed for magnetizing iron or steel depend upon the use of the electric current as described later.

Poles of a magnet. - Certain parts only of a magnet possess the power of attracting iron. These parts are called the poles of the magnet. The poles of a bar magnet, for example, are usually situated at the ends of the bar.

Compass. Naming of poles. - A horizontal magnet free to turn about a vertical axis places itself approximately north and south. This fact is made use of in the compass, which consists of a pivoted horizontal magnet playing over a divided circle. The north-pointing end of a magnet is called its north pole and the south-pointing end of a magnet is called its south pole.

Mutual action of two magnets. - The north pole of one magnet attracts the south pole of another magnet, and the north poles of two magnets or the south poles of two magnets repel each 
other; that is, unlike magnetic poles attract each other, and like magnetic poles repel each other.

Distributed and concentrated poles. - The poles of a magnet, that is, the seats of the attracting or repelling forces, are distributed over considerable portions of the bar, generally the end portions. This is especially the case with short, thick bars. In the case of long, slim magnets, however, the poles are usually more nearly concentrated at the ends of the bar. In the former case the poles are said to be distributed, in the latter case the poles are said to be (approximately) concentrated.

The elementary theory of magnetism in its simplest aspects applies to long slim magnets; that is, to magnets with concentrated poles and the following discussion applies primarily to such magnets. The various definitions may be applied, however, to magnets with distributed poles although rigorous statements are in this case very complex, involving elaborate use of differential and integral calculus.

2. Strength of pole. - The poles of a magnet attract iron with greater or less force, according to the size of the magnet and according to the thoroughness with which the magnet has been magnetized. The poles of a magnet are said to be strong when they attract iron or steel with relatively great force.

Unit pole. Measure of pole strength. - A magnet pole is said to have unit strength, or to be a unit pole, when it exerts an attraction or repulsion of one dyne upon an equal $*$ pole at a distance of one centimeter, and the force in dynes with which a unit pole acts upon a given pole at a distance of one centimeter is adopted as the numerical measure of the strength of the given pole.

A magnet pole of $m^{\prime}$ units strength at a distance of one centimeter from a pole of unit strength is, according to the above statement, attracted or repelled with a force of $m^{\prime}$ dynes. If the unit pole is replaced by a pole of $m^{\prime \prime}$ units strength the force be-

* For the purpose of this definition the poles of two entirely similar magnets which have been similarly magnetized, may be considered to be equal. 
comes $m^{\prime \prime}$ times as great, or $m^{\prime} m^{\prime \prime}$ dynes. That is, a pole of $m^{\prime}$ units strength at a distance of one centimeter from another pole of $m^{\prime \prime}$ units strength is attracted or repelled with a force of $m^{\prime} m^{\prime \prime}$ dynes.

3. Coulomb's law. - The force of attraction or repulsion of two magnet poles is inversely proportional to the square of the distance between them. This fact was established experimentally in I 800 by Coulomb who measured the force of attraction of two magnet poles at various distances apart. A long slim magnet was suspended horizontally by a wire, thus forming a torsion pendulum. One pole of another long slim magnet when brought near to one of the poles of the suspended magnet caused a twist of the suspending wire and from the observed angle of twist the value of the attracting or repelling force was calculated.

Complete expression for the force of attraction of two magnet poles. - According to the previous article two poles $m^{\prime}$ and $m^{\prime \prime}$ attract or repel each other with a force of $m^{\prime} m^{\prime \prime}$ dynes when they are one centimeter apart, therefore, according to Coulomb's law, the poles attract or repel with a force of $m^{\prime} m^{\prime \prime} / r^{2}$ dynes when they are $r$ centimeters apart. That is

$$
F=\frac{m^{\prime} m^{\prime \prime}}{r^{2}}
$$

in which $m^{\prime}$ and $m^{\prime \prime}$ are the respective strengths of the magnet poles, $r$ is their distance apart in centimeters, and $F$ is the force in dynes with which they attract or repel each other.

Algebraic sign of magnet pole. - The poles $m^{\prime}$ and $m^{\prime \prime}$ are alike in sign when both are north poles or when both are south poles. In either case the product $m^{\prime} m^{\prime \prime}$ is positive, and in this case the force $F$ in equation (I) is by experiment a repulsion; therefore it is convenient to consider the force $F$ as a positive force when it is a repulsion. On the other hand $m^{\prime}$ and $m^{\prime \prime}$ are opposite in sign when one is a north pole and the other a south pole. In this case the product $m^{\prime} m^{\prime \prime}$ is negative and the force $F$ 
in equation (I) is an attraction. It is customary to consider a north pole as positive and a south pole as negative.

Equality of north and south poles of a magnet. - The two poles of a magnet are always equal in strength though opposite in sign. When a magnet is broken in two, each piece is a complete magnet with a north pole and a south pole.

A bar of steel may be irregularly magnetized so as to have one or more north poles and one or more south poles; one or more of the poles, sometimes called consequent poles, being near the middle of the bar. In such a case the sum of the strengths of all the north poles is equal to the sum of the strengths of all of the south poles.

It is often convenient to speak of an isolated magnet pole, meaning one pole of a very long slim magnet, the other pole of which is so far away as to be negligible in its effects.

4. Magnetic field. - A magnetic field is any region in which a magnet pole if present is acted upon by a force tending to pull it in one direction or another. For example, the region surrounding a magnet is a magnetic field; the region surrounding a wire carrying an electric current is a magnetic field; the behavior of a compass needle shows that the entire region surrounding the earth is a magnetic field.

A magnetic field has other important effects besides the force action which it exerts upon a magnet pole; however, this force action is the effect which is usually employed as a basis for the discussion of the ideas of direction and intensity of a magnetic field at a point. Given a magnetic field, the field surrounding a wire carrying electric current for example ; in order to direct attention to the nature of this field at a given point we imagine a magnet pole placed at that point, and we consider the force with which the field acts upon this pole. A magnet pole which is used in this way as an indicator of magnetic field we call a test pole.

Intensity of a magnetic field at a point. - A magnetic field is said to be intense when it exerts a relatively great force upon a 
given test pole. A given magnetic field usually varies greatly in intensity from point to point. Thus the magnetic field due to a magnet $A$ is much more intense at points near the poles of the magnet than it is at points remote from the poles, for a test pole is acted upon by a much greater force when it is near one of the poles of $A$ than when it is at points remote from both poles of $A$.

The force, $\mathcal{H}$, in dynes which acts upon a unit test pole when the pole is placed at a given point in a magnetic field is adopted as the numerical measure of the intensity of the magnetic field at the given point. This force-per-unit-test-pole, $\mathscr{H}$, is hereafter spoken of simply as the intensity of the magnetic field at a point.

The force $F$ with which a magnetic field acts upon a test pole of $m$ units strength is $m$ times as great as the force $\mathscr{H}$ with which the field acts upon a unit test pole placed at the same point. Therefore

$$
F=m \mathscr{H}
$$

in which $F$ is the force in dynes which acts upon a test pole of strength $m$ when the pole is placed in a magnetic field of which the intensity is $\mathscr{H}$.

The International Electrical Congress of 1900 adopted the name gauss for the unit field intensity.

Direction of a magnetic field at a point. - The force with which a magnetic field acts upon a north pointing test pole is opposite in direction to the force with which the same field acts upon a south pointing test pole placed at the same point. The direction of the force with which a magnetic field acts upon a north pointing test pole is adopted conventionally as the direction of the field at the point.

Homogeneous fields and non-homogeneous fields. - A magnetic field generally varies in intensity and in direction from point to point. Such a field is said to be non-homogeneous. A magnetic field which has everywhere the same intensity and the same direction is called a homogeneous or uniform field. 
Hydraulic analogy of magnetic field. - (a) A steadily moving liquid has at each point a definite velocity in a definite direction and the trend of a steadily moving liquid may best be pictured in the mind by imagining curved lines drawn through the liquid, these lines being at each point in the direction of the velocity of the liquid at that point. Such lines are called stream lines. Where the stream lines are crowded together the velocity of the liquid is great, and where the stream lines are spread apart the velocity of the liquid is small. When the stream lines are parallel straight lines the velocity of the liquid is everywhere the same in value and in direction, and the motion of the liquid is said to be homogeneous or uniform.

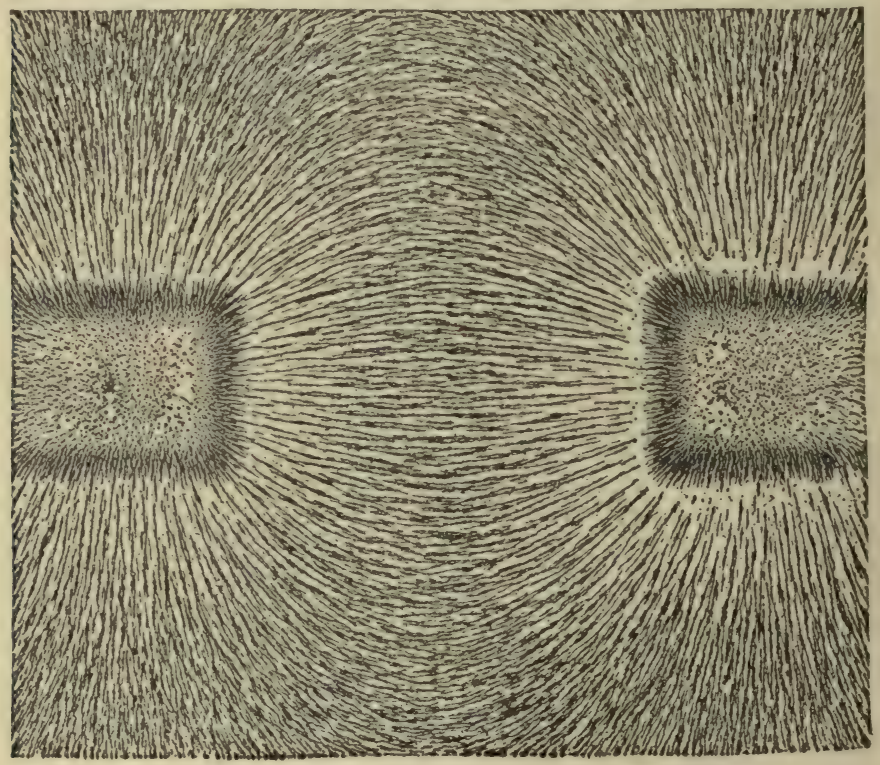

Fig. 1.

(b) A magnetic field has at each point a definite intensity in a definite direction, and the trend of a magnetic field may best be pictured in the mind by imagining curved lines drawn through the field, these lines being at each point in the direction of the 
field at that point. Such lines are called lines of force. Where the lines of force are crowded together the intensity of the field is great, and where the lines of force are spread apart the intensity of the field is small. When the lines of force are parallel straight lines the intensity of the magnetic field is everywhere the same in value and in direction, and the field is said to be homogeneous or uniform.

Magnetic figures. - The trend of the lines of force in the neighborhood of a magnet is beautifully shown by placing a pane of glass over the magnet and dusting iron filings upon it. The filings, becoming magnetized, tend to arrange themselves in fila-

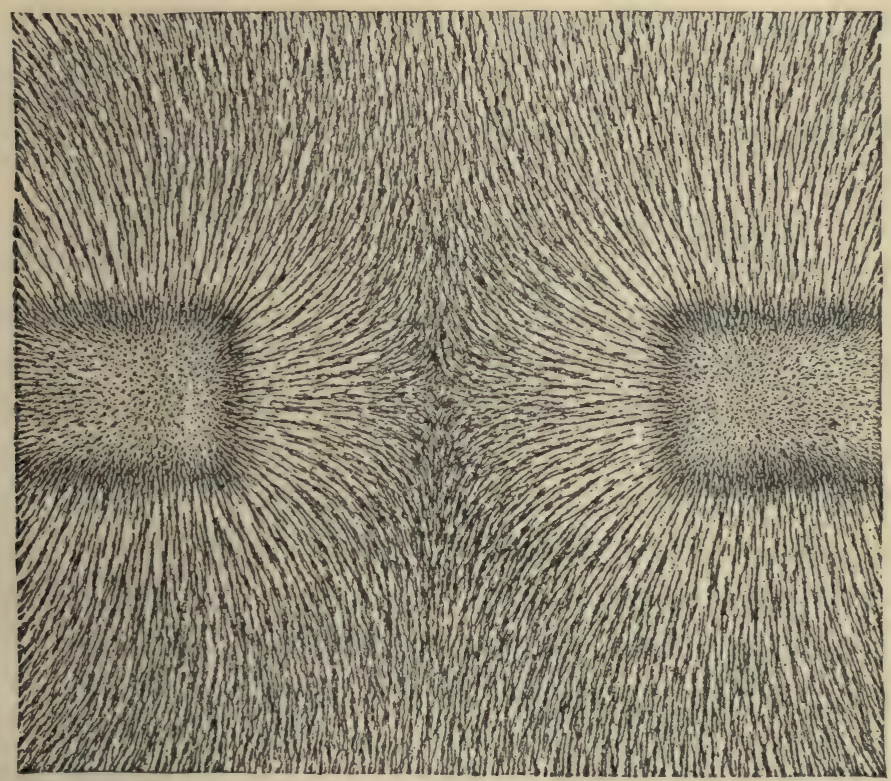

Fig. 2.

ments along the lines of force. Slight tapping of the glass facilitates this arrangement of the filings. Figs. I, 2, 4, 5, 6 and 7 are photographic reproductions of magnetic figures obtained in this way. Fig. I shows the trend of the lines of force in the neighborhood of two unlike poles and Fig. 2 shows the trend of the lines of force in the neighborhood of two like poles. 
The force of attraction of the two unlike magnet poles of Fig. I may be attributed to a tendency of the lines of force to shorten, very much as if the lines of force were stretched elastic filaments. This idea of tension along the lines of force of a magnetic field is also useful in giving an insight into the cause of the side push which a magnetic field exerts upon a wire carrying an electric current as explained later.

Not only do the lines of force in a magnetic field have a tendency to shorten, but they push each other apart sidewise. That is, there is a tension along the lines of force and a pressure at right angles to the lines of force. The repulsion of the two like poles in Fig. 2 may be attributed to this side pressure of the lines of force.

5. Direction and intensity of field near an isolated magnet pole. - Inasmuch as a test pole of north polarity is attracted directly towards a given south pole and repelled straight away from a given north pole, it is evident that the lines of force of the magnetic field surrounding an isolated magnet pole are radiating straight lines, that the magnetic field near a north pole is directed away from it, and that the magnetic field near a south pole is directed towards it.

Imagine a test pole of strength $m^{\prime}$ placed at a point distant $r$ from a given pole of strength $m$. The force with which the pole $m$ acts upon the test pole $m^{\prime}$ is: $F=m m^{\prime} / r^{2}$, according to equation ( $\mathrm{r}$ ) ; but the force acting upon the test pole $m^{\prime}$ is equal to the product of the strength of the test pole into the field intensity at the test pole due to the given pole $m$, according to equation (2). Therefore

$$
\frac{m m^{\prime}}{r^{2}}=m^{\prime} \mathscr{H}
$$

or

$$
\mathscr{H}=\frac{m}{r^{2}}
$$

6. Magnetic flux. - Consider a plane surface, $s$ square centi- 
meters in area, stretched across and at right angles to a uniform magnetic field of intensity $\mathcal{A}$. The product $s \mathcal{H}$ is called the magnetic flux across the surface. That is :

$$
\Phi=s \mathscr{H}
$$

in which $\Phi$ is the magnetic flux across a plane surface of area $s$ square centimeters at right angles to a uniform magnetic field of intensity $\mathscr{H}$. When the plane surface is not at right angles to the uniform magnetic field then :

$$
\Phi=s \mathscr{H} \cos \theta
$$

in which $\theta$ is the angle between $\mathscr{H}$ and the normal to the surface. When the field is not uniform or when the surface is curved then :

$$
\Delta \Phi=\mathfrak{A} \cos \theta \cdot \Delta s
$$

in which $\Delta \Phi$ is the flux across an element of the surface of which the area is $\Delta s, \mathscr{H}$ is the intensity of the field at the element of surface, and $\theta$ is the angle between $\mathscr{H}$ and the normal to the element of surface. In this case the total flux across a finite surface is found by integrating equation (4c) over the finite surface.

The unit flux is the flux across one square centimeter at right angles to a magnetic field of unit intensity, or it is the flux across I $/ n$th of a square centimeter at right angles to a magnetic field of $n$ units of intensity. The International Electrical Congress of I900 adopted the name maxwell for the unit flux; however, the unit flux is almost universally called the line, and a given flux is usually expressed as so many lines.

The manner in which magnetic field is distributed in space is mathematically identical with the manner in which velocity is distributed throughout a moving incompressible liquid. The stream lines in a moving incompressible liquid are closed or reëntrant lines.* Similarly, the lines of force of a magnetic field

* Unless the motion extends to infinity. 
are closed or reëntrant lines. ${ }^{*}$ A closed surface, such as a sphere, may be imagined to be drawn in a moving liquid, and a certain amount of liquid may flow into the enclosed region across a portion of the bounding surface, if so, then an exactly equal amount of liquid must flow out of the enclosed region across some other portion of the bounding surface. Similarly, if magnetic flux enters an enclosed region through one portion of the bounding surface, an exactly equal flux must pass out of the enclosed region across some other portion of the bounding surface, or in other words, the total magnetic flux across a closed surface is zero.

A clear idea of the manner in which magnetic flux is distributed in space may be obtained as follows: Imagine a net stretched across any given magnetic field and imagine the meshes of the net to be large in area where the field is weak or where the field is inclined to the netted surface so that the product $s \mathcal{H} \cos \theta$ is unity for each mesh of the net.

Imagine lines of force to be drawn through the magnetic field starting from every point in the periphery of one of the meshes of the net. These lines of force form a tube-like surface having

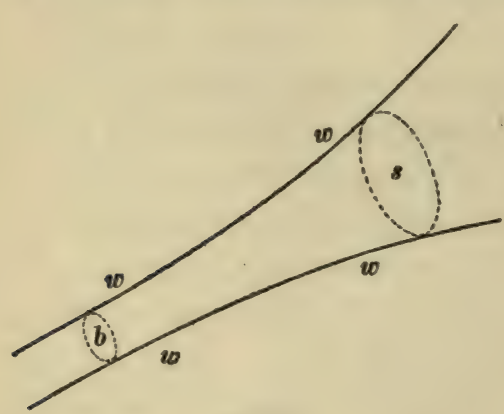

Fig. 3. the given mesh for a base. The magnetic flux across the base of this tube, $b$, Fig. 3 , is unity by supposition. The magnetic flux through any given surface $s$ stretched across the tube anywhere is also unity For the given surface $s$, the base $b$, and the walls $w 20$ of the intervening portion of the tube constitute a closed surface across which the total flux inwards is zero; but the flux across the walls wze is everywhere zero since these walls are everywhere parallel to the lines of force; therefore the flux inwards across $b$ is equal to the

* Unless the field extends to infinity. 
flux outwards across $s$. The tube is therefore called a tube of flux, or since the flux through it is everywhere unity it is often called a unit tube.

Similar unit tubes may be formed by lines of force passing out from the peripheries of the other meshes of the net. In this way the entire region of the magnetic field may be split up into unit tubes and the magnetic flux across any surface anywhere in the field will be equal to the number of these unit tubes which cross the surface.

These unit tubes may be represented each by a single line of force drawn along its axis. Then the magnetic flux across any surface anywhere in the field will be equal to the number of these lines (unit tubes) which cross the surface. Lines of force are always imagined to be drawn in a magnetic field so that each line may represent a unit tube.

7. Magnetic flux from a magnet pole of strength m. - Proposition. - The number of lines of magnetic flux emanating from a magnet pole of strength $m$ is equal to $4 \pi m$. That is :

$$
\Phi=4 \pi m
$$

in which $\Phi$ is the magnetic flux emanating from a magnet pole of strength $m$. If the pole is a north pole, the flux $4 \pi m$ passes out or diverges from it. If the pole is a south pole, the flux $4 \pi m$ passes in or converges towards it.

Proof of equation (5). - Imagine a spherical surface of radius $r$ drawn with the pole $m$ at its center. The area of this spherical surface is $4 \pi r^{2}$, the field intensity at every point on this spherical surface $^{*}$ due to the pole $m$ is $m / r^{2}$ according to equation (3), and this field is everywhere at right angles to the spherical surface. Therefore the magnetic flux across the spherical surface is equal to $m /\left(r^{2}\right) \times 4 \pi r^{2}$ which is equal to $4 \pi m$.

Remark. - The poles of a magnet are the places where magnetic flux passes from iron into air (north pole) or from air into

* Except of course the small part of the surface where the bar of the magnet passes through it. 
iron (south pole). When the magnetic flux is confined wholly to air as in case of the magnetic field surrounding a wire carrying an electric current there are of course no magnet poles. When the magnetic flux is confined wholly to iron as in case of an endless iron rod in the form of a ring there are likewise no magnetic poles.

8. Magnetization. - When a piece of iron or other magnetic substance is placed in a magnetic field it becomes a magnet. Thus a bar of iron or steel placed in the intense magnetic field inside of a coil of wire through which an electric current is flowing, becomes strongly magnetized. A neutral or unmagnetized bar of iron when held in the direction of the earth's magnetic field shows north polarity at the north-pointing end and south

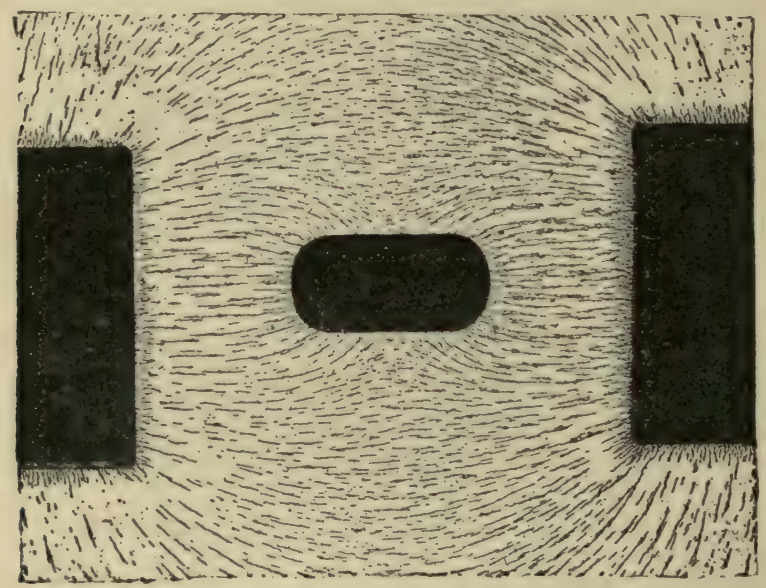

Fig. 4.

polarity at the other end. If the bar is turned end for end, the magnetism of the bar is reversed. A sharp blow with a hammer renders the bar more susceptible to the weak magnetizing action of the earth's field. The polarity of the ends of the bar may be easily determined by means of a compass needle. This action of a magnetic field upon iron and steel is called magnetization. 
When a piece of iron is placed in a magnetic field the original trend of the lines of force in the field is greatly altered. Thus Fig. 4 shows the effect of a small piece of iron upon the magnetic field between two flat-ended magnet poles. In the absence of the small piece of iron the field is as shown in Fig. 5.

The effect of a piece of iron in a magnetic field is always such as to suggest that "iron is a better carrier of lines of force than

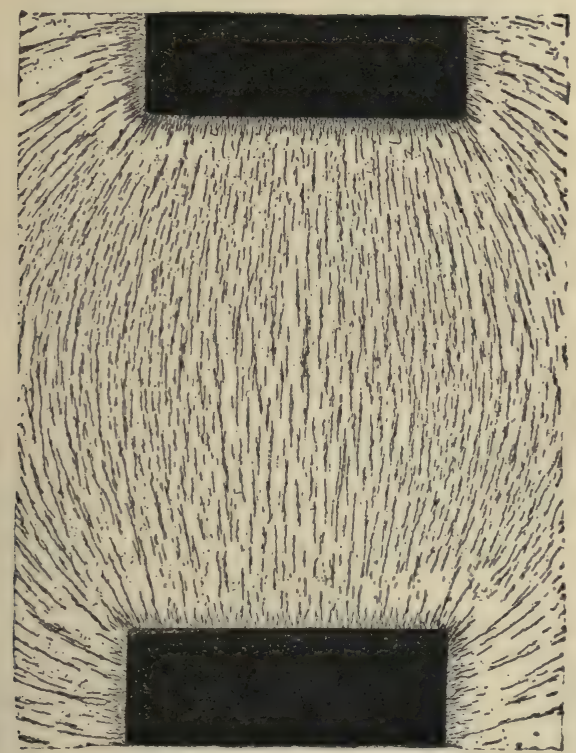

Fig. 5.

air." The lines of force tend to converge into the piece of iron and pass through it.

9. The magnetic field due to an electric wire. - A wire through which an electric current is flowing is called an electric wire for brevity. The lines of force of the magnetic field produced by an electric wire encircle the wire. A north-pointing test pole tends to move round the wire in one direction and a south-pointing test pole tends to move round the wire in the opposite direction. 
Fig. 6 shows the lines of force encircling a straight electric wire. The black circle at the center of the figure is a section of the wire and the wire is perpendicular to the plane of the figure.

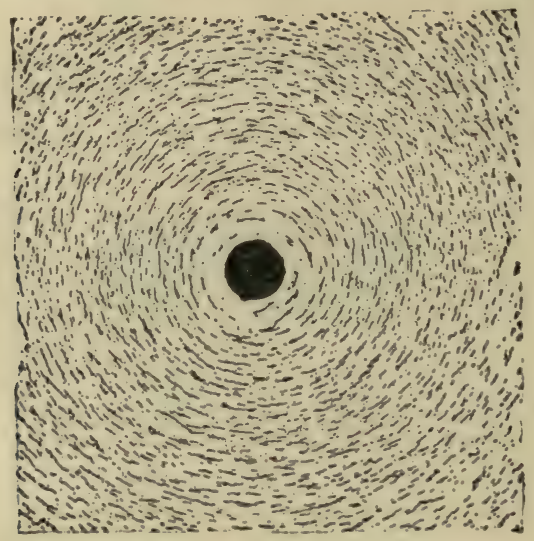

Flg. 6.

10. The composite magnetic field produced when a straight electric wire is stretched across a magnetic field, which, but for the

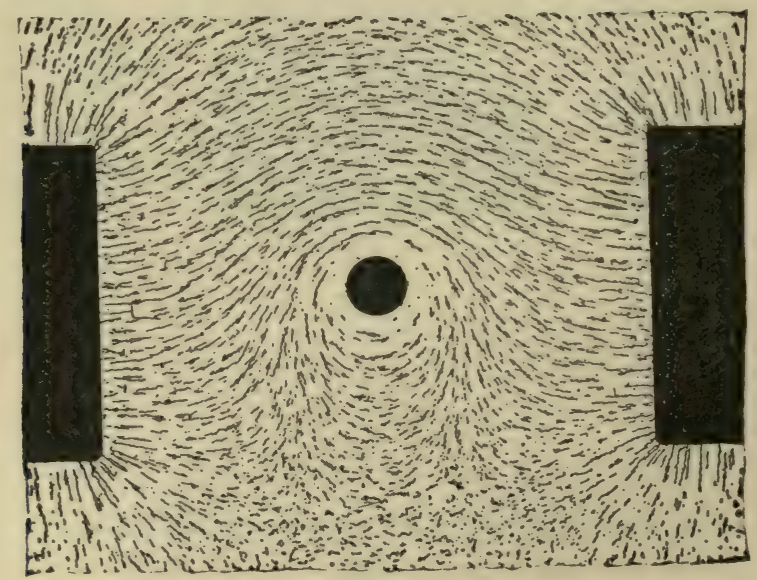

Fig. 7.

presence of the electric wire, would be a uniform field. - The magnetic field between the flat-ended magnet poles, Fig. 5, is nearly 
uniform. Let an electric wire be stretched between the poles perpendicular to the plane of the paper. The composite field due to the combined action of the magnet poles and the electric wire is represented in Fig. 7, which is from a photograph. The

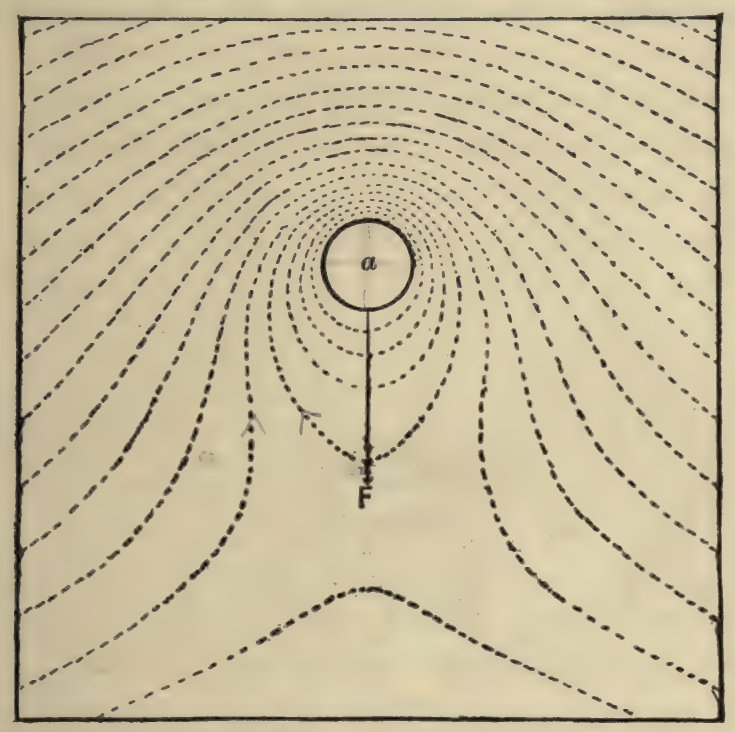

Fig. 8.

black circle at the center of the figure is a section of the wire. The trend of the lines of force near the wire in Fig. 7 is clearly shown by the dotted lines in Fig. 8, which is from a drawing.

Side push on an electric wire stretched across a uniform magnetic field, that is, a field which would be uniform but for the presence of the wire. The electric wire $a$, Fig. 8 , is pushed sidewise by the magnetic field as indicated by the arrow $F$. This force is at right angles both to the magnetic field and to the wire, and it may be ascribed to the tendency of the lines of force to shorten themselves.

11. Strength of current. - Consider a straight electric wire stretched across a uniform magnetic field of unit intensity, the wire being at right angles to the field as described in the forego- 
ing article. The force in dynes with which the field pushes sidewise on one centimeter of this wire is adopted as the numerical measure of the strength of the current in the wire. This force-perunit-length-of-wire-per-unit-field-intensity is hereafter called, simply, the strength of the current in the wire; let it be represented by $I$. The force pushing sidewise on $l$ centimeters of wire in unit field is $I l$ dynes, and if the field intensity is $\mathscr{H}$ units intensity instead of one unit intensity, the force is multiplied $\mathscr{H}$ times, becoming IlöH dynes. Therefore

$$
F=I l \mathscr{H}
$$

in which $F$ is the force in dynes pushing sidewise on $l$ centimeters of wire which is stretched across a magnetic field of $\mathscr{H}$ units intensity, and $I$ is the strength of current in the wire.

The c.g.s. unit of current and the ampere. - When, in equation (6), $F$ is expressed in dynes, $l$ in centimeters, and $\mathscr{H}$ in c.g.s. units or gausses, then the current strength $I$ is expressed in c.g.s. units of current. That is, the c.g.s. unit of current is one dyne-per-centimeter-per-unit-field-intensity, or it is a current of such strength that one centimeter of a wire in which it flows is pushed sidewise with a force of one dyne when the wire is stretched at right angles across a magnetic field of unit intensity. The so-called practical unit of current, the ampere, is one tenth of a c.g.s. unit of current. $\dagger$ The c.g.s. unit of current is called the abampere.

* When $l$ and $\mathscr{H}$ are not at right angles, then

$$
F=I l \mathscr{H} \sin a
$$

in which $a$ is the angle between $l$ and $\mathscr{H}$. In this case $F$ is at right angles to both $l$ and $\mathscr{A P}$ as before.

$\nmid$ The definition of current strength as dynes-per-unit-field-per-centimeter-lengthof-wire, the wire being at right angles to the field, is essentially a very concise statement of the fundamental method of measuring current. This fundamental method of measuring current is a very difficult operation. It has, however, been carried out with slight modifications and the c.g.s. unit current has been found to deposit 0.01118 gram of metallic silver per second from pure silver nitrate. On the basis of 
Direction of current. - A magnet pole tends to move round an electric wire as stated in Art. 9. The current is by common consent considered to flow along the wire in the direction in which a right-handed screw coaxial with the wire would travel if turned in the direction in which a north pole tends to move round the wire.

The relative directions of a current in a wire, the magnetic field $\mathscr{H} \mathfrak{H}$ in which the wire is placed, and of the side push $F$ acting on the wire may be determined as follows: The field due to the current circles round the wire in a direction which is easily determined from the above convention as to the direction of current, when the direction of the current is given. This field due to the current works against or weakens the field $\mathscr{H}$ on one side of the wire, and strengthens the field $\mathscr{H}$ on the other side of the wire, as shown in Fig. 8. The wire is pushed away from the side upon which the composite field is intense.

\section{Intensity of the magnetic field at the center of a circular coil of} wire. - Fig. 9 shows a circular coil of wire of radius $r$ through which a current of strength $I$ is flowing. It is required to determine the intensity, at the center of the coil, of the magnetic field due to the current in the coil. Imagine a test pole of strength $m$ placed at the center of the coil. This test pole produces a magnetic field which is everywhere at right angles to the

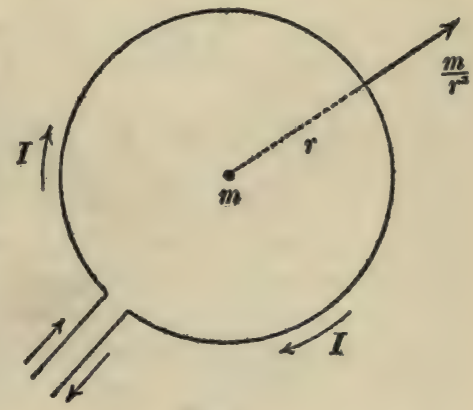

Fig. 9. wire of the coil, and of which the intensity at the wire is $m / r^{2}$, by equation (3). The action of this field on the wire is to push the wire sidewise (towards the reader in Fig. 9), and, by equation (6), the force with which the wire is pushed is $I \times$ length of wire

this determination the International Electrical Congress of 1893 defined the ampere as that current which will deposit 0.001 I 18 gram of silver per second from a solution of pure silver nitrate. 
in coil $\times m / r^{2}$; but the length of wire in the coil is $2 \pi r Z$, where $Z$ is the number of turns of wire in the coil. Therefore,

$$
F=I \times 2 \pi r Z \times \frac{m}{r^{2}}=\frac{2 \pi Z I}{r} \times m
$$

This is the force with which the test pole acts on the coil of wire. The coil of wire acts upon the test pole with an equal and opposite force, but the force with which the coil acts on the test pole is equal to $\mathscr{H} m$, where $\mathscr{H}$ is the intensity, at the test pole, of the field due to the coil. Therefore

$$
\mathscr{H} m=F=\frac{2 \pi Z I}{r} \cdot m
$$

or :

$$
\mathscr{H}=\frac{2 \pi Z I}{r}
$$

in which $\mathscr{H}$ is the intensity in gausses of the magnetic field at the center of a circular coil containing $Z$ turns of wire, $r$ is the radius of the coil in centimeters, and $I$ is the current in the wire in c.g.s. units or abamperes.

The intensity of the magnetic field at a point in the axis of a circular coil at a distance $d$ centimeters from the plane of the coil is

$$
\mathscr{H}=\frac{2 \pi Z r^{2} I}{\left(r^{2}+d^{2}\right)^{2}}
$$

in which $Z$ is the number of turns of wire in the coil, $r$ is the radius of the coil in centimeters, $I$ is the current strength in c.g.s. units, and $d$, as above stated, is the distance from the plane of the coil to the point where $\mathscr{H}$ is reckoned.

Proof of equation (8). Imagine a test pole of strength $m$ placed at the given point. The field intensity at the wire due to this pole is $m /\left(r^{2}+d^{2}\right)$. The component of this field in the plane of the coil is

$$
\frac{m}{r^{2}+d^{2}} \times \frac{r}{\sqrt{r^{2}+d^{2}}}
$$

and this component pushes the coil sidewise with a force equal to

$$
I \times 2 \pi r Z \times \frac{r m}{\left(r^{2}+a^{2}\right)^{\frac{3}{2}}} .
$$

This same force reacts on the pole and is equal to $m \mathscr{H}$ so that

$$
\mathscr{H}=\frac{2 \pi Z r^{2} I}{\left(r^{2}+d^{2}\right)^{\frac{3}{3}}} .
$$


13. Magnetic field inside of a long solenoid. A winding of wire is called a coil. The usual form of coil consists of wire wound on a spool. When the spool is very short and large in diameter the coil is called a circular coil. The intensity of magnetic field at the center of a circular coil is discussed in the foregoing article. When the spool is very long in comparison with its diameter the coil is called a solenoid. The solenoid is usually made by winding one or more layers of wire on a long tube.

The magnetic field in a long solenoid is uniform, that is, the field has the same intensity at every point inside of the solenoid and the same direction, namely, parallel to the axis of the solenoid.

The intensity in gausses of the magnetic field inside of a long solenoid is :

$$
\mathscr{H}=4 \pi z I
$$

in which $z$ is the number of turns of wire per centimeter length of solenoid, and $I$ is the current in abamperes. Equation (9) may be written :

$$
\mathscr{H}=4 \pi \cdot \frac{Z}{l} \cdot I
$$

in which $Z$ is the total number of turns of wire on the solenoid and $l$ is the length of the solenoid in centimeters.

Discussion of equation (9). - The equation gives the intensity of the magnetic field at every point in a long solenoid, except at points near the ends of the solenoid, and it is true whatever the shape of the section of the solenoid. The following proof of equation (9) applies however to points in the axis of a cylindrical solenoid.

Let Fig. Io represent a portion of a long cylindrical solenoid of radius $r$, having $z$ turns of wire per centimeter of length and carrying current $I$. Let $p$

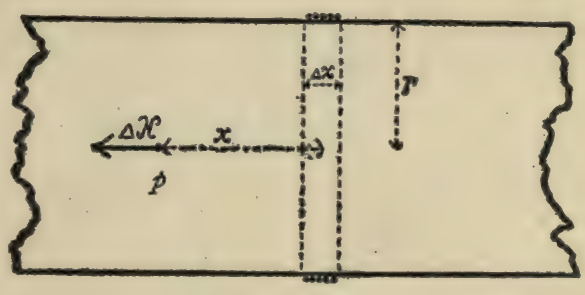

Fig. 10. be the point, in the axis of the solenoid, at which the field intensity is to be determined. Each element of the solenoid contributes its share to the intensity of field 
at $p$. Let $\Delta \mathscr{H}$ be the field intensity at $p$ due to that element of the coil which is at a distance $x$ to the right of $p$, which element is of length $\Delta x$ and has $z \Delta x$ turns

of wire. Then from equation (8) we have :

$$
\Delta \mathscr{H}=\frac{2 \pi I r^{2} z \cdot \Delta x}{\left(r^{2}+x^{2}\right)^{\frac{3}{2}}}
$$

Integrating this expression from $x=+\infty$ to $x=-\infty$ gives $\mathscr{H}=4 \pi z I$.

14. Electrical resistance. Joule's law. - When an electric generator produces an electric current in a circuit a part of the work expended in driving the generator reappears as heat in the various parts of the circuit. The electric current seems to be opposed by a kind of resistance somewhat analogous to the resistance which a pipe offers to the flow of water. A portion of a circuit is said to have more or less resistance according as more or less heat is generated in it by a given current.

Joule's law. - The rate at which heat is generated in a given wire is proportional to the square of the current flowing in the wire, or the amount of heat generated in a given wire is proportional to the square of the current and to the elapsed time, that is

$$
H=R I^{2} t
$$

in which $H$ is the amount of heat generated by the current $I$ in $t$ seconds, and $R$ is a constant for the given wire. The constant $R$, units-of-heat-per-(unit current) ${ }^{2}$-per-second, is adopted as the numerical measure of the resistance of the wire, and it is called the resistance of the wire.

The c.g.s. unit of resistance. - When in equation (I I), $H$ is expressed in ergs, * $I$ in c.g.s. units, and $t$ in seconds, then $R$ is expressed in terms of a unit called the c.g.s. unit of resistance, or the abohm.

The Ohm. - When in equation (I I), $H$ is expressed in joules, * $I$ in amperes, and $t$ in seconds, then $R$ is expressed in ohms.

* Heat is best expressed in terms of its energy value. Thus one erg of heat is the quantity of heat which is the equivalent of one erg of work; one joule of heat is the quantity of heat which is the equivalent of one joule of work. There are 4.2 joules in one calorie. 
That is, a wire has a resistance of one ohm when one joule of heat is generated in it per second by one ampere of current. One ohm is equal to $10^{9}$ abohms.

Specific resistance. - The resistance of a wire is proportional to the length of the wire and inversely proportional to the sectional area of the wire. That is :

$$
R=\rho \cdot \frac{l}{s}
$$

in which $R$ is the resistance of a wire of given material, $l$ is the length of the wire, $s$ is the sectional area of the wire, and $\rho$ is a constant which is called the resistivity or specific resistance of the material of which the wire is made. The specific resistance is the resistance of a wire of unit length and of which the sectional area is unity. Electrical engineers ordinarily express length of wire in feet and sectional area in circular mils. In this case $\rho$ is the resistance of a wire one mil in diameter and one foot long; the "mil-foot" of wire as it is sometimes called. The resistance of a mil-foot of ordinary commercial copper wire at ordinary room temperature is about $10.8 \mathrm{ohms}$.

Influence of temperature on resistance. - The resistance of a wire depends not only upon length, size and material, but also upon temperature. Most metallic wires increase in resistance with rise of temperature.

The increase of resistance of a given wire due to a rise of temperature is proportional to the initial resistance and approximately proportional to the rise of temperature ; that is, if $R_{0}$ is the resistance of a wire at some standard temperature, say $\mathrm{O}^{\circ} \mathrm{C}$., then the increase of resistance when the wire is heated to $t^{\circ} \mathrm{C}$. is equal to $\beta R_{0} t$, where $\beta$ is a proportionality factor. Therefore the total resistance $R$, of the wire at $t^{\circ} \mathrm{C}$. is $R_{t}=R_{0}+\beta R_{0} t$, or

$$
R_{t}=R_{0}(\mathrm{I}+\beta t)
$$

The quantity $\beta$ is called the temperature coefficient of resistance of the given material. For many pure metals $\beta$ has nearly 
the same value, namely, 0.0037 , that is, the resistance of a pure metal is very nearly proportional to the absolute temperature as measured by an air thermometer. The value of $\beta$ for high grade commercial copper is about 0.004 per centigrade degree.

Solutions of acids and salts, graphitic carbon, and nearly all so-called insulating substances such as glass and rubber, decrease in resistance with rise of temperature ; in other words their temperature coefficient of resistance is negative.

15. Power required to maintain a current in a circuit expressed in terms of resistance and current. - When the work which is spent in maintaining a current all appears in the circuit as heat, then the rate at which heat appears in the circuit is equal to the rate at which work is done in the maintenance of the current. Therefore, the power $P$ required to maintain a current $I$ through a wire having a resistance $R$ is equal to $R I^{2}$ if no portion of the power is used for producing mechanical or chemical effects. That is :

$$
P=R I^{2}
$$

in which $P$ is the power in watts (joules per second) required to maintain a current of $I$ amperes through a wire having $R$ ohms of resistance. Of course equation (14) holds true when all quantities are expressed in c.g.s. units. ( $P$ in ergs per second, $I$ in abamperes, and $R$ in abohms.)

16. Electromotive force. - It may seem from equation (14) that the power delivered by a generator should be proportional to the square of the current, but the current delivered by a given generator under fixed conditions can be increased only by reducing the resistance of the circuit to which the generator delivers current. In fact the power delivered by a given generator under fixed conditions of running is very nearly proportional to the current so that the ratio $P / I$ is nearly constant. This ratio $P / I$, whether it is constant or not, is called the electromotive force of a generator. That is :

$$
P=E I
$$


in which $P$ is the power delivered by an electric generator, $I$ is the current, and $E$ is the electromotive force between the generator terminals. The electromotive force between the terminals of a generator is analogous to the pressure-difference between inlet and outlet of a fan blower. An increase of current delivered by a generator * usually causes a decrease of electromotive force between the terminals of the generator very much as an increased delivery of air by a fan, by opening the outlet for example, causes a decrease of pressure difference between inlet and outlet of the fan.

Unsatisfactory character of fundamental definition of electromotive force. - The definition of a physical quantity consists in every case of a concise statement of the fundamental method of measuring that quantity, and when this fundamental method of measuring a quantity involves operations which are not feasible under the ordinary conditions of practical work, the definition always seems more or less unsatisfactory. Thus the above definition of electromotive force, as units-of-work-per-second-per-ampere, assumes that the rate of doing work in pushing a current through a circuit is to be measured directly in mechanical units. This can indeed be done, but it is an operation of great difficulty and one that is seldom performed.

Definition of electromotive force based on Ohm's law. - When all the work which is delivered to an electrical circuit is used to heat the circuit in accordance with Joule's law, a simple relation, called Ohm's law, exists between current, resistance, and electromotive force, and the simplest method of measuring electromotive force (and therefore the simplest definition of electromotive force) is based on Ohm's law.

17. Ohm's law. - An important relation between electromotive force, current strength, and resistance exists in the case of a circuit in which all of the work delivered by the generator is used in heating the circuit in accordance with Joule's law. Thus the

* Such as a separately excited dynamo, or a shunt dynamo. 
rate at which work is delivered by the generator is $E I$, and the rate of generation of heat in the circuit is $R I^{2}$. When the whole of $E I$ is used in the generation of heat we have $E I=R I^{2}$ or

$$
E=R I
$$

or

$$
I=\frac{E}{R}
$$

This relation was established by $\mathrm{Ohm}$ in 1827 and it is called Ohm's law.

The volt. - When $R$ in equations (16) is expressed in ohms and $I$ in amperes then $E$ is expressed in terms of a unit called the volt. That is, one volt is an electromotive force sufficient to maintain one ampere of current through a wire having a resistance of one ohm. When, in equation (15), electromotive force is expressed in volts, and current in amperes, then the power $P$ which is delivered to a circuit is expressed in watts.

When, in equations (16), current is expressed in abamperes and resistance in abohms, then electromotive force is expressed in c.g.s. units. The c.g.s. unit of electromotive force is called the abvolt. When, in equation (I 5), electromotive force is expressed in abvolts and current in abamperes then the power $P$ is expressed in ergs per second.

It is to be particularly noted that equations (I4), (I 5), (I6a), and $(16 b)$ apply to the whole or to any portion of an electrical circuit. Thus if $R$ is the resistance of a transmission line delivering a current $I$ to a receiving circuit such as an electric motor, then $R I^{2}$ is the power lost in the line in watts, and $R I$ is the electromotive force lost in the line. This electromotive force lost in the transmission line on account of line resistance is called line drop of electromotive force or pressure, or simply line drop. Similarly the electromotive force $R I$ used to overcome the resistance of the armature of an electric generator, is called armature drop.

18. Series and parallel connections. - When two portions of an electric circuit are so connected that the entire current in the cir- 
cuit passes through both portions, the portions are said to be connected in series. When two portions of an electrical circuit are so connected that the current in the circuit divides and a part of it flows through each portion, the portions are said to be connected in parallel.

The ordinary arc lamps which are used to light city streets are connected in series and the entire current delivered by the lighting generator flows through each lamp. $\mathrm{Or}_{\mathbf{1}}$ the other hand, if the electromotive force of the generator is, say, 4,000 volts, and if there are 80 similar lamps in series, the electromotive force between the terminals of each lamp will be 50 volts. The electromotive force of a generator is subdivided among a number of receiving devices connected in series.

The ordinary glow lamps which are used for house lighting are connected in parallel between copper mains which lead out from the terminals of the generator, and, except for a slight loss of electromotive force in the mains, the full electromotive force of the generator acts upon each lamp. On the other hand, if the generator delivers, say I,000 amperes, and if there are 2,000 similar lamps connected between the mains, the current in each lamp will be $1 / 2$ ampere. The current delivered by a generator is subdivided among a number of receiving devices connected in parallel.

Electric generators may be connected in series. When this is done the electromotive force available for the maintenance of current is equal to the sum of the electromotive forces of the individual generators.

Electric generators which have equal electromotive forces may be connected in parallel. When this is done the total current delivered by the set is equal to the sum of the currents delivered by the individual generators.

Combinations of series and parallel connections are most frequently used in grouping voltaic cells and storage batteries. Series-connected-groups of cells may be arranged in parallel, as shown in Fig. II, or parallel-connected-groups of cells may be 
arranged in series as shown in Fig. 12. The electromotive force of such an arrangement is equal to $n E$, where $n$ is the number of cells in series, and $E$ is the electromotive force of a single cell.

The combined resistance of a number of resistances connected in series is equal to their sum.

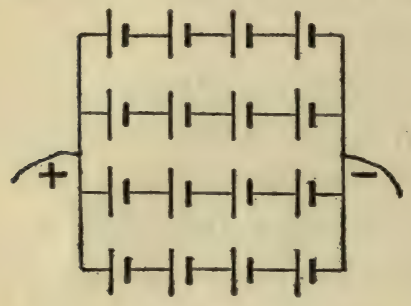

Fig. 11.

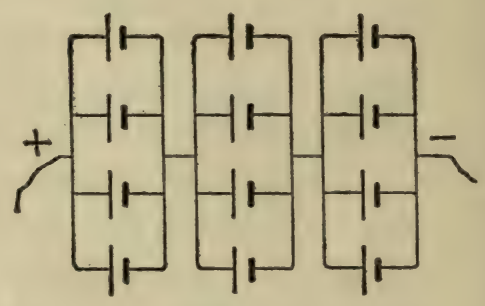

Fig. 12.

The combined resistance of a number of resistances connected in parallel is equal to the reciprocal of the sum of the reciprocals of the individual resistances.

19. To determine the current in each of two branches of a circuit when the total current and the resistance of each branch are given. - The solution of this problem depends upon two principles as follows :

(a) The current in the undivided part of a circuit is equal to the sum of the currents in the respective branches into which the circuit divides. (Kirchhoff's law.)

(b) Let $A$ and $B$ be the two points at which two or more branches of a circuit unite. The product of the resistance $R$ of any one of the branches into the current $I$ flowing in that branch is equal to the electromotive force between the branch points $A$ and $B$. Therefore the product $R I$ has the same value for every branch terminating in the points $A$ and $B$.

In the case of two branches we have from $(a)$

and from $(b)$ we have

$$
I=I_{1}+I_{2}
$$

$$
R_{1} I_{1}=R_{2} I_{2}
$$


in which $I$ is the total current and the subscripts refer to the respective branches. Solving these two equations for $I_{1}$ and $I_{2}$, we have :

$$
\begin{aligned}
& I_{1}=\frac{R_{2}}{R_{1}+R_{2}} \cdot I \\
& I_{2}=\frac{R_{1}}{R_{1}+R_{2}} \cdot I
\end{aligned}
$$

To determine the combined resistance of a number of coterminous branches of a circuit. Let $I$ be the total current flowing in the circuit, $E$ the electromotive force between the branch points, and $R_{1}, R_{2}, R_{3}, \ldots$ the resistances of the respective branches. Then $E / R_{1}, E / R_{2}, E / R_{3}$, etc., are the currents in the respective branches, and from Kirchhoff's law we have

$$
I=\frac{E}{R_{1}}+\frac{E}{R_{2}}+\frac{E}{R_{3}}+\cdots
$$

Now, the combined resistance $R$ of all the branches is defined as that resistance through which the electromotive force $E$ between the branch points may maintain the total current $I$; so that $R I=E$ or $I=E / R$. Substituting this value of $I$ in the above equation we have

whence

$$
\frac{E}{R}=\frac{E}{R_{1}}+\frac{E}{R_{2}}+\frac{E}{R_{3}}+\cdots
$$

$$
R=\frac{\mathrm{I}}{\frac{\mathrm{I}}{R_{1}}+\frac{\mathrm{I}}{R_{2}}+\frac{\mathrm{I}}{R_{3}}+\cdots}
$$

20. General solution of a network. - Consider a network of wires consisting of the branches $2,3,4,5$, and 6 connected through a wire I I to a battery of which the electromotive force is $E$, as shown in Fig. I 3 . Let the long arrows, arbitrarily chosen, represent the directions in which the currents in the various wires are to be considered as positive in sign; should a current in one of the branches, as found by calculation, be negative 
in sign, its actual direction will be opposite to the direction indicated by the arrow. Let it be required to find the currents $i_{1}, i_{2}$,

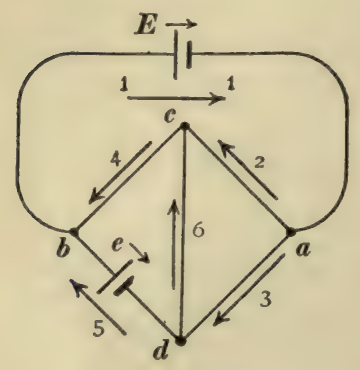

Fig, 13. $i_{3}, i_{4}, i_{5}$, and $i_{6}$ in the respective branches $I, 2,3,4,5$ and 6 , having given the resistances $r_{1}, r_{2}, r_{3}, r_{4}, r_{5}$ and $r_{6}$ of the respective branches, and the electromotive forces $E$ and $e$. The directions of these given electromotive forces are indicated by the short arrows. This problem is to be solved with the help of two distinct principles as follows :

(a) The algebraic sum of all the currents flowing towards a branch point is equal to zero. (Kirchhoff.)

(b) The algebraic sum of all the electromotive forces acting around a closed circuit, or a mesh of a network of conductors, is equal to the sum of the products $r i$ around the mesh. (Kirchhoff.)

Applying the first principle to the branch points, $a, b, c$, and $d$, Fig. $\mathrm{I} 3$, in succession, with due consideration of algebraic signs, we have the following four equations :

$$
\begin{array}{r}
i_{1}-i_{2}-i_{3}=0 \\
-i_{1}+i_{4}+i_{5}=0 \\
i_{2}-i_{4}+i_{6}=0 \\
i_{3}-i_{5}-i_{6}=0
\end{array}
$$

Consider any mesh, for example the one formed by the branches I, 2 and 4, Fig. I3. The sum of the products $r i$ taken in a clock-wise direction around this mesh is equal to the sum of all the electromotive forces acting in a clock-wise direction around the mesh, a counter-clock-wise electromotive force being reckoned as negative. Applying the second principle in this way to the mesh formed by branches I, 2 and 4, we have:

$$
r_{1} i_{1}+r_{2} i_{2}+r_{4} i_{4}=E
$$


Applying the second principle in a similar manner to the mesh 2-3-6 gives :

$$
r_{6} i_{6}-r_{2} i_{2}+r_{3} i_{3}=0
$$

Applying the second principle in a similar manner to the mesh 4-6-5 gives :

$$
-r_{4} i_{4}-r_{6} i_{6}+r_{5} i_{5}=-e
$$

Applying the second principle in a similar manner to the mesh I-3-5 gives :

$$
r_{1} i_{1}+r_{3} i_{3}+r_{5} i_{5}=E-e
$$

For convenience let us call the equations (i), (ii), (iii) and (iv), which refer to branch points, point equations ; and the equations (v), (vi), (vii) and (viii) which refer to meshes, mesh equations. In problems of this kind there is always one superfluous point equation and one superfluous mesh equation, and any one of the point equations together with

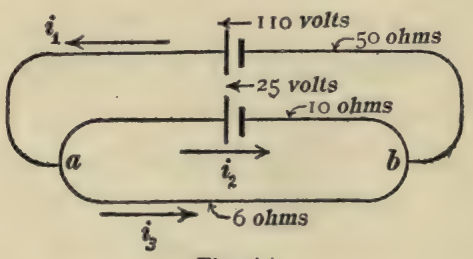

Fig. 14. any one of the mesh equations are to be dropped, thus leaving as many independent equations as there are unknown quantities $i_{1}, i_{2}, i_{3}, i_{4}, i_{5}$, and $i_{6}$.

Example. - A IIO-volt battery is connected to two branch points $a$ and $b$, Fig. I4, between which are two branches ( 2 and 3). Branch 2 has Io ohms resistance and contains a 25-volt battery as shown in Fig. 14. Branch 3 has a resistance of 6 ohms. It is required to find the currents $i_{1}, i_{2}$ and $i_{3}$.

Considering the point $a$ we have the equation :

$$
i_{1}-i_{2}-i_{3}=0
$$

Considering the mesh $\mathrm{I}-2$ we have the equation :

$$
50 i_{1}+10 i_{2}=110-25
$$

Considering the mesh $\mathrm{I}-3$ we have the equation :

$$
50 i_{1}+6 i_{3}=\mathrm{IIO}
$$


Substituting the value of $i_{2}$ from (ii) and the value of $i_{3}$ from (iii) in (i) we have :

$$
i_{1}=+1.87 \text { amperes }
$$

whence from equations (ii) and (iii) we have:

$$
\begin{aligned}
& i_{2}=-0.86 \text { amperes } \\
& i_{3}=+2.73 \text { amperes }
\end{aligned}
$$

21. Faraday's discovery. Induced electromotive force and induced current. - In I83 I Faraday discovered that a momentary electric current is produced in a coil of wire when a magnet is pushed into or withdrawn from the opening of the coil, or when an iron rod around which the coil is wound is magnetized or demagnetized. The motion of the magnet in the first case, or the changing magnetism of the iron rod in the second case, produces a momentary electromotive force in the coil, and this electromotive force produces a momentary electric current in the coil if the coil forms a portion of a closed circuit. Electromotive force and electric current produced in this way are called induced electromotive force and induced current.

22. Lenz's Law. - An induced current always opposes the action which produces it, and the work done in overcoming this opposition is the work which is expended in producing the induced current.

Examples. - (a) When a magnet is pushed into a coil the induced current is in such a direction as to tend to push the magnet out of the coil ; more work must, therefore, be done in pushing the magnet into the coil than would be done if the circuit of the coil were open so that no induced current could be produced; this extra work is the work which is expended in producing the induced current.

(b) When an iron rod wound with wire is magnetized, the current induced in the winding of wire opposes the magnetization, and more work must be done to magnetize the rod than would 
be done if the induced current did not exist. This additional work is the work which is expended in producing the induced current.

A more general aspect of Lenz's law is given in Art. 26 where the relation between the electric generator and the electric motor is discussed.

23. Electromotive force induced in a straight wire moving sidewise across a uniform magnetic field. - With the help of Lenz's law the fundamental law of induced electromotive force may be derived. For this purpose it is sufficient to consider the simplest case, namely, the motion of a straight wire sidewise across a uniform magnetic field.

A straight wire $A B$, Fig. 15 , slides sidewise at a velocity of $v$ centimeters

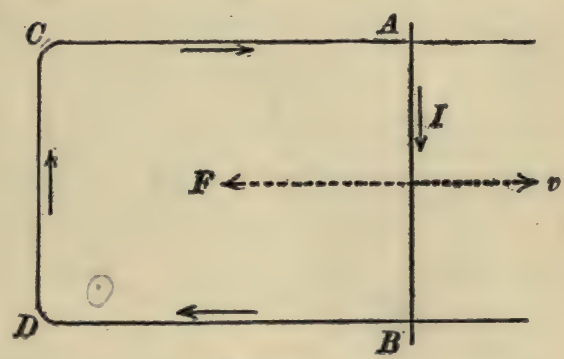

Fig. 15. per second along two straight wires or rails $C A$ and $D B$ distant $l$ centimeters from each other. The rails are connected at $C D$ so that $A B C D$ is a closed circuit. The whole arrangement is placed in a uniform magnetic field of intensity $\mathscr{H}$, the direction of the field being perpendicular to the plane $A B C D$ and towards the reader.

The motion of the wire $A B$ induces in it an electromotive force; this electromotive force produces a current in the circuit $A B C D$, and because of this current the wire $A B$ is pushed sidewise by the magnetic field with the force $F$, of which the value in dynes is given by the equation

$$
F=I l \mathscr{H}
$$

in which $I$ is the strength of the induced current in abamperes.

Now $F v$ is the rate in ergs per second at which work is done in moving the wire $A B$ against the opposing force $F$, and, from Lenz's law, this must be equal to the rate $E I$ at which work is 
done by the induced electromotive force in maintaining the induced current $I$. That is :

$$
F v=E I
$$

in which $E$ is the value of the induced electromotive force in abvolts.

Substituting the value of $F$ from equation (6) in equation (ii) and solving for $E$, we have:

$$
E=l \mathscr{H V}
$$

That is, the electromotive force $E$, in c.g.s. units, induced in a straight wire $l$ centimeters long moving sidewise at a velocity of $v$ centimeters per second across a uniform magnetic field of intensity $\mathscr{H}$, is equal to the product $l \mathfrak{F}$ V. This result is expressed in abvolts and it is to be divided by $10^{8}$ to reduce to volts, that is

$$
E=\frac{l \mathscr{H} v}{10^{8}} \text { volts }
$$

24. Expression of induced electromotive force in terms of number of lines of force cut per second. During $t$ seconds the slidingwire $A B$, Fig. I 5 , moves over a distance $v t$ and sweeps over $l v t$ square centimeters of area. Multiplying this area by $\mathscr{H}$ gives the magnetic flux $\Phi(=l v t \mathcal{H} \mathcal{H})$ which passes through the area, and this flux $\Phi$ is the number of lines of force cut by $A B$ during the $t$ seconds, so that $\Phi / t(=l \mathfrak{H} \mathscr{f} v=E)$ is the number of lines of force cut per second by $A B$. That is, the electromotive force in c.g.s. units induced in a wire is equal to the number of lines of force cut by the wire per second. This proposition is here derived for a straight wire moving sidewise across a uniform magnetic field, but it is true for any wire straight or curved moving in any manner in any magnetic field uniform or non-uniform.

25. Expression of induced electromotive force in terms of the rate of change of the magnetic flux enclosed by or passing through a circuit. As the wire $A B$, Fig. 15 , moves to the right the enclosed area $A B C D$ increases, the magnetic flux through $A B C D$ 
increases, and the increase of this magnetic flux during a given time is equal to the number of lines of flux swept over by $A B$ during that time; or in other words, the rate of increase of the magnetic flux through $A B C D$ is equal to the number of lines of force cut by $A B$ per second. Therefore the induced electromotive force in a circuit is equal to the rate at which the magnetic flux through the circuit is changing, electromotive force being expressed in abvolts. This result is here derived for the very special case represented in Fig. I 5, but it is true for any shape of circuit in any magnetic field uniform or non-uniform, and it is true whether the change of flux through the circuit be due to motion or to changing magnetism or to both.

Let the magnetic flux through a circuit be represented by $\Phi$, then its rate of change is represented by $d \Phi / d t$, and we have

$$
E=\frac{d \Phi}{d t}
$$

in which $E$ is the induced electromotive force in a circuit expressed in abvolts.

If the circuit has $Z$ turns of wire then the electromotive force induced in each turn is $d \Phi / d t$, and the total induced electromotive force expressed in abvolts is :

$$
E=Z \cdot \frac{d \Phi}{d t}
$$

Equations $(20 a)$ and $(20 b)$ are more properly written with the negative sign, thus :

$$
E=-\frac{d \Phi}{d t} \text { and } E=-Z \cdot \frac{d \Phi}{d t}
$$

inasmuch as an increasing flux produces an induced electromotive force which is in the direction in which a left handed screw would have to be turned to cause the screw to travel in the direction of the magnetic field upon which the flux depends.

In any given case an induced electromotive force may be thought of as being produced by either $(a)$ the cutting of lines of force at a definite rate by a wire, or $(b)$ the change at a definite rate of the magnetic flux enclosed by a loop or coil of wire. These 
two actions are fundamentally identical, and the one or the other may be assumed as the basis of the discussion of any given case according to convenience. Thus in discussing the action of the alternator in article 27 the notion of the rate of change of flux is employed, whereas the notion of the cutting of lines of force is employed in the discussion of the direct current dynamo in article 28.

\section{TABLE OF Units.*}

EleCtric.

\begin{tabular}{|c|c|c|c|c|}
\hline Name of Quantity. & Symbol & $\begin{array}{l}\text { Name of } \\
\text { Practical Unit. }\end{array}$ & $\begin{array}{l}\text { Name of } \\
\text { c.g.s. Unit. }\end{array}$ & $\begin{array}{l}\text { Practical Unit } \div \\
\text { c.g.s. Unit. }\end{array}$ \\
\hline Current............... & $I, i$ & ampere. & (abampere.) & $10^{-1}$ \\
\hline Resistance....................... & $\vec{R}, r$ & ohm. & (abohm.) & $10^{9}$ \\
\hline Resistivity ....................... & $\boldsymbol{p}$ & $\begin{array}{l}\text { ohms of a } \\
\text { centimeter } \\
\text { cube. }\end{array}$ & $\begin{array}{l}\text { abohms of a } \\
\text { centimeter } \\
\text { cube. }\end{array}$ & $10^{9}$ \\
\hline Conductance .................. & $\mathbf{r} / R$ & (mho.) & ${ }^{-}$ & $10^{-9}$ \\
\hline Conductivity ...................... & $\mathbf{I} / \rho$ & & & $10^{-9}$ \\
\hline Electromotive force ........... & $E, e$ & volt. & (abvolt.) & $10^{8}$ \\
\hline Inductance....................... & $L$ & henry. & centimeter. & $10^{9}$ \\
\hline Capacity............................ & $C$ & farad. & - & $10^{-9}$ \\
\hline Inductivity........................ & $k$ & - & & - \\
\hline Dielectric strength........... & & $\begin{array}{l}\text { volts per } \\
\text { centimeter. }\end{array}$ & $\begin{array}{l}\text { abvolts per } \\
\text { centimeter. }\end{array}$ & $10^{8}$ \\
\hline
\end{tabular}

Magnetic.

\begin{tabular}{|c|c|c|c|c|}
\hline 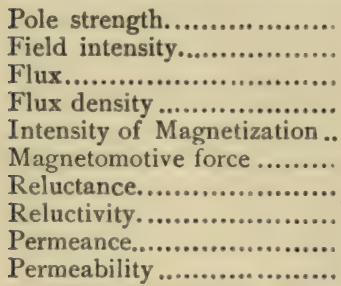 & $\begin{array}{c}m \\
\mathscr{O} \\
\Phi, \phi \\
\mathscr{B} \\
\mathscr{J} \\
\mathscr{F} \\
\mathscr{R} \\
\mathbf{I} / \mu \\
\mathbf{1} / \mathscr{R} \\
\mu\end{array}$ & $\bar{\square} \bar{\square}$ & $\begin{array}{l}\text { gauss. } \\
\text { maxwell. } \\
\text { gauss. } \\
\text { (gilbert.) } \\
\text { (oersted.) } \\
-\end{array}$ & $\bar{\square}$ \\
\hline
\end{tabular}

* Appendix A, on the magnetism of iron, is essentially a continuation of this introductory chapter on elementary theory, and the units of magnetomotive force, reluctance, permeability, etc., are tabulated here for convenience. Names in parentheses have not yet been adopted by the International Electrical Congress. 


\section{CHAPTER II.}

\section{THE DYNAMO.}

26. The dynamo. - The dynamo electric machine, or, simply, the dynamo, is a machine for the production and maintenance of an electric current when the machine is supplied with mechanical power, or conversely for the development of mechanical power when the machine is supplied with an electric current. When used for the former purpose the dynamo is called an electric generator or simply a generator; when used for the latter purpose the dynamo is called an electric motor or simply a motor.

The action of the dynamo as a generator is essentially as follows: A wire $l$ centimeters in length, see Fig. 15 , is forced by an external source of mechanical power to move sidewise at a velocity of $v$ centimeters per second across a magnetic field of intensity $\mathscr{H}$. This motion induces an electromotive force $E$ in the wire in accordance with equation (19a), namely,

$$
E=l \mathscr{f}(v \text { abvolts }
$$

This electromotive force produces a current of $I$ abamperes in the wire, if the wire is a portion of a closed circuit, and because of this current the wire is pushed sidewise by the magnetic field with the force:

$$
F=I l \mathscr{H} \text { dynes }
$$

which opposes the motion of the wire. Work is done by the external mechanical agent at the rate of $F v$ ergs per second in moving the wire against the opposing force $F$, and the work so spent goes to maintain the current in the wire.

The action of the dynamo as a motor is essentially as follows: A current of $I$ abamperes is forced by an external source of electric current through a wire $l$ centimeters in length which is 
stretched across a field of intensity $\mathscr{H}$. This current causes the field to push the wire sidewise with the force

$$
F=I l \mathscr{H} \text { dynes }
$$

This force moves the wire sidewise at a velocity of $v$ centimeters per second and develops mechanical power. The motion of the wire induces in it the electromotive force

$$
E=l \mathscr{H} v \text { abvolts }
$$

which opposes the flow of current in the wire. Work is done by the external source of electric current at the rate of EI ergs per second in forcing the current against the electromotive force $E$, and the work so spent goes to maintain the motion of the wire.

There are two distinct types of dynamo electric machines, namely $(a)$ alternating current machines, and $(b)$ direct current machines. The alternating current generator delivers what is called an alternating current, that is, a current which is subject to rapid periodic reversals of direction. The direct current generator, on the other hand, delivers a current which is not reversed in direction and which is more or less steady in value. All direct current machines, except the so-called homopolar dynamos, are essentially alternating current machines with the addition of a commutator instead of collecting rings.

27. The alternating current dynamo is the simplest form of dynamo electric machine. It consists essentially of an arrangement in which a loop of wire is moved in front of the projecting north and south poles of a magnet in such a way that the magnetic flux due to the magnet poles passes through the loop first in one direction and then in the other direction repeatedly. This repeated reversal of the magnetic flux through the loop of wire induces an electromotive force in the wire in one direction while the flux is increasing (from negative to positive), and in the other direction while the flux is decreasing (from positive to negative). This alternating electromotive force produces an alternating cur- 
rent in the loop of wire and in any outside circuit which is connected to the terminals of the loop. An alternating current dynamo is usually called, simply, an alternator.

A common type of alternator consists of a stationary multipolar electromagnet, called the field magnet, of which the poles $N, S$, $N, S$, etc., project radially inwards as shown in Fig. I6 towards

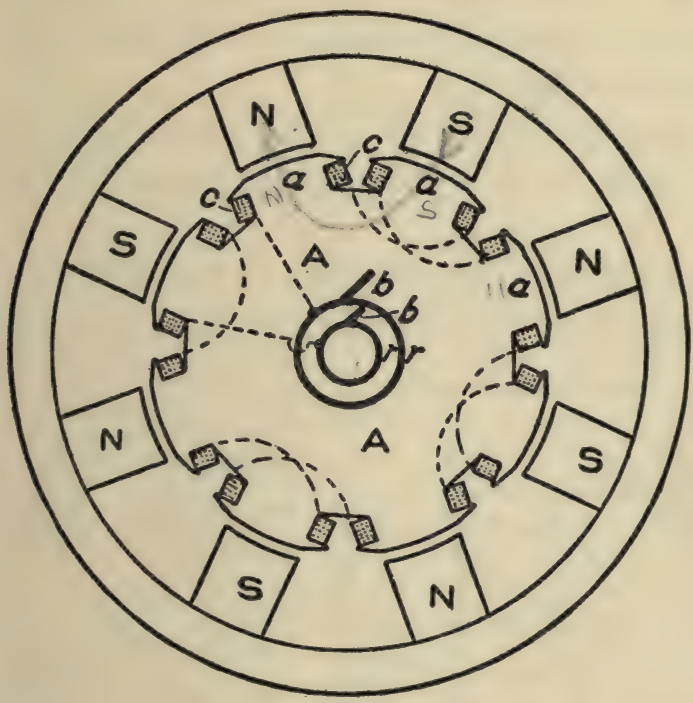

Fig. 16.

the passing teeth $a, a$ of a rotating structure $A$ called the armature. On the armature shaft at one end of the armature are fixed two metal rings $r r$ called collecting rings. These rings are insulated from each other and from the shaft. The ends of the armature wire are connected to these rings as shown, and the wire is wound around the armature teeth in the form of coils, which are shown in section by the dotted squares $c, c$ in Fig. I6. In this type of alternator it is convenient to attribute the induced electromotive forces to the changing of the magnetic flux through the armature teeth and coils as they pass the field poles. As the armature rotates the magnetic flux passes through a given armature coil in one direction when the tooth on which the coil is 
wound is squarely under a north pole, and in the reverse direction when the tooth is squarely under a south pole. The induced electromotive force is always opposite in direction around adjacent coils, and the armature coils are all connected in series in such a manner* that their electromotive forces are added, thus giving an alternating electromotive force of large value between the collecting rings. The external circuit which is to receive alternating current from the machine is connected to metal brushes $b b$ which rub on the collecting rings.

The armature body, or core, $A$, is built up of sheet-iron stampings to prevent the formation of eddy currents as explained in Art. 32 .

The field magnet of the alternator is magnetized or excited by a continuous or steady current, or, as it is technically called, a

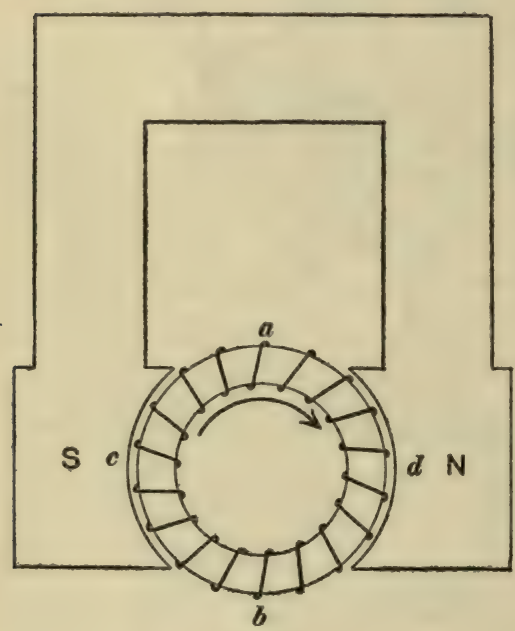

Fig. 17. direct current. This current flows through coils of wire (not shown in Fig. I6) which are wound on the polar projections $N, S, N, S$, etc. The direct current for exciting the field magnet of an alternator is usually obtained from a small auxiliary direct-current dynamo called the exciter. Alternators used in practice give from 50 to 250 or more reversals of current per second.

\section{The direct current dyna-} mo.--The following description applies to the direct-current dynamo having an armature of the ring type. The relation of the ring to the drum armature and a full discussion of armature windings are taken up in Appendix C.

* The coils are connected so that in following along the armature wire from one collecting ring to the other, one would pass around adjacent armature teeth in opposite directions. 
An iron ring $a b$, Fig. I 7 , which is built up of sheet-iron stampings, is wound uniformly with insulated wire as indicated in the figure, the ends of the wire being spliced together and soldered so that the winding is endless. This iron ring with its winding of wire is called the armature; it rotates, as indicated by the curved arrow, between the poles $N S$ of a strong field magnet.

The wires on the outside of the iron ring have electromotive forces induced in them as they move across the pole faces of the field magnet and cut the lines of force. These electromotive

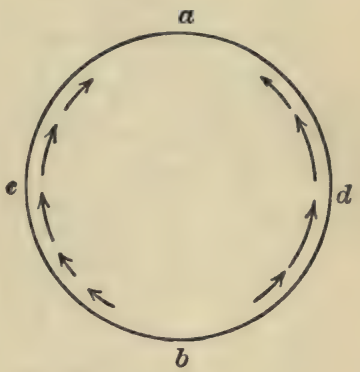

Fig. 18. forces cannot, however, produce current in the endless wire that is wound on the armature, inasmuch as exactly equal and opposite electromotive forces are induced on the opposite sides, $c$ and $d$, of the ring, as shown diagrammatically in Fig. 18 , in which figure the circle $a d b c$ represents the endless wire winding on the ring.

A steady, or very nearly steady, current can be taken from the winding on the ring through an outside receiving circuit $l$, Fig. I9, by keeping the terminals of this circuit in metallic contact with the windings on the ring at $a$ and $b$ as shown in Fig. 19.

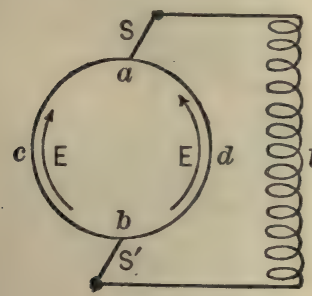

Fig. 19.

For this purpose the insulation may be removed from the outer portions of the wire windings on the ring, and the two stationary metal or carbon brushes SS, Fig. I9, arranged to rub at $a$ and $b$ as the ring rotates.

In practice, wire leads are soldered to the various turns of wire on the ring and connected to insulated copper bars near the axis of rotation, as shown in Fig. 20. Sliding contact is then made with these copper bars instead of with the turns of wire at $a$ and $b$ directly. This set of copper bars constitutes what is called the commutator. 
Armature current and armature resistance. From Fig. I9 it is evident that, of the total current which flows out of the armature

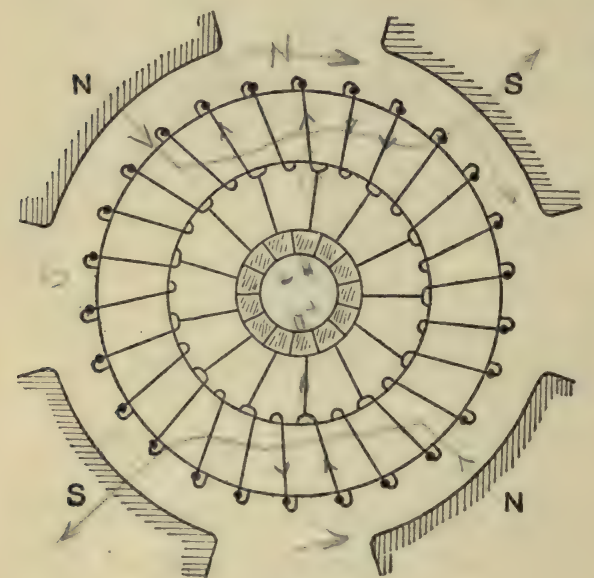

Fig. 20. to the receiving circuit $l$, half flows through the windings on one side of the ring, and half through the windings on the other side of the ring. That is, the windings on the ring present two paths for the flow of current from brush $b$ to brush $a$, and the current in each path is half of the current which is delivered to the receiving circuit.

The resistance of the armature winding between the brushes is one half the resistance of each separate path, since the two paths are similar and in parallel. Furthermore, the resistance of the armature between brushes is one quarter of the resistance of the winding considered as one long coil.

Closed coil and open coil armatures. - The armature winding shown in Fig. 17 is called a closed coil winding, inasmuch as the winding itself forms a closed circuit. Fig. 2 I shows the simplest example of what

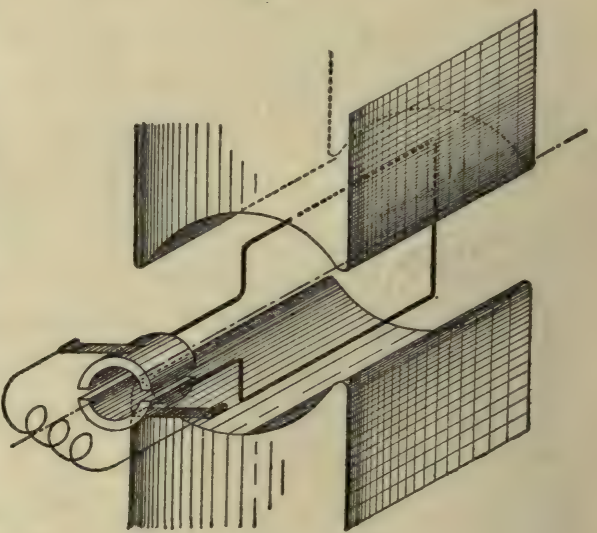

Fig. 21 . is called an open coil winding. It consists of a single coil of wire of which the ends are connected to the two segments 
of a two-part commutator. The current which is produced in the armature winding of Fig. $2 \mathrm{I}$ is an alternating current, and the two-part commutator serves to reverse the connections of the armature to the external receiving circuit twice during each revolution of the armature, so that the current, which is an alternating current in the armature winding, becomes a uni-directional current in the external circuit. The Brush arc lighting dynamo and the Thomson-Houston arc dynamo are examples of machines having open coil armatures.

The current in any given turn of wire on a closed coil armature is also an alternating current, inasmuch as it flows in one direction through the turn when the turn is on the side $d$ of the armature (see Fig. I 7), and in the opposite direction through the turn when the turn is on the side $c$ of the armature.

The above discussion applies to the simplest form of direct-current dynamo; namely, a two-pole dynamo with a simple ring winding. Commercial forms of direct-current dynamos, however, may be bipolar or multipolar, they may have ring or drum armatures, and they may have any one of a variety of armature windings, including open coil windings, according to the size of the machine and the conditions of service.

29. The multipolar directcurrent dynamo. - Fig. 22 shows a ring armature rotating inside of a crown of six inwardly projecting field magnet poles, NSNSNS. The

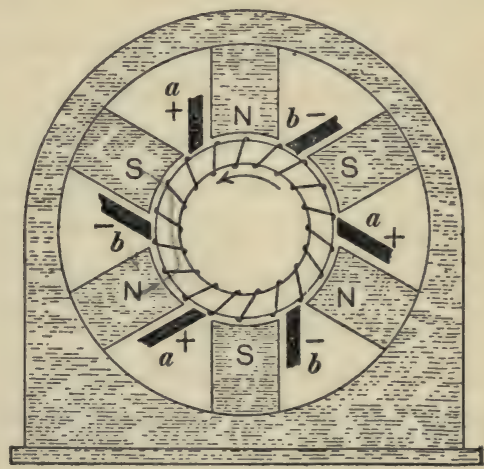

Fig. 22. electromotive forces induced in the windings as they sweep across the pole faces cannot produce current in the endless wire that is wound on the ring, inasmuch as the electromotive forces induced 
under the north poles are just balanced by the electromotive forces induced under the south poles, as is shown diagrammatically in Fig. 23. To utilize the induced electromotive forces $e$ e e e e $e$, Fig. 23, for the production of direct current, six brushes $a$ a $a$ and $b b b$, Fig. 22, should be used. Three of these brushes

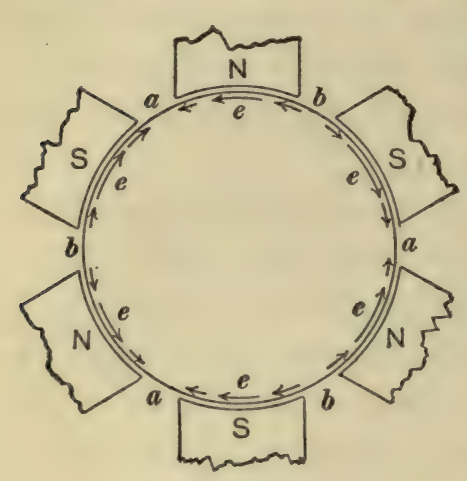

Fig. 23. maintain contact with the windings at $a \quad a \quad$, and through all three of these brushes current flows out of the armature to one terminal of a receiving circuit. The other three brushes $b b b$ maintain contact with the windings at $b b b$, Fig. 23 ; all three of these brushes are connected to the other terminal of the receiving circuit, and current flows into the armature through all three. The three brushes $a$ a $a$ together constitute, therefore, the positive terminal of the armature, and the brushes $b b b$ constitute the negative terminal of the armature.

Armature current and armature resistance. - The total current flowing out of the armature of the six-pole dynamo shown in Fig. 22 flows out of the three brushes $a a a$, so that one third of the total current flows out of each of the positive brushes. An inspection of Fig. 23 shows, furthermore, that current flows in the armature winding to each positive brush from both sides; that is through two paths. Therefore, in the six-pole dynamo with a simple ring armature, there are six paths (in parallel) in the armature winding from negative to positive brushes, and therefore one sixth of the current output of the machine flows through each path.

The resistance of this six-pole armature between positive and negative brushes is equal to one sixth of the resistance of any one path, since the six paths are similar and in parallel. Therefore the resistance of the armature between positive and negative 
brushes is one thirty-sixth of the resistance of the whole winding considered as one long coil.

Another type of multipolar armature winding, namely the wave winding, is described in Appendix C. The wave winding has but two paths between positive and negative brushes irrespective of the number of poles and brushes.

When an armature provides $p^{\prime}$ similar paths in parallel between positive and negative brushes, then evidently I $/ p^{\prime}$-th of the armature windings are in series in each path.

30. The fundamental equation of the direct-current dynamo. The equation which expresses the relation between the induced electromotive force of a dynamo, the amount of magnetic flux from each field pole, the number of field poles, the number of conductors on the outside of the armature, the number of paths in the armature winding between brushes, and the speed of the armature, is called the fundamental equation of the dynamo on account of its importance in the theory of the design and operation of generators and motors.

Let $\Phi=$ magnetic flux which enters the armature from each north pole of the field magnet and leaves the armature at each south pole of the field magnet. $p=$ number of field poles.

$Z=$ number of conductors on the outside of the armature.

$p^{\prime}=$ number of electrical paths in parallel between the brushes.

$E_{a}=$ total electromotive force induced in the armature. This is the same as the electromotive force between the brushes, as measured by a voltmeter, when the current in the armature is negligibly small.

Then

$$
n=\text { speed of armature in revolutions per second. }
$$

$$
E_{a}=\frac{p \Phi Z n}{p^{\prime}} \text { abvolts }
$$

or

$$
E_{a}=\frac{p \Phi Z n}{p^{\prime} \times 10^{8}} \text { volts }
$$


Proof. - During I/pn-th of a second a given armature conductor sweeps past a field pole, from $a$ to $b$, Fig. 22, and cuts $\Phi$ lines of force. This conductor therefore cuts lines of force at an average rate which is equal to $\Phi \div \mathrm{I} / p n$, or $\Phi p n$ lines of force per second, and this is the average electromotive force in the given conductor while it is moving from $a$ to $b$, Fig. 22 ; also this is the average electromotive force per conductor in all the conductors between $a$ and $b$ at any instant. Now, there are $Z / p^{\prime}$ conductors $*$ in series in each path between the brushes, and since $\Phi p n$ is the average electromotive force per conductor, therefore $Z / p^{\prime} \times \Phi p n$ is the electromotive force between the brushes. That is, $E_{a}=p \Phi Z n / p^{\prime}$, in which $E_{a}$ is, of course, expressed in abvolts.

Equations (2 I) apply to bipolar or multipolar machines, and to machines having ring or drum armatures with any kind of winding except open-coil windings, and these equations give the electromotive force induced in the windings between the brushes, provided the brushes are at the neutral points as shown in Fig. 22 and as explained in article $5 \mathrm{I}$.

31. Field excitation of generators. - Some very small generators, such as the "magnetos," which are used for ringing telephone call bells, are provided with field magnets of hardened steel, which are permanently magnetized. Large generators and motors, on the other hand, are always provided with electrically excited field magnets (electromagnets).

Separate excitation and self-excitation. - The field magnet of an alternator must be excited by direct current, usually from an outside source, as stated in Art. 27. $\dagger$ The field magnets of cer-

* The conductors which constitute a given path are always distributed evenly over the space $a b$, Fig. 22, so that the average electromotive force per conductor for all the conductors of a path is the same as the general average for the whole armature. In the simple ring-wound armature, $p=p^{\prime}$ and the conductors which at any instant lie between the points $a$ and $b$, Fig. 22, constitute one path.

† In the so-called composite field alternators this separate excitation is supplemented by a direct current supplied by the alternator itself through a rectifying commutator. 
tain types of direct-current generators are usually excited by current from an outside source; thus the low voltage generators used for electroplating are excited in this way. This method of field excitation is called separate excitation. Direct-current generators, however, usually supply their own field current. This method of field excitation is called self-excitation.

The shunt generator. - The winding on the field magnet of a direct-current generator may consist of many turns of comparatively fine wire having considerable resistance. In this case the terminals of the field winding are connected directly to the brushes of the machine, and from 2 to Io per cent. of the permissible current output of the generator is taken to magnetize or excite the field, the remainder being available for use in the external circuit. In this case the field winding and the outside receiving circuit are in parallel with each other between the brushes, so that the field winding is in the relation of a shunt to the outside receiving circuit. A generator with its field windings arranged in this way is called a shunt generator.

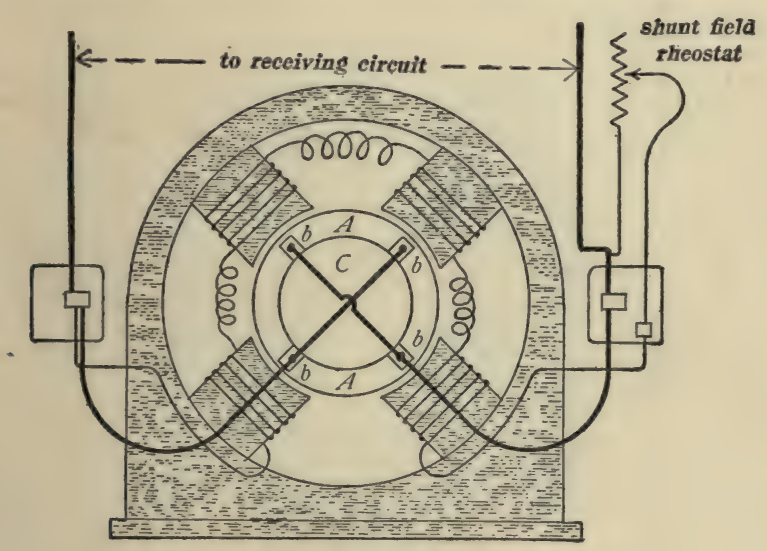

Fig. 24.

In the shunt generator an adjustable resistance (rheostat) is usually connected in the field circuit for controlling the value of the field current and thereby controlling the voltage of the machine. 
Fig. 24 shows the arrangement of a shunt generator with its field rheostat, $A A$ is the armature, $b b b b$ are the brushes, and $C$ is the commutator. The brush leads are connected to terminal blocks to which the receiving circuit and the shunt field

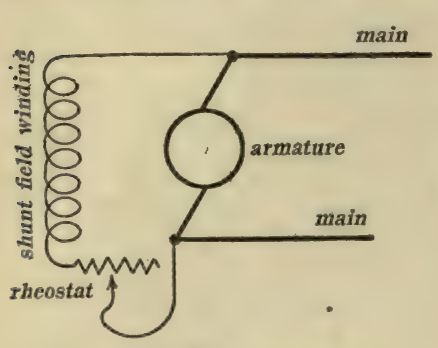

Flg. 25. winding are also connected. Fig. 25 shows the conventional diagram of connections of a shunt generator.

The series generator.-The winding on the field magnet of a directcurrent generator may consist of comparatively few turns of heavy wire having a low resistance. In this case the field winding is connected in series with the external receiving circuit, the whole current delivered by the machine flows through the field winding, and from 2 to 10 per cent. of the electromotive force of the machine is used to force the current through the field winding, the remainder being available for forcing

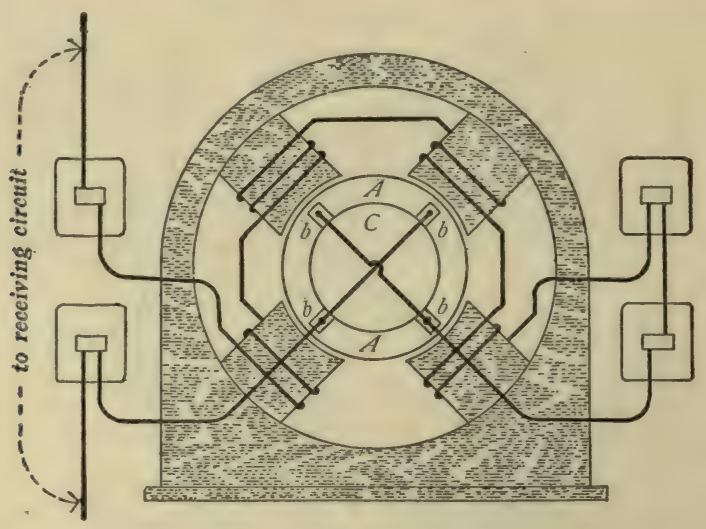

Fig. 26.

current through the external receiving circuit. A generator with its field windings arranged in this way is called a series generator. In this type of generator the control of the current in the field winding for a given current output of the machine is accomplished by 
the use of an adjustable low resistance shunt $B$, connected between the terminals of the field winding as shown in Figs. 27 and 68.

Fig. 26 shows the arrangement of a series generator without regulating field shunt, and Fig. 27 shows the conventional dia-

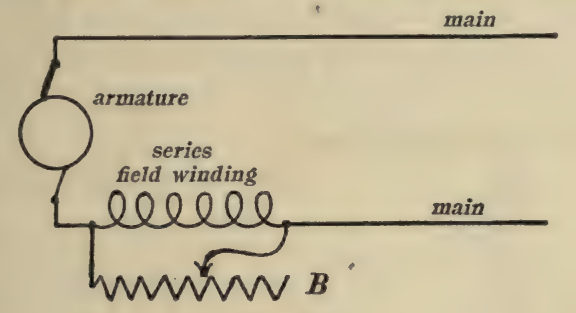

Fig. 27.

gram of connections of a series generator, including the regulating field shunt above mentioned.

The compound generator. - The winding on the field magnet of a direct-current generator is frequently in two parts, one part consisting of many turns of fine wire, the other part consisting of relatively few turns of heavy wire. In this case the fine wire

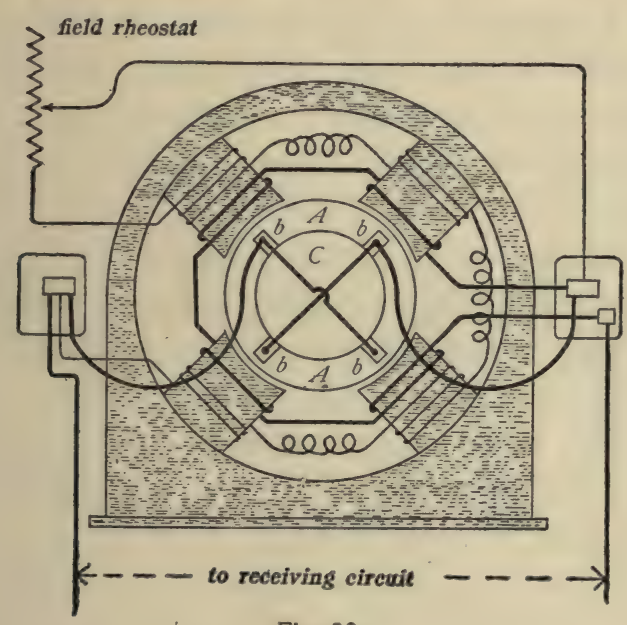

FIg. 28.

winding is connected directly to the brushes, or to the terminals of the machine, and the coarse wire winding is connected in series with the outside receiving circuit. This method of field excitation 
is a combination of the two previous methods, and a generator with its field windings so arranged is called a compound generator.

Fig. 28 shows the arrangement of a compound generator with a rheostat in circuit with the shunt field winding, Fig. 29 shows the conventional diagram of connections of a "long shunt" compound generator, and Fig. 30 shows the conventional diagram

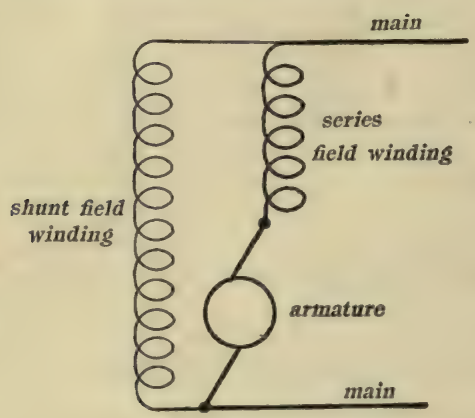

Fig. 29.

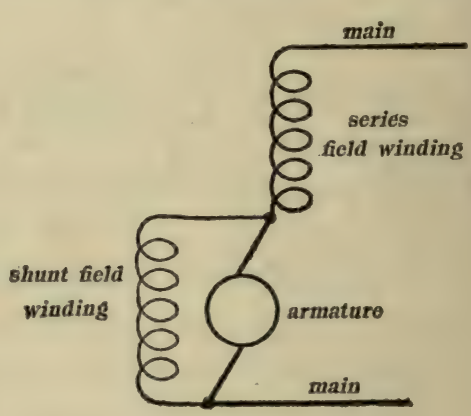

Fig. 30.

of connections of the "short-shunt" compound generator. In practice it is a matter of but little consequence whether a compound generator is connected "long shunt" or "short shunt." The calculations of currents, electromotive forces, losses, and efficiencies are however slightly different in the two cases, as explained in Art. 65 .

Shunt and compound generators are always used for generating direct current for lighting and power purposes when the current is to be delivered to lamps and motors at approximately constant voltage, which is the most usual condition of supply. The series generator, on the other hand, is seldom used except for constant current, series, arc lighting and for long distance direct-current power transmission as exemplified by a few European plants.

Field excitation of motors. - Inasmuch as a motor always receives current from an outside source there is no such thing as self-excitation of a motor, the field excitation being necessarily what is called separate excitation. A shunt motor has a fine wire field winding which is connected in parallel with the armature to 
the supply mains. A series motor has a coarse wire field winding which is connected in series with the armature to the supply mains. A compound motor has a field winding in two parts; one part is of fine wire connected in parallel with the armature, and the other part is of coarse wire connected in series with the armature. The connections of motors to the mains differ from the connections of generators on account of the starting resistance which must be used with a motor. Motor connections are discussed in detail in Chapter IV.

Shunt motors are almost always used for constant speed driving when the current is taken from constant voltage mains. The series motor is used for variable speed driving when the current is supplied at approximately constant voltage as exemplified by the use of motors for hoisting and for propelling street cars. Compound motors have characteristics which adapt them to special kinds of service as explained later.

32. Eddy currents. Lamination. - When a piece of iron is magnetized or demagnetized, the changes of the magnetic flux through the central portions of the iron induce electromotive forces in the surrounding portions of the iron, and these electromotive forces produce what are called Foucault currents or eddy currents in the iron. Eddy currents are also produced in a mass of metal, iron or copper, which is near a moving magnet, or which moves in the neighborhood of a stationary magnet.

Those parts of a dynamo which are subject to rapid and frequent changes of magnetization are built up of thin sheets of iron so as to leave the iron continuous in the direction of magnetization, but discontinuous in the direction in which the eddy currents tend to flow. Such a mass of iron is said to be laminated. The iron cores of dynamo armatures are always laminated, inasmuch as any given part of the iron core of an armature is subject to magnetic reversals as it moves past the field magnet poles.

Eddy currents are produced in the solid iron of a dynamo field magnet when the flux through it varies in value. Eddy currents are also produced in the surface layers of the pole pieces when 
the armature core has projecting teeth, inasmuch as the lines of force between the pole face and the armature core are concentrated in the form of tufts or brushes, as shown at $b b b$ in Fig. $3 \mathrm{I}$, and as the armature rotates these tufts sweep across the pole face and

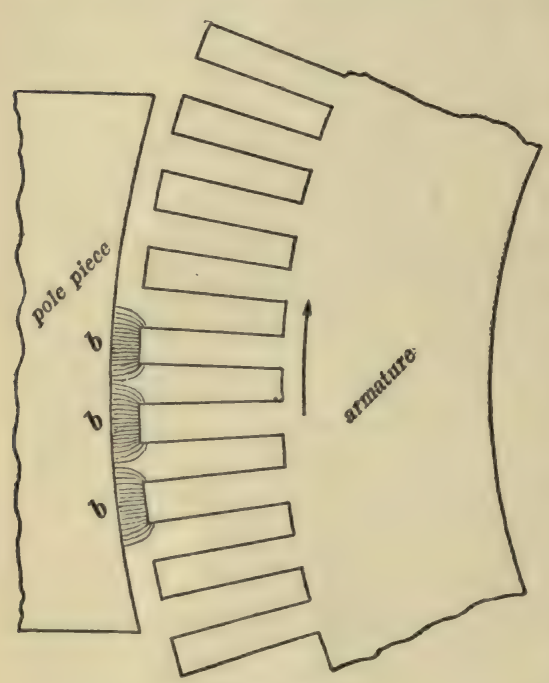

Fig. 31 . thus tend to produce eddy currents. Pole pieces are therefore sometimes laminated.

The prevention of eddy currents is the more complete the thinner the laminations and the better the insulation between them. When a mass of iron is subject to very rapid and frequent magnetic reversals the laminations are made very thin and insulated by a coating of hard varnish before being assembled on the armature shaft. For example, in an experimental alternator recently constructed by the Westinghouse Electric \& Manufacturing Company $*$ the armature core is subject to 20,000 magnetic reversals per second and the laminations are only $0.00 \mathrm{I}$ inch in thickness. In direct-current dynamos of moderate size the armature core is subject to from I 5 to 60 or more magnetic reversals per second, and the laminations are usually made from 0.020 to 0.035 inch in thickness. Large copper wires on smooth core armatures have eddy currents induced in them on account of the difference of intensity of the magnetic field in the air gap at the two edges of the moving conductor. These eddy currents flow up along one side and down along the other side of the conductor. The result is that the actual total current is crowded over to one side of the

* Trans. A. T. E. E., B. G. Lamme, 1904. 
conductor, as it were, and the effective resistance of the conductor is increased, so that the $R I^{2}$ loss in the conductor is increased. This production of eddy currents in the armature conductors may be greatly reduced by using stranded conductors, or by placing the conductors in slots in the armature core.

33. Examples of typical dynamos. - Fig. 32 shows in outline a typical bipolar dynamo, and Fig. 33 shows a typical multipolar dynamo. The regions $s s$ are called the air gaps, ff the

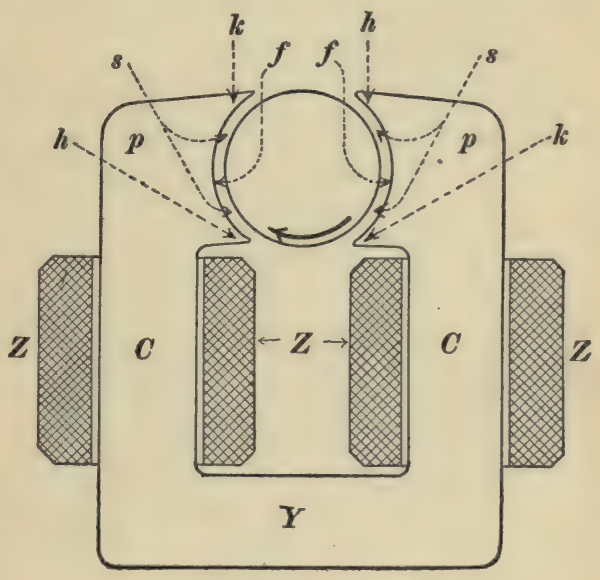

Fig. 32. pole faces, $p p$ the pole pieces, and $h h k k$ are called the pole tips or horns; $h h$ are called the leading pole tips and $k k$

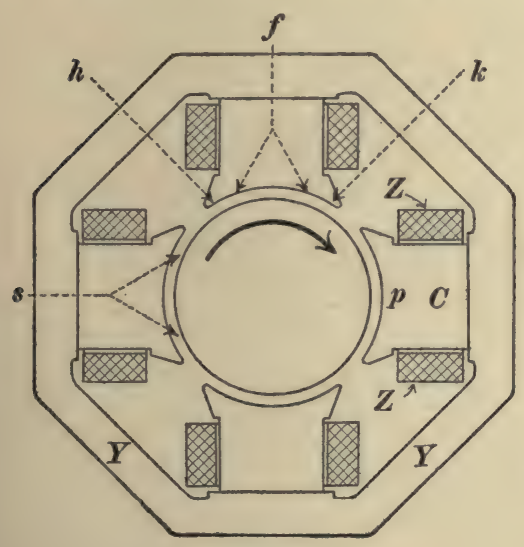

Fig. 33. are called the trailing pole tips with reference to the motion of the armature as indicated by the curved arrow. The coils of wire $Z Z$ are called the field coils, and the portions $C C$ of the field magnet iron around which the field coils are wound, are called the field magnet cores. The portion $Y$ is called the yoke of the field magnet.

Figs. 32, 34, 35, 36 and 37 show typical forms of bipolar field magnet structures, and Figs. 33, 38 and 39 show typical forms of multipolar field magnet structures. The form shown in 
Fig. 39 is used chiefly in large alternators in which the field magnet rotates inside of a large stationary ring-shaped armature.

Fig. 40 is a general view of an early type of bipolar dynamo manufactured by the CrockerWheeler Company. The base plate of the machine serves as the field yoke, and the bearing pedestals and base plate are cast in one piece. Each field magnet core and pole piece is forged from a single piece of wrought iron, ${ }^{*}$ and the two

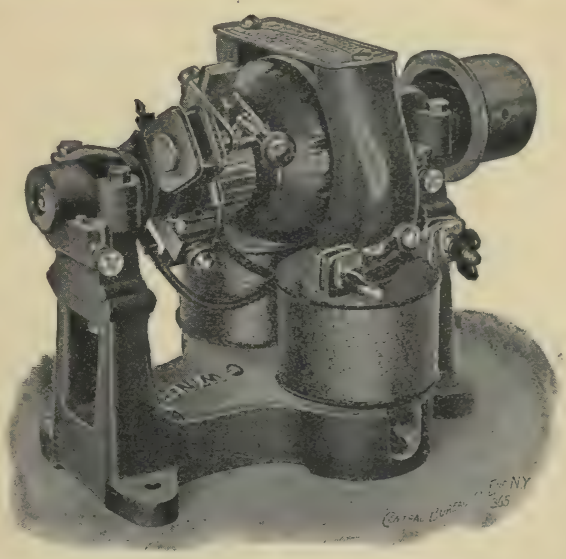

Fig. 40. pole pieces are connected at the top by a non-magnetic brass plate which serves as a protective cover for the armature. The armature is of the ring type, the windings of which are concentrated in a number of coils which lie in deep slots on the outside surface of the armature as shown in Fig. 4I. The armature core is, of course, built up of sheet iron stampings, and in order to facilitate the winding of the armature coils, the two halves (right and left as seen in Fig. 4I) of the armature core are built up separately and put together after the coils are wound upon them. The two halves of the armature core are held together by small bolts $B B$ which pass through the interleaved stampings of the two halves of the core. The armature core is supported by small bolts like $B B$ which pass through the armature teeth and connect to two end plates of brass which are attached to the shaft. The brass plate at the commutator end is provided with apertures through which the commutator leads

* A more usual construction is that in which the field magnet cores are wrought iron or cast steel, and the pole pieces are separate pieces of cast iron or cast steel bolted to the ends of the cores. 
pass from the armature coils to the commutator bars. The brushes are carried by two insulated metal studs fixed to an arm which is supported upon the projecting end of one of the bearings. This arm may be turned about the axis of the armature at will, and clamped so as to hold the brushes in any desired position.

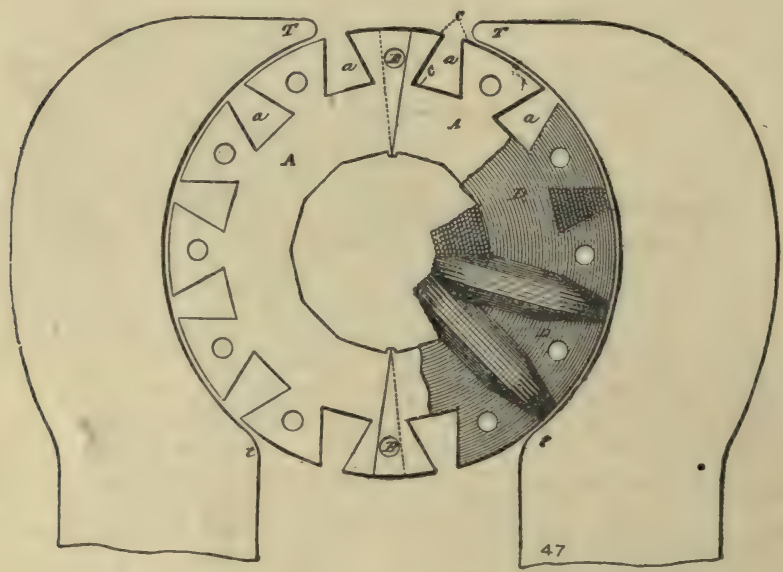

Fig. 41.

The brushes of direct-current dynamos are always carried upon a supporting arm or frame which may be turned about the axis of the armature at will. This adjustable brush support is called a rocker-arm or rocker-frame.

Fig. 42 is a general view of a multipolar dynamo manufactured by the Fort Wayne Electric Works. The field yoke, that is, the external ring of the field magnet, is cast in two parts which are bolted together, and the upper part of the field may be lifted off when it is desired to remove the armature. Fig. 43 is a view of the field structure and base, with the armature and all field windings removed. One of the laminated structures which constitutes a combined field magnet core and pole piece, is shown in Fig. 44. These pole pieces are set into the mould and castwelded to the field ring. An enlarged area of pole face is obtained by the use of an open frame of cast iron, shown in Fig. 


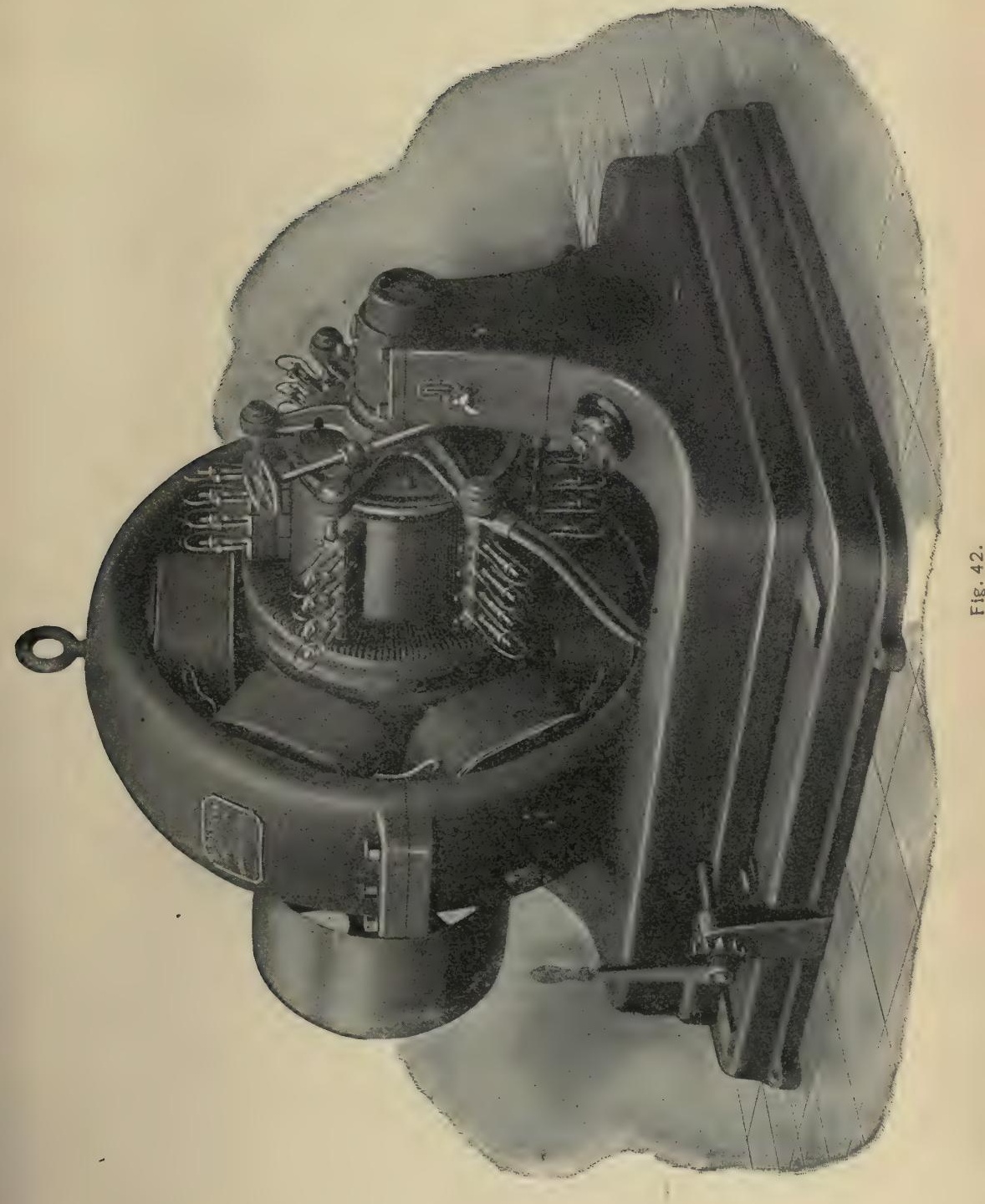


56 ELEMENTS OF ELECTRICAL ENGINEERING.

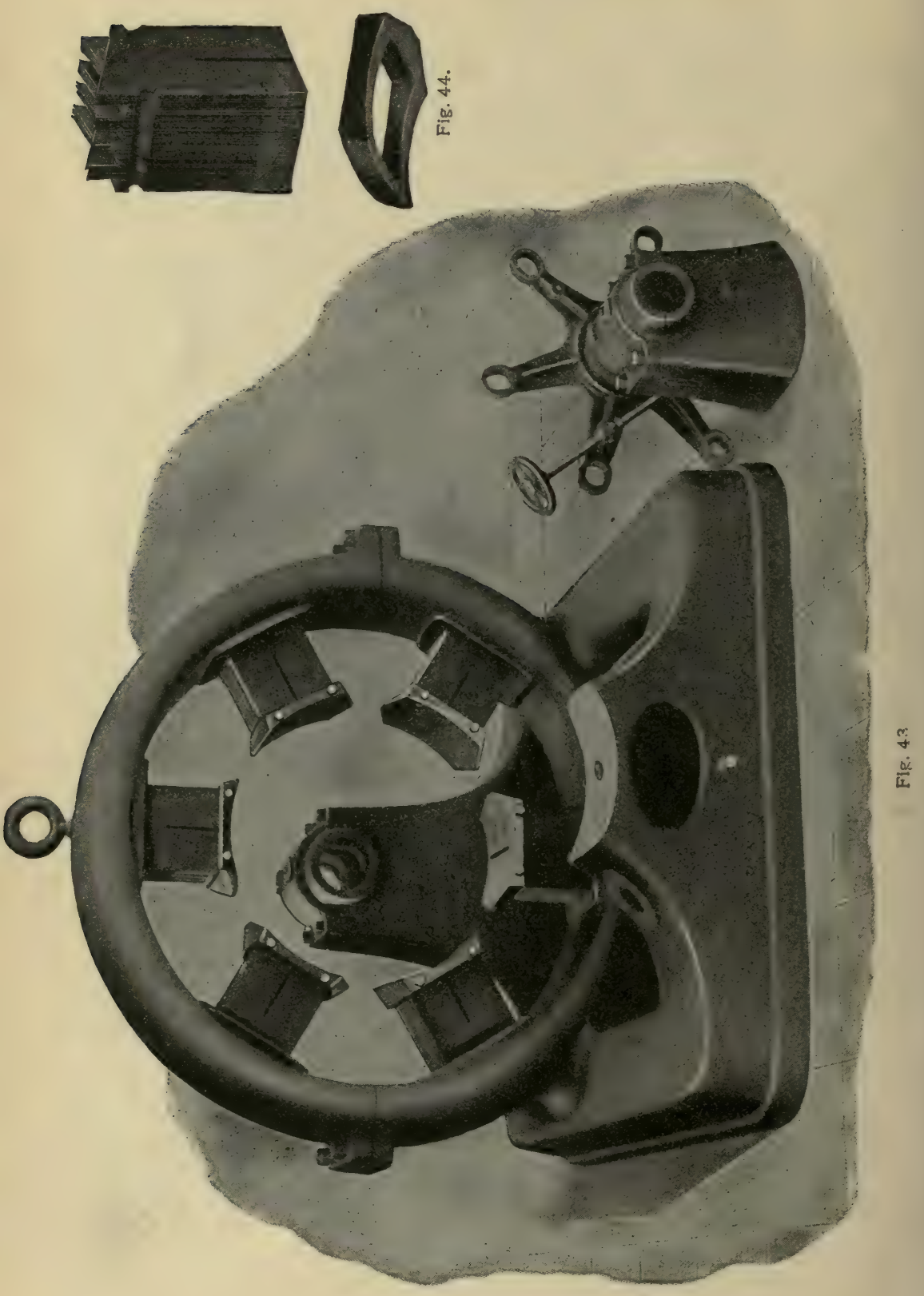


THE DYNAMO.

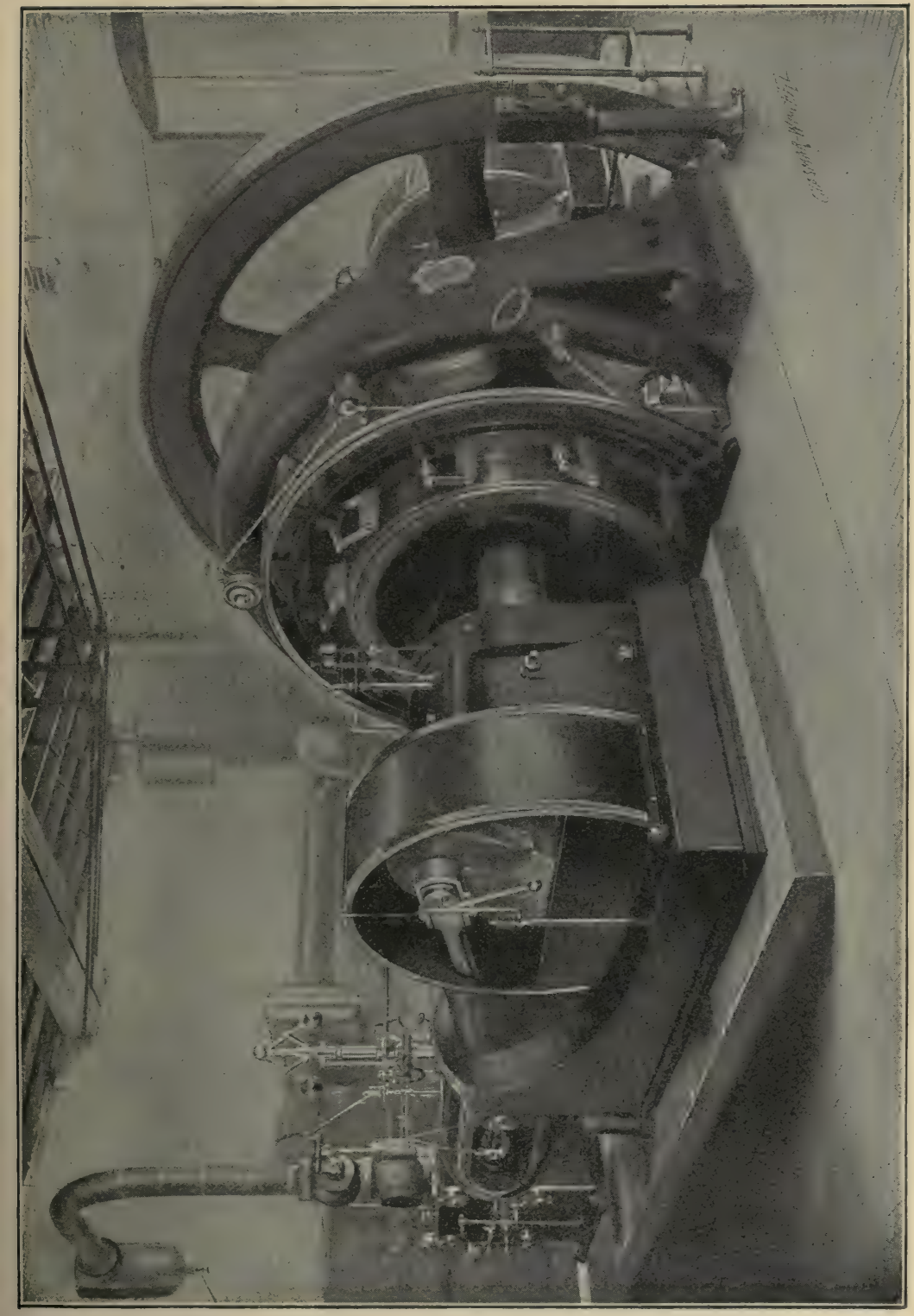

$\dot{10}$
$\dot{0}$
$\dot{a}$ 
44, which is called the pole shoe. This pole shoe is put in place and fixed by bolts, after the field coils have been slipped into position on the projecting field cores.

Fig. 45 is a general view of a ten-pole direct-current generator of the Bullock Electric Manufacturing Company, connected directly to the main shaft of the driving engine. The figure shows the rocker-frame, which supports the ten sets of brushes, and the mechanism for adjusting the brushes by turning the rockerframe by means of the small hand wheel on the right. Fig. 46

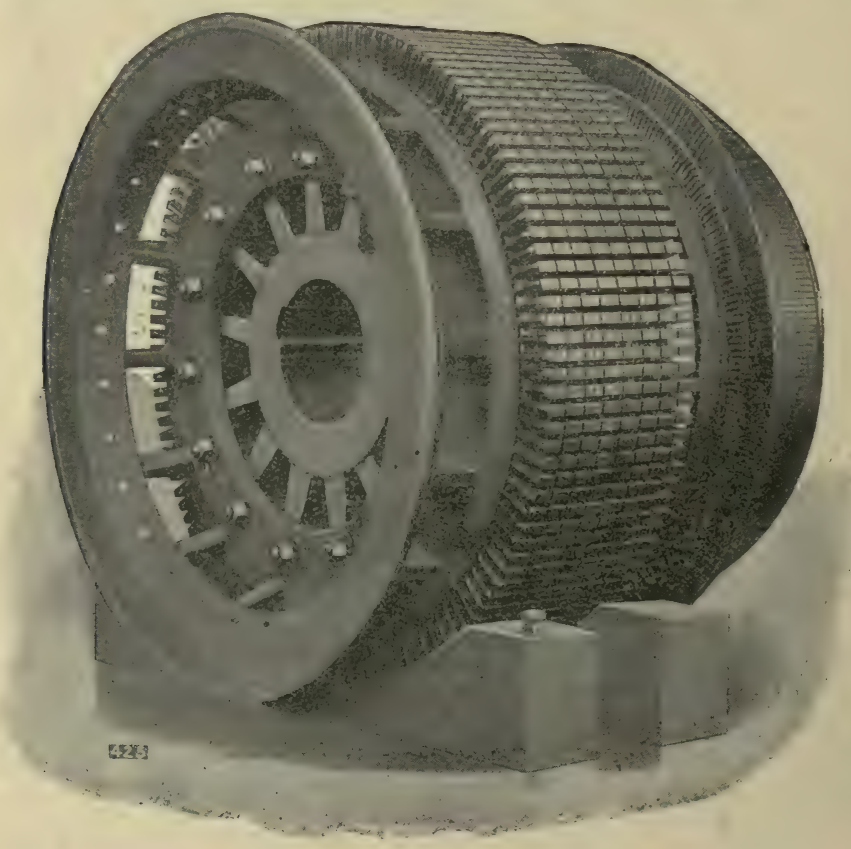

Fig. 46.

shows the armature core and commutator of the generator which is shown in Fig. 45. The manner in which the sheet-iron armature core stampings are supported on what is called a spider, is shown in Fig. 47. The partly wound armature is shown in Fig. 48 , and two of the armature coils are shown in Fig. 49. The space between the armature core and the flange at one end of 
THE DYNAMO.

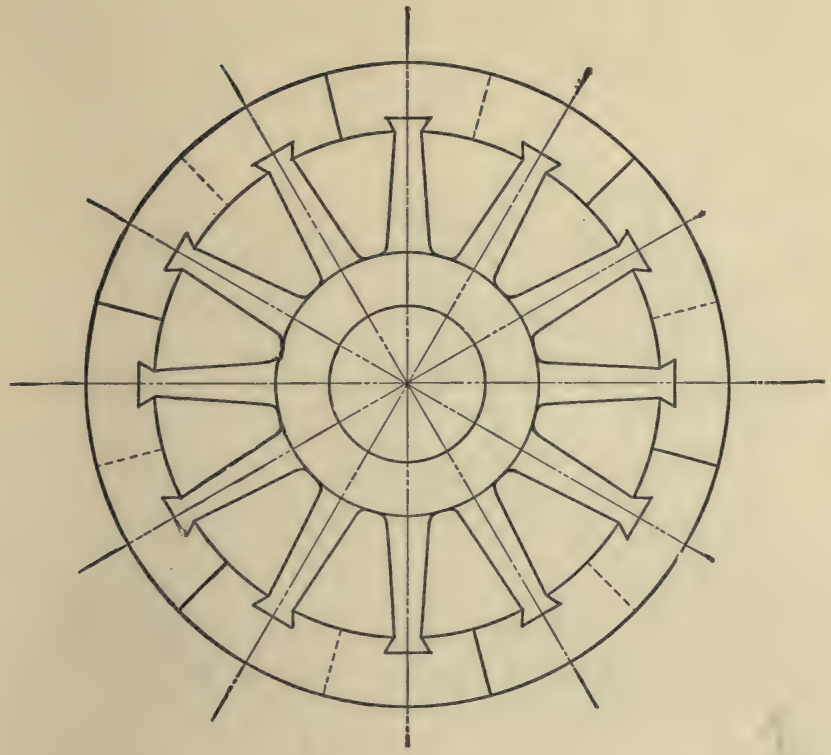

Fig. 47.

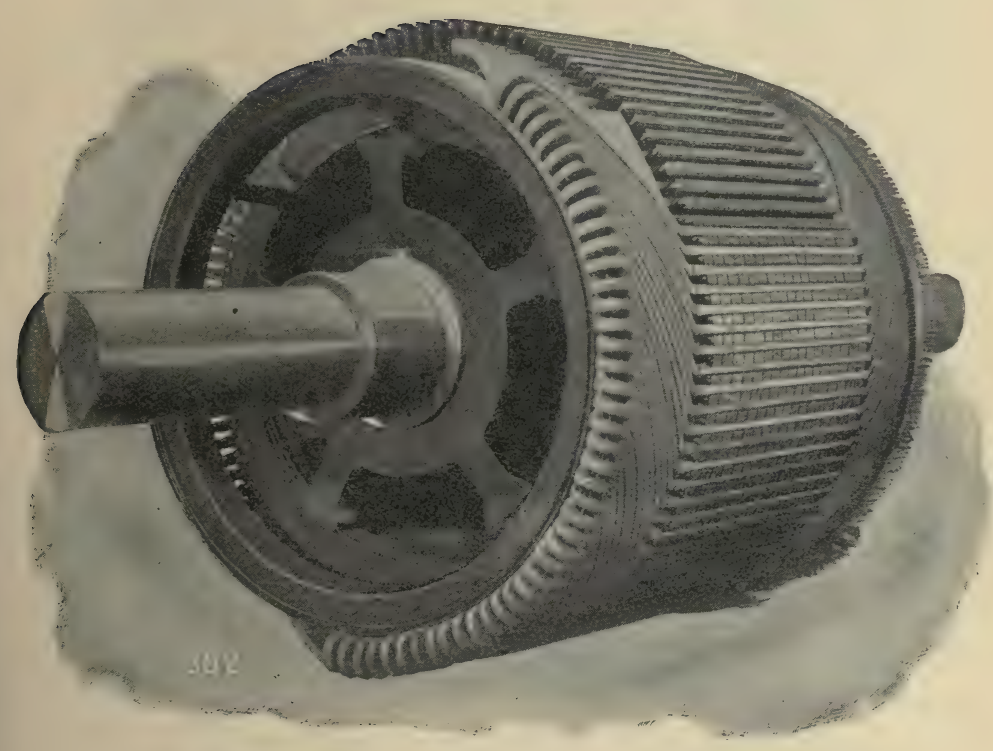

Fig. 48. 
the armature core, and the space between the armature core and the commutator at the other end, see Fig. 46, are for receiving the overlapping ends of the armature coils. Both of these spaces

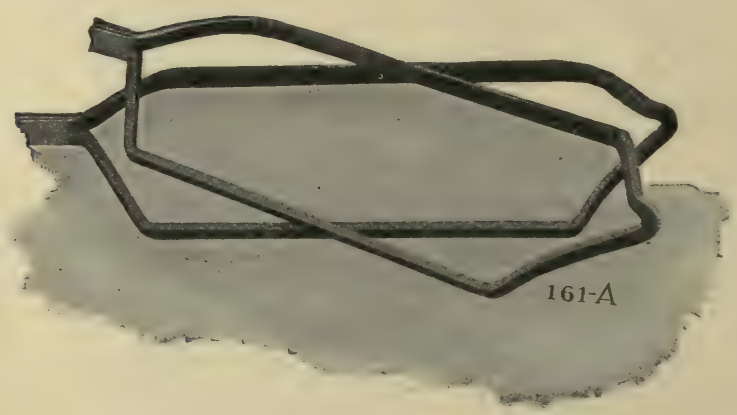

Fig. 49.

are left more or less open, so that the cool air may be drawn in at one end of the rotating armature and driven out through the overlapping ends of the coils, as shown by the arrows in Fig. 50.

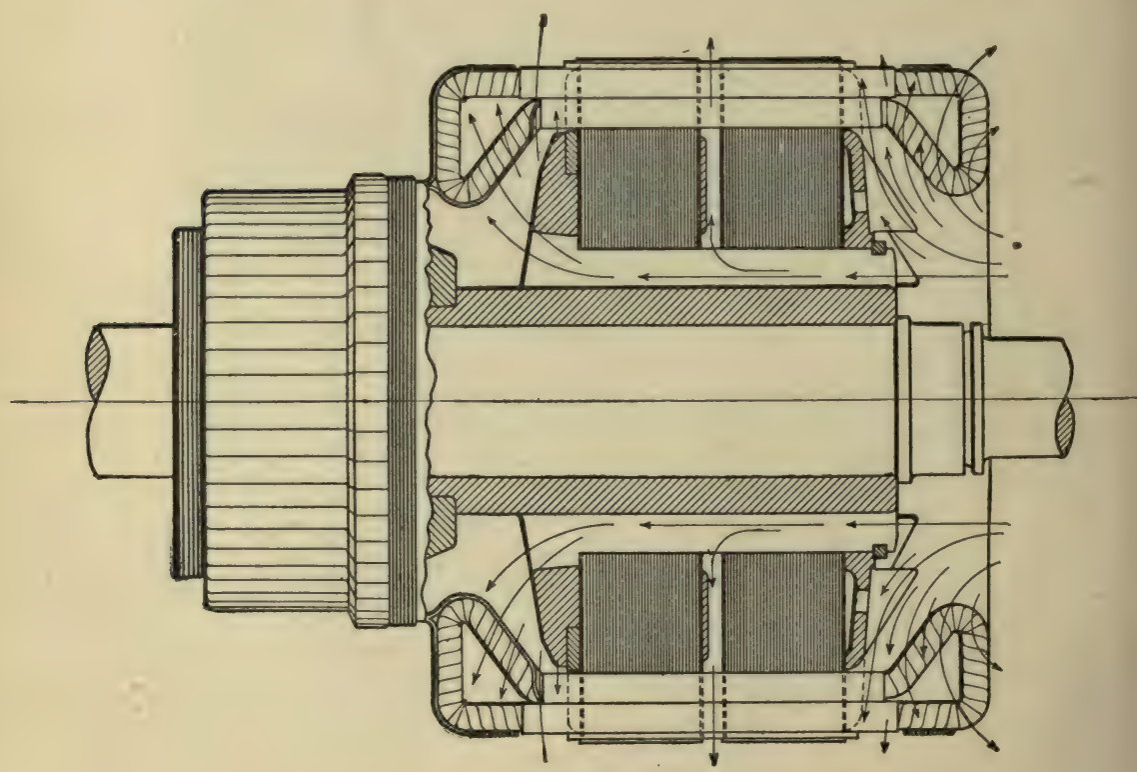

Fig. 50. 
Furthermore, the laminations of the armature core are separated at intervals, thus forming air ducts from the interior to the exterior of the armature core. Fig. 46 shows seven places where the laminations are thus separated, and Fig. 50 shows one place where the laminations are separated. The object of this thorough ventilation of the armature is to avoid the high temperatures

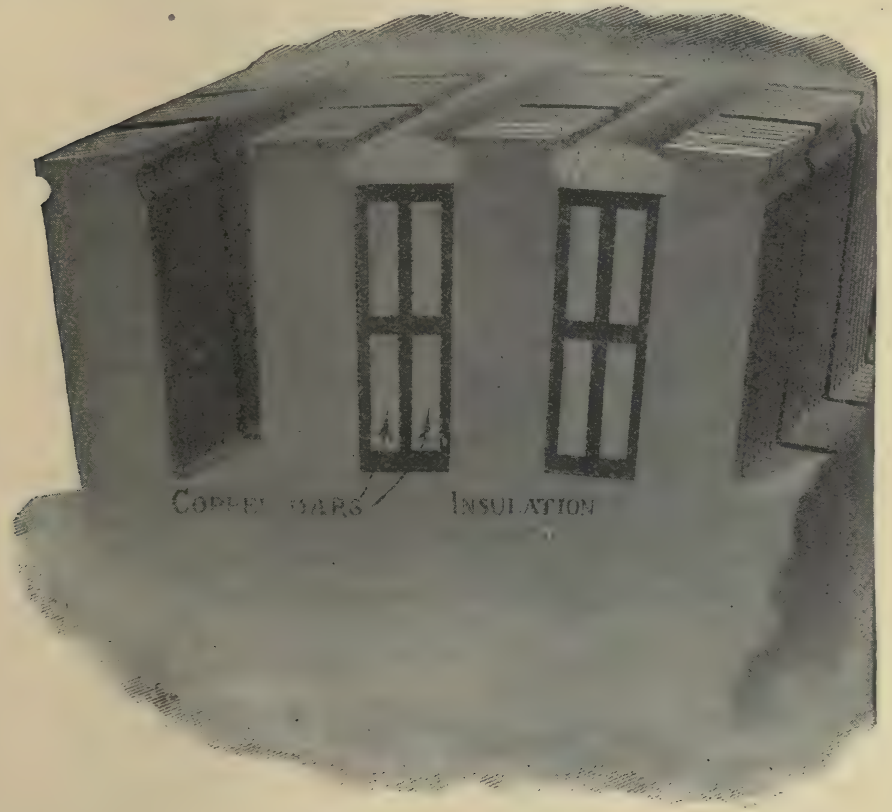

Fig. 51.

which would otherwise be produced by the very considerable heat which is generated in the armature core by eddy currents and magnetic hysteresis, and in the armature windings by the armature current. Fig. $5 \mathrm{I}$ is a view of a portion of the armature core showing two slots filled with conductors which are held in place by wooden wedges, and also showing several of the ventilating ducts between the sheet-iron laminations. The armature conductors shown in Fig. 5 I consist of massive rectangular bars of copper. Wire-wound armatures are, in general, used for 
machines giving small current outputs, and bar-wound armatures for machines giving large current outputs.

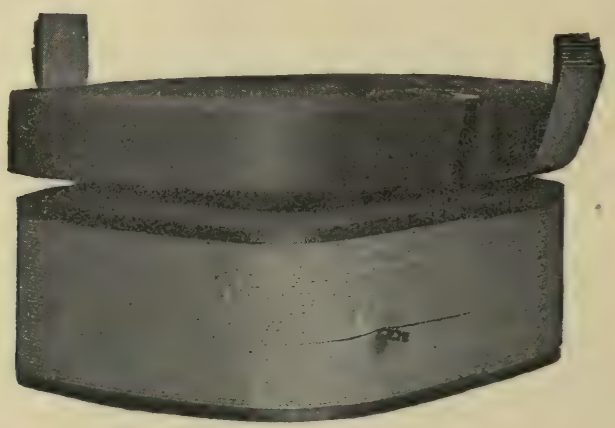

Fig. 52.

Fig. 52 shows one pair of field coils (shunt coil and series coil) of the generator which is shown in Fig. 45. The manner

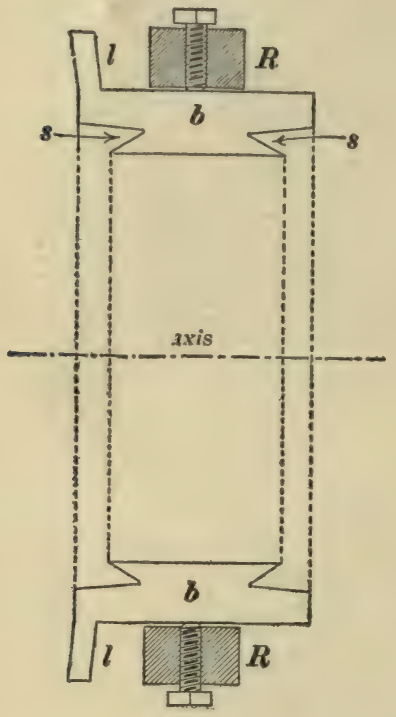

Fig. 53.

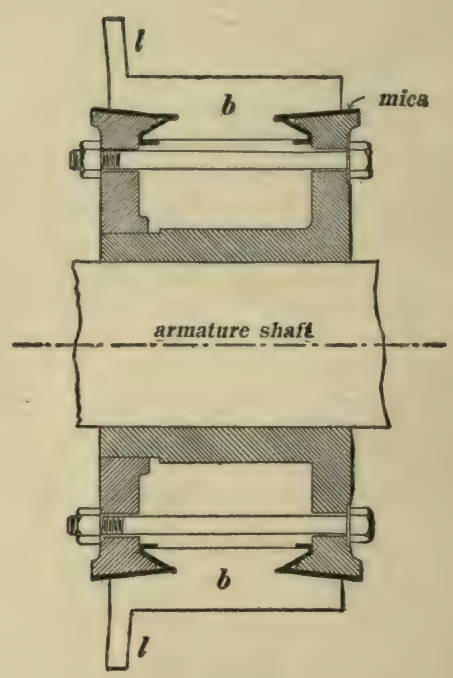

Fig. 54.

in which the various shunt coils are connected together, and in which the various series coils are connected together, is shown in 
Fig. 28. In dynamos of earlier design, the shunt and series windings were wound one over the other on a single spool. Present practice favors the separation of these windings for the sake of more perfect ventilation, and on account of greater accessibility for repairs.

34. Details of Commutator. The commutator, the function of which is explained in Art. 28, is always made in the form of a rigid self-contained structure. Forged copper bars, bb, Fig. 53, slightly thinner at one edge than at the other, are built up in the form of a hollow cylinder with accurately gauged mica plates between the bars. This built up structure is clamped in a massive ring $R R$, placed in a lathe, and the conical seats ss are turned out. A steel hub is provided with conical lips which fit into the grooves ss, Fig. 53, and the ends of the hub are drawn together by bolts, as shown in Fig. 54. A thin conical washer of moulded mica separates the steel hub

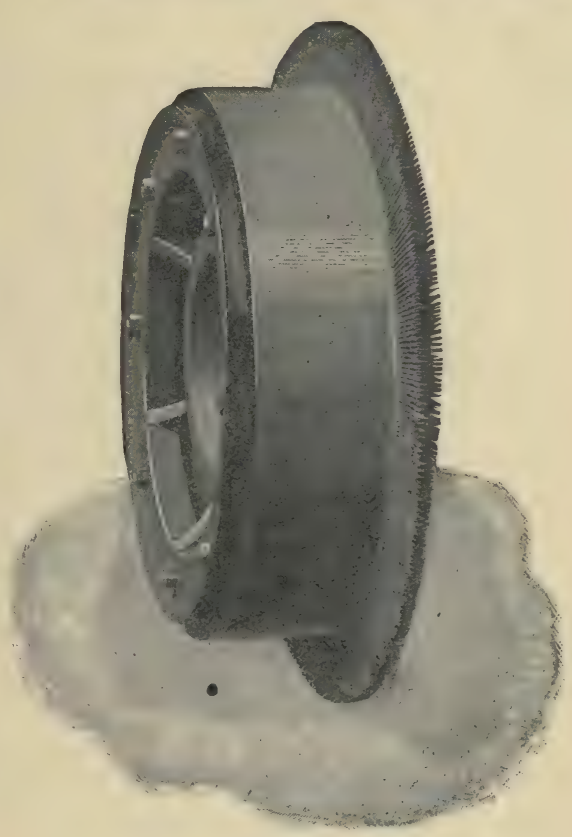

Fig. 55. from the copper bars, as shown in Fig. 54. The radial lugs $l$, one on each commutator bar, or segment, serve for the attachment of the wire leads which are tapped into the armature windings. Fig. 55 is a general view of a completed commutator manufactured by the General Electric Company.

35. Brushes and Brush Holders. Metal brushes were formerly almost exclusively used for dynamos. Such brushes were made 
of compact bundles of thin metal leaves or wires, so as to insure many points of contact with the commutator. Metal brushes are however objectionable for two reasons: $(a)$ It is very difficult to keep the surface of the commutator smooth when metal brushes are used, inasmuch as the soft copper bars and the soft metal brushes do not wear well mechanically. There is always a tendency for soft metals to become rough when rubbed together, especially if any extraneous cause, such as the slight sparking at the brushes of a dynamo, starts the roughening process.

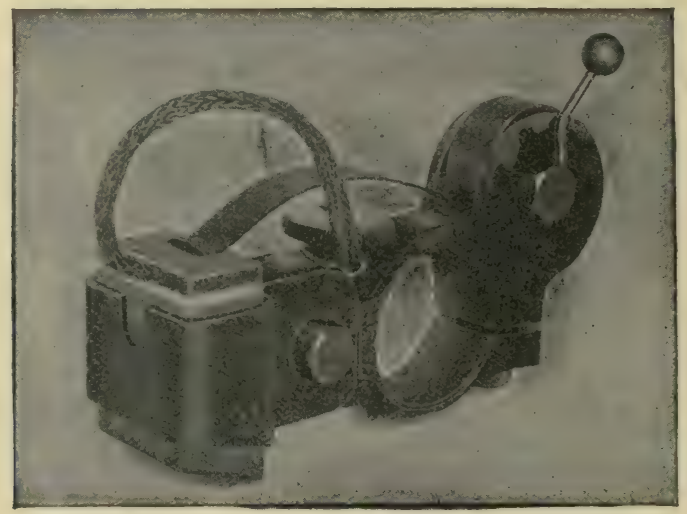

Fig. 56.

(b) The tendency of a dynamo to spark at the brushes is greatly lessened by using brushes made of comparatively high resistance material, such as carbon, instead of low resistance material like metal. Dynamo brushes are now almost universally made of hard blocks of graphitic carbon. Such brushes wear well mechanically without other lubrication than that supplied by the graphite of the brush.

The function of the brush holder is to press the solid blocks of carbon which are used as brushes firmly against the commutator, and at the same time to give sufficient freedom to the carbon blocks to enable them to seat themselves snugly against the commutator so as to ensure a large area of contact. Fig. 56 
shows a carbon brush holder manufactured by the General Electric Company. The current is led from the carbon block to the metal part of the holder by the flexible cable of fine copper wire which is called a pig tail, thus relieving the spring from doing duty as an electrical conductor. The use of the spring as a conductor would heat it and cause it to lose its temper. The small lever at the back serves to adjust the pressure with which the spring presses the carbon block against the commutator.

36. Bipolar and multipolar dynamos compared. - In the early days of electrical engineering comparatively small sized dynamos only were used, and the bipolar field structure was almost universal. The increase in size of the machines involved in recent electrical engineering developments has led to the adoption of the multipolar field structure. The advantages of the multipolar over the bipolar field structure for large-sized machines are as follows :

(a) Economy of iron. - The magnetic flux is available for the production of electromotive force only where it passes across the air gap between the pole faces and the armature core. Therefore

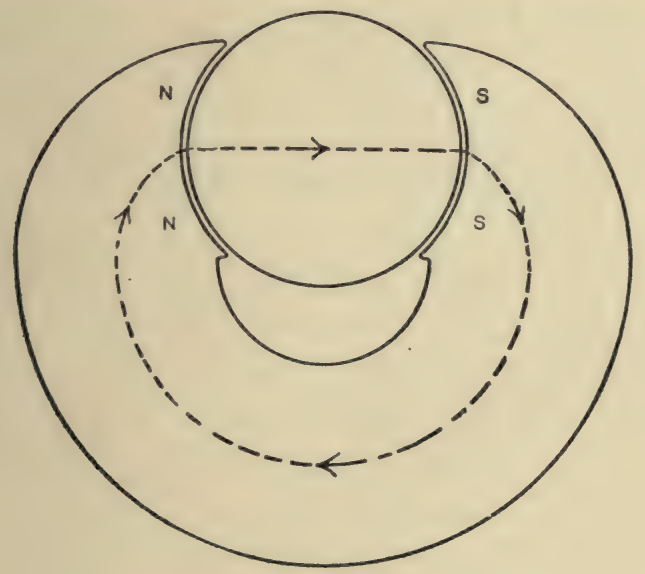

Fig. 57,

that distribution of flux around the armature is most desirable which gives the shortest possible paths in the armature iron from 
the places where the flux enters to the places where the flux leaves the armature, and the shortest possible paths in the field iron from the places where the flux leaves the armature to the places where the flux enters the armature. Shortness of paths in armature and field iron means a great saving in the amount of iron required in the machine for a given amount of useful flux.

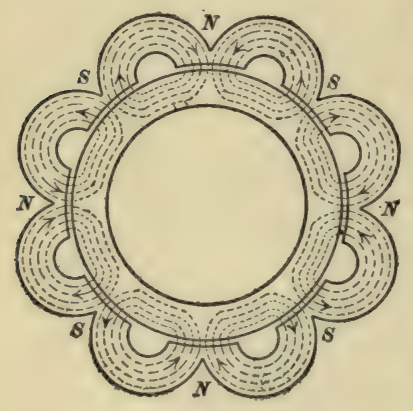

Fig. 58.

The difference between the bipolar field and the multipolar field in regard to length of path of magnetic flux, is shown in Figs. 57 and 58 . These figures show a given sized armature with seven-tenths of its surface covered by pole faces and therefore with seventenths of its windings effective at each instant in the production of electromotive force. The length of each magnetic circuit in Fig. 58 is very much less than the length of the magnetic circuit shown in Fig. 57. The figures show in a striking way that a much greater amount of iron is required for the bipolar than for the multipolar structure.

(b) Economy of field copper. - Shortness of path in iron with given flux density means a saving in the amount of field copper for a given amount of power expended in field excitation $\left(R I^{2}\right.$ loss), or a saving in the amount of power expended in field excitation for a given amount of field copper. The dependence of field excitation (ampere turns) upon the length and sectional area of the magnetic circuit is discussed in Appendix A.

(c) Reduction of sparking. - The sparking of a dynamo at the brushes depends largely upon the magnetizing action of the armature current. This magnetizing action of the armature current is called armature reaction, and it is proportional to the product of the current in the armature conductors and the number of armature conductors under a pole face. An increase in the number of poles, therefore, reduces the armature reaction, and thereby reduces the tendency to spark at the brushes. 
The following disadvantages of the multipolar construction outweigh the above advantages when the number of poles is increased beyond the proper limit.

(a) Magnetic leakage. - A considerable portion of the magnetic flux, which is forced through the field magnet cores by the field winding, crosses from pole tip to pole tip without passing through the armature. This flux, called the leakage flux, does not contribute to the generation of electromotive force in the armature conductors. When a great number of field magnet poles surround an armature of given diameter, the tips of adjacent poles which are of opposite polarity are very close together and the magnetic leakage is excessive. The effect of this excessive magnetic leakage is to reduce the useful magnetic flux, and hence to reduce the electromotive force generated in the armature, unless the field excitation is very greatly increased at the expense of an increase of field copper, or an increase of power expended in field excitation. The percentage of armature surface actually covered by the pole faces is usually made smaller as the number of poles is increased, in order to avoid excessively short distances between adjacent pole tips.

(b) Multiplicity of parts. - A multipolar machine usually has as many brush sets as poles, and as many field coils as poles. Therefore the cost of manufacture increases with the number of poles because of the increase in the number of parts required.

\section{Smooth core and slotted armatures compared.-Dynamo} armatures were formerly made with smooth cores built up of circular disks of sheet iron, the armature windings being placed upon the armature surface and bound with bands of phosphorbronze or steel wire. With this type of armature there is necessarily a thick layer of non-magnetic material, air and copper, between the pole face and the armature core. The armature wires are exposed to mechanical injury, and the sidewise drag on the wires due to the side push of the magnetic field between the pole face and armature core not infrequently causes the armature 
conductors to become loose, especially when an excessive current flows through the armature on account of an accidental short circuit. Dynamo armatures are now constructed with deep slots, as shown in Figs. 4I, 46, 48, and $5 \mathrm{I}$. In this type of armature the conductors are well protected mechanically, and the side force, which in a smooth core armature acts upon the conductors, acts chiefly on the armature teeth of a slotted armature. Furthermore, the air gap, or distance from the iron of the pole face to the iron of the armature core, may be made very much smaller in the slotted than in the smooth core armature. In Chapter VI it is shown that, on account of sparking, the air gap should sometimes be greater than mechanical clearance alone would require, so that the possibility of reducing the air gap is not always of prime importance.

The manner of cutting the lines of force by the conductors which lie in the deep slots of an armature is as follows: Consider the tuft of lines of force which emanate from an armature tooth. This tuft moves across the pole face as the armature rotates. This motion of the tuft of lines of force is, in a certain sense, only apparent, since it is due to the addition of new lines of force to the forward edge of the tuft by the very rapid flitting of the lines of force across the slot which is ahead of the tooth in question, and the taking away of lines of force from the backward edge of the tuft by the very rapid flitting of the lines of force across the slot which is behind the tooth in question. The velocity at which the lines of force flit across the slots and cut the armature conductors is much greater * than the mean velocity of the armature conductors. The fundamental equation of the dynamo, see Article 30, applies to smooth core and slotted core armatures alike.

* In the ratio of the flux density in the slot to the mean flux density in the gap space. The above apparent motion of a tuft of lines of force is similar to the apparent motion of a tuft of the bristles of an ordinary brush when the finger is rubbed over it, and the rapid flitting of the lines of force across a slot is similar to the rapid flitting of the bristles across the nearly vacant space behind the finger. 
38. Special designs of dynamos. - The essential features of a dynamo are the same whatever the use for which the dynamo is intended; whether it is to be used as a generator or as a motor ; whether it is to be driven as a generator by a belt or by being directly connected to an engine or to a water-wheel ; or whether it is operated as a motor and drives a machine by a belt or by being constructed as an integral part of the machine which it drives.

There are, however, important special points in the design of a generator which is to be directly connected to an engine or to a water-wheel, and in the design of a motor which is to be directly connected to the machine which it drives. These special points

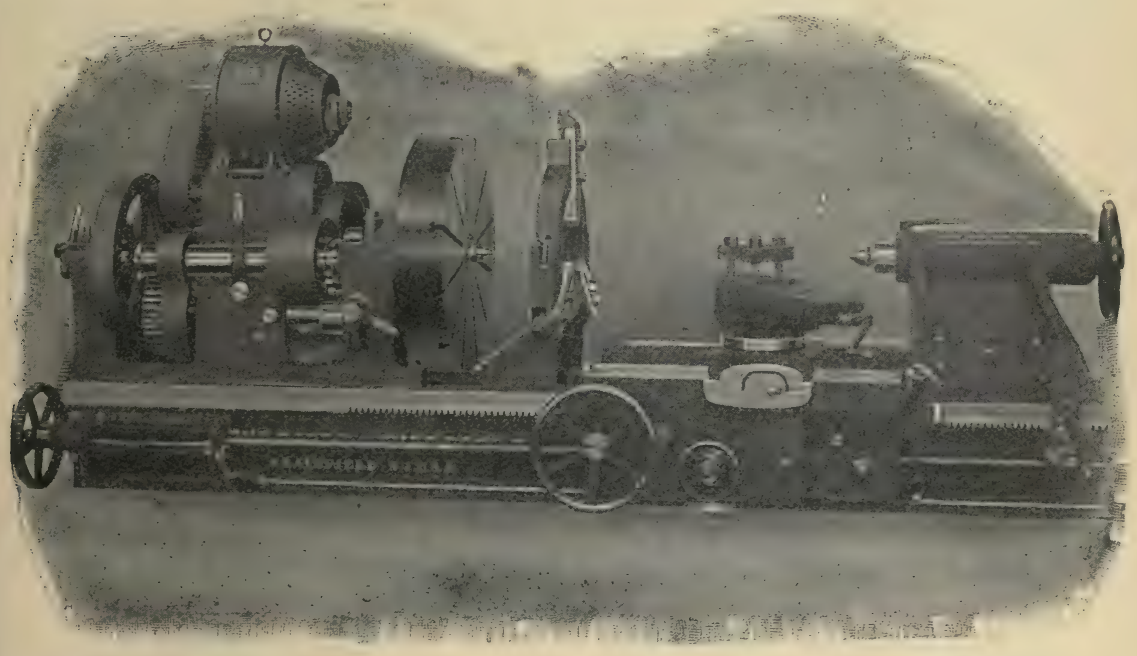

Fiz. 59. Westinghouse Va-iable Speed Motor Driving a 48-Iuch Lathe.

in design relate chiefly to the arrangements of the shaft, bearings and pedestals, and to the shape of the frame which serves as a base for the machine.

Thus Fig. 4I shows a typical belted generator, Fig. 45 shows a typical generator mounted on the crank shaft of the driving engine, and Fig. 59 shows a lathe with a specially designed motor geared directly to it. 
Electric motors for use in very dusty places are usually built with an enclosing dust-proof case. Such an enclosed motor is shown in Fig. 6o. This type of motor is usually called a mill

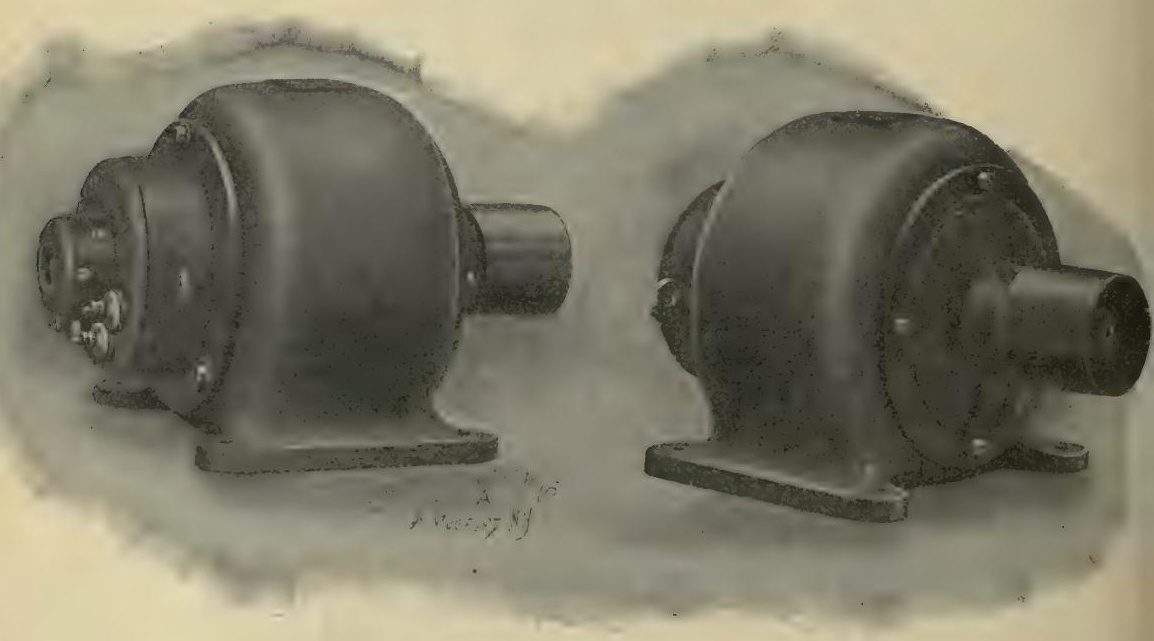

Fig. 60 .

type motor. Street car motors are also enclosed. A typical street car motor is shown in Fig. $9 \mathrm{I}$.

A given motor carrying a given load runs much cooler when it is entirely open to the air than when it is enclosed, or, for a given running temperature it will carry a heavier load if it is open than if it is enclosed.

39. The dynamotor. - It is often necessary to take current from lighting mains at, say, I I volts for use in charging a few cells of storage battery. To do this a large resistance may be connected in series with the storage battery. The objection to this is that a great deal of power is lost in the resistance. A method, which is usually more economical, is to operate a standard I IOvolt motor with current taken from the I I o-volt mains and use this motor to drive a generator which is designed to give the low voltage required for charging the storage battery. This combination of motor and generator is called a motor-generator set. 
The motor and generator of a motor-generator set may be built as one machine, that is, a machine having one field magnet and one armature structure but with two distinct windings, one of many turns of fine wire connected to a commutator at one end, and another of fewer turns of coarse wire connected to a second commutator at the other end. This machine can then be run as a motor by a small current delivered to its fine wire windings from the supply mains, and at the same time it will operate as a generator and deliver a large current at a reduced voltage from its coarse wire windings. Such a machine is called a dynamotor. Fig. 6 I shows a dynamotor made by the Crocker-Wheeler Com-

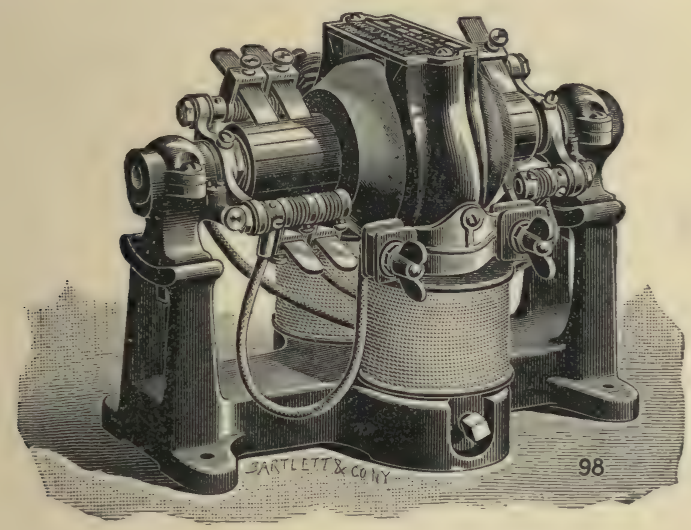

Fig. 61.

pany and designed to take current from I Io-volt mains and to deliver current from its coarse wire armature winding at 25 volts.

40. The homopolar dynamo. - A simple type of direct-current dynamo, hitherto but little used on account of its very low electromotive force, is the so-called homopolar dynamo (sometimes called the unipolar dynamo, and sometimes the acyclic dynamo). Fig. 62 shows a longitudinal section and an end view of a simple type of homopolar dynamo.

The armature is a solid cylindrical piece of soft iron or steel $A A$ which rotates inside of a hollow cylindrical field yoke $Y Y$. 
The field flux, the trend of which is shown by the closed dotted lines $\Phi \Phi$, is produced by a field winding $Z Z$ which is fixed to the inside of the hollow cylindrical yoke, and surrounds the rotating
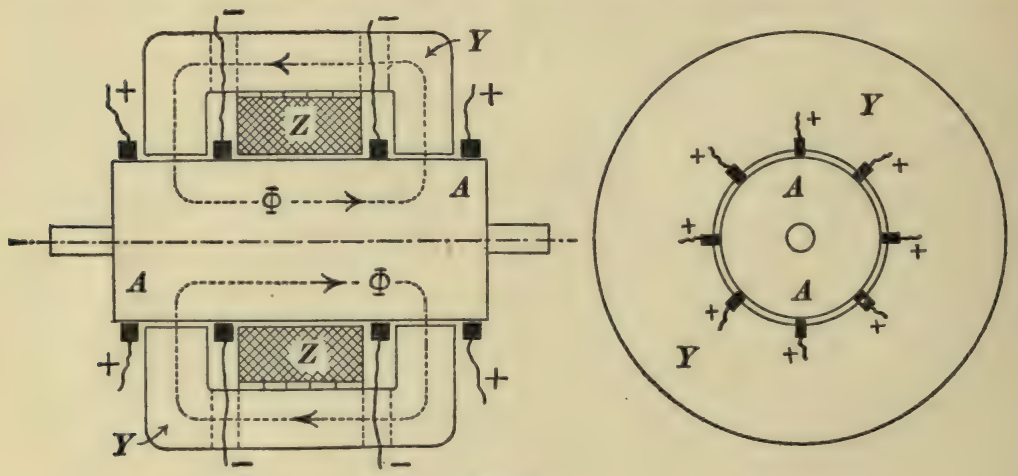

Fig. 62.

cylindrical armature $A$. There are two sets of positive brushes which are arranged to rub upon the extreme ends of $A A$, and two sets of negative brushes which are arranged to rub on $A A$ at any convenient place inside of $Y Y$. The leads which connect

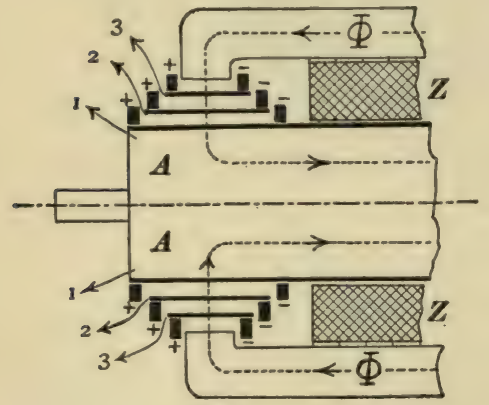

Fig. 63. to the brushes inside of $Y Y$ pass through a series of holes through $Y Y$, which are shown by the short dotted lines.*

The low electromotive force of the unipolar dynamo is due to the fact that the armature consists necessarily of a single element in which electromotive force is induced. To obtain a high electromotive force therefore, a number of distinct machines must be connected in series. $\dagger$ These distinct machines may have, however, a common mag-

* A modified form of unipolar dynamo is described by H. E. Heath in the Electrical World and Engineer, February 10, 1900, Vol. 35, p. 210.

† See paper, "Acyclic Homopolar Dynamos," by T. E. Noeggerath, Trans. A. I. E. E., January $27, \mathbf{1} 905$. 
netic circuit as shown in Fig. 63. This figure shows one end of a machine with three distinct armatures, each having its own positive and negative brushes. One armature is the solid cylinder $A$, the second armature is a cylindrical metal shell 2 , 2 insulated from $A$, and the third armature is an insulated cylindrical metal shell 3, 3. To connect these three armatures in series so that their electromotive forces may be added, the positive brushes of No. I are connected to the negative brushes of No. 2, the positive brushes of No. 2 to the negative brushes of No. 3 and so on, exactly as in the series connections of voltaic cells.

Fig. 62 shows that each set of brushes consists of a number of individual blocks of metal or carbon arranged symmetrically around the periphery of the rotating armature. 


\section{CHAPTER III.}

THE OPERATION OF THE DYNAMO AS A GENERATOR.

41. Constant voltage and constant current supply. - When a number of lamps, or motors, or both, are connected in parallel with each other across the supply mains, it is necessary to maintain an unvarying electromotive force between the mains in order that the various lamps and motors may operate independently of each other. This method of supply is called constant voltage distribution. In this system a given lamp or motor is put out of service by disconnecting it from the mains or by simply opening its circuit. When a lamp or motor is put out of service in this way, each remaining lamp or motor takes the same current as before, and the generator delivers a total current which is less than before by the amount of current which was taken by the disconnected lamp or motor.

When a number of lamps connected in series are supplied with current, it is necessary to maintain a constant current in this circuit in order that the various lamps may operate independently of each other. This method of supply is called constant current distribution. In this system a given lamp is put out of service, not by breaking its circuit, but by short-circuiting it, that is by closing a switch which establishes a by-pass for the current. The closing of this switch generally reduces the total resistance of the circuit of lamps as a whole, and the maintenance of a constant current in the circuit requires a decrease of the electromotive force of the generator when lamps are put out of service, and an increase of the electromotive force of the generator when additional lamps are put into service.

The constant voltage system of supply is almost universally employed nowadays, both for direct-current distribution and for 
alternating current distribution; for long distance power transmission, for electric railways, and in general for the supply of current to motors and to lamps.

The only case in which it is distinctly advantageous to use the constant current system is for street lighting in cities where a fixed number of widely distributed lamps, arc or incandescent, are to be operated.

The shunt generator driven at constant speed, and especially the compound generator, maintains automatically an approximately constant voltage even though the current output varies.

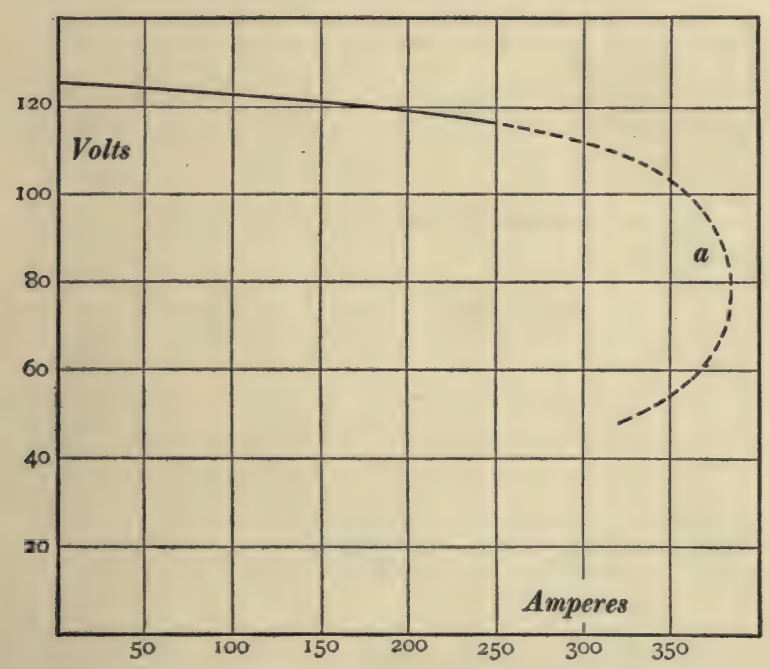

Fig. 64.

This is shown by the so-called characteristic curves in Figs. 64 and 65. The ordinates of the curve in Fig. 64 represent the voltages of a shunt generator, and the abscissas represent the corresponding current outputs. The voltage of a shunt generator decreases slightly as the current output increases, as is evident from Fig. 64. The ordinates of the curve in Fig. 65 represent the voltages of a compound generator (flat-compound), and the abscissas represent the corresponding current outputs. The voltage of a flat- 
compound generator is very nearly independent of the current output as is evident from Fig. 65 .

In practice the voltage of a shunt or compound generator is under the control of an attendant who, by manipulating a shunt field rheostat, may keep the voltage as nearly constant as desired.

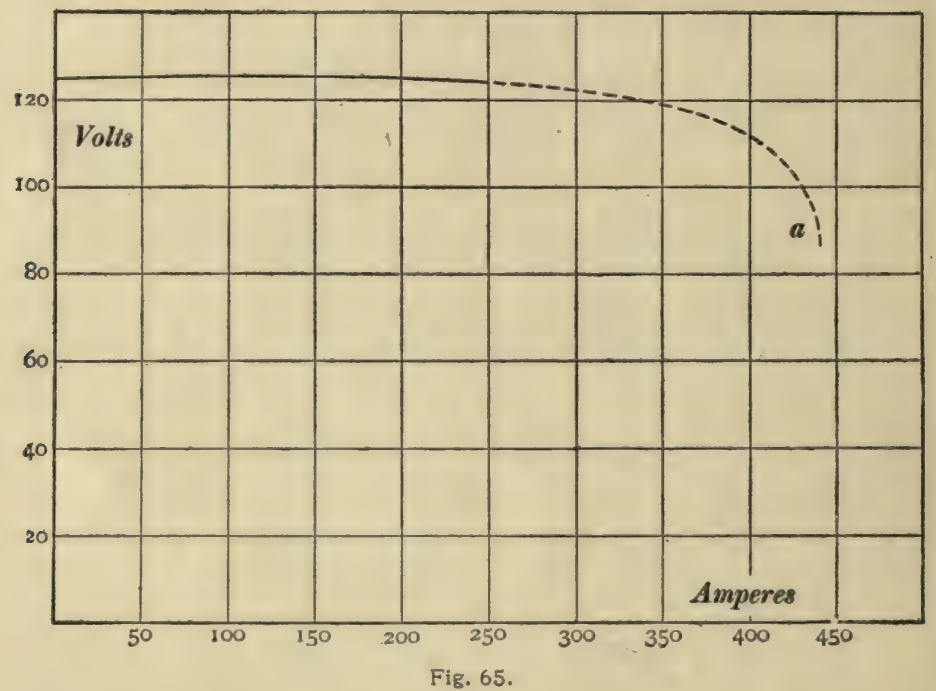

The ordinates of the curve in Fig. 66 represent the voltages of a series generator, and the abscissas represent the corresponding current outputs. The voltage of a series generator increases rapidly with increase of current output as is evident from Fig. 66.

The dotted portions of the curves in Figs. 64,65 and 66 show the trend of the characteristic curves for current outputs greater than the normal full load values for which the machines are designed.

The adaptability of the various types of generator to the requirements of the constant current system, or rather the inherent lack of complete adaptability may be made evident by replotting the data on which the characteristic curves of Figs. 64 and 66 are based, so as to show the current outputs of the different generators as ordinates, and the resistances of the receiving circuit as 
OPERATION OF THE DYNAMO AS A GENERATOR. 77 abscissas. Fig. 67 shows these current-resistance curves of a shunt generator and of a series generator. The dotted portions of the

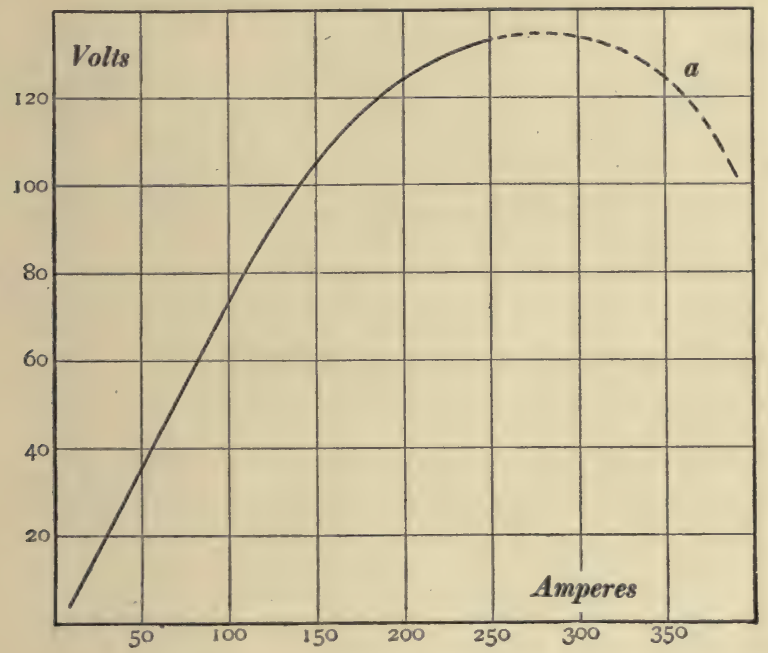

Fig. 66.

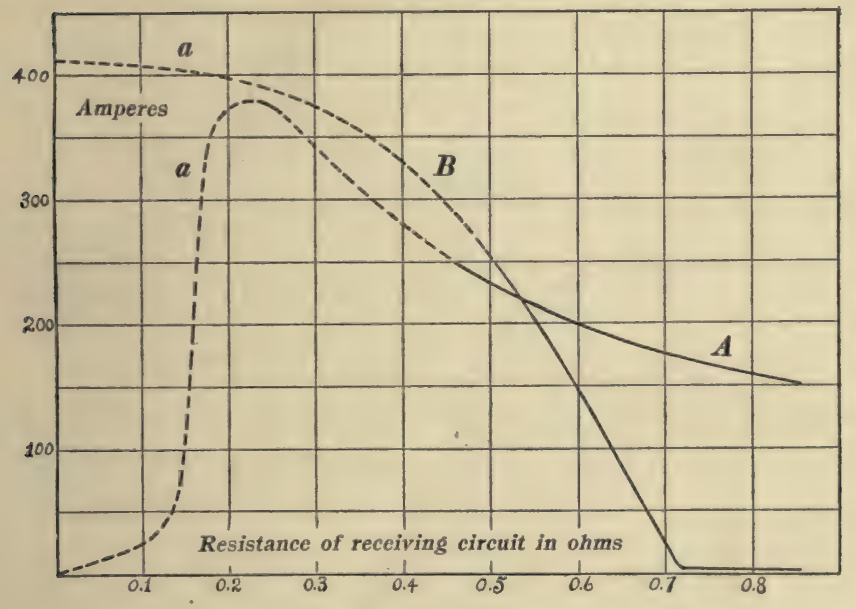

Fig. 67.

curves in Fig. 67 correspond to the dotted portions in Figs. 64 and 66. An ideal constant current generator would have for its 
current-resistance curve a horizontal straight line. The dotted part of the shunt generator curve $A$ in Fig. 67 is very far from being a horizontal straight line, while a large portion of the undotted part of the shunt generator curve is not greatly inclined. On the other hand the dotted part of the series generator curve $B$ is not greatly inclined, while the undotted portion is greatly inclined. Therefore, the nearest approximation to an automatic constant current generator would be a shunt generator designed so as to operate normally on the undotted portion of its characteristic curve as shown in Figs. 64 and 67 , or a series generator designed so as to operate normally on the dotted part of its characteristic curve as shown in Figs. 66 and 67.

As a matter of fact, however, the series generator is always used for constant direct-current supply for the following reason : The voltages used for series constant current arc lighting are high, ranging from 1,000 to 6,000 volts per lamp-circuit. A high voltage shunt generator is expensive to construct because of the great quantity of very fine and very highly insulated wire required for its field winding, whereas the high voltage series generator has a comparatively coarse wire field winding which need not be so thoroughly insulated as the shunt winding.

Generators which are used for constant current distribution are always provided with automatic devices for keeping the current

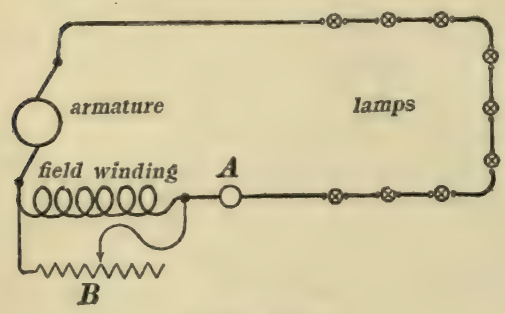

Fig. 68. output constant, in spite of changes of resistance of the receiving circuit.*

\section{Connections of a series} generator and its receiving circuit. Fig. 68 is a diagram of connections of a series generator, some of its accessory apparatus, and a circuit of arc lamps. An ammeter $A$ is

* See chapter on Arc-Lighting Dynamos in S. P. Thompson's “Dynamo-Electric Machinery." 
usually placed in the circuit so that the station attendant can see when the current has the proper value. In Fig. 68 an adjustable resistance $B$ is shown connected in parallel with the field winding; by varying this resistance, that portion of the fixed current output of the generator which flows through the field winding may be changed at will, and thus the electromotive force of the generator may be controlled so as to cause it to deliver a constant current in spite of variations in the resistance of the receiving circuit. This hand controlling device is not used in commercial arc lighting plants, and it is only mentioned here to give a clear idea of a possible method for controlling a series generator.

43. Connections of a shunt or compound generator and its receiving circuit. Fig. 69 is a diagram of connections of a compound generator (short-shunt) with its field rheostat $R$, main fuses at $b$

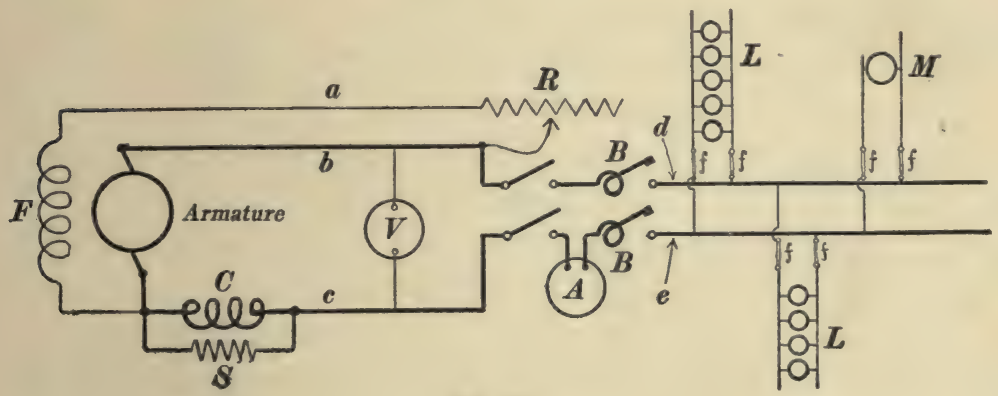

Fig. 69.

and $c$, main switch, ammeter $A$, voltmeter $V$, and circuit breaker $B B$. These accessory appliances are always mounted in convenient positions on a panel of insulating material, slate or marble, called a switch-board. The wires $a, b$, and $c$ lead from the generator to the switch-board, and the wires $d$ and $e$ lead from the switch-board to the lamps. The wires leading to the individual groups of lamps $L L$, and to the motor $M$, in Fig. 69 are, of course, smaller than the mains leading out from the switch-board. It is wise to insert fuses $f f$, as shown in Fig. 69, at every point 
where a smaller wire attaches to a larger one, otherwise a short circuit might occur in one of the small groups of lamps, taking enough current to make the small copper wires red hot, but not enough to open the circuit breaker $B B$, or to melt the main fuses in the wires $b$ and $c$, thus giving rise to a serious fire risk.

The connections of a shunt generator are the same as in Fig. 69 except that there is no series field winding.

44 The building up of a generator at starting. - When a selfexcited generator is started, the slight residual magnetism in the field structure induces a small electromotive force in the armature windings. This electromotive force produces a small current in the field winding which strengthens the residual magnetism. This strengthened residual magnetism induces an increased electromotive force; this produces an increased current in the field winding which in turn still further increases the magnetism, and so on until the machine is in full action. This process is called building $u p$; it usually takes half a minute or more for this building up process to bring a machine up to its full voltage.

If a generator has no residual magnetism it cannot begin the building up process. To get such a machine into operation it is necessary to excite its field magnet temporarily, by direct current from an outside source. This generally leaves enough residual magnetism to start the building up process.

It frequently happens that the current produced in the armature by the residual magnetism flows through the field winding in such a direction as to weaken the residual magnetism. In this case the machine cannot build up at all. This failure to build up does not depend upon the direction of the residual magnetism ; for if the field current produced by given residual magnetism strengthens (or weakens) the residual magnetism, then if the residual magnetism be reversed, it will produce a reversed current, and this reversed current will strengthen (or weaken) the reversed residual magnetism as before.

Whether the current produced by the residual magnetism flows through the field winding in the direction to strengthen or in the 
direction to weaken the residual magnetism, depends only upon the direction of driving of the generator, and upon which terminal of the field winding is connected to a given brush.

If under given conditions, a generator does build up, it cannot build up if its direction of driving is reversed, or if its field connections are reversed; if, however, its direction of driving and its field connections are both reversed, then it will build up. If, under given conditions, a generator cannot build up, it may build up if its direction of driving is reversed, or if its field connections are reversed.

A generator driven in a given direction may build up so that either the one or the other of its brushes is the positive brush, according to the initial direction of the residual magnetism. A generator which is repeatedly started and stopped usually builds up each time in the same direction; but some outside disturbance, such as a momentary current from a lightning discharge, may reverse the residual magnetism of a machine; in this case the machine will build up in a reverse direction the next time it is started.

From the above description of the building up process it may perhaps seem that there is no limit to the field strength and voltage reached by a given self-excited generator driven at a given speed. There is however a definite limit in every case, as is explained in Appendix B on characteristics.

45. Output of a generator. (a) Shunt or compound generator.Shunt and compound generators are used for service in which an approximately constant electromotive force is to be maintained between the terminals of the machine. Under these conditions the current delivered by the machine is inversely proportional to the resistance of the receiving circuit. When the resistance of the receiving circuit is large the current is small, and when the resistance of the receiving circuit is small the current is large, according to Ohm's law.

(b) Series generator. - The relation between the current output of a series generator and the resistance of the receiving circuit is complicated by the fact that the electromotive force of a series 
generator is not constant but increases very rapidly as the current output increases. 'When the resistance of the receiving circuit is large, the electromotive force is small and the current output is very small. When the resistance of the receiving circuit is small, the electromotive force is large and the current is very large.

Power output of a shunt or compound generator. - The power output of a generator, being equal to the product of the terminal voltage of the generator and the current output, is small when the current is small and large when the current is large. Furthermore, since the generator armature is driven in opposition to the side push which the field magnet exerts upon the armature wires, and since this side push is proportional to the current, it follows that the amount of power required to drive a generator increases with its current output. When the current output (to external receiving circuit) is zero, the only power required to drive a shunt generator, for example, is the power required to supply the field current and to supply the friction and magnetic losses in the armature.

Power rating. - From the above discussion it may appear that any desired amount of power however great can be delivered by a given generator. In a certain limited sense this is true, but when the current output of a generator is increased more and more, the temperature of the machine increases more and more, on account of the heat generated by the current in the windings, and the tendency to spark at the brushes increases. The deterioration of the insulating materials caused by heat sets a more or less definite limit to the permissible rise of temperature of a generator, and the smooth running and durability of the commutator depend upon sparkless commutation. Therefore, there is a practical limit to the output of a given generator beyond which either the heating or the sparking becomes excessive. This practical limit to the output of a generator determines what is called its rated output.

46. Conditions which affect the electromotive force of a generator. (a) Primary conditions. Speed and field excitation. - The 
electromotive force of a given generator depends upon its speed $n$, and upon the armature flux $\Phi$ according to equation (2I). The armature flux depends in its turn upon the degree of excitation of the field magnet. Therefore, aside from the comparatively small disturbing action of the current in the armature, the voltage of a generator depends upon its speed and its field excitation.

The dependence of the voltage of a generator upon the value of the armature flux, $\Phi$, is strikingly shown by the following experiment: Given a generator driven at a constant speed, with a voltmeter connected across its terminals, the receiving circuit being disconnected. When a heavy slab of iron is laid across the pole pieces, the voltage, as indicated by the voltmeter, drops considerably on account of the fact that a portion of the flux from the field magnet poles now passes through the iron slab instead of passing through the armature, and $\Phi$ is therefore decreased. The iron slab acts as a magnetic shunt in parallel with the armature core of the generator. When the slab is removed the voltage rises to its original value.

(b) Secondary condition. - The disturbing action of the current in the armature of a generator is comparatively small in its effect upon the voltage of the generator, and therefore it is permissible to "consider the voltage of a generator as primarily dependent upon its speed and its field excitation, and as only secondarily dependent upon the armature current. The effect of armature current upon voltage is twofold. In the first place the current in the armature of a generator, under ordinary operating conditions, opposes * the magnetizing action of the field windings, and thus tends to lessen the armature flux $\Phi$, and thereby to lessen the actual electromotive force induced in the armature. In the second place a portion of the electromotive force actually induced in the armature is used to overcome the resistance of the armature (and of the series field winding) through which the current

* The demagnetizing action of the armature current is here referred to. The crossmagnetizing action of the armature current is discussed in Chapter VI. The magnetic action of the armature current is called armature reaction. 
flows before it reaches the terminals of the machine. Electromotive force lost in this way is called internal drop, and because of this internal drop, the electromotive force between the terminals of the generator is less than the electromotive force actually induced in the armature.

The effect of internal drop upon the terminal voltage of a generator may be easily formulated as follows :

Let $I_{a}$ be the current flowing through the armature;

$I_{s}$ the current flowing through the shunt field winding;

$I_{c}$ the current flowing through the series field winding of

a series or compound machine;

$I_{x}$ the current flowing through the external circuit;

$E_{a}$ the total electromotive force induced in the armature;

$E_{x}$ the electromotive force between the terminals of a generator ;

$R_{a}$ the resistance of the armature, including resistance of brushes and of contacts between the brushes and the commutator ;

$R_{s}$ the resistance of the shunt field winding; and

$R_{c}$ the resistance of the series field winding;

$R_{x}$ the resistance of the external circuit.

The loss of electromotive force in the armature due to armature resistance is $R_{a} I_{a}$, and the loss of electromotive force in the series field winding on account of its resistance is $R_{c} I_{e}$, so that the total internal drop is $R_{a} I_{a}+R_{c} I_{c}$ and the relation between $E_{x}$ and $E_{a}$ is :

$$
E_{x}=E_{a}-R_{a} I_{a}-R_{c} I_{c}
$$

A voltmeter connected to the terminals of a generator indicates $E_{x}$. The value of $E_{a}$ cannot be directly measured by a voltmeter except when $I_{a}$ and $I_{c}$ are negligibly small, in which case $E_{a}=E_{x}$.

In case of the shunt generator there is no series field winding, so that $R_{c}$ is zero and the term $R_{c} I_{c}$ drops out of equation (22).

In case of the, series generator $I_{a}=I_{c}=I_{x}$ since there is but one electric circuit. 
In case of the long shunt compound generator

$$
I_{a}=I_{c}=I_{x}+I_{s}
$$

as is evident when we consider, referring to Fig. 29, that the whole armature current flows through the series field winding, and then divides between the shunt field winding and the external circuit.

In case of the short-shunt compound generator

$$
I_{x}=I_{c}=I_{a}-I_{s}
$$

as is evident when we consider, referring to Fig. 30, that the armature current divides at the brushes, flowing partly through the shunt field winding, and partly through the series field winding and the external circuit.

47. Comparison of separately excited generator, shunt generator, compound generator and series generator in regard to variations of terminal voltage. Separately excited generator. - The field current of a separately excited generator is constant, so that the armature flux $\Phi$ is constant except for the comparatively slight decrease due to armature reaction when the machine is delivering current. Therefore :

(a) The total induced voltage $E_{a}$, of a separately excited generator falls off in proportion to the speed at which the machine is driven, and the terminal voltage, $E_{x}$ which is always approximately equal to $E_{a}$, likewise falls off nearly in proportion to the speed. This is evident from the fundamental equation $E_{a}=\Phi Z^{\prime} n$.

(b) The external voltage $E_{x}$, of a separately excited generator falls off with increase of current, on account of internal drop $R_{a} I_{a}$, and on account of the lessening of $\Phi$ by armature reaction.

Shunt generator. - The field current of a shunt generator falls off when the terminal voltage, $E_{x}$, decreases, inasmuch as the shunt field windings are connected between the terminals of the machine. Therefore :

(a) A decrease in speed of a shunt generator causes a much greater decrease in $E_{a}$ and $E_{x}$ than in the case of the separately 
excited generator, because of the decrease of $I$, which accompanies the decrease of $E_{x}$.

(b) An increase in current output of a shunt generator causes a much greater decrease in $E_{x}$ than in the case of the separately excited generator, because of the decrease of $I_{s}$ which accompanies the decrease of $E_{x}$.

The compound generator. - (a) When a generator has a compound field winding, the current output of the machine flows through the series field coil,* and the field excitation of the machine is therefore increased with increased current output. If the series field coil has a sufficient number of turns of wire, this increase of field excitation may not only counterbalance the demagnetizing action of the current in the armature, but it may more than counterbalance this demagnetizing action and cause $\Phi$ to actually increase with increase of current output, thus increasing the total induced electromotive force $E_{a}$.

I. This increase of $E_{a}$ can be made sufficient to compensate for the internal drop, $R_{a} I_{a}+R_{c} I_{c}$, thus keeping $E_{x}$ approximately constant with increase of current output. In this case the machine is said to be flat-compounded.

2. This increase of $E_{a}$ can be made more than sufficient to compensate for the internal drop. In this case the voltage between the terminals of the machine increases with increase of current output, and the machine is said to be over-compounded.

(b) The dependence of the voltage of a compound generator upon speed is, for a given current output, nearly the same as in the case of the shunt generator.

Series generator. - (a) The voltage of a series generator is zero or nearly zero, $f$ when the current output is zero, and it increases rapidly with the current output. This is evident when we consider that the field winding is in series with the external circuit.

(b) For a given current output, the total induced voltage, $E_{a}$,

* This statement applies specifically to the short-shunt compound dynamo.

† Residual magnetism produces a small electromotive force even when the series field current is zero. 
of a series generator is proportional to the speed, and the terminal voltage, $E_{x}$, is very nearly proportional to the speed.

Adaptability of the various methods of field excitation to special purposes. - Separate excitation is very often used in dynamo testing. When it is desired to obtain a voltage which fluctuates as little as possible with changes of speed, separate excitation is used in commercial installations.

Generators which are used for charging storage batteries, and generators which are used for electroplating, are liable to have their field magnetism reversed when the machine is stopped without disconnecting it from the external receiving circuit. In such service the reversal of field magnetism is very much less likely to occur when the generator is separately excited. Therefore separately excited generators are sometimes used for charging storage batteries, and usually for electroplating.

Direct-current generators in central stations for lighting or power are nearly always self-excited, inasmuch as modern engines and water wheels give very nearly constant-speed driving, and therefore the use of an auxiliary generator for supplying current for exciting the field magnets of the main generators is not usually warranted by the greater constancy of voltage obtainable thereby. Alternating current generators are always separately excited, since the alternating current delivered by an alternating current generator is not suitable for field excitation, unless rectified by a special commutator as in the "composite field" alternator.

When electric current is to be supplied at a constant, or approximately constant, voltage, shunt or compound generators are used, as has been previously pointed out. Series generators are not suited to the delivery of a varying current at a constant voltage. When a constant current is to be supplied at varying voltage, series generators are generally used. Such generators are provided with special regulating devices to vary the voltage, so as to keep the current output constant when the resistance of the external circuit changes. 
48. Voltage regulation of generators. - A generator which is designed for supplying current at constant voltage is called a "constant voltage" generator. Thus a shunt or compound generator is called a "constant voltage" generator, although the maintenance of a strictly constant voltage by such a machine is accomplished by the manipulation of a field rheostat. The range of variation of the terminal voltage of such a machine with variation of current output, when the field rheostat is left wholly unchanged, is an important practical matter. The change of voltage from full load to no load, expressed as a percentage of full load voltage, is called the "voltage regulation" of the generator, speed of driving and resistance of field circuit being kept constant.

Example. - The field rheostat of a certain shunt generator is adjusted so that its terminal voltage is IIO volts when it is delivering its rated full load current of 250 amperes. When the main switch is opened so as to reduce the current output to zero, the terminal voltage of the generator is observed to rise to 126.5 volts. The regulation of this machine is :

$$
\frac{\text { I } 26.5-\text { I IO }}{\text { I IO }}=0.15 \text { or I } 5 \text { per-cent. }
$$

It is to be noted that the word regulation is applied to changes of voltage inherent in the machine itself, while the word control is applied to changes of voltage due to deliberate adjustments by an attendant.

\section{Control of voltage of shunt or compound generator by manip-} ulation of field rheostat. - The "field rheostat" is the rheostat which is in circuit with the shunt field winding, as indicated in Fig. 69. If for any reason the voltage between the terminals of the generator is greater or less than the desired value, resistance may be added to or taken from the shunt field circuit by manipulating the field rheostat, thus decreasing or increasing the current in the shunt field winding, and thereby decreasing or increasing the field excitation, so as to bring the terminal voltage to the desired value. 
Manipulation of the field rheostat is of course necessary in case of a shunt generator, when it is desired to keep the terminal voltage constant with varying current output. In case of the flatcompound generator, also, the terminal voltage always varies to some extent with variation of current output, and the compound generator is therefore always provided with a field rheostat in its shunt field circuit so as to enable an attendant to control its terminal voltage at will.

The usual form of field rheostat consists of coils or zigzags of wire or metal ribbon connected in series, and arranged with a number of contact points over which a metal arm may be moved, so as to include any desired portion of the rheostat in series with the field winding. The insulation of the rheostat is generally made of fireproof material, such as mica or asbestos. Special provision is made for cooling, either by ventilating or by great expanse of radiating surface.

There is a wide variety in the design of commercial field rheostats. Fig. 70 is a general view of a field rheostat manufactured by the Wirt Electric Company. Part of the front plate is cut away to show the resistance strips, EEE. This resistance strip is a long ribbon of high resistance alloy, coated on one side with fireproof insulation, such as flake mica, and wound in a long helix on a mandril of flat-oval section. This flat helix is then slipped off the mandril, and arranged in a circle between two flat circular plates, $M J$, and insulated from the plates by sheets of mica, $F G$. The two plates are then clamped together with considerable pressure, and a steel band $N$ is spun over the outer e:dge of the plates to hold them together. The metal plates are deeply ribbed, front and back, to facilitate the radiation of heat. One end of the resistance helix is connected to one of the terminal binding posts, shown at the bottom of the figure, and the other binding post connects to the rotating metal arm $C$ which makes contact with the inner ends of the convolutions of the flat helix. The rheostat is connected in series with the shunt field winding of the generator by wires attached to the rheostat binding 
posts, and it is mounted in a convenient position on the switchboard panel.

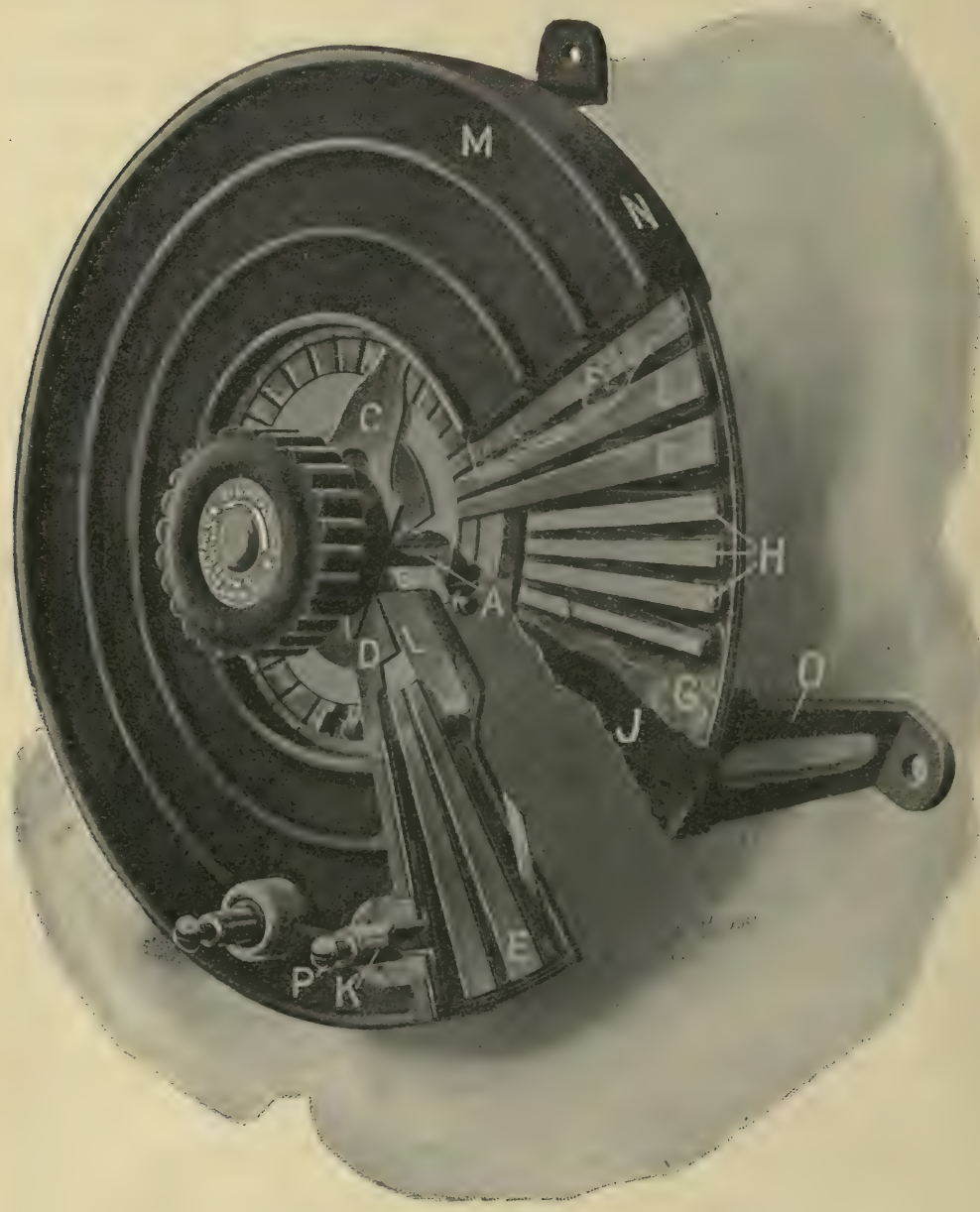

Fig. 70.

In the rheostat manufactured by the Ward-Leonard Company, the zigzags of resistance wire are embedded in a thick layer of glazing or enamel on the back of a deeply-ribbed cast-iron plate.

50. Adjustment of a compound generator.-A compound generator always has a low resistance shunt, usually made of Ger- 
man silver ribbon, connected across the terminals of its series field winding. By adjusting the resistance of this shunt once for all, any desired fractional part of the current output of the machine may be made to flow through the series field winding. The machine is manufactured with a greater number of turns of wire in its series field winding than are strictly necessary, and by changing the resistance of the above-mentioned shunt, the machine may be adjusted in the factory for flat-compounding, or for any desired degree of over-compounding. This adjustment should be made when the machine is at its normal running temperature. Fig. 69 shows a compound generator (short-shunt) with the abovementioned shunt, $S$, between the terminals of the series field winding.

Over-compound generators are used where current is to be delivered to lamps or motors over a long transmission line and where it is desired to maintain an approximately constant voltage at the terminals of the lamps or motors in spite of the increased loss of electromotive force in the line (line drop) with increase of current. For example, railway generators are usually over-compounded so as to give 500 volts at the generator terminals when the current output is zero, and 550 volts at the generator terminals when the current output is at the normal full load value. In this case the machine is said to be IO per cent. over-compounded, inasmuch as the full load voltage is I Io per cent. of the no load voltage.

51. Brush lead. - Fig. 7 I represents a two-pole generator with ring armature. The fine curved lines show the trend of the magnetic flux from the north pole of the field magnet into the armature core, and from the armature core into the south pole of the field magnet.

The turns of wire as they pass the points $a$ and $b$, Fig. 7 I, have zero electromotive force induced in them, whereas the electromotive force induced in the individual turns of wire becomes greater and greater on either side of the points $a$ and $b$ up to the pole tips, and approximately equal electromotive forces are 
induced in every turn which is under the pole faces. Fig. 72 is a diagram, similar to Fig. I 8, in which the circle represents the endless circuit formed by the windings on the ring armature. The arrows in Fig. 72 show the directions of the electromotive

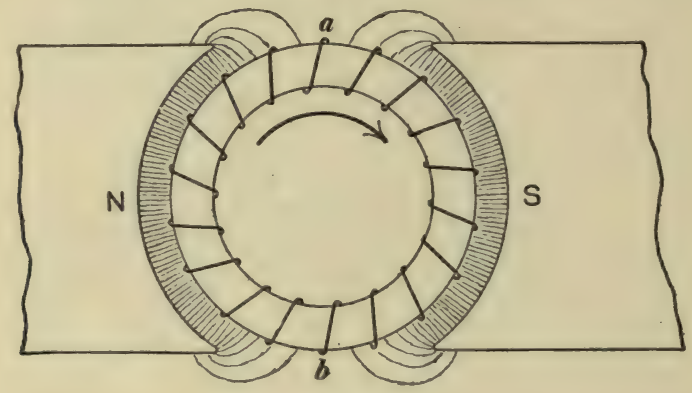

Fig. 71 .

forces induced in the various turns of wire on the armature, and the lengths of these arrows are intended to show roughly the relative values of these induced electromotive forces.

Figs. 71 and 72 show the state of affairs when the current in the armature windings is small. When the armature current

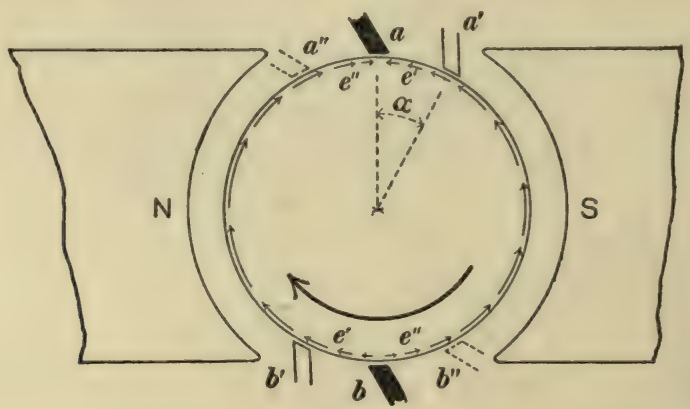

Fig. 72 .

is large, the trend of the magnetic flux is altered from that shown in Fig. $7 \mathrm{I}$ to that shown in Fig. 73, and the electromotive forces in the various turns are altered from what is shown in Fig. 72 to what is shown in Fig. 74 . 
The line $a b$, Figs. 71, 72, 73 and 74, is called the neutral axis of the armature. The line connecting the brushes is called the axis of commutation.* When the brushes of a generator are

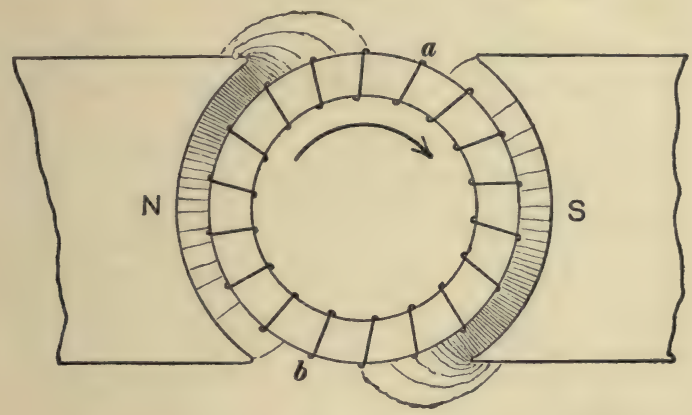

Fig. 73.

shifted by moving the rocker arm, the electromotive force between the brushes, as indicated by a voltmeter, reaches a maximum value when the axis of commutation coincides with the

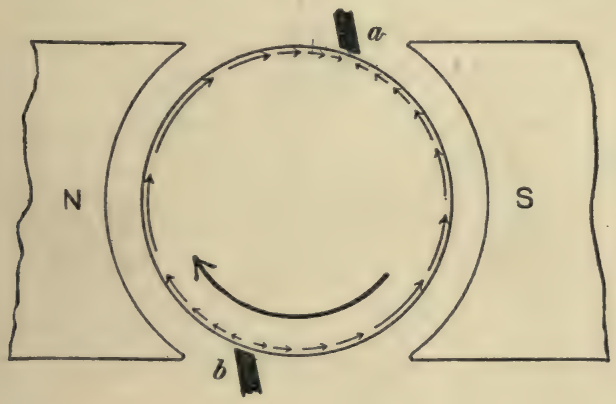

Fig. 74.

neutral axis. In fact the whole of the electromotive force, $E_{a}$, which is induced in each half of the armature, is available between the brushes when the brushes are at the points $a$ and $b$, Figs. 72 and 74 .

* The leads which connect the armature conductors to the commutator bars are supposed to be radial, for the sake of simplicity of statement. In commercial machines these leads are nearly always curved like segments of a spiral. 
When the brushes are shifted from the neutral axis to the points $a^{\prime}$ and $b^{\prime}$, Fig. 72 , for instance, the available electromotive force between them is reduced in value to $\left(E_{a}-2 e\right)$, where $e$ is the electromotive force induced in the portion of the armature winding between $a$ and $a^{\prime}$, or in the portion between $b$ and $b^{\prime}$.

The angle between the neutral axis and the axis of commutation is called the angle of lead of the brushes. When the shift of the brushes from the neutral axis is in the direction of rotation of the armature, the brushes are said to have a forward lead, and when the shift of the brushes is in the opposite direction, the brushes are said to have a backward lead. Thus the angle $\alpha$, Fig. 72 , is the angle of forward lead of the brushes when they are at the points $a^{\prime}$ and $b^{\prime}$.

The sparking at the brushes of a generator is a minimum when the brushes have a slight forward lead, the amount of lead depending upon the current output. The sparking at the brushes of a motor is a minimum when the brushes have a slight backward lead, the amount of lead depending upon the current intake of the motor, that is to say, upon the motor load. A poorly designed dynamo usually requires continual adjustment of the brushes forwards or backwards to keep them at the minimum sparking point with changes of load, otherwise the sparking will be excessive. A well-designed dynamo, however, will operate satisfactorily from zero load to full load with fixed brushes.

The fundamental equation of the dynamo, equation (2I), was derived on the assumption that the angle of lead of the brushes was zero. 


\section{CHAPTER IV.}

THE OPERATION OF THE DYNAMO AS A MOTOR.

52. Conditions which determine the direction of running of a motor.-Precisely the same conditions which determine the direction in which a self-exciting dynamo with given connections of field to brushes, must be driven, in order that it may build up as a generator, determine the direction in which the machine will run as motor when it is supplied with current.

The shunt dynamo, with given connections of field to brushes, runs as a motor in the same direction in which it must be driven in order to build up as a generator. This may be made evident às follows :

The heavy arrow in Fig. 75 shows the direction of the electromotive force and current in the armature of a shunt generator, and the dotted arrows show the direction of the currents in field

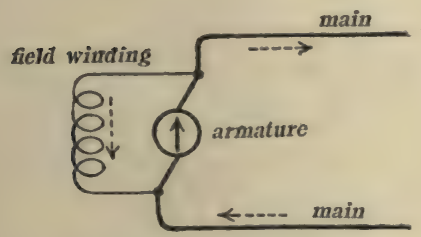

Fig. 75. Shunt Generator.

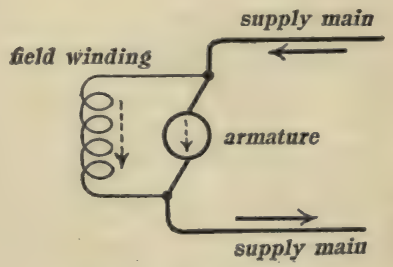

Fig. 76. Shunt Motor.

and in mains ; the heavy arrows in Fig. 76 show the direction of the current delivered to a shunt motor, and the dotted arrows show the direction of the currents in field and armature. Figs.* 75 and 76 show at a glance that the current in the armature of a shunt machine is reversed with respect to the field current, when

* There are four possible combinations of arrows in Figs. 75 and 76, and each combination has the property here specified. 
the function of the machine is changed from generator to motor, so that the mechanical force with which the field acts upon the armature, is reversed; but the force action opposes the motion of the generator, and helps the motion of the motor. Therefore since the force action is reversed, the motion must be in the same direction, whether the machine operates as generator or as motor.

The series dynamo, with given connections of field to brushes, runs as a motor in a direction opposite to that in which the machine must be driven to enable it to build up as a generator. This is evident from Figs. 77 and 78 which show that, with given

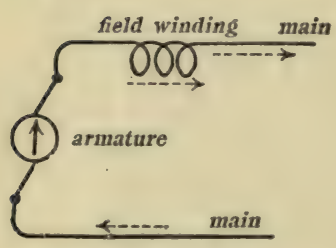

Flg. 77. Series Generator.

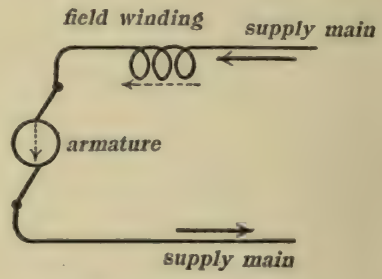

Fig. 78. Series Motor.

connections of field to brushes, the relative direction of current in armature and in field is the same, whether the machine is operating as a generator or as a motor. Therefore the mechanical force with which the field acts upon the armature is unchanged in direction when the function of the machine is changed from generator to motor; but the force action opposes the motion of the generator and helps the motion of the motor. Therefore the motion must be reversed, since the force action is unchanged.

Direction of running of a motor. - The direction of running of a motor, with given connections of field winding to brushes, is the same, irrespective of the direction of the current supplied to the machine. This is evident when we consider that the reversal of the supply current, which is represented by the heavy arrows in Figs. 76 and 78 , reverses the current both in the armature and in the field, and that the reversed field of course exerts a force 
upon the reversed armature currents in the same direction as before, thus leaving the direction of rotation unchanged. To reverse the direction of running of a motor the connections must be changed so that the current through armature (or field) is reversed while the direction of the current in the field (or armature) remains unchanged. This requires the reversal of the armature connections, or a reversal of the field connections, but not of both.

53. Fundamental equation of the motor. - Let $E_{x}$ be the electromotive force applied at the terminals of a motor. This is called the impressed electromotive force. This electromotive force is partly used to overcome the resistance $R_{a}$ of the armature. The part so used is equal to $R_{a} I_{a}$, where $I_{a}$ is the current flowing through the armature. The remainder of the impressed electromotive force balances the electromotive force, $E_{a}\left(=\Phi Z^{\prime} n\right)$ which is induced in the rotating armature by the field flux, and it is numerically equal thereto. Therefore:

$$
E_{x}=\Phi Z^{\prime} n+R_{a} I_{a}
$$

in which $E_{x}$ is the impressed electromotive force acting on a motor, $R_{a}$ is the armature resistance, ${ }^{*} I_{a}$ is the current flowing through the armature, $\Phi$ is the magnetic flux entering the armature from one field pole, $n$ is the speed of the motor in revolutions per second, and $Z^{\prime}$ is written for the factor $p Z \mid p^{\prime} \mathrm{IO}^{8}$. (See Art. 30.)

The electromotive force, $\Phi Z^{\prime} n$, which is induced in the rotating armature of a motor, is opposite in direction to the impressed electromotive force, $E_{x}$ and to the current, $I_{a^{*}}$. Therefore $\Phi Z^{\prime} n$ is called the counter electromotive force of the motor.

In the generator the voltage is determined by the fundamental equations (2I) and (22), inasmuch as all the other quantities in these equations are fixed in value by the conditions under which

* In the long-shunt compound motor the combined resistance of armature and series field coil, $R_{a}+R_{c}$, is to be used for $R_{a}$. It is hardly necessary to formulate the very slightly different behavior of the short-shunt compound motor. 
the generator is used. In the case of the motor, on the other hand, the speed is determined by these fundamental equations, inasmuch as all the other quantities in these equations are, as a rule, fixed in value by the conditions under which the motor is used. Therefore, the most convenient form of equation $(23 a)$ is that which gives speed in terms of the other quantities. Solving equation (23a) for speed, we have

$$
n=\frac{E_{x}-R_{a} I_{\alpha}}{\Phi Z^{\prime}}
$$

Another useful form of this equation is that which expresses $I_{a}$ in terms of the other quantities, namely:

$$
I_{a}=\frac{E_{x}-\Phi Z^{\prime} n}{R_{\mathrm{u}}}
$$

From this equation it is evident that the current $I_{a}$ is not in general equal to $E_{x}$ divided by $R_{a}$, inasmuch as a small part only of $E_{x}$ is used to overcome resistance. When the motor is not running, that is when $n=0$, then $I_{a}$ is equal to $E_{x}$ divided by $R_{a}$, and the value of $I_{a}$ is excessively large. To avoid this excessive flow of current when $n$ is small, an auxiliary resistance must be connected in series with the armature as explained in Art. 59.

Torque. - Another equation of considerable importance in the discussion of the motor is the expression for torque. The value of the torque with which the field magnet acts upon the armature, may be derived by considering the side push on each armature wire, multiplying each side push by its lever arm, namely the radius of the armature, and adding these results together. This method of derivation, however, repeats many of the essential considerations involved in the derivation of the fundamental equation of the dynamo as given in Art. 30. It is simpler to derive the expression for torque directly from the Sundamental equation (23) with the help of Lenz's law as follaws :

The rate at which work is done, in watts, in forcing the current $I_{a}$ through the armature in opposition to the counter elec- 
tromotive force $\Phi Z^{\prime} n$, is equal to the product $I_{a} \times \Phi Z^{\prime} n$; and, according to Lenz's law, this is equal to the mechanical power developed by the torque $T$ with which the field magnet acts upon the rotating armature. Now, the mechanical power in watts developed by a torque of $T$ pound-inches acting upon a body making $n$ revolutions per second is :

$$
P=\frac{2 \pi \times 2.54 \times 453.6 \times 980}{\mathrm{IO}^{7}} . n T \text { watts }
$$

or

$$
P=0.7 \text { I } n T \text { watts. }
$$

Therefore by Lenz's law we have

whence

$$
I_{a} \times \Phi Z^{\prime} n=0.7 \mathrm{I} n T
$$

$$
T=\mathrm{I} .4 \mathrm{I} \Phi Z^{\prime} I_{a} \text { pound-inches }
$$

in which $Z^{\prime}=p Z \mid p^{\prime} 10^{8}, I_{a}$ is the armature current in amperes, $\Phi$ is the magnetic flux entering the armature from one field magnet pole, $p$ is the number of magnet poles, $p^{\prime}$ is the number of paths in parallel through the armature between brushes, and $Z$ is the total number of conductors on the armature.

The net effective torque at the pulley of a motor is less than $T$ in equation (25) by the amount of the torque required to overcome friction and other so-called "stray power" losses.

If the net or useful torque is used for $T$ in equation (24) the power $P$ will be the power actually delivered by the motor pulley.

54. Siemens' law of efficiency. - The dependence of the efficiency of a motor upon the conditions of driving is complicated by the loss of power in field excitation, by friction, and by eddy currents and hysteresis. This matter is fully discussed in Chapter V. A very simple expression for the efficiency of a motor, due to Siemens, is especially useful for purposes of general discussion. The formula is only approximate, however, inasmuch as it is based on the assumption that the only loss of power in the 
motor is that which is expended in heating the armature windings by the armature current; that is to say, field loss and friction losses are ignored. The total power delivered to a motor, neglecting field loss, is equal to $E_{x} I_{a}$, where $E_{x}$ is the impressed voltage, and $I_{a}$ is the current-flowing through the armature. Part of this power is consumed in heating the armature windings, and part is used in forcing the current through the armature in opposition to the electromotive force, $E_{a}$ which is induced in the armature windings by the motion of the armature. This latter part is equal to $E_{a} I_{a}$ and it is, according to Lenz's law, all converted into the mechanical power which turns the armature.

Therefore, ignoring the power used for field excitation, the intake of electrical power by the motor is $E_{x} I_{a}$; and, ignoring the losses of mechanical power in the motor, by bearing friction, air friction, and magnetic friction, $*$ the output of mechanical power is $E_{a} I_{a}$, so that the approximate efficiency is: $E_{a} I_{a} \div E_{x} I_{a}$ or $E_{a} / E_{x}$. This expression for efficiency, known as Siemens' law, always gives an overestimate of the efficiency of a motor; and when a motor is running under a small load the ratio $E_{a} / E_{x}$ may be many times as great as the actual efficiency.

55. Constant voltage and constant current motor driving. - The discussion of the electromagnetic behavior of a generator, that is, the discussion of the variation of its voltage with its current output, is impossible unless the mechanical conditions of driving are specified. In fact, constant speed driving is nearly always taken as the basis for the discussion of generator characteristics.

Similarly, the discussion of the mechanical behavior of a motor, that is, the discussion of the variation of its speed with its power output or with its torque, is impossible unless the electrical conditions of driving are specified. Usually the discussion of the mechanical characteristics of a motor is based upon the assumption, either of a constant voltage between the motor ter-

* The effect of eddy currents and hysteresis in a dynamo armature is to create a drag which opposes the motion of the armature, very much like ordinary mechanical friction. 
minals or of a constant current flow through the motor. In practice motors are always used under conditions which approximate to constant voltage driving; constant current driving is never used. Therefore, the following discussion of the behavior of motors is based upon the assumption of constant, or approximately constant, voltage between the supply mains. In this discussion the shunt motor is considered first and in detail ; the compound motor is treated as a modification of the shunt motor ; and the series motor is considered last.

When a motor is delivering mechanical power, it is said to be loaded, and its output of mechanical power is called its load. The rated full load of a motor is the load for which the armature and field currents are as large as permissible without excessive rise of temperature in any of the motor parts, or excessive sparking.

56. The shunt motor. - The essential connections of a shunt motor to constant voltage supply mains are shown in Fig. 79. It is to be particularly noted that Fig. 79 shows the connections of the motor after it has been started and brought up to speed. Special connections are made at starting as will be explained later.

The shunt field coils are connected directly to the supply mains, usually without including a field rheostat, and the field

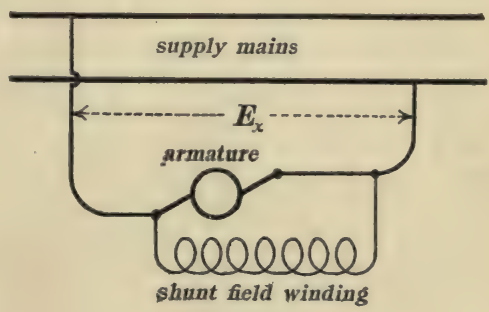

Fig. 79. current is therefore constant, so that the only variation of the armature flux, $\Phi$, is the slight variation due to the demagnetizing action of the armature current.

Shunt motor unloaded. - When a motor is unloaded the torque necessary to drive it is small, and therefore the armature current is small, according to equation (25). Consequently the part, $R_{a} I_{a}$, of the impressed voltage which is used to overcome the resistance of the armature, is negligibly small, since both $I_{a}$ and $R_{a}$ 
are small. Therefore, neglecting $R_{a} I_{a}$, equation $\left(23^{b}\right)$ may be written :

$$
n=\frac{E_{x}}{\Phi Z^{\prime}} \text { at zero load }
$$

Inasmuch as $\Phi Z^{\prime} n$ is the counter electromotive force of the motor, we see from equation (26) that a shunt motor at zero load runs at a speed such as to make its counter electromotive force, $E_{a}$, sensibly equal to the impressed electromotive force, $E_{x}$.

Shunt motor loaded. - When a load is thrown on a shunt motor which has been previously running at zero load, its tendency is to lower the speed slightly and thereby to decrease the counter electromotive force, $\Phi Z^{\prime} n$. As the counter electromotive force decreases, the armature current increases according to equation $(23 c)$, and the torque increases according to equation (25), thus enabling the motor to carry its load. The drop in speed of a shunt motor, from zero load to full load, ranges from 2 per cent. of zero load speed for large-sized motors, to Io per cent. or more for small motors.

Tendency, in a shunt motor, for the demagnetizing action of the armature current to counteract the tendency of the speed to decrease with increase of load. When a shunt motor is loaded it is necessary for the counter electromotive force to decrease sufficiently to permit enough current to flow through the armature to develop the torque required to carry the load. Now, if $\Phi$ were invariable, the necessary decrease of counter electromotive force could be produced only by a drop in speed, but as a matter of fact the flux $\Phi$ is decreased slightly by the demagnetizing action of the current in the armature, and consequently the necessary decrease of counter electromotive force $\left(\Phi Z^{\prime} n\right)$ is brought about in part by this decrease of $\Phi$, and in part by a decrease in speed, so that the actual decrease in speed is less than it would be if $\Phi$ were invariable.

The dependence of the speed of a shunt motor upon the value of the armature flux, $\Phi$, is strikingly shown by the following ex- 
periment. A shunt motor is supplied with current from constant voltage supply mains and runs at a certain speed, as indicated by a tachometer. A slab of iron is now laid across the pole pieces so that a considerable portion of the flux $\Phi$ passes through the slab, and the armature flux is consequently reduced. The speed of the motor immediately increases as indicated by the tachometer. This rise in speed is due to the fact that the reduction of $\Phi$ reduces the counter electromotive force, $\Phi Z^{\prime} n$, of the motor. A sudden increase of current through the armature results, according to equation (23c). This increased current produces more torque than is required to carry the load on the motor, so that the motor speeds up until the increase of its counter electromotive force reduces the current to the value corresponding to the required torque.

An interesting and important fact in connection with a shunt motor is that such a motor runs at a lower speed when first started than after it has been running for some time. This is due to the fact that the rise of temperature of its field coils increases their resistance, decreases the field current, and decreases $\Phi$. This rise of speed often amounts to as much as 5 per cent. in commercial machines.

Dependence of speed upon brush lead. - It was pointed out in Art. 5 I that the electromotive force between the brushes of a generator running at a given speed with given field excitation, is reduced in value by shifting the brushes from the neutral axis, and that the electromotive force is a maximum when the brush lead is zero. In the case of a shunt motor supplied from constant voltage mains, the speed is a minimum when the brushes are in the neutral axis, and the effect of shifting the brushes either forwards or backwards, is to increase the speed. This effect is particularly noticeable at zero load. When the motor is heavily loaded the matter is greatly complicated by the magnetizing or demagnetizing action of the armature current.

The explanation of this variation of speed of a shunt motor with brush lead is as follows: When the brushes are shifted from 
the neutral axis, the net counter-electromotive force between the brushes is decreased, speed remaining unchanged. As a result, the impressed voltage, $E_{x}$, forces an increased current through the armature, thus producing an increased torque which causes the motor speed to increase until the counter-electromotive force reaches a value sufficiently large to reduce the current to the value required to supply the necessary driving torque.

57. Speed regulation of the shunt motor. - A motor which is designed for supplying mechanical power at constant speed is called a "constant speed" motor. Thus a shunt motor is called a "constant speed" motor, although the maintenance of a strictly constant speed by such a motor can be accomplished only by the manipulation of a rheostat. The range of variation of speed of a "constant speed" motor with change of load, when the rheostat is left wholly unchanged, is an important practical matter. The change of speed from full load to no load expressed as a percentage of full load speed, is called the "speed regulation" of the motor, voltage of supply and resistance of armature circuit and of field circuit being kept constant. Thus, if the speed of a motor rises from $\mathrm{I}, \mathrm{OOO}$ revolutions per minute at full load to $\mathrm{I}, \mathrm{O} 5 \mathrm{O}$ revolutions per minute at no load, its speed regulation is 5 per cent.

It is to be noted that the word

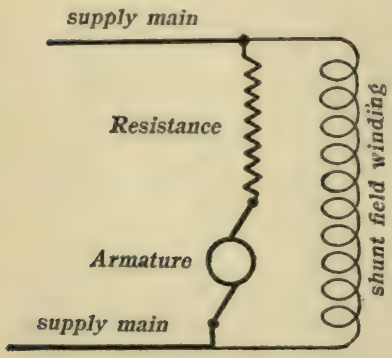

Fig. 80 . regulation is applied to changes of speed inherent in the machine itself, while the word control is applied to changes of speed due to deliberate adjustments by an attendant.

Influence of armature resistance upon the speed regulation of a shunt motor. - When the resistance of the armature of a shunt motor is large, or when a considerable resistance is connected in series with the armature as shown in Fig. 80, then the speed of the motor falls off greatly with increase of load. The cause of this great drop in speed under load is evident from equations 
(23c) and (25). The very considerable current which is necessary to develop torque sufficient to enable the motor to carry a load, demands a large value in the numerator of equation (23c), when $R_{a}$ is large, and this large value of $\left(E_{x}-\Phi Z^{\prime} n\right)$ necessitates a great drop in speed in order that $\Phi Z^{\prime} n$ may be considerably less than $E_{x}$. It is to be especially noted, however, that the zero load speed of a shunt motor is not perceptibly affected by a moderately large value of $R_{a}$, inasmuch as $I_{a}$ is very small at zero load. It is for this reason that the speed of a shunt motor cannot be satisfactorily controlled by inserting resistance in series with the armature. The difficulty is that, when the speed is cut down in this way while the motor is loaded, the speed increases greatly when more or less of the load is thrown off.

58. Speed control of the shunt motor. - The peculiar and valuable properties of the shunt (and compound) motor are: (a) that it runs at a definite speed at zero load, that is to say, it has no tendency to race when the load is thrown off; and (b) that its speed drops but little below zero load speed even when the motor is fully loaded. For many purposes, for example, in the driving of lathes by motors, it is desirable to run the motor fast or slow at will. This speed control is accomplished in the case of the shunt motor by five different methods, as follows :

(I) Armature rheostat method. - When the load on a motor is constant, its speed may be controlled by inserting resistance in series with its armature, as explained in the last article.

(2) Field rheostat method. - From equation (26) it is at once evident that the zero load speed (and consequently the full load speed to nearly the same extent) may be raised by decreasing $\Phi$, or lowered by increasing $\Phi$. These changes of $\Phi$ may be accomplished by means of a rheostat in circuit with the field winding of the motor. By means of this rheostat the resistance of the shunt field circuit may be raised or lowered, thus decreasing or increasing the field current, and thereby decreasing or increasing $\Phi$. 
This method of speed control is limited as follows: (a) It is not feasible to increase $\Phi$ much beyond a certain value on account of what is called the magnetic saturation of the iron through which this flux $\Phi$ passes. To increase $\Phi$ to this saturation value requires an excessive value of field current and may involve excessive heating of the field magnet coils. (b) When $\Phi$ is decreased more and more, the operation of the motor becomes unsatisfactory; the torque that can be developed by the machine [see equation (25)] becomes very small when $\Phi$ is small, and the magnetizing action of the current in the armature begins to preponderate over the weakened magnetizing action of the field windings, and serious sparking at the brushes results. Experience shows that the maximum practicable range of speed control of the shunt motor by means of the field rheostat is about one hundred per cent., that is, a shunt motor which runs at speed $n$ when its field magnet is near saturation (largest feasible value of $\Phi$ ) will operate more or less satisfactorily down to a value of $\Phi$ equal to about half the saturation value, and the corresponding speed is of course $2 n$.

(3) Method by altering the reluctance of the magnetic circuit. If the reluctance of the magnetic circuit of a shunt motor is increased, the flux $\Phi$ is decreased, the field excitation being constant. The speed of the motor will, therefore, be increased as explained above. If the increase of reluctance is produced by widening the gap space, then the sparking difficulties which usually accompany a decrease of $\Phi$ are largely obviated by the increased width of the gap space as explained in Arts. 77 and 78 .

This method of speed control is exemplified by the "multispeed" motors of the Stow Manufacturing Company. The details of one of these motors are shown in Fig. 8I $a$ and a general view of the same motor is shown in Fig. $81 b$. Each of the four field magnet cores in Fig. $8 \mathrm{I} a$ is hollow and contains a solid cylindrical plunger of iron $A$. In motors of large size the ends of these plungers are the central parts of the pole faces, whereas in the smaller motors of the Stow Company a thin shell of iron 
THE OPERATION OF THE DYNAMO AS A MOTOR. IO7
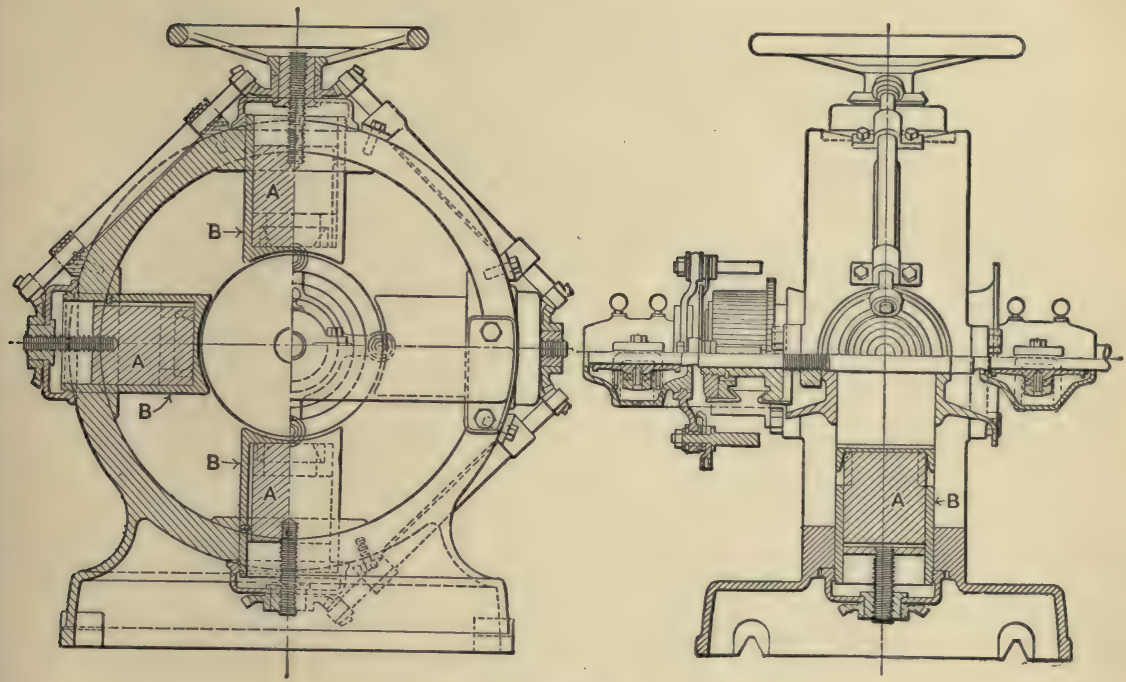

Fig. $81 a$.

covers the end of each plunger as shown in Fig. $8 \mathrm{I} a$. In both cases, however, the increase of reluctance produced by the withdrawal of the plungers takes place chiefly in the gap spaces.

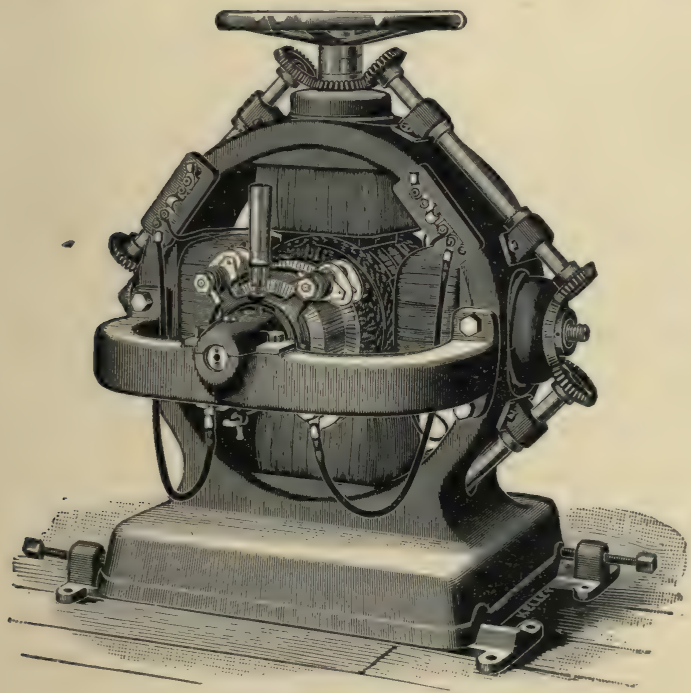

Fig. $81 b$. 
The withdrawing of the plungers diverts the larger part of the reduced flux into the pole tips where it is most effective in the prevention of sparking, and at the same time interposes a greatly increased reluctance in the path of the cross-flux, CC, Fig. Io8.

(4) Multivoltage speed control. - From equation (26) it is evident that the zero load speed (and consequently the full load speed to nearly the same extent) may be made high or low by supplying current to the armature at high or low voltage $E_{x}$, the field flux $\Phi$ being kept constant. The range of speed control of a shunt motor by this method is limited only by the speed the motor can stand mechanically, and by the safe limit of impressed

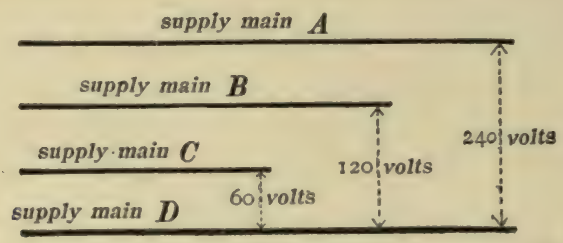

Fig. 82.

voltage $E_{x^{*}}$ In carrying out this method of speed control, current is supplied over a number of mains, for example, $A, B, C$ and $D$, Fig. 82 , between which constant electromotive forces of any chosen values are maintained by a set of generators. The field winding of the shunt motor is permanently connected to one pair of these supply mains, and arrangements are made, by means of a suitable "controller," for easily and quickly connecting the motor armature to any pair of mains at will.

This multivoltage method of speed control gives a series of distinct and widely separate speeds. For example, with the voltages indicated in Fig. 82 the available speeds would be $n, 2 n$ and $4 n$, where $n$ is the speed corresponding to $E_{x}=60$ volts. The multivoltage method is usually combined with the field rheostat method, so that by the latter method the intermediate speeds may be obtained.

(5) The Ward Leonard method of speed control. - A wide range of speed control of a shunt motor by means of a field rheostat can- 
not be realized, inasmuch as very low speeds cannot be reached because of the saturation of the iron parts of the motor as explained above, and inasmuch as very high speeds are not practicable because of the trouble from sparking when the field excitation of the motor is very small; the impressed voltage $E_{x}$ being constant in value. Now, on the other hand, a generator does not tend to spark badly when run at low field excitation, because when the field excitation is low, the voltage between commutator segments is low also. Therefore a given generator may be satisfactorily used to give a range of values of electromotive force from zero, when its field excitation is zero, to a maximum value, when its field magnets are saturated. The Ward Leonard method of speed control makes use of this property of a generator as follows: An auxiliary constant speed motor takes current from the supply mains and drives a generator, $G$, at constant speed. The field winding of this generator, $G$, is provided with a field rheostat which has a sufficient range of resistance to change the field excitation of the generator from its full value to nearly zero. The main motor, which is to be driven at variable speed, has its field connected to the supply mains so that its field excitation is maintained at a constant value, while its armature is supplied with current from the generator $G$. With this arrangement it is practicable to supply the main variable speed motor with current at any desired voltage from zero up to the greatest voltage obtainable from the generator $G$ when the field of the latter is fully excited.

Comparison of methods of speed control. - The armature rheostat method of speed control is fairly satisfactory when the motor load does not vary rapidly. The disadvantage of this method, aside from the great fluctuation of speed with load, is that the use of a rheostat in series with the armature involves a very considerable waste of power due to the $I^{2} R$ loss in the rheostat. The advantages of this method are : that it is simple and cheap, that it can be arranged to give fine gradations of speed control by having many resistance steps in the rheostat, and that it permits 
of a speed control ranging from full speed to zero speed, provided the motor load is fairly constant.

The control of speed by a field rheostat does not involve much waste of power, inasmuch as the field current is very small in comparison with the armature current of a shunt motor. Additional advantages of this method are : simplicity and cheapness, the absence of great fluctuations of speed with load, and the possibility of fine gradations of speed control when a finely subdivided rheostat is used. The disadvantage of this method is that it has a limited range, as already explained.

The control of speed by the change of reluctance method possesses all of the advantages of the field rheostat method using a very finely subdivided rheostat, and it saves the cost of such a rheostat and gives a wider range of speed control because of the partial elimination of sparking as before explained. On the other hand the change of reluctance method requires a motor of a special and rather expensive design.

The multivoltage method of speed control has the great disadvantage that it is expensive, requiring as it does, several generators, * an elaborate system of wiring, and a complex controller for each motor. The disadvantage that it does not provide for a finely graded speed control is entirely overcome by combining with it the field rheostat method for giving intermediate speeds, as already explained. The combined multivoltage and field rheostat system of speed control is extensively used in machine shops for driving machine tools where it is desired to make arrangements quickly to drive the tool at that speed which will accomplish a particular kind of work in the shortest time.

The advantage of the multivoltage method over the armature rheostat method of speed control in securing a greater constancy of speed under variations of load, is well illustrated by the experimental data in the following table. These observations were

* In practice a single main generator is used, and this main generator operates one or more motor-generator sets, technically called balancers, for subdividing the voltage of the main generator, as explained in the chapter on electrical distribution. 
made upon a motor-driven lathe turning a piece of steel $3 \frac{3}{4}$ inches in diameter. The speed, which has been reduced to a low value by an armature rheostat, rises from 60.8 to 2 IO feet per minute when the depth of cut is reduced from $1 / 8$ inch to zero ; whereas, when the speed has been reduced to the desired low value by using $E_{x}=60$ volts instead of $E_{x}=236$ volts, the speed rises only from 60 to $7 \mathrm{I}$ feet per minute when the depth of cut is reduced to zero.

\begin{tabular}{|c|c|c|c|c|c|c|c|c|}
\hline \multirow{3}{*}{ 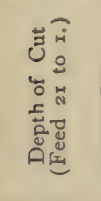 } & \multicolumn{4}{|c|}{$\begin{array}{l}\text { ARMATURE RHEOSTAT CONTROL. } \\
\text { (Rheostat Adjusted to Give a Desired } \\
\text { Speed with the Full Depth of Cut ) }\end{array}$} & \multicolumn{4}{|c|}{$\begin{array}{l}\text { MULTivoltagr Control. } \\
\text { (Speed Reduced to Desired Value with the } \\
\text { Full Cut by Using a 6o-Volt Supply } \\
\text { lnstead of a } 236-\text { Volt Supply. }\end{array}$} \\
\hline & \multirow{2}{*}{$\begin{array}{l}\text { Amperes. } \\
\text { Armature. }\end{array}$} & \multicolumn{2}{|c|}{ Volts. } & \multirow{2}{*}{$\begin{array}{c}\text { Cutting Speed } \\
\text { in F eet per } \\
\text { Minute. }\end{array}$} & \multirow{2}{*}{$\begin{array}{l}\text { Amperes. } \\
\text { Armature. }\end{array}$} & \multicolumn{2}{|c|}{ Volts. } & \multirow{2}{*}{$\begin{array}{l}\text { Cutting Speed } \\
\text { in F Feet per } \\
\text { Minute. }\end{array}$} \\
\hline & & Armature. & Line. & & & Armature. & Line. & \\
\hline \multirow{6}{*}{$\begin{array}{l}\frac{1}{8} \text { inch. } \\
\frac{1}{16} \quad 6 \\
\frac{1}{32} \quad 6 \\
\frac{1}{64} \text { 66 } \\
\text { Zero. }\end{array}$} & & 53 & 236 & 60. & 48 & 5 & $6 c$ & 6 \\
\hline & & 105 & 236 & 12 & 34 & 5 & 6 & \\
\hline & & 143. & 23 & 16 & 2 & 5 & 6 & \\
\hline & & 146.6 & 23 & 172 & 2 & 5 & & 6 \\
\hline & & 179 & 236 & 210 & 28 & 57 & 60 & 71 \\
\hline & \multicolumn{4}{|c|}{ Field current $0.82 \mathrm{amp}$. } & \multicolumn{4}{|c|}{ Field current $0.82 \mathrm{amp}}$. \\
\hline
\end{tabular}

The Ward Leonard system of speed control is perhaps more expensive than the multivoltage system, but it is the most complete method of all, giving as it does a finely graded control of speed from full speed down to zero speed. This system is used to a considerable extent for operating guns and turrets on warships where completeness and fineness of control is of prime importance, and where the question of cost is secondary.

A disadvantage which is common to all methods of speed control of an electric motor, where the motor is to supply approximately the same amount of power at all speeds, is that the motor must be very large to be able to deliver the requisite amount of power when it runs at the lowest speed, and that the capacity of the motor is not fully utilized when it runs at higher speeds. A much smaller and cheaper motor could take the place of the large variable speed motor, if the small motor were driven at constant full speed, and if the variations of speed of the driven machine were accomplished by mechanical means; and the 
smaller motor would run at a higher efficiency. The use of variable speed motors is justified, however, in many cases because of the great ease and quickness with which speed changes can be accomplished.

59. The shunt motor starting rheostat. - From equation $(23 c)$ it is evident that an excessively large current, $I_{a}\left[=\left(E_{x}-\Phi Z^{\prime} n\right) / R_{a}\right]$ will flow through the motor armature at starting if the armature terminals are connected directly to the supply mains, inasmuch as $\Phi Z^{\prime} n$ is zero at starting, since $n$ is then zero, and the resistance $R_{a}$ of the armature is small. It is necessary to prevent this exces-

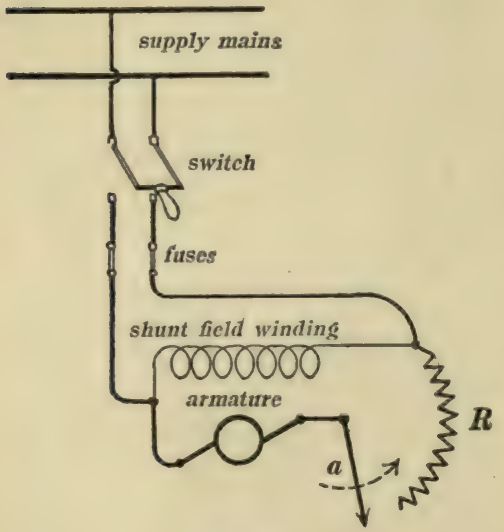

Fig. 83. sive flow of current at starting by connecting an auxiliary resistance in series with the motor armature (not in series with the field). While the motor is speeding up, the value of $\Phi Z^{\prime} n$ increases, and the current tending to decrease, it is permissible to gradually cut out the auxiliary resistance. When the motor has reached nearly full speed, the auxiliary resistance is wholly cut out. This auxiliary resistance, when conveniently arranged for being put in series with the armature and slowly cut out as the motor speeds up, is called a starting rheostat.

Fig. 83 shows the simplest arrangement of a shunt motor with a starting rheostat, the motor being connected to the supply mains through a double-pole single-throw switch. The closing of this switch connects the field circuit of the motor to the mains, the first movement of the rheostat arm $a$ connects the armature to the supply mains through the whole of the starting resistance $R$, and the continued movement of the rheostat arm slowly cuts out the starting resistance. 
Starting rheostat with automatic overload release and dead line* release. - The most frequent causes of trouble with a shunt motor are as follows: $(a)$ The motor may be running normally when, for some reason or other, the supply of current fails, the line wires becoming "dead." When this happens the motor of course stops, but the connections to the supply mains remain intact, with all of the resistance cut out of the starting rheostat. When the supply of current is again established under these conditions, an excessive amount of current flows through the motor armature and it is likely to be burned out. In service, a motor should be protected from damage in this way by a dead line release which automatically throws the arm of the starting rheostat back to the starting position when the supply of current fails.

(b) The motor may be greatly overloaded. When this happens the motor takes excessive current from the supply mains, and the motor may be overheated or even burned out. In service, a motor should be protected from damage in this way by an overload release which automatically throws the arm of the starting rheostat to the starting position when an excessive current flows through the motor armature.

The electrical connections of a shunt motor starting box with automatic dead line release and overload release, are shown in Fig. 84. The motor armature is represented by $A$, and the shunt field winding by $F$. To start the motor, the switch is closed, and the rheostat arm $C$ is slowly moved in the direction of the dotted arrow until it reaches $B$. The first move of the arm $C$ connects the shunt field winding directly to the mains, and at the same time it connects the armature to the mains through the resistances $R R$. The continued movement of the arm cuts the resistance out of the armature circuit. In the particular arrangement shown in Fig. 84, the resistances $R R$ are left in series with

* The name applied to this device by the manufacturers is the "starting rheostat with overload and no-load release." This name is, however, misleading, inasmuch as what is called the "no-load release" operates only when the line becomes dead, but does not operate when the load on the motor is reduced to zero. 
the field winding as they are cut out of the armature circuit, but this has but little effect upon the field excitation, inasmuch as the resistances $R R$ are very small in comparison with the resistance

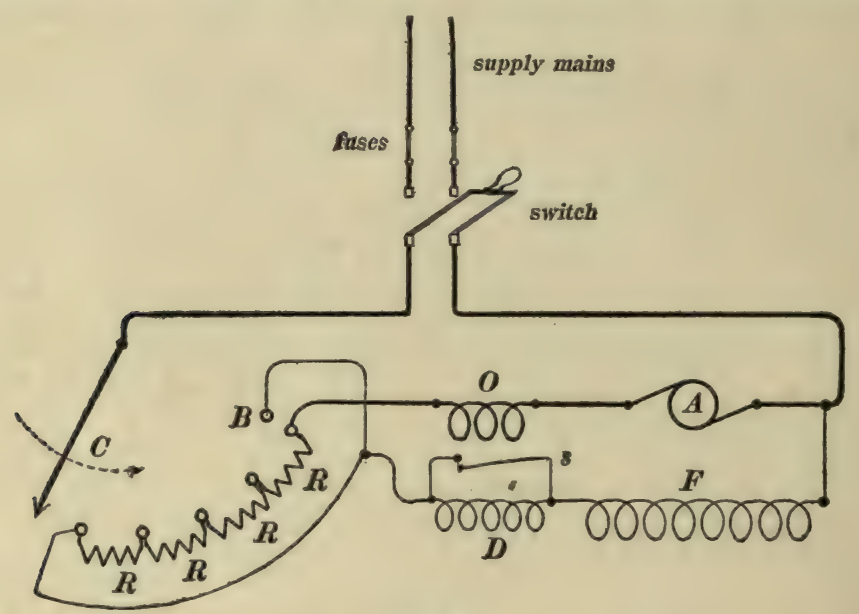

Fig. 84 .

of the shunt field winding, and the resistances $R R$ are cut out of the field circuit when the arm $C$ touches the contact point $B$.

When the rheostat arm $C$ is pushed to the right in starting the motor, it stretches a spring and the rheostat arm is held in the running position by an electromagnet, of which the winding $D$ is in series with the field winding $F$ of the motor. When the lines become dead, current ceases to flow through $D$ and $F$, the electromagnet releases the rheostat arm, and the stretched spring pulls the arm back to the starting position.

The overload release is actuated by the electromagnet $O$, of which the winding is in series with the motor armature. The armature of this electromagnet $O$ is held back by a spring, the tension of which is so adjusted that the lever may be moved by a prescribed value of current through $O$. When this value of current is reached, the movement of the armature of $O$ actuates a small switch $s$ which short-circuits the winding $D$. This causes the electromagnet $D$ to lose its magnetism, and release the arm $C$ as before. 
Fig. $85 a$ is a general view of one of the General Electric Company's shunt motor starting rheostats with an automatic dead line release, and Fig. $85 b$ is a complete diagram of the connections of this starter to a motor, and, through a double pole switch and two fuses, to the supply mains. Fig. $85 a$ shows the rheostat arm in the starting position, and Fig. $85 b$ shows the arm in the running position. To start the motor, close the switch, and move the rheostat arm slowly to the right until the block of

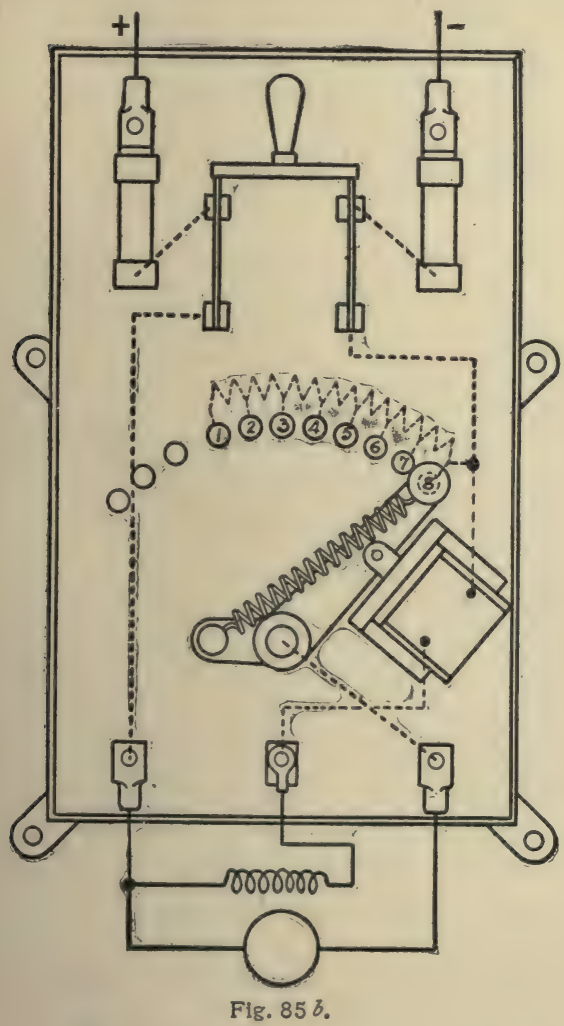

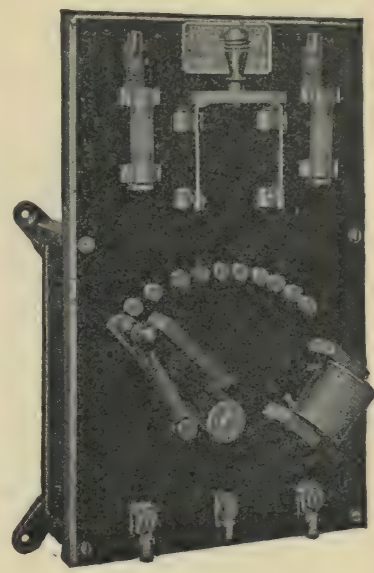

Fig. $85 a$.

iron on the rheostat arm comes against the retaining electromagnet, of which the winding is in series with the field winding of the motor, as shown in Fig. $85 b$.

Fig. 86 is a view of one of the General Electric Company's automatic double release (dead line and overload) starting rheostats for a shunt motor. The figure shows the rheostat arm in the starting position. This starter is usually connected to the supply mains through a doublepole switch and two fuses. To start the motor the switch is closed, and the rheostat arm is moved 
slowly to the running position where it is held by the retaining electromagnet. The connections of this starting box are shown

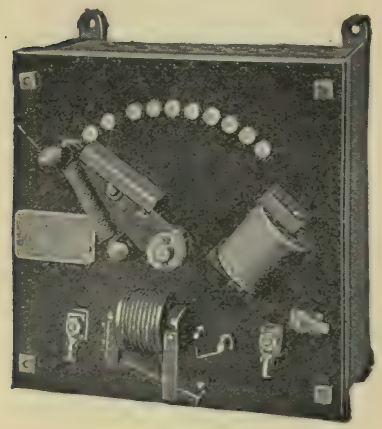

Fig. 86. in Fig. 84, and the discussion of that figure gives the details of its action.

Precautions to be observed in the operation of a shunt motor. - Always start cautiously. If the motor does not start promptly with the first move of the arm, it is likely that the field circuit is broken, or that the load on the motor is excessive. Do not allow the motor to run long with the rheostat arm not in the running position, as the resistance in a starting rheostat overheats if it is left permanently in circuit with the armature. To stop the motor, open the switch and leave the starter arm to set itself automatically at the starting position. Further instructions for the operation of generators and motors are given in Chapter VII.

60. The compound motor. - There are two arrangements of the compound motor, according to whether the current in the series winding opposes the magnetizing action of the shunt winding, or helps it. The first arrangement is called the differential compound winding, and the second arrangement is called the cumulative compound winding.

The differential compound motor. - When a differential compound motor is loaded, the current through the armature and the series field winding increases, and, by opposing the magnetizing action of the current in the shunt field winding, reduces the armature flux $\Phi$, and thus decreases the counter electromotive force $\Phi Z^{\prime} n$, if the speed $n$ remains constant. If this decrease of $\Phi$ is just sufficient to give the requisite decrease of counter electromotive force to permit enough current to flow through the armature to enable the motor to carry its load, then the motor speed will not fall off with increase of load, at least not until the load 
becomes excessive. The object of the differential compounding of a motor is to prevent the decrease of speed with increase of load, or even to cause an actual increase of speed with increase of load up to full load. The shunt motor, however, runs at a speed which is nearly enough constant for most purposes, and therefore the differential compound motor is seldom used.

The cumulative compound motor. - The speed of this motor decreases very considerably with increase of load. Such a motor has, however, the great advantage that at starting, when the current through the armature and series field winding is large, the total field excitation is large and hence the torque is large. That is to say, the cumulative compound motor has a large starting torque like the series motor, as explained later.

At starting, when the current through the armature and series field winding of this motor is large, the total field excitation is very large. The advantage of this large field excitation (large $\Phi$ ) at starting is twofold: $(a)$ the torque is large $\left(=\mathrm{I} .4 \mathrm{I} \Phi Z^{\prime} I_{a}\right.$ according to equation 25$)$, and (b) the counter electromotive force, $\Phi Z^{\prime} n$, is larger than it would be with a smaller value of $\Phi$. The result is $(a)$ that the motor accelerates more rapidly, and (b) that during the time that the motor is speeding up its efficiency is greater than it would be if its counter electromotive force were smaller in value.

The cumulative compound motor has the disadvantage that its running speed decreases very considerably with increase of load, on account of the increase of $\Phi$ produced by the series field winding. This disadvantage may, however, be obviated by providing a device for automatically cutting out the series field winding by short-circuiting it after the motor is fairly started. The machine then runs as a simple shunt motor.

Cumulative compound motors are frequently used for operating elevators and hoists, rolling mills, and other machinery where large starting torque is required, and where constancy of speed under variations of load is not necessary. The advantage of the cumulative compound motor over the series motor for service of 
the kind above specified (where the load may not be always and inseparably connected to the motor) is, that the former motor will not speed up indefinitely ( $r a c e$ ) when its load is thrown off.

The simple shunt motor may be made to operate more or less like a differential compound motor by giving the brushes a backward lead (see Art. 5 I), or like a cumulative compound motor by giving the brushes a forward lead. This is due to the fact that with a backward lead of the brushes of a motor, the armature current opposes the magnetizing action of the shunt field winding, and that with a forward lead of the brushes, the armature current helps the magnetizing action of the shunt field winding.

61. The series motor. - Series motors are used (with constant or approximately constant voltage supply mains) only where the motor load is always and inseparably connected to the motor, and where constancy of speed with variations of load is not

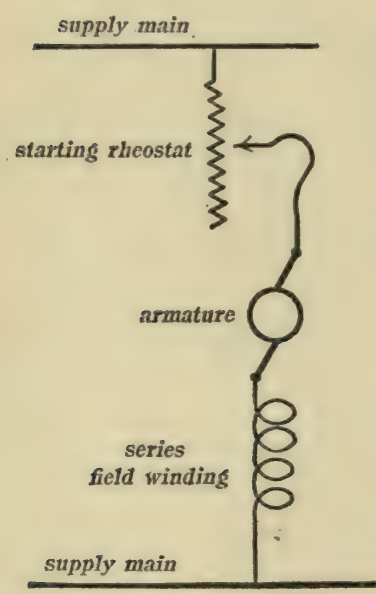

Fig. 87. especially desired. Thus series motors are much used for driving fans, where the fan is inseparably attached to the motor, and for driving automobiles and street cars. The necessity of having the load of a series motor attached to it inseparably is due to the fact that if a series motor, connected between constant voltage supply mains, were to have its load reduced more and more, its speed would rise indefinitely, and the enormous centrifugal forces would in all probability wreck the armature.

Fig. 87 shows the connections of a series fan motor with its starting rheostat. At starting considerable resistance is connected in series with the motor, and as the motor speeds up this resistance is slowly cut out.

When a street car is operated by a single series motor, the 
connections of the motor to trolley wire and rail are essentially the same as the connections shown in Fig. 87, except that provision is made for quickly reversing the armature connections (or the field connections) in order to reverse the direction of running of the motor.

In street car service, where stopping and starting is frequent, the motor runs for a great portion of the time at low speed with a considerable resistance in series with it. Under these conditions most of the energy supplied to the motor circuit is lost as heat in the rheostat, and the efficiency of the motor is very low.

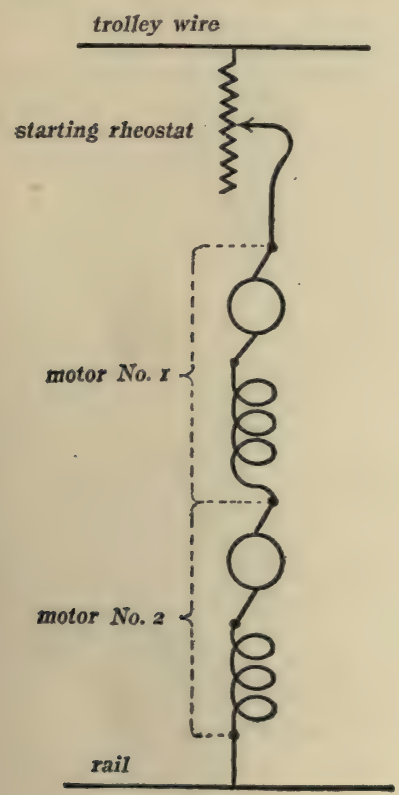

Fig. 88

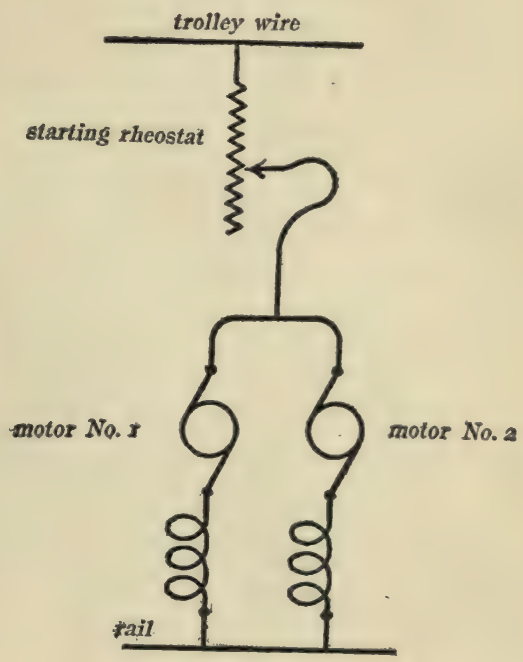

Fig. 89.

Some idea of the efficiency may be obtained with the help of Siemens' law. (See Art. 54.) The approximate efficiency $E_{a} / E_{x}$ is small at starting, inasmuch as the counter electromotive force, $E_{a}\left(=\Phi Z^{\prime} n\right)$, is small when the speed is small. By using two motors on a car, and arranging to have them connected in series at starting, the counter electromotive force is doubled, and the efficiency is nearly doubled. 
The two motors in series are of course started with resistance in circuit as shown in Fig. 88, and this resistance is slowly cut out in steps as the speed increases. When the speed has reached, say, Io miles per hour and the resistance is all cut out, the connections are quickly changed $*$ to those shown in Fig. 89. That is, the motors are quickly connected in parallel and the starting resistance is connected in series with them. The speed then continues to rise, and as it rises the starting resistance is again slowly cut out.

The use of two series † motors in this way is called the " seriesparallel system of control." All of the successive connections described above are made in the proper order by turning a crank which actuates a spindle upon which metal sectors are mounted as shown in Fig. 90. This figure shows a controller spindle with eleven sectors (some of which are hidden behind the spindle) and eleven contact-fingers which touch the sectors when the spindle is turned. The small cylinder at the upper right hand in the figure is arranged to make the necessary changes of connections of the armature terminals for reversing the direction of driving of the motors, by turning the small lever at the top. The large oval structure is an electromagnet, one pole piece of which is spread out in the region behind the controller spindle, and the other pole piece is hinged to the core of the electromagnet, so that it may be swung back, as shown in the figure,

* This change of connections is actually carried out in four distinct steps made in rapid succession as follows: $(a)$ Some resistance is reconnected in series with the two motors. (b) One motor, No. 2, Fig. 88, is then short-circuited, and this short-circuit constitutes a connection from motor No. I to the rail. (c) The rail terminal of motor No. 2 is then disconnected. (d) This terminal of motor No. 2 is then connected to the rheostat terminal of motor No. I, giving the connection shown in Fig. 89. During operation $(b)$ motor No. 2 cannot build up as a generator, inasmuch as it is running in the wrong direction.

† Sometimes four series motors are used on one car. With such a four-motor equipment it is customary to connect the motors as two units, each unit consisting of two motors permanently connected in parallel. These two units are then treated as two single motors, and operated by the series-parallel controllers exactly as explained above; that is, at starting the two units are in series and at full speed the two units are in parallel. 
THE OPERATION OF THE DYNAMO AS A MOTOR. I2I

to give access to the controller spindle and contact fingers. This swinging pole piece carries twelve plates of a compressed asbestos insulating compound which serve as fire-proof partitions between the various pairs of sectors and fingers. The object of the electromagnet is to "blow out" the arcs which form between the sectors and fingers when connections are broken by turning

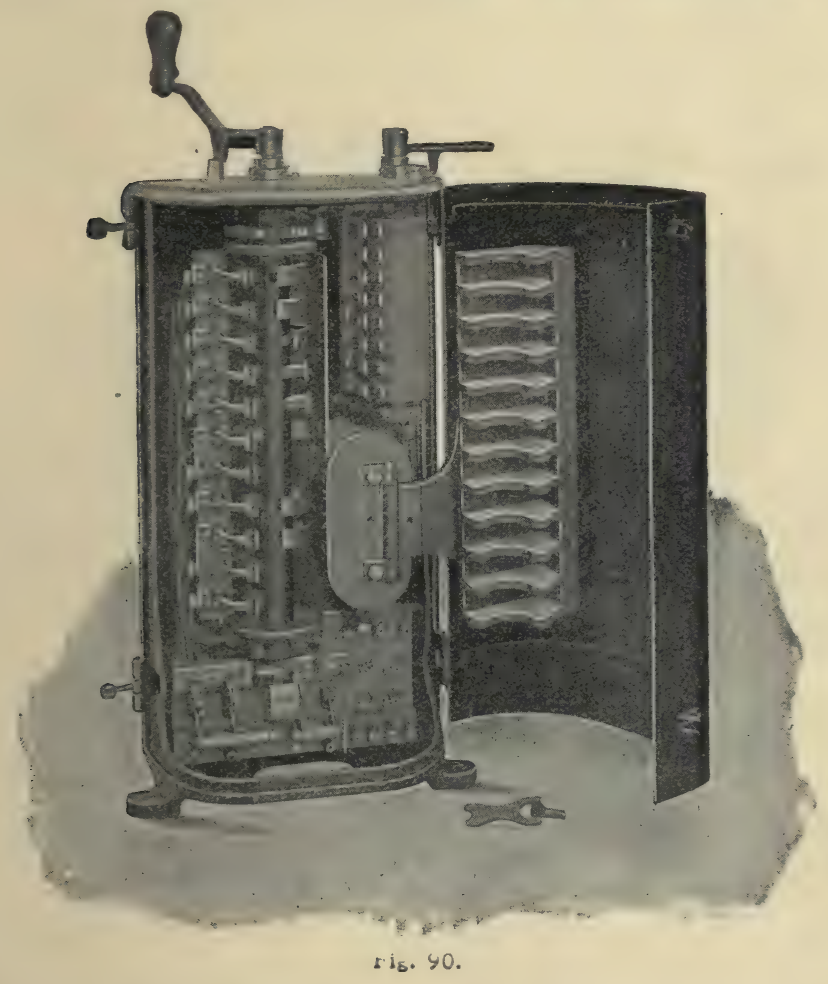

the spindle, and the asbestos plates serve to prevent the arcs from flashing from sector to sector or from finger to finger. The apparatus as a whole is called a scries parallel controller. Fig. 90 shows a series-parallel controller of the General Electric Company.

Fig. 9I shows a typical street railway motor. The motor, commutator and all, is enclosed in a steel case to protect it from 
dust and moisture. The field poles, usually four in number, project inwardly from this steel case which serves as a field magnet yoke. The figure shows the lower half of the motor case dropped, thus exposing the armature and commutator to view. The motor case is supported at one side by the car axle, the two axle bearings being shown in Fig. 9I, and on the opposite side the case is supported by a spring which rests on the truck frame. This gives the heavy motor structure that freedom of motion

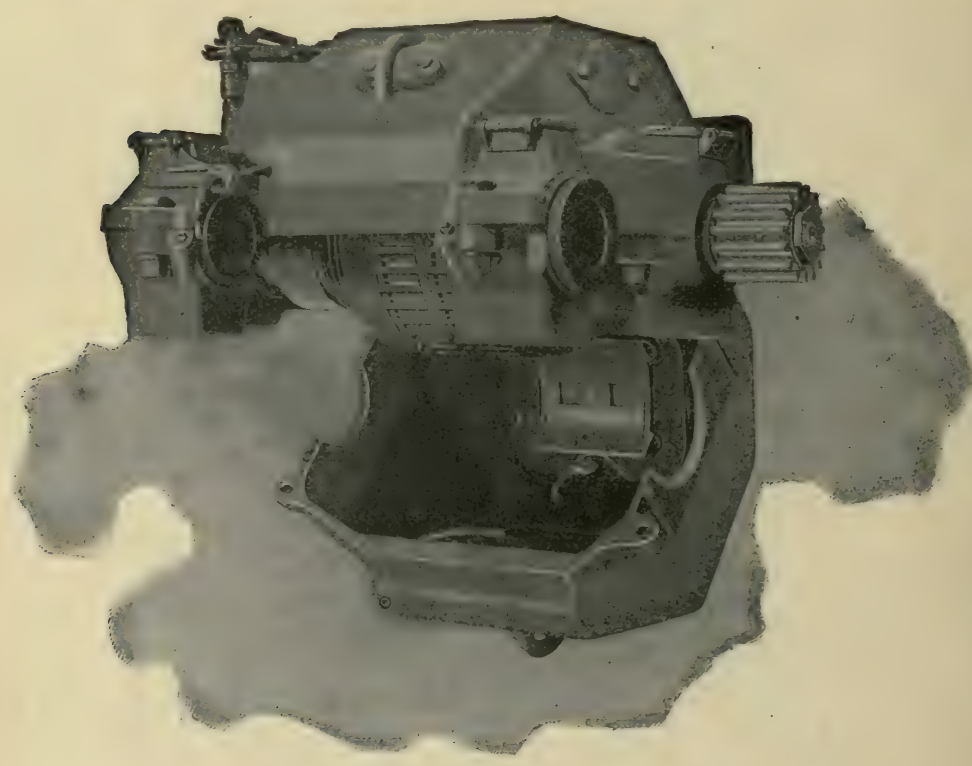

Fig. 91.

which is necessary when the car is running over a rough track at high speed, but at the same time the car axle and the armature shaft are at a fixed distance apart, so as to accommodate the spur gears which transmit power from the armature to the axle. The spur gear shown in Fig. 9I is attached to the projecting end of the armature shaft. The wire-leads from the brushes and from the series field windings are brought out of the steel case through rubber bushings and connect through flexible insulated cables to 
the rheostats and controlling devices. The brushes are not supported in a rocker arm but in rigid holders attached to the inside of the case, and when the brushes are badly worn and need to be replaced, they may be reached through a hand hole which is covered by a metal lid one end of which shows in Fig. 9I.

62. Characteristic curves of motors. The behavior of a motor under given electrical conditions of driving is most clearly represented by a curve showing the relationship between any two of the following variable quantities : speed, torque, and mechanical power output. When the current is supplied from constant vol-

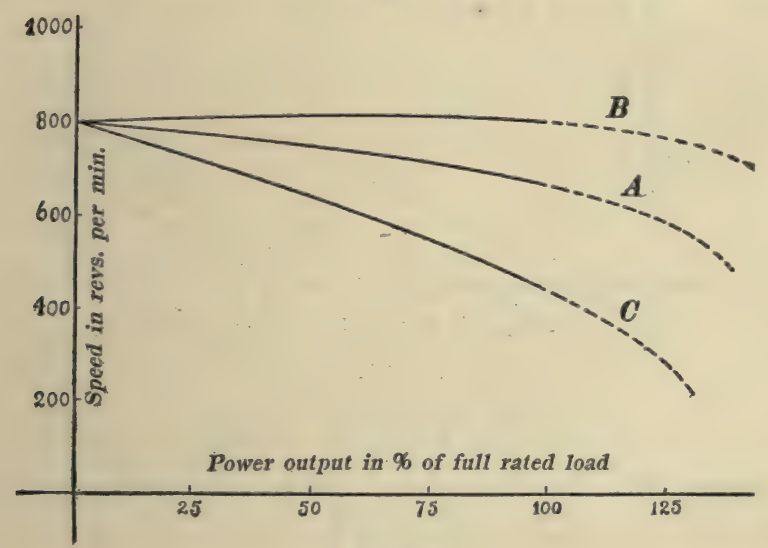

Fig. 92.

tage mains, it is often convenient to show by a curve the relationship between the current and any one of the three mechanical factors : speed, torque, and power output. Such a curve is called, in general, a characteristic curve of the motor. In speaking of a characteristic curve it is customary to designate it in terms of the quantities which are represented by its coördinates, always naming its ordinates first, and its abscissas second. For example, a speed-torque characteristic would be a curve of which the ordinates represent speeds, and the abscissas represent torques.

(a) Shunt and compound motor characteristics. - The curve $A$, Fig. 92 , is a speed-power characteristic of a simple shunt 
motor, curve $B$ is a speed-power characteristic of a differential compound motor, and curve $C$ is a speed-power characteristic of a cumulative compound motor.

(b) The series motor. - Fig. 93 shows a speed-torque characteristic of a series motor. This curve shows the enormous rise in speed at light loads (very small torques).

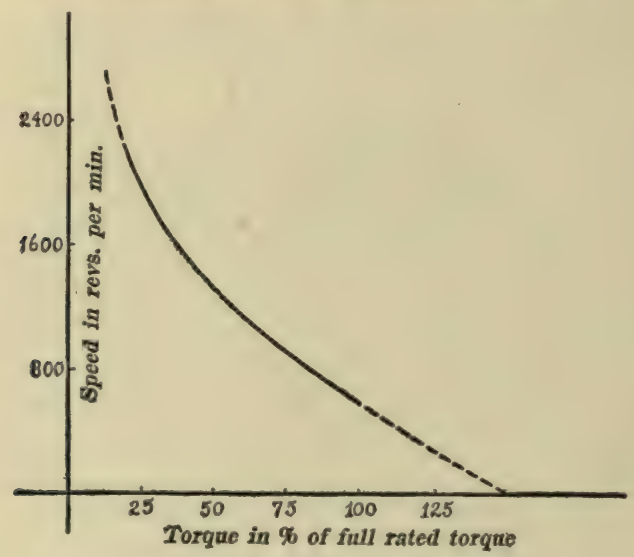

Fig. 93.

The characteristics of street railway motors, as usually plotted, are as follows: (a) The speed-current curve in which the ordinates represent the speed of the car in miles per hour, instead of the speed of the motor armature in revolutions per minute; and (b) the tractive-effort-current curve in which the ordinates represent the net tractive effort in pounds weight exerted, instead of the torque in pound-inches developed by the motor armature. Such characteristic curves involve the gear ratio of the motor, and the diameter of the drive wheels on the car.

The curve marked "miles per hour" in Fig. 94 is a speedcurrent curve of a 25 -horse power Westinghouse railway motor, and the curve marked " tractive-effort 33 -inch wheel" is the tractive-effort-current curve of the same motor under the following conditions: The electromotive force between trolley wire and rail is 500 volts, and the motor is connected between the trolley 
THE OPERATION OF THE DYNAMO AS A MOTOR. I25

wire and the rail without any rheostat in its circuit. Of course if two of these motors are used in parallel without any resistance connected in series with them, the total tractive effort will be twice as great at a given speed as that shown in Fig. 94.

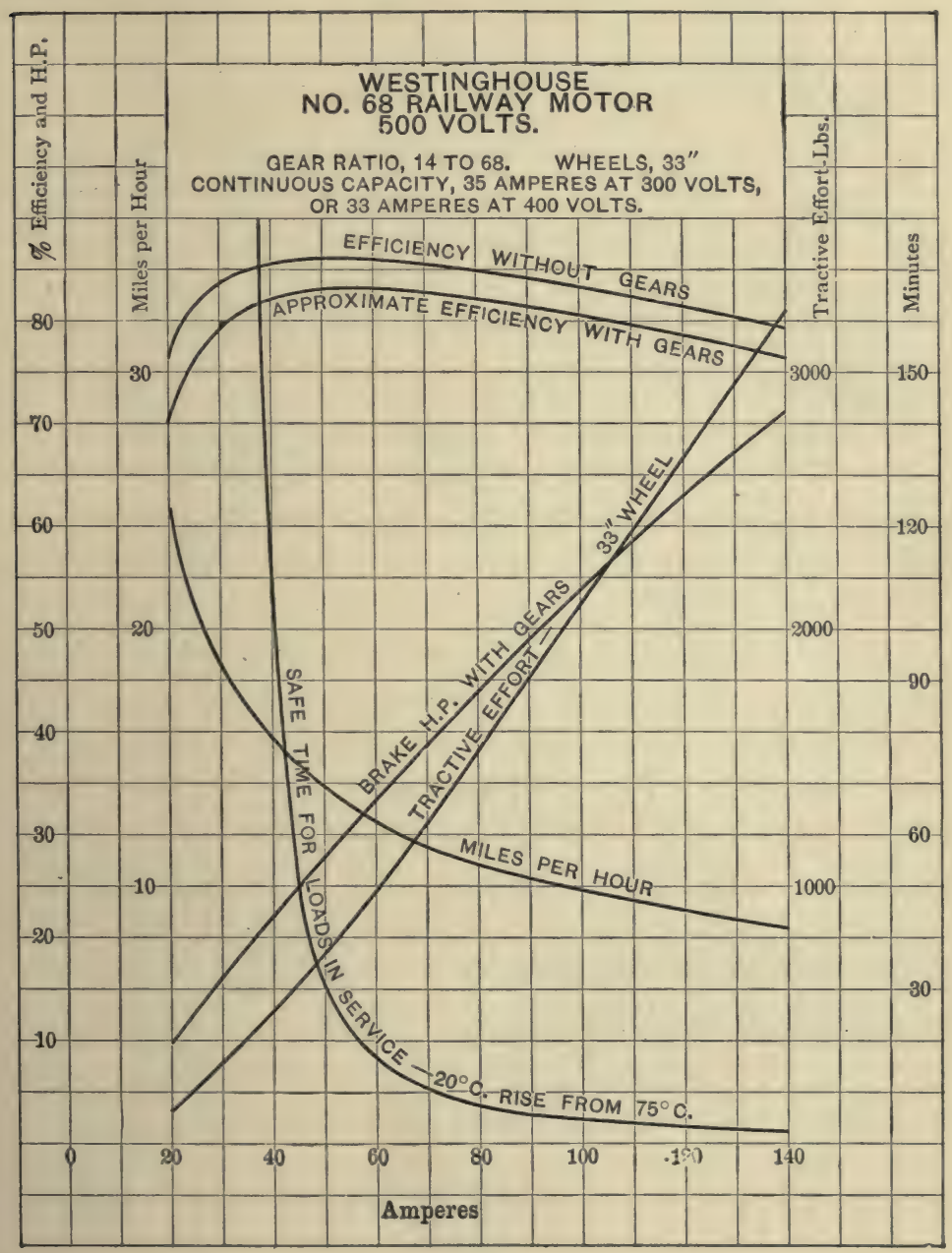

Fig. 94.

The general trend of the speed-torque curve of the series motor supplied from constant voltage mains, as shown in Fig. 93 , 
can be explained as follows: When the motor speed is small, the counter-electromotive force developed in the armature is small, so that a large current flows through the series field winding and through the armature. Therefore the field excitation is large, the flux $\Phi$ is large, and the torque is large according to equation (25). As the speed increases, the torque falls off on account of decrease of current due to increase of counter-electromotive force, and on account of the decrease of $\Phi$ due to the decrease of current. If the torque required to turn the armature is small, as it is when the load on the motor is zero, then the motor speed will increase greatly.

There is in the case of the series motor, no well-defined zeroload speed as there is in the case of the shunt motor. Any motor at zero-load approaches that speed for which its counterelectromotive force, $\Phi Z^{\prime} n$, is equal to the electromotive force of supply. A motor at zero-load approaches this limiting speed more and more nearly the smaller the resistance of its armature, or, for a given armature resistance, the smaller its power losses. In the shunt motor the armature flux $\Phi$ is nearly constant irrespective of speed and of load, and therefore a perfectly definite speed (the zero-load speed) gives a counter-electromotive force equal to the electromotive force of supply. In the series motor, on the other hand, the armature flux $\Phi$ approaches zero with decrease of load on account of the decrease of current with decrease of load, so that an indefinitely high speed would be necessary at zero load, to make $\Phi Z^{\prime} n$ equal to $E$ the electromotive force of supply. 


\section{CHAPTER V.}

POWER LOSSES IN GENERATORS AND MOTORS. EFFICIENCY.

63. Motor and generator losses. - The losses of power in a generator or in a motor are: the field loss $F$, the armature loss $A$, and the stray power loss $S$. The field loss is due to the heat generated in the field windings by the field current, that is, no power is required to excite the field magnet except the power used to overcome the resistance of the field winding. The armature loss, as here defined, is due to the heat generated by the armature current in the brushes, in the brush contacts, and in the armature windings. Field and armature losses can be accurately calculated as explained below. The stray power loss includes eddy current and hysteresis losses, chiefly in the armature core, and losses due to friction in the bearings and at the brushes, and to air friction. Stray power loss cannot be accurately calculated from any simple data. This loss is usually determined by experiment.

(a) Ficld loss. - A certain amount of power is consumed in maintaining the field current of a dynamo. All this power goes to heat the field coils in accordance with Joule's law, that is, no power would be consumed in field excitation if it were possible to make the field windings of a material having zero resistance. The field loss in watts may be calculated by means of the following equations.

(I) For a shunt dynamo.

or

$$
F=R_{s} I_{s}^{2}
$$

$$
F=E_{x} I
$$

or 


$$
F=\frac{E_{x}^{2}}{R}
$$

in which $R_{s}$ is the resistance of the shunt winding including the field rheostat, $I_{s}$ is the current in the shunt winding, and $E_{x}$ is the electromotive force between the terminals of the machine. The resistance of the shunt winding increases considerably after the machine is started, on account of rise of temperature. In calculating field loss, the resistance of the winding at the steady running temperature of the machine should be used; this is frequently called the hot resistance, for brevity.

The field loss of a shunt dynamo (generator or motor) is approximately constant. Thus the field winding of a shunt motor is usually connected directly to the constant voltage mains, and, after the steady running temperature has been reached, the field loss is strictly constant whatever the load on the motor may be. On the other hand, the terminal voltage of a shunt generator varies more or less with its current output and, inasmuch as the field winding is connected across the terminals of the machine, the field current, and therefore the field loss also, vary slightly. When the terminal voltage of a shunt generator is kept constant with increase of current output by the manipulation of a field rheostat, the field current and also the field loss change considerably.

(2) For a compound dynamo:

$$
F=R_{s} I_{s}^{2}+R_{c} I_{c}^{2}
$$

in which $R_{s}$ and $I_{s}$ have the same significance as in equation (27a), $R_{c}$ is the resistance of the series field winding, and $I_{c}$ is the current in the series field winding.

In applying equation (28) to "long shunt" or "short-shunt" compound dynamos, careful attention must be given to the diagram of connections to insure that the values of $I_{s}$ and $I_{c}$ are correctly derived from such data as may be given.

(3) For a series dynamo equation (28) may be used by dropping 
the term $R_{s} I_{s}^{2}$. The field loss of a series dynamo (generator or motor) varies greatly with the load.

(b) Armature loss. - The power losses in the armature of a dynamo include the losses in the armature core due to eddy currents and hysteresis. These core losses are, however, usually included under the term "stray power" loss. By armature loss is here meant simply the power which is consumed in the armature windings in accordance with Joule's law, together with the power lost at the brushes on account of contact resistance. The armature loss in watts may be calculated from the equation :

$$
A=R_{a} I_{a}^{2}
$$

in which $R_{a}$ is the resistance of the armature from positive to negative brushes; $R_{a}$ includes the resistance (hot) of the armature windings, the contact resistances of the brushes, and the resistances of the brushes themselves; and $I_{a}$ is the total current leaving the armature at the positive brushes, or entering the armature at the negative brushes.

Armature loss varies greatly with the current output of the generator, and with the amount of load on a motor.

(c) Stray power loss. - This term includes all losses * which cannot be satisfactorily calculated from simple data. These losses are -

(I) Eddy current and hysteresis losses, chiefly in the armature core, due to reversals of magnetization as the armature rotates. Considerable eddy current loss may occur in the massive copper conductors of a bar-wound armature is explained in Art. 32.

(2) Friction losses in the bearings and at the brushes, and

(3) Air friction loss, or windage, as it is called, due to the fanlike action of the rotating armature.

These losses cannot be separately measured with accuracy. The stray power loss which is the sum of these losses can, however, be quite accurately determined for a given machine by an experimental test.

* That is, all losses except $R I^{2}$ losses. 
When a dynamo runs at constant speed with constant field excitation, the stray power loss is approximately constant, irrespective of the current output of the machine if it is acting as a generator, and irrespective of the load on the machine if it is acting as a.motor. Therefore the stray power loss of a shunt generator driven at constant speed or of a shunt motor supplied from constant voltage mains, is approximately constant irrespective of the load on the machine. The stray power loss of a series generator or motor varies greatly with its load.

It is often desired to find the stray power $S^{\prime \prime}$, in a dynamo (generator or motor), when run at such speed and field excitation as to give an armature voltage $E_{a}^{\prime \prime}$, having given the experimentally determined value $S^{\prime}$ of the stray power loss of the dynamo when run at such speed and field excitation as to give an armature voltage $E_{a}{ }^{\prime}$. Now the various component parts of the stray power loss are very different functions of field excitation and speed, and the dependence of $S$ upon field excitation and speed cannot be accurately represented by any simple formula. For small variations of field excitation and speed, however, it is sufficiently exact to assume that the stray power loss is proportional to the electromotive force induced in the armature, from which we obtain the equation :

$$
S^{\prime \prime}=S^{\prime} \frac{E_{a}^{\prime \prime}}{E_{a}^{\prime}}
$$

64. Experimental determination of stray power loss. - The stray power loss of a dynamo cannot be satisfactorily calculated from any easily obtainable data. The simplest method for determining the stray power loss of a dynamo at a prescribed speed and prescribed degree of field excitation, is to observe the power $P$ required to drive the dynamo as a motor, without any load, at the prescribed speed, and with its field excited to the prescribed degree. This power $P$ exceeds the stray power loss $S$ by a very small amount, which can be calculated as explained below. 
The prescribed degree of field excitation may be specified in three different ways : $(a)$ In terms of the current that is to be used in the field winding; $(b)$ in terms of the voltage that is to be applied to the terminals of the field winding; or $(c)$ in terms of the electromotive force that is to be generated in the armature at the prescribed speed.

Let us consider, for example, a shunt dynamo of which the stray power loss is to be determined at a speed of, say, 1,000 revolutions per minute with its field excited by applying an electromotive force of I I o volts to the terminals of the field winding.

Let the machine be separately excited by connecting its field winding to I IO-volt supply mains, and let current be supplied to the armature through an ammeter and an adjustable resistance. By adjusting this resistance the machine is made to run as a motor at zero load at the prescribed speed. The armature current, $I_{a}$, is then read off the ammeter, and the voltage $E$ is read off a voltmeter connected between the brushes.

Inasmuch as the machine is driven as a motor without load, all of the power, $E I_{a}$, which is delivered to the armature, is used to supply the stray power loss $S$, and the armature loss $R_{a} I_{a}^{2}$, so that

$$
S=E I_{a}-R_{a} I_{a}^{2}
$$

This gives the stray power loss under the prescribed conditions as to field excitation and speed. The stray power loss at full load (slightly different value of $E_{a}$ from that which obtains at zero load) may be approximately calculated from the above value of $S$ by using equation (30).

Example.-A given dynamo, rated as a roo-volt, 50-ampere generator, when driven at a speed of $\mathrm{I}, 200$ revolutions per minute, is tested for stray power loss as follows: The field is separately excited by being connected to I IO-volt mains in series with an adjustable rheostat. The armature terminals are connected to the I I O-volt mains also, through an ammeter and an adjustable rheostat, and the machine starts, running as a motor. A voltmeter 
is connected to the armature terminals, and the two rheostats are adjusted until the machine runs at I,200 revolutions per minute, and shows an electromotive force of 100 volts between the brushes. The ammeter then shows that 2.4 amperes is flowing through the armature. The resistance of the armature (hot) is found by measurement to be $0.25 \mathrm{ohm}$.

From these data the power $P$ delivered to the armature is found to be $P=2.4$ amp. $\times 100$ volts $=240$ watts, and the armature loss corresponding to the current of 2.4 amperes is $(2.4 \mathrm{amp} .)^{2} \times$ $0.25 \mathrm{ohm}=\mathrm{I} .44$ watts, so that the stray power loss under the condition of the test is 240 watts - I.44 watts, or 238.56 watts. A slight correction to this result may be applied to find the value of $S$, corresponding to a speed of $\mathrm{I}, 200$ revolutions per minute and $E_{a}$ equal to 100 volts, as follows: Under the conditions of the test, IOO volts $=E_{x}=E_{a}+R_{a} I_{a}$, where $R_{a}=0.25 \mathrm{ohm}$ and $I_{a}=2.4$ amperes, so that $E_{a}$ of the test is equal to 99.4 volts. Therefore, according to equation (30), the value of $S$, corresponding to $E_{a}=100$ volts, is approximately

$$
238.56 \text { watts } \times \frac{100}{99.4}=239.8 \text { watts }
$$

If it were desired to find from the above test the approximate value of $S$ for a speed of I, IOO revolutions per minute and a field excitation which gives with this speed $E_{a}=105$ volts, equation (30) would give

$$
S=238.56 \text { watts } \times \frac{105}{99.4}=251.8 \text { watts }
$$

65. Efficiency of a Generator.-The efficiency of a generator is defined as the ratio : output of power divided by intake of power. This ratio is sometimes called the true efficiency or the commercial efficiency to avoid confusing it with the two partial efficiencies defined later.

Calculation of true efficiency.-When the stray power loss of a dynamo has been determined for a given speed and a given volt- 
age, it is easy to calculate the true efficiency of the dynamo for a specified output of power as a generator at the given speed and voltage, the resistances of the various windings of the dynamo being known. This calculation is based upon the equation

$$
\text { efficiency }=\frac{\text { power output }}{\text { power output }+ \text { losses }}
$$

It is much more instructive to discuss this matter of efficiency calculation by means of several typical numerical examples, than by deriving a general formula.

Example I. The shunt generator. - Given a shunt generator which delivers 50 amperes of current at I IO volts between its terminals. It is required to calculate the efficiency of the generator under these conditions having given the following data:

$R_{s}=44 \mathrm{ohms}$ (hot) including the portion of the field rheostat which must be in circuit to bring the voltage between the terminals of the machine to the specified value.

$R_{\alpha}=0.14 \mathrm{ohm}$ (hot).

Stray power loss at given speed and voltage $=700$ watts Solution :

(a) Power output $=\mathrm{I}$ IO volts $\times 50$ amperes $=5,500$ watts

(b) Field loss $\quad=R_{s} \times\left(\frac{E_{x}}{R_{s}}\right)^{2}=275$ watts

(c) Armature loss $=R_{a}\left(50+\frac{E_{x}}{R_{s}}\right)^{2}=386$ watts

(d) Efficiency $=\frac{5,500}{5,500+275+386+700}=0.80 \mathrm{I}$

Example 2. The compound generator (long-shunt). - Given a long-shunt compound generator which delivers 50 amperes of current at I I volts between its terminals. It is required to calculate the efficiency of the generator under these conditions having given the following data : 


$$
\begin{aligned}
& R_{c}=55 \mathrm{ohms} \text { (hot) } \\
& R_{c}=0.02 \mathrm{ohm} \text { (hot) } \\
& R_{a}=0.14 \mathrm{ohm} \text { (hot) }
\end{aligned}
$$

Stray power loss at given speed and voltage $=700$ watts. Solution :

(a) Power output $=$ I IO volts $\times 50$ amperes $=5,500$ watts

(b) Shunt field loss $=R, \times\left(\frac{E_{x}}{R_{s}}\right)^{2}=220$ watts

(c) Series field loss $=R_{c}\left(50+I_{s}\right)^{2}=54$. I watts

(d) Armature loss $=R_{a}\left(50+I_{s}\right)^{2}=379$ watts

(e) Efficiency

$$
=\frac{5,500}{5,500+220+54 \cdot I+379^{\circ}+700}=0.803
$$

Example 3. The compound generator (short-shunt). - Given a short-shunt compound generator which delivers 50 amperes of current at I IO volts between its terminals. It is required to calculate the efficiency of the generator under these conditions, having given the following data :

$$
\begin{aligned}
& R_{a}=55 \mathrm{ohms} \text { (hot) } \\
& R_{c}=0.02 \mathrm{ohm} \text { (hot) } \\
& R_{a}=0.14 \mathrm{ohm} \text { (hot) }
\end{aligned}
$$

Stray power loss at given speed and voltage $=700$ watts. Solution :

(a) Power output $=$ I IO volts $\times 50$ amperes $=5,500$ watts

(b) Shunt field loss $=R_{s} I_{s}^{2}=224$ watts

$$
\left[E_{s}=E_{x}+R_{c} I_{c}=\text { I I I volts so that } I_{s}=2.018\right.
$$
amperes]

(c) Series field loss $=R_{c} I_{c}^{2}=50$ watts

(d) Armature loss $=R_{a} I_{a}^{2}=379$ watts

(e) Efficiency $\quad=\frac{5,500}{5,500+224+50+379+700}=0.803$

Example 4. The series generator. - Given a series generator which delivers 50 amperes of current at I IO volts between its 
terminals. It is required to calculate its efficiency under these conditions, having given the following data :

$$
\begin{aligned}
& R_{c}=0.12 \mathrm{ohm} \text { (hot) } \\
& R_{a}=0.15 \mathrm{ohm} \text { (hot) }
\end{aligned}
$$

Stray power loss at given speed and voltage $=700$ watts. Solution :

(a) Power output $=50$ amperes $\times$ I 10 volts $=5,500$ watts

(b) Series field loss $=R_{c} I_{c}^{2}=300$ watts

(c) Armature loss $=R_{a} I_{a}^{2}=375$ watts

(d) Efficiency

$$
=\frac{5,500}{5,500+300+375+700}=0.800
$$

66. Efficiency of conversion and electrical efficiency of a generator. - The total mechanical power expended in driving the armature of a generator is used: $(a)$ to supply the stray power loss $S$, that is, to overcome the opposition to the rotation of the armature due to friction, windage, eddy currents, and hysteresis ; and (b) to overcome the opposition to the rotation of the armature due to the action of the field magnet on the armature conductors on account of the current flowing in them. The portion $(b)$ of the total power is, according to Lenz's law, equal to the total electrical power $E_{a} I_{a}$, developed in the armature. The ratio of the total electrical power, $E_{a} I_{a}$, developed in the armature to the total mechanical power supplied to the armature, is called the efficiency of conversion of the generator. From the above discussion the total mechanical power delivered to the armature is evidently $\left(E_{a} I_{a}+S\right)$, so that

$$
\text { Efficiency of conversion }=\frac{E_{a} I_{a}}{E_{a} I_{a}+S}
$$

A portion, $A$, of the total electrical power developed in the armature of a generator is used to overcome armature resistance, a portion, $F$, is expended in field excitation, and the remainder $E_{a} I_{a}-A-F$ is delivered as useful power to the external re- 
ceiving circuit. The ratio of the useful electrical power delivered by a generator, to the total electrical power developed in the armature, is called the electrical efficiency* of the generator. That is

$$
\text { Electrical efficiency }=\frac{E_{a} I_{a}-A-F}{E_{a} I_{a}}=\frac{E_{x} I_{x}}{E_{a} I_{a}}
$$

The values of $A$ and $F$ may be calculated with the help of equations (27), (28) and (29).

The product of the two partial efficiencies is equal to the true or commercial efficiency. That is, the efficiency of conversion is the fraction of the total mechanical power which is converted into electrical power, and the electrical efficiency is the fractional part of the latter which is delivered to the receiving circuit.

\section{Variation of the efficiency of shunt and compound generators} with power output. - The shunt field loss and the stray power loss of a shunt or compound generator driven at constant speed are approximately constant. On the other hand, the armature loss plus the series field loss is nearly proportional to the square of the current output. The efficiency of the generator is therefore very small when the power output is small, inasmuch as the constant losses, namely shunt field loss and stray power loss, are then large as compared with the power output. The efficiency of the generator increases with increase of output, passes through a maximum value for a certain output, and then with further increase of output, the efficiency decreases because of the rapid increase of armature loss.

The efficiencies of a shunt or compound generator may be calculated for a series of assigned values of power output by the method explained in Art. 65, and a curve may then be plotted showing power outputs as abscissas, and efficiencies as ordinates. Such a curve is called the efficiency curve of the generator. Efficiency curves are frequently plotted showing efficiencies as ordinates, and current outputs in amperes as abscissas.

* Sometimes called the economic coefficient of a generator. 
The full line curve in Fig. 95 is a typical efficiency curve of a shunt generator. The efficiency curve of a compound generator is very similar in form to the efficiency curve of the shunt generator. A generator is usually designed to give its maximum efficiency at its rated full load output. The ordinate of the horizontal dotted curve represents the approximately constant loss due to stray power and $R_{s} I_{s}^{2}$ loss in the shunt field winding. The ordinates of the curved dotted line, measured from the horizontal dotted line, represent the $R_{u} I_{a}^{2}$ loss in the armature.

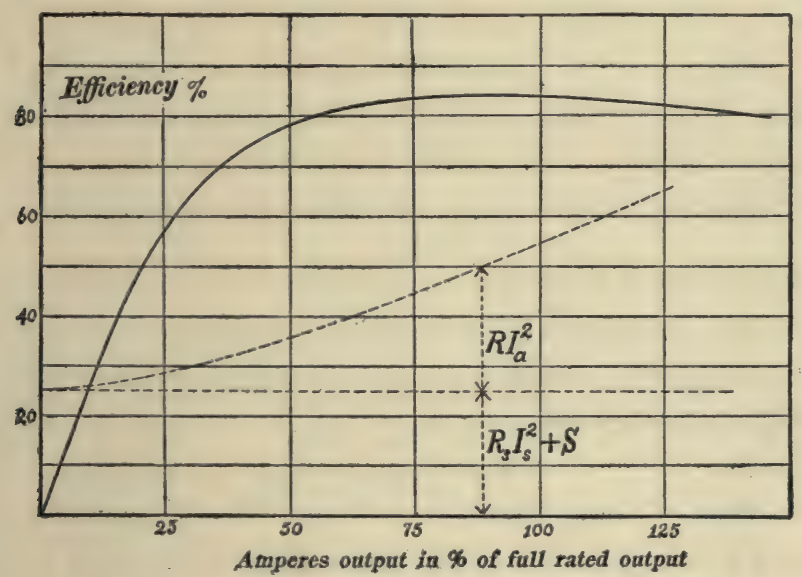

Fig. 95. Efficiency and loss curves of a shunt generator.

The maximum efficiency of a shunt or compound generator occurs for that particular current output for which the sum of the variable losses (series field loss and armature loss) is equal to the sum of the constant losses (shunt field loss and stray power loss).

Proof. - Let $L$ be the sum of the constant losses, and let $E_{x}$ be the terminal voltage of the machine. For the sake of simplicity let us assume $I_{a}=I_{c}=I_{x}$ and let us represent this common current by $I$. This assumption is approximately true, inasmuch as the shunt field current is small as compared with the current output, except when the current output is very small. Then we have :

Power output $=E_{x} I$.

Armature loss + series field loss $=\left(R_{a}+R_{c}\right) I^{2}=R I^{2}$, where $R$ is written for $R_{a}+R_{c}$. 
Therefore :

$$
\text { Efficiency }=\frac{E_{x} I}{E_{x} I+R I^{2}+L}
$$

Differentiating this expression with respect to $I$, assuming $L$ to be constant, and placing the differential coefficient equal to zero, we find :

$$
L=R I^{2}
$$

which shows that the efficiency is a maximum when the sum of armature and series field losses is equal to the sum of shunt field and stray power losses.

The efficiency of a series generator is also zero at zero current output, it increases with increase of current output, reaches a maximum, and falls off with further increase of current output.

The efficiency of a generator depends also upon its size. A generator of one or two kilowatts rated capacity would have an efficiency of about 65 per cent. at best, while very large generators have efficiencies as high as 95 per cent., or even higher.

68. Efficiency of a motor. - The efficiency of a motor is defined as the ratio: output of power divided by intake of power. This ratio is sometimes called the true efficiency or the commercial efficiency to avoid confusing it with the two partial efficiencies defined later.

Calculation of true efficiency. - When the stray power loss of a dynamo has been determined for a given speed and voltage, it is easy to calculate the efficiency of the dynamo when operating as a motor at the given speed and voltage, and supplied with a specified intake* of electrical power; the resistances of the various windings of the dynamo being known.

This calculation is based upon the equation :

$$
\text { efficiency }=\frac{\text { power intake }- \text { losses }}{\text { power intake }}
$$

It is more instructive to discuss this matter of efficiency calculation by means of several typical examples than by deriving a general formula.

* The calculation of the efficiency of a motor for a specified output of power is more complicated than for a specified intake of power or current. 
Example I. Shunt motor.-A given shunt motor is supplied with 50 amperes of current from I IO-volt mains, and it is required to calculate the efficiency of the motor, having given the following data :

$R_{s}=44$ ohms (hot),

$R_{a}=0.14 \mathrm{ohm}$ ( hot),

Stray power loss $=700$ watts at the given voltage and speed. Solution :

(a) Power intake $=$ I 10 volts $\times 50$ amp. $=5,500$ watts.

(b) Shunt field loss $=R_{s}\left(\frac{E_{x}}{R_{s}}\right)^{2}=275$ watts.

(c) Armature loss $=R_{a}\left(50-\frac{E_{x}}{R_{s}}\right)^{2}=316$ watts.

(d) Efficiency $=\frac{5,500-275-316-700}{5,500}=0.765$.

Example 2. Compound motor (long-shunt). - A given compound motor is supplied with 50 amperes of current from I IOvolt mains, and it is required to calculate the efficiency of the motor, having given the following data:

$R_{s}=55$ ohms (hot),

$R_{e}=0.078$ ohm (hot),

$R_{\alpha}=0.09$ ohm (hot),

Stray power loss $=700$ watts at given voltage and speed.

Solution :

(a) Power intake $=\mathrm{I}$ IO volts $\times 50$ amperes $=5,500$ watts.

(b) Shunt field loss $=R_{s}\left(\frac{E_{x}}{R_{s}}\right)^{2}=220.0$ watts.

(c) Series field loss $=R_{c}\left(50-\frac{E_{x}}{R_{s}}\right)^{2}=179.7$ watts.

(d) Armature loss $=R_{u}\left(50-\frac{E_{x}}{R_{n}}\right)^{2}=207.4$ watts.

(e) Efficiency $=\frac{5,500-220-179.7-207.4-700}{5,500}=0.762$. 
Example 3. Compound motor (short-shunt). - A given compound motor is supplied with 50 amperes of current from I IOvolt mains, and it is required to calculate the efficiency of the motor, having given the following data :

$$
\begin{aligned}
& R_{s}=55 \text { ohms (hot), } \\
& R_{c}=0.078 \text { ohm (hot), } \\
& R_{a}=0.09 \text { ohm (hot), }
\end{aligned}
$$

Stray power loss $=700$ watts at given voltage and speed. Solution :

(a) Power intake $=$ I 10 volts $\times 50$ amperes $=5,500$ watts.

(b) Series field loss $=0.078 \times(50 \mathrm{amp} .)^{2}=195$ watts.

(c) Shunt field loss $=R_{s} \times\left(\frac{E_{x}-R_{c} \times 50}{R_{s}}\right)^{2}=204.7$ watts.

(d) Armature loss $=R_{a}\left(50-\frac{E_{x}-R_{c} \times 50}{R_{s}}\right)^{2}=208$ watts.

(e) Efficiency $=\frac{5,500-195-204.7-208-700}{5,500}=0.762$.

Example 4. Series motor. - A given series motor takes 50 amperes of current with an electromotive force of I IO volts between its terminals. Under these conditions the motor has a definite speed and a definite stray power loss. It is required to calculate the efficiency of the motor under the given conditions, having given the following data :

$R_{\mathrm{o}}=0 . \mathrm{I} 2 \mathrm{ohm}$ (hot),

$R_{a}=$ O.I $5 \mathrm{ohm}$ (hot),

Stray power loss $=700$ watts at the given speed and voltage. Solution :

(a) Power intake $=$ I IO volts $\times 50 \mathrm{amp} .=5,50$ n watts.

(b) Series field loss $=R_{c} \times(50)^{2}=300$ watts.

(c) Armature loss $=R_{a} \times(50)^{2}=375$ watts.

(d) Efficiency $=\frac{5,500-300-375-700}{5,500}=0.75$.

It is important to remember that the stray power loss of a series motor varies greatly with the load on the motor, or in 
other words, with the intake of current by the motor, and that the stray power loss varies also with the speed.

69. Efficiency of conversion and mechanical efficiency of a motor. - The total electrical power, $E_{x} I_{x}$, delivered to a motor is used: (a) to supply the power lost in heating the field windings, and to supply the armature loss, $R_{a} I_{a}{ }^{2} ;$ and $(b)$ to force the current through the armature in opposition to the induced electromotive force $E_{a}$. The part $(b)$ is equal to $E_{a} I_{a}$ and, according to Lenz's law, it is all converted into the actual mechanical power which supplies the stray power loss and the useful power delivered at the pulley. The ratio of the mechanical power, $E_{a} I_{a}$, developed in the armature to the total electrical power, $E_{x} I_{x}$, delivered to the motor, is called the efficiency of conversion of the motor. That is :

$$
\text { Efficiency of conversion }=\frac{E_{a} I_{a}}{E_{x} I_{x}}
$$

A portion $S$ of the total mechanical power developed in the motor armature is used to overcome bearing friction, air friction, and magnetic friction, and the remainder, $\left[E_{a} I_{a}-S\right]$, is delivered at the motor pulley as useful power. The ratio of the useful power delivered by a motor, to the total mechanical power developed in the motor armature, is called the mechanical efficiency of the motor. That is

$$
\text { Mechanical efficiency }=\frac{E_{a} I_{a}-S}{E_{a} I_{a}} \quad .
$$

The actual or commercial efficiency of a motor is equal to the product of its efficiency of conversion and its mechanical efficiency, inasmuch as the efficiency of conversion is the fractional part of the delivered electrical power which is converted into mechanical power, and the mechanical efficiency is the fractional part of this total mechanical power which is available at the motor pulley.

70. Variation of the efficiency of shunt and compound motors with load. - The shunt field loss and the stray power loss of a 
shunt or compound motor supplied with current from constant voltage mains, are nearly constant. On the other hand, the armature loss plus the series field loss is nearly proportional to the square of the current intake of the motor. The efficiency of the motor is therefore very small when the load is small, inasmuch as the constant losses, namely, field loss and stray power loss, are then a large part of the motor intake. The efficiency of the motor increases with increase of load, passes through a maximum value for a certain definite load, and then, with further increase of load, the efficiency decreases because of the rapid increase of armature loss.

The efficiencies of a shunt motor or a compound motor may be calculated for a series of assigned values of current or power intake, and from these data a curve may be derived showing the relation between power output and efficiency. Fig. 96 shows a

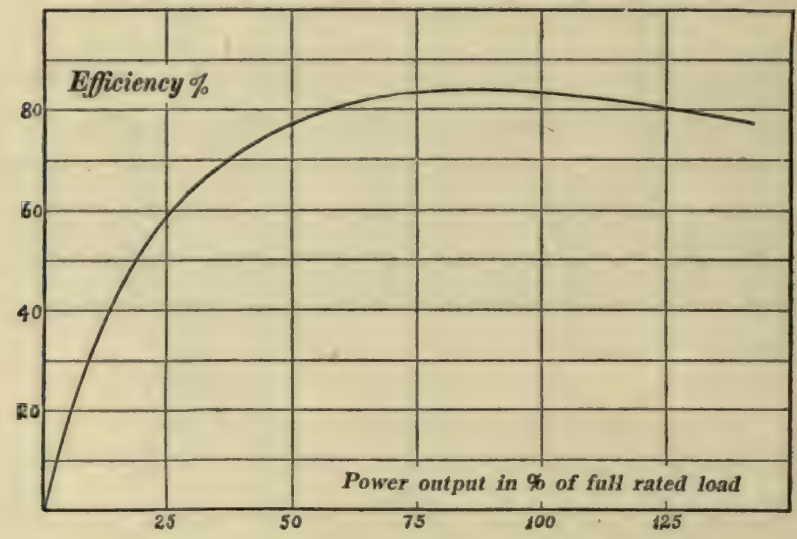

Fig. 96. Efficiency Curve of a Shunt Motor.

typical efficiency curve of a shunt motor. The efficiency curve of a compound motor is very similar to the efficiency curve of a shunt motor shown in Fig. 96.

The upper curves in Fig. 94 show the efficiency of a street railway motor at various loads (current intakes). A street railway motor is run for a great portion of the time at about $\frac{1}{3}$ full 
load, and such a motor is therefore designed to give its maximum efficiency at about $\frac{1}{3}$ full rated load, as shown in Fig. 94. When a motor is to be used most of the time at full load, it is always designed to give its maximum efficiency at or near full load.

The maximum efficiency of a shunt motor (in which the stray power loss is approximately constant) occurs for that particular load for which the armature loss, $R_{a} I_{a}^{2}$, is equal to the constant loss, namely stray power loss plus field loss. The proof of this proposition is very similar to the proof of the corresponding proposition concerning the efficiency of a shunt generator (see article 67).

The efficiency of a motor depends also upon its size. Thus a very small motor of $\frac{1}{4}$-horse power may have an efficiency under the best conditions of about 60 per cent., while a 20 - or 30-horse power motor may have an efficiency of 90 per cent. or more.

\section{The use of the separately excited motor as a dynamometer.} - One of the most convenient methods for determining the amount of mechanical power required to drive a machine of any kind, is to drive the given machine by a separately excited motor, measure the electrical input of power to the armature of the motor, and subtract from this observed input the stray power loss and the armature loss in the motor, thus finding the net mechanical power delivered to the given machine.

Example. - The power required to drive a certain ventilating fan is to be determined. With this end in view, the fan is belted to a shunt motor. The field winding of the motor is connected to the I IO-volt supply mains. The armature of the motor is also connected to the supply mains with an ammeter and a rheostat in series with it. The rheostat is adjusted until the fan is driven at the desired speed when the armature current is observed to be 16.4 amperes, and the electromotive force between the brushes, as measured by a voltmeter, is observed to be 92.5 volts.

The belt is then thrown off, a much larger resistance is placed in series with the armature, and this resistance is adjusted until 
the motor speed is the same as before. The ammeter reading is then observed to be 0.85 ampere, and the electromotive force between the brushes is observed to be gi.6 volts. The resistance (hot) of the armature, as determined by separate measurements, is found to be $0.4 \mathrm{ohm}$.

Solution :

(a) Total power delivered to motor armature to drive the fan $=92.5$ volts $\times 16.4 \mathrm{amp} .=\mathrm{I}, 5 \mathrm{I} 7$ watts.

(b) Stray power loss $=91.6$ volts $\times 0.85$ amp. $-0.4 \mathrm{ohm}$ $\times(0.85 \text { amp. })^{2}=77.6$ watts.

(c) Armature loss when motor is driving the fan $=0.4 \mathrm{ohm}$ $\times(\text { I6.4 amp. })^{2}=$ I07.6 watts.

(d) Mechanical power delivered to the fan $=1,5 \mathrm{I} 7$ watts -77.6 watts -107.6 watts $=1,332$ watts. 


\section{CHAPTER VI.}

\section{RATINGS AND GUARANTEES.}

72. Dynamo speeds. - The capacity (power output) of a given sized dynamo, whether it is to be used as a generator or as a motor, depends upon the speed at which the machine is to be run. Roughly speaking the capacity of a dynamo is proportional to its speed. Thus, a given sized dynamo might be rated as a IO-horse power motor or a I 5-horse power motor according to whether it is to be run at a speed of 1,000 revolutions per minute or at a speed of $\mathrm{I}, 500$ revolutions per minute; and another dynamo might be rated as a 50-kilowatt generator or as a 75-kilowatt generator according to whether it is to be driven at a speed of 500 revolutions per minute or at a speed of 750 revolutions per minute.

It is of prime importance in making a fair comparison of prices, from different manufacturers, on generators or motors of a given rated output, to consider the speeds at which the machines are to be driven. One manufacturer may very properly charge, for a large, slow-speed machine, a higher price than that charged by another manufacturer for a small, high-speed machine of the same rated output.

The speed at which a dynamo may be driven depends partly upon mechanical conditions, such as completeness of balance of the rotating part, strength of the rotating part to withstand the stresses due to rotation, and satisfactory running of the bearings and brushes; and if a dynamo is to be directly connected to an engine as a generator, or to a printing press or other machine as a motor, the speed at which the dynamo must run is fixed by the speed of the machine to which it is to be connected.

The speed at which a dynamo may be driven depends also to some extent upon electromagnetic conditions as follows : 
A shunt dynamo has an inherently proper speed. Thus a shunt generator should be driven at that speed which will develop sufficient electromotive force in the armature to produce enough current through the shunt field winding to magnetize the field magnet nearly to saturation. If a shunt generator is driven at a speed much lower than this, its field excitation will be low, and its maximum permissible output of power will be considerably less than it would be if its field winding were redesigned to give full field excitation at this reduced speed.

A shunt motor should have its field winding connected to supply mains giving the proper electromotive force to fully excite its field magnet, and under these conditions the armature when connected to the same supply mains, will run at a certain definite speed.

A series dynamo does not have an inherently proper speed. Thus, a series generator may be driven at any desired speed, and, if the receiving circuit has a suitable resistance, the full rated current will be delivered by the machine, its field will be fully excited, and the electromagnetic action of the machine will be at its best. Driven at high speed a series generator will develop a high electromotive force, and driven at low speed the machine will develop a low electromotive force, when the current is at its full load value.

A series motor does not have an inherently proper speed. Such a motor may be run at full current intake (and consequently at full field excitation) at any speed, although, of course, a high electromotive force will be needed to drive the machine at high speed with full current intake.

Correlation of speed and voltage ratings of shunt dynamos. From what has been said above, it is evident that a given dynamo, even a given shunt dynamo, may be run more or less satisfactorily at a speed very considerably above or below the rated speed of the machine, but the use in commercial work of certain widely separated standard voltages of supply, I I o volts, 220 volts, and 500 volts, leads to very definite speed ratings of generators and motors. 
Thus a motor which has a field winding designed to give full field excitation from 220-volt mains, and an armature that is well enough insulated to withstand a 220-volt supply, and strong enough mechanically to run at the speed given by a 220 -volt supply, will run at a definite speed, say I,200 revolutions per minute, under these conditions. Such a motor would be rated as a 220-volt motor at a speed of I,200 revolutions per minute, and although it would hardly be permissible to run this motor from I IO- or 500-volt mains, still the motor would perhaps run satisfactorily from, say, 250-volt mains at a speed, say, of 1,350 revolutions per minute. A shunt dynamo has a fairly definite, inherently proper speed, which fact, taken in conjunction with the widely separate standard voltages used in practice, limits a shunt machine (generator or motor) to a certain very definite speed.

\section{Limitation of output of a dynamo as a generator or as a} motor. - The output of a motor or generator is limited in practice by three distinct things: $(a)$ By overheating and consequent destruction of the insulating materials used in the construction of the machine; (b) by excessive sparking at the brushes and the consequent rapid wear, unsatisfactory running, and excessive heating of the commutator; and $(c)$ by excessive drop in voltage of a generator or excessive drop in speed of a motor, at excessive loads. That is to say, a dynamo is rated for that load for which it neither heats nor sparks excessively, and for which neither its voltage, if it is to be used as a "constant voltage" generator, nor its speed, if it is to be used as a "constant speed" motor, falls off more than a certain small amount. Heating rather than sparking is the thing that usually limits the output of a machine, especially in the case of completely enclosed machines like the enclosed motors shown in Figs. 60 and 91; while closeness of regulation (voltage regulation in case of a generator and speed regulation in case of a motor) sets a limit to the permissible rating of a machine only in rare special cases where very close regulation is demanded. 
Estimation of ratings from dimensions and speed. - It is often important to be able to make an approximate estimate of the proper rating of a machine from its specified speed, and its dimensions; for example in the purchase of machines which are not fully guaranteed, such as second-hand machines. There is of course a considerable difference between the legitimate ratings of well designed and poorly designed machines of the same size, so that no accurate general rule can be given for finding ratings. The following formula is as good, perhaps, as can be devised. :

$$
P=k l d^{2} n
$$

in which $l$ is the gross length of the armature core in inches, $d$ is the external diameter of the armature core in inches, $n$ is the speed in revolutions per minute, $P$ is the power rating of a motor in horse-power, or of a generator in kilowatts, and $k$ is a factor the approximate values of which are as follows: For motors, to give $P$ in horse-power, $k$ ranges from about 0.00002 for a 5 horsepower motor to about 0.00004 for a 50 horse-power motor. The rating of an enclosed motor is about 65 per cent. of that of an open motor of the same size and speed. For generators, to give $P$ in kilowatts, $k$ ranges from about 0.000015 for a 5 kilowatt generator, to about 0.000030 for a 50 kilowatt generator, to about 0.000032 for a 200 kilowatt generator. Ratings obtained from the above equation may very properly be slightly increased or decreased according to a general estimate of the degree of perfection of design of a given machine.

74. Heating. - The ultimate steady running temperature of any part of a dynamo is the temperature at which the part gives off heat (by radiation, convection and conduction) to its surroundings as fast as heat is generated in the part by friction, by eddy currents and hysteresis, or by the heating effect of the electric current. This ultimate steady running temperature is not reached in less time than from six to eighteen hours of steady running under full load, according to the size of the machine. Small 
machines require a short time, and large machines a long time, to reach this ultimate temperature.

On account of the time required for a dynamo to reach its final steady running temperature, it is evident that a machine may be run for a short time under excessive load without causing undue rise of temperature.

The rated capacity of a machine which is intended for continuous service is always based upon ultimate steady running temperature, and operating engineers usually exact from the manufacturers a definite short period over-load guarantee, for example, a 50-per cent. over-load for one hour, or a 25 -per cent. over-load for three hours, without exceeding a prescribed rise of temperature.

The rated capacity of a machine which is intended for a specific kind of intermittent service may properly be based upon a short period run. Thus street car motors are usually rated on the basis of a one-hour run, and a street railway motor rated at 25horse power would overheat if it were operated for much more than one hour at full load.

It is of prime importance in making a fair comparison of prices from different manufacturers on generators or motors of a given rating, to consider this time element which should always be specified by the manufacturer. One manufacturer may very properly charge for a large machine which will give I oo kilowatts output steadily (or say 125 kilowatts for three hours) a higher price than that charged by another manufacturer for a generator which he rates at Ioo kilowatts, if the second manufacturer bases his rating. on a three-hour run at full load.

75. The heat run. - Inasmuch as dynamo ratings and overload guarantees are usually based upon rise of temperature of the machine, it is evidently important to check these guarantees by actual test. This test is called the heat run.

Temperatures of armature and field windings, as specified in rating and overload guarantees, are understood to be calculated from the measured resistances, cold and hot, of these windings. 
The temperature of a machine part, other than windings, is understood to be its surface temperature as indicated by a thermometer of which the bulb is laid flat against the part and covered by a small wad of clean, dry cotton waste. A large wad of waste would interfere too much with the free cooling of the part under test.

The heat run is made by operating the machine under full load conditions for the specified time, or until a constant running temperature has been reached. The machine is then shut down, and the temperatures of the various parts are determined by thermometers as above explained. Also the "hot" resistances of the various windings are measured, from which the mean temperatures of the windings may be calculated, the resistances at room temperature having been measured beforehand.

Important practical points concerning this heat test are given in Art. 79.

The commutator and brushes may be safely run at a much higher temperature than the armature and field magnet windings. This is because the mica insulation of the commutator is nearly fireproof, while the cotton and varnish insulation of the armature and field windings can not withstand a temperature beyond $100^{\circ} \mathrm{C}$. without charring. The bearings should be run at a comparatively low temperature to ensure satisfactory lubrication. The allowable temperatures, as specified in the report of the Committee on Standardization of the American Institute of Electrical Engineers, ${ }^{*}$ are partly determined by the above considerations and partly by differences in the methods used for determining temperatures. Temperatures calculated from resistance measurements are accurate mean temperatures, whereas temperatures by thermometer refer to the surface layers, which are always cooler than the interior. Thus a magnet winding may show a temperature rise of $50^{\circ} \mathrm{C}$. by a thermometer laid on its surface when a temperature rise of $75^{\circ} \mathrm{C}$. is obtained by calculation from resistance measurements.

* See Art. 79. 
76. Sparking. - One effect of the sparking of a dynamo is to roughen the commutator and cause the brushes to chatter and to wear the commutator rapidly; another effect is to cause excessive heating of the commutator and brushes. It is not possible to specify precisely the degree of sparking that is permissible, nor is there any satisfactory quantitative sparking test to which a finished machine may be subjected. It is for this reason that ratings and guarantees are based specifically upon rise of temperature. It may be stated in a general way that a dynamo should not show any visible sparking when its output is changed from zero load to full load, or from full load to zero load, the brushes being left in a fixed position.

A dynamo will bear a maximum output rating, with a minimum cost of materials, when it reaches its heating and sparking limits simultaneously.

The cause of sparking, and the means adopted by the designer to obviate sparking are discussed in the following articles on armature reaction and commutation.

77. Armature reaction. - The magnetizing action of the current in a dynamo armature is called armature reaction. Armature reaction has a two-fold effect. (a) It causes the magnetic flux which enters the armature from the field magnet poles to be crowded toward the trailing pole tips of a generator, or towards the leading pole tips of a motor, as shown in Figs. 97, 98 and 99. (b) It opposes (generally) the passage of the flux through the armature and thus reduces the amount of flux.

The part $(a)$ of armature reaction is called cross-magnetizing action, and the part $(b)$ is called demagnetizing action. The crossmagnetizing action has an important influence upon the sparking of a dynamo, and therefore it has to do more or less with the rating of a given machine. The demagnetizing action, inasmuch as it reduces the flux through the armature, affects the voltage regulation of a generator and the speed regulation of a motor. 
Demagnetizing action and cross-magnetizing action.-The nature of armature reaction and its resolution into the two parts, demagnetizing action and cross-magnetizing action, is most clearly

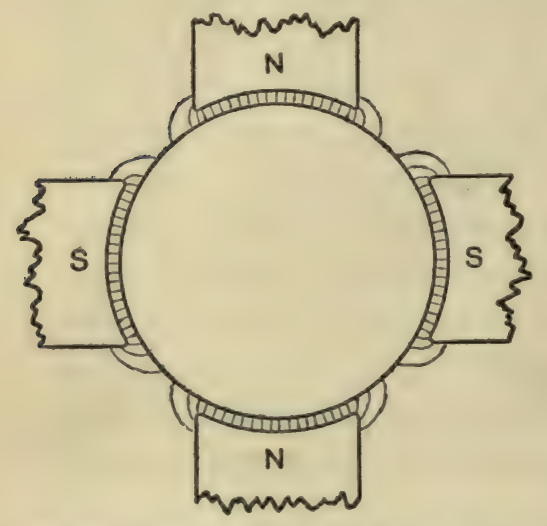

Fig. 97. Undistorted field of a dynamo when armature current is small.

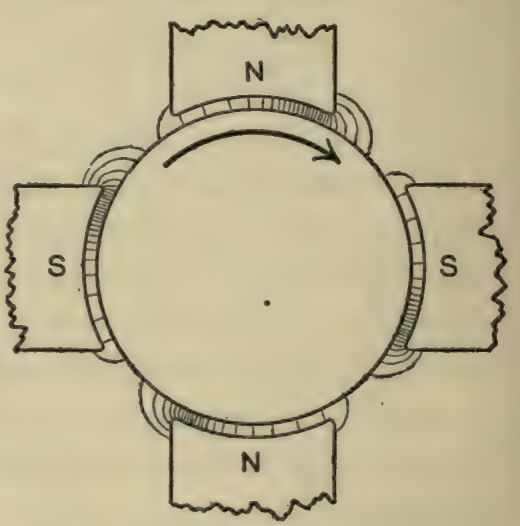

Fig. 98. Distorted field of a generator when its armature current is large.

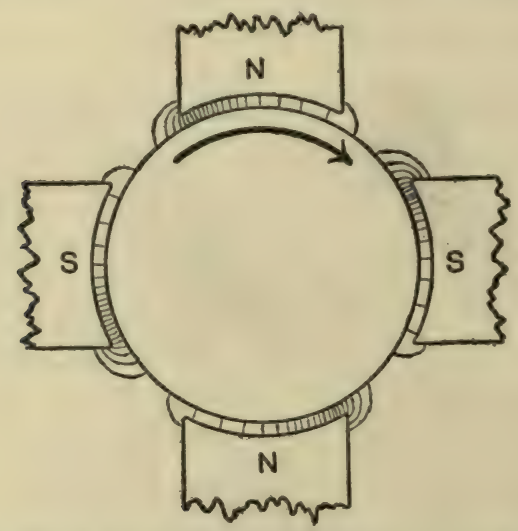

Fig. 99. Same as Fig. 98 , but showing motor distortion for same direction of running.

brought out by considering a bipolar dynamo as shown in Fig. IOO. The brushes are shown at an angular distance $a$ ahead of the line $A B$, which is at right angles to the axis of the field. All of the conductors marked with a dot are supposed to carry outward flowing current (towards the reader), and all conductors 
marked with a cross are supposed to carry inward flowing current (away from the reader).

Fig. IOI is like Fig. IOO, but it shows only those armature conductors which lie within the angular distance $\pm a$ from the

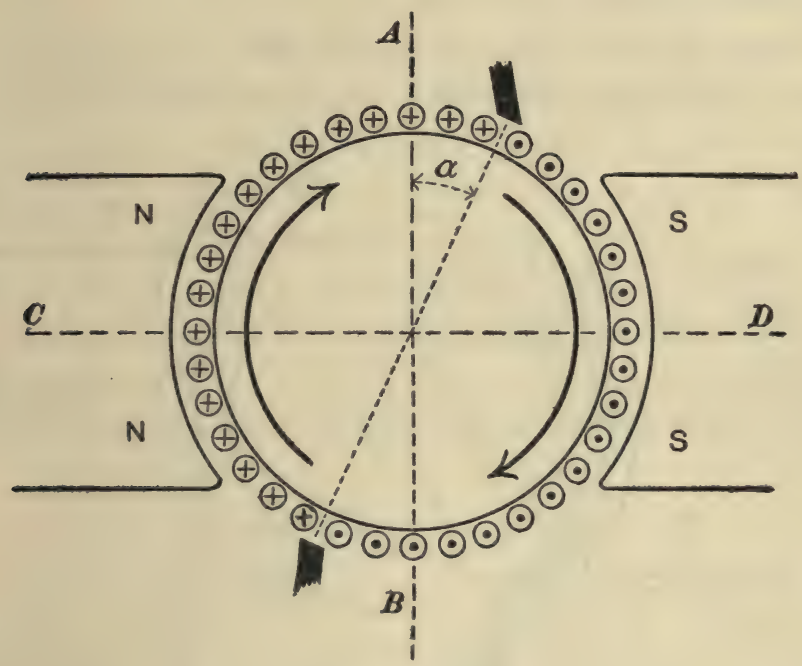

Fig. 100 .

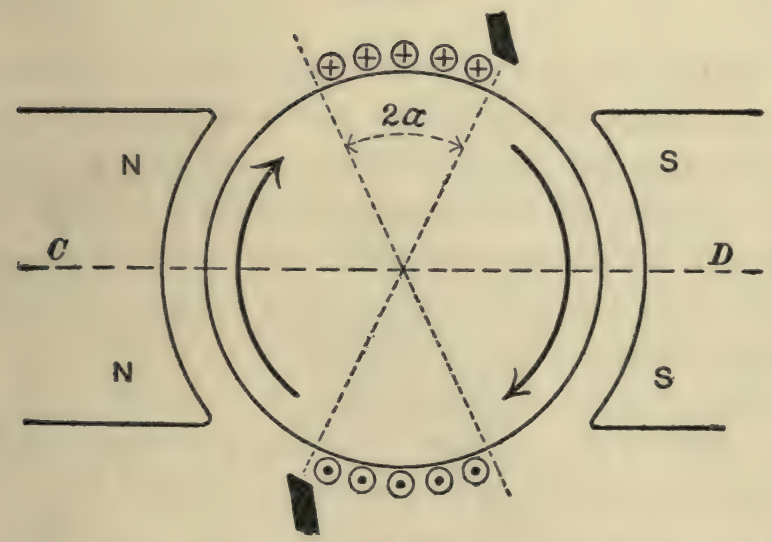

Fig. 101.

line $A B$. Each of the conductors in the top group in Fig. IOI may be thought of as paired with a conductor in the bottom 
group, and the pair may be looked upon as a complete turn of wire, or in other words, all the conductors shown in Fig. IOI may be looked upon as a magnetizing coil surrounding the line $C D$ as an axis. The magnetic action of this coil is the demagnetizing action of the armature.

Fig. IO2 is like Fig. IOI except that it shows only those armature conductors which lie at a greater angular distance than

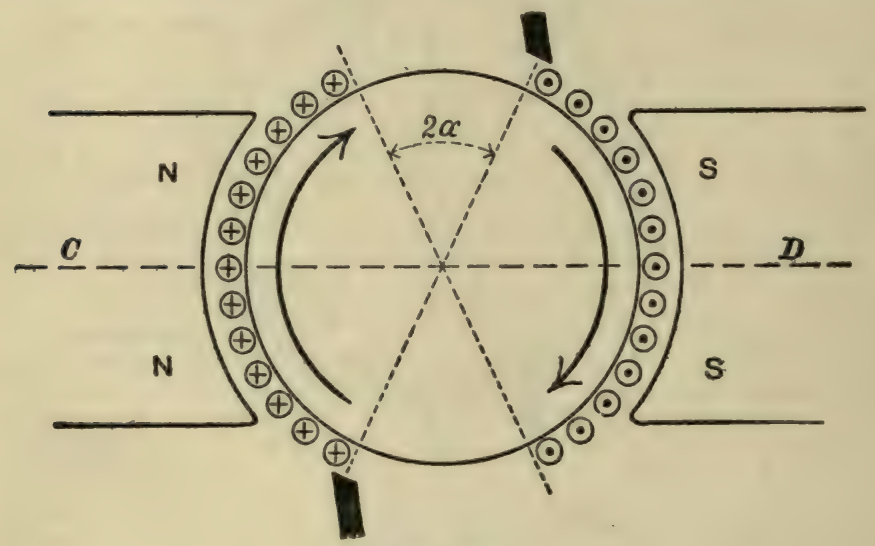

Fig. 102.

$\pm \boldsymbol{\alpha}$ from the line $A B$. Each of the conductors on the right in Fig. IO2 may be thought of as paired with a conductor on the left, and the pair may be looked upon as a complete turn of wire, or in other words, all the conductors shown in Fig. 102 may be looked upon as a magnetizing coil surrounding the line $A B$ (Fig. IOO) as an axis. The magnetizing action of this coil is the cross-magnetizing action of the armature.

The state of affairs shown in Figs. IOO, IOI and IO2 obtains in a generator, as may be seen by applying the rule which correlates direction of a current in a wire, direction of field in which the wire is placed, and direction of side-push on the wire. Furthermore, the demagnetizing action of the conductors shown in Fig. IOI is positive, that is, it is a genuine demagnetizing action which opposes the passage of the flux $\Phi$ through the armature, as may 
be seen by applying the rule which correlates direction of current in a coil and direction of magnetizing action of the coil. To summarize, we have in Fig. Ioo a generator of which the brushes have a forward lead from the line $A B$ and the armature has a demagnetizing action. In a generator with brushes having a backward lead from the line $A B$, the armature has a magnetizing action which helps the passage of the flux $\Phi$ through the armature. In a motor, on the other hand, forward lead of brushes gives magnetizing action, and backward lead of brushes gives demagnetizing action of the armature.

The effect of the cross-magnetizing action in the armature of a dynamo may be made more easily intelligible with the help of Figs. 103, 104 and 105. Fig. 103 shows the magnetic flux through armature and field poles due to the magnetizing action

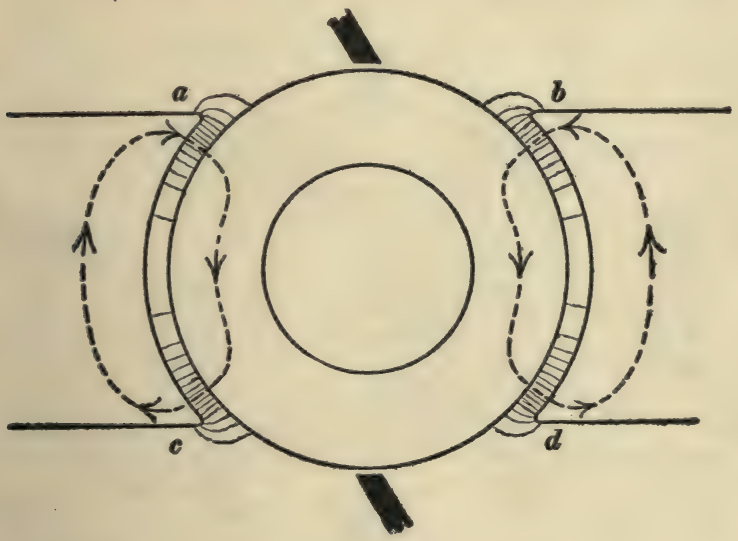

Fig. 103.

of the armature alone (field excitation zero), Fig. IO4 shows the magnetic flux due to the action of the field coils alone (armature current zero), and Fig. I05 shows the composite effect due to field coils and armature acting together. At the pole tips $a$ and $d$ the flux is in the same direction, in Figs. 103 and 104, and therefore the resultant flux density under these tips is great, as shown in Fig. 105. At the pole tips $b$ and $c$ the flux is in opposite 
directions in Figs. 103 and 104, and therefore the resultant flux density under these tips is small, as shown in Fig. I05.

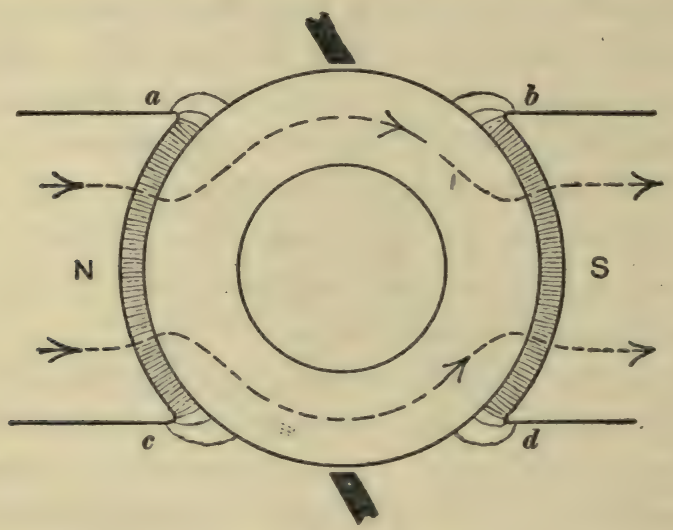

Fig. 104.

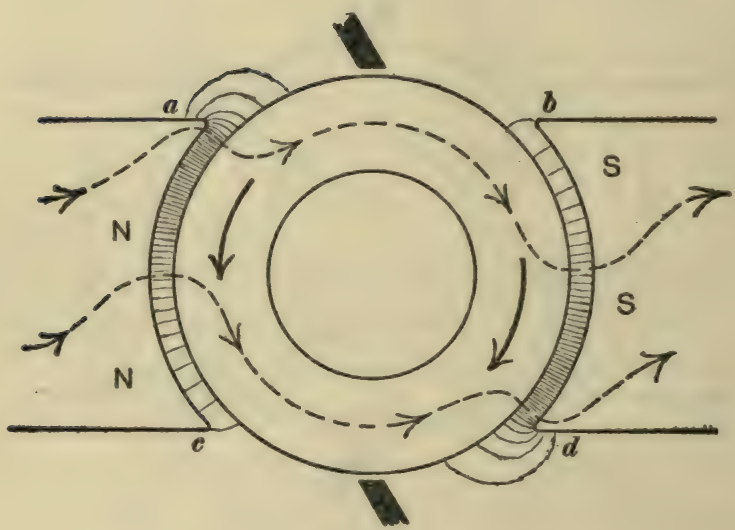

Fig. 105.

Fig. I06 shows a multipolar machine with the brushes at an angle $\boldsymbol{a}$ ahead of the diagonal lines $A B *^{*}$ The conductors at a greater angular distance than $\pm a$ from the line $A B$ balance each other's magnetizing action on the magnetic circuit, the mean path through which is shown by the heavy dotted line $\Phi$.

* Which bisect the angles between the lines $C D$ which are drawn from the center of the armature to the middle points of the pole pieces. 
That is to say, to each conductor in the group $g$ there is a corresponding symmetrically located conductor in the group $h$ with an oppositely flowing current in it. On the other hand, the magnetizing action of the conductors which lie within the angular distance $\pm a$ from the line $A B$ is unbalanced, and this unbal-

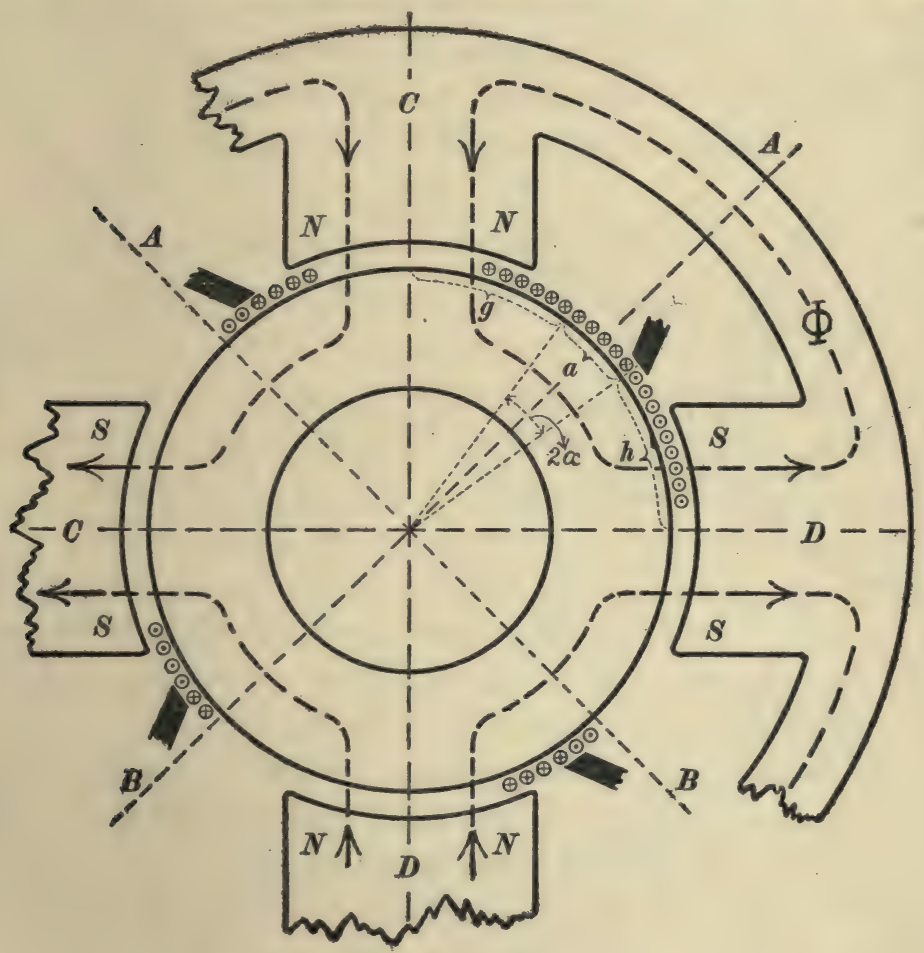

Flg. 106.

anced magnetizing action is the demagnetizing action of the armature on the magnetic circuit represented by the dotted line $\Phi$.

The value of this demagnetizing action, expressed in ampereturns, is found by multiplying the number of conductors * within the space $2 a$ by the current flowing in each. That is :

* If a wire encircles an iron rod which constitutes a magnetic circuit, then the iron rod encircles the wire. In reckoning an ampere-turn, one must be sure either that the wire encircles the iron rod which constitutes the magnetic circuit, or that the magnetic circuit encircles the wire. 


$$
D=\frac{a}{180} \cdot \frac{Z I_{a}}{p^{\prime}}
$$

in which $D$ is the demagnetizing ampere-turns (per magnetic circuit) of a dynamo armature, $\boldsymbol{a}$ is the angle of forward lead of the brushes, $Z$ is the total number of armature conductors, $I_{a}$ is the total armature current, and $p^{\prime}$ is the number of paths into which the current $I_{a}$ divides in flowing through the armature. Equation (35) is true whatever the number of field magnet poles may be.

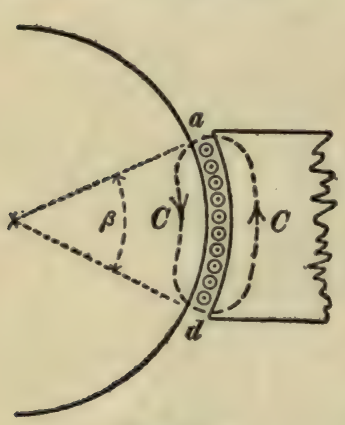

Fig. 107.

The cross-magnetizing action of the armature is represented in the above discussion as being due to all the armature conductors which lie at a greater distance than $\pm a$ from the lines $A B$. It is evident, however, that the conductors which are beyond the pole tips cannot contribute to the magnetic flux shown under the pole tips in Fig. I03. In fact the cross-magnetizing action of the armature depends only upon the number of armature conductors which lie under a pole face as shown in Fig. I07.

The intensity $\mathscr{H}^{\prime}$, of the magnetic field under the pole tips in Fig. IO3 due to the armature alone, may be quite easily calculated as follows: Consider the magnetic circuit CC, Fig. 107, which encircles all of the conductors under a pole face. The number of these conductors is $Z \times \beta / 360$, and the current in each conductor is $I_{a} / p^{\prime}$, so that the magnetomotive force around $C C$ is $Z \times \beta / 360 \times I_{a} / p^{*}$ ampere-turns, or $4 \pi / 10 \times Z \times \beta / 360 \times$ $I_{a} / p^{\prime} \quad$ c.g.s. units. $\dagger$ Now the iron of the pole face and of the armature core may be considered to have zero magnetic reluctance as compared with the air in the gap space, so that approximately

* This gives the cross-magnetizing ampere-turns (per magnet pole) of a dynamo armature.

† See Appendix A for a full discussion of magnetomotive force and of the magnetization of iron. 
the whole of the magnetomotive force around the circuit $C C$ is brought into action at the two points $a$ and $d$ where the circuit crosses the gap space. Therefore one half of the whole magnetomotive force is brought into action at $a$, and one half at $d$, Fig. 107, so that, dividing the magnetomotive force across the gap at $a$ or $d$ by the distance $l$ in centimeters across the gap, we have the desired value of $\mathscr{H}^{\prime}$, namely,

$$
\mathscr{H}^{\prime}=\frac{\pi \beta}{1,800} \cdot \frac{Z I_{a}}{p^{\prime} l}
$$

The various devices and schemes for reducing the effect of armature reaction are chiefly of interest to the, dynamo builder, but they are of some importance to the operating engineer also, and they are briefly as follows :

In the first place the demagnetizing action of the armature can be reduced to a very small value by making the brush lead small, a condition which is realized, without excessive sparking, in most modern machines. In the second place, the disturbing effect of cross-magnetizing action depends upon the ratio $\mathscr{H}^{\prime} / \mathscr{H}$, where $\mathscr{H}^{\prime}$ is the field intensity under the pole tips due to the armature alone, and $\mathscr{H}$ is the field intensity in the gap space due to the field winding alone. The disturbing effect of armature reaction is never troublesome when the value of this ratio is small. (a) A small value of the ratio $\mathscr{\mathcal { H } ^ { \prime }} / \mathscr{H}$ may be obtained by using a very large value of $\mathscr{H}$ or, in other words, by using a very strong field excitation. (b) A small value of the ratio $\mathscr{H}^{\prime} / \mathscr{H}$ may be realized by keeping $\mathscr{H}^{\prime}$ small; and to keep $\mathscr{H}^{\prime}$ small for a given current in each path in the armature and for a given number of armature conductors, requires, according to equation (36), the use of narrow pole faces (small value of $\beta$ ), and of a wide air gap (large value of $l$ ).

The angular breadth $\beta$ of the pole faces is never reduced below about 65 per cent. of $360 / p$ degrees, where $p$ is the number of field magnet poles.*

* The ratio $\beta \div 360 / p$ (= pole span/pole pitch) is usually made about 0.7 in multipolar dynamos. 
The value of $\mathscr{H}^{\prime}$ may be reduced below the value given by equation (36) by constructing the pole pieces as shown in Fig. 108, so that the magnetic circuit $C C$ includes, not only the air

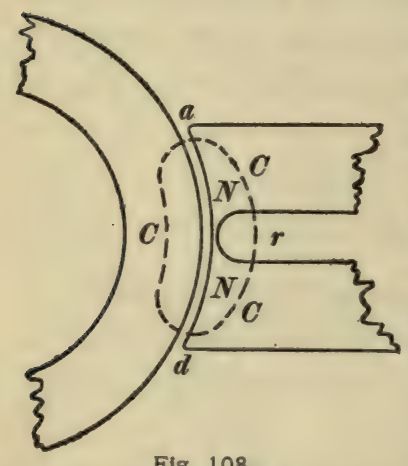

FIg. 108. gaps at $a$ and $d$, but also the very considerable air space at $r$, which does not interfere with the flow of the useful flux $\Phi$.

The effect of a long gap space may be produced by using narrow teeth and wide slots on the armature. These narrow teeth are then highly saturated by the flux $\Phi$, so that their magnetic reluctance is great, and this high reluctance is the exact equivalent of a long air gap.

A high reluctance in the path, CC, Fig. 108, of the cross-flux, but not in the path of the useful flux $\Phi$, may be produced by lengthening the gap space under the pole tips, leaving a short gap space under the middle of the pole face. This effect may be accomplished most advantageously in a laminated pole piece by cutting away the tips of half of the laminations, thus greatly reducing the sectional area of the iron in the pole tips. Under these conditions the least overcrowding of the flux under a pole tip, as shown in Fig. 105, tends to push the iron of the pole tip beyond magnetic saturation, thus vastly increasing its reluctance. The nearly sparkless operation under all loads, of dynamos of recent design, is largely due to the use of pole pieces of the kind here described.

The best method of all for reducing armature reaction is by the use of the so-called compensating winding, which consists of a layer of wires, parallel to the armature shaft, embedded in the pole faces, as shown in Fig. I09, and so connected that the total current, or a definite fraction of the current, from the brushes of the machine flows through each wire, as indicated by the crosses and dots in Fig. I09. The use of this compensating winding is not 
ordinarily warranted on account of its cost. It is, however, an essential feature of the ordinary commutator motor when driven

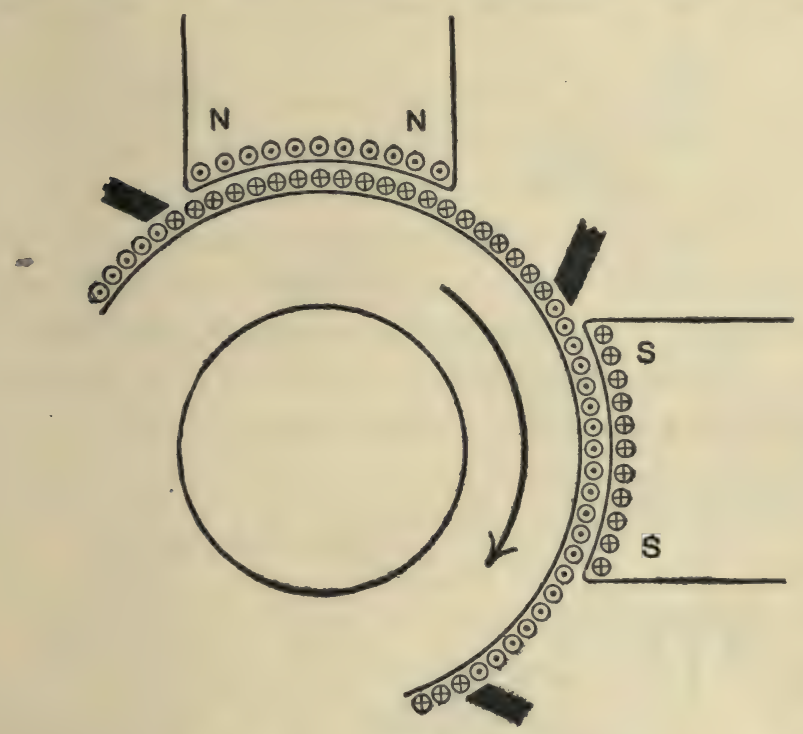

Fig. 109.

by alternating current, as exemplified in the recent development of the single phase alternating current motor for railways.

78. The phenomena of commutation. Non-sparking condition. The cause of sparking, and a clear idea of the means available for reducing sparking, may be obtained by a careful consideration of what takes place in a section of the armature winding and at the two commutator bars to which it is connected, when the two bars pass under a brush.

Fig. I IO shows the positive brush of a generator, a portion of the commutator with its segments or bars $c, d, e, f$ and $g$, a portion of the ring winding of the armature with its sections $T, U, V$, $W$ and $X$, and the leading tip of one of the pole pieces. The line $n n^{\prime}$ represents the neutral axis (see Art. 5I)

Before a given section of the armature winding reaches the positive brush, current is flowing through it towards the brush, as 
indicated by the small arrows in Fig. I IO, and after the given section of the armature winding has passed the brush, the current in the section is reversed in direction, still, of course, flowing towards the brush.

The figure shows the instant when the section $V$ is short-circuited by the contact of the brush with the two commutator bars $e$ and $f$. This state of affairs lasts for a very brief time, and if an action could be brought about whereby the previously existing current in this section could be reduced to zero and an equal and opposite current established in the section during this brief time, then there would not be the least tendency for a spark to form between the brush and the bar $e$ at the instant they separate.

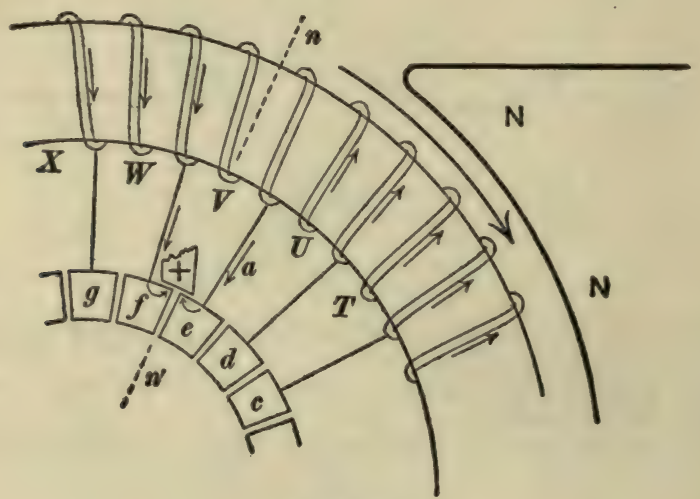

Fig. 110.

(a) As to the establishing of a reversed current in the section $V$ while it is short-circuited, it is to be noted that electromotive forces are induced in the armature windings everywhere except exactly in the neutral axis, as explained in Art. $5 \mathrm{I}$. Therefore, if we give the brush shown in Fig. I Io a forward lead from the neutral axis, the sections as they are short-circuited by the brush will be in a region where they have electromotive forces induced in them in the proper direction to establish the reversed current above mentioned; and the greater the forward lead, the greater these electromotive forces will be, so that an angle of 
lead may be found by trial for which the condition of sparkless commutation above stated, is completely realized.

(b) The use of high resistance brushes of carbon tends not only to cause a decrease to zero of the current existing in the sections before they reach a brush, but also to establish the necessary reversed current during the time that a section is short-circuited by the brush. The decrease to zero is due mainly to the resistance of the brush material which forms a part of the short circuit. The establishment of the reversed current in the sections is due mainly to the contact resistance between the brush and the commutator, as follows: when the commutator has moved a little beyond the position shown in Fig. I ro, the increased resistance between bar $e$ and the brush, due to decreased area of contact, diverts the current $a$ through the section $V$ as a reversed current in $V$.

It is impracticable to depend solely upon the action described above under $(a)$ for producing sparkless commutation, inasmuch as to do so would require the lead of the brushes to be readjusted with every change of load. Therefore the action described above under $(b)$ must be depended upon to a greater or less extent.

In the case of generators and of motors which are driven always in the same direction, the brushes are adjusted to give the best commutation at, say, half load, and at greater (or smaller) loads the action $(a)$ is deficient (or excessive) and the deficiency (or excess) must be counteracted by action (b).

In motors for cranes and electric cars the direction of running is repeatedly reversed and the brushes are fixed permanently in the diagonal axes $A B$, Fig. I06. In this case the cross-magnetizing action of the armature current shifts the neutral axis so that the action $(a)$ tends to maintain rather than to reverse the current in the short-circuited sections. In this case the action described above under $(b)$ is not only the sole cause of the reversal of the current in the short-circuited sections, but in producing this reversal the action $(b)$ has to counteract the opposing 
action (a). Motors which are repeatedly reversed would not operate satisfactorily with low resistance brushes of metal.

A current in a circuit of large inductance has great electrical momentum and the quick reversal of a current in such a circuit requires a very considerable electromotive force. Therefore the reversal of current in the short-circuited sections of an armature during the brief time that the short-circuit continues, is vitally dependent upon a small value of the inductance of the sections. Small inductance is especially important inasmuch as the action (b) above described is chiefly relied upon, and this action is equivalent to a very small electromotive force. The inductance of an armature section, wound in slots of a given size, is proportional to the square of the number of turns of wire in the section. Therefore a small inductance necessitates few turns of wire in each armature section.

79. Standard rules for ratings and guarantees. - In the early days of electrical engineering, different manuiacturers followed widely divergent rules for fixing the ratings of their machines with the result that ratings were almost meaningless. In addition to this, the terminology of electrical engineering, and especially the mathematical notation employed in electrical engineering literature was badly confused. To remedy this state of affairs, a Committee on Standardization was appointed by the American Institute of Electrical Engineers in 1898 and their report, ${ }^{*}$ some of the details of which have since been revised, was laid before the Institute in June, 1899. The revised report is dated May, 1902.

The recommendations of this committee on rise of temperature, insulation, and rating of dynamo machines are given below. The remainder of the report of the committee is devoted chiefly to recommendations concerning notation, to definitions, and to the enunciation of principles. This part of the report is adequately represented in the general mode of treatment adopted in this text.

* Trans. A. I. E. E., Vol. XVI., pp. 255-268. This report has been issued by the Institute as a separate publication. 


\section{RISE OF TEMPERATURE.}

\section{General Principles.}

26. Under regular service conditions, the temperature of electrical machinery should never be allowed to remain at a point at which permanent deterioration of its insulating material takes place.

27. The rise of temperature should be referred to the standard conditions of a room-temperature of $25^{\circ} \mathrm{C}$, , a barometric pressure of $760 \mathrm{~mm}$. and normal conditions of ventilation; that is, the apparatus under test should neither be exposed to draught nor enclosed, except where expressly specified.

28. If the room temperature during the test differs from $25^{\circ} \mathrm{C}$, the observed rise of temperature should be corrected by $x / 2$ per cent. for each degree C. Thus with a room temperature of $35^{\circ} \mathrm{C}$., the observed rise of temperature has to be decreased by 5 per cent., and with a room temperature of $15^{\circ} \mathrm{C}$., the observed rise of temperature has to be increased by 5 per cent. The thermometer indicating the room temperature should be screened from thermal radiation emitted by heated bodies, and from draughts of air. When it is impracticable to secure normal conditions of ventilation on account of an adjacent engine, or other sources of heat, the thermometer for measuring the air temperature should be placed so as fairly to indicate the temperature which the machine would have if it were idle, in order that the rise of temperature determined shall be that caused by the operation of the machine.

29. The temperature should be measured after a run of sufficient duration to reach practical constancy. This is usually from 6 to 18 hours, according to the size and construction of the apparatus. It is permissible, however, to shorten the time of the test by running a lesser time on an overload in current and voltage, then reducing the load to normal, and maintaining it thus until the temperature has become constant.

In apparatus intended for intermittent service, as railway motors, starting rheostats, etc., the rise of temperature should be measured after operation under as nearly as possible the conditions of service for which the apparatus is intended, and the conditions of the test should be specified.

In apparatus which by the nature of their service may be exposed to overload, as railway converters, and in very high voltage circuits, a smaller rise of temperature should be specified than in apparatus not liable to overloads or in low voltage apparatus. In apparatus built for conditions of limited space, as railway motors, a higher rise of temperature must be allowed.

30. In electrical conductors, the rise of temperature should be determined * by their increase of resistance where practicable. For this purpose the resistance may be measured either by galvanometer test, or by drop-of-potential method. A temperature coefficient of 0.42 per cent. per degree C., from and at $0^{\circ} \mathrm{C}$., may be assumed for copper. Temperature elevations measured in this way are usually in excess of temperature elevations measured by thermometers.

* By the formulas

$$
R^{t}=R_{0}(\mathrm{I}+0.0042 t) \text { and } R_{t+\theta}=R_{0}[\mathrm{I}+0.0042(t+\theta)]
$$


When thermometers are applied to the free surface of a machine, it is desirable that the bulb of the thermometer should be covered by a pad of definite area. A convenient pad may be formed of cotton waste in a shallow circular box about one and a half inches in diameter, through a slot in the side of which the thermometer bulb is inserted. An unduly large pad over the thermometer tends to interfere with the natural liberation of heat from the surface to which the thermometer is applied.

31. With apparatus in which the insulating materials have special heat-resisting qualities, a higher temperature elevation is permissible.

32. In apparatus intended for service in places of abnormally high temperature, a lower temperature elevation should be specified.

33. It is recommended that the following maximum values of temperature elevation should not be exceeded :

Commutating machines, rectifying machines and synchronous machines.

Field and armature, by resistance, $50^{\circ} \mathrm{C}$

Commutator and collector rings and brushes, by thermometer, $55^{\circ} \mathrm{C}$.

Bearings and other parts of machine, by thermometer, $40^{\circ} \mathrm{C}$.

Rotary induction apparatus :

Electric circuits, $50^{\circ} \mathrm{C}$, , by resistance.

Bearings and other parts of the machine, $40^{\circ} \mathrm{C}$., by thermometer.

In squirrel-cage or short-circuited armatures, $55^{\circ} \mathrm{C}$, , by thermometer, may be allowed.

Transformers for continuous service - electric circuits by resistance, $50^{\circ} \mathrm{C}$, , other parts by thermometer, $40^{\circ} \mathrm{C}$., under conditions of normal ventilation.

Reactors, induction and magneto-regulators - electric circuits by resistance, $50^{\circ} \mathrm{C}$, other parts by thermometer, $40^{\circ} \mathrm{C}$.

Where a thermometer, applied to a coil or winding, indicates a higher temperature elevation than that shown by resistance measurement, the thermometer indication should be accepted. In using the thermometer, care should be taken so to protect its bulb as to prevent radiation from it, and, at the same time, not to interfere seriously with the normal radiation from the part to which it is applied.

34. In the case of apparatus intended for intermittent service, except railway motors, the temperature elevation which is attained at the end of the period corresponding to the term of full load, should not exceed $50^{\circ} \mathrm{C}$., by resistance in electric circuits. In the case of transformers intended for intermittent service, or not operating continuously at full load, but continously in circuit, as in the ordinary case of lighting transformers, the temperature elevation above the surrounding air-temperature should not exceed $50^{\circ} \mathrm{C}$. by resistance in electric circuits and $40^{\circ} \mathrm{C}$. by thermometer in other parts, after the period corresponding to the term of full load. In this

where $R_{t}$ is the initial resistance at room temperature $t^{\circ} \mathrm{C}$.

$R_{t+\theta}$ is the final resistance at temperature elevation $\theta^{\circ} \mathrm{C}$.

$R_{0}$ is the inferred resistance at $0^{\circ} \mathrm{C}$.

These combine into the formula

$$
\theta=(238 . \mathrm{I}+t)\left(\frac{R_{t+\theta}}{R_{t}}-\mathrm{I}\right) \text { degrees } \mathrm{C} .
$$


instance, the test load should not be applied until the transformer has been in circuit for a sufficient time to attain the temperature elevation due to core loss. With transformers for commercial lighting, the duration of the full-load test may be taken as three hours, unless otherwise specified. In the case of railway, crane and elevator motors, the conditions of service are necessarily so varied that no specific period cor. respoding to the full-term load can be stated.

35. The commercial rating of a railway motor should be the h.p. output, giving $75^{\circ} \mathrm{C}$ rise of temperature, above a room temperature of $25^{\circ} \mathrm{C}$, after one hour's continuous run at 500 volts terminal pressure on a stand, with the motor covers removed.

For determining the service temperature of a railway motor, the temperature rise should be determined by operating the motor on a straight and level track and under specified conditions :

(I) As to the load carried in tons per motor.

(2) The schedule speed in miles per hour.

(3) The number of stops per mile.

(4) The duration in seconds of the stops.

(5) The acceleration to be developed in miles per hour per second.

(6) The braking retardation to be developed in miles per hour per second.

These specifications should be determined or agreed upon, as equivalent to the actual service, and the motors to be closed or open, according to the way in which they are to be operated in service.

The tests should be made in both directions over the same track.

By a "level track" should be understood a track in which the gradient does not exceed one-half per cent. at any point.

By a "straight track" should be understood a track in which the radius of curvature is nowhere less than the distance travelled by the car in 30 seconds, at the maximum speed reached during the run.

The wind velocity during a test should not exceed ro miles per hour in any direction.

\section{INSULATION.}

36. The ohmic resistance of the insulation is of secondary importance only, as compared with the dielectric strength, or resistance to rupture by high voltage.

Since the ohmic resistance of the insulation can be very greatly increased by baking, but the dielectric strength is liable to be weakened thereby, it is preferable to specify a high dielectric strength rather than a high insulation resistance. The high-voltage test for dielectric strength should always be applied.

\section{INSULATION RESISTANCE.}

37. Insulation resistance tests should, if possible, be made at the pressure for which the apparatus is designed.

The insulation resistance of the complete apparatus must be such that the rated voltage of the apparatus will not send more than $\mathbf{1} / \mathbf{1}, 000,000$ of the full load current, at the rated terminal voltage, through the insulation. Where the value found in this way exceeas I megohm, I megohm is sufficient.

\section{Dielectric Strength.}

38. The dielectric strength or resistance to rupture should be determined by a continued application of an alternating e.m.t. for one minute The source of alternating 
e.m.f. should be a transformer of such size that the charging current of the apparatus as a condenser does not exceed 25 per cent. of the rated output of the transformer.

39. In alternating-current apparatus, the test should be made at the frequency for which the apparatus is designed.

40. The high-voltage test should not be applied when the insulation is low, owing to dirt or moisture, and should be applied before the machine is put into commercial service.

The high potential test should be made at the temperature assumed under normal operation, as specified, in Paragraph 2 under "Efficiency."

4I. It should be pointed out that tests at high-voltages considerably in excess of the normal voltages, to determine whether specifications are fulfilled, are admissible on new machines only.

42. The test for dielectric strength should be made with the completely assembled apparatus and not with its individual parts, and the voltage should be applied as follows :

Ist. Between electric circuits and surrounding conducting material, and

2d. Between adjacent electric circuits, where such exist, as in transformers.

The tests should be made with a sine wave of e.m.f., or where this is not available, at a voltage giving the same striking distance between needle points in air, as a sine wave of the specified e.m.f., except where expressly specified otherwise. As needles, new sewing needles should be used. It is recommended to shunt the apparatus during the test by spark gap of needle points set for a voltage exceeding the required voltage by to per cent.

43. The following voltages are recommended for apparatus not including transmission lines or switchboards.

Rated Terminal Voltage.

Not exceeding 400 volts.

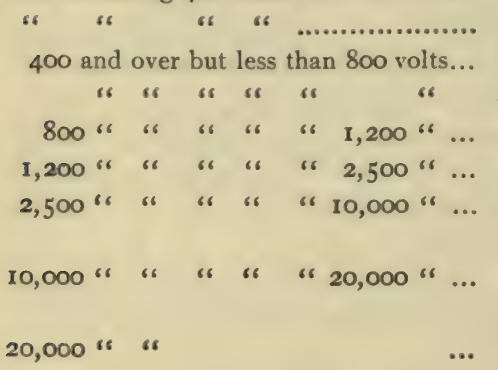

Rated Output.

Under $10 \mathrm{k}$. w.

Testing Voltage.

w. and over.............. I,500 "6

Under ro k. w................ I, 500 "

10 k.w. and over............ 2,000 "6

Any ........................... 3,500 6

Any ........................... 5,000, 6

Any..... Double the normal rated voltages.

Any...... 10, o0o volts above normal rated voltages.

Any...... 50 per cent. above normal rated voltages.

Except that transformers of 5,000 volts or less, directly feeding consumption circuits, should be tested at I0,000 volts.

Synchronous motor fields and fields of converters started from the alter-

nating current side

5,000 volts. 
Alternator field circuits should be tested under a breakdown test voltage corresponding to the rated voltage of the exciter, and referred to an output equal to the output of the alternator; i. e., the exciter should be rated for this test as having an output equal to that of the machine it excites.

Condensers should be tested at twice their rated voltage and at their rated frequency.

The values in the table above are effective values, or square roots of mean square, reduced to a sine wave of e.m.f.

44. In testing insulation between different electric circuits, as between primary and secondary of transformers, the testing voltage must be chosen corresponding to the high-voltage circuit.

45. In transformers of 20,000 volts upwards, it should be sufficient to test the transformer by operating it at 50 per cent. above its rated voltage ; if necessary, with sufficiently higher frequency to induce this voltage.

46. The test of the insulation of a transformer, if no testing transformer is available, may be made by connecting one terminal of the high voltage winding to the core and low-voltage winding, and then repeating the test with the other terminal of the high-voltage winding so connected. The test of dielectric resistance between the low-voltage winding and the core should be in accordance with the recommendation in Section 43, for similar voltages and capacities.

47. High voltage tests on transformers or other apparatus should be based upon the voltages between the conductors of the circuit to which they are connected.

48. When machines or apparatus are to be operated in series, so as to employ the sum of their separate e m.f.'s, the voltage should be referred to this sum, except where the frames of the machines are separately insulated both from ground and from each other.

The insulation between machines and between each machine and ground should be tested, the former referred to the voltage of one machine, and the latter to the total voltage of the series.

49. Underground cables, and line switches, should be tested by the application of an alternating e.m.f. for one minute at twice the voltage at which the cable or switch is to be operated.

\section{RATING.}

75. Both electrical and mechanical power should be expressed in kilowatts, except when otherwise specified. Alternating-current apparatus should be rated in kilowatts on the basis of non-inductive condition; i. e., with the current in phase with the terminal voltage.

76. Thus the electric power generated by an alternating-current apparatus equals its rating only at non-inductive load, that is, when the current is in phase with the terminal voltage.

77. Apparent power should be expressed in kilovolt-amperes as distinguished from real power in kilowatts.

78. If a power-factor other than 100 per cent. is specified, the rating should be expressed in kilovolt-amperes and power-factor, at full load.

79. The full-load current of an electric generator is that current which with the 
rated full-load terminal voltage gives the rated kilowatts, but in alternating-current apparatus only at non-inductive load.

8o. Thus, in machines in which the full-load voltage differs from the no-load voltage, the full-load current should refer to the former.

If $P=$ rating of an electric generator and $E=$ full-load terminal voltage, the fullload current is :

$I=\frac{P}{E}$ in a continuous-current machine or single-phase alternator.

$I=\frac{P}{E \sqrt{3}}$ in a three-phase alternator.

$I=\frac{P}{2 E}$ in a quarter-phase alternator.

8r. Constant-current machines, such as series arc-light generators, should be rated in kilowatts based on terminal volts and amperes at full load.

82. The rating of a fuse or circuit breaker should be the current-strength which it will continually carry. In addition thereto, the current-strength at which it will open the circuit should be specified.

\section{Classification of Voltages and Frequencies.}

83. In direct-current, low-voltage generators, the following average terminal voltages are in general use and are recommended :
125 volts.
250 volts.

550 to 600 volts.

84. In direct-current and alternating-current low-voltage circuits, the following average terminal voltages are in general use and are recommended :

$$
\text { I } 10 \text { volts. }
$$

220 volts.

In direct-current power circuits, for railway and other service, 500 volts may be considered as standard.

85. In alternating-current, constant-potential, primary-distribution circuits, an average e.m.f. of 2,200 volts, with step-down transformers of ratios $1 / 10$ and $1 / 20$, is in general use, and is recommended :

86. In alternating-current, constant-potential, high-pressure circuits, at the receiving end, the following voltages are in general use, and are recommended :

6,000. 10,000. 15,000. 20,000. 30,000. 40,000. 60,000.

87 . In alternating-current generators, or generating systems, a range of terminal voltage should be provided from no-load voltage to Io per cent. in excess thereof, to cover drop in transmission. If a greater range than ten per cent. is specified, the generator should be considered as special.

88. In alternating-currrent circuits, the following approximate frequencies are recommended as desirable:

25 cycles per second. 60 cycles per second. $\quad$ I 20 cycles per second. *

These frequencies are already in extensive use and it is deemed advisable to adhere to them as closely as possible.

* The frequency of 120 cycles per second may be considered as covering the already existing commercial frequencies between 120 cycles per second and 140 cycles per second. 


\section{Overload Capacities.}

89. All guarantees on heating, regulation, sparking, etc., should apply to the rated load, except where expressly specified otherwise, and in alternating-current apparatus to the current in phase with the terminal e.m.f., except where a phase displacement is inherent in the apparatus.

90. All apparatus should be able to carry the overload specified in Section 92, without self-destruction by heating, sparking, mechanical weakness, etc., and with an increase in temperature elevation not exceeding $15^{\circ} \mathrm{C}$. above those specified for full loads, the overload being applied after the apparatus has acquired the temperature corresponding to full-load continuous operation. (See sections 30 to 34.)

91. Overload guarantees should refer to normal conditions of operation regarding speed, frequency, voltage, etc, and to non-inductive conditions in alternating apparatus, except where a phase displacement is inherent in the apparatus.

92. The following overload capacities are recommended :

Ist. In direct-current generators and alternating-current generators, 25 per cent. for two hours.

2d. In direct-current motors, induction motors and synchronous motors, not including railway motors and other apparatus intended for intermittent service, 25 per cent. for two hours, and 50 per cent. for one minute, for momentary overload capacity.

3d. Synchronous converters. 50 per cent. for one half hour.

4th. Transformers. 25 per cent. for two hours. Except in transformers connected to apparatus for which a different overload is guaranteed, in which case the same guarantees shall apply for the transformers as for the apparatus connected thereto.

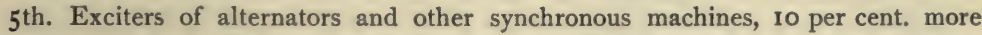
overload than is required for the excitation of the synchronous machine at its guaranteed overload, and for the same period of time.

7 th. All exciters of alternating-current, single-phase or polyphase, generators should be able to give at constant speed, sufficient voltage to excite the alternator, at the rated speed, to the full-load terminal voltage, at the rated output in kilovolt-amperes and with 50 per cent. power factor. 


\section{CHAPTER VII.}

THE PRACTICAL OPERATION OF DYNAMOS. STATION EQUIPMENT.

80. General instructions for managing generators and motors.* Dynamos are usually provided with an eye-bolt to which ropès or chains for lifting may be attached. In handling a dynamo, or in removing an armature for repairs, great care must be exercised to avoid abrasion of the windings. An armature should never be laid upon a hard floor but should always rest upon a soft bed of sacking.

The bearings should be kept thoroughly lubricated with a fine grade of oil. Carbon brushes do not need much oil lubrication, and in any case oil must be used very sparingly on the commutator, since the sparking tends to char it, and furthermore, the oil collects dust and retains the worn-off particles of carbon and copper producing a gummy coating which leads to poor electrical contact between the brushes and the commutator.

The commutator should be cleaned occasionally with a tightly woven cloth (free from lint), and, after this operation, a mere trace of oil, or preferably vaseline, may be applied to the cloth and thence to the commutator.

The tension of the brush springs should be ordinarily adjusted to give a pressure of about I.5 pounds per square inch of contact surface between the brushes and the commutator. Too little pressure leads to chattering of the brushes and consequent sparking and roughening of the commutator. Excessive brush pressure gives rise to excessive brush friction and undue heating of the commutator and brushes.

* This discussion, and the discussion of dynamo diseases or faults, is adapted from the excellent little book " Practical Management of Dynamos and Motors," by F. B. Crocker and S. S. Wheeler. 
A dynamo should be kept perfectly clean and dry, and it should not be exposed to dust. A water-proof covering should be thrown over the machine when it is not in use.

When a dynamo, generator or motor, is started for the first time (or after it has been standing unused for a long time), it should be very closely watched for an hour or more, inasmuch as an unexpected defect may develop.

If there is the remotest possibility that a generator is wet or even moist, it should be driven for several hours without exciting its field; the idea being to accelerate the drying process by the fan-like action of the rotating armature. The field is then to be gradually and cautiously excited by cutting out resistance in the field rheostat, and finally the current output is to be cautiously increased to its full rated value.

When a motor has been exposed to dampness it should be run as a generator and treated as above described, the idea being to drive the machine for a number of hours and dry it thoroughly before any electromotive force is brought into action between the various windings. In the case of an isolated motor, however, it is not feasible to drive it as a generator. If the machine is very damp it should be dried by gentle and long-continued heating. In many cases, however, it is allowable to excite the field of the motor, start it, and run it for several hours on zero load; the motor may then be gradually loaded until full load is reached.

Unless a dynamo is positively known to be dry it must be assumed to be moist and treated accordingly. A new machine may become damp in transit from the factory.

In the opening of a switch a destructive arc or spark is likely to be formed, especially if the current is large, or the inductance of the circuit is high, as in the case of a field winding. Properly constructed switches have carbon blocks so arranged as to take this arc or spark off the metal parts of the switch, thus lessening the wear on the switch. In any case a switch must be opened and closed with a vigorous and unhesitating movement.

A belted dynamo, generator or motor, should be provided 
with a screw device for adjusting the tension of the belt by moving the machine back and forth on slide rails.

81. General directions for starting. - Before starting a dynamo, generator or motor, see that the machine is clean, especially the commutator and brushes. The metallic dust which comes from the wear of the commutator and brushes is likely to make a short circuit, and it should therefore be removed with special care. Examine the whole machine closely to be sure that all screws and contacts are tight. Fill the oil cups, adjust the oil feed, and see that the oil ducts are clear. In the case of ring oiling bearings, the rings should work freely and they should at all times dip into the oil in the reservoir. See that the belt is in place and has the proper tension. If it is the first starting of the machine, it should be turned a few times by hand, or very slowly by power, to make sure that the machine rotates freely, and that the belt runs in the center of the pulleys. Examine the brushes; see that they press with sufficient pressure against the commutator, and adjust them to the proper position by moving the rocker-arm. If the proper position for the brushes is not indicated by a mark, this last adjustment must be left until after the machine is running and its field excited. Then the proper position of the brushes is indicated by minimum sparking, and by maximum voltage in the case of a generator, or minimum speed in the case of a motor.

The machine should then be started and brought up to full speed, gradually if possible, all switches, in the case of a generator, being left open. While starting, the attendant should be in instant readiness to stop if anything should seem to go wrong.

82. Directions for starting and stopping a single shunt or compound generator.* - The arrangement of a single shunt or compound generator for supplying current to lamps or motors is shown in Fig. 69. In the starting of a single generator plant like this the procedure is simple, there being no necessity for the special precautions required in the starting and stopping of one

* The management of the various types of series generators used for arc lighting is described in special books of instruction issued by the manufacturers. 
of a set of generators in parallel, as pointed out in a subsequent article. To start the generator shown in Fig. 69 open the main switch, if it is not already open, turn the hand wheel of the field rheostat so as to include all of its resistance in the field circuit, start the engine and generator and bring them slowly up to full speed, cut out resistance from the field rheostat until the generator builds up to nearly full voltage, close the main switch and finally adjust the field rheostat to give the exact voltage desired. Raising the voltage above the proper steady value is likely to damage the incandescent lamps, and is to be carefully avoided. To stop the generator, turn the hand wheel of the field rheostat so as to include all of the resistance of the rheostat in the field circuit, open the main switch and stop the engine. In case of an over-load, due to short circuit out on the mains or to the connecting of too many lamps or motors, the circuit breaker will open the circuit. Before closing the circuit breaker again, open the main switch, then close the circuit breaker, and finally close the main switch; by proceeding in this way the circuit breaker will operate instantly if the short-circuit on the line still exists.

Directions for starting and stopping motors are given in articles 59 and $6 \mathrm{I}$.

83. Diseases of dynamos. - Difficulty in getting a dynamo to operate is most frequently due to a broken connection or a loose contact. Always make sure that the connections are properly made and that the various circuits are free from loose and uncertain contacts. Troubles in the dynamo itself may be classified according to their symptoms as follows :

I. Sparking at the commutator.

II. Heating of commutator and brushes

III. Heating of armature.

IV. Heating of field magnet.

V. Heating of bearings.

VI. Noise.

VII. Symptoms peculiar to generators.

VIII. Symptoms peculiar to motors. 
Sparking and noises are, of course, readily perceived. The overheating of a dynamo may be most easily detected by applying the hand to the various parts of the machine, or by holding the hand in the stream of air that is thrown off from the rotating armature. In general a temperature that cannot be borne by the hand is to be considered excessive, although carbon brushes and a commutator in which there is no solder may be safely run at a higher temperature than can be borne by the hand. Of course, the hand can bear a much higher temperature in a non-conducting substance like wood or varnish than it can in a conducting substance like metal, so that a little experience is necessary to enable one to judge whether the temperature of any part of a dynamo is excessive. An odor of burning varnish is indicative of serious overheating, and a machine which shows this symptom should be stopped at once. Never use water or ice to cool a dynamo.

I. Sparking at the commutator. - This may be due to the following causes :

I. Excessive current on account of a simple overload on a motor or generator, or to a short-circuit on the supply mains leading out from a generator.

2. Brushes not in proper position. (See Art. 78.)

3. Commutator surface rough or uneven causing brushes to jump. This defect may be remedied by using a fine file on the commutator while the armature is in motion and the field unexcited, or by using fine sand-paper applied by a block of wood which is hollowed out to fit the commutator ; care must be taken to remove sand and copper dust when the job is finished. Never use emery paper or emery cloth on a commutator.

If the commutator surface is very uneven, the armature, if not too large, must be removed, placed in a lathe, and the commutator turned true and smooth, making one or more fine cuts with a diamond-pointed tool.

4. Very poor balance of the armature may also cause sufficient vibration to make the brushes jump.

5. Brush contact poor. This may be due to insufficient brush 
pressure, or to the fact that the ends of the brushes are not worn to the exact shape of the commutator so that the brush makes contact at a few points only. When the commutator becomes foul with grease and dust the ends of the brushes become dirty and the contact between the brushes and the commutator is poor.

6. A short-circuited coil, or an open-circuited (broken) coil in the armature may cause sparking. This sparking is always located at one point on the armature, namely at the commutator segments in which the defective armature coil terminates. A short-circuited coil is distinguished from an open-circuited coil partly by less violent sparking, and, partly by excessive local heating in the former case. Sometimes a short-circuit or break occurs on account of the displacement of the armature conductors by centrifugal force while the machine is in motion. Such a fault $\mathrm{i}_{\mathrm{S}}$ often very perplexing inasmuch as it cannot be detected by test on the machine when it is standing still.

7. "Grounds" in armature winding, that is, accidental electrical contact between two or more points of the armature conductors and the armature core, will produce sparking.

8. Weak field excitation. This cause of sparking may be identified in any one of three ways, namely: $(a)$ by the low voltage which is associated with it in a generator, or the high speed in a motor; $(b)$ by the weakness of the magnet poles as indicated by their attraction for a bit of iron held in the hand (care being taken that the bit of iron is not pulled out of the hand and thrown against the rotating armature); (c) by the abnormally large angle of lead of the brushes required to give sparkless running. This defect, weak field excitation, is usually due, in a shunt or compound dynamo, to an excessively high resistance in the shunt field circuit due to a faulty contact either in the field winding itself or in the field rheostat

9. Chatter of brushes. When the brushes are improperly held in the brush-holders the vibration may cause them to chatter.

II. Heating of commutator and brushes. - This may be due to the following causes : 
I. Conduction of heat from another and hotter part of the dynamo.

2. Sparking. Sometimes a degree of sparking that is scarcely visible may cause overheating of the brushes and commutator.

3. Arcing or short-circuit between the commutator segments across the mica insulation. This may be due to the collection of metallic particles. The remedy is to clean the commutator thoroughly.

4. Imperfect electrical connections between the brushes and the brush holder. This frequently happens with carbon brushes. In any case, carbon brushes heat more than metallic brushes; this is especially the case if the ends of the carbon brushes do not fit the commutator accurately, thus giving a surface of contact which is far from uniform. Carbon brushes should not be depended upon to carry much more than 40 amperes per square inch of contact surface.

III. Heating of the armature. - This may be due to the following causes :

I. Conduction of heat from another and hotter part of the dynamo.

2. Overload as explained under cause (I) for sparking. This always causes an overheating of the entire armature.

3. Poor insulation of armature windings due to moisture. This may cause local heating if the moisture is localized. It is indicated by the steaming of the armature. The wet armature should be baked for several hours at about $240^{\circ} \mathrm{F}$. in an oven, near a fire, or by passing full-load current through it. In the latter case the armature may be left in the machine and kept in motion, but the field should not be excited.

4. Short-circuited coils. This produces local heating. See discussion of cause (5) of sparking.

IV. Heating of field coils. - This may be due to :

I. Conduction of heat to the field coils from another hotter part of the dynamo.

2. Excessive current in the field coils caused either by an exces- 
sive voltage, or by the short-circuiting of a portion of the field winding.

3. Moisture in the field coils. See discussion of cause (3) of armature heating.

V. Heating of bearings. - This may be due to :

I. Conduction of heat to the bearings by another and hotter part of the dynamo.

2. Any one of the common mechanical defects; lack of oil ; grit ; roughness of shaft ; too tight a fit between shaft and bearing ; crooked shaft; poor alignment of bearings ; end thrust of collar, or pulley, or shoulder on shaft against the bearing; or excessive belt tension.

3. Side pull of field magnet upon the armature when the armature is nearer to one pole-piece than to the other or others.

VI. Noise. - The sound produced by almost any machine is perhaps the best indication to an experienced attendant of its condition. The legitimate sounds of a dynamo are the humming sounds due chiefly to the armature teeth as they pass the tips of the pole pieces, and to the slight movement of the iron parts, especially of the laminations, as they are magnetized and demagnetized by pulsating or alternating currents. The abnormal noises are the following:

I. The fluttering noise accompanied by more or less violent vibration due to unbalanced armature or pulley.

2. The noise due to the striking of the armature against the pole pieces, or to the striking of the shaft collar, or pulley, or belt against the bearings. At low speed this is a scraping noise, and at full speed it is like No. I. The striking of the armature against the pole pieces may be detected by the scraping noise at low speed, by the abrasion of the armature surface, or by looking through the gap space in front of the various pole pieces.

3. Rattling noise due to loose parts, such as screws. This noise is most perceptible at moderately low speeds.

4. Flapping of belt or the pounding of the laced or cemented joint against the pulley. A certain amount of flapping and 
pounding noise is unavoidable and legitimate, but a whip-like crack as the joint passes over the pulley indicates a loose lacing.

5. A sharp screeching noise, like that produced by rubbing the hand over a varnished table, indicates the slipping of the belt due either to overload or to insufficient belt tension.

6. A squeaking or hissing noise is produced by the brushes. When this becomes very prominent, a little vaseline is needed. The source of this noise may be determined by pressing the finger against one brush at a time, or, if there is more than one brush in each set, one brush at a time may be lifted from the commutator. The chattering of the brushes is indicated by noise intermediate between a rattle and a screech.

VII. Symptoms of trouble peculiar to a generator are those which have to do with its voltage. The generator may not build up at all, it may develop only a weak electromotive force, or it may become reversed.

I. Failure to build up. This is perhaps the trouble most frequently encountered in the starting of a generator. The following brief statements of the causes of failure to build up are based upon the full discussion in Art. 44 .

(a) Excessive resistance in the field circuit due to a poor contact or an actual break. The brush contacts often have an excessively high resistance when a generator is first started, and a momentary pressure of the fingers on the brush or brushes may enable the machine to build up.

(b) A short circuit in the machine or in the external circuit prevents a shunt generator from building up.

(c) Residual magnetism too weak. In this case the field must be disconnected from the armature and excited for a moment from an outside source.

(d) Reversed connections or reversed direction of rotation. (See Art. 44.)

(e) Brushes not in proper position. In all of the discussion in this treatise the brushes are represented as in their proper positions when they are nearly midway between the field poles. This 
however is seldom or never the case in actual machines, because the connections from the armature conductors to the commutator segments are seldom or never radial. That position of the brushes which gives a maximum electromotive force between them as indicated by a voltmeter is the proper position.

2. Excessively low voltage of a shunt generator which is driven at full speed is due to an excess of resistance in the shunt field circuit.

3. Reversal of a generator. When a machine is standing idle its residual magnetism may be reversed by proximity to another dynamo, or by stray current from some outside source. When the generator is next started it will build up in a direction the reverse of that desired. This reversal of a generator may be corrected by stopping the machine, exciting its field for a moment in the proper direction from some outside source, and then starting it up again.

When a number of generators are to be run in parallel, the shunt field windings of all of them should be connected to the bus * bars, for then it is not possible for one of the machines to become reversed with respect to the others.

VIII. Symptoms of trouble peculiar to a motor are abnormal variations of speed, and, inasmuch as the speed of a series motor varies greatly under ordinary running conditions, the following simple statements apply primarily to the shunt motor.

I. Failure to start. This is most likely to be due to a faulty connection, but it may be due to excessive starting friction, or to a combination of moderate starting friction with an under-excitation of the field magnet.

2. Running at low speed. This might conceivably be due to a greatly over-excited field, but it is more probably due to an over-load, or to the combination of a heavy load with an underexcited field.

3. Running at excessive speed. This never occurs on a very

* Omnibus bars, the bars which run along the back of the switchboard panels and which receive all the current from all of the generators. 
heavy load. It is generally due to under-excitation of field. The displacement of the brushes from their proper positions causes a shunt motor to run faster than its normal speed if its load is not too great, but this is always accompanied by sparking and this trouble, therefore, is covered by the discussion under class $\mathrm{I}$.

84. Tests for faults in armatures.* - In the four tests described below, the field of the machine is not excited and the armature is not run.

(a) Tests for broken leads. - Clean the commutator and substitute for one of the regular brushes a piece of sheet metal which touches one commutator bar at a time. Connect a low voltage and low resistance battery through an ammeter to the metal brush and to the carbon brush, and turn the armature very slowly by hand causing the metal brush to make good contact with each commutator bar in succession. Usually there are two leads soldered to each commutator bar. A break in any one of these leads will be indicated by a reduced deflection of the ammeter, and a break in both leads will be indicated by zero deflection of the ammeter when the metal brush touches the commutator segment to which the faulty leads belong.

(b) If no broken lead is found by test $(a)$, a broken circuit in the armature winding itself may be located as follows: Connect the low resistance battery through an ammeter to two metal strips which touch adjacent commutator bars, and turn the armature very slowly by hand. An armature section which is broken, or which has an abnormally high resistance, will be indicated by a reduced deflection of the ammeter. In this test a short-circuited armature section will be indicated by an increased ammeter deflection.

In both of these tests $(a)$ and $(b)$ the ammeter circuit will be repeatedly broken as the metal brush (or brushes) rides over the

* See paper on "Location of Grounds in Armatures, Fields, etc.," by C. E. Gifford, Trans. Am. T. E. E., Vol. XII., pages 260-267. 
mica insulation between the commutator bars, and care must be taken to distinguish these breaks from the partial or complete breaks which may be found while the brushes make good contact with the commutator bars.

(c) Test for short-circuit. - A short-circuited armature section may perhaps be best located as follows: Pass a current from a battery through the armature, using the regular brushes of the machine. Then take a very low reading voltmeter (or a galvanometer), connect wire leads to it, and touch these wire leads to one pair after another of adjacent commutator bars. A zero or greatly reduced deflection of the voltmeter indicates a shortcircuited section.

(d) Test for ground. - A ground in the armature winding, that is, a connection between the armature winding and the armature core may be located as follows: Connect a battery to the dynamo brushes. Bring two leads from a very low reading voltmeter (or a galvanometer) and connect one of these leads to the armature shaft and the other to one after another of the commutator bars. A deflection of the voltmeter indicates a ground, and the ground is located near the commutator bar that gives the least deflection.

85 Rules for personal safety. - Personal injury may result from the passage of an electric current through the body, or from severe burns due to a short circuit of the mains by a screw-driver or other metal tool held in the hands. To avoid the first danger the body must not be made a portion of an electric circuit, to avoid the second danger never use a metal tool on live wires as, for example, in the replacing of a burned out fuse.

To make the body a portion of an electric circuit requires, of course, two points of contact with the body. To avoid this it is important to use one hand, only, in manipulating apparatus which is connected with live wires, the other hand being put in the pocket or held behind the back. It must not be forgotten, in this connection, that the greatest risk lies in making a circuit through the hand to the feet when one stands on a moist floor 
and when there is a ground on one main. A workman should, therefore, always stand on a dry wooden box or platform when working with live wires. Rubber overshoes, if they are whole, insulate a person effectively from the ground, and the use of rubber gloves and of tools with insulated handles is sometimes advisable. It is better, however, not to use such devices on account of the habit of carelessness they develop.

The idea that voltages above a certain minimum, only, are dangerous is erroneous. Everything depends upon the nature of the two contacts with the body and their proximity. Voltages as low as IIO volts are dangerous if the contacts with the body are broad metal plates pressed against the moist skin.

An electrical shock often produces death indirectly by stopping the action of the respiratory organs, and in every case a prompt attempt should be made to restore respiration by the method of artificial breathing employed as a first aid to a person who is taken from the water in a drowning condition.

86. The operation of generators in parallel. - A generator operates at its maximum efficiency at or near full load. Therefore when the total output of a station varies from hour to hour, as it always does in electric lighting and street railway service, it is desirable to use several generators which may be put into service one after the other as the station output increases, and disconnected one after the other as the station output decreases; the object being to maintain nearly full load at all times upon the generators which are in service. This is especially advisable when each generator is driven by a separate engine, inasmuch as the engines also may then be operated under the most economical conditions. This use of a number of generating units in a station is also advantageous in that a spare unit (engine and generator) may be installed at a moderate cost to serve in case of a breakdown, whereas a single-unit station would have to be completely duplicated in order to provide for such an emergency.

When a number of shunt or compound generators are installed in a station as above explained they are always operated in 
parallel. Series generators are seldom used in combination, but when they are so used they are always operated in series.

The proper division of the total load among a number of shunt generators connected in parallel depends simply upon the adjustment of their respective shunt field rheostats. On the other hand, a number of compound generators connected in parallel as shown in Fig. I I constitute an unstable system. To obviate this instability and ensure the proper distribution of load among a number of compound generators operating in parallel, the so-called equalizing arrangement of connections is used. This equalizing arrangement

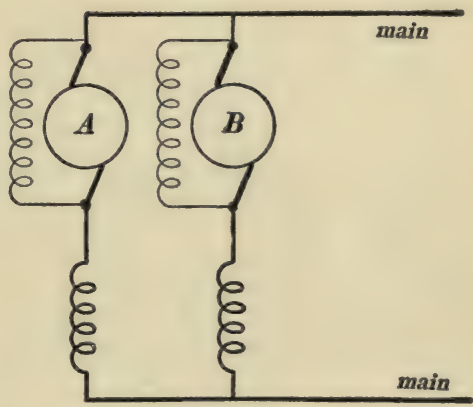

Fig. 111. consists simply of the connection of the series field coils of all the generators in parallel with each other, independently of the parallel connections of the generator armatures, as shown in Fig. I I2. Two distinct conditions must be satisfied by the arrange-

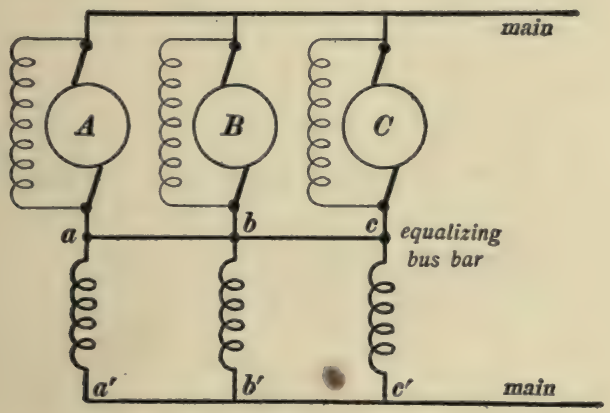

Fig. 112.

ment shown in Fig. I 2 to ensure the proper division of the load among the several generators, namely:

(a) Each compound generator must be separately adjusted to give the same degree of compounding, by means of a German 
silver shunt $S$ (not shown in Fig. I I 2) connected in parallel with its series field coil as explained in Art. 50.

(b) The total resistances $a a^{\prime}, b b^{\prime}$ and $c c^{\prime}$, Fig. I I 2, must be inversely proportional to the full load current outputs of the respective generators. This ensures the proper division of the total current among the several series field coils.

The use of the equalizing arrangement makes it possible to operate in parallel compound generators which are very different in size and in design.

The instability of two or more compound generators connected in parallel may be shown as follows: Consider two over-compounded generators connected in parallel as shown in Fig. I I I. Suppose that the machines $A$ and $B$ are running steadily and that each is giving half of the total current that is being delivered to the mains. If machine $A$ then runs momentarily at a slightly increased speed a momentary increase of its induced electromotive force is produced, which causes an increase in the current which it delivers and a corresponding decrease in the current delivered by $B *^{*}$ The increase of load current in machine $A$ causes a further increase of the induced voltage of this machine, inasmuch as it is understood to be over-compounded, and this increase of induced electromotive force causes a further increase of load current in $A$. At the same time the lessened current in $B$ causes a decrease of its induced electromotive force which tends to decrease its current still more, and so on. This unequal distribution of load between $A$ and $B$ when once started goes to an extreme and leads to an excessive load current in machine $A$ (or $B$ ) and a negative load current in machine $B$ (or $A$ ), that is one machine becomes a motor and takes poyer from the other.

Generator panels. - The accessory apparatus belonging to each one of a number of generators operating in parallel is mounted on

* This is due to the fact that the two machines form together a closed circuit of very low resistance, and a slight difference in the electromotive forces of the two machines causes a large current to flow around this circuit adding to the load current of one machine and subtracting from the load current of the other. 
a switch-board panel, and these panels, called generator panels, are arranged side by side as shown in Fig. II3. The dotted lines represent the panels and the full lines represent the electrical connections. Fig. I I 4 shows a special form of switch for opening the shunt field circuit of a generator. This switch is not shown in Fig. I I3, inasmuch as Fig. I I 3 is drawn to too small a scale.

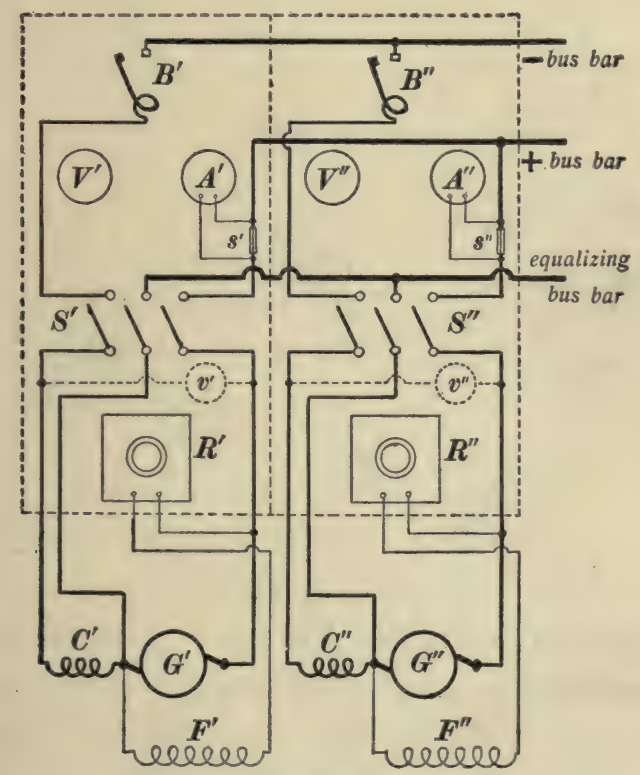

Fig. 113.

$G^{\prime}$ and $G^{\prime \prime}$ are two compound generators, $F^{\prime}$ and $F^{\prime \prime}$ their shunt field windings, $R^{\prime}$ and $R^{\prime \prime}$ their shunt field rheostats, and $C^{\prime}$ and $C^{\prime \prime}$ their series field windings. $S^{\prime}$ and $S^{\prime \prime}$ are two triple-pole switches, $A^{\prime}$ and $A^{\prime \prime}$ are ammeters, $s^{\prime}$ and $s^{\prime \prime}$ are ammeter shunts, and $B^{\prime}$ and $B^{\prime \prime}$ are circuit breakers. The small dotted circles $v^{\prime}$ and $v^{\prime \prime}$ and the dotted lines show the electrical connections of the two voltmeters $V^{\prime}$ and $V^{\prime \prime}$.

When the shunt field circuit of a dynamo is broken the current persists in flowing across the break in the form of a long arc or spark on account of the great electrical momentum of a current in a field winding of many turns of wire. This arc, occurring every time the field switch is opened, destroys the switch contacts, and, what is more serious, the high electromotive force developed 
across the break and between the terminals of the field winding is likely to puncture the insulation of the winding. The arrangement shown in Fig. I I 4 obviates these difficulties in the following manner :

The two wires $p$ and $p^{\prime}$ connect to the armature terminals, the field rheostat being placed in the field circuit at any convenient

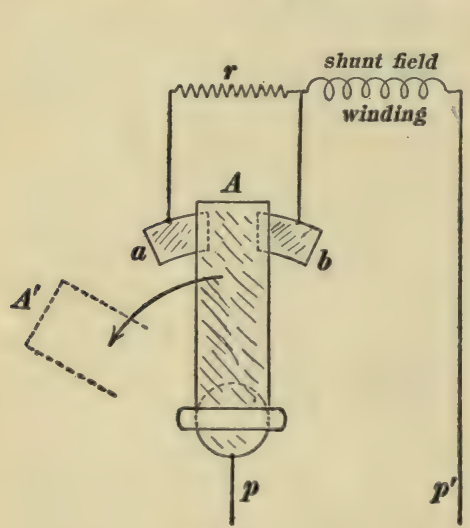

Fig. 114. point. To open the shunt field circuit the metal arm $A$ is turned slowly to the position $A^{\prime}$. At first the arm $A$ breaks contact with the metal block $b$ and connects the comparatively high resistance $r$ in series with the field winding. This leaves the shunt field circuit unbroken so that the current can continue to flow, but, on account of the high resistance $r$, the current dies away slowly, and by the time the arm breaks contact with the metal block $a$, the current is already so small that serious arcing across the break is obviated.

Feeder panels. - In a large lighting or power station a number of separate circuits are usually supplied with current from a set of parallel-connected generators. Thus Fig. I I 5 shows the essential connections of three compound generators in parallel supplying current to three circuits $F, G$ and $H$. The pairs of wires $F, G$ and $H$ are called feeders. The apparatus for controlling the feeders is usually mounted upon one or more switch-board panels, called feeder panels. These feeder panels are arranged along side of the generator panels, and the + and - bus bars run along behind all of the panels.

Fig. I 6 shows a front, back and side elevation, and the diagram connections of a feeder panel designed by the General Electric Company for two pairs of feeders. Each feeder circuit is controlled by a double-pole switch and a circuit breaker, and an 
ammeter is arranged to indicate the current delivered to each pair of feeders. In the diagram of connections in Fig. I 16 each ammeter is shown as a millivoltmeter connected to a shunt, and an arrangement is provided whereby each millivoltmeter can be connected across the bus bars in series with a high resistance so as to serve as a voltmeter. The four short lines at the bottom of the diagram of connections in Fig. I 16 are the feeders.

Fig. I 7 is a front view of a complete switch-board, of the General Electric Company's design, consisting of two generator panels and two feeder panels, and Fig. I 8 is a back view of the

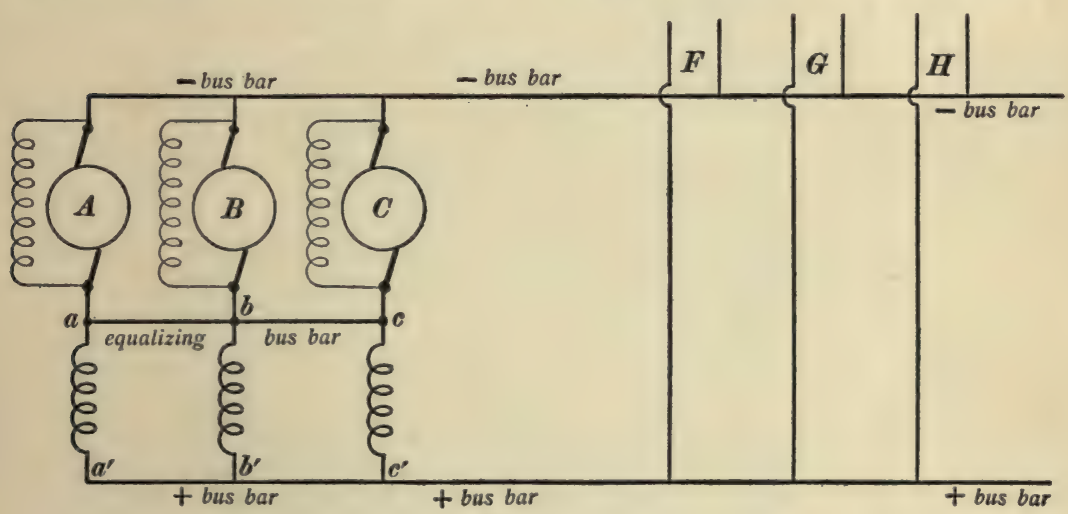

Fig. 115.

same. The large horizontal bars shown near the top in Fig. I I 8 are the bus bars. The details of one of the generator panels (except the circuit breaker at the top) are given in Fig. I I9, which shows a front, back, and side elevation of the panel and a diagram of the connections. This panel is shown without a voltmeter, and the "potential buses" are simply voltmeter leads running along back of the switch-board, so that a single voltmeter placed anywhere on the board may be connected through these "potential buses" to any machine, or to any pair of feeders, at will, by means of a plug switch which is pushed into the " receptacle." Fig. I I 9 shows a pair of fuses instead of a circuit breaker for protecting the generator. The ammeter is shown in 
190 ELEMENTS OF ELECTRICAL ENGINEERING.
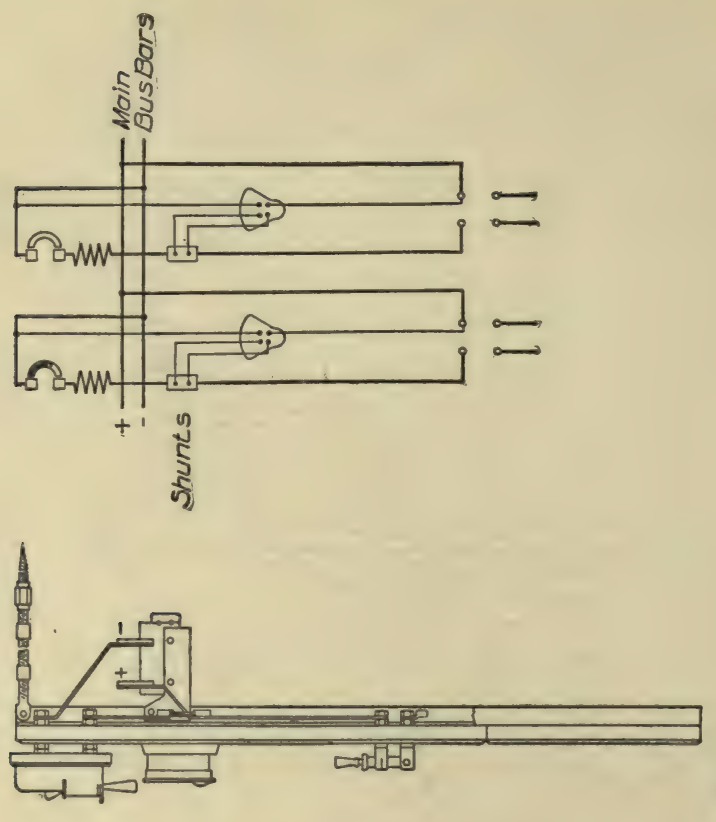

$\stackrel{\circ}{\frac{0}{10}}$

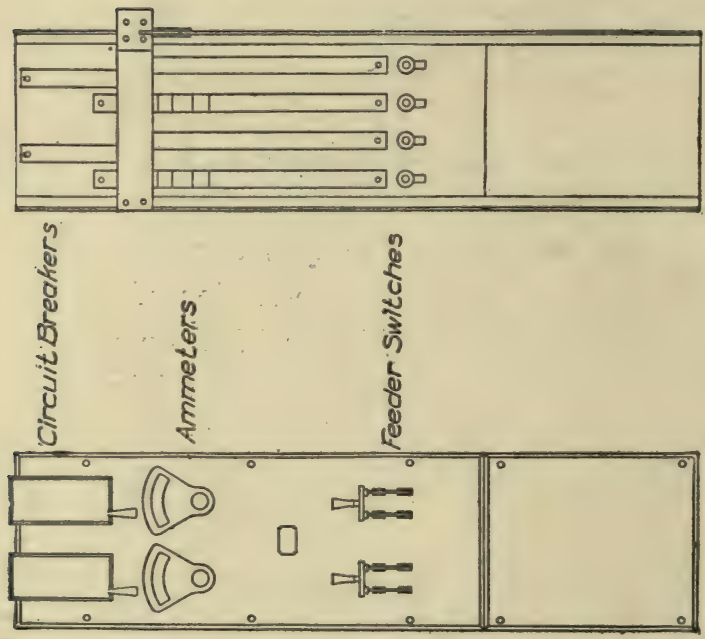


Fig. I I9 as a millivoltmeter connected to a shunt and also arranged to be connected through a high resistance to the bus bars so that it may be used as a voltmeter if desired. The dotted lines in the diagram of connections in Fig. I I 9 show a voltmeter (not on the panel) which may be connected either to the main bus bars or to the "potential buses."

One of the feeder panels in Fig. I I 7, having eight two-pole switches, controls eight pairs of feeders and the other controls

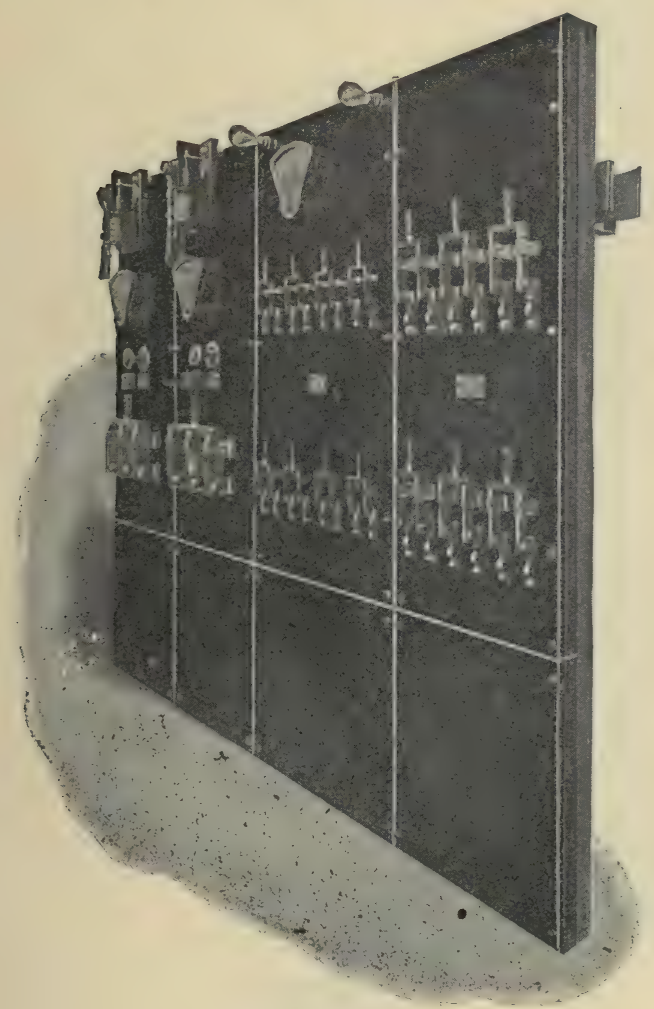

Fig. 117.

six pairs of feeders. The feeders on both of these panels are protected not by circuit breakers but by fuses as shown in Fig. I 20 , which figure gives a front, back, and side elevation and a diagram of connections of that panel in Fig. I I 7 which controls eight pairs 
of feeders. The eight pairs of feeders in the diagram of connections in Fig. I 20 are the short vertical lines beneath the sixteen small semi-circles which represent the fuses. Two lamps and a ground connection are provided on the panel shown in Fig. I 20 for use as a ground detector as explained in a subsequent article.

87. Starting and stopping of generators which are operated in parallel. - The first generator that is put into operation is started

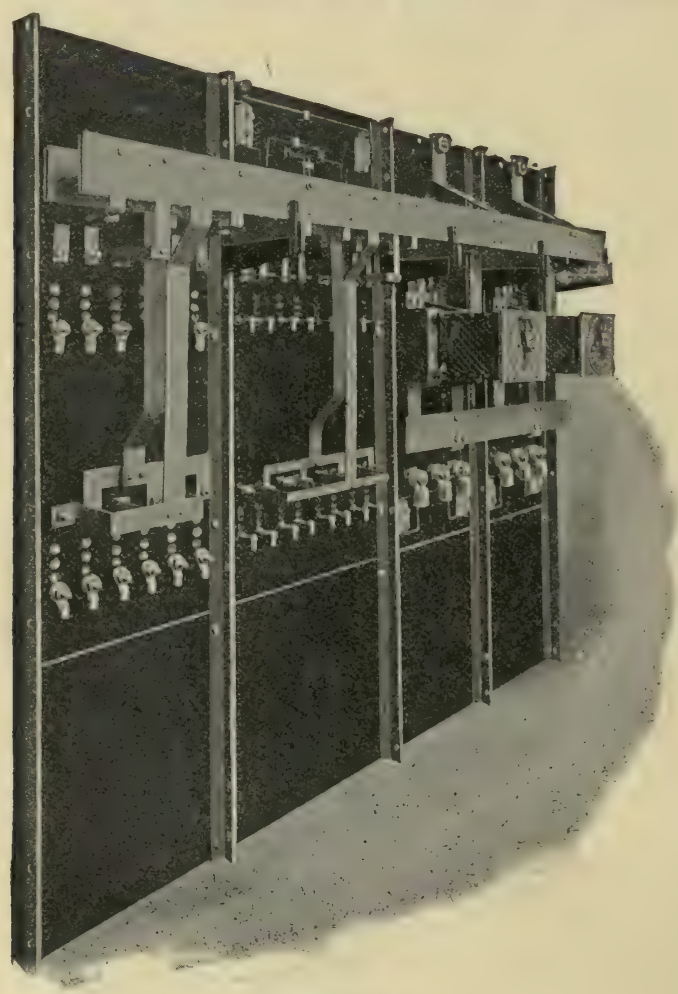

Fig. 118.

as explained in Art. 82. After the first generator, say $G^{\prime}$, Fig. I I 3 , is in operation, the others are started one at a time, as follows: The driving engine is started and the generator, $G^{\prime \prime}$, Fig. I I 3, is slowly brought up to full speed. The shunt field switch, Fig. I I4, is then closed and the machine is brought up to a volt- 
age equal to that of the generator or generators already in operation, by adjusting the shunt field rheostat, $R^{\prime \prime}$, Fig. II 3. Then the triple-pole switch $S^{\prime \prime}$ is closed and the field rheostat $R^{\prime \prime}$ is again finally adjusted to make the generator $G^{\prime \prime}$ take its share of the load.
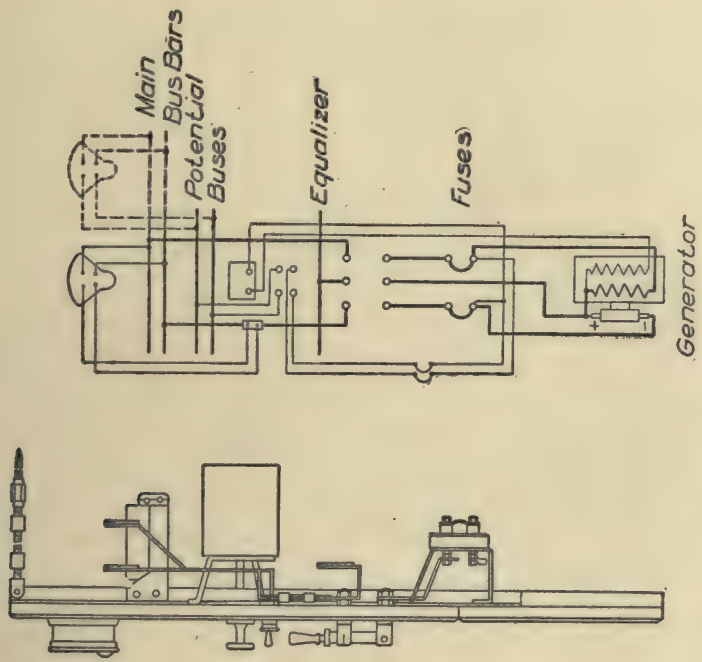

$\stackrel{\Xi}{\stackrel{\circ}{0}}$
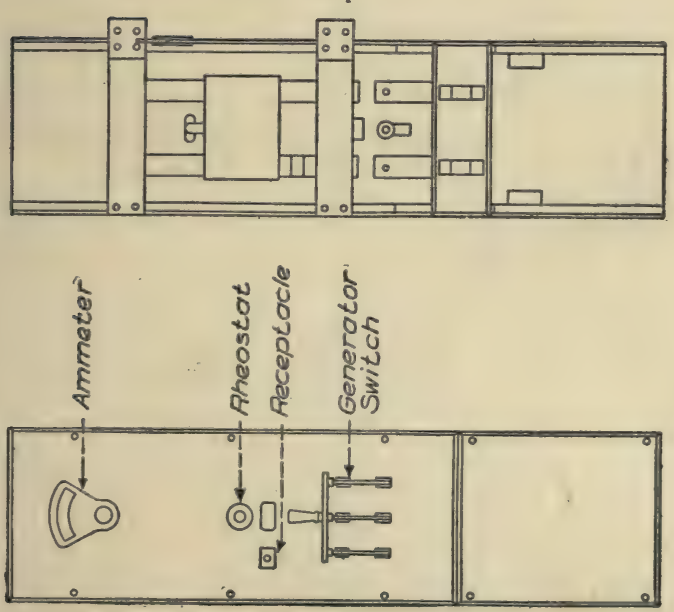
If on starting the generator $G^{\prime \prime}$, it should build up in a reversed direction, which is sometimes the case, then the closing of the switch $S^{\prime \prime}$ would be disastrous, inasmuch as the two generators, $G^{\prime}$ and $G^{\prime \prime}$ would act together to produce an excessive current
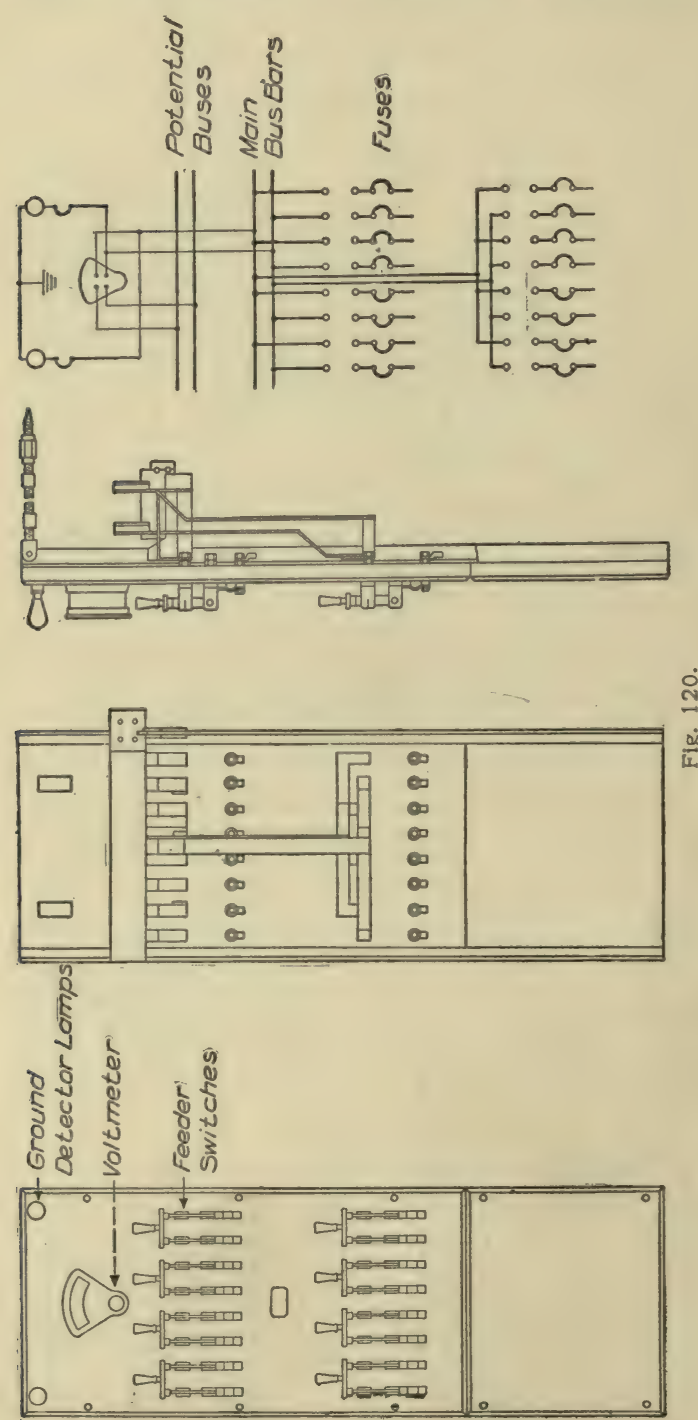
through the local circuit formed by the two machines. It is, therefore, very important, in starting a generator which is to be connected in parallel with others already in operation, to be sure that the generator builds up in the proper direction. This condition is ensured $(a)$ by leaving the series field coils permanently connected in circuit, as explained below, or $(b)$ by connecting the shunt field windings of the generators, not to the brushes of the respective machines, as shown in Figs. II I, I I 2, I I 3 and II9, but to the bus bars. This is called "bus excitation."

Never open the shunt field switch of a generator which is operating in parallel with others. To do this makes the generator armature a short-circuit for the other machines and the armature is likely to be burned out.

To stop a generator which is operating in parallel with others increase the resistance in its shunt field circuit by manipulating its shunt field rheostat until its load is very small as indicated by the ammeter. Then open the triple pole switch belonging to the generator, and stop the driving engine.

Feeder control. - A central station usually delivers current over a number of feeder circuits to a number of "distributing points," from each of which a small district is supplied with current through a network of mains. It is desirable, especially in the distribution of current for incandescent lighting, to maintain an unchanging voltage at every distribution point. The importance of this requirement is due to the fact that a slight excess of voltage shortens the life of incandescent lamps, and that a slight deficiency of voltage greatly reduces their candle power.

A constant voltage can be maintained at any one of a number of centers of distribution by proper adjustment of the voltage between the bus bars at the station; but to maintain constant voltages at two or more independent centers of distribution the voltage between each pair of fecders at the station must be adjusted separately. Let $A$ and $B$ be the constant voltages to be maintained at two centers $a$ and $b, R$ the resistance of the $a$ feeders, $R^{\prime}$ the resistance of the $b$ feeders, $I$ the current demanded at $a$, 
and $I^{\prime}$ the current demanded at $b$. Then $A+R I$ is the necessary voltage between the $a$ feeders at the station, and $B+R^{\prime} I^{\prime}$ is the necessary voltage between the $b$ feeders at the station, and, since $I$ and $I^{\prime}$ vary independently, $A+R I$ cannot in general be equal to $B+R^{\prime} I^{\prime}$.

The maintenance of a prescribed constant voltage at each center of distribution depends upon the proper control of the voltage between each pair of feeders at the switch board. The following methods of feeder control are feasible in direct-current stations. Methods of feeder control employed in alternating stations are described in a subsequent volume of this text.

(I) Rheostat method. One method of feeder control is to insert a low resistance rheostat in each feeder circuit at the switch board, to keep the voltage between the bus bars at the highest value required by any of the feeders, and to lower the voltage between each pair of feeders to the desired value by adjusting these rheostats.

(2) Combination three bus and rheostat method. The rheostat method of feeder control involves a considerable loss of energy in the rheostats when many of the feeders require voltages much below the bus bar voltage. This loss of energy may be greatly reduced by operating the generators in two independent sets, each set consisting of one or more generators operating in parallel, so that one set may be operated at a voltage somewhat higher than that of the other set. Then all of the feeders requiring higher voltages may be supplied from the first set of generators, and all of the feeders requiring lower voltages may be supplied from the second set of generators. In this arrangement the controlling rheostats for adjusting the feeder voltages to the exact desired values absorb but little power.

The operation of the generators in two independent sets requires only three bus bars inasmuch as one bus bar can serve as the common positive (or negative) terminal of all of the generators.

When the combination method of feeder control is used, one wire of each pair of feeders is connected to the common positive 
(or negative) bus bar, and the other wire is connected to a changeover switch, so that it may be connected at will to either of the two negative (or positive) bus bars. Arrangements must also be provided for quickly altering the connections on the switch board so that any given generator may be operated with either set of generators at will.

(3) The booster method. A third method of feeder control is to connect an auxiliary generator in series with each feeder circuit at the station, so that by changing the field excitation of this generator the feeder voltage may be raised to any desired extent above the bus bar voltage. This method of feeder control is not adapted to a direct current lighting station where the feeder voltages differ from the bus bar voltage by only a few volts, inasmuch as the auxiliary generators would be too expensive and the losses of power would be excessive, much greater perhaps than in the simple rheostat method of feeder control. The booster method is, however, frequently used in electric railway power stations. For example, suppose that a power station is erected for supplying current to four or five miles of electric railway, and suppose that it becomes necessary to supply current from this station to an extension of the railway. In such a case either an excessive amount of capital must be invested in a heavy copper feeder, or a very large drop amounting to one or two hundred volts must be permitted. The latter alternative is usually chosen and it is desirable to make up for the excessive voltage drop by increasing the feeder voltage at the station. This is done by connecting the armature of a one or two hundred volt generator in the feeder circuit at the station. This auxiliary generator, which may be either engine or motor driven, is called a booster. The booster used as here described would have series field excitation, so that its electromotive force would be roughly proportional to the current flowing through it, thus keeping the voltage at the distant end of the feeder circuit approximately constant irrespective of the amount of current delivered.

The use of the booster as outlined in the above example would 
be commercially economical in spite of the great loss of power in the feeder, if the demand for power at the distant section of the railway lasts but two or three hours each day, otherwise the use of the booster should be looked upon as a temporary expedient, inasmuch as a continuous loss of power in the feeder might warrant either the use of a much larger feeder or the installation of the alternating-current long-distance transmission system which is described in a subsequent volume of this text.

Modifications of the connections shown in Figs. II2, II3 and 119. - It is sometimes considered desirable to leave the series field windings of all the compound generators which operate in parallel as a set, always in circuit, whether all of the generators are delivering current or not. One reason for this may be best explained by an example.

Given two similar compound generators arranged for parallel operation. Suppose that it is desired to provide a ten per cent. increase of voltage at the station at full-load to compensate for a ten per cent. line drop at full-load so as to provide for a constant voltage at the end of the line, that is, at the lamps. Now if the station output is half full-load value (equal to full-load on one machine), it is not desirable to have the voltage more than five per cent. in excess of the zero-load value. But if this half of full station load is carried by one generator and if the series field coil of the other is disconnected, then the full current output will flow through the series field coil of the generator which is in action, and this generator will give its normal full-load voltage which is ten per cent. in excess of the zero-load voltage. If, on the other hand, both series field coils are left connected, then only one half of the current output of the station (and of the one generator) flows through each series field coil and the voltage does not rise to I Io per cent. of the zero-load value, nor indeed to ro5 per cent. for that matter. See problems on Chapter VII.

Another advantage of leaving all the series field coils permanently in circuit is that the field excitation due to these coils always causes each generator to build up in the right direction at start- 
ing. A third advantage of leaving the series field coils permanently in circuit is that the series field excitation is not suddenly added to the shunt field excitation on closing the switch. In this case the newly connected machine does not take any appreciable load until its shunt field rheostat is adjusted after the machine is connected. On the other hand, the series field coils being disconnected and the machine being of course brought up to the full station voltage by the shunt field excitation before it is connected, the sudden increase of its field excitation which is produced by connecting the series field coil, tends to make it take its load suddenly, and perhaps to make its load excessive until the shunt field rheostat is again adjusted.

When the series field coils of all the generators are left permanently in circuit, the main switches $S^{\prime}$ and $S^{\prime \prime}$, Fig. I I 3 , are of course two-pole switches, and each series field coil is provided with a single-pole switch for opening or closing its circuit at will ; but the procedure in starting is essentially as above described.

88. The operation test. - Most electric stations and equipments are installed under contracts which are completed only when the station or equipment has been put into satisfactory operation by the constructing engineer. This of course implies an operation test of the electrical installation under the supervision of the constructing engineer. This test is a most important one, inasmuch as it frequently brings to light essential details which have been overlooked, and inasmuch as it gives an opportunity for the person who is to take charge of the apparatus to work for several days under the direction of a more experienced engineer. The operation test is carried out by following the procedure involved in the regular management of the installation as explained in the foregoing articles, the various machines being started with the utmost caution and a sharp watch being kept for symptoms indicative of trouble.

The performance test. - Aside from the testing of individual machines to make sure that they meet the guaranteed regulation (see Art. 48), efficiency (see Arts. 64,65 and 68), and rating 
(see Art. 75), it is usual to make a test of a completed station, including boilers and engines to determine the economy of the station as a whole to make sure that a certain station economy, if guaranteed, is realized.

89. Ammeters. - An ammeter is a galvanometer with a pointer which plays over a divided scale and indicates, directly, the value of the current flowing through the instrument. In many cases a small but definite fractional part, only, of the current flows through the ammeter, the remainder flowing through a low resistance shunt. The numbers on the ammeter scale may be made to give the value of the total current including that which flows through the shunt. If desired, the instrument may be provided with several interchangeable shunts of different resistances, so that the instrument may be used to measure large or small currents at will. In such a case the scale readings must be multiplied by a factor to give the total current, and this factor has a large value for a low resistance shunt and a small value for a high resistance shunt.

There are five distinct types of ammeters, namely :

(a) The tangent-galvanometer type in which the pointer is attached to a small permanent magnet which is deflected by the current to be indicated. This type is now seldom used.

(b) The D'Arsonval-galvanometer type in which the current to be indicated (or a definite fractional part of it) flows through a small suspended or pivoted coil which is deflected by a permanent steel magnet. The pointer is attached to the pivoted coil. This type is much used for precision ammeters for direct currents. The Weston direct-current ammeters are of this type.

(c) The electrodynamometer type, in which the current to be indicated (or a definite fractional part of it) flows through a stationary coil and a movable (pivoted) coil connected in series. The force action between the two coils deflects the pivoted coil and causes an attached pointer to play over a divided scale. This type is much used for precision ammeters for alternating current ; it is suitable also for direct current, but it has the disadvantage 
that the deflecting forces are small, so that the instrument must be very finely constructed. Another disadvantage of this type when used for direct currents is that the direction of deflection is not reversed by a reversal of the current.

(d) The hot wire type in which the current to be indicated flows through a fine wire, which by its rise of temperature and consequent expansion, actuates a pointer which plays over a divided scale. This type of instrument, although very cheap in construction, has not yet come into very general use on account of its inconstancy.

(e) The plunger type. This type includes all of the great variety of ammeters in which a piece of soft iron is magnetized and deflected by a coil of wire through which the current to be indicated, flows. The simplest form of the plunger type of ammeter is that from which it takes its name, namely a coil of wire and a soft iron plunger which is drawn into the coil by the current, in opposition to the pull of a spring or to the pull of gravity. In the most approved form of plunger ammeter a soft iron vane is attached to a pivot which is controlled by hair springs, and the instrument is so designed as to magnetize the soft iron vane strongly, even when the current to be indicated is a small fraction of that which gives a full deflection of the instrument. This accomplishes the double purpose of making the deflections more nearly proportional to the current, and of eliminating the errors due to magnetic hysteresis, that is, to the tendency of the iron vane to retain its magnetism with a decreasing current.

The plunger type of instrument is used more than any other for switch-boards, especially for alternating currents. As a directcurrent instrument it has the disadvantage that the direction of its deflection is not reversed by a reversal of the current.

90. Voltmeters. - The voltmeter is a high-resistance ammeter, the scale of which, instead of giving the value $i$ of the current flowing through the instrument, gives the value of the electromotive force $r i$ between the terminals of the instrument, $r$ being 
the resistance of the instrument. There is but one type of voltmeter, namely; the electrostatic voltmeter, which is not essentially an ammeter. In this instrument the electromotive force to be indicated is connected to two insulated metal plates. The plates then attract each other, and one plate which is delicately suspended carries a pointer which plays over a divided scale.

From the above statement it might seem that there is no difference between ammeters and voltmeters, whereas the failure on the part of a novice to appreciate the very important difference leads to many serious accidents in the laboratory. An ammeter, including its shunt, always has a very low resistance, so that when it is placed in a circuit the current may flow through the instrument with the least possible opposition due to its resistance. A voltmeter, on the other hand, is always designed to give its full deflection with a very small current, and its resistance is therefore always high. When the instrument is connected to the terminals of a dynamo or to supply mains the current which it takes is so small that its effect on the system is negligibly small.

The tangent-galvanometer type of voltmeter is seldom used. The D'Arsonval galvanometer type is frequently used for direct current circuits. The electrodynamometer type of voltmeter is used for precision measurements of alternating voltages. The plunger type is most frequently used for switch-board instruments, especially for alternating currents.

Voltmeter multiplying coils. - If an auxiliary resistance be connected in series with a voltmeter, the instrument still indicates the electromotive force between its terminals, and the total electromotive force which is forcing current through the auxiliary resistance and the instrument is greater than the electromotive force indicated by the instrument in the ratio $(x+R) / r$, where $r$ is the resistance of the instrument and $R$ is the auxiliary resistance. This auxiliary resistance is called a multiplying coil. Multiplying coils are frequently used in this way to adapt a low reading voltmeter to higher voltages.

91. The wattmeter. - Power delivered by direct-current mains 
is usually calculated from ammeter and voltmeter readings. This method is not however applicable in case of alternating current. A method for measuring power which is always applicable is by means of the wattmeter. The wattmeter is a special form of electro-dynamometer, the connections of which are shown in Fig. I 21. A coil of fine wire, $A$, is connected to the mains in series with a non-inductive resistance $R$.

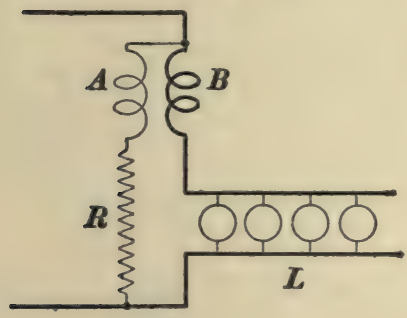

Fig. 121. This fine wire coil $A$ is delicately suspended inside of a stationary coarse wire coil, $B$, through which flows the current that is delivered to the lamps or other receiving units $L$. The force action between the two coils $A$ and $B$ is definite for a definite value of power delivered to $L$, be the voltage high and the current small, or the voltage low and the current large. Therefore the deflection of the movable coil, $A$, depends only upon the power delivered, so that the scale of the instrument may be arranged to indicate watts directly. The wattmeter is sometimes called the indicating wattmeter to distinguish it from the watt-hour meter which is sometimes improperly called a recording wattmeter.

A wattmeter connected as shown in Fig. I2 I, indicates the

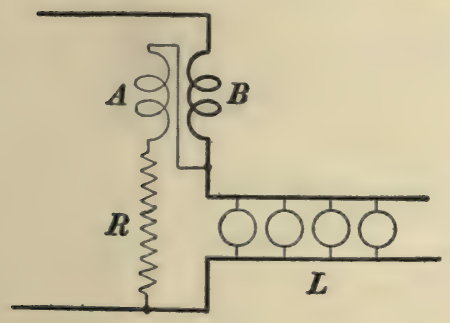

Fig. 122.

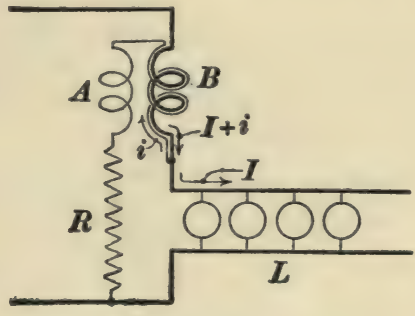

Fig. 123.

power delivered to $L$ plus the power lost in heating the coil $B$. On the other hand, a wattmeter connected as shown in Fig. I 22 indicates the power delivered to $L$ plus the power delivered to the 
circuit $A R$. The compensated wattmeter of the Weston Electrical Instrument Company is designed to eliminate these sources of error. This wattmeter is connected as shown in Fig. 122, but the wire leading to the coil $A$ is carried back through the coil $B$ and laid alongside of the windings of $B$ as shown in Fig. I23.

Let $I$ be the current flowing through $L$, Fig. 122 ; let $E$ be the voltage between the terminals of $L$, and let $i$ be the current flowing through $A$ and $R$. Then the current flowing through $B$ is $(I+i)$, and the force action between the two coils is proportional to $i \times(I+i)$. By laying the fine wire along side of the coarse wire in coil $B$, as shown in Fig. 123 , the electromagnetic action of coil $B$ is reduced to what it would be if the current $I$, only, were flowing in it, so that the force action between the two coils in Fig. 123 is proportional to $i \times I$, or to $E \mid R \times I$, or to $E I$. That is, the force action is proportional to the power delivered to $L$ so that the scale may be arranged to indicate this power correctly.

92. The watt-hour meter is an instrument for summing up or integrating the total work or energy delivered to a circuit. It is used chiefly as a meter for recording the amount of energy delivered to a customer by a central station. The Thomson watt-

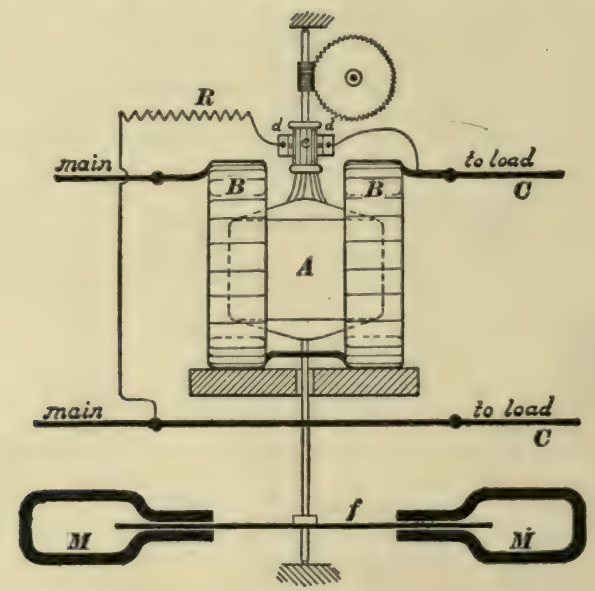

Fig. 124 .

hour meter of the General Electric Company is suitable for both direct and alternating current service; the induction watt-hour meter is suitable only for alternating current service. The induction watt-hour meter is described in a later volume of this text. 
The Thomson meter is a small electric motor without iron, the armature and field coils of which constitute an electrodynamometer. It is connected to the mains and to the receiving circuit in the same way as the wattmeter shown in Fig. I22. The field coils, $B B$, Fig. I24, of this motor correspond to the coil $B$, Fig. I 22, and the armature $A$ and non-inductive resistance $R$, Fig. 124, correspond to the coil $A$ and the resistance $R$ of Fig. 122. Current is led into the rotating armature $A$ by means of the metal brushes $d d$ which rub very lightly upon a small silver commutator $e$. The armature $A$ is mounted on a vertical spindle which is as nearly frictionless as possible, and the opposition to rotation is due mostly to the electromagnetic drag of the permanent steel magnets $M M$ upon the copper disk $f$ which is attached to the armature spindle.

The integrating action of the watt-hour meter is as follows: In the first place, the armature rotates at a speed which is proportional to the driving torque, because the retarding torque with which the magnets $M M$ act upon the disk $f$, is proportional to the speed. In the second place, the driving torque is proportional to $E I$ exactly as in the case of the force action between the coils of a wattmeter as explained in Art. 9I. Therefore, the speed of the armature is proportional at each instant to the power delivered; that is the revolutions per hour of the spindle is proportional at each instant to the watt-hours of work delivered per hour, so that the total number of revolutions of the spindle in a given time is proportional to the watt-hours of work delivered during that time.

The starting coil. - In the above discussion it is assumed that the torque which opposes the rotation of the armature is proportional to the speed. This is not strictly true inasmuch as the retarding torque consists of two parts, namely, mechanical friction, which is nearly constant irrespective of speed, and electromagnetic drag which is strictly proportional to the speed. If a constant driving torque in addition to the torque due to the action of the two coils $A$ and $B$ could be provided to overcome mechanical friction, then the speed of the meter would be proportional to the delivered power, and the indications of the instrument would be more nearly correct. This is accomplished in the Thomson meter by using an auxiliary field coil having a few turns of fine wire connected in circuit with $A$ and $R$, Figs. 122 and 124 . This is called the starting coil, and if the electromotive force between the mains is nearly constant, the current through this starting coil and through $A$ and $R$ is nearly constant, and an approximately constant driving torque is produced by the electromagnetic action of the starting coil on the armature $A$.

93. Fuses and circuit breakers. - A fuse is a link of fusible metal placed in an electric circuit so that when the current ex- 
ceeds a certain value, the link is heated to its fusing point, is melted, and thereby causes the circuit to be broken. Fuse wire is generally made of an alloy of tin and lead and it is rated at about 80 per cent. of the greatest current it can carry for an indefinite time. Thus a rated IO-ampere fuse wire is one which can carry no more than I 2.5 amperes steadily without melting.

The melting of a fuse link is always followed by an electric arc which, although of short duration, generates a quantity of hot vapor, the sudden expansion of which tends to scatter the melted metal. This blowing of a fuse, as it is called, is likely to set fire to nearby inflammable materials, and therefore a fuse link should always be enclosed in a fire-proof receptacle. This receptacle is usually made of porcelain. Furthermore, when two fuse links are mounted side by side, as shown in Fig. 69, they should be placed in separate compartments in the porcelain receptacle so that the arc cannot flash across and short-circuit the mains. A fuse link is sometimes enclosed in a fire-proof tube of insulating material.

Fuse wire or a fuse link is more or less sluggish in action inasmuch as a considerable amount of heat is required to raise the temperature of the wire or link to the fusing point. This is especially true of large fuse links. Furthermore, the amount of current that a fuse link will carry steadily without melting varies greatly with the mode of mounting. Thus a fuse link lying flat against the porcelain of a receptacle is cooled thereby, and its current carrying capacity is therefore increased; again a fuse link which is mounted in a thoroughly ventilated receptacle has a greater current carrying capacity than it would have if mounted in a receptacle which does not permit of the circulation of air.

The terminals to which the fuse wire or link is connected absorb considerable heat, and therefore influence the rating of a fuse of given size, especially if the fuse is short.

For the above reasons the rating of a fuse is a somewhat indefinite and uncertain matter.

The electromagnetic circuit breaker, on the other hand, is very 
prompt in action and when once adjusted, it is opened by a current of a perfectly definite value. The electromagnetic circuit breaker has the further great advantage over a fuse link in that connections can be reëstablished at once after the circuit has been opened by an excess of current, whereas the replacing of a fuse is a tedious operation and it is attended by serious risk as explained in Art. 85 .

The circuit breaker is a metal switch, single-pole or doublepole, which is arranged to open automatically when the current is in excess of a certain value. The closing of this switch puts a strong spring in tension tending to open it. The switch is kept closed by a latch which is controlled by a trigger, and this trigger is released by an electromagnet (through which the main current flows) when the current reaches a certain value.

Inasmuch as a circuit breaker always opens when an excessive current flows, it is evident that an intense arc will always flash

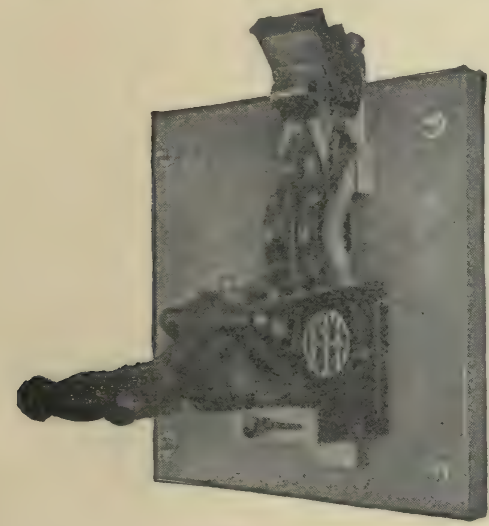

Fig. 125.

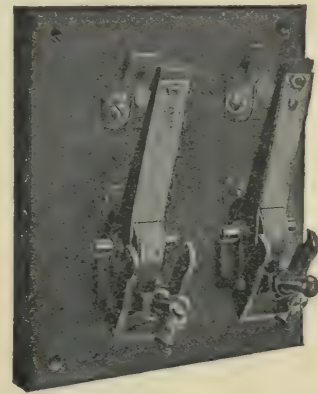

Fig. 126.

across the break. It is necessary to thoroughly protect the metal contacts of the circuit breaker from the destructive action of this arc by having a pair of auxiliary contacts in parallel with the main contacts and so arranged that the main contacts open first (without an arc) and the auxiliary contacts open a moment later (with an intense arc). 
The auxiliary contacts are sometimes made of carbon blocks. In this case one carbon block is mounted at the extreme end of the movable arm of the breaker so that when the breaker opens and the arm falls back, the carbon blocks are separated widely enough to put out the arc. Fig. 125 shows a single-pole carbon

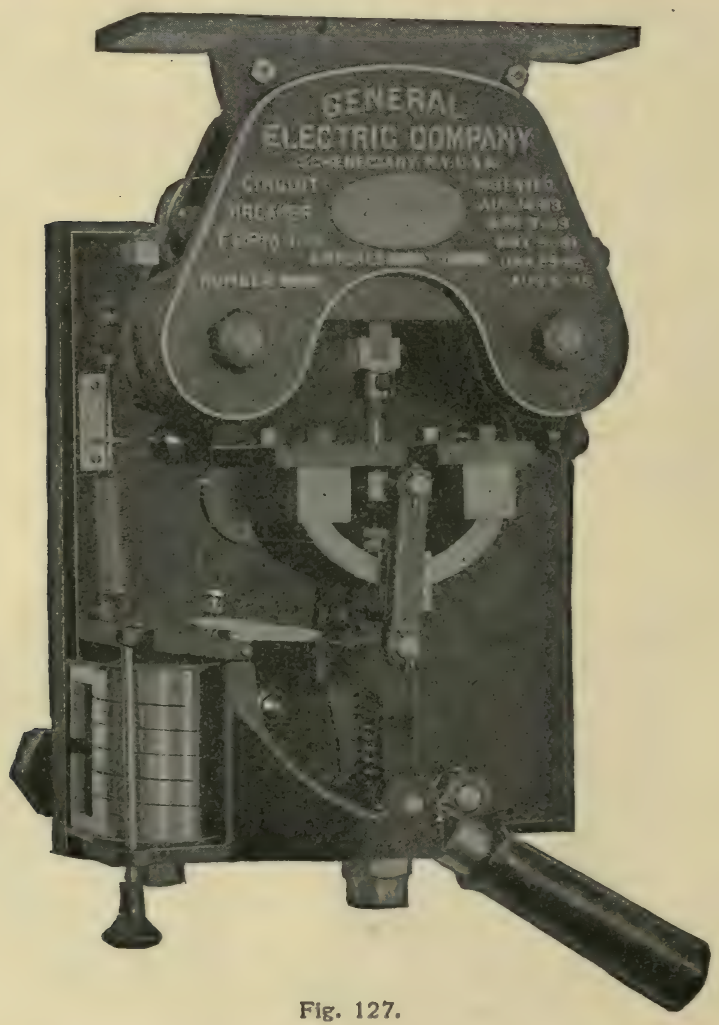

block circuit breaker manufactured by The Cutter Company, and Fig. I 26 shows two single-pole carbon block breakers of the Switchboard Equipment Company mounted side by side as a double-pole breaker.

Fig. 127 shows a direct-current magnetic blow-out circuit breaker of the General Electric Company. In this breaker the auxiliary contacts lie between the poles of an electromagnet 
which blows out the arc almost instantaneously when the auxiliary contacts are separated. In Fig. I 27 the structure at the top is the blow-out magnet, and the rod which is shown immediately below the center of this blow-out magnet actuates the auxiliary contacts which are hidden by the large flat front pole-piece of the blow-out magnet.

The main contacts of a circuit breaker are usually made of a bundle of thin sheets of spring copper and a massive block or blocks of copper against which the ends of the thin sheets are pressed when the circuit breaker is closed, as shown in Fig. I 27. This figure also shows very distinctly the electromagnetic device which actuates the trigger and opens the breaker. The coil of this electromagnetic device consists of a massive copper bar wound in the form of a helix. The rod which terminates in the small handle at the lower left hand corner in Fig. 127 is for tripping the trigger by hand when, it is desired to open the breaker. The large handle is the lever for closing the breaker; the figure shows the breaker closed. The breaker can be adjusted to be opened by any desired value of current within certain limits, by adjusting the tension of the small helical spring shown at the left in Fig. I 27.

94. Grounds and ground detectors. - A ground on one wire of a pair of supply mains is unsafe because an accidental ground on the other wire will complete the circuit. If this second ground is through a human body, a personal injury may result, and if either ground connection is through a wire or other conductor, the conductor may become hot enough to start a fire. In any case a ground connection on both mains involves a waste of power. Since accidental ground connections frequently occur on electric mains, a device called a ground detector is usually mounted upon the switchboard in a generating station for showing the existence of serious grounds on the line wires.

A ground detector usually employed on low voltage lines (up to five or six hundred volts) consists of two incandescent lamp units* connected in series between the mains with the middle

* Each unit consists of one or more lamps in series according to the voltage. 
point between the lamps connected to earth as shown in Fig. I 20. Whenever both line wires have grounds of the same resistance, high or low, both lamps burn with equal brightness. Whenever one line wire has a fairly low resistance ground, and the other a high resistance ground, the lamps are unequal in brightness, and the main which connects to the dim lamp is the main with the low resistance ground. In order to distinguish between the unsafe condition with both mains grounded through nearly equal low resistances, and the safe condition with both mains grounded through very high resistances, the lamps should be arranged to be disconnected one at a time. If $\operatorname{lamp} A$ is $\operatorname{dim}$ when $\operatorname{lamp} B$ is disconnected from main $B$, then main $B$ has a high resistance ground; and if lamp $A$ is bright when lamp $B$ is disconnected then main $B$ has a low resistance ground. The reason for these statements can be easily worked out by the reader by making a diagram showing the relations of the two ground resistances to the lamp or lamps.

95. Lightning and lightning arresters. - When a lightning stroke occurs in the neighborhood of a transmission line, a sudden rush of current takes place over the line due to one or more of the following causes: (a) Electric charge accumulated on the line is suddenly released and tends to flow to earth; (b) the magnetic action of the lightning discharge induces a sudden pulse of current in the line; $(c)$ when the lightning actually strikes the line, an enormous rush of current takes place as the total electric charge of the lightning flash flows to earth.

The damage to electrical apparatus by a very intense lightning stroke may be a more or less complete destruction due to the heat generated by the lightning discharge itself. Weak lightning discharges are however very much more frequent than very intense ones, and the damage done by a weak lightning discharge is usually due to a puncturing of the insulation of the windings of a machine and a consequent short-circuit in the machine which causes it to be burned out by its own current.

When the sudden rush of current which accompanies a light- 
ning discharge encounters a portion of the circuit which has considerable electrical inertia or inductance, a very great electromotive force is created between the terminals of that portion of the circuit, in the same way that an enormous mechanical force is created when a moving body strikes a heavy stationary body. Thus a sudden rush of current coming into a station over a transmission line, encounters the highly inductive windings of wire on the field or armature of a dynamo. The electrical inertia (inductance) of the winding dams up the rush of current, as it were, and the current rush is almost sure to break through the insulation at the very entrance to the winding, passing from the copper wire to any metal that is connected more or less thoroughly to earth.

The lightning arrester is a device for shielding a dynamo or other electrical machine from the rushes of current which come into a station on an overhead line during a thunderstorm.

Fig. I 28 is a diagram showing the essential features of a light-

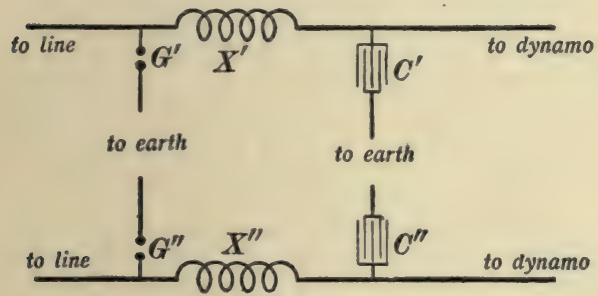

Fig. 128. ning arrester, or rather, of two distinct lightning arresters, one for each line wire. Such a pair of lightning arresters when mounted on one base plate is called a double-pole arrester.

Each of the line wires upon entering the station building is connected to a coil of wire, called a choke coil or reactance coil, whence the wire leads on to the dynamo or other machine. Just in front of the choke coils each line wire has a ground connection broken by a short spark gap $G$, and just behind the choke coils each line wire should have a ground connection through a condenser $C$ with very thick insulation. Fig. I 28 shows every essential feature of a lightning arrester except the device for extinguishing the arc which tends to persist at the spark gaps $G^{\prime}$ and $G^{\prime \prime}$ after the lightning discharge has passed to earth. The action of the apparatus shown in Fig. I 28 is as follows: A rush 
of current entering the station over either line wire is dammed up in front of the choke coil causing an electromotive force sufficiently high to break across the spark gap $G^{\prime}$ or $G^{\prime \prime}$, thus establishing a conducting path to earth. The function of the condenser may be best explained in terms of a mechanical analogue as follows: A wall is to be shielded from the excessive force of a hammer blow by allowing the hammer to strike against a heavy ball of iron which rests against the wall. The ball must move a little to take up the momentum of the hammer. If the ball and wall were both perfectly rigid the ball would fail to protect the wall. Some slight degree of elasticity in the ball or wall is necessary, or an elastic cushion must be interposed between the ball and the wall. Each condenser shown in Fig. I 28 acts like this elastic cushion, permitting some movement through the choke coil of current which does not necessarily flow on to and through the dynamo, but merely charges the condenser. The condenser is not often used with lightning arresters, inasmuch as the dynamo and the connecting wires usually have sufficient electro-elasticity or capacity. Lightning arresters installed out on a transmission line do not require choke coils, but a choke coil should always be inserted between the spark gap of a lightning arrester and the generator or motor or other apparatus which is to be protected.

The choke coil of a lightning arrester must be extremely well insulated. The use of an iron core does not increase the inductance of a coil with respect to excessively quick rushes of current, inasmuch as the iron does not have time to become magnetized. Lightning arrester choke coils are therefore usually made of a single layer of coarse insulated wire wrapped on a porcelain core, or of an insulated copper ribbon wound like a roll of tape.

When a lightning discharge jumps across both gaps $G^{\prime}$ and $G^{\prime \prime}$, Fig. 128, the gaps constitute for an instant a low resistance path between the line wires, and electric arcs, started by the lightning discharge, are maintained by current from the generator. This virtually short-circuits the generator, hence provision must be made for extinguishing the arc or arcs above described. The 
various types of lightning arresters in use differ chiefly in the means employed for extinguishing the arc.

The extinguishing of an arc across the spark gaps of a lightning arrester is accomplished by one or more of the following means :

(a) By the magnetic blow-out. An electromagnet may be used to extinguish the arc which persists across the spark gap of a lightning arrester in the same way that it is used to blow out the arc in a circuit breaker. The spark gap need only be placed between the poles of a fairly strong electromagnet. The magnetic blow-out is not suitable for alternating current circuits.

(b) By cooling the arc. An alternating current arc does not persist across even a very short gap between massive blocks of metal unless the electromotive force is very large. Thus an alternating electromotive force of 500 volts effective value cannot maintain an arc across a $\frac{3}{64}$ inch gap between massive blocks of brass. This is due no doubt to the cooling of the vapors of the arc by the metal blocks at the time of a reversal of the alternating current, when the current is nearly zero in value; the cooled vapors being a sufficiently strong insulator to withstand the succeeding wave of electromotive force.

(c) By smothering the arc. An arc cannot be maintained, except by an excessively high electromotive force, in a narrow enclosed space. This fact is utilized in several types of lightning arresters by enclosing the spark gap in a narrow space surrounded by insulating material.

(d) By using resistance. The maintenance of an arc depends upon the flow of considerable current. Hence an arc cannot be maintained across the spark gaps of a lightning arrester if sufficient resistance is connected in series with the gaps. This resistance should of course be non-inductive, and it should be made of bulky material so as to absorb, without excessive rise of temperature, the heat generated in it by the lightning discharge. The best form for this resistance is a solid rod of nonconducting material, such as burned clay, coated with a thin layer of conducting material, such as graphite. Such a coated rod 
conducts a current rush nearly as well as a solid rod of graphite, inasmuch as a very sudden rush of current flows only through the surface layers, or skin, of a conductor ; but it offers an enormously greater resistance than a solid graphite rod to a current which is steady or which changes slowly* in value. Therefore a rod of clay coated with graphite offers an easy path for a lightning discharge, but prevents the flow of the generator current which tends to maintain the arc.

Fig. I 29 shows a singlepole magnetic blow-out direct current lightning arrester manufactured by the General Electric Company.

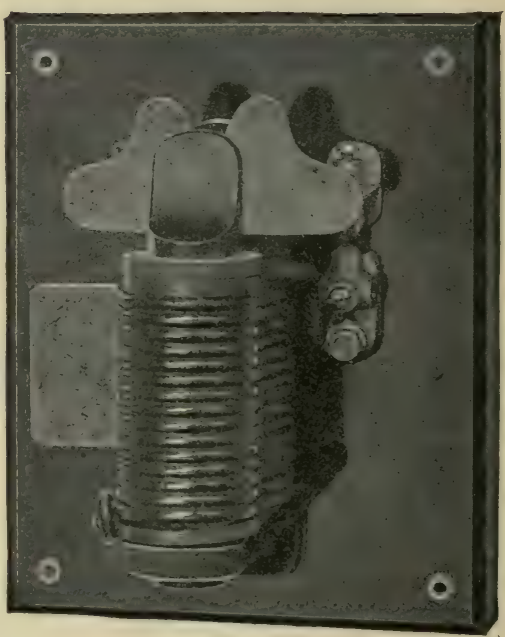

Fig. 129. This particular arrester has two spark gaps in series. The main spark gap is between the two wing-shaped pieces of brass, and it lies between the poles of an electromagnet. The auxiliary spark gap is between the two rounded brass points shown at the right in Fig. I29. The winding of the electromagnet forms a shunt around the auxiliary spark gap, and the current which continues to flow across the two gaps of the arrester after a lightning discharge has passed, quickly establishes itself in the magnet winding, excites the magnet, and blows out the arc in the main gap.

Figs. I3O, I3 I and I32 show a compact form of magnetic blow-out lightning arrester enclosed in a porcelain receptacle.

* The current rush which constitutes a lightning discharge sometimes grows at a rate exceeding 500,000,000 amperes per second during the few millionths of a second that it continues to grow. Compared with this the maximum rate of growth of an ordinary commercial alternating current, namely, a few thousand amperes per second, is very slow indeed. 
PRACTICAL OPERATION OF DYNAMOS.

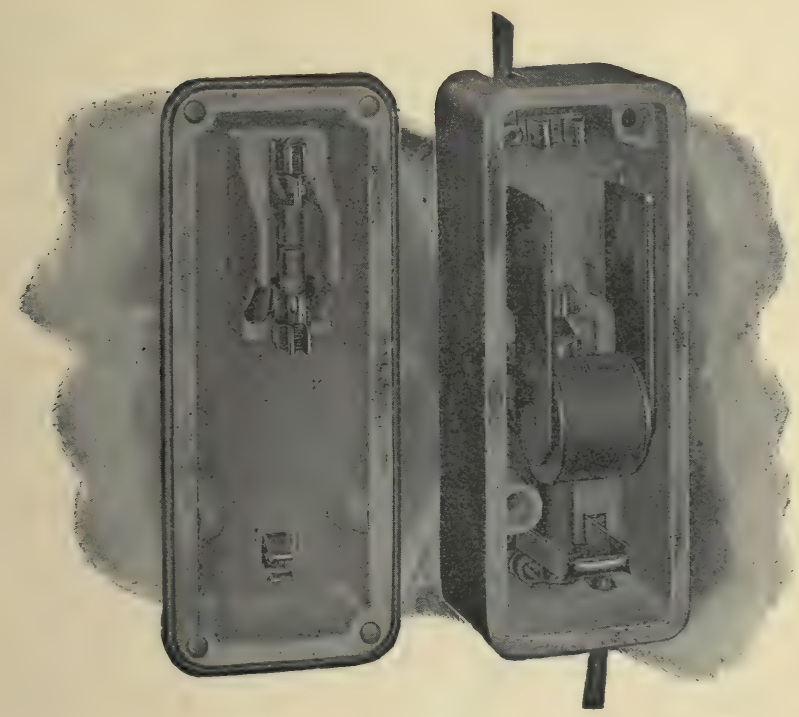

Fig. 130.

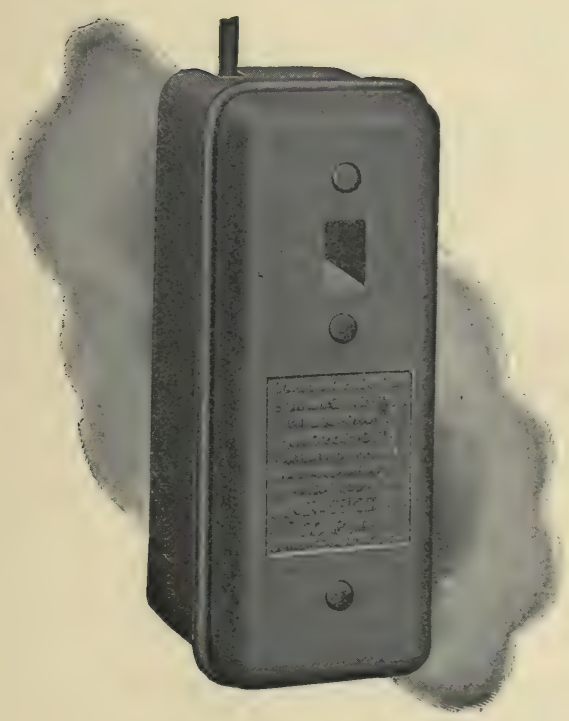

Fig. 131. 
This arrester is made by the General Electric Company. It is suitable for direct current circuits only, and, being compact and enclosed, it is specially suitable for protecting the motors on street cars. The spark gap in this arrester is between the ends of two short brass rods which are shown attached to a porcelain lug on the inside of the cover in Fig. I 30. A carbon rod resistance is connected in series with this spark gap, and the winding of the blow-out magnet is connected as a shunt around the whole or a portion of this carbon resistance. The magnet blows the arc out through a hole (shown in Fig. I3I) in the porcelain lug and cover.

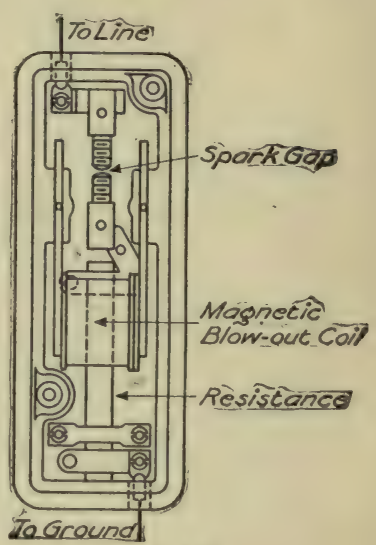
Fig. 132.

Fig. I 32 shows the details of the arrester and the manner in which it is connected to the line and to the ground. Fig. I33 shows four of these arresters connected to two pairs of feeders, and two additional arresters connected to the generator mains.

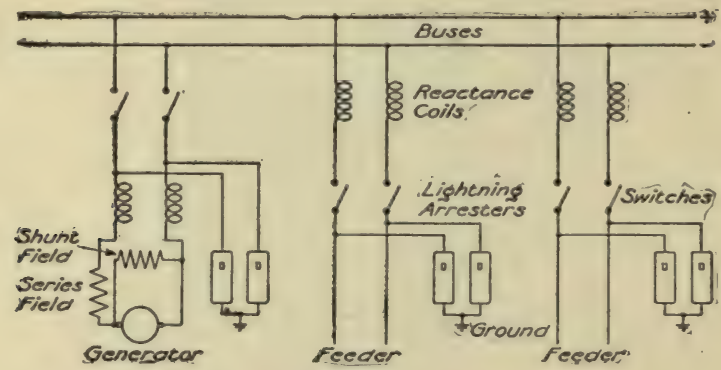

Fig. 133.

Fig. I34 shows a Wurts alternating current lightning arrester manufactured by the Westinghouse Company. It consists simply of seven massive brass cylinders held in a porcelain clamp, and presents seven spark gaps in series between the end cylinders which are connected to the terminals $G$ and $L$. The. point $L$ is connected to the line and the point $G$ to ground. Fig. 
I 35 shows two arresters like Fig. I 34 connected to the two mains of a 2,000-volt alternator. The Wurts arrester depends solely upon the cooling effect of the massive brass cylinders for extinguishing the arc across the spark gaps.

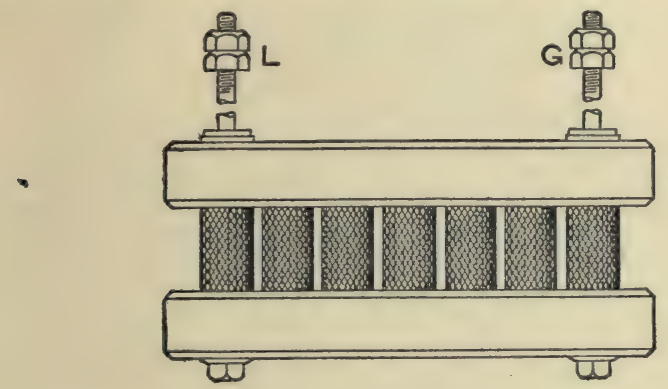

Fig. 134.

The "type $K$ " arrester of the Westinghouse Company extinguishes the arc by smothering it. This arrester, now superseded by the "MP" arrester of the same company, consists of a block of hard wood to one flat face of which two metal plates are attached. The spark gap is between the edges of these metal

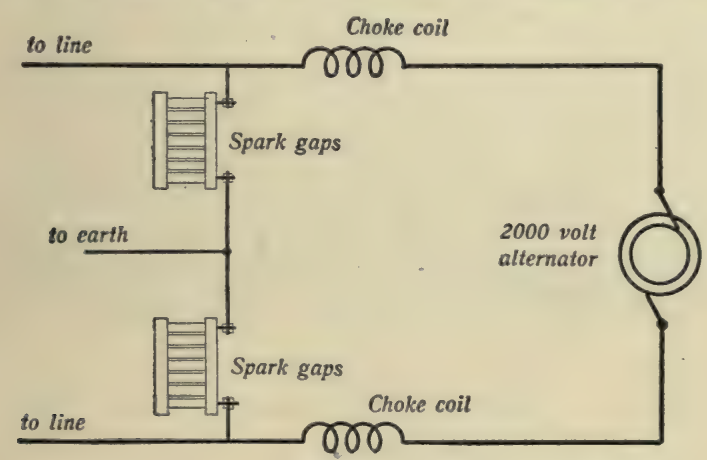

Fig. 135.

plates, and the rather wide gap is bridged by the charred surface of the wood. Another block of wood, with a small hole through it for the escape of the hot vapors of the arc, is placed over the spark gap as a cover, and serves to smother the arc. The charred surface of the wood enables the lightning discharge to pass across 
the rather wide gap of this arrester without an excessive rise of electromotive force.

Fig. I36 shows a two-pole alternating current lightning

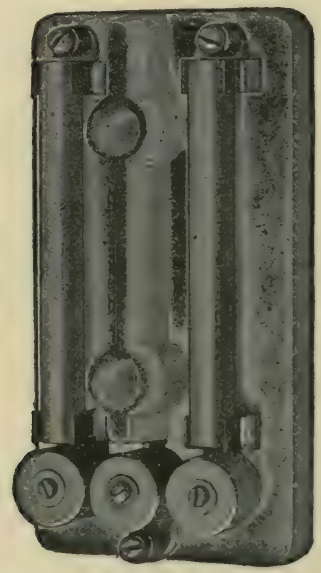

Fig. 136.

arrester of the General Electric Company. In this arrester the arc is extinguished partly by the cooling effect of the massive brass cylinders between which the spark gaps lie, and partly by the effect of resistances connected in series with the spark gaps. The middle one of the three short brass cylinders, which are shown at the bottom in Fig. 136 , is connected to ground, and the brass cylinders at the sides are connected through the long graphite resistance rods to the line wires. The double-pole arrester shown in Fig. I 36 is suitable for 1,000 -volt alternating current mains. For higher voltages several spark gaps and resistance rods are connected in series between each line wire and the earth.

Fig. I 37 shows the parts of the "MP" lightning arrester of the Westinghouse Company. This arrester, aside from the choke coil which is of course separate, consists of a block of porous material filled with small hard grains of carbon. This block is clamped between two flat metal plates and connection is 
made from the line through a very short air gap to one of these plates, and from the other plate to earth. A lightning discharge passes through the block in the form of innumerable microscopic sparks between the carbon grains, and no arc is developed. The

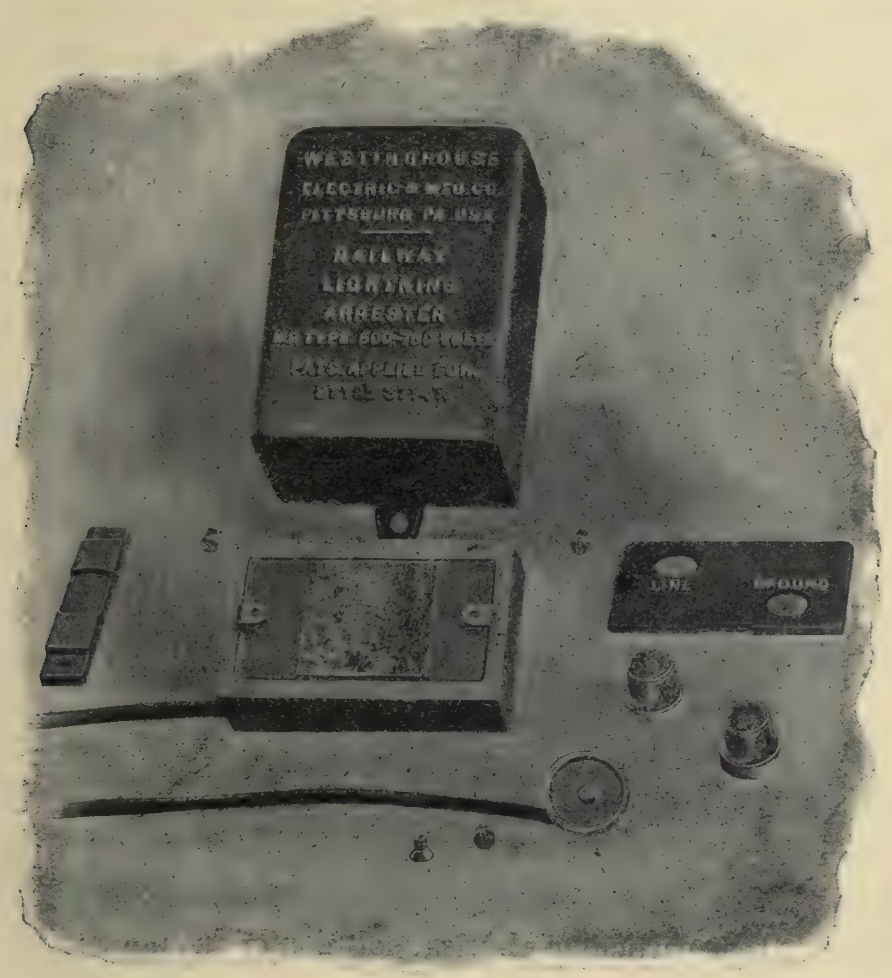

Fig. 137.

spark gap between the line and the first metal plate serves as a complete break in the circuit from the line to ground, except of course at the instant of a lightning discharge. This arrester is suitable for both direct and alternating current circuits.

96. Fire risks and the Underwriters' rules. Rules governing the installation of electrical apparatus of all kinds have been formulated by a National Conference on Standard Electrical 
Rules * with the object of minimizing fire risks and risks of personal injury. These rules are published in convenient form and at a nominal price by the National Board of Fire Underwriters $†$ under the title "The National Electrical Code," and a supplement, which is revised semi-annually, contains a list of all kinds of electrical wires and fittings which are approved by the Underwriters. Any person who assumes any degree of responsibility for the laying out, or even the maintenance, of electrical installations should be familiar with the National Electrical Code.

The rules and requirements which constitute the National Electrical Code are classified in the edition of 1905 as follows :

Class A. Rules applying to stations and dynamo rooms.

Class B. Rules applying to outside work.

Class C. Rules applying to inside work.

Class D. Rules applying to fittings, materials, and details of construction.

Class E. Miscellaneous rules.

Class F. Rules applying to marine work.

97. Installation and operation costs. - From one point of view engineering is a composite of all the physical sciences, and from another point of view it is a branch of the science of economics. Any elementary treatise on engineering must, however, be chiefly devoted to purely physical problems inasmuch as the student must become familiar with engineering as a branch of physical science before he can possibly undertake as a practicing engineer to choose that particular physical solution of an engineering problem which will best meet the requirements of economy.

* The following is a list of the Associations composing this National Conference : American Institute of Architects, American Institute of Electrical Engineers, American Society of Mechanical Engineers, American Institute of Mining Engineers, American Street Railway Association, Associated Factory Mutual Fire Insurance Companies, Association of Edison Illuminating Companies, International Association of Fire Engineers, International Association of Municipal Electricians, National Board of Fire Underwriters, National Electric Light Association, National Electrical Contractors' Association, Underwriters' National Electric Association.

† General Agency 34 Nassau St., New York City. 
The economic problem is in every case to produce the best results at a minimum of cost and this is always a very complicated problem, inasmuch as the cost of erection and maintenance of engineering works and the value of the service rendered thereby are both dependent upon minute variations of local conditions, they both fluctuate from year to year with the varying stress of business activity, and they both change with every improvement in industrial processes.

The first estimate of the cost of any engineering undertaking is usually based upon general statistics of the cost of more or less similar undertakings, and the final cost is determined by the bids of contractors who have had more or less experience in the particular line of work and whose margin of profit must in general be great enough to cover the many uncertain items that always appear in any new undertaking.

The following data on the cost of installing and operating steam and electric plants may be used for making a preliminary estimate of the costs of an electric station. These data are averages based upon the records of a large number of actual cases, and the cost of a given station may depart more or less widely from these figures on account of peculiar local and temporary conditions.

Cost of steam power.* - The ordinates of the curve $A$ in Fig. I 38 give the approximate total cost per horse-power capacity, including buildings, chimneys and all accessories, of boiler and engine plants of various sizes, and the ordinates of curve $B$ give the coal consumption in pounds per horse-power-hour.

The ordinates of the curves $C, D, E, F$ and $G$ in Fig. I 39 give the costs per horse-power-year ( 308 days of io hours each) of the following items :

* The data from which the curves in Figs. 138 and I 39 are plotted are taken from a paper by Wm. O. Webber, The Engineer (Cleveland), Vol. 40, p. 145, February 2, 1903. Important data on the cost of steam power are given by C. E. Emery, Trans. Am. Inst. Elec. Eng., Vol. 12, pp. 358-389, 1895; and by H. A. Foster, Trans. Am. Inst. Elec. Eng., Vol, 14, pp. 385-421, 1897. 
222 ELEMENTS OF ELECTRICAL ENGINEERING.

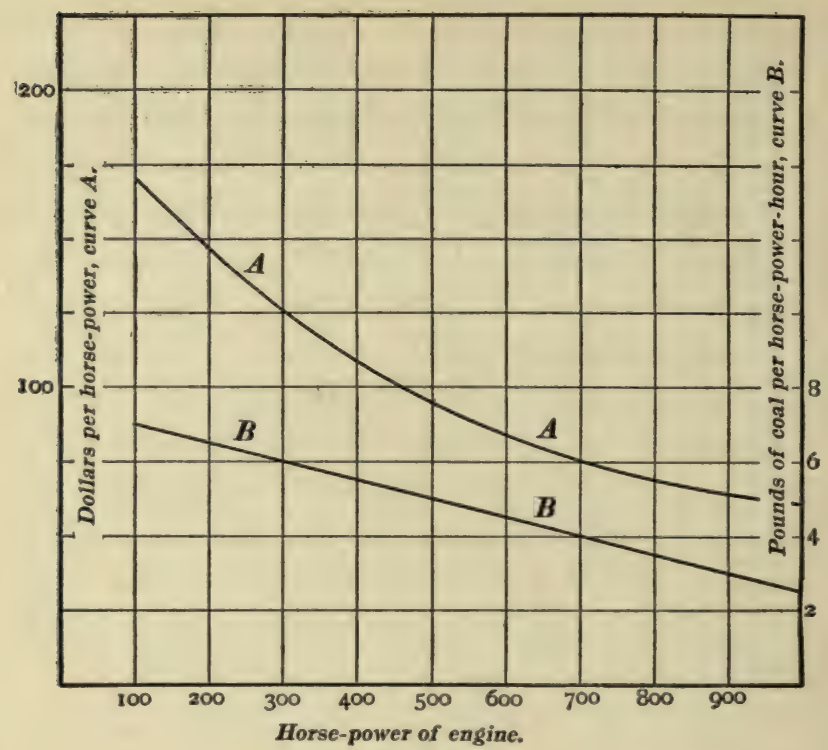

Fig. 138.

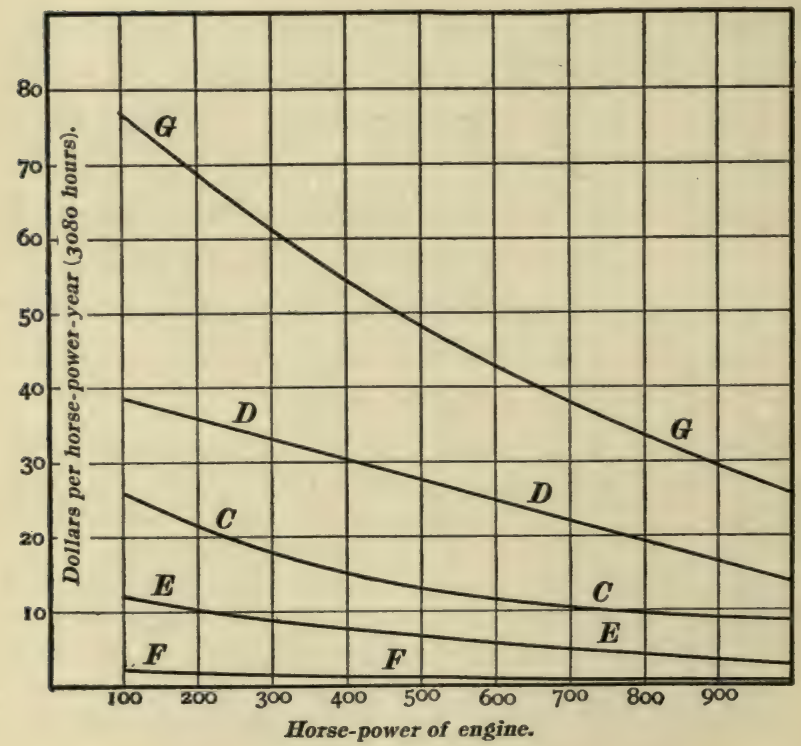

Fig. 139. 
Curve $C$. Fixed charges per horse-power-year. This item is reckoned at 14 per cent. on the total cost, and it includes interest at 6 per cent., depreciation 4 per cent., repairs 2 per cent., insurance I per cent., and taxes I per cent.

Curve $D$. Cost of coal per horse-power-year at $\$ 4.00$ per ton of 2,00o pounds.

Curve $E$. Wages per horse-power-year.

Curve $F$. Cost of oil and supplies per horse-power-year.

Curve $G$. Total cost per horse-power-year.

These curves are based on the assumption that the smaller sizes of engines are simple non-condensing engines of the Corliss type, that the medium and large-sized engines are run condensing, and that the largest sizes are compound engines.

The item of wages in a 100 horse-power plant would include an engineer at $\$ 2.50$ per day and a fireman at $\$ 1.50$ per day, which would amount to about $\$ \mathrm{I}, 200$ per year. In a I,000 horse-power plant the item of wages would include one engineer at $\$ 3.00$ per day, one assistant engineer at $\$ 2.00$ per day, three men in the boiler room during the day and one night watchman at $\$$ I.5O per day, which would amount to about $\$ 3,500$ per year.

Where the cost of coal is more or less than $\$ 4.00$ per ton, the cost of coal per horse-power-year may be easily determined from curve $D$, Fig. 139, the ordinates of which represent the cost of coal per horse-power-year at $\$ 4.00$ per ton, and the total cost per horse-power-year, as given by curve $G$, may be corrected accordingly.

It is important to remember that the cost of power as given by curve $G$, Fig. I 39, is based on the assumption that the engines are working at full load for Io hours per day, 308 days in the year. When a plant operates at a fraction of its full-load capacity the cost per horse-power-hour is increased as explained in Art. 97.

The cost of electrical power at the switchboard. - The load factor $*$ of an ordinary electric light station varies from 0.15 for 
plants in small towns where there is but little lighting during the day, to as much as 0.40 or more for plants in large cities where there is a considerable demand for current during the day. The load factor of a plant which supplies current for an electric railway may be as high as 0.50 or 0.60 . The following estimate of the cost of electrical power at the switchboard is based upon the cost of steam power as given in Figs. I 38 and 139 and upon the assumption that the plant operates at full-load for Io hours per day for 308 days per year.

The electrical equipment of a station costs about $\$ 30$ or $\$ 35$ per kilowatt of capacity for large stations and somewhat more for small stations. The ordinates of the curve $J$ of Fig. I4 I, which represent the annual fixed charges on the electrical equipment

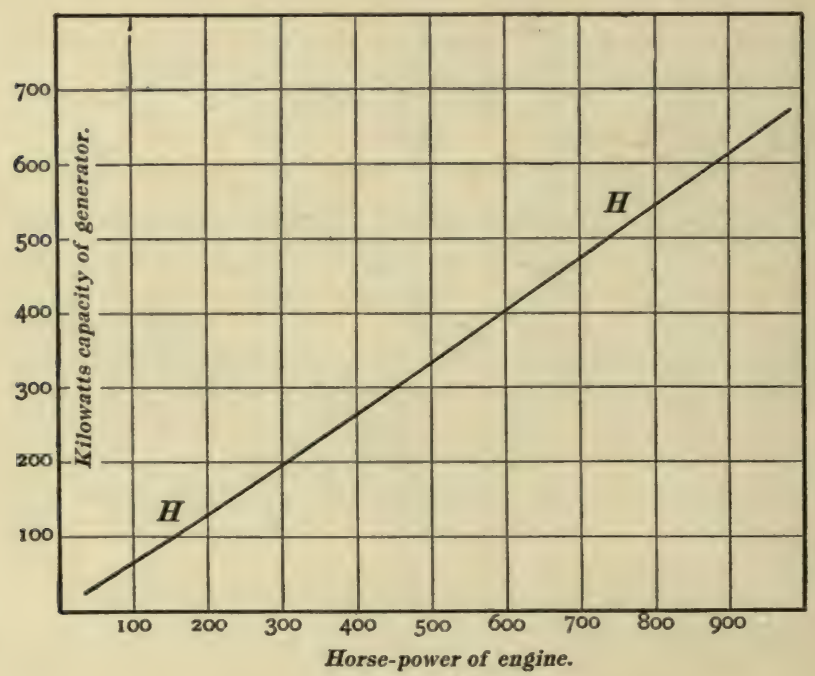

Fig. 140.

per kilowatt of station capacity, are based on a cost of the electrical equipment which ranges from $\$ 45$ per kilowatt for a 100 kilowatt station to $\$ 35$ per kilowatt for a 700 kilowatt station.

The cost of the additional labor required by the electrical plant is about 25 per cent. of the cost of labor for the steam plant alone. 
The additional cost of oil and supplies is about 40 per cent. of the cost of this item for the steam plant alone.

The ordinates of the curve $H$ in Fig. I 40 give the capacity in kilowatts of the electrical equipment corresponding to various horse-power capacities of engine plants. This curve is not exactly a straight line inasmuch as the efficiency of an electrical generator increases with its size.

The curves in Fig. I4I show an approximate analysis of the cost of electrical power when the station is operated at full-load for 3,080 hours each year.

Curve $I$ shows the cost of steam power per kilowatt-year of electrical output.

Curve $J$ shows the fixed charges on the electrical equipment per kilowatt-year. This item is reckoned as I4 per cent. on the

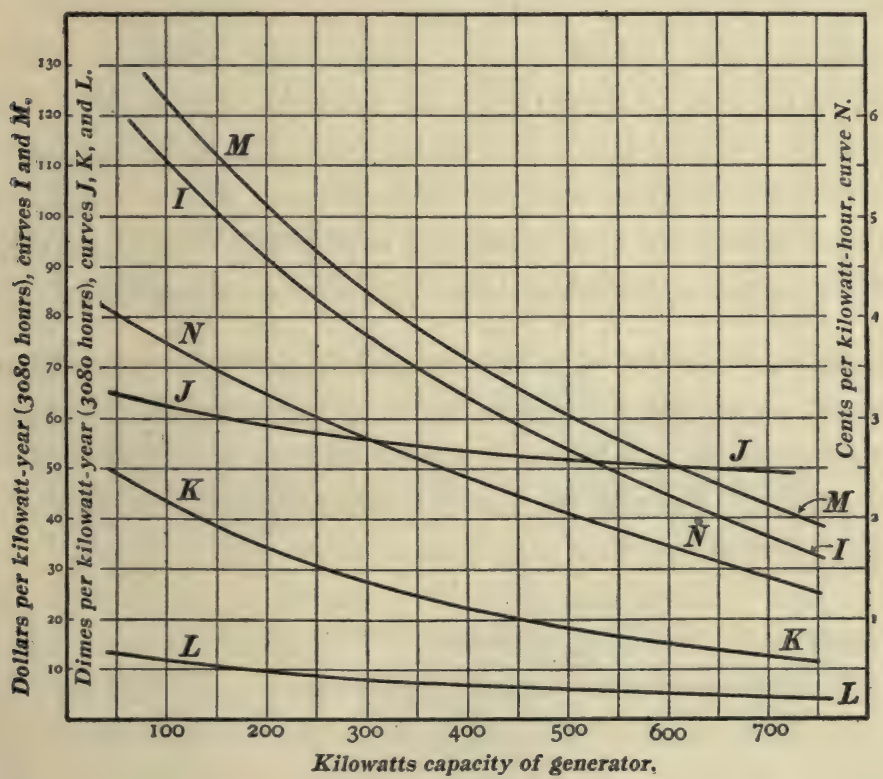

Fig. 141.

cost of the electrical equipment and it includes interest at 6 per cent., depreciation 4 per cent., repairs 2 per cent., insurance I per cent. and taxes I per cent. 
Curve $K$ shows the cost of attendance of electrical equipment in addition to attendance of steam plant.

Curve $L$ shows the cost of oil and supplies for electrical equipment.

Curve $M$ shows total cost per kilowatt-year of electrical output; and

Curve $N$ shows the total cost per kilowatt-hour of electrical output.

Cost of electrical power delivered to the consumer. - The curves in Fig. I4I give a general idea of the cost of electrical power at the switchboard on the assumption of unity load factor. The cost of electrical power delivered to the consumers is greater than the cost at the switchboard by an amount sufficient to cover the cost of the following items: (a) The power lost in the distributing system; (b) interest on the cost of the distributing system; (c) depreciation and repairs on the distributing system; and $(d)$ taxes. The sum of these items for a whole year divided by the number of kilowatt-hours delivered per year often amounts to more than the original cost per kilowatt-hour at the switchboard, especially where the consumers are scattered over a large district or where current is distributed through costly underground cables.

98. The load factor of a station and its influence upon the cost of power. - In most electric stations the demand for current fluctuates greatly, so that a station which is designed to deliver the maximum amount of current demanded is operated for a large portion of the time at a fraction of its rated capacity.

Fig. $142 *$ shows the daily load curves of an electric lighting station in Christiania, Norway. The upper curve $A A$ is the load curve for midwinter when the days are extremely short and dark, the lower curve $B B$ is for midsummer when the days are very long, and the dotted curve $C C$ is the mean daily load curve for the whole year. The difference between the winter and sum-

* Taken from an article by Norberg-Schulz, Electrotechnische Zeitschrift, October 5, 1905, pp. 919-921. 
mer demand for current is very considerable even in middle latitudes and, as Fig. I 42 shows, it is excessively great in Christiania.

The sharp maximum of station load, which occurs at $6 \mathrm{P}$. M. in Christiania in winter time, and usually between 7 and $8 \mathrm{P}$. M. in middle latitudes in winter time, is called the "peak of the load." The boiler, engine, and generator capacity of a station must be sufficient to meet the excessive demand for current that constitutes the peak of the load, although it is usually permissible, during the short time that the excessive demand lasts, to force the boilers and overload the engines and generators to some extent.

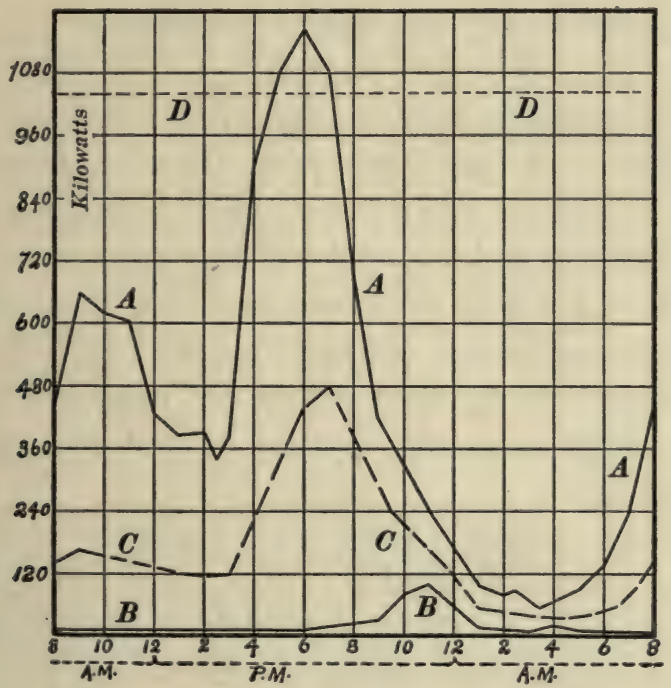

Fig. 142.

The actual output of a station in kilowatt-hours during a given period, a month for example, divided by the output that could be obtained during that period by operating the station night and day at its full rated capacity, is called the load factor * of the station. Thus if the Christiania plant, referred to in Fig. 142, is a

* The load factor of a station which operates a certain number of hours each day is sometimes defined as the actual output during the day's run divided by the output that would be obtained were the station operated at full-load for the whole time of the run. 
I, O40 kilowatt plant, its load factor during midwinter would be equal to the area under the curve $A A$ divided by the area under the dotted line $D D$, and its load factor during midsummer would be equal to the area under the curve $B B$ divided by the area under the dotted line $D D$.

When a station is operated at light load the item of wages may be somewhat reduced, especially if the station is shut down during certain hours each day; also the item of fuel is reduced, but neither of these items is reduced in proportion to the reduction of load. On the other hand the fixed charges, that is, the interest on the investment, depreciation, repairs, and taxes are approximately constant. Therefore a reduction in the output of a station does not reduce the operation costs very much, so that the cost per kilowatt-hour increases greatly with decrease of load.

The influence of load factor upon the cost of power can be estimated in a given case when we know how many hours each day the station is to be shut down, how great a reduction will be effected in the item of wages, and how great the increase of coal consumption per kilowatt-hour will be. The following table, ${ }^{*}$ although based upon rather old data, will serve as an illustration of the decreasing cost per kilowatt-hour with increasing load factor.

Example. - Consider a I,000 horse-power electric lighting station which, with its distributing system, represents a total investment of $\$ 150,000$, and the equipment of which includes four 250 horse-power engines each driving a I60 kilowatt generator.

The fixed charges (interest, depreciation, repairs, insurance, and taxes), estimated in round numbers as 14 per cent. on the total investment, are $\$ 2 \mathrm{I}, 000$ per year.

* From the Electrical World, Vol. 24, p. 120, Aug. 11, 1894. A very complete. table of costs of electric power at the switchboard, showing a wide range of load factors, is given by R. W. Conant in a paper entitled The Cost at the Switchboard of Electric Power for Street Railways, Electrical World, Vol. 32, pp. 313-319, September 24, 1898. See also Cost of Electric Railway Power by A. S. Richey, Street Railway Journal, Vol. 25, pp. 126-128, Jan. 21, 1905, and Vol. 18, p. 827, Dec. 7, I901 ; and Pittsburg Street Railway System, Street Ry. Rev., Vol. 13, pp. 185-198, April, 1903. 
Table Showing Effect of Load Factor on Cost of Power.

Cents per Kilowatt-hour.

\begin{tabular}{|c|c|c|c|c|}
\hline Load Factor. & 0.2 . & 0.4 . & o.6. & 0.8 . \\
\hline Interest on investment........................ & 1.70 & 0.85 & 0.57 & 0.42 \\
\hline Depreciation................................... & 1.20 & 0.66 & 0.44 & 0.33 \\
\hline Management and taxes ...................... & I. 50 & 0.75 & 0.50 & 0.40 \\
\hline Repairs.......................................... & 1.00 & 0.55 & 0.40 & 0.30 \\
\hline Fuel ........................................... & I. 50 & I. 40 & I. 30 & I. 20 \\
\hline Labor................................................ & 1.50 & I. 20 & I.00 & 0.90 \\
\hline Petty stores................................... & 0.20 & 0.15 & 0. 10 & 0.08 \\
\hline Cost per kilowatt-hour in cents... & 8.60 & $5 \cdot 5^{6}$ & $4 \cdot 3^{I}$ & 3.63 \\
\hline
\end{tabular}

If the station were run at full-load day and night the year round the running expenses would be approximately as follows : (a) Wages of one chief engineer at $\$ 5$ per day, two assistant engineers at $\$ 2.50$ per day (one to have charge at night the other during the day), one lineman at $\$ 2.50$ per day, one lineman at $\$ 1.50$ per day, three helpers in the engine room (one at night and two during the day) at \$I.50 per day, and six men in the boiler room (three at night and three during the day) at \$I.50 per day, making a total of $\$ 10,000$ per year; $(b)$ Coal at 6.3 pounds per horsepower-hour and at $\$ 4$ per ton would amount to $\$$ I I, 400 per year; and $(c)$ Petty stores would amount to about $\$ 6,500$ per year. The total annual expense of $\$ 147,900$ would be the cost of $6,000,000$ kilowatt-hours, which would be at the rate of 2.46 cents per kilowatt-hour.

If the station were run night and day the year round at an average load equal to 0.20 of its full-load capacity, the running expenses would be approximately as follows: (a) Wages (two engine room helpers and three firemen less than before) would amount to $\$ 7,260$ per year; $(b)$ Coal at 8 pounds per horsepower-hour and at $\$ 4$ per ton would amount to $\$ 29,440$ per year; and $(c)$ Petty stores would be, say, $\$ 5,500$ per year. The total annual expense of $\$ 63,200$ would be the cost of $1,200,000$ kilowatt-hours, which would be at the rate of 5.26 cents per kilowatt-hour.

It would certainly pay in this plant to use a more economical 
type of engine than is assumed as a basis for Figs. I 38 and I 39, so as to reduce the cost of coal.

99. Examples of installation and operation costs. - The general averages of cost which are given in Figs. I38, I 39 and I4I would be misleading without a few actual examples to show how widely costs vary in particular cases.

Installation costs. - The accompanying table of costs gives actual cost data on seven electric power stations not including any part of the distributing system. Total costs are all given in thousands of dollars. The figures which follow Max. and Min. are the extremes of variation of cost per kilowatt capacity under ordinary conditions. Wide deviations from these figures in the actual plants are due to unusual conditions. Thus the land for plant No. 5 which is in a large city cost $\$ 43,000$; this plant has very expensive foundations of concrete on piling, and its engine capacity aggregates 4,500 indicated horse-power when perhaps 3,500 horse-power would be sufficient. The cost per kilowatt of the various parts of the station equipment is in each case the total cost divided by the capacity in kilowatts of the generators installed.

Operation costs. ${ }^{*}$ - The accompanying table of operation costs is taken from the records of two lighting stations for one year. Station A is a 225 kilowatt plant owned and operated by a city of 7,000 inhabitants in Connecticut. The equipment includes a I 50 kilowatt alternator and a 75 kilowatt alternator each belted to a separate engine. The entire plant represents an investment of $\$ 62,5$ I 2 of which $\$ 37,674$ is in the building and the equipment of the station, and $\$ 24,838$ is in the distributing system including the transformers. The total number of lamps installed is 9,000 I6 candle-power incandescent lamps and I07 arc lamps. The cost of trimming and cleaning the arc lamps (\$230.79) is not

* The Electrician (London) publishes each year a digest of the data on operation costs which electric lighting stations are required by law to report to the Board of Trade. These digests contain much valuable information. See also "Cost of Generation and Distribution of Electrical Energy" by Robert Hammond, Journal Institution of Electrical Engineers, Vol. 27, pp. 246-437, 1898. 

included in the tabulated costs of operation of the station and distributing system. The cost of repairs is the average for three years. A portion of the fixed charges, namely, repairs and insurance, are tabulated in accordance with actual expenditure; and the remainder, namely, interest, depreciation, and taxes, are estimated at I I per cent., that is, interest 6 per cent., depreciation 4 per cent., and taxes I per cent., although as a matter of fact the investment is covered by municipal bonds which bear interest at 3.5 per cent. and the plant pays no taxes. This plant supplies current to consumers on a sliding scale of flat rates, that is so much per month for each lamp installed, the rate being less the greater the number of lamps installed by an individual consumer. This station is operated I 5 hours per day, 365 days per year.

Station B is a 405 kilowatt plant owned and operated by a city of 7,500 inhabitants in Connecticut. The equipment in-

Expense Accounts for One Year of Two Electric Plants.

$A=225$ kilowatt plant. $B=405$ kilowatt plant.

\begin{tabular}{|c|c|c|}
\hline & Plant A. & Plant B. \\
\hline Total investment................. & $\$ 62,512.00$ & $\$ 89,880.00$ \\
\hline Kilowatt-hours output for the year......................... & $356,575.00$ & $525,346.00$ \\
\hline Labor and superintendence... & $\$ 4,064.71$ & $\$ 7,029.91$ \\
\hline Repairs .............................. & 754.00 & $1,142.00$ \\
\hline Coal (at about $\$ 4$. Io per ton at both plants)............. & $7,225.53$ & $6,956.84$ \\
\hline 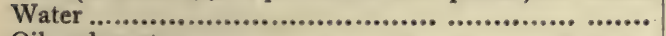 & 31.00 & \\
\hline 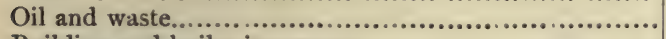 & 105.00 & \\
\hline Building and boiler insurance............................... & 188.00 & $2,686.72$ \\
\hline Liability insurance.......................................... & 187.00 & \\
\hline 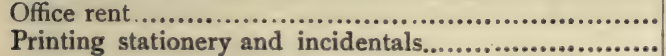 & $\begin{array}{r}40.00 \\
883.86\end{array}$ & \\
\hline Total disbursements............ & $\$ 13,479.10$ & $\$ 17,815.47$ \\
\hline $\begin{array}{l}\text { Cost per kilowatt-hour (cents) including } 3 \mathrm{~T} / 2 \text { per cent. } \\
\text { interest and } 4 \text { per cent. depreciation.................... }\end{array}$ & 5.10 & 4.78 \\
\hline $\begin{array}{l}\text { Cost per kilowatt-hour (cents) including } 6 \text { per cent. } \\
\text { interest, } 4 \text { per cent. depreciation, I per cent. taxes... }\end{array}$ & $5 \cdot 71$ & 5.46 \\
\hline
\end{tabular}

cludes four 60 kilowatt direct current generators each direct connected to a 100 horse-power engine, one 165 kilowatt direct 
current generator direct connected to a 300 horse-power engine, and a Brush arc generator driven by a 90 horse-power electric motor. The entire plant represents an investment of $\$ 89,880$ of which $\$ 60,165$ is in the building and equipment of the station and $\$ 29,7$ I 5 is in the distributing system. The total number of lamps installed is 6,300 I6 candle-power incandescent lamps and I09 arc lamps. A separate account is not kept in this station of the cost of trimming and cleaning the arc lamps. The cost of repairs is the average for three years. The same remarks concerning interest, depreciation, and taxes apply to this station as to station A. This plant supplies current to consumers on a meter basis, the monthly bill being made out at the following net prices :

For lighting and for fan motors :

First Ioo kilowatt-hours at 9.0 cents per kilowatt-hour.

$\begin{array}{lllllll}\text { Next } 200 & \text { " } & & \text { " } & 7.2 & \text { " } & \text { " } \\ \text { Over } 300 & \text { " } & & \text { " } & \text { " } & \text { " }\end{array}$

For motive power (not including fan motors) :

First 200 kilowatt-hours at 4.5 cents per kilowatt-hour.

$\begin{array}{llllllll}\text { Next } 400 & \text { " } & \text { " } & 3.6 & \text { " } & \text { " } & \text { " } & \text { " } \\ \text { Over } 600 & \text { " } & 2.7 & \text { " } & \text { " } & \text { " }\end{array}$

The plant is operated continuously except from one hour after sunrise to one hour before sunset on Sundays.

It is seen from the table that the average cost of generation of electrical energy is between 5 and 6 cents per kilowatt-hour and it may seem that station B supplies energy for motive power below cost. This is, however, not the case ; the station is run day and night irrespective of the demand for current for motive power, and the added cost of operating the station due to the supply of current to motors is probably less than one cent per kilowatt-hour. This matter is discussed in the next article.

100. Systems of charging for electrical energy. (I) The flat rate system. - Many small electric light stations supply current 
to consumers at a certain price per month for each lamp installed. The 16 candle-power incandescent lamp is the unit generally employed, and the price in many cases is fifty cents per month per lamp, with a scale of discounts increasing with the number of lamps. This system of charging is called the flat rate system. The use of this system simplifies the station book-keeping and it avoids the cost of meters, but it is very unsatisfactory inasmuch as a wasteful customer pays no more than an economical one.

2. The meter system. - The selling of electrical energy by the kilowatt-hour as indicated by a watt-hour meter is almost universal in large electric plants, and it is coming to be the rule among small plants also. It may seem that the use of the watt-hour meter would, like the use of the gas meter, insure an equitable system of charging customers, but it is not so. In supplying gas for lighting, a large storage * reservoir makes the gas generating plant independent of the irregular consumption of the gas. In an electric plant, on the other hand, the electrical energy must, in most cases, be generated as used and the capacity of the plant must be sufficient to meet the maximum demand for current. That is to say, the size and cost of the plant is determined by the maximum demand, so that the greater value of the electrical energy at the time of maximum demand must be considered in any equitable schedule of prices. Two simple cases will serve as a basis for the discussion of prices as follows :

Case 1. - If the demand for current by each customer of a central station were uniform day and night, or if all the customers were to use current according to one fixed daily regime, $†$ then it would be entirely equitable to charge a uniform price per kilowatthour, inasmuch as the same fraction of the total energy supplied to each would be supplied during the time of maximum demand. This uniform schedule of prices should be subject to a scale of

* The use of the storage battery for equalizing the load of an electric station is discussed in a subsequent chapter.

† Imagine a curve of which the abscissas represent the hours of the day and the ordinates the current used by a customer. This curve represents the "daily regime" of the customer. 
discounts provided the station management wishes to increase the general demand for current, which will be the case so long as the demand is less than can be met by the station. When the demand reaches the full capacity of the station the scale of discounts should be rearranged so as to be less favorable to larger users than before, unless it is possible at once to secure funds for increasing the size of the station.

Case II. - If there are several distinct classes of customers differing as to their daily regime in the use of current, then [on the assumption that the total cost of operation of a station may be approximately separated into two parts, namely, a constant part $A$ which depends upon the size (that is the capacity) of the station, and a variable part $B$ which is proportional to the station load] the proper basis upon which to establish an equitable schedule of prices is as follows :

(a) Each customer should be charged so much per year on the rate at which he uses electrical energy during the period of excessive station load. This charge should of course be subject to a scale of discounts and the income from it should cover the constant part $A$ of the total cost of operating the plant, with a margin of profit.

(b) Each customer should be charged, in addition to (a), a certain amount per kilowatt-hour for the total electrical energy supplied to him. This charge should also be subject to a scale of discounts, and the income from this charge should cover the variable part $B$ of the total cost of operating the plant, with a margin of profit.

There are three practicable methods for carrying out this system of charging, as follows :

I. By classification of rates. - The usual method is to classify customers according to their daily regime in the use of current, and to establish a distinct rate per kilowatt-hour for each class. For example, there are three more or less distinct classes of service in most electric plants, namely, (a) domestic lighting service with its negligible demand for current during the day and 
its excessive demand for current from 5 or 6 or 7 o'clock to Io or I I o'clock P. M., (b) store and factory lighting service with its almost uniform demand for current from 3 or 4 o'clock to 6 or 8 o'clock P. M., and $(c)$ motive power service with a nearly uniform demand for current from 7 A. M. to 6 P. M. Customers of the first class should be charged at a high rate, those of the second class at a lower rate, and those of the third class at a still lower rate. It is to be understood that these statements refer to differences in scheduled selling price and not to differences in discounts; the same scale of discounts may in fact be applied to all. This system of class rates is exemplified by the scale of prices which are given in Art. 98.

2. By the use of the two-rate meter. - The two-rate meter is an ordinary watt-hour meter with two sets of dials, and a clock which throws into gear one set of dials during the period of excessive station load, between 7 and Io P. M. for example, and the other set of dials during the remainder of the 24 hours of each day. When this meter is used the customer is charged at a high rate per kilowatt-hour for the energy indicated by the first set of dials, and at a low rate for the energy indicated by the second set of dials.

3. By the use of the maximum demand meter. - The maximum demand meter is a device, generally an ammeter in its essential features, of which the indicator, which is too sluggish to be affected by a momentary short-circuit, is left standing at the point to which it is carried by the greatest current used by the customer during the month. In the use of this device the monthly charge to the customer is so much for the maximum rate and so much per kilowatt-hour for the energy indicated by an ordinary watthour meter.

The minimum charge. - There is a certain amount of service rendered to a customer by the mere fact that the current from a central station is at his disposal at any time night or day; on the other hand the interest on the cost of the watt-hour meter, the cost of its maintenance, and a portion of the cost of the station 
book-keeping represents a corresponding expense to the station, and this expense justifies a certain fixed charge against each customer for the above-mentioned service. This fixed charge is usually made in the form of a minimum monthly charge.

Fluctuating and intermittent service. - If the demand for current by each of the customers of a station varies in a purely erratic way from hour to hour and from day to day, the resultant demand of a large number of customers will approximate very nearly to a perfectly uniform demand day and night. If there are several distinct classes of customers and if the demand for current by a customer in either class departs in a purely erratic way from a certain mean daily regime which is characteristic of that class, then the resultant demand of a large number of customers will be very nearly the same as if each customer were to use current in strict accordance with the mean regime of his class. In both of these cases the system of charging outlined above under cases I. and II. is applicable when the station is large and the number of individual customers great.

In the case of a small station with a small number of individual customers, erratic variations in daily regime by individuals becomes a serious matter, inasmuch as the resultant demand shows considerable erratic variation. In such a case any service, such as motor service for elevators, which is peculiarly subject to wide variations should be charged for at a high rate which should be determined partly by a consideration of the mean daily regime and partly by a consideration of the amplitude of the departures from this mean. There is no very satisfactory method for determining an equitable charge in this case, in fact a small station is justified in making the charge for elevator service, for example, prohibitory.

Advantages of the meter system as compared with the flat rate system. - The equitable distribution of charges which is realized by the use of the meter system leads to an economical use of current and enables a station of given capacity to supply current to an increased number of customers. 
Frequently a newly installed electric light plant starts out on the flat rate system, which it retains until the increasing demand for current exceeds the capacity of the station. Then the meter system is adopted to curtail the waste of current and so relieve the situation.

The pecuniary saving incident to the use of the meter system is great enough to give the customer the service he needs at a reduced total cost, to cover the cost of installing and maintaining the meters and the cost of the extra book-keeping involved, and to yield, in addition, an increased margin of profit to the station management. The advantage to the station may not appear, however, unless the demand for current is such that it would exceed the station capacity if the flat rate system were used. This is evident when we consider that a very slight saving in the cost of operating the station would be effected by decreasing a moderate station load by adopting the meter system; whereas the large income that is in sight when the demand for current under the flat rate system exceeds the station capacity may be to some extent realized by the adoption of the meter system. 


\section{- CHAPTER VIII.}

STORAGE BATTERIES.

101. Electrolysis. - When an electric current passes through a conducting liquid which is not a chemical element the liquid is decomposed. Thus melted salts, and acids and salts in solution, are decomposed by the electric current. This chemical decomposition is called electrolysis, and the liquid in which electrolysis takes place is called an electrolyte.

Electrolytes generally have very high specific resistances as compared with the metals, so that electrolysis is usually carried out in a vessel provided with two flat plates of metal or carbon which serve to lead the current into and out of the electrolyte. Such an arrangement is called an electrolytic cell and the plates of metal or carbon are called the electrodes. The electrode at which the current enters the electrolyte is called the anode, and the electrode at which the current leaves the electrolyte is called the cathode.

Consider a solution of hydrobromic acid $(\mathrm{HBr})$. When an electric current is passed through this solution, hydrogen $(\mathrm{H})$ is liberated at the cathode and bromine $(\mathrm{Br})$ is liberated at the anode. In general the molecule of any dissolved salt or acid is separated into two parts by electrolysis; one part is liberated at the cathode and is called the cathion, and the other part is liberated at the anode and is called the anion. Thus hydrogen $(\mathrm{H})$ is the cathion and bromine $(\mathrm{Br})$ is the anion of hydrobromic acid. In all metallic salts the metal constitutes the cathion, and the acid radical or halogen constitutes the anion. In acids the hydrogen constitutes the cathion, and the acid radical or halogen constitutes the anion. Thus the cathion of copper sulphate $\left(\mathrm{CuSO}_{4}\right)$ is copper $(\mathrm{Cu})$, and the anion is the acid radical $\left(\mathrm{SO}_{4}\right)$. 
In many cases the cathion and anion are not actually liberated at the electrodes, because of what are called secondary reactions. Thus in the electrolysis of an aqueous solution of sodium chloride $(\mathrm{NaCl})$, the cathion $\mathrm{Na}$, when it is liberated at the cathode, immediately reacts upon the water forming $\mathrm{NaOH}$ and free hydrogen; in the electrolysis of copper sulphate $\left(\mathrm{CuSO}_{4}\right)$ between copper electrodes, the anion $\mathrm{SO}_{4}$ combines with the copper of the anode forming fresh $\mathrm{CuSO}_{4}$ which goes into solution, or which is deposited in crystals on the anode if the solution is saturated; in the electrolysis of $\mathrm{H}_{2} \mathrm{SO}_{4}$ between inert electrodes the hydrogen is liberated at the cathode as a gas, and the anion $\mathrm{SO}_{4}$ reacts on the water according to the formula $\mathrm{SO}_{4}+\mathrm{H}_{2} \mathrm{O}$ $=\mathrm{H}_{2} \mathrm{SO}_{4}+\mathrm{O}$, and the free oxygen escapes as a gas. The reason for taking the unfamiliar substance, hydrobromic acid, in the above example is that in the electrolysis of hydrobromic acid there are no secondary reactions at the electrodes.

102. The voltaic cell.* - The chemical action that is caused by the flow of current through an electrolytic cell is confined wholly to the immediate neighborhood of the electrodes, and this chemical action is usually forced, that is, work has to be done to bring it about, or, in other words, an outside electromotive force is required to push the current through the cell.

When, however, secondary chemical actions take place at one or both electrodes, it frequently happens that the total chemical action that is brought about by the flow of current through an electrolytic cell is a source of energy. In such a case the electrolytic cell itself can maintain a current through the electrolyte from electrode to electrode and through an outside circuit of wire which connects the electrodes. Such an electrolytic cell is called a voltaic cell or primary battery.

Examples. - When a strip of clean zinc and a strip of copper or carbon are dipped into dilute sulphuric acid, no chemical action takes place. When the plates are connected together by a wire,

* A number of voltaic cells connected together constitute a voltaic battery. The word battery is, however, frequently applied to a single cell. 
a current immediately starts to flow through the circuit, leaving the cell at the copper or carbon electrode (the cathode) and entering the cell at the zinc electrode (the anode). This current decomposes the sulphuric acid $\left(\mathrm{H}_{2} \mathrm{SO}_{4}\right)$, the hydrogen is liberated at the copper or carbon cathode and escapes from the cell as a gas, and the sulphuric acid radical $\left(\mathrm{SO}_{4}\right)$ which is set free at the zinc anode combines with the zinc and forms zinc sulphate $\left(\mathrm{ZnSO}_{4}\right)$ which goes into solution. The combination of $\mathrm{Zn}$ and $\mathrm{SO}_{4}$ develops more energy than is required for the decomposition of the $\mathrm{H}_{2} \mathrm{SO}_{4}$ so that the chemical action in this cell is a source of energy.

The available energy of the reaction which takes place in the above cell may be greatly increased by providing an oxidizing agent in the neighborhood of the cathode so that the hydrogen may be oxidized and form water $\left(\mathrm{H}_{2} \mathrm{O}\right)$ at the moment of its liberation by the current. The energy of this oxidation is then added to the available energy of the total chemical action in the cell.*

103. Voltaic action and local action. - Two kinds of chemical action are to be distinguished in a voltaic cell : (a) The chemical action which depends upon the flow of current, and does not exist when there is no current; and $(b)$ the chemical action which is independent of the flow of current, and which exists whether the current is flowing or not.

The chemical action which depends on the current is proportional to the current, it is essential to the operation of the voltaic cell as a generator of current, its energy is available for the maintenance of the current, and it is called voltaic action.

The chemical action in a voltaic cell which is independent of the flow of current does not help in any way to maintain the current, it represents absolute waste of materials, and it is called local action. Local action takes place more or less in every type

* The student is referred to Professor H. S. Carhart's Primary Batteries, published by Allyn and Bacon, Boston, Mass., for full information on primary batteries, and primary battery tests. 
of voltaic cell. It is especially great in cells of the Grenet type, and it may be reduced to a minimum in a given type of voltaic cell by coating the zinc with a thin layer of mercury.

The feature of the Grenet type of cell which favors local action is that no provision is made in this cell to keep the oxidizing agent, which in this case is potassium bichromate or chromic acid, confined to the neighborhood of the carbon cathode, where it is needed to oxidize the free hydrogen; but it is allowed to mix with the whole of the electrolyte thus coming into contact with the zinc anode. Under these conditions the zinc dissolves rapidly in the electrolyte. whether a current is flowing or not. In a well cared for Grenet cell, even while it is being used to give a large current, about 80 per cent. of the zinc is consumed by local action, and only 20 per cent. by voltaic action.

The useful consumption of zinc by voltaic action, while a voltaic cell is delivering a given current for a specified time, is equal to the amount of zinc that would be deposited by the given current during the specified time upon the cathode of an auxiliary electrolytic cell containing a solution of a zinc salt, zinc sulphate, for example.

An essential feature of voltaic action is that it is reversed if a current is forced backwards through a voltaic cell by an outside agent, provided that no material that has played a part in the previous voltaic action has been allowed to escape from the cell. Thus, in the operation of the simple voltaic cell consisting of a zinc anode and carbon cathode in dilute sulphuric acid, the $\mathrm{H}_{2} \mathrm{SO}_{4}$ is decomposed, $\mathrm{ZnSO}_{4}$ is formed at the anode, and hydrogen is liberated at the cathode. If the current is reversed so that the carbon plate becomes the anode and the zinc plate the cathode, then the $\mathrm{ZnSO}_{4}$ previously formed will be decomposed, metallic zinc will be deposited upon the zinc cathode, and $\mathrm{SO}_{4}$ will be liberated at the carbon anode where it will combine with the trace of hydrogen that is clinging to the carbon plate and form $\mathrm{H}_{2} \mathrm{SO}_{4}$. In this cell the greater part of the liberated hydrogen has of course escaped and the reversed chemical action, due to a 
reversed current cannot long continue. Local action, on the other hand, being independent of current is not affected by a reversal of the current.

104. The storage cell. - A voltaic cell that is free from local action and in which all of the materials which take part in the voltaic action are conserved in the cell, may be regenerated after use by sending through it a reversed current. This regeneration is due to the reversed chemical action that is produced by the reversed current, as explained in the previous article. A voltaic cell that is adapted to be thus regenerated is called a storage cell. The process of regeneration is called charging and the use of the cell as an electric generator is called discharging.

A good storage battery must be free from local action, the materials which take part in the voltaic action must be kept in the cell, and the electrodes must not crumble to pieces with frequent charging and discharging of the cell.

A storage cell always requires a larger voltage between its electrodes to charge it than it is able to maintain while it is being discharged. This is due in part to the resistance* drop $R i$ in the cell, inasmuch as this resistance drop opposes the flow of current both in charging and in discharging; and it is due in part to variations in the concentration of the electrolyte at the electrodes during charging and discharging as explained later. Consequently more work must be done in charging a storage cell than can be regained by discharging it.

105. The lead storage cell. $\dagger$ - The voltaic cell which, up to the present time, has been found to meet most satisfactorily the

* The resistance of the cell is here intended to include what is sometimes called transition resistance at the surface of the electrodes, together with the effect at the surface of the electrodes which is sometimes called irreversible polarization.

$\dagger$ The voltaic cell having a cathode of lead peroxide, an anode of zinc, and an electrolyte of dilute sulphuric acid has been used to some extent as a storage cell; see Secondary Batteries by E. J. Wade, pages 118-126, The Electrician Company, London. The Edison storage cell (spongy iron anode, nickel peroxide cathode in a solution of caustic potash) is described by A. E. Kennelly in the Trans. A. I. E. E., for May, 1901. 
requirements of a storage cell, as above specified, is a voltaic cell having a cathode of lead peroxide $\left(\mathrm{PbO}_{2}\right)$, an anode of spongy metallic lead, and an electrolyte of dilute sulphuric acid. The lead peroxide and the spongy metallic lead are both converted into insoluble lead sulphate $\left(\mathrm{PbSO}_{4}\right)$ when this cell delivers current, and this lead sulphate is converted back into lead peroxide and spongy lead respectively when a reversed current is forced through the cell. The lead peroxide and the spongy lead are called the active materials of the cell. These active materials are mechanically weak and porous, and they are usually supported in the interstices of massive grids of metallic lead. Furthermore, the active material being a rather poor electrical conductor, the lead grids serve not only as mechanical supports, but also to deliver current to or receive current from the active material which constitutes the real electrodes.

Action of the coll while discharging. - When the lead storage cell delivers current, the electrolyte $\mathrm{H}_{2} \mathrm{SO}_{4}$ is split up by the current into $\mathrm{H}_{2}$ and $\mathrm{SO}_{4}$. The hydrogen is liberated at the cathode, where it reduces the lead peroxide to $\mathrm{PbO}$, and this $\mathrm{PbO}$ combines with a portion of the $\mathrm{H}_{2} \mathrm{SO}_{4}$ of the electrolyte forming $\mathrm{PbSO}_{4}$ and water. The $\mathrm{SO}_{4}$ which is liberated at the anode combines with the spongy lead and forms $\mathrm{PbSO}_{4}$.

During this process the active material expands because the lead sulphate is more bulky than the spongy lead and the lead peroxide; and the electrolyte grows less concentrated (and of course increases in resistance) because of the absorption of $\mathrm{SO}_{4}$ by the active material. This decrease of concentration is especially great in the pores of the active material.

Action of the cell while being charged. - When the lead storage cell is regenerated by forcing a reversed current through it, the above-described action is reversed. The lead sulphate on one electrode is converted back to lead peroxide, the lead sulphate on the other electrode is reduced to spongy metallic lead, the electrolyte grows more dense, especially in the pores of the active material, and the active material contracts. 
It is the usual practice among electrical engineers to call that terminal of an electric generator out of which current flows, the positive terminal, and that terminal into which current flows, the negative terminal. In conformity with this usage, that electrode of a storage cell which is cathode during discharge is called the positive grid and the other the negative grid. The positive grids are of a pale salmon color and the negative grids are a neutral gray.

The following arrangement gives a clear idea of the action of the lead storage cell while discharging and while being charged :

\section{Discharging, *}

Positive grid. $\mathrm{PbO}_{2}+\mathrm{H}_{2} \mathrm{SO}_{4}+\mathrm{H}_{2}=2 \mathrm{H}_{2} \mathrm{O}+\mathrm{PbSO}_{4}$
Negative grid. $\mathrm{Pb}+\mathrm{H}_{\text {Direction of current through the cell }}^{\text {(negative to positive grid). }}$

Charging.*

Positive grid. $\quad \mathrm{PbSO}_{4}+2 \mathrm{H}_{2} \mathrm{O}+\mathrm{SO}_{4}=2 \mathrm{H}_{2} \mathrm{SO}_{4}+\mathrm{PbO}_{2}$

1 Direction of current through the cell (positive to negative grid).

Negative grid. $\mathrm{PbSO}_{4}+\mathrm{H}_{2}=\mathrm{H}_{2} \mathrm{SO}_{4}+\mathrm{Pb}$

Variation of voltage during charge and discharge. - The electromotive force between lead peroxide and spongy lead immersed in dilute sulphuric acid, increases with increasing concentration of the acid. Therefore the voltage of a lead storage cell, on open circuit, is large when the cell is completely charged, because of the great concentration of the acid; and it grows smaller and smaller with decreasing concentration of the acid as the cell is discharged. Furthermore, while the cell is being charged, the concentration of the electrolyte in immediate contact with and especially in the pores of the active material is greater than the

* The above-described action of the lead storage cell constitutes the simple working theory of the cell. These actions do take place but they are no doubt accompanied by more complex actions such as the formation of per-sulphates at the anode and of sub-sulphates at the cathode. See The Theory of the Lead Accumulator by Friedrich Dolezalek (English translation by C. L. von Ende, published by John Wiley \& Sons). 
mean concentration of the entire electrolyte; and while the cell is discharging the concentration of the electrolyte in contact with the active material is less than the mean. It is for this reason largely that a greater electromotive force is required to charge a lead storage cell than the cell maintains while discharging.

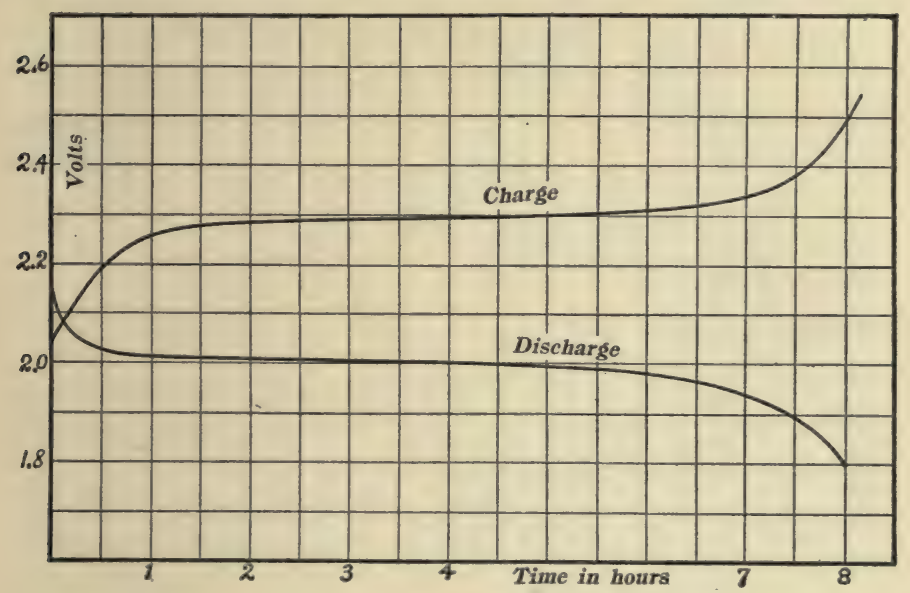

Fig. 143.

The two curves in Fig. I43, show the variation of the voltage between the terminals of a lead storage cell while it is discharging and while it is being charged. The shapes of these charge and discharge curves depend greatly upon the thickness of the electrodes, the temperature of the cell, the concentration of the electrolyte, and the value of the charging or discharging current.

Practical limit to discharge. - The extent to which a lead storage cell may be discharged in practice is determined by the following considerations: (a) The lead peroxide and the spongy metallic lead which constitute the active materials of a fully charged lead storage cell, are fairly good electrical condưctors; whereas the lead sulphate into which these materials are converted by discharge is an extremely poor electrical conductor, much too poor to act as electrode material even in small pieces 
packed in the interstices of the lead grids. The active material on the positive grid must always contain a large proportion of lead peroxide to give it the necessary electrical conductivity, and the active material on the negative grid must always contain a large proportion of spongy lead for the same reason. Therefore it is not permissible to discharge a cell so completely as to convert a large portion of the lead peroxide and spongy lead into lead sulphate. (b) The conversion of all of the lead peroxide on the positive grid and of all of the spongy lead on the negative grid to lead sulphate would involve an excessive expansion of the active materials which would be more likely than a moderate degree of expansion to detach the active materials from the grids. (c) When a storage cell is discharged beyond a certain limit its electromotive force falls off excessively.

Usually a lead storage cell is not discharged beyond the point at which its voltage falls to I.8 volts while giving its full rated current, and in the usual types of cells this degree of discharge corresponds to the conversion, of from $\frac{1}{6}$ to $\frac{1}{4}$ of the active material into lead sulphate.

Limit to charge. - When all of the active material on the positive and negative grids is converted into lead peroxide and spongy lead respectively, then a continuation of the charging current causes hydrogen gas to be liberated at the negative grids and oxygen gas to be liberated at the positive grids without producing any further chemical change in the active material. This evolution of gas is not especially harmful to the cell unless it becomes so violent as to cause the disintegration of the active material by the production of bubbles within the pores, or between the active material and the solid metal of the grid.

Capacity. - The capacity of a storage cell is expressed in ampere-hours, that is to say, in terms of the product of the current in amperes times the number of hours that the cell can deliver the current when it is operated between the practical limits of charge and discharge. The capacity of a storage cell varies greatly with the discharge rate as explained later. 
Efficiency. - The energy efficiency of a storage battery is the ratio of the energy delivered by the cell during discharge to the energy delivered to the cell during charge. The efficiency ranges from 70 to 95 per cent. according to the conditions of use of the battery. If the battery is discharged to $\mathrm{r} .8$ volts per cell, then completely charged, then discharged to I.8 volts per cell, and so on, the efficiency varies from about 70 per cent. with high rates of charge and discharge to about 80 per cent. with low rates of charge and discharge. If the battery is charged and discharged through a very narrow range during short intervals of time, for example if a battery is charged for one minute then discharged for one minute repeatedly, its efficiency may be as high as 93 or 94 per cent.

Rates of charge and discharge. - The current delivered by a storage cell, or the current used in charging it, measures its rate* of discharge or charge. Thus one may properly speak of a tenampere rate of charge or discharge. When a storage cell is discharged at a high rate the voltage of the cell tends to fall off rapidly, partly on account of the large $R i$ drop in the cell, but chiefly because of the fact that the rapid absorption of the sulphuric acid in the formation of lead sulphate makes the acid in the pores of the active material very dilute. On the other hand, when a cell is charged at a high rate the voltage tends to rise excessively.

Furthermore, in rapid charging or discharging the tendency is for the surface layers, only, of the active material to take part in the voltaic action, so that the ampere-hour capacity of the cell is reduced, and the rapid and non-uniform expansion and contraction of the active material tends to cause disintegration.

106. Examples of storage battery grids. - There are two general processes for making storage battery grids, namely: $(a)$ The Planté process and $(b)$ the Faure process. In the Planté process

* A discharge rate is usually specified in practice by giving the number of hours required to discharge the cell at the given rate. Thus engineers speak of a two-hour rate, a five-hour rate, etc. 
a lead plate with a finely corrugated surface is subjected to the action of a suitable acid bath which converts the surface layers of the metal into active material by corrosion. Planté's original process was to expose the lead plates to the action of dilute sulphuric acid, the corroding action of which he accelerated by making the plates alternately anode and cathode. In the modified Planté processes * now used the corrosion is accelerated by the addition of a small percentage of a lead dissolving acid, such as nitric acid or acetic acid.

In the Faure process the active material is manufactured in bulk by any suitable chemical process and introduced mechanically into the interstices of the lead grids. $\dagger$ Faure's original process which is still extensively used was to apply the active material

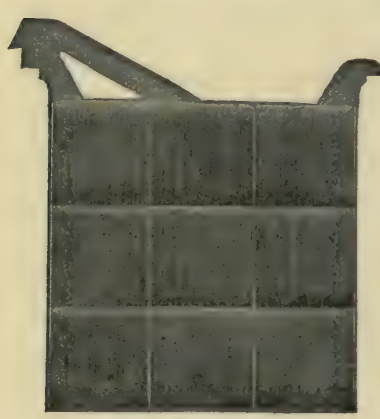
in the form of a paste made by wetting Fig. 144. litharge or a mixture of litharge and red lead with dilute sulphuric acid.

The grid of the Gould storage battery, which is shown in Fig. I44, is an example of a grid made by the accelerated Planté process. Thick plates of metallic lead of the desired shape are placed between rapidly rotating spinning rolls which raise thin fins of the metal on the faces of the plate. A central web and cross-ribs of untouched lead are left to give the grid the requisite strength and conductivity, and the active material is formed over the greatly increased surface of the fins by a process of corrosion. Each spinning roll consists of a large number of thin steel disks with thin spacing washers between, all clamped rigidly together on a shaft. The positive grid always gives more trouble from buckling and it is weakened by corrosion much more rapidly

* See Storage Battery Engineering, by Lamar Lyndon, Chapter XVI.

† A variety of grids made by modifications of the Faure process are described by Lyndon, Storage Battery Engineering, Chapter XVII. 
than the negative grid and therefore a greater number of crossribs is left on the positive grid than on the negative grid.

The grids of the stationary type of storage cell of the Electric Storage Battery Company, which is known as the "Chloride" cell, are shown in Fig. I45. This figure also shows one of the thin corrugated wooden plates which are used as separators between the grids. The positive grid of this cell is a plate of leadantimony alloy cast with numerous holes into which circular plugs or coils of pure corrugated lead tape are placed. These plugs are then converted into active material by an accelerated Planté process. The negative grid is made by, inserting small

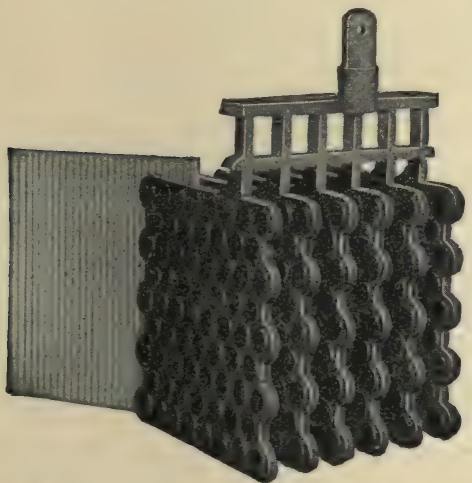

Positive grids.

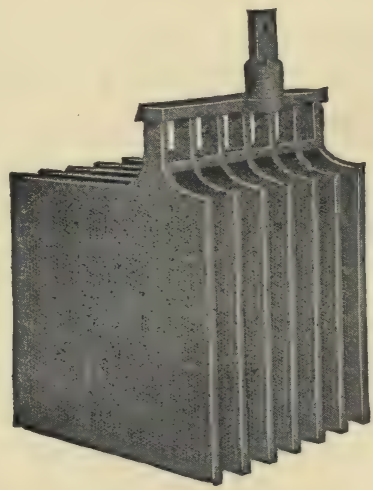

Negative grids.

Fig. 145.

blocks of active material into holes in a grid of pure lead. This grid is made in two halves which are pressed together over the blocks of active material and riveted together under pressure.

The grids of the portable type of storage cell of the Electric Storage Battery Company, which is known as the "Exide" cell, are grids cast of a lead-antimony alloy. The interstices in the positive grid are filled with a paste of red lead and sulphuric acid, and the interstices of the negative grids are filled with a paste of litharge and sulphuric acid.

Storage battery grids made by the Planté process are usually much heavier than Faure grids of the same capacity, but the 
active material which is formed by the Plante process is more compact and more firmly attached to the grids than the active material that is applied to the grids mechanically in the Faure process. Heavy stationary batteries usually have Planté grids, and light portable batteries usually have Faure grids, or, what is perhaps better, Planté positive grids and Faure negative grids.

The following quotations from a large manufacturer give some idea of the weights and costs of storage batteries :

\section{Heavy Stationary Type.}

Weights and costs of a Ioo-volt (50-cell) storage battery rated at 400 ampere hours on an eight-hour discharge and capable therefore of delivering 40 kilowatthours.

\begin{tabular}{|c|c|c|c|c|}
\hline & \multicolumn{2}{|c|}{ Weight. } & \multicolumn{2}{|c|}{ Cost. } \\
\hline & Pounds. & $\begin{array}{c}\text { Pounds per } \\
\text { kilowatt hour. }\end{array}$ & Dollars. & $\begin{array}{l}\text { Dollars per } \\
\text { kilowatt-hour. }\end{array}$ \\
\hline In glass jars. & 10,300 & 258 & 1,400 & 35.00 \\
\hline In lead-lined wood tanks. & 14,600 & 365 & 1,650 & 41.25 \\
\hline
\end{tabular}

Depreciation 6 or 7 per cent. per annum when properly cared for.

\section{Light Portable Type.}

Weights and costs of a IOO-volt (50-cell) storage battery of the carriage type rated at 200 ampere-hours on a four-hour discharge and capable therefore of delivering 20 kilowatt-hours.

\begin{tabular}{l|c|c|c|c}
\hline \hline & \multicolumn{2}{|c|}{ Weight. } & \multicolumn{2}{c}{ Cost. } \\
\cline { 1 - 5 } & Pounds. & $\begin{array}{c}\text { Pounds per } \\
\text { kilowatt-hour. }\end{array}$ & Dollars. & $\begin{array}{c}\text { Dollars per } \\
\text { kilowatt-hour. }\end{array}$ \\
\hline In covered rubber jars. & 2,340 & II & 800 & 40.00 \\
\hline
\end{tabular}

Depreciation 15 per cent. per annum or more.

\section{Management and care of the lead storage cell.* - The} lead storage cell deteriorates rapidly in service, especially when it is not properly cared for ; and, therefore, since the first cost of a

* The reader is referred to Chapter XIV. of Lyndon's Storage Battery Engineering for a very full discussion of this subject. 
storage battery is high, it is important that it should have proper care.

The electrolyte should be made of very pure sulphuric acid. The least trace of platinum (from the platinum still used in the manufacture of the acid) is very harmful. In general a metallic salt of any kind is objectionable, inasmuch as the foreign metal is deposited on the grids and gives rise to local voltaic action. Also the presence of lead dissolving acids such as nitric or acetic is objectionable, as is also the presence of chlorides. The effect of these impurities is to accelerate the corrosion of the solid lead of the grid converting it into active material.

High concentration of the electrolyte reduces the internal resistance of the cell, and it facilitates to some extent the diffusion of acid into and out of the active material. On the other hand, high concentration of the electrolyte tends to cause trouble from sulphatation as explained later. Therefore a cell which is likely to stand for a long time unused should have a low density electrolyte, and a cell that is cared for properly may have a higher density electrolyte. The usual densities range from I.2 I to I.24 charged, and from 1.185 to 1.195 discharged.

The over-concentrated acid at the surface of the grids during charge tends to flow to the bottom of the cell and the underconcentrated acid at the surface of the grids during discharge tends to flow to the top of the cell. In large and especially in deep cells the electrolyte should be continuously circulated by pumping a small stream of air to the bottom of the cell.

When it is desired to test the electrolyte it should be thoroughly stirred by blowing air through it.

Water must be occasionally added to make up for evaporation. This should be poured to the bottom of the cell through a long rubber tube attached to a funnel. The electrolyte should always cover the grids.

The grids should be frequently inspected with a view to the discovery of any sulphatation, or buckling, and a thin wooden stick should be occasionally passed between the grids to make 
sure that the grids are not connected by a block of detached active material. Sulphatation and buckling are usually due to longcontinued standing at discharge, which is brought about by an unnoticed internal short-circuit of the cell.

A storage cell should not be allowed to stand discharged for any length of time.

A storage battery standing unused should be partly discharged and immediately recharged at least once per week.

When a storage battery is to be put out of service for a long time, it should be partly discharged, the electrolyte should then be drawn off from each cell and pure water poured in immediately, then the battery should be short-circuited until the voltage (on open-circuit) falls to about 0.5 volt per cell. The cells should then be rinsed with several changes of water, allowing the plates to soak thoroughly in each, and the final washing water should be drawn off and the plates allowed to dry. When the battery is again put into commission it is only necessary to pour in electrolyte and give the battery a long-continued over-charge.

Sulphatation of the grids of a lead storage cell consists * of the conversion of portions of the active material wholly into lead sulphate. This pure sulphate is a very poor conductor and, once it is formed, it is difficult to make it act as anode or as cathode and thus reconvert it to lead peroxide or to spongy lead respectively. A layer of pure lead sulphate sometimes forms between the active material and the metallic lead of the grid, and sometimes the external surface of the active material becomes covered with a crust of pure sulphate. Pure lead sulphate is white and whenever white spots appear on the grids of a lead storage cell, the cell should be subjected to a very long-continued over-charge in the attempt to reduce the pure lead sulphate into active material.

The normal discharge rate of a lead storage cell, on the basis of an eight-hour discharge, is from 6 to 7 amperes per square foot

* It is claimed by some authorities that sulphatation consists in the formation of hydrated lead sulphate. 
of positive grid area, where positive grid area is equal to $2 l b n$, $l$ being the length, $b$ the breadth of each grid, and $n$ the number of positive grids. The reason for referring the discharge rate to the area of the positive grids rather than to the area of the negative grids is that the positive grids are always one less in number than the negative grids, so that both sides of every positive grid are active.

The discharge rate of a storage battery in amperes should never exceed four or five times the normal rate of discharge (eight-hour basis). If a greater current must be taken from the battery it should be for a few minutes, only, and the battery should be at full charge.

The rate of charge may be high when the battery is nearly discharged, but it should be low when the battery approaches full charge, especially after the evolution of gas begins. A good rule for rapid charging is to deliver to the battery 35 per cent. of the total ampere-hours during the first hour ; 52 per cent. during the next two hours, and I4 per cent. during the fourth hour. Thus a IOO ampere-hour cell may be completely charged in four hours by using a charging current of 35 amperes during the first hour, 26 amperes during the second and third hours, and I 4 amperes during the fourth hour.

Very slow charging is injurious. The charging current should never be less than about one fourth of the normal discharge rate of the cell (on an eight-hour basis).

The variation of the ampere-hour capacity with the rate of discharge depends greatly upon the thickness of the active material and the freedom of circulation and diffusion of the electrolyte. The values for the stationary batteries of the Electric Storage Battery Company are as follows: A cell that has Ioo amperehours capacity on an eight-hour discharge (I 2.5 ampere discharge rate) has a capacity of 87.5 ampere-hours on a five-hour discharge (I7.5 ampere discharge rate), 75 ampere-hours on a three-hour discharge ( 25 ampere discharge rate), and 50 amperehours on a one-hour discharge (50 ampere discharge rate). 
108. The use of storage batteries.*-Storage batteries are used for the one fundamental purpose of storing electrical energy at a given time and place in order that it may be used when and where it may be desired. The large first cost of storage cells and their rapid depreciation, which amounts to from 5 to Io per cent. per annum even when they are properly cared for, limits their commercial use to those cases in which the advantages of storage are very great.

Portable cells. - Storage cells which are intended to be carried about are generally made as light as possible by using thin grids and hard rubber containing vessels; and even then the weight is very great. Thus a storage battery designed for car lighting, and capable of operating thirty I6-candle-power incandescent lamps for 8 hours, weighs about 3,240 pounds. The necessity of recharging a battery promptly after it has been used is also a serious matter, and storage cells which are used in small sets for driving small motors and induction coils are almost never properly cared for, and therefore they last but a very short time.

Stationary cells. - Storage batteries are most extensively used in connection with central stations :

(a) For supplying the station output during the hours of small demand. In this case the battery is charged while the station is in operation, and discharged during the remainder of the day, thus obviating the expense of operating the station continuously.

(b) For equalizing a rapidly fluctuating station load. In this case provision is made for the battery to charge while the station load is below the average and to discharge while the station load is above the average. This is the most important use of large storage battery installations, and the cost of installing and maintaining the battery is set over against the saving in the first cost of the station and the saving in the cost of operating the station.

(c) As a reserve. In alternating-current generating stations a small direct-current generator is used to excite the field magnets

* A very complete discussion of the uses of storage batteries is given by Lamar Lyndon, in his Storage Battery Engineering. 
of the alternators, and it is desirable either to duplicate this direct-current generating machinery or install a storage battery so that the station may not be thrown out of service by a slight accident to a comparatively insignificant part of the station machinery.

Controlling Devices. - When a storage battery is used for operating motors, as in case of automobile batteries for example, no attempt is made to compensate for decrease of battery voltage during discharge. When, however, a battery is used for operating incandescent lamps, the decrease of battery voltage during discharge must be compensated so as to give a constant voltage between the lighting mains.

When a storage battery is used for equalizing a rapidly fluctuating station load, provision must be made for automatically causing the battery to discharge when the station load is large and to charge when the station load is small.

109. The use of the storage battery for supplying the station output during the hours of small demand. - When a storage battery is used for this purpose it is nearly always required to deliver current at constant voltage. A sufficient number of storage cells is used to give the required voltage when the battery is discharged and has I.8 volts per cell, and the controlling device is arranged to take up the excess of voltage when the battery voltage is higher than the desired value.

Control of voltage by rheostat. The current, $i$, delivered by the battery is made to flow through an adjustable resistance, $R$, Fig.

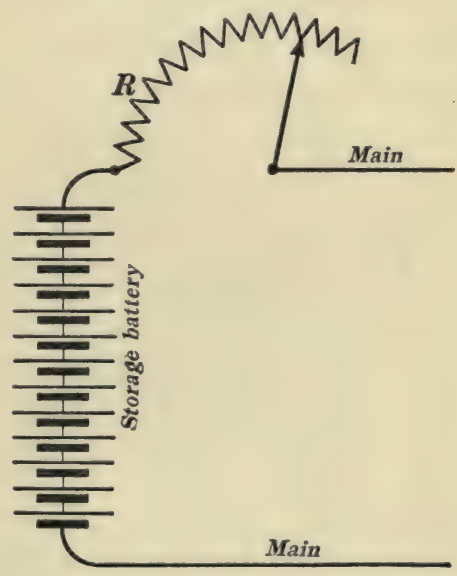

Fig. 146. I46, so that the excess of battery voltage may be used up as the voltage drop, $R i$, in the resistance. When the lamp load is con- 
stant this method of control is fairly satisfactory, for, in this case, the resistance has to be adjusted only as the battery voltage falls off. When the lamp load fluctuates, however, the rheostat requires constant attention, inasmuch as the voltage drop, $R i$, in the rheostat may change suddenly with a sudden change of load.

Control of voltage by counter-electromotive-force cells. When current flows through a low-resistance electrolytic cell consisting of plain lead plates in dilute sulphuric acid, the voltage drop through the cell varies from about 2. I to 2.4 volts according to the value of the current. The excess voltage of a discharging storage battery may be taken up by causing the current to flow through a number of such cells connected in series, the number being reduced as the battery voltage decreases. The advantage of this arrangement is that the voltage which is lost in these controlling cells does not vary greatly with the current. This method of voltage control is seldom used in practice. It has no advantage over the rheostat method when the load is constant, and the end-cell method is usually preferred when the load is variable.

Control of voltage by end-cells. This method of control will be explained by giving an actual example of a battery to be used for delivering current at I Io volts. The lowest permissible voltage at the end of the discharge is usually taken to be 1.8 volts per cell. Therefore the number of cells required to give a minimum of $\mathrm{I} I 0$ volts is $110 \div 1.8$, which is equal to $6 \mathrm{I}$. The highest voltage is about 2.15 volts per cell at the very beginning of the discharge (see Fig. I43), and 5 I cells are therefore required at the very beginning of the discharge to give I ro volts. Therefore, the entire battery being fully charged, 5 I cells are used at the beginning of the discharge, and as the voltage of the battery falls off the number of cells is increased, by connecting-in additional cells at one end of the set, until, when the battery reaches the limit of discharge, all of the 6 I cells are in service. Under these conditions it is evident that the end-cells, which are in service only a portion of the time during the delivery of current by 
the battery, are not completely discharged. Therefore, when the battery is recharged, the end-cells are placed in circuit at the start and cut out one by one when they become fully charged, as indicated, for example, by the copious evolution of gas.

An important detail in the carrying out of the end-cell method of voltage control is the design of the switch for connecting and disconnecting the end-cells without interrupting the delivery of current, and without momentarily short-circuiting the individual cells. The essential features of this end-cell switch are shown in Fig. 147. The terminals of the end-cells are brought out to a

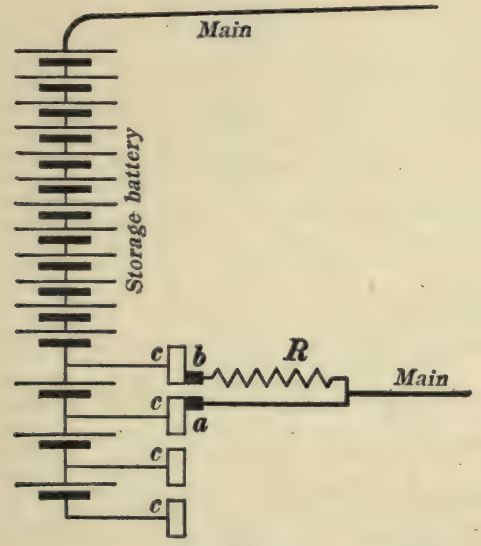

Fig. 147.

series of contact blocks, $c c c c$, and the movable contact consists of two pieces, $a$ and $b$, which together bridge across between the blocks, $c c$, when they are moved along and short-circuit a cell through a resistance, $R$, which is large enough to limit the shortcircuit current to about the value of the normal discharge current of the cells.

The shunt booster. - In the use of a storage battery as above described, a voltage greatly in excess of the normal station voltage is required in charging, especially when the battery approaches complete charge. Thus the 6 I cells, required to give i I o volts at 1.8 volts per cell, require about 2.5 volts each, or a total of 
I 52.5 volts, when they are nearly charged, see Fig. 143. This high voltage for charging is usually obtained by connecting the batteries to the station mains through a small shunt-wound gen-

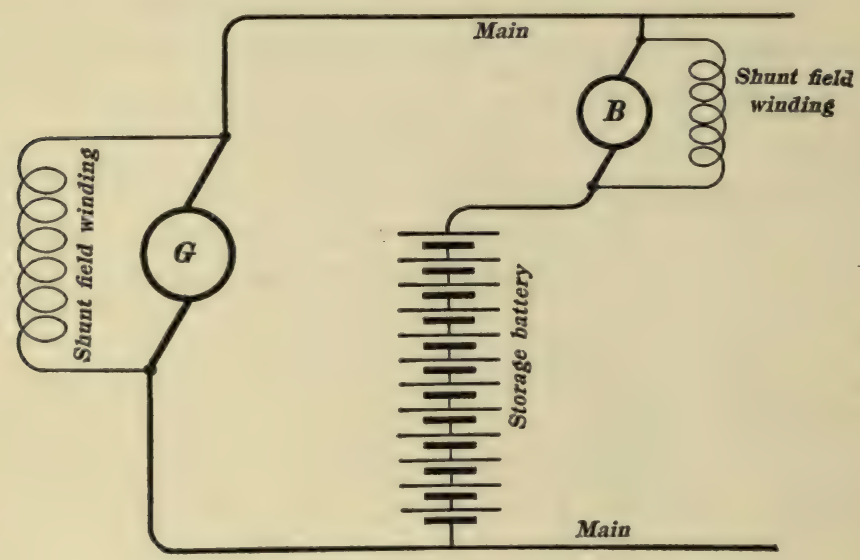

FIg. 148.

erator, which is called a booster, as shown in Fig. 148 , in which $G$ is the main generator of the station and $B$ is the booster.

110. Automatic boosters. - When the station load changes slowly, as is usually the case in an electric lighting station, there is ample time for an attendant to connect up a shunt booster and charge a storage battery when the station load is small, and to disconnect the booster and make the necessary arrangements for discharging the battery when the peak of the load comes on. When, however, the station load fluctuates rapidly and irregularly as is usually the case in an electric railway power station, hand control of the storage battery is impossible. In such cases automatic boosters must be used.

The floating battery. - The simplest arrangement for causing a storage battery to operate automatically and tend to equalize a station load, is that which is frequently employed in connection with long feeders over which a considerable drop of voltage takes place when a large current is delivered. This arrangement is shown in Fig. 149, in which $G$ is the main generator and $B a$ is 
the storage battery. Any great demand for current causes the voltage, $E$, to decrease, so that the battery can discharge, and when the demand for current is small the voltage, $E$, rises and the battery is charged. A battery connected as shown in Fig. I49

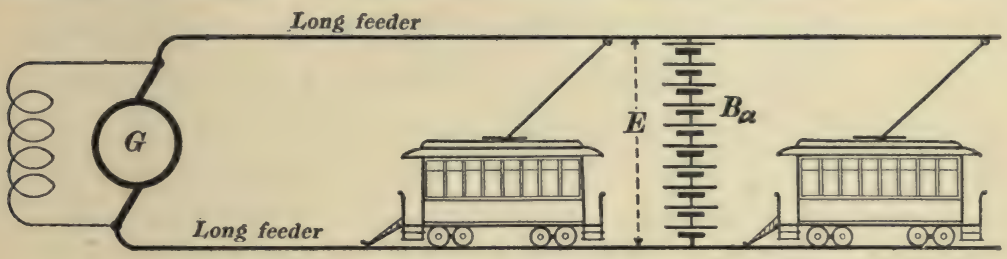

Fig. 149.

is called a floating battery. Such a floating battery cannot completely equalize the demand on the station, inasmuch as the rise and fall of the voltage, $E$, depends upon some decrease and increase of the current flowing through the long feeders.

The negative booster. - It frequently happens that a small generating plant in a modern office building supplies current for lighting and also for operating an elevator motor. The lamps require constant voltage and the motor operates most satisfactorily if provision is made for a great decrease in voltage when the motor starts and takes excessive current. In this case the voltage, $E_{1}$, at the lamps may be kept nearly constant, and a large delivery of current to the elevator motor may be made to cause a great decrease in the voltage, $E_{2}$, see Fig. I 50 , by interposing an auxiliary

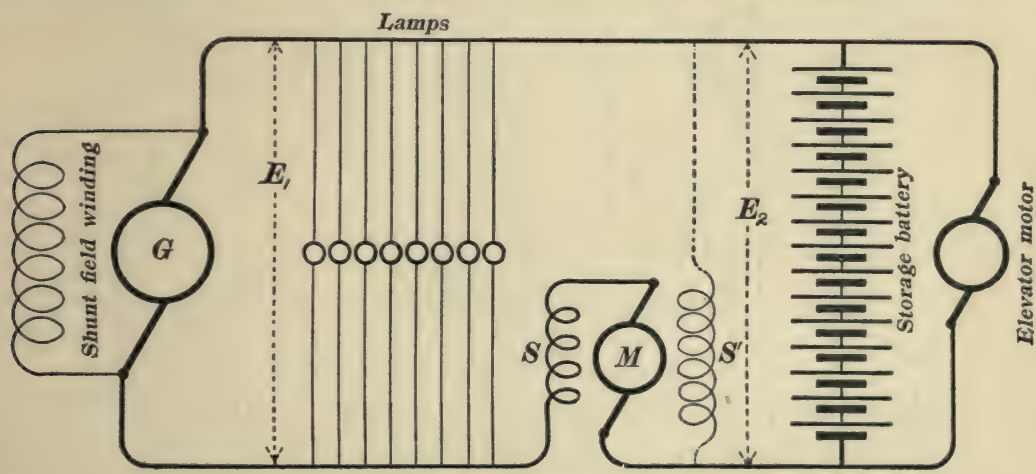

Fig. 150 . 
series motor (a negative booster), $M$, as shown. The power developed by $M$ is belted back to the main generator $G$. When the elevator motor takes but little current the voltage, $E_{2}$, is high and the battery is charged. When the elevator motor takes a large current, the current flowing in the series field coil, $S$, of the auxiliary motor, $M$, causes it to develop an increased counter electromotive force so that $E_{2}$ is decreased and the battery discharges. The motor, $M$, produces an effect which is equivalent to a voltage drop in a long pair of feeders, and the battery operates exactly like a floating battery.

The motor, $M$, keeps the output of the main generator, $G$, much more nearly constant if it is provided with two field windings, $S$ and $S^{\prime \prime}$, which oppose each other. In this case the windings, $S$ and $S^{\prime}$, may be made to balance each other when the elevator motor takes its average current. Then if the elevator motor takes less than its average current the coil, $S^{\prime}$, predominates, $M$ becomes a generator, increases $E_{2}$, and causes the battery to be charged; if the elevator motor takes more than its average current the coil,

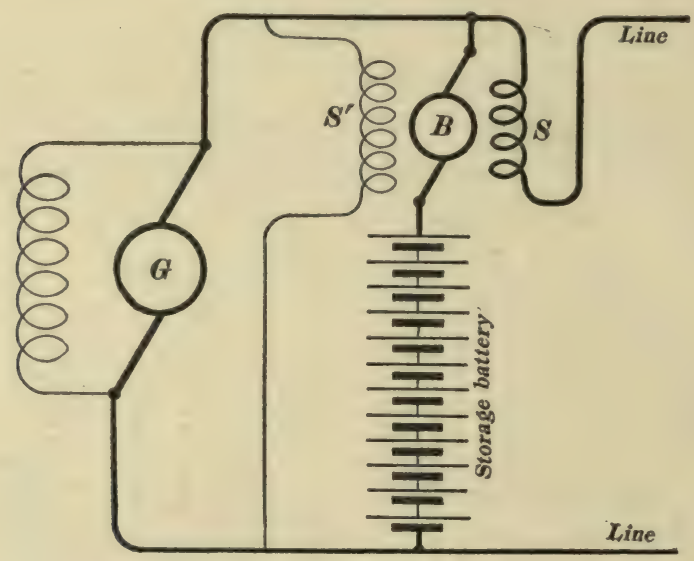

Fig. 151 .

$S$, predominates, $M$ becomes a motor, decreases $E_{2}$, and causes the battery to discharge. The use of the additional field winding, $S^{\prime}$, which opposes $S$, makes it possible to use many more turns 
of wire in the winding, $S$, so that a very small variation of current in $S$ will produce the variations of voltage required to control the storage battery.

The differential booster. - Fig. I 5 I shows an arrangement, due to Mailloux, in which a booster, $B$, is actuated by variations of line current. The booster has two opposing field windings, $S$ and $S^{\prime}$. When the demand for current is at its average value the windings, $S$ and $S^{\prime}$, balance each other, the small generator, $B$, develops no electromotive force, and the battery neither charges nor discharges. When the line current is excessive the winding, $S$, predominates, and the voltage of $B$ helps the battery to discharge; when, however, the line current is small the winding, $S^{\prime}$, predominates and the reversed voltage of $B$ helps the line voltage to charge the battery.

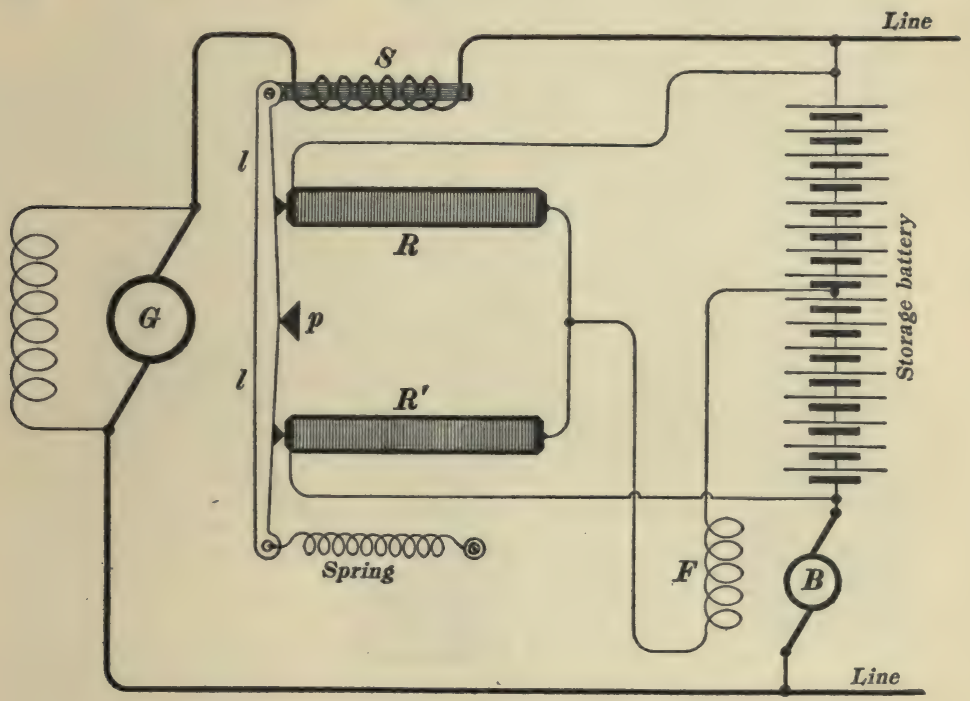

Fig. 152.

Booster with automatic carbon rheostat control. - Fig. I 52 shows a carbon rheostat, $R R^{\prime}$, connected across the terminals of the storage battery, and the field winding, $F$, of the booster, $B$, connected from the middle of the rheostat to the middle of the 
battery. A solenoid, $S$, pulls on an iron plunger which is attached to one end of the lever, $l l$. Two lugs on this lever press on the two piles of carbon plates, $R$ and $R^{\prime}$, which constitute the rheostat. The current in the field winding, $F$, is zero when the resistances, $R$ and $R^{\prime}$, of the piles of carbon plates are equal, the current through $F$ is in one direction when $R$ is greater than $R^{\prime}$, and in the other direction when $R$ is less than $R^{\prime}$. Any

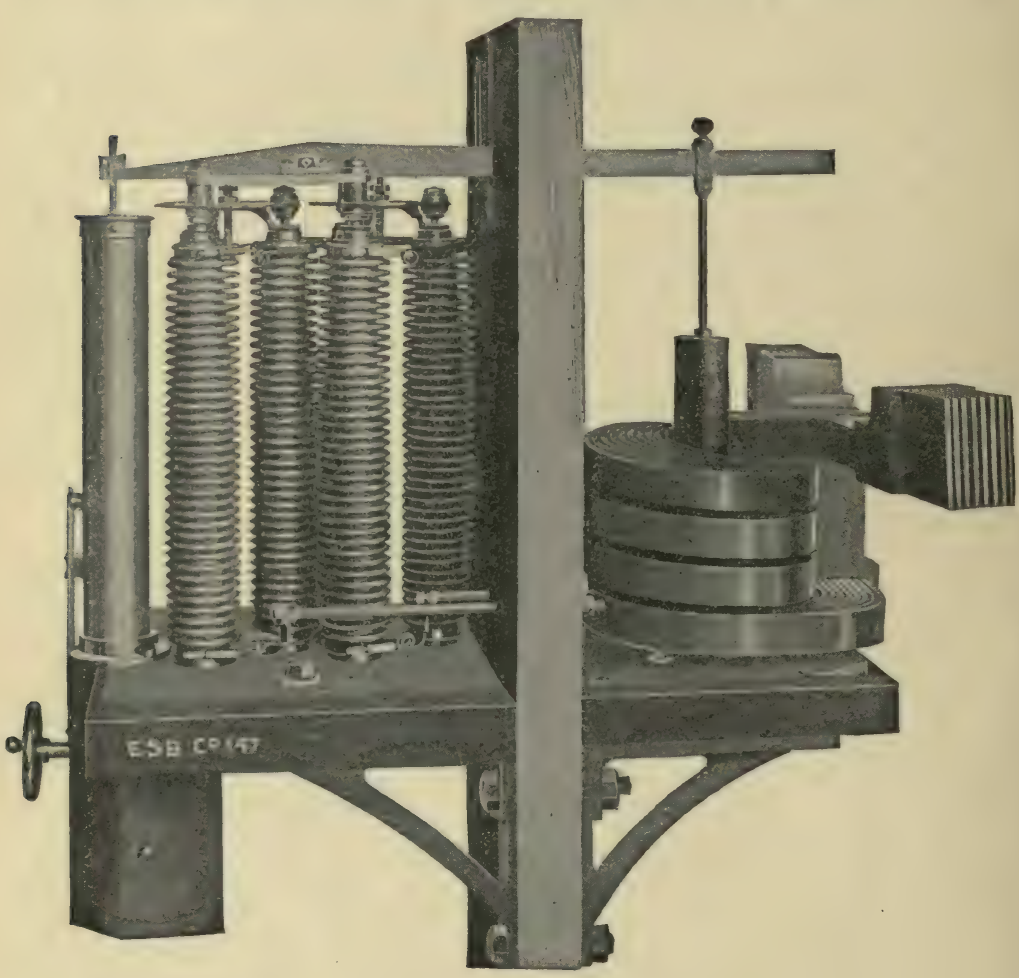

Fig. 153.

increase in the line current above its mean value produces a very slight increase of current in $S$ which increases the pull on the lever, reduces $R$ and increases $R^{\prime}$, and so excites the field of the booster as to cause the battery to discharge. In a similar manner a decrease of line current below its mean value causes the bat- 
tery to charge. In practice the current controlled by the rheostat, $R R^{\prime}$, excites the field magnet of a very small auxiliary generator which delivers current to the field winding of the booster. The loss of power in the rheostat, $R R^{\prime}$, is thus greatly reduced.

A general view of the automatic carbon rheostat, as manufactured by the Electric Storage Battery Company, is shown in Fig. I 53 .

Example showing the equalizing effect of an automatically regulated storage battery upon the generator load in a street railway plant. - The ordinates of the extremely irregular curve in Fig. I 54 represent during an interval of ten minutes the fluctuating demand for current at a typical railway power station. Without a storage battery the generators would have to meet this extremely irregular demand, varying from a minimum of about 180 amperes to a maximum of about 850 amperes. The ordinates of the slightly undulating dotted curve show the values of generator output when an adequate storage battery is installed. In this particular case the battery was regulated by a booster of which the field excitation was under the control of a carbon rheostat as shown in Fig. I 52. When the total load curve is above the dotted curve the battery discharges and when the total load curve is below the dotted curve the battery charges.

The general average of the generator load must be slightly greater than the general average of the station output inasmuch as some energy is lost in the battery, but the average generator load during a short period may be much greater or much less than the average station output during the period. Thus the average station output during the ten-minute run shown in Fig. I 54 was evidently greater than the average generator load during that time so that the battery was on the whole being discharged. Example of a storage battery and booster installation.* - A power station supplies current at a constant electromotive force

* An example of a large storage battery installation is described by Franklin E. Moore in the Street Railwuy Journal for Sept. 21, 1901, "The Application of Storage Batteries to the System of the Brooklyn Heights Railroad Company." 
264 ELEMENTS OF ELECTRICAL ENGINEERING.

of 500 volts to a street railway and the demand for current varies irregularly from zero to a maximum value of 400 amperes, having

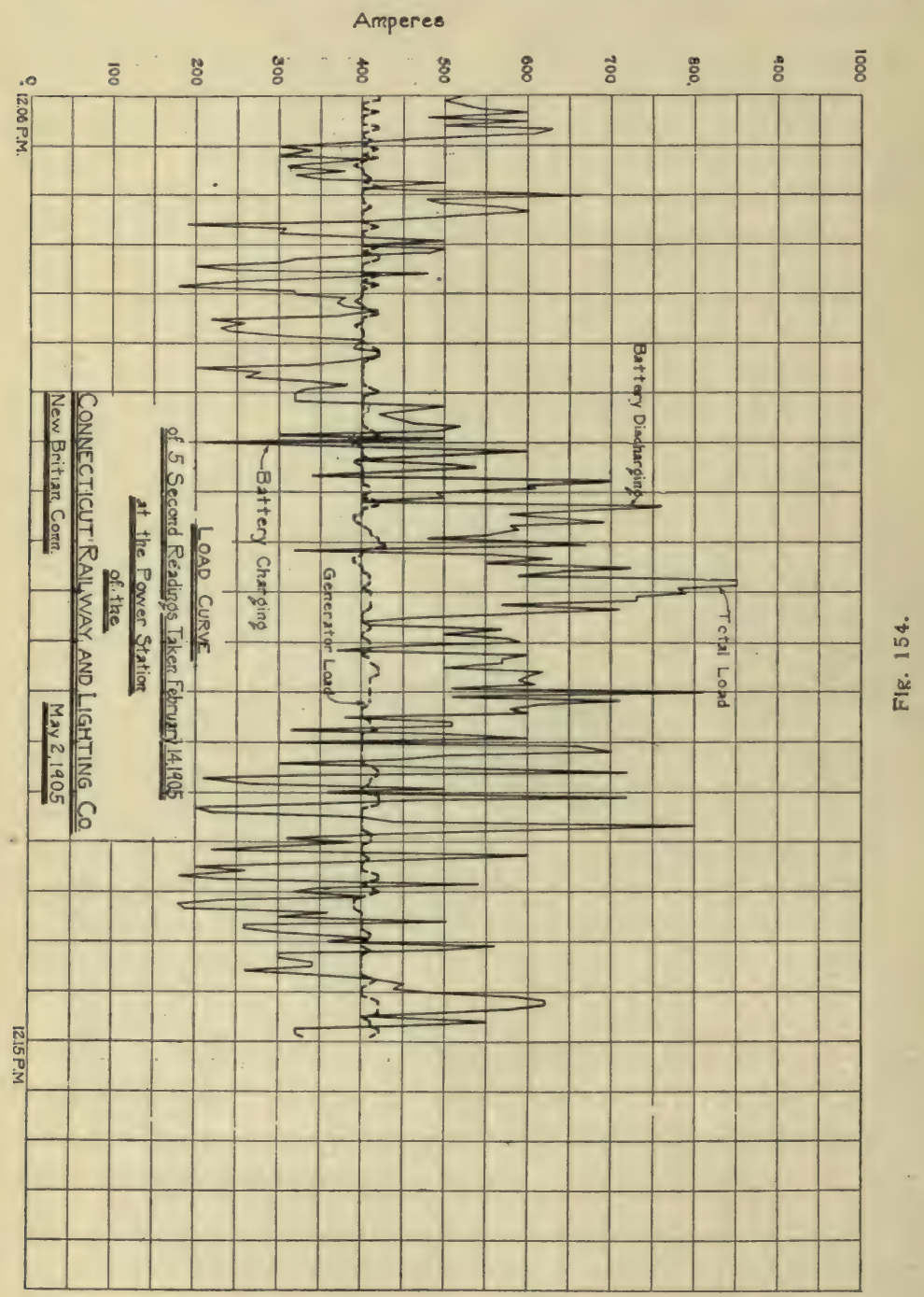

a mean value of 150 amperes. The boiler, engine and generator are designed to deliver a little more than the mean station load 
of 75 kilowatts, and the variations of load are equalized by a storage battery which has to deliver a maximum current of 250 amperes (= 400 amperes - I 50 amperes) on discharge, and absorb the entire generator output of 150 amperes when the station load is zero. On account of the greater values of current on discharge than on charge, the maximum duty of the booster is lessened by choosing the number of storage cells so as to have the mean voltage of the battery greater than the station voltage. The storage battery in fact consists of 234 cells, which require a maximum charging electromotive force of about 562 volts (2.4 volts per cell), and the battery is never discharged below 445 volts ( 1.9 volts per cell). The duty required of the booster varies between wide limits. The heaviest duty required for charging the battery comes when the battery is at nearly full charge and the demand for current zero; then the booster must develop 62 volts with 150 amperes of current flowing through its armature. The heaviest duty required for discharging the battery comes when the battery is nearly discharged and the demand for current a maximum; then the booster must develop 55 volts with 250 amperes in its armature. The booster would therefore be rated, say, as a 55-volt 250-ampere generator, inasmuch as the voltage of such a machine could be easily pushed up to 62 volts with an armature current of only i 50 amperes. This booster differs from an ordinary generator in having a very large space provided for its field windings so as to give room for the two opposing windings $S$ and $S^{\prime}$ of Fig. I $5 \mathrm{I}$. This booster may be driven by the same engine that drives the main generator, or, as is more usual, by a shunt motor which is supplied with current from the station bus bars. 


\section{CHAPTER IX.}

\section{ELECTRIC DISTRIBUTION AND WIRING.}

111. Series and parallel systems of distribution. - The constant-voltage method of electrical distribution is briefly discussed in Art. 4I and the system of connecting lamps and motors in parallel between the supply mains in the carrying out of the constant-voltage method is shown in Fig. 69. This is called the parallel system of distribution. The constant-current method of electrical distribution is also briefly discussed in Art. 4I, and the system of connecting lamps in series in the carrying out of the constant-current method is shown in Fig. 68. This is called the series system of distribution. The parallel system of distribution is used in the great majority of electric installations both for direct current and for alternating current. In the case of alternating-current distribution the use of transformers, and especially the employment of several phases of voltage and current, complicates the scheme of connections to some extent as described in the second volume of this text. The series system of distribution is generally used for street lighting in cities and in some cases for power transmission by series generators and motors.*

Combinations of series and parallel connections.

(a) Connection of series-groups in parallel. - When the voltage of supply in the constant-voltage method of distribution is greater than can be conveniently used for operating single lamps, the lamps are usually arranged in groups, each group consisting of a number of lamps

* Thury's System of Direct-current Power Transmission. See London Electrician. Vol. 38, pages 683-687, March 19, 1897; Zeitschrift für Electrotechnik (Vienna), Vol. 16́, pages 5-10, 1898; Bulletin de la Société International des Electriciens, Vol. 17, pages 9-93, January, I900; and Electrical Review (New York), January, I90I. 
connected in series, and these groups of lamps are connected in parallel with each other across the mains. This arrangement is exemplified in the lighting of electric cars where the standard supply-voltage is 550 volts and where the lamps for lighting the cars are usually I I O-volt lamps connected in series-groups of five lamps each, these groups being connected in parallel with each other between the trolley and the rail. A similar arrangement is employed for the very low voltage osmium lamps* which are now coming into extensive use abroad. Thus three 37-volt osmium lamps are connected in a series-group and such groups are connected in parallel with each other across standard I IO-volt mains.

(b) The connection of parallel-groups of lamps in series. - In the early days of electric lighting the constant-current method of supplying arc lamps for street lighting was quite common. Many towns which were provided with this series-system of distribution were not provided with any other means for supplying incandescent lamps, and the only feasible method for operating incandescent lamps was to connect a group of such lamps in parallel and to connect this group in series in the arc-lamp circuit. This arrangement is now seldom or never used.

Advantages and disadvantages of the connection of series-groups of lamps in parallel. - In order to clearly understand the advantages of grouping electric lamps in series it is necessary to keep in mind the fact that in the earlier days of electric-lighting electric lamps could not be made satisfactorily for higher voltages than about IOO or I IO volts; and that, at the present time, the higher-voltage carbon-filament lamps are less efficient or shorter lived than low-voltage lamps because of the excessively small size of the filament of a high-voltage lamp. This may be summed up in the general statement that the electric lamp is essentially a low-voltage device, so that if one wishes to use a high voltage in order to reduce the amount of copper required for a given installation the lamps must be arranged in series-groups. The saving

* See London Electrician, Vol. 55, p. 141, May 12, 1905. 
of copper by using a high voltage * is evident when we consider that the delivery of a certain amount of power can be accomplished by half as much current when the voltage is doubled, that the doubling of the voltage permits in general a double voltage-drop in the line wires, and that this double voltage-drop with half as much current means that the wires may be one quarter as heavy as before.

The disadvantage of the series-grouping of lamps is that each group must be turned off and on as a unit, unless a special device is used to connect an equivalent resistance in place of a lamp which is to be turned off.

The grouping of incandescent lamps in series on highvoltage supply mains is exemplified in the lighting of streets by incandescent lamps from a I, I OO- or 2,200-volt alternating-current supply. In this case the lamps are arranged in series-groups of IO or 20 lamps each and provision is made for automatically inserting an equivalent resistance in place of a lamp that happens to break down.

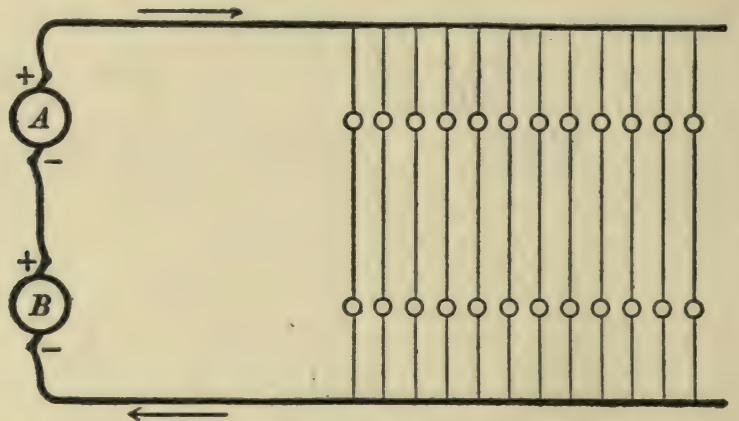

Fig. 155.

112. The Edison three-wire system of distribution. - Fig. I 55 shows a number of I IO-volt lamps connected, in seriesgroups of two lamps each, to 220-volt mains, and supplied with current from two I IO-volt generators connected in series; and Fig. I 56 shows an arrangement which is the same as Fig. I 55 ,

* See equation (43), Art. I17. 
except that a third main, $C D$, is added as shown. The addition of this third main makes it possible to turn single lamps on and off, and if the lamps in use by each consumer are judiciously placed, some in the $A$-set and some in the $B$-set, there will always be nearly the same number of lamps in each set, even when entire freedom is allowed in the turning off and on of single lamps, so that the middle main need never carry much current. In fact the current in the middle main will be a small incoming current when the $A$-set contains a few more lamps than the $B$-set, or a small outgoing current when the $B$-set contains a few more lamps than the $A$-set.

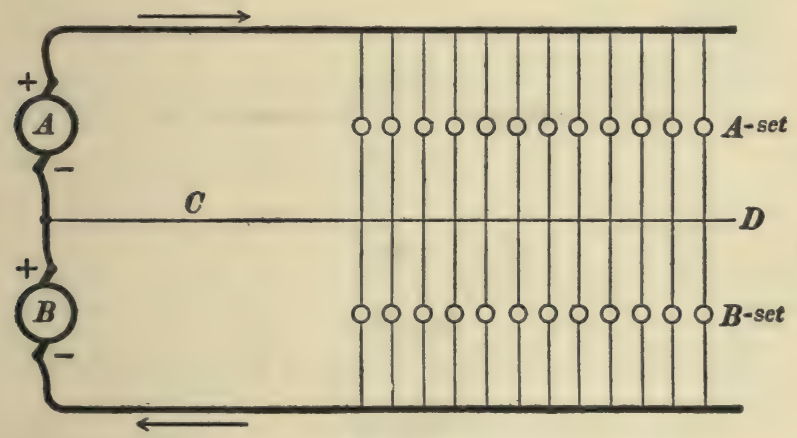

Fig. 156.

The arrangement shown in Fig. I 56 is called the Edison threewire system of distribution. In practice the middle main is usually made of the same size wire as each outside main, and each outside main need be only one quarter as heavy as would be required to supply the same number of lamps in the simple parallel system using I I volts ; therefore to supply a given number of lamps in the Edison three-wire system requires only three-eighths as much copper as would be required in the simple system with the same per cent. drop of voltage.

When the number of lamps in the $A$-set in Fig. I 56 is different from the number of similar lamps in the $B$-set the system is said to be unbalanced. When the system is unbalanced the middle main carries current, and the effect of the voltage-drop in the 
middle main is to tend to increase the voltage which acts on one set of lamps and to decrease the voltage which acts on the other set of lamps. These voltage relations are clearly represented for a particular case in Fig. I 57. This figure shows the state of

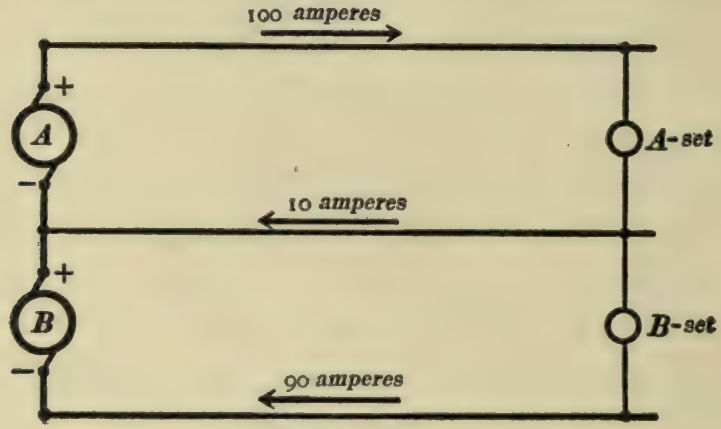

Fig. $157 a$.

affairs when the $A$-set of lamps takes Ioo amperes, and the $B$-set. 90 amperes, each main having $\frac{1}{20}$-ohm resistance, and the lamps being supposed to be bunched at the ends of the mains for the sake of simplicity. Electric current may always be considered

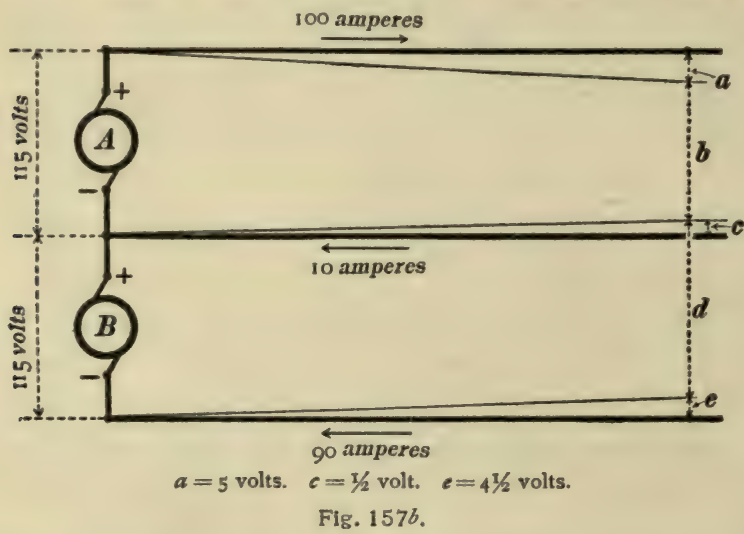

as flowing down hill so that the electric level (or potential) is to be thought of as falling off along each main in the direction of the current, as indicated by the fine inclined lines in Fig. $157 b$, 
and the distances between these inclined lines at the ends of the mains represent the voltages acting on the two sets of lamps. Thus the voltage $b$ acting on the $A$-set is I I 5 volts -5 volts $-1 / 2$ volt $=109.5$ volts, and the voltage $d$ acting on the $B$-set is I I 5 volts $-4 \mathrm{~T} / 2$ volts $+\mathrm{I} / 2$ volt $=$ I I I volts.

113. Special three-wire generators and three-wire balancers. The use of two generators as indicated in Figs. I 56 and 157 involves an added expense for machinery in the generating station, and the extensive use of the Edison three-wire system has given rise to several special types of three-wire generators which can be used singly for supplying a three-wire system, and to several types of an auxiliary device, called a three-wire balancer, which enables a single 220-volt generator of the ordinary type to supply an Edison three-wire system.

(a) The split-pole three-wire generator. - Fig. I $58 a$ shows a two-pole 220-volt generator with each pole, $N N^{\prime}$ and $S S^{\prime}$, sepa-

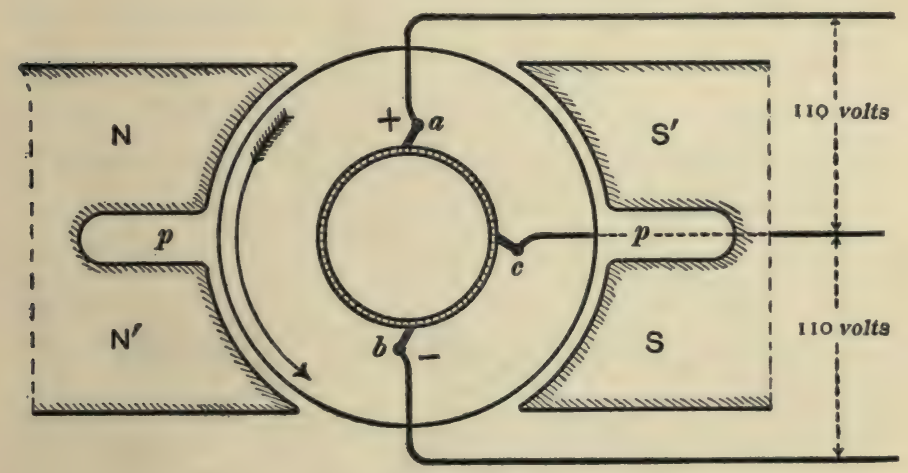

Fig. $158 a$.

rated into two parts by deep slots, $p p$. The main brushes, $a$ and $b$, collect current at 220 volts, an auxiliary brush, $c$, makes contact with the commutator midway between $a$ and $b$, and the middle main of the three-wire system is connected to this auxiliary brush, c. The auxiliary brush, $c$, cannot be used on an ordinary solid pole generator because the sections of the armature winding as 
they are short-circuited by the auxiliary brush, $c$, would have excessive short-circuit currents produced in them by the large induced electromotive forces in the sections. The sections must, therefore, be out of the inducing field in the neutral spaces, $p p$, when they are short-circuited by the auxiliary brush. When the generator shown in Fig. I $58 a$ is heavily loaded the armature current tends to crowd the magnetic flux into the portions $N^{\prime}$ and $S^{\prime}$ of the pole pieces thus causing the voltage between brushes, $a$ and $c$, to become greater and the voltage between brushes, $b$ and $c$, to become less. This tendency must be to some extent counteracted, and it must be possible for the station attendant to control the voltages, $a$ to $c$ and $c$ to $b$, separately, in a practicable three-wire generator. This is accomplished in the three-wire generator of Dettmar* as follows : A four-pole field structure is excited so as to be essentially' a two-pole field magnet acting on

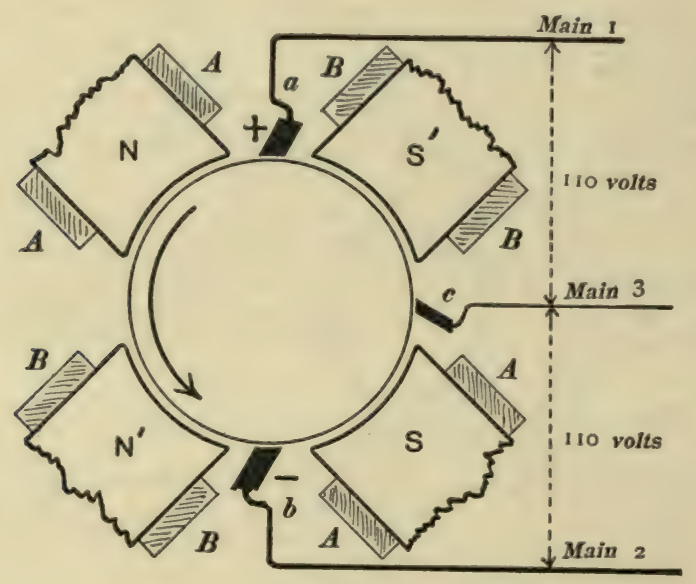

Fig. $158 b$.

an armature properly wound for a two-pole field as shown in Fig. I $58 b$. The magnetic flux out of $N$ (and into $S$ ), Fig. I $58 b$, produces the voltage, $b$ to $c$, and this flux is produced by the exciting coils, $A A$ and $A A$, the current in which can be controlled by

* Electrotechnische Zeitschrift, Vol. 18, pp. 55 and 230, 1897. 
a field rheostat. Similarly the magnetic flux out of $N^{\prime}$ (and into $S^{\prime}$ ) produces the voltage, $a$ to $c$, and this flux is produced by the exciting coils, $B B$ and $B B$, the current in which can be controlled by a second field rheostat.

The tendency of the armature current to increase the flux out of $N^{\prime}$ (and into $S^{\prime}$ ) and to decrease the flux out of $N$ (and into $S$ ) may be to some extent counteracted by connecting the field coils, $A A$ and $A A$, between brushes, $a$ and $b$, and connecting the field coils, $B B$ and $B B$, between brushes, $c$ and $b$. The best method, however, for counteracting this tendency is to place a field winding in series with either of the outside mains, I or 2, so that it acts to increase the flux out of $N$ (and into $S$ ) and to decrease the flux out of $N^{\prime}$ (and into $S^{\prime}$ ).

(b) The double-current generator. - The synchronous converter, or rotary converter as it is sometimes called, is an ordinary directcurrent dynamo with the addition of two or more collector rings tapped into equidistant points of the armature winding. When such a machine is driven by mechanical power it can supply direct current from the brushes that rub on the commutator, or alternating current from the brushes that rub on the collector rings, or it can supply direct current and alternating current simultaneously. Therefore such a machine is called a double-current generator. This machine is fully described in the second volume of this text.

An arrangement, due to Dobrowolsky, for using a doublecurrent generator for supplying direct current to an Edison threewire system is also described in the second volume of this text.

(c) The use of rheostats for balancing a three-wire system. - If a three-wire system is kept exactly balanced, an ordinary 220volt generator having two brushes can be used to supply the system. When such a generator is used, approximate balance is maintained by arranging the lights in and near the station in groups which may be transferred by the station attendant from the $A$-set to the $B$-set or vice versa, at will, by throwing switches on the main switch-board, and the approximate balance so ob- 
tained is made exact by the use of a pair of adjustable resistances connected from the middle main to the outside mains.

(d) The motor-generator balancer. - The two generators shown in Fig. I 56 may be replaced by a single 220-volt generator of the ordinary type and the current that comes into or flows out of the station on the middle main may be taken care of by a small motor-generator, consisting of two small shunt-wound dynamos, $P$ and $Q$, with their armatures mounted on one shaft and connected electrically, as shown in Fig. 159. Consider the

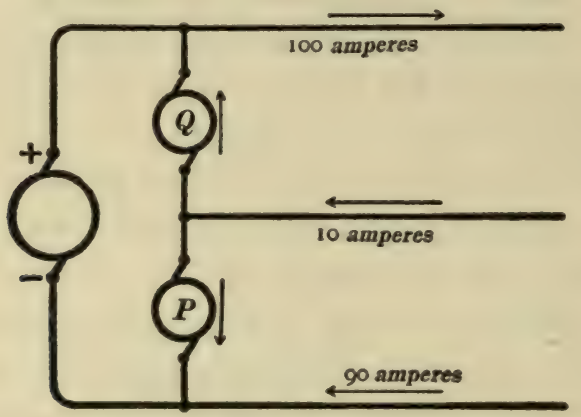

Fig. 159.

particular case in which the upper main carries an outward current of 100 amperes, the middle main a return current of 10 amperes, and the lower main a return current of 90 amperes as shown in Fig. I 59. Part of the current which enters the station on the middle main will then flow down hill, as it were, through $P$ to the negative terminal of the large generator, thus causing $P$ to to act as a motor and deliver mechanical power to $Q$ which will act as a generator and pump the remainder of the current in the middle main up hill, as it were, to the positive terminal of the large generator. When an outward current flows in the middle main, $Q$ is operated as a motor by current that flows down hill from the positive terminal of the large generator, and $P$ operates as a generator and pumps current up hill from the negative terminal of the large generator. In order to keep the potential of the middle main at the proper value so as to divide the electro- 
motive force of the large generator into two equal parts, $P$ and $Q$ must have carefully adjusted compound field windings, or the field rheostat of $P$ or $Q$ must be repeatedly adjusted as the current in the middle main changes in value.

In the use of a motor-generator balancer it is desirable to keep the system approximately balanced as explained above under (c) and thus greatly reduce the duty of the motor-generator. By careful grouping of the consumers' lamps and motors, the unbalancing of a three-wire system may, in practice, be kept within eight or ten per cent., so that the rated output capacity of each of the dynamos of a motor-generator balancer need be only eight or ten per cent. of that of the main generator.

\section{Factors which determine the size of wires in practice. -} There are five conditions which should be considered in selecting the size of wires for distributing electric current, namely: $(a)$ The wire must have sufficient strength to withstand the mechanical stresses to which it may be subjected. This condition applies especially to wires strung on poles. (b) The wire must be large enough to carry the prescribed current without becoming so hot as to damage its insulation or to ignite adjacent inflammable materials. This condition applies especially to wires in a building. (c) The wire must be large enough to keep the variations of voltage at the lamps, or other receiving units, within certain limits. This variation of voltage is briefly discussed on page 195 , from the point of view of feeder control, and it is more fully discussed in Art. I 17. This condition, $c$, applies only to the distributing wires of a constant-voltage system. (d) The size of a wire should be chosen so as to give an economic balance between the cost of the copper and the cost of the power lost in the wire. This condition is discussed in Art. I 2O. (e) In extreme cases the size of a wire may be determined by a consideration of the electric strength of the air or other insulating substance surrounding the wire, inasmuch as the strength of an insulating medium to withstand the electric stress between two wires due to a given voltage 
between them, depends in part upon the size and shape of the wires as explained in Art. I $2 \mathbf{I}$.

Whenever, in a given case, any one of these conditions demands a larger wire than would be required by any of the other conditions, the larger wire should be used. Frequently an engineer is guided by one only of the above conditions in laying out the preliminary plans for a distributing system. When this is the case the preliminary plans should be examined carefully to see that all of the conditions are satisfied before the plans are finally adopted.

\section{Mechanical stresses in aerial wires and their supports.*} Stresses in the supports. - The stresses in the insulator pins, cross-arms, and poles are: (a) The stresses due to the weight of the wire plus the weight of an occasional coating of ice; this weight is to be considered as resting directly upon the insulators and constituting a force acting vertically downwards. $t$ (b) The stresses due to the unbalanced tensions $\ddagger$ of the wire on the opposite sides of an insulator. The tensions of the wire on the opposite sides of an insulator are in nearly every case sensibly equal in value and unbalancing occurs only where the wire terminates or changes its direction. In the case of a straight pole-line on a slope the tension of the wire is generally greater on the down-hill side of the pole, but the unbalanced force is in this case a force acting vertically downwards, that is, a given insulator supports a large part of the weight of the lower span of wire and a correspondingly small part of the weight of the upper span of wire. (c) Stresses due to wind pressure.

(I) The weight of wire and ice produces, in the poles and pins, stresses of simple compression, which stresses may nearly always be neglected, inasmuch as poles and

* A discussion of the details of pole-line construction is beyond the scope of this text. Information concerning these details may be found in "Electrical Transmission of Energy," A. V. Abbott, 1905 edition, Chapter III.

$\dagger$ This force is the sum of the vertical components of the tension of the wire on the two sides of an insulator.

$\ddagger$ Horizontal components of the tensions, inasmuch as vertical components are considered under $(a)$. 
pins which are strong enough to withstand the bending stresses to which they are subjected are not perceptibly affected by these slight stresses of compression.

The weight of wire and ice produces a bending stress in the cross-arms, and the breadth, $b$, and depth, $d$, of the cross-arms must be sufficient to sustain this bending stress, the length of the cross-arms being determined by the number of wires and their required distance apart. The simplest case is that shown in Fig 160, which

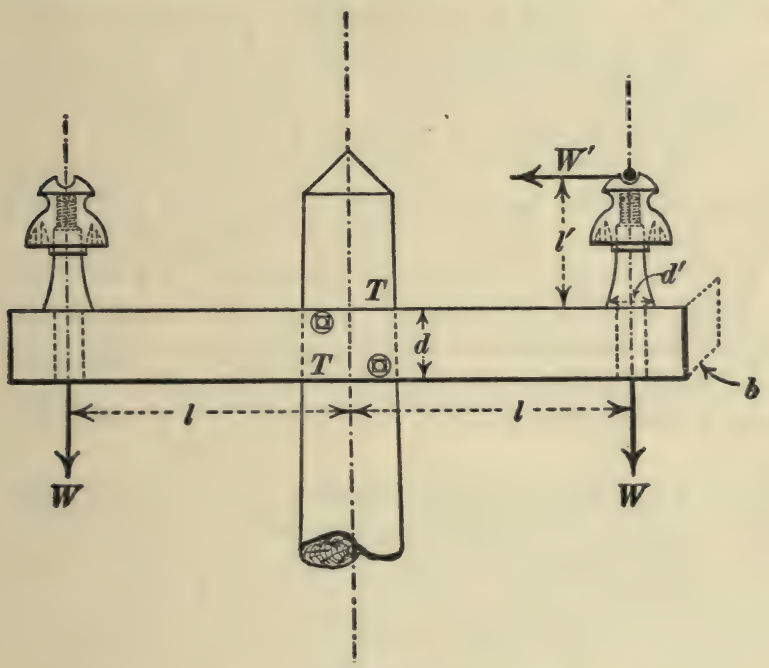

Fig. 160.

shows a cross-arm carrying two wires. In this case the dimensions, $b, d$, and $l$, as shown in the figure must satisfy the equation :

$$
S=\frac{6 W l}{b d^{2}}
$$

in which $S$ is the permissible fiber stress of the cross-arm material in pounds per square inch at the points, TT, Fig. 160 , and $W$ is the total weight in pounds resting on one pin. The dimensions, $b, d$, and $l$, are expressed in inches.

A coating of ice one-eighth of an inch thick is seldom exceeded, and it is cheaper to repair the line after an excessively severe sleet storm than it is to make it strong enough to sustain much more than one-eighth of an inch of ice on the wires.

The permissible values of $S$ may be taken from the table of tensile strengths of timber.

(2) The stresses due to unbalanced tensions are the most important stresses to be considered in pins and poles. Having given the value of the tension and the angle turned at a corner, the side force, $W^{\prime}$, Fig. 160 , is easily determined, and the dimensions, $l^{\prime}$ and $d^{\prime}$ (diameter of pin at base), Fig. I60, must satisfy the equation :

$$
S^{\prime}=\frac{32 W^{\prime} l^{\prime}}{\pi d^{\prime 3}}
$$


in which $S^{\prime}$ is the maximum permissible fiber stress in pounds per square inch, $W^{\prime}$ is the resultant horizontal force in pounds acting on the insulator, and $l^{\prime}$ and $d^{\prime}$ are expressed in inches.

The cross-arms on a corner pole are usually set so as to be parallel to the resultant force due to wire tensions, and hence, except at the end of a line, this resultant force does not produce a bending stress in the cross-arms.

The unbalanced tensions of the wires produce a bending stress in the poles; and the diameter, $d^{\prime}$, of the pole at the ground and the height, $l^{\prime \prime}$, of the pole, both in inches, must satisfy equation $\left(3^{8}\right)$, using for $W^{\prime}$ the resultant horizontal force due to all of the wires. In most cases a corner pole is guyed or braced so that the bending stress in the pole is to a great extent eliminated.

(3) Stresses due to wind pressure vary with the direction as well as the velocity of the wind. When the wind blows parallel to the line its effect is slight because the wires are parallel to the wind. It is considered sufficient in practice to provide the necessary strength to withstand a side wind giving a maximum pressure of from 20 to 30 pounds per square foot of surface, according to the degree of exposure of the line. In calculating the force of a side wind on a cylinder like a pole or wire, the effective exposed area is taken as two thirds of the product of the diameter of the cylinder times its length.

The effect of a side wind is to produce bending stresses in the insulator pins and in the poles, and the dimensions of the pin in inches, as shown in Fig. 160, must satisfy equation $\left(3^{8}\right)$, where $W^{\prime}$ is the total force of the wind on the wire in pounds, and $S^{\prime}$ is the maximum permissible fiber stress in pounds per square inch. Also the height of $l^{\prime \prime}$ of the pole and its diameter, $d^{\prime}$, at the ground, both in inches, must satisfy equation $\left(3^{8}\right)$, in which case $W^{\prime}$ is the force of the wind on all the wires plus about half or two-thirds of the force of the wind upon the pole and crossarms.

In estimating the stresses on pin, cross-arm and pole due to weight of wire and ice, or the stresses due to wind pressure on the wires, a length of wire equal to the distance between adjacent poles must be assumed to be supported by each insulator.

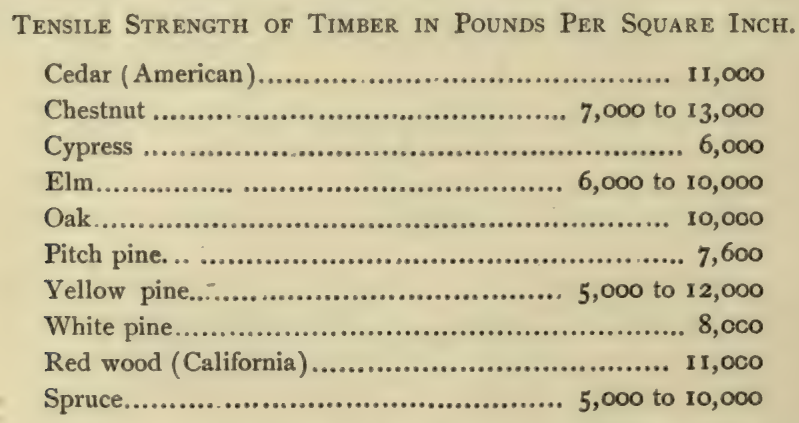

The usual factor of safety being 4 to 6 , the permissible fiber stress in pounds per square inch is from one-sixth to one-fourth of the values given in this table. 
Stresses in the wire. - In stringing a wire on poles two things in particular should be provided for, namely, $(a)$ an approximate equality of wire tension on the two sides of each insulator, and (b) a certain maximum tension in the wire when it is shortened by the coldest winter weather.

The first condition is desirable not only because it relieves the pins, cross-arms, and poles from unnecessary stress, but also because it is difficult to tie a line wire to an insulator so that it cannot slip lengthwise through the tie, unless the line wire is bent which it should not be if it can be avoided. The horizontal components of the wire tension can always be made equal on the two sides of an insulator; but in the case of a pole line on a grade the vertical component of the wire tension will be somewhat greater on the down-hill side of an insulator when the horizontal components are equal.

The second condition is explained in the following discussion.

Pole line on a level. - The calculation of the tension in a span of wire in terms of length of span, vertical sag at the center of the span, and weight of the wire, or the calculation of the sag corresponding to a prescribed tension, is based upon the equation to the curve formed by the wire. When the sag is a small fraction of the length of the span, say one twentieth or less, the curve formed by the wire is sensibly a parabola and the working formulæ are:

and

$$
T=\frac{l^{2} w}{8 h}
$$

$$
s=l+\frac{8 h^{2}}{3^{l}}
$$

in which $T$ is the tension of the wire in pounds, $l$ is the length of the span in feet, $h$ is the sag at the center of the span as shown in Fig. 16r, $s$ is the length in feet of the wire in a span, and $w$ is the weight of the wire in pounds per foot. Equation (39) gives the tension of the wire at the center of the span. The tension at the ends of the span is wh pounds greater than at the center; but this difference amounts to only 2 per cent when the sag is one twentieth of the length of the span, and it is always negligible. The important use of equation (40) is in making allowance for the effects of changes of temperature.

Equation (39) when solved for $l$ gives :

$$
l=\sqrt{\frac{8 \hbar T^{y}}{w}}
$$

where $T^{\prime}$ represents the maximum safe tension of the wire in pounds, which is equal to the breaking tension $T_{b}$ in pounds divided by the factor of safety. See following 
tables. From this equation it is evident that the poles may be spaced farther and farther apart the greater the value of $T^{\prime}$, the greater the permissible sag, and the less the weight of the wire; but it is to be noted that the ratio $T^{\prime} / w$ is independent of the size of the wire, inasmuch as $T^{\prime \prime}$ and $w$ are both proportional to the sectional area of the wire.

The spacing of the poles is usually chosen tentatively as the first step in the design of a pole line. When a great deal depends upon the permanence of a line, as in a transmission line supplying power to many customers, the poles are placed close together in order to make the line substantial and in order that the sag may be small enough to avoid the possibility of the wires swaying into contact. Close spacing is especially necessary in the case of heavy wires so as to distribute the weight of the heavy wire over a large number of insulators, the insulator being one of the weakest elements in the construction. Poles are usually spaced as follows on straight-pole lines: (a) Heavy power transmission lines about 80 feet, which is the spacing on the Niagara-Buffalo transmission line; $(b)$ Ordinary electric-lighting circuits in city or suburban districts, from 100 to 125 feet; $(c)$ Telegraph and telephone lines 125 to 150 feet. In every case the poles should be placed near together where the pole line follows a curve, thus making the line turn a very obtuse corner at each pole, in order to avoid excessive stresses in the supporting structure due to unbalanced tensions of the wire. Furthermore pole spacing is often determined by surrounding local conditions such as the presence of obstacles or the recurrence of cross-streets in cities.

The amount of sag in a span of line wire should be small in order to prevent the swaying of the wire by the wind. This swaying is objectionable because it tends to break the wire where it is fastened to the insulators and because it is likely to bring adjacent wires into contact. Once the spacing of the poles is chosen, the minimum permissible sag is determined as explained in the next paragraph; although the amount of sag that may be allowed has a great deal to do with the choice of the pole spacing. Very long spans, * such as spans across rivers, have a sag equal to one twentieth or one thirtieth of the span. In ordinary pole-lines the sag seldom exceeds one one-hundred-and-fiftieth of the length of span, in coldest weather.

Effects of temperature. - Wires are usually strung on poles during warm weather, the wire grows shorter as the temperature falls, and the tension of the wire is therefore greatly increased during cold winter weather. Hence, it is important to string a wire with sufficient sag (and a correspondingly low tension) so that the coldest weather may not increase the tension of the wire beyond the safe value, $T^{\prime}$. Knowing the temperature, $t$, of the wire when it is strung, and the lowest winter temperature, $t^{\prime}$, the calculation of the necessary sag, $h$, and tension, $T$, at temperature, $t$, is carried out as follows: Take the values of $T^{\prime}\left(=T_{b} / n\right)$ and $w$ from the following tables, and from these, together with the chosen distance, $l$, between poles, calculate the winter sag, $h^{\prime}$, using equation (39), and calculate the corresponding length of wire, $s^{\prime}$, in a

* "Long spans for transmission lines," by F. O. Blackwell, Trans. American Institute of Electrical Engineers, June, 1904.

"Conductors for long spans," by F. O. Blackwell, Trans. International Electrical Congress, Vol. 2, St. Louis, 1904. 
span using equation (40). Then calculate the length of the wire at summer temperature, $t$, by the equation

$$
s=s^{\prime}\left[\mathrm{I}+\beta\left(t-t^{\prime}\right)\right]
$$

in which $\beta$ is the coefficient of linear expansion of the wire as given in the following tables. From the value of $s$, so calculated, the value of the sag, $h$, at temperature, $t$, may be calculated from equation (40), and then finally the tension, $T$, at summer temperature, $t$, may be calculated from equation (39).

It is to be noted that as a line wire cools and shortens, its tension increases, so that its thermal contraction is accompanied by an elastic elongation due to the increase of tension; but this effect is generally neglected in practical line calculations, inasmuch as the error is always on the safe side, that is, the actual winter tension is less than that anticipated in the calculations.

Pole line on a grade. - It is usual to make the horizontal component of the tension of the wire the same in value all along a pole line on a grade, so that the actual tension of the wire is slightly greater on the down-hill side than on the up-hill side of each pole. The problem of determining the sag corresponding to a given horizontal tension, and the problem of allawing for the effects of temperature are treated in the same way as in case of a pole line on a level except that the following equations are used instead of equations (39) and (40):

$$
\begin{gathered}
T=\frac{l^{2} H z w}{2 d^{2}}\left(\mathrm{I}-\sqrt{\mathrm{I}-\frac{d}{H}}\right)^{2} \\
s=l+\frac{2 H d}{3^{l}\left(\mathrm{I}-\sqrt{\mathrm{I}-\frac{d}{H}}\right)}+\frac{2(H-d)^{2} d}{3 l d+3 l H\left(\mathrm{I}-\sqrt{\mathrm{r}-\frac{d}{H}}\right)}
\end{gathered}
$$

in which $T, l, w$, and $s$ represent the same quantities as in equations (39) and (40), $d$ is the difference in level between the ends of the span, and $H$ is the sag of the wire below the upper end of the span, as shown in Fig. 162.

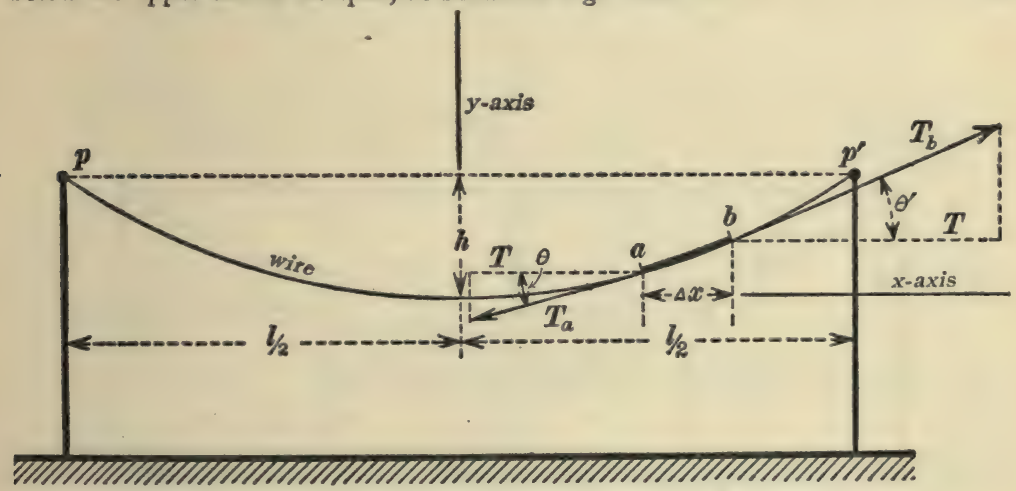

Fig. 161.

Derivation of equations (39) to (42). - Consider a wire, Fig. 161, suspended between two points, $p$ and $p^{\prime}$. If the wire is nowhere greatly inclined the actual 
length of any element, $a b$, of the wire is very nearly equal to the horizontal projection, $d x$, of the element. Therefore the weight of the element is very nearly equal to $w \cdot d x, w$ being the weight of the wire per unit length. Furthermore, the horizontal component of the tension of the wire has necessarily the same value, $T$, all along the span of wire.

Consider the element, $a b$, of the wire of which the coordinates of the end, $a$, are $x$ and $y$, and the coördinates of the end, $b$, are $x+d x$ and $y+d y$. Let $d y \mid d x$ be the value of the first differential coefficient of $y$ at the end, $a$, then $d y\left|d x+a^{2} y\right| d x^{2} \cdot d x$ is its value at the end, $b$. The force, $T_{a}$, pulling at the end, $a$, of the element is the tension of the wire at $a$, its horizontal component is $T$, and its component vertically downwards is $T \tan \theta$ or $T d y \mid d x$. The force, $T_{b}$, pulling at the end, $b$, of the element is the tension of the wire at $b$, its horizontal component is $T$, and its component vertically upwards is $T \tan \theta^{\prime}$ or $T\left(d y\left|d x+d^{2} y\right| d x^{2}-d x\right)$. Therefore the unbalanced force pulling upwards on the element, $a b$, is $T d^{2} y \mid d x^{2} \cdot d x$, and this unbalanced force is equal to the weight of the element, $w \cdot d x$, so that:

$$
T \frac{d^{2} y}{d x^{2}}=w
$$

whence

$$
T y=\frac{1}{2} w x^{2}+c x+c^{\prime}
$$

but, since $y=0$ and $d y \mid d x=0$ when $x=0$, the constants, $c$ and $c^{\prime}$, must be each equal to zero, so that :

$$
y=\frac{w}{2 T} \cdot x^{2}
$$

From Fig. 161 it is evident that $y=h$ when $x=l / 2$; therefore, substituting these values in equation (ii), we have equation (39).

The second member of equation (40) consists of the first two terms of the infinite series which expresses the length of the arc of a parabola in terms of its chord, l, and the distance, $h$, of the middle of the arc from the chord.

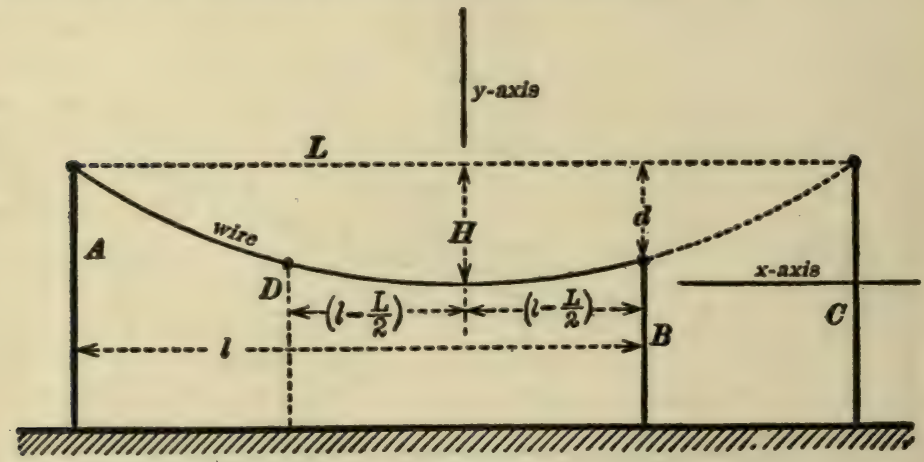

Fig. 162 .

Equations (4I) and (42) are derived from equations (39) and (40). Consider a given span of wire between two poles, $A$ and $B$, Fig. 162, at a horizontal distance, 
$l$, from each other, $d$ being the difference in level of the tops of the poles, and $H$ the sag of the wire below the top of pole, $A$, as shown. The given span, $A B$, may be considered as part of a longer span, $A C$, of which the length is $L$, as shown in the figure; and the portion, $B D$, of the given span may be looked upon as a span also. Let $S$ be the length of wire in the long span, $A C$, and $P$ the length of wire in the short span, $B D$. Then the length of wire in the given span, $A B$, is :

$$
s=\frac{S}{2}+\frac{P}{2}
$$

Furthermore, applying equations (39) and (40) to the span, $A C$, we have

and

$$
T=\frac{L^{2} 2 v}{8 H}
$$

$$
S=L+\frac{8 H^{2}}{3 L}
$$

Applying equation (40) to the span, $B D$, gives :

$$
P=(2 l-L)+\frac{8(H-d)^{2}}{3(2 l-L)}
$$

The equation to the parabolic curve formed by the wire is

$$
y=\frac{4 H}{L^{2}} \cdot x^{2} ;
$$

and at the top of the pole, $B, y=H-d$, and $x=l-L / 2$, so that :

$$
H-d=\frac{4^{H}}{L^{2}}\left(l-\frac{L}{2}\right)^{2}
$$

\begin{tabular}{|c|c|c|c|}
\hline & $\begin{array}{c}\text { Tensile Strength } \\
\text { in Pounds per Circular } \\
\text { Mil }=a .\end{array}$ & $\begin{array}{l}\text { Density in Pounds } \\
\text { per Mil-Foot }=b \text {. }\end{array}$ & $\begin{array}{c}\beta \text {-Coeffic ent of Linear } \\
\text { Expansion per } \\
\text { Degree } F \text {. }\end{array}$ \\
\hline Steel & 0.0785 & $2.65 \times 10^{-6}$ & 0.0000064 \\
\hline 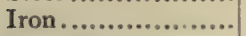 & 0.0417 & $2.65 \times 10^{-6}$ & 0.0000064 \\
\hline Hard-drawn copper & 0.0300 & $3.03 \times 10^{-6}$ & 0.0000094 \\
\hline Aluminum............. & 0.0204 & $0.91 \times 10^{-6}$ & 0.0000128 \\
\hline
\end{tabular}

Equations $\left(4^{I}\right)$ and $\left(4^{2}\right)$ are obtained by eliminating $L, P$ and $S$ from (iii) and (iv) by'means of equations (v), (vi) and (vii).

Tensile Strengths, Weights and Coefficients of ExpanSION OF WIRES.

Usual factor of safety from 2 or 3 in warm climates to 6 or 7 in cold climates.

Factor of safety for aluminum must be larger than for other metals on account of low elastic limit of aluminum.

Breaking tension of wire $T_{b}=a d^{2}$ in pounds.

Weight of wire $w=b d^{2}$ in pounds per foot, where $d$ is the diameter of the wire in mils.

Length at $t^{\circ} \mathrm{F} .=$ length at $t^{\prime} \times\left[\mathrm{r}+\beta\left(t-t^{\prime}\right)\right]$.

For weight of galvanized iron or steel wire add about 6 per cent. to weight of plain wire. 
116. Safe carrying capacity. - An electric wire rises in temperature until it gives off heat to its surroundings as fast as heat is generated in it by the current. Therefore the rise of temperature for a given current, or the current corresponding to a prescribed rise of temperature depends upon the facility with which the wire gives off heat; and this facility depends greatly upon the degree of ventilation of the region in which the wire is placed, and upon the nature of the adjacent materials, whether they be wood, or plaster, or stone, or metal. Wires covered by wooden mouldings or enclosed in the narrow air space inside of the walls of a building are located most unfavorably with regard to rise of temperature because they cannot give off heat readily, and because any great rise of temperature involves a serious risk of fire.

\section{Table of Carrying Capacity of Wires.}

\section{(From National Electrical Code.)}

For insulated aluminum wire the safe carrying capacity is eighty-four per cent. of that given in the following tables for copper wire with the same kind of insulation.

\begin{tabular}{|c|c|c|c|}
\hline $\begin{array}{c}\text { Brown and Sharpe } \\
\text { Gauge. }\end{array}$ & $\begin{array}{l}\text { Sectional Area in } \\
\text { Circular Mils. }\end{array}$ & $\begin{array}{l}\text { Rubber Insulation. } \\
\text { Amperes. }\end{array}$ & $\begin{array}{l}\text { Other Insulation. } \\
\text { Amperes. }\end{array}$ \\
\hline 18 & 1,624 & 3 & 5 \\
\hline 16 & 2,583 & 6 & 8 \\
\hline 14 & 4,107 & 12 & 16 \\
\hline 12 & 6,530 & 17 & 23 \\
\hline 10 & 10,380 & 24 & 32 \\
\hline 8 & 16,510 & 33 & 46 \\
\hline 6 & 26,250 & 46 & 65 \\
\hline 5 & 33,100 & 54 & 77 \\
\hline 4 & 41,740 & 65 & 92 \\
\hline 3 & 52,630 & 76 & I10 \\
\hline 2 & 66,370 & 90 & I3I \\
\hline I & 83,690 & 107 & 156 \\
\hline 0 & 105,500 & 127 & 185 \\
\hline 00 & 133,100 & 150 & 220 \\
\hline 000 & 167,800 & 177 & 262 \\
\hline 0000 & 211,600 & 210 & 312 \\
\hline & 400,000 & 330 & 500 \\
\hline - & 600,000 & 450 & 680 \\
\hline - & $1,000,000$ & 650 & $1, O C O$ \\
\hline[ & $1,500,000$ & 850 & 1,360 \\
\hline- & $2,000,000$ & 1,050 & 1,670 \\
\hline
\end{tabular}

The lower limit is specified for rubber covered wires to prevent gradual deterioration of the rubber by the heat of the wires, but not from fear of igniting the insulation. The question of voltage-drop is not taken into consideration in the above tables. 
117. Voltage regulation as a factor determining the size of wires. - The so-called constant-voltage system of current distribution is the system that is generally used in electric-light and power installations, and the sizes of feeders, mains, and service wires used in such a system are generally determined on the basis of a prescribed voltage-drop. This is especially the case when current is supplied to incandescent lamps, the satisfactory operation of which depends upon approximate constancy of voltage. The voltage-drop in the feeders which lead out from a central station to a center of distribution is usually compensated for by what is called feeder control at the station as explained on page 195. On the other hand, the voltage-drop in the mains which lead out from a center of distribution and the voltage-drop in the service wires which lead from the street mains to the lamps are not compensated for, and, as they affect the value of the voltage at the lamps, these voltage-drops must be small. The total drop between a center of distribution and the lamps is generally limited to a certain percentage of the voltage at the lamps. A total drop of about 5 per cent., 2 per cent. in the mains and 3 per cent. in the service wires, is frequently allowed; although a greater or less drop may be advisable if the distance between the center of distribution and the lamps is very great or very small respectively.

Dependence of total weight of wire upon the voltage at the lamps and upon the distance of the lamps from the center of distribution. - If a given amount of power, $P$, is to be supplied to lamps at voltage, $E$, with a given percentage drop, $p$, by a separate pair of wires leading from the center of distribution to the lamps, then the weight of the copper required is proportional to $l^{2} / E^{2}$, where $l$ is the distance between the center of distribution and the lamps. This is evident when we consider that the current is $P / E$, that the drop $p E /$ Ioo is equal to $R \times P / E$, that $R=10.8 \times 2 l / d^{2}$, and that $W=0.00000303 \times 2 l d^{2}$, so that

$$
W=0.00654 \frac{P l^{2}}{p E^{2}},
$$


where $R$ is the resistance of the two wires in ohms, $W$ is the weight in pounds of the two copper wires required to deliver $P$ watts of power to a receiving unit at $E$ volts, $p$ is the percentage drop in the wires (actual drop $=p E / I 00$ ), and $l$ is the distance in feet from the center of distribution to the lamps. The constant in equation (43) applies only to copper wires.

Inasmuch as the weight of the copper is inversely proportional to $E^{2}$, according to equation (43), it is evident that a very great saving in copper may be effected by using a high voltage at the lamps. The permissible voltage at the lamps is limited, however, $(a)$ by the fact that incandescent lamps cannot be made to operate satisfactorily at voltages higher than about 220 volts, and $(b)$ by the danger that is involved in the use of high voltages.

The saving in copper by the use of high voltage, combined with the practical necessity of low-voltage delivery, has led to the use of the Edison three-wire system as explained in Art. II2. In the alternating-current system of distribution power can be transmitted at any desired high voltage and cheaply and efficiently transformed near the place of consumption to any desired low voltage. Therefore the alternating-current system permits of very great economy of copper in the transmission lines and does not involve any of the difficulties or dangers incident to the utilization of high voltages at lamps and motors.

When the voltage-drop in a transmission line is not limited by the necessity of maintaining an approximately constant voltage at the lamps, or other receiving units, the size of wire should be determined on the basis of economic considerations as explained in Art. I 20 ; and it is to be particularly noted that the weight of copper, demanded by economic considerations, for the delivery of a given amount of power is not proportional to $l^{2} / E^{2}$ but to l/E.

118. Wiring calculations in constant-voltage systems for a motor or for a concentrated group of lamps. - Two important cases arise in the laying out of wiring plans in a constant-voltage system, 
namely, (a) the case in which current is delivered at one point to a motor or to a group of lamps, constituting what is called a concentrated load; and $(b)$ the case in which current is delivered to a scattered group of lamps or motors, constituting what is called a distributed load.

The problem of determining the size of wire required to deliver a specified amount of power, $P$, to a concentrated load at a specified voltage, $E$ (at the lamps), with a specified percentage drop, $p E / \mathrm{IOO}$, in the wires, is solved as follows : (I) The current, $I$, is equal to $P / E$. Sometimes the current is given directly, as when a specified number of lamps is to be supplied with a specified current per lamp. (2) Given the percentage drop, $p$, the actual drop in volts is known. (3) This actual drop is equal to $R I$, so that the resistance, $R$, of the two line-wires is thus determined. Knowing the value of $R$ and the length, $2 l$, of the wire in feet, the diameter, $d$, of the wire in mils may be calculated from the equation $R=10.8 \times 2 l / d^{2}$.

The result of these separate steps may be most conveniently represented in the formula

$$
d^{2}=\text { circular mils }=\frac{2,160 l I}{p E}
$$

in which $d$ is the diameter in mils of copper wires required to deliver $I$ amperes to a concentrated load distant $l$ feet from the center of distribution, $p$ is the drop of voltage expressed in per cent.; thus $p=6$ for 6 per cent., and $E$ is the voltage at the lamps.

It is preferable to avoid the specification of voltage-drop as a percentage; and in fact the drop is usually specified in volts.

* In laying out the wiring for a complete electric-lighting system it is a great saving of time to make use of what are called wiring charts, which give at a glance the solution of equation (44) $a$ or $(44) b$ for any particular case. The construction and use of such charts are fully explained in a small book, entitled The Wiring Computer, by Carl Hering (The McGraw Publishing Company.) 
In this case the sectional arca of the wire in circular mils is given by the formula :

$$
d^{2}=\text { circular mils }=\frac{21.6 l I}{e}
$$

in which $e$ is the drop expressed in volts, and $d, l$ and $I$ have the same meanings as in equation (44) $a$.

In a great many cases the size of wires to be used to deliver current to a uniformly distributed load with a specified drop of voltage is determined by equations (44), by assuming a concentrated load equal to the given distributed load and located near the center of the given distributed load.

119. Wiring calculations for constant-voltage systems with distributed loads. - When a number of widely distributed lamps is supplied with current by one pair of service wires, or when a number of widely distributed customers is supplied by one pair of street mains, we have what is called a distributed load. The problem of determining the size of street mains to supply a number of scattered customers is the same as the problem of determining the size of service wires to supply a number of scattered lamps. In the first case the voltage-drop between the center of distribution and the various service points is the important consideration, and in the second case the voltage-drop between a service point and the individual lamps is the important consideration ; a service point is understood to be the point of attachment of a pair of service wires to the street mains.

In a distributed load two kinds of variation of voltage occur, namely, $(a)$ the variation of voltage from lamp to lamp when the number of lamps in operation is fixed, and $(b)$ the variation of voltage at any given lamp as the number of lamps in operation is increased or decreased.

Concerning the first type of variation it may be stated in general that the lamp voltage is less and less the more remote the lamp is from the service point, the most remote lamp having always the lowest voltage. 
Concerning the second type of variation it may be stated in general that the voltage at every lamp falls off to some extent when additional lamps are turned on, and rises when lamps already in operation are turned off. The range of variation in voltage at a given lamp is from a lowest value, when all the lamps are in operation, to a value very nearly equal to the voltage at the service point, when the given lamp, only, is in operation. Therefore, the lamp that is most remote from the service point is subject to the greatest range of variation of voltage as other lamps are turned on and off.

There are two clearly defined cases that arise in the laying out of wires for distributed loads, namely, Case I., in which the lamps supplied by a given pair of service wires are turned on and off separately, and Case II., in which all of the lamps supplied by a given pair of service wires are turned on and off together. In the first case the wiring must be laid out so as to keep the voltage variations of both types $(a)$ and $(b)$ within certain limits; and in the second case the wiring may be laid out with reference to the limitation of voltage variations of the first type only, that is variations of voltage from lamp to lamp, inasmuch as voltage variations of the second type $(b)$ do not exist in Case II.

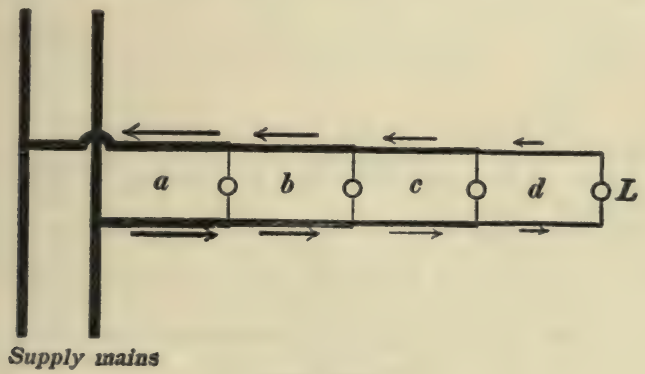

Fig. 163.

Case I. - An example of a distributed load is shown in Fig. I63. When all of the lamps are in operation the end lamp, $L$, has the lowest voltage of any lamp in the group, and the voltage at this lamp varies through the greatest range when other lamps are 
turned off and on. Therefore, if the voltage at the end lamp is to be kept within, say, 3 per cent. of its normal value, $E$, which is the value when all the lamps are in operation, then the voltagedrop in the wires must not exceed 3 per cent. of $E$ when all the lamps are in operation.

To secure a specified drop out to the end lamp, $L$, when all the lamps are in operation, with the minimum weight of copper in the wires, the sectional area of each portion, $a, b, c$ and $d$, Fig. $16_{3}$, of the wires must be $*$ proportional to the square root of the current in that portion. $\dagger$ Thus, if each lamp in Fig. I63 takes the same amount of current, then the current values in the portions $a, b, c$ and $d$, are as $4: 3: 2: 1$, and the sectional areas of the respective portions of the wires should be as $\sqrt{4}: \sqrt{3}: \sqrt{2}: \sqrt{\mathrm{I}}$ in order to give a minimum voltage-drop at the end lamp, $L$, with a given amount of copper, or to give a minimum amount of copper for a specified voltage-drop at the end lamp, $L$.

In laying out street mains to supply a group of scattered customers it is generally advisable, on account of the large amount of copper involved, to taper the mains in steps in going farther and farther from the center of distribution; but, as a rule, the successive steps should be made longer than the distance between adjacent customers, in order to avoid an excessive number of joints in the mains.

In laying out service wires to supply current to a scattered group of lamps, it is generally not advisable to taper the wires in steps, because the amount of copper involved may not be large ; whereas the expense of making many joints, together with the

* It should be kept in mind that the fundamental condition here is a minimum amount of copper for a given voltage-drop. A minimum amount of copper for given watts lost in the line requires the sectional area of the wires to be proportional to the current at each point; that is, the number of circular mils per ampere must be the same throughout the system to give a minimum amount of copper for a given loss of power in watts.

† The general proof of this proposition involves the highly elaborate methods of the calculus of variations and therefore the proof of the proposition is not given here. 
expense of inserting fusible cut-outs at each point where wires of unequal size are joined, as required by the insurance rules, may be considerable.

Rule $I_{\text {. }}$ - When it is desired to reduce the size of a pair of street mains (or service wires) in steps so as to secure the greatest economy of copper, the size of each portion of the mains is determined as follows. Having given the total drop to be allowed out to the end of the line, calculate the factor $s$ from the equation :

$$
s=\frac{2 \times 10.8 \times\left(a \sqrt{i_{1}}+b \sqrt{i_{2}}+c \sqrt{i_{3}}+\cdots\right)}{\text { total drop in volts }}
$$

in which $a, b, c \ldots$ are the lengths in feet of the respective portions of the pair of mains, and $i_{1}, i_{2}, i_{3} \ldots$ are the currents in amperes in the respective portions. The sectional areas of the various portions of the mains in circular mils are then equal to $s \sqrt{i_{1}}, s \sqrt{i_{2}}, s \sqrt{i_{3}} \ldots$ respectively.

Rule 2. - When service wires of uniform size are to be used for supplying current to a scattered group of lamps, the size of the wire to give a prescribed drop out to the end lamp may be determined as follows: Estimate the distance, $L$, of the "center of gravity" of the group of lamps by the formula:

$$
L=\frac{l^{\prime}+l^{\prime \prime}+l^{\prime \prime \prime}+\cdots}{n}
$$

where $l^{\prime}, l^{\prime \prime}, l^{\prime \prime \prime}$, etc., are the distances in feet of the individual lamps* from the service point and $n$ is the total number of lamps in the group. Then calculate the size of the wire that would be required to supply the $n$ lamps as a concentrated group at the prescribed total drop and at the distance $L$ from the service point.

Case II. - When the lamps of a group are always turned on and off together the variation of voltage from lamp to lamp can

* If the lamps are arranged in subgroups it is easier to take $l^{\prime}$ as the product of the distance of the first subgroup times the number of lamps in that group, $l \prime l$ as the product of the distance of the second subgroup times the number of lamps in that group, and so on. 
of course be kept within bounds by limiting the voltage-drop between the service point and the end lamp of the group. In fact a group of lamps which is to be operated as a unit is generally wired according to the same rules as a group that is not to be operated as a unit, that is the wiring is generally laid out according to rules $I$ and 2 of Case I.; but a special wiring scheme, called the return loop scheme, * may be used to eliminate voltage variations of the first type $(a)$ in a group of lamps that is operated as a unit, whatever the total voltage-drop may be.

The fundamental idea of the return loop scheme may be seen with the help of Fig. 164a. The current in the wire, $a b$, at any

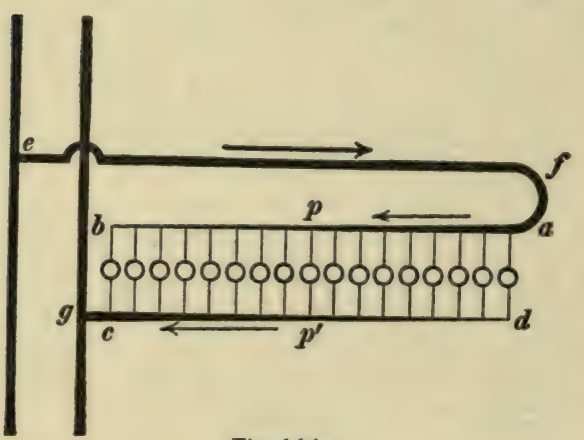

Fig. 164a. point, $p$, is proportional to the distance, $p b$, the lamps being assumed to be uniformly distributed; and the current at any point, $p^{\prime}$, in the wire, $c d$, is proportional to the distance, $p^{\prime} d$. If the wires, $a b$ and $c d$, are tapered so as to have sectional areas proportional to the current at each point, then the value of $R i$ is the same in both wires between any pair of lamps; but $R i$ is a drop of voltage in a given direction along one wire and a rise of voltage in the same direction along the other wire, therefore the lamp voltage is constant throughout the group of lamps, whatever the total voltage-drop between the service point and the lamps may be.

The use of tapered wires is of course impracticable and the return loop scheme is always carried out either with wires tapered in steps or with wires of uniform size, usually the latter. Under such conditions the voltage varies to some extent from lamp to lamp but the range of this variation is very much less than the total drop.

* Sometimes called the anti-parallel scheme. 
The return loop scheme of wiring evidently requires three wires of a given length instead of two, and therefore it requires much more copper than the simple parallel wiring scheme for the same total voltage-drop. The advantage of the return loop scheme however is that a very large total drop is permissible, so that the size of the wires may always be determined from economic considerations.

The return loop scheme is usually employed in the wiring of churches, lecture halls and theaters, where the lamps are either all in

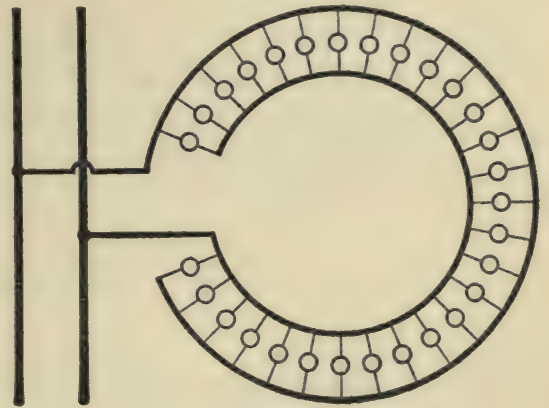

Fig. 165 . use or all out of use, or where the lamps in certain groups are either all in use or all out of use.

In many cases the lamps in a group are arranged in a circular or reëntrant row. In such a case the return loop scheme is carried out as shown in Fig. 165, or as shown in Fig. 166.

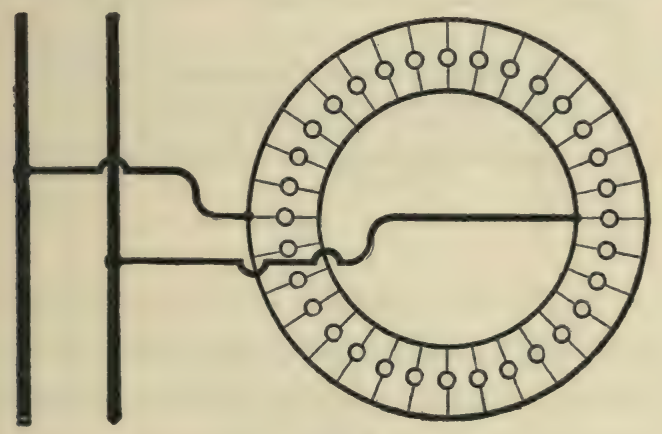

Fig. 166.

Return loop scheme with wires of uniform size. - When the wires used in the return loop scheme are of uniform size (not tapered) the middle lamp, $p$, Fig. $164 b$, has the lowest voltage of 
any lamp in the group, and the size of the wires, $e f a b$ and $g c d$, is usually determined with reference to the voltage-drop between the service point, $e g$, and the middle lamp, $p$. Let $I$ be the total current delivered to the group of lamps, and let the lamps be assumed to be uniformly distributed as shown in Fig. 1646 ; then the current in the element, $\Delta x$, is $I(X-x) / X$, and the voltage-drop in the element, $\Delta x$, is $\rho \cdot \Delta x$ times $I(X-x) / X$, where $\rho$ is the resistance per unit length of the wire, $c d$. Therefore the voltage-drop along $c d$ from $c$ to the middle lamp is :

$$
\frac{\rho I}{X} \int_{x=0}^{x=X / 2}(X-x) d x=\frac{3}{8} \rho X I
$$

Also the voltage-drop along $a b$ from $a$ to the middle lamp is $3 / 8 \rho X I$, so that the total drop between the service point, $e g$, and the middle lamp, $p$, is $I\left(r^{\prime}+r^{\prime \prime}\right)$, where $r^{\prime}$ is the resistance of $g c$ plus the resistance of $e f a$, and $r^{\prime \prime}$ is equal to $3 / 4 \rho X$, where $\rho X$ is the resistance of one of the wires, $a b$ or $c d$.

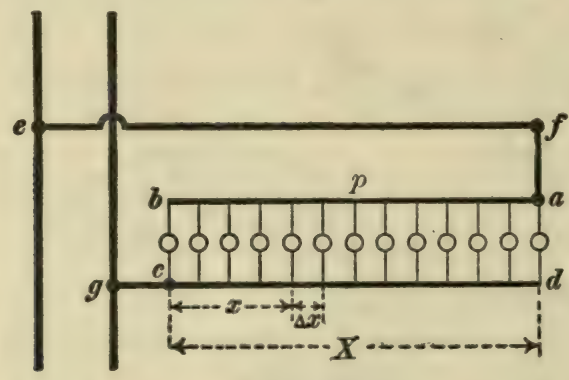

Fig. $164 b$.

Rule 3. - To give a prescribed voltage-drop between the service point and the middle lamp of a row, which is connected according to the return loop scheme, make the wire of such size that the total current delivered to the group of lamps would give the prescribed drop over a length $l^{\prime}+l^{\prime \prime}$ of the wire, where $l^{\prime}$ is the sum of the distances, $g c$ and $e f a$, in Fig. $164 b$, and $l^{\prime \prime}$ is $3 / 4$ of the distance $a b$. 
Modifications of Cases I. and II. - Every practical case of wiring in the constant-voltage system of distribution can be treated as a slight modification of Cases I. and II. above described. Thus Fig. I67 shows two groups of lamps each exactly like the single

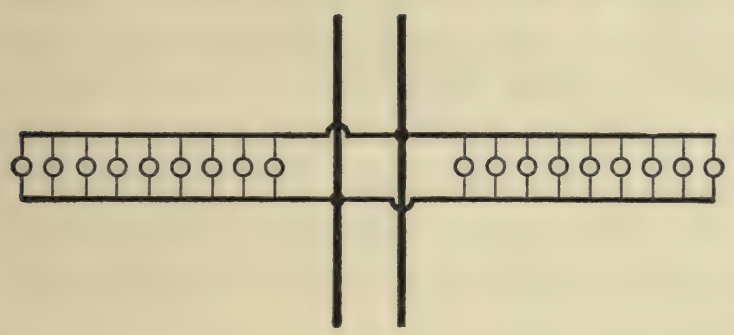

Fig. 167.

group in Fig. $16_{3}$; and Fig. 168 shows a combination of Figs. 163 and 164 , that is, the portion, $p a$, of the group of lamps in Fig. 168 is arranged in conformity with Fig. I63 and the portion, $p b$, is arranged in accordance with the return loop scheme.

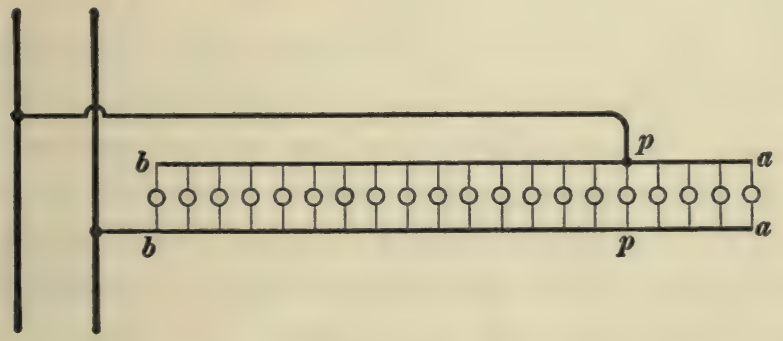

Fig. 168.

120. The economic balance between loss of power and the cost of copper in the distribution of electric current. - The original cost of erection of a distributing line consists of two nearly independent parts, namely $(a)$ the cost of the copper and $(b)$ the cost of poles, cross-arms, pins and insulators and the cost of erection. That is to say, even if one were to double the size of wires to be used the cost of item $(b)$ would not be increased to any considerable extent. The disadvantage of using large wires lies, there- 
fore, almost wholly in the annual charge, including interest on the cost of the wire, depreciation of the wire, and taxes thereon. The advantage $*$ of using large wires, on the other hand, lies in the decreased loss of power in the wires. Therefore, the most economical size of wire is that for which the additional annual charge on a larger wire would exceed the annual value of the power saved by the use of the larger wire, or, in other words, the most economical size of wire is that for which the sum of the annual charge on the total copper plus the annual value of the power lost in the wires is a minimum. The annual charge may be estimated at: interest 5 per cent., depreciation 3 per cent., and taxes 1.5 per cent.

The economic balance between loss of power and cost of copper always leads to a definite number of circular mils of sectional area of wire per ampere of current, without regard to the voltage or to the distance of transmission.

Electric power is to be supplied for $h$ hours each year to a customer. The cost of power at the switchboard is $p$ dollars per kilowatt-hour, the cost of copper is $c$ dollars per pound, and the interest charge on invested capital (including a small percentage to cover the depreciation of copper wires and taxes) is $t$ per cent. per annum. It is required to find the sectional area of the copper wire in circular mils per ampere of current on the condition that any increase in the amount of copper would effect a saving of power of which the annual value would be less than the interest on the cost of the additional copper. Let $2 l$ be the length of the wire in feet (equal to twice the length of the line), $s$ its sectional area in circular mils, $R$ its resistance in ohms, $W$ its weight in pounds, and $I$ the current in amperes. Then $R=10.8 \times 2 l / s$ so that the lost power in kilowatts is $\frac{2 \mathrm{I} .6}{\mathrm{I}, \mathrm{OOO}} \cdot \frac{l}{s} I^{2}$, and the annual loss of energy is $\frac{2 \mathrm{I} .6}{\mathrm{I}, \mathrm{OOO}} \cdot \frac{l}{s} I^{2} h$ kilowatt-hours, of which the value at $t$

* It is to be kept in mind that we are not here considering the fact that in the constant-voltage system the wires must be large enough to limit the voltage-drop. 
dollars per kilowatt-hour is $\frac{2 \mathrm{I} .6}{\mathrm{I}, \mathrm{000}} \cdot \frac{l}{s} \cdot I^{2} p h$ dollars per year. On the other hand $W=0.00000303 \times 2 l s$ pounds, of which the cost is 0.00000606 lsc dollars, the interest on this cost is 0.0000000606 lsct dollars per year, and the quantity to be made a minimum by choosing $s$ is $\frac{21.6}{1,000}-I^{2} p h+0.0000000606 / s c t$. Differentiating this expression with respect to $s$ and placing the differential coefficient equal to zero gives :

$$
-\frac{21.6}{1,000} \frac{l}{s^{2}} \cdot I^{2} p h+0.0000000606 l c t=0
$$

from which $l$ cancels out, and we find :

$$
\frac{s}{I}=\text { circular mils per ampere }=597 \sqrt{\frac{p h}{c t}}
$$

or

$$
s=597 I \sqrt{\frac{p h}{c t}}
$$

The meanings of the symbols, $s, I, h, p, c$ and $t$, are specified above. When the delivered current, $I$, is not constant, the average value of the current must not be used, but the square-root-of-theaverage-value-of-the-square should be used in equation (46).

Example 1. - The cost of power at the switch-board of an electric-power station is 1.6 cents per kilowatt-hour ( $p=0.016)$, the interest on invested capital is 5 per cent., and the annual depreciation and taxes is three per cent. $(t=8)$, the cost of copper wire is 16 cents per pound $(c=0.16)$, and a current of 200 amperes is delivered to a customer for I, 000 hours each year. Considerations of economy would lead, under these conditions, to the use of transmission wires 650 mils in diameter, whatever the distance of the customer from the station may be.

If the distance from the station to the consumer is 538 feet, then the total length of wire is 1,076 feet, its resistance is 0.0275 ohm, and the voltage-drop with 200 amperes is 5.5 volts. That 
is, if current is to be supplied to the customer at I IO volts, the size of wire required on the basis of a 5 per cent. drop of voltage is the same as the size of wire required to give an economic balance between the loss of power and the cost of copper under the specified conditions. If the distance is greater than 538 feet, then considerations of economy would give a smaller wire than would be required by a 5 per cent. drop in voltage; and, if the distance is less than 538 feet, then considerations of economy would give a larger wire than would be required by a 5 per cent. drop in voltage.

Example 2. - Cost of power, rate of interest and cost of copper being the same as in example $I$, it is required to find the most economical size of wire for carrying 100 amperes for 400 hours each year and 300 amperes for 600 hours. The average square of the current is

$$
\frac{\left(100^{2} \times 400\right)+\left(300^{2} \times 600\right)}{400+600}=58,000 \text { amperes }^{2}
$$

and the square-root-of-average-square is $24 \mathrm{I}$ amperes. Therefore using $h=400+600$ hours and $I=24 I$ amperes in equation (46), we have $s=509,200$ circular mils, or the diameter of the wire must be 7 I 3 mils.

Dependence of total weight of copper on voltage of delivery and distance of customer from station. - A given amount of power, $P$, is to be delivered to a customer at a distance, $l$, from the station and at a voltage, $E$. The current is $P / E$ so that from equation (46) we have :

$$
s=597 \frac{P}{E} \sqrt{\frac{p h}{c t}}
$$

which, substituted in the formula $W=0.00000303 \times 2 l s$, gives :

$$
W=0.0036 \text { г } 8 P \frac{l}{E} \sqrt{\frac{p^{h}}{c t}} .
$$

which shows that the amount of copper required by economic 
considerations is proportional to the distance, $l$, and inversely proportional to the voltage of delivery.

Limitations of Kelvin's law.-The economic balance between the loss of power in transmission wires and the cost of copper was first pointed out by Lord Kelvin, and the condition expressed by equation (46) is sometimes called Kelvin's law of economy.* In the derivation of equation (46) it was assumed, first, that the cost of poles, cross-arms and pins, and the cost of erection of the pole line are the same whatever the size of the wire may be, and second, that the cost of the wire is so much per pound. The first assumption is approximately true only for wires of moderate weight. For very heavy wires the supporting structure must be very strong and therefore expensive. The second assumption is approximately true only for bare wires. For insulated wires the cost per pound varies considerably with the size of the wire.

The sum of the three items: (a) Annual value of energy lost in a line, reckoned on the basis of its cost per kilowatt-hour at the switchboard; (b) Annual interest on the entire cost of the line, and taxes; (c) Annual cost of line depreciation and repairs, makes the difference between the cost of the energy at the switchboard and its cost delivered to a customer.

121. Electric strength of line insulation as a factor in the determination of the size of wires. - The two diagrams, $A$ and $B$, Fig. 169, represent in section two lead-encased cables with the same radial thickness of insulating substance between the central wire and the lead sheath, and the fine radial lines represent the lines of force of

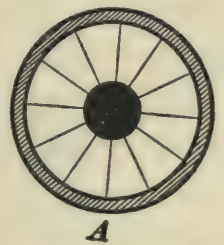

Fig. 169.

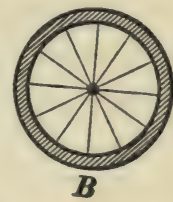

$B$ the electric field in the insulating medium for the same voltage between the wire and the lead-covering in each case.

* A very complete discussion of Kelvin's law is given by Dr. F. A. C. Perrine in his book entitled Conductors for Electrical Distribution(D. Van Nostrand, 1903), pages $161-178$. 
The voltage determines the average intensity of the electric field between the core and the casing, and the figure shows that the electric field is much more intense near the surface of the small wire in $B$ than it is near the surface of the large wire in $A$. Therefore the strength of a given insulating medium to withstand the electric stress between the core and sheath of a cable depends not only upon the radial thickness of the insulation but also upon the radius of the central wire.
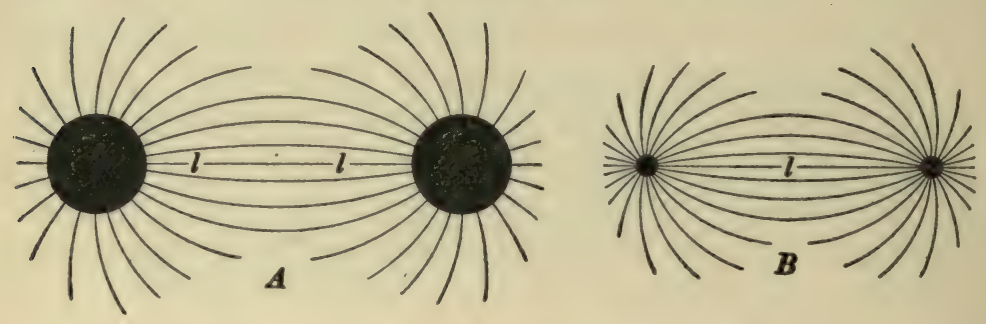

Fig. 170.

Each of the diagrams, $A$ and $B$, Fig. I 70 , represents in section the two wires of a transmission line, and the fine curved lines represent the lines of force of the electric field in the insulating medium for the same voltage between the wires in each case. The voltage determines the average intensity of the electric field along the straight lines, $l$, and the figure shows that the electric field is much more intense near the small wires in $B$ than it is near the large wires in $A$. Therefore the strength of a given insulating medium to withstand the electric stress between two line wires depends not only upon the distance from surface to surface of the wires, but also upon the size of the wires.

This dependence of the strength of the line insulation upon the size of the wires may be made more intelligible in terms of a mechanical analogue. Thus a block of glass is much more severely strained when it is squeezed between two fine-pointed tools than when it is squeezed with the same total force between two blunt-pointed tools, and it is in the immediate neighborhood of the fine points that the excessive strain occurs. 
Maximum electric stress in the insulation of a cable. - Let $R_{1}$ be the radius of the central wire of a cable and $R_{2}$ the inside radius of the sheath. The electric

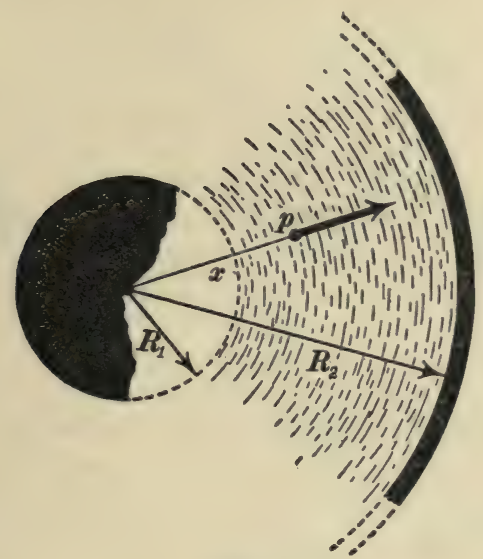

Flg. 171.

field intensity (volts per centimęter) at a point $p$, Fig. I 7 I, distant $x$ from the axis of the cable, is proportional to $\mathbf{I} / x$. Therefore we may write

$$
f=\frac{k}{x}
$$

where $f$ is the electric field intensity at a point distant $x$ centimeters from the axis, and $k$ is a constant to be determined. The electromotive force, $E$, between the central wire and the sheath is :

$$
E=\int_{R_{1}}^{R_{2}} f \cdot d x
$$

whence, substituting the value of $f$ from (i), we have:

Therefore :

$$
E=k \log _{e} \frac{R_{1}}{R_{2}}
$$

$$
k=\frac{E}{\log _{e} \frac{R_{2}}{R_{1}}}
$$

Now the electric field intensity at the surface of the central wire is $k / R_{1}$ from equation (i) or, using the value of $k$ from (iv), we have

$$
f_{\max }=\frac{E}{R_{1} \log _{\theta} \frac{R_{2}}{R_{1}}}=\frac{E}{2.3 R_{1} \log _{10} \frac{R_{2}}{R_{1}}}
$$

in which $f_{\max }$ is the maximum electrical stress (volts per centimeter) in the insulation of a cable, $R_{1}$ is the radius of the central wire in centimeters, $R_{2}$ is the inside 
radius of the metal sheath, $E$ is the electromotive force in volts between the centra wire and the sheath, and $\log _{e} R_{2} / R_{1}$ is the Naperian logarithm of the ratio $R_{2} / R_{1}$.

Maximum electric stress in the medium between parallel wires. - Let $R$ be the radius of each wire, and $d$ the distance from center to center. The electric field intensity (volts per centimeter) at a point, $p$, on the line, $l$, distant $x$ from the center of $W^{\prime}$ and distant $d-x$ from the center of $W^{\prime \prime}$, see Fig. I72, consists of two parts, $f^{\prime}$

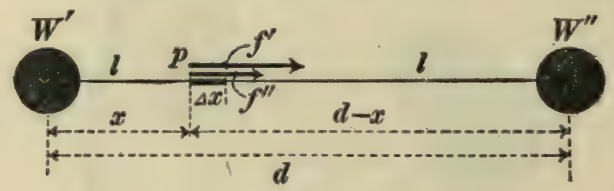

Fig. 172.

and $f^{\prime \prime}$, which are proportional to $\mathrm{I} / x$ and to $\mathrm{I} /(d-x)$ respectively, so that we may write :

$$
f^{\prime}=\frac{k}{x}
$$

and

$$
f^{\prime \prime}=\frac{k}{d-x}
$$

These two equations are only approximately true unless the distances $x$ and $d-x$ are measured from points a very little closer together than the axes of the two wires. When, however, $R$ is small in comparison with $d$, it is sufficiently exact to measure $x$ and $d-x$ from the axes of the wires.

The electromotive force $E$ between the wires is :

$$
E=\int_{x=R}^{x=d-R}\left(f^{\prime}+f^{\prime \prime}\right) d x
$$

Therefore, using the values of $f^{\prime}$ and $f^{\prime \prime}$ from equations (i) and (ii), we find, by integration :

$$
E=2 k \log _{\Theta} \frac{d-R}{R}
$$

or, since $R$ is usually small in comparison with $d$, this equation may be written :

whence

$$
E=2 k \log _{e} \frac{d}{R}
$$

$$
k=\frac{E}{2 \log _{\odot} \frac{d}{R}}
$$

Now the region of greatest intensity of the electric field is at the surface of one (or the other) of the wires where $x=R$, and where

$$
f^{\prime}+f^{\prime \prime}=\frac{k}{R}+\frac{k}{d-R}
$$


Therefore, using the value of $k$ from (iv), and neglecting $k /(d-R)$ which is very small in comparison with $k \mid R$, we have :

$$
f_{\max }=\frac{E}{2 R \log _{e} \frac{d}{R}}=\frac{E}{4.6 R \log _{10} \frac{d}{R}}
$$

in which $f_{\max }$ is the maximum electric stress (volts per centimeter) in the medium between two parallel wires each of radius $R$ centimeters, $E$ is the electromotive force between the wires in volts, $d$ is the distance in centimeters between the axes of the wires, and $\log _{e} d \mid R$ is the Naperian logarithm of the ratio, $d / R$.

Example. - The electrical strength of air is about 26,000 volts per centimeter.* Therefore, substituting 26,000 for $f_{\max }$ in equation (49), we find that 3,014 volts is the greatest electromotive force that the air can sustain between two wires 6 mils in diameter and 6 inches apart; and that 104,800 volts is the greatest electromotive force that the air can sustain between two wires 500 mils in diameter and 6 inches apart center to center. It is worthy of note that when the voltage between two very fine wires but slightly exceeds the sustaining strength of the intervening air, the air breaks down only in the immediate neighborhood of the wires, and discharge from wire to wire takes place mainly by convection currents of electrically charged air.

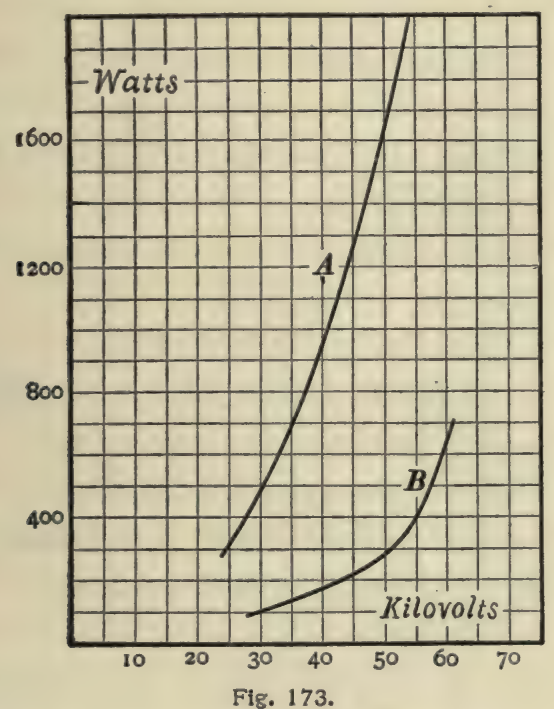

The dependence of the safe value of voltage between wires upon the size of the wires is shown in a very interesting way by the curves $\dagger$ in Fig. 173. Curve $A$ shows

* Dielectric Strength of Air, by C. P. Steinmetz, Transactions A. I. E. E., Vol. XV., pp. 281-326, 1898 .

†From a paper by Charles F. Scott on High-Voltage Power Transmission, Transactions A. I. E. E., Vol. XV., pp. 531-576. 
the watts lost by discharge between two bare wires each 0.0126 inch in diameter and $\mathrm{I}, \mathrm{O} 40$ feet long stretched side by side at a distance of 48 inches apart; and curve $B$ shows the watts lost by discharge between two bare wires each 0.128 inch in diameter, 1,040 feet long and 48 inches apart. The abscissas in Fig. I73 represent effective values of the alternating electromotive forces used. An alternating electromotive force reaches a maximum value equal to about I.4 times its effective value.

122. Pole-line insulation. - When the voltage between two line wires is less than that required to cause a break-down of the intervening air, the leakage of current through the air from wire to wire is entirely negligible. The only perceptible leakage of current is at the supporting insulators and through branches of trees and other objects which happen to touch the wires.

If the insulators are made of glass or thoroughly vitrified porcelain the leakage of current through the material of the insulator is always negligible, unless the insulator is ruptured, but the leakage of current over the surface of the insulator may be considerable. This leakage over the surface of the insulators is reduced to a minimum by designing the insulators so that the length of the leakage path measured along the surface may be as great as possible, and so that a portion of the surface may be shielded from rain and mist. This is accomplished by making a series of deep grooves around the bottom of the insulator as shown by the sectional view of the insulators in Fig. 160. This type of insulator is called the petticoat type; single, double or triple petticoat as the case may be. An objection to the petticoat insulator is that insects are apt to build their nests in the deep recesses between the petticoats and thus impair the insulation. This is especially the case when the insulators are opaque and the grooves dark.

The strength of an insulator to withstand a high voltage without rupture has nothing directly to do with its insulation resistance. Analogously, the strength of a porous earthenware jar to withstand pressure without bursting has nothing directly to do with the facility with which the porous walls of the jar permit the water to flow through them. Insulators for high-voltage lines are 
usually tested for electric strength by subjecting them to a voltage about twice as great as they are to withstand in service.

Wires on pole lines are provided with an insulating covering only when it is desired to reduce the risk of accidental contacts with adjacent wires. Thus the high-voltage lines which supply arc lamps in city streets should have insulating coverings to reduce the danger of accidental contacts with telephone and telegraph wires. On the other hand the wires of a very high-voltage transmission line are usually bare, inasmuch as any ordinary insulation would be inadequate to ensure protection in case of accidental contacts with other wires or with persons.

123. Insulation of underground, house and station wires. - In the installation of underground, house and station wires, two things should be kept in mind, namely : (a) The insulation of the wires, and especially the employment of insulating material that will be absolutely water-proof, and $(b)$ the protection of the wires and their insulating covering from mechanical injury. The only water-proof insulation is a covering of rubber or a covering of fibrous insulation encased in a seamless lead sheath, and the most satisfactory mechanical protection is that which is afforded by an iron pipe or by a vitrified clay conduit laid in concrete underground, or built in the walls and floors of an office building.

124. Supplementary references. - A full description of the great variety of structural details involved in pole lines and underground distributing wires and cables, and a full discussion of the details of station- and house-wiring including the questions of location and design of junction boxes, switches and cut-outs, are beyond the scope of this text. A very complete treatment of pole lines and underground wires and cables is given by A. V. Abbott in his book entitled Electrical Transmission of Energy, Van Nostrand, 1905. A wealth of practical information concerning outside and inside wiring is given in the National Electrical Code which is described on page 220 of this text. Valuable information concerning wires of all kinds, iron, steel and copper, bare and insulated, may be found in the little book entitled Wire in 
Electical Construction, which is published by John A. Roebling's Sons Company, of Trenton, N. J.

Weights and Resistances of Copper Wires.

Brown and Sharpe Gauge.

\begin{tabular}{|c|c|c|c|c|c|c|}
\hline \multirow{2}{*}{$\begin{array}{l}\text { Gauge } \\
\text { Num- } \\
\text { bers. }\end{array}$} & \multirow{2}{*}{$\begin{array}{l}\text { Diameters } \\
\text { in } M \text { Mils }=d .\end{array}$} & \multirow{2}{*}{$\begin{array}{l}\text { Areas in Cir- } \\
\text { cular Mils } \\
=d^{2}\end{array}$} & \multicolumn{2}{|c|}{ Weights. } & \multicolumn{2}{|c|}{$\begin{array}{l}\text { Resistances per } 1,000 \text { Feet in } \\
\text { International Ohms. }\end{array}$} \\
\hline & & & $\begin{array}{l}\text { Per } x, 000 \\
\text { Feet }\end{array}$ & Per Mile. & At $60^{\circ} \mathrm{F}$. & At $75^{\circ} \mathrm{F}$. \\
\hline 0000 & 460 & 211,600 & $64 I$ & 3,382 & $.048 \mathrm{II}$ & .04966 \\
\hline 000 & 410 & I68, 100 & 509 & 2,687 & .06056 & $.0625 \mathrm{I}$ \\
\hline$\infty$ & 365 & 133,225 & 403 & 2,129 & .07642 & .07887 \\
\hline o & 325 & 105,625 & 320 & 1,688 & .09639 & .09948 \\
\hline $\mathbf{I}$ & 289 & 83,521 & 253 & $\mathbf{1}, 335$ & .1219 & $.125^{8}$ \\
\hline 2 & $25^{8}$ & 66,564 & 202 & 1,064 & .1529 &.$\times 579$ \\
\hline 3 & 229 & $52,44^{I}$ & 159 & 838 & $.194 I$ & .2004 \\
\hline 4 & 204 & 41,616 & 126 & 665 & .2446 & .2525 \\
\hline 5 & 182 & 33,124 & 100 & 529 & .3074 & $\cdot 3172$ \\
\hline 6 & 162 & 26,244 & 79 & 419 & $\cdot 3879$ & .4004 \\
\hline 7 & 144 & 20,736 & 63 & 331 & $.49 \mathrm{I}$ & .5067 \\
\hline 8 & I 28 & $16,3^{8} 4$ & 50 & 262 & .6214 & .6413 \\
\hline 9 & II 4 & 12,996 & 39 & 208 & .7834 & .8085 \\
\hline Io & 102 & 10,404 & 32 & 166 & .9785 & I.OI \\
\hline II & 91 & $8,28 \mathrm{I}$ & 25 & 132 & 1. 229 & 1.269 \\
\hline 12 & $8 I$ & $6,56 r$ & 20 & 105 & I. 552 & 1.601 \\
\hline 13 & 72 & 5,184 & 15.7 & 83 & 1.964 & 2.027 \\
\hline 14 & 64 & 4,096 & 12.4 & 65 & 2.485 & 2.565 \\
\hline 15 & 57 & 3,249 & 9.8 & $5^{2}$ & 3.133 & 3.234 \\
\hline 16 & $5 \mathrm{I}$ & $2,60 \mathrm{I}$ & 7.9 & 42 & 3.914 & 4.04 \\
\hline 17 & 45 & 2,025 & 6.1 & 32 & 5.028 & 5.189 \\
\hline I8 & 40 & 1,600 & 4.8 & 25.6 & 6.363 & 6.567 \\
\hline 19 & 36 & 1,296 & 3.9 & 20.7 & 7.855 & 8.108 \\
\hline 20 & 32 & 1,024 & 3.1 & 16.4 & 9.942 & 10.26 \\
\hline 21 & 28.5 & 812.3 & 2.5 & 13 & 12.53 & 12.94 \\
\hline 22 & 25.3 & 640.1 & I. 9 & 10.2 & 15.9 & 16.41 \\
\hline 23 & 22.6 & 510.8 & I. 5 & 8.2 & 19.93 & 20.57 \\
\hline 24 & 20.1 & 404 & I. 2 & 6.5 & 25.2 & 26.01 \\
\hline 25 & 17.9 & 320.4 & .97 & 5.1 & 31.77 & 32.79 \\
\hline 26 & 15.9 & 252.8 & .77 & 4 & 40.27 & 41.56 \\
\hline 27. & 14.2 & 201.6 & $.6 \mathrm{I}$ & 32 & 50.49 & 52. II \\
\hline 28 & 12.6 & I58.8 & .48 & 2.5 & 64.13 & 66.18 \\
\hline 29 & II. 3 & 127.7 & $\cdot 39$ & 2 & 79.73 & 82.29 \\
\hline 30 & 10 & 100 & $\cdot 3$ & I. 6 & IOI. 8 & 105.1 \\
\hline $3^{1}$ & 8.9 & 79.2 & .24 & I. 27 & 128.5 & I32.7 \\
\hline 32 & 8 & 64 & .19 & 1.02 & I 59.1 & 164.2 \\
\hline 33 & 7.1 & 50.4 & .15 & $.8 \mathrm{r}$ & 202 & 208.4 \\
\hline 34 & 6.3 & 39.7 & .12 & .63 & 256.5 & 264.7 \\
\hline 35 & 5.6 & 31.4 & .095 & .5 & 324.6 & $335 . \mathrm{I}$ \\
\hline 36 & 5 & 25 & .076 & .4 & 407.2 & 420.3 \\
\hline
\end{tabular}


This table is based on a resistance of $10.5 \mathrm{I}$ ohms per mil-foot at $75^{\circ} \mathrm{F}$, a temperature coefficient of resistance of 0.0022 per degree Fahrenheit, and a density of $3.03 \times 10^{-6}$ pound per milfoot, or 555 pounds per cubic foot.

Aluminum. - Resistances in above table are to be multiplied by $\mathrm{r}$.6I for aluminum wire.

Weights in above table are to be multiplied by 0.30 for aluminum wire.

Iron and Steel. - Resistances in above table are to be multiplied by about 7 for iron and steel wires.

Weights in above table are to be multiplied by 0.876 for iron and steel wires. 


\section{CHAPTER $\mathrm{X}$.}

\section{PHOTOMETRY AND ELECTRIC LIGHTING.}

125. Radiant heat. Light. - The radiation from a hot body may be resolved into simple parts each of which is a train of ether waves of definite wave-length. All of these simple parts of the total radiation have one common property, namely, they generate heat in a body which absorbs them. Therefore every portion of the radiation from a hot body is properly called radiant heat. The intensity of a beam of radiant heat is measured by the heat it delivers per second to an absorbing body. Thus the radiation emitted by a standard candle represents a flow of about 450 ergs * per second across one square centimeter of area at a distance of one meter from the candle.

Radiant heat of which the wave-length lies between 39 and 75 millionths of a centimeter affects the optic nerves and gives rise to sensations of light. Therefore radiant heat of which the wavelength lies between these limits is called light. These limits, which are called the limits of the visible spectrum, are not sharply defined, but they vary considerably with different persons and with the degree of fatigue of the optic nerves.

The physical intensity of a beam of light is measured by its perfectly definite thermal effect, that is by the heat energy it delivers per second to an absorbing body. Thus those parts of the radiation of a standard candle which lie within the visible spectrum represent a flow of about 9.3 ergs per second across an area of one square centimeter at a distance of one meter from the candle. Comparing this with the flow of energy which is represented by the total radiation from a standard candle, namely, 450 ergs per

* Heat is properly expressed in energy units. One erg of heat is the amount of heat that is equivalent to one erg of mechanical energy. 
second across an area of one square centimeter at a distance of one meter from the candle, it follows that only about 2 per cent. of the energy radiated by a standard candle lies within the limits of the visible spectrum, that is, only about 2 per cent. of the radiation from a standard candle is light.

The luminous intensity of a beam of light is presumably measured by the intensity of the light sensation it can produce, but the intensity of the light sensation produced by a given beam of light is extremely indefinite. A given beam of light entering the eye may produce a strong or a weak sensation depending upon manifold individual peculiarities of the person and upon the degree of fatigue of the retina, and the vividness of the sensation depends upon the extent to which it is enhanced by attention. Our sensations are really not quantitative in the physical meaning of that term ; in fact they enable us merely to distinguish objects, to judge whether things are alike or unlike, and the certainty and precision with which we can do this is exemplified in every outward aspect of our daily life. The ratio of the luminous intensities of two beams of light is measured by using a device to alter in a known ratio the physical intensity of one beam until it gives, as nearly as one can judge, a degree of illumination on a screen which is equal to (like) the illumination produced by the other beam.

126. Photometry. - The measurement of the light emitted by a lamp is called photometry. This measurement is always made by comparing a beam of light from the given lamp with a beam of light from a standard lamp, as explained in the previous paragraph, and the physical device there referred to is called a photometer. The comparison of the total light in a beam from a given lamp with the total light in a beam from a standard lamp is called simple photometry; whereas the comparison wave-length by wavelength throughout the spectrum is called spectro-photometry. A fundamental difficulty* in simple photometry is that different

* A method for overcoming this difficulty by means of what is called the flickerphotometer is described in Nichols \& Franklin's Elements of Physics, Vol. III., p. 128. 
lamps usually show differences of color, and these differences of color do not disappear when the attempt is made to adjust a photometer to equality of brightness.

The results of the spectro-photometric * comparison of gas light, lime light, and day light are shown by the curves in Fig. I 74. $\dagger$ The curves refer to beams of gas light, lime light and - day light all of which have the same intensity at Fraunhofer's $D$

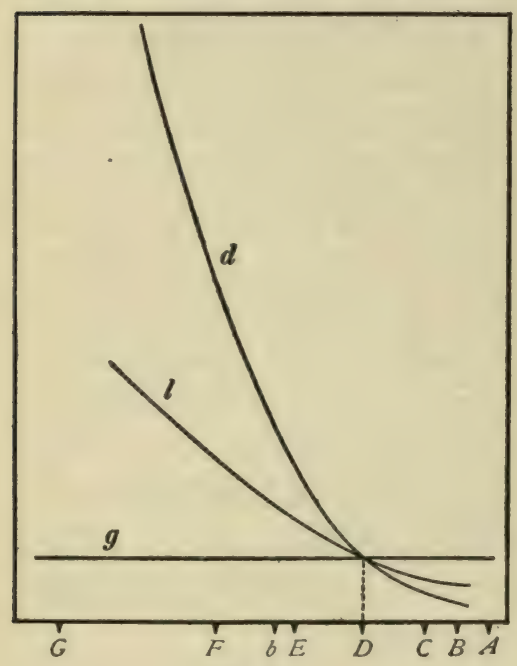

Fig. 174. line (the middle of the yellow region of the spectrum); and the curves show, for example, that the beam of day light is about six times as bright as the beam of gas light at Fraunhofer's $F$ line (in the blue region of the spectrum), and only about one fourth as bright as the gas light at Fraunhofer's $B$ line (in the red region of the spectrum).

\section{Standard lamps. -} The Britush standard candle is a sperm candle made according to exact specifications and burning I 20 grains of sperm per hour. The Hefner lamp, so called from its inventor, is a lamp which burns pure amyl acetate. The wick and its containing tube are of prescribed dimensions and the wick is turned up to give a flame of prescribed height. $\ddagger$

* A simple form of spectro-photometer is described in Nichols and Franklin's Elements of Physics, Vol. III., p. izo.

† See Nichols and Franklin. A spectro-photometric comparison of sources of artificial illumination, American Journal of Science, Vol. 38, pp. 100-114, December, 1889.

‡ See Zeitschrift für Instrumentenkunde, Vol. XVIII., p. 257, July, 1893. A very satisfactory discussion of the Hefner lamp may be found in Photometrical Meas. urements, by Wilbur M. Stine, The Macmillan Co., 1900, pp. 145-160. 
The intensity of a horizontal beam of light from a Hefner lamp is called a hefner unit or a hefner. If a lamp were to give one hefner unit of light intensity in every direction, the amount of light, or the so-called flux of light emitted by the lamp, would be what is called one spherical-hefner.*

The great reliability of the Hefner lamp as compared with the standard sperm candle has led to the definition of the candle in terms of the hefner unit. The candle or the candle unit is a beam of light of which the intensity is I.1 36 hefner units and the spherical-candle is equal to I.I 36 spherical-hefners. The standard sperm candle is no longer used in photometric work.

128. Conical intensity and sectional intensity of light. - The expression, intensity of a beam of light, which is used in the above definitions of the hefner and the candle, refers to the amount of light in a unit-sized cone of rays. This conical intensity, which it may be called for brevity, is expressed in hefners or candles ; it is independent of distance, since the light in a given cone of rays remains in that cone; and it depends only upon the area and brightness of the luminous surface of the lamp.

The intensity of a beam of light may also refer to the amount of light per unit sectional area of the beam. This sectional intensity of a beam of light, which it may be called for brevity, decreases as the square of the distance from the lamp increases, and it is expressed in terms of a unit called the lux, which is the sectional intensity of a horizontal beam from a Hefner lamp at a distance of one meter from the lamp. The sectional intensity, $I$, of a given beam in luxes at a distance of $d$ meters from a lamp is given by the equation :

$$
I=\frac{h}{d^{2}}
$$

in which $h$ is the conical intensity of the beam in hefner units.

* The spherical-hefner is not a kind of hefner. The word spherical is not used as an adjective, but the term stands for a distinct conception; it is one word, and it should be written with a hyphen. 


\section{Intensity of illumination. - The amount of light per unit sec-} tional area of a beam, that is, the sectional intensity of the beam, measures the intensity of illumination of a surface upon which the beam falls perpendicularly. Therefore the intensity of illumination of a surface may be expressed in luxes. Thus the intensity of illumination required for easy reading is the intensity of illumination at a distance of one foot from a standard candle, which intensity is sometimes called the "candle-foot," and it is equal to I.I $36 /(0.305)^{2}=12.21$ luxes according to equation (50).

A complete statement of the various photometric units depends upon a clear understanding of what is called solid angle. Consider a cone, and a sphere with its center at the apex of the cone. The solid or spherical angle of the cone is measured by the ratio of the area of the portion of the spherical surface within the cone to the square of the radius of the sphere. Thus the unit of solid angle is subtended by one square centimeter of the surface of a sphere of one centimeter radius, and the complete surface of a sphere represents $4^{\pi}$ units of solid angle.

Consider a lamp placed at the center of a sphere of unit radius so that one unit of area of this sphere may represent one unit of solid angle or one unit-cone. Imagine the lamp to give one hefner of conical intensity of light in every direction. Then the amount of light (light-flux) passing out in one unit-cone (through unit area of the sphere) is called one lumen of light-flux.

The given lamp emits one spherical-hefner ( 0.88 of a spherical-candle) of light-flux, because the conical intensity is assumed to be the same in every direction; but the whole spherical surface represents $4 \pi$ units of solid angle or $4 \pi$ unit cones. Therefore the lamp emits $4 \pi$ lumens of light-flux. That is to say, a spherical-hefner of lightflux is equal to $4 \pi$ lumens.

The lux, which is defined above as the intensity of illumination at a distance (horizonta!ly) of one meter from a Hefner lamp, represents of course a certain amount of light flux falling upon each square centimeter of the illuminated surface. Now the area of a sphere of one meter radius is $40,000 \pi$ square centimeters and if the lamp gave out light equally in all directions, one spherical-hefner or $4 \pi$ lumens would pass out from it. Therefore one lux represents one ten-thousandth of a lumen per square centimeter or $\frac{1}{4000 \delta \bar{\pi}}$ of a spherical-hefner per square centimeter.

As an illustration of the significance of the terms hefner, lumen and lux, consider a beam of light emitted by a glow lamp. Let the conical intensity of this beam be $\mathbf{1} 8.2$ hefners ( 16 candles). Let the solid angle of this beam be 0.01 of a unit, that is to say, the solid angle subtended by o or of a square centimeter of the surface of a sphere of which the radius is one centimeter. Then the number of lumens of light-flux in the beam is 18.2 hefners multiplied by o.or unit of solid angle, which is equal to 0.182 lumen. The sectional intensity of this beam at a distance of one meter from the lamp is 18.2 luxes. 
The so-called search-light presents some very interesting features in regard to conical intensity. The numerical example given below will serve as an illustration. To understand this example one must remember that light which emanates from a point in the focal plane of a lens or mirror is transformed by the lens or mirror into a beam of rays which are all parallel (ignoring errors of spherical and chromatic aberration and astigmatism ) to a line drawn from the point to the center of the lens or to the center of curvature of the mirror. Therefore the light which emanates from a small luminous surface in the focal plane is transformed into a series of parallel beams all the rays of which lie (at a great distance from the search-light) within the cone formed by drawing lines from every point of the small luminous surface to the center of the lens or to the center of curvature of the mirror, and the solid angle of this cone is equal to the area of the small luminous surface divided by the square of the focal length of the lens (or mirror).

The powerful arc lamp of a certain search-light emits light of 10,000 candles conical intensity towards every part of a lens (or mirror) which subtends one unit of solid angle as seen from the arc which is at its focus. The luminous surface of the lamp is one quarter of a square centimeter and the focal length of the lens (or mirror) is 30 centimeters. Therefore the solid angle of the cone which contains the search-light beam is $1 / 4$ divided by $30^{2}$ or $\frac{1}{3600}$ of a unit, and, if we assume that no light is lost in the lens (or on the mirror) by absorption, the conical intensity of the search-light beam must be 3,600 times as great as the conical intensity of the light direct from the lamp, or $36,000,000$ candles. One who considers this example carefully will be impressed with the important fact that the candle is not a unit of quantity of light. The candle and the hefner are units of conical intensity.

129. The Bunsen photometer is a device for measuring the conical intensity of a beam of light from a given lamp in hefners or in candles. It is the photometer that is almost universally used in simple photometric measurements. The given lamp and the standard lamp are placed at the ends of a horizontal bar and a screen of thin unsized paper is moved along the bar until the two sides of the screen are equally illuminated by the two lamps. The intensities of illumination (sectional intensities of the beams of light) due to the respective lamps are $h / d^{2}$ and $h^{\prime} / d^{\prime 2}$, according to equation (50), and since these are equal we have :

$$
\frac{h}{h^{\prime}}=\frac{d^{2}}{d^{\prime 2}}
$$

in which $h$ and $h^{\prime}$ are the conical intensities in hefners or in candles of the light sent towards the screen by the respective lamps, and $d$ and $d^{\prime}$ are the distances of the lamps from the screen. 
An irregular grease spot on the thin paper screen enables one to judge better when the illumination is the same on the two sides. This spot should be made with clean paraffin and the excess of paraffin should be drawn out of the screen by placing it between folds of absorbent paper and applying a hot flat-iron.

In the use of the Bunsen photometer a carbon-filament electric lamp is generally used as a working standard. This lamp is previously standardized for a particular voltage and in a particular direction, by comparing it with a Hefner lamp; and when used it is operated at this particular voltage. Carefully standardized carbon-filament lamps are offered for sale by the United States Bureau of Standards, Washington, D. C.

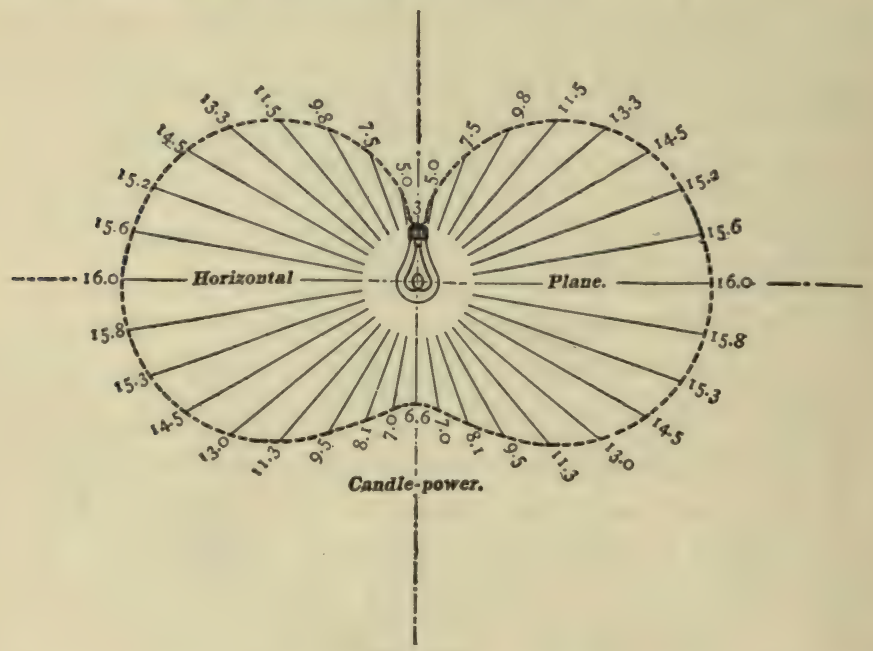

Fig. 175.

130. Distribution of light around a lamp. - In the definition of the spherical-hefner the idea of uniformity of distribution of light around the lamp was introduced for the sake of simplicity. In fact, however, no lamp gives complete uniformity of distribution, but the conical intensity in hefners or candles is always greater in certain directions and less in other directions. Thus Fig. 175*

* Taken from a paper by J. R. Cravath and V. R. Lansingh on Reflectors, Shades and Globes, Electrical World and Engineer, Vol. 46, pp. 907, 947, 991, I033 and 1074, November 25 to December 23, 1905. 
shows the distribution of light about a typical " 16 -candlepower" carbon-filament lamp without a shade, and Fig. I 76 shows the distribution of light about the same lamp when it is placed in an aluminum cone reflector. In these figures the conical intensity of the light in each direction in candles is represented to scale by the length of the corresponding radius vector of the dotted curve.

The distribution of light about a lamp, which, like a carbon-filament electric lamp, can be held in any position, may be determined by mounting the lamp in a universal holder at one end of the photometer bar, turning it step by step into various positions, and taking the photometer reading for each position.

In some cases a lamp is symmetrical with respect to an axis,

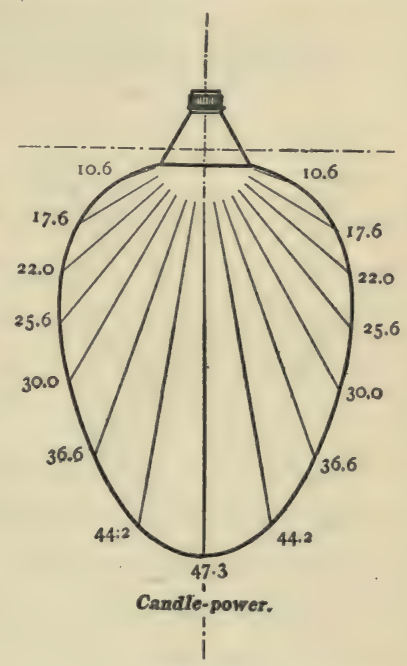

Fig. 176. so that a complete knowledge of the distribution of light about the lamp may be obtained by determining the intensities of the light in different directions in a single plane which contains the axis of symmetry.

In many cases a lamp is approximately symmetrical with respect to an axis so that the slight variations of the intensity of the light around the axis of approximate symmetry are of no importance. In such a case the lack of symmetry may be averaged out, as it were, by rotating the lamp at a speed of three or four revolutions per second about its axis of approximate symmetry while the photometric readings are being taken. The data for Figs. 175 and 176 were obtained in this way.

In the case of a lamp which must be held in a fixed position, one or more mirrors are used to reflect the different beams from 
the lamp along the photometer bar. Thus Fig. I 77 shows three mirrors, $A A, B B$ and $C C$, arranged to reflect the light from a fixed lamp, $L$, along a photometer bar. The three mirrors are supported in a rigid frame which may be rotated about the line, $D E$, as an axis. The figure shows the mirrors in the position to reflect the downward beam from the lamp to the photometer screen.

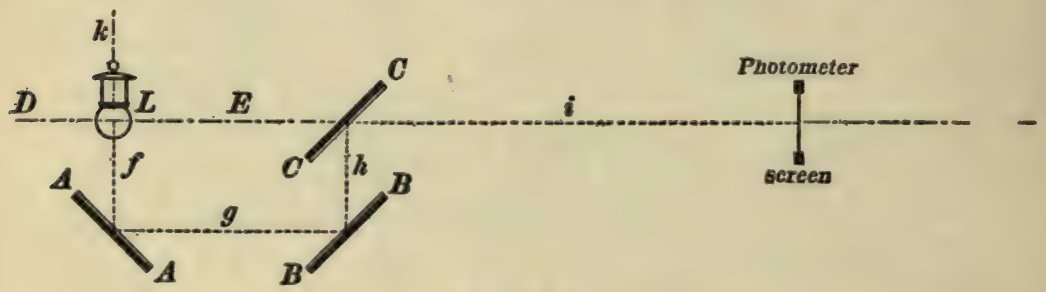

Fig. $17 \%$.

The mirrors, $A A, B B$ and $C C$, Fig. 177 , must be large enough so that with the eye placed at the photometer screen one can see the entire luminous surface of the lamp including the globe or shade; and the distance of the lamp from the screen must be taken as the sum of the distances, $f, g, h$ and $i$, Fig. I 77. The mirrors reflect a certain fractional part, only, of the light from the lamp, and, therefore, the photometer reading must be multiplied by a correction factor. This correction factor is found by observing the photometer readings of the horizontal beam from the lamp with and without the mirrors, making due allowance for the effective distance from lamp to screen in each case.

If it is feasible the lamp should be rotated steadily about the vertical axis, $k f$, in Fig. 177 , while the photometer readings are being taken.

131. Measurement of total light flux from a lamp. - If a lamp were to emit light of the same conical intensity in all directions, then the conical intensity of the light in hefners (or candles) would be numerically equal to the total light flux from the lamp in spherical-hefners (or spherical-candles), and a single measurement of such a lamp by means of a Bunsen photometer would give not only the conical intensity of the light in hefners (or 
candles) but also the total light flux in spherical-hefners (or spherical-candles).*

In general, however, light is emitted by a lamp unequally in different directions and it is necessary to distinguish between conical intensity and total light flux. The total light flux in spherical-hefners emitted by a lamp is determined by measuring the conical intensity in hefners in every direction and taking the average, which gives the light flux in spherical-hefners. If this average is to be calculated in the ordinary way by adding and dividing, the directions in which the separate readings are taken must be distributed uniformly over the surface of a sphere with its center at the lamp. This sphere is called the reference sphere for brevity. If the readings are not so distributed, each reading must be multiplied by the spherical area which is to be properly assigned to it, and the sum of such products must be divided by the total area of the reference sphere to give the correct average.

When a lamp can be rotated at a speed of three or four revolutions per second about its axis of approximate symmetry,

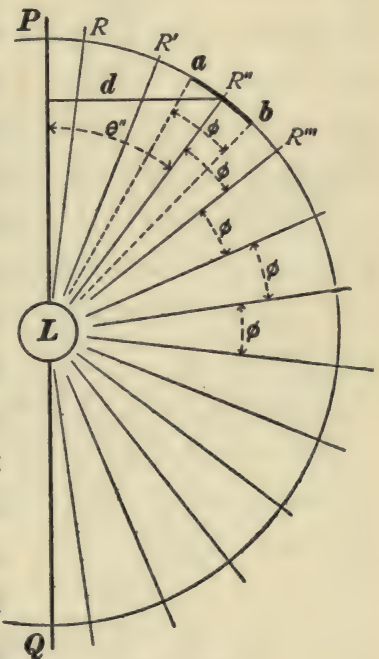

Fig. 178. the total light flux may be determined accurately by taking readings of the conical intensity in different directions in one plane only, namely, a plane which includes the axis of rotation. Thus the lamp, $L$, Fig. I 78 , is rotated about the vertical axis, $P Q$, and the

* There is a widespread tendency among electrical engineers to confuse the unit of conical intensity, the candle, with the unit of light flux, the spherical-candle. This is due to the fact that, in the absence of exact data concerning the distribution of light around a given lamp, the irregularities of distribution are ignored and the lamp is thought of as giving out light uniformly in all directions. That is to say, electrical engineers have been inclined to ignore that property of a lamp (the irregularity of distribution of the light of the lamp) which makes the distinction between the candle and the spherical-candle important. 
conical intensities, $R, R^{\prime}, R^{\prime \prime}, R^{\prime \prime \prime}$, etc., at equal angular distances, $\phi$, are measured. On account of the rotation of the lamp each setting of the photometer gives the average conical intensity along a parallel of latitude, as it were. Each reading, $R, R^{\prime}, R^{\prime \prime}$, etc., represents, therefore, the conical intensity over a zone of the reference sphere; so that the readings must be multiplied by the areas of the respective zones and the sum of these products divided by the total area of the reference sphere to give the average conical intensity in all directions, which is equal of course to the total light flux in spherical-units, hefners or candles as the case may be. Thus the reading, $R^{\prime \prime}$, Fig. 178 , refers to the spherical zone, $a b$, of which the area is sensibly equal to the length of the arc, $a b(=r \phi)$, multiplied by the mean circumference $\left(=2 \pi d=2 \pi r \sin \theta^{\prime \prime}\right)$, of the zone. The radius, $r$, of the reference sphere cancels out when one divides the above-mentioned sum by the total area of the reference sphere $\left(=4 \pi r^{2}\right)$, as above explained.

Example. - The conical intensities are given in Fig. I75 for every $10^{\circ}$. The area of each of the two polar zones corresponding to conical intensities 3.0 and 6.6 is, $\pi \times\left(\frac{10}{360} \text { of } 2 \pi r\right)^{2}$; the area of each of the zones corresponding to conical intensities 5.0 and 7.0 is, $2 \pi r \sin 10^{\circ} \times\left(\frac{10}{360}\right.$ of $\left.2 \pi r\right)$; the area of each of the zones corresponding to conical intensities 7.5 and 8.1 is, $2 \pi r \sin 20^{\circ} \times\left(\frac{10}{360}\right.$ of $\left.2 \pi r\right)$; and so on. A calculation of the mean conical intensity in all directions from the data given in Fig. I 75 gives I 3.33 spherical-candles as the light flux emitted by the lamp.

132. The Matthews integrating photometer. - Two ingenious arrangements of mirrors have been devised by Professor C. P. Matthews by means of which the light flux from a lamp in spherical-candles can be determined by a single setting of a photometer.* A photometer provided with such an arrangement of mirrors is called an integrating photometer. The essential features of one of Professor Matthews' arrangements are shown in Fig. I79. The lamp, $L$, to be tested is rotated about the vertical axis, $V V$, and a number of equidistant beams from $L$, all lying in the plane, $Q L V$,

* The Integrating Photometer, C. P. Matthews, Transactions of American Institute of Electrical Engineers, Vol. XVIII., pp. 677-697, I901 ; Vol. XX., pp. 59-70, 1902. 

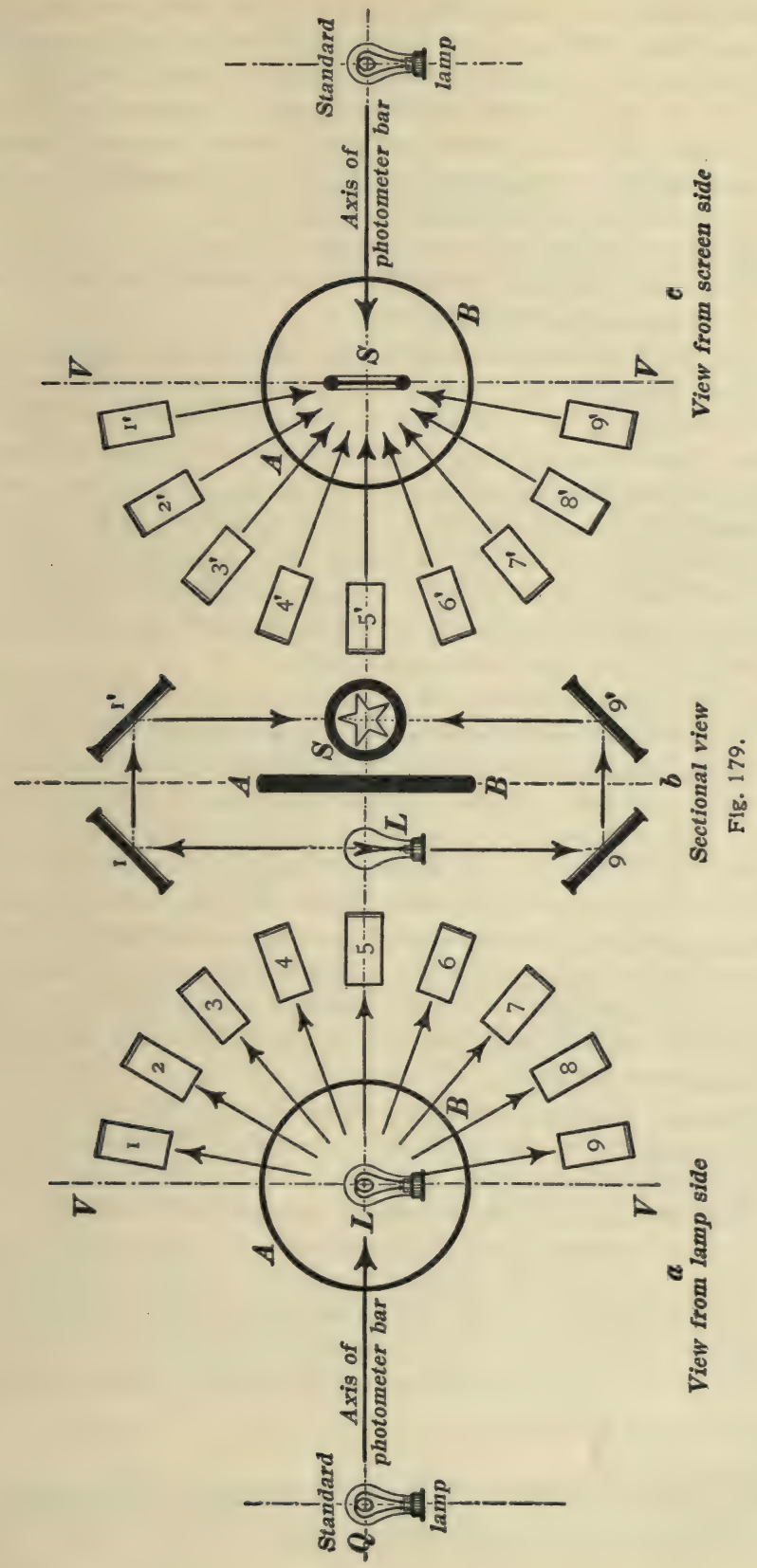
and each representing a zone of the reference sphere, ${ }^{*}$ are reflected to the photometer screen, $S$, as shown. This photometer screen is stationary and the photometer setting is made by moving the standard lamp nearer to or farther from the screen.

If the beams of light from all of the mirrors struck the photometer screen at right angles, the reading of the photometer would be the sum of the conical intensities of all the beams, that is, the photometer reading would be proportional to the simple average of the conical intensities of all of the beams.

The spherical-candle power of a lamp is found, however, not by taking the simple average of the conical intensities in various directions, but by taking what is called a weighted average as explained in Art. 13 1 .

If the illumination of the screen by each beam alone were $z^{\prime} / z$ as large as it would be if the beam struck the screen at right angles, $z$ being the area of the equatorial zone of the reference sphere which is associated with the beam that is at right angles to $V V$, and $z^{\prime}$ being the area of that zone of the reference sphere which is associated with any given beam, then the reading of the photometer, Fig. 179, would be the sum $\Sigma\left(z^{\prime} I / z\right)$ that is, the photometer reading would be proportional to the true sphericalcandle power of the lamp. In fact the reading of the photometer would be $n$ times the spherical-candle power $\left(=\Sigma_{z^{\prime}} I / 4 \pi r^{2}\right.$ ), where $n$ is equal to the ratio of the area of the entire reference sphere, $4 \pi r^{2}$, to the area of the equatorial zone, 2 . The incomplete reflection of light by the mirrors is considered later.

If the photometer screen were entirely free from gloss an oblique beam of light would produce a degree of illumination inversely proportional to the area of screen over which unit sectional area of the oblique beam is spread, that is, a degree of illumination proportional to the sine of the angle between the beam and the plane of the screen. This is precisely the reduction of illumination specified above in terms of the ratio $z^{\prime} / z$. It is impossible however to make a photometer screen without gloss, that is a screen that does not show regular reflection to some extent, and the more oblique the incident light the larger the proportion of the light which is reflected regularly and the less the proportion that is reflected diffusely by the screen. Therefore the mirrors near the axis, $V V$, in Fig. 179 must be somewhat nearer to $L$ and $S$ so as to lessen the optical distance from $L$ to $S$, and thus intensify slightly the beams that strike the screen obliquely.

The Matthews integrating photometer is adjusted as follows so as to eliminate errors due to gloss of screen and errors due to absorption of light by the mirrors. Put an auxiliary standard lamp in place of $L$, cover all of the mirrors except the pair that receives the horizontal beam from the auxiliary standard, that is mirrors 5 and $5^{\prime}$ in Fig. 179, and take the reading $k \dagger$ of the photometer. Then cover all of the mirrors except any given pair, turn the auxiliary standard lamp so that its standard face or aspect is towards the uncovered pair, set the photometer arbitrarily at reading, $\mathrm{kz}^{\prime} / \mathrm{z}$, and adjust the uncovered pair of mirrors inwards or outwards until the screen shows equal illumination on its two sides. Proceed in like manner with each pair of mirrors until the adjustment is complete.

* Compare Art. I3I.

$\dagger$ The quantity $\mathrm{I} / k$ is the factor by which any reading of the photometer must be multiplied to correct for loss of light due to incomplete reflection by the mirrors. 
When the photometer has been thus properly adjusted its reading must be multiplied by the factor $z / 4 \pi k r^{2}$ to give the true spherical-candle power of a lamp, $L$, which is being measured, $z$ being the area of the zone of the reference sphere which is associated with the horizontal beam, and $4 \pi r^{2}$ being the total area of the reference sphere.

The theory of the Matthews integrating photometer is here given in full on account of the fact that this photometer is destined to come into general use.

133. The problem of illumination. - A room may be said to be well lighted when the eye is easily able to distinguish, in minute detail of perception, the various objects in the room. Completeness of visual perception depends upon three conditions, namely, (a) a sufficient brightness of the illumination; (b) a proper location of the light sources, so as to bring out that combination of soft shadows which is so essential to the perception of form, and (c) a proper composition * of the light, so as to bring out those physical differences in objects which the eye perceives as variations of color.

A room may be intensely illuminated by a single arc lamp, but such illumination is ineffective, even when the eye is shaded from the direct light of the lamp, because the excessive harshness of the shadows renders the perception of form almost impossible; on the other hand a sufficiently intense and properly distributed light, which contains certain wave-lengths in great excess, may be ineffective because of the unusual or weak color effects produced thereby. For example, the light of an ordinary kerosene lamp is very deficient in the shorter wave-lengths ; these shorter wavelengths have much to do with the bringing out of blue and violet tints; and, consequently, a deep blue or violet piece of cloth appears almost black by kerosene lamp light. False color values are produced in a very striking way by the light from the mercury-vapor lamp on account of the almost complete absence of the longer wave-lengths (red) in the light from this lamp. The brilliant white light of the carbon arc lamp, on the other hand, contains all wave-lengths in about the same proportion as

* The composition of light refers to the relative intensities of the various wavelength components of the light. 
sun light, and all colors show up well by the light of such an arc lamp.

Glare. - Excessive contrast of light and shade in the field of vision tends to hinder visual perception. The eye adapts itself automatically to the brightest lights in the field of view and all perception of detail in the shadows is lost. This blotting out of detail in the shadows by excessively brilliant lights in the field of vision, which is called glare, is especially marked when the field of vision includes a bright unshaded lamp. The explanation of glare is as follows: In the first place, the pupil of the eye contracts greatly when there is a bright light in the field of vision and this contraction lessens the effective brightness not only of the "high lights" but also of the deep shadows; in the second place the sensitiveness of the retina seems to be greatly reduced in bright light, and, while this reduction of sensitiveness may leave the eye able to perceive detail in the more brilliantly illuminated portions of the field of vision it tends to obliterate all detailed perception in the shadows; and in the third place an intense beam of light entering the eye from a bright source illuminates the whole interior of the eye, just as a beam of sunlight entering a window illuminates a room, and this diffused light in the eye illuminates and excites the portions of the retina where the images of the shadows fall, and thereby obliterates all detail of perception.

An interesting case involving excessive contrast is that in which a workman at a loom, for example, has his immediate work illuminated to a fair degree of brightness while the remainder of the room is left in darkness. If the workman could keep his eyes fixed upon his work incessantly, it is conceivable that this kind of illumination might be satisfactory; but the eye moves about in spite of everything one can do, and under the assumed conditions the workman would be unable to see when he glanced about the room and he would be blinded when he glanced at his work. To avoid this impracticable situation a general illumination of the room is necessary. 
It is very important in arranging for the illumination of a room to place the lamps outside of the field of vision, if possible, so that no light can enter the eye directly from the lamps and render the eye insensible to the delicate shading of surrounding objects. The excessive discomfort that is produced by the glare of improperly located lamps, such for example as the exposed foot-lights of a poorly arranged stage, is due not only to the physical pain that is associated with long continued looking at a bright light, but more especially to the incessant strain of trying to peer into the dark region beyond.

Where a lamp cannot be removed from the field of vision the bad effects of glare may be greatly reduced by enlarging the effective luminous surface of the lamp by means of a translucent globe. A translucent globe always absorbs a considerable portion of the light of a lamp, but the effectiveness of the globe in eliminating glare is due primarily to the fact that a given amount of light coming from a small brilliant source produces a much greater glare than the same amount of light coming from a large faint source.

Dim lamps versus brilliant lamps. - A much more satisfactory distribution of light in a space to be illuminated may be obtained by using several lamps of moderate brightness than by using one or two lamps of great brightness. Thus very bright lamps are not suitable for illuminating small rooms because the one or two lamps required to produce the desired quantity of light give a very unsatisfactory distribution, and the number of lamps required to give a satisfactory distribution would produce an excessive amount of light. To give a satisfactory distribution of light over the field of vision in the lower portion of a room, very bright lamps should be raised to a considerable height over head. In general, therefore, very bright lamps are unsatisfactory. except for lighting very large high rooms and for street lighting; such lamps may, however, be used satisfactorily in moderately small rooms when the indirect system of illumination is employed. In this system all of the direct light from the lamp or lamps is thrown 
upon the ceiling of the room and the diffused light reflected from the ceiling produces a beautiful soft illumination in the lower portion of the room.

Infuence of absorption upon illumination. - An illuminated surface, such as the wall, ceiling, or floor of a room, or the surface of an object in the room, absorbs a definite fractional part of the light which falls upon it. This fraction is called the coefficient of absorption of the illuminated surface. The lamps in a room emit a given flux of light and at the instant the lamps are . turned on the intensity of the illumination in the room is quickly increased by repeated reflections of the light from the illuminated surfaces until the rate of absorption of light by the illuminated surfaces is equal to the rate of emission of light by the lamps. Given, for example, two rooms, $A$ and $B$, illuminated by the same number of lamps. Suppose that the two rooms have the same area of walls and objects to be illuminated, but suppose that the illuminated surfaces in room $A$ absorb an average of 40 per cent. of the light which falls upon them, whereas the illuminated surfaces in room $B$ absorb an average of 80 per cent. In both rooms the same amount of light is emitted by the lamps and therefore the same amount of light is absorbed by the illuminated surfaces ; but this absorbed light is only 40 per cent. of the mean intensity of illumination in room $A$ whereas it is 80 per cent. of the mean intensity of illumination in room $B$; therefore the mean intensity of illumination in room $A$ is twice as great as it is in room $B$.*

Flux of light required for effective illumination. - The total flux of light, in spherical-candles, required for the effective illumination of a given-sized room depends upon the manner in which the light is distributed, upon the composition (color) of the light, and upon the mean coefficient of absorption of the illuminated surfaces as pointed out above. Interior lighting is usually accomplished by lamps which give a soft yellow light, the lamps are usually

* In general the total flux of light required to produce a given mean intensity of illumination in a room is proportional to $a b$, where $a$ is the combined area of all the surfaces to be illuminated and $b$ is the mean coefficient of absorption of these surfaces. 
distributed over-head so as to be as much as possible out of the field of vision, and walls and ceiling are usually yellowish-white. Under these conditions about 0.2 of a spherical-candle is required for each square foot of floor area to give a degree of illumination that would be considered satisfactory in a reception room or in a lecture hall. If the ceiling is very high a greater candle-power is required inasmuch as the area of the walls is increased. If the ceiling and walls are very dark, if they are made of stained oak - or cherry paneling, for example, effective illumination may require 0.4 or 0.5 of a spherical-candle per square foot of floor area.

Street lighting by arc lamps.* - Arc lamps consuming about 500 watts each are usually placed 200 or 300 feet apart in American cities, and the height of the lamps above the street is usually 20 or 30 feet. In many cases, especially in streets having shade trees, smaller units placed nearer together give much better illumination.

Old style open-arc street lamps for all night burning have two sets of carbons, and when one set burns out the other set is thrown into circuit automatically. Such lamps are trimmed daily, one man being able to trim about 80 lamps per day.

The number of hours of operation per year of street lamps varies greatly in different cities. Thus the all-night schedule, lamps operated every night from 30 minutes after sunset to 30 minutes before sunrise, aggregates 3,950 hours per year in New York City; the aggregate time varies slightly with latitude. In the so-called moonlight schedule the lamps are operated about 2,100 or 2,200 hours per year.

134. The electric lamp. - The production of light by means of the electric current depends upon the heating by the current of a portion of an electric circuit to incandescence, and the electric lamp consists essentially of an element which is so heated, and in some cases, of special regulating devices. Electric lamps may

* See Electrical Engineer's Pocket Book, H. A. Foster, D. Van Nostrand, I903 (third edition), for information on arc lamps and on arc-lighting schedules, pages 393-402 and 414-422. 
be conveniently classified under three heads, namely, $(a)$ glow lamps, in which the heated element is a solid electrical conductor in the form of a rod or filament; $(b)$ vapor lamps, in which the light is given off by a highly incandescent or luminescent column of vapor through which the electric current flows; and $(c)$ the ordinary carbon-arc lamp, in which the light is given off chiefly by the tips of the carbons which are raised to an extremely high temperature by the faintly luminous but excessively hot column of conducting vapor between them.

Under the head of glow lamps are included the ordinary carbon-filament lamp, several varieties of metal-filament lamps, and the Nernst lamp in which the glower is a rod of porcelain-like material made of a mixture of the rare earths.

Under the head of vapor lamps are included the recent types of luminous-arc lamps in which the electric arc is charged with metallic vapor and is highly luminous, the mercury-vapor lamp, and the Geissler-tube lamp of Macfarlane Moore.

135. Lamp efficiency. - In practice the efficiency of a lamp is always specified by giving the watts consumed in the lamp per spherical-candle of light flux emitted. Thus the carbon-filament glow lamp of the kind that is at present most widely used consumes about 3.6 watts per spherical-candle. The actual efficiency of a lamp is of course the ratio of the light energy emitted by the lamp to the total energy supplied to the lamp. The actual efficiency is the greater the smaller the so-called efficiency in watts consumed per spherical-candle.

Ängström * has found that that part of the horizontal radiation of a Hefner lamp which lies within the limits of the visible spectrum represents a flow of 8.I ergs per second across one square centimeter at a distance of one meter from the lamp, so that one spherical-hefner corresponds to a flow of $4 \pi \times \mathrm{IOO}^{2} \times 8$. I ergs per second or $\mathrm{O} . \mathrm{IO} 2$ watt. This corresponds to O.I I 5 watt for one spherical-candle. The power value of the sphericalhefner (or candle) depends, however, to some extent upon the

* Physikalische Zeitschrift, Vol. III., p. 257, 1902. 
composition (color) of the light. The value of 0 . I 5 watt per spherical-candle applies to the orange-colored light from the Hefner lamp, and this value can be considered only as an approximation to the power value of one spherical-candle of yellowish light, or of pure white light, or of bluish light.

Taking O.II 5 watt as the approximate power value of one spherical-candle, it follows that the ordinary carbon-filament glow lamp which takes 3.6 watts per spherical-candle (about 3.I watts per mean horizontal candle) has an actual efficiency of about 3 per cent., an ordinary enclosed-arc lamp which takes 2.2 watts per spherical-candle has an actual efficiency of about 5.2 per cent., a luminous-arc lamp which takes I. 2 watts per sphericalcandle has an actual efficiency of about 9.6 per cent., and a mercury-vapor lamp which takes 0.6 watt per spherical-candle has an actual efficiency of about 19.2 per cent.

The thermodynamics of radiation. - If the light-giving element of a lamp were to conform to the laws of radiation which apply to an ideal black body, then the efficiency of the lamp would be a definite and well-known function of the temperature* of the element, increasing with the temperature and reaching a maximum of 90 or 95 per cent. at a temperature of about $4600^{\circ} \mathrm{C}$. This temperature is far greater than any known solid or liquid substance can stand.

All substances depart more or less from the laws of radiation which apply to an ideal black body. This is especially the case with gaseous substances, and the light efficiency of the radiation emitted by such substances is not a well defined function of the temperature. Indeed, the idea of temperature applies strictly to substances in thermal equilibrium, only, and the column of luminescent vapor in a mercury-vapor lamp, for example, cannot be said to have any temperature at all, that is to say, the luminescent state of the vapor could not be reproduced by raising the vapor to any assignable temperature whatever.

136. The carbon-arc lamp. - The arc lamp is the earliest form of electric lamp. It consists of two carbon rods arranged so that an electric current may flow from the one to the other forming what is called an electric arc between them, and a more or less elaborate mechanism for moving one or both of the carbon rods so as to keep the arc fairly steady. The electric arc consists of

* A very good resumé of the thermodynamics of the electric lamp is given by $\mathrm{E}$. F. Roeber in the Transactions of the American Electrochemical Society, Vol. VIII., pp. $243-267,1905$. 
a column of intensely heated vapor which conducts the electric current across from one carbon rod to the other.

An interior view of a typical direct-current enclosed-arc lamp is shown in Fig. 180, and the same lamp is shown in Fig. 181 with its casing and outer globe complete. This lamp is designed to operate across constant-voltage mains, it has a resistance coil connected in series with the arc, and the arc is enclosed in a

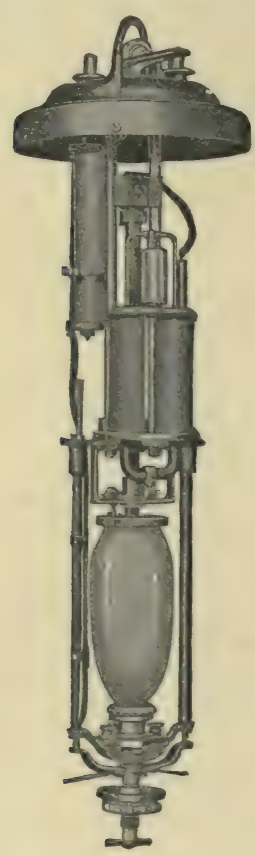

Fig. 180 .

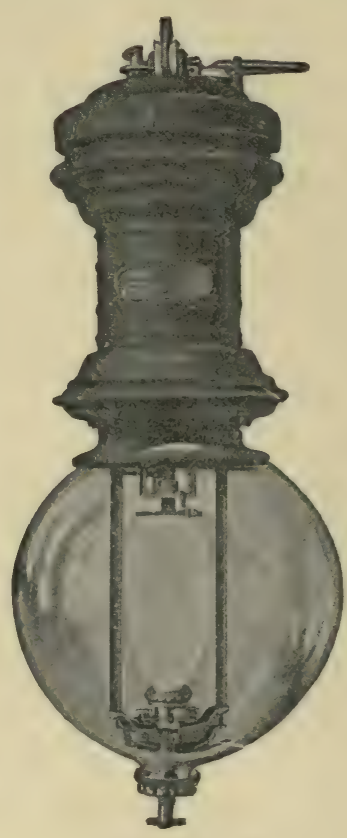

Fig. 181.

nearly air-tight bulb of thin opal glass into the ends of which the carbon rods project.

The mechanism of this lamp is operated by a plunger-type electromagnet with its winding in series with the arc. The plunger of this electromagnet actuates a lever to which is fixed a clutch that grips the upper carbon. When the lamp switch is open the clutch lies against a trigger which releases the carbon and allows it to fall and make contact with the lower carbon. 
When the lamp switch is closed the current which starts to flow draws in the plunger and the clutch catches the carbon and lifts it, thus forming the arc. As the carbons waste away by combustion due to the oxygen of the air, the arc lengthens slightly, the current decreases and the consequent movement of the plunger lowers the upper carbon slowly. When the limit of this downward movement is reached the clutch touches the trigger and allows the carbon to fall when the sudden increase of current pulls the plunger and lifts the carbon again as at the start, and so on.

The alternating-current arc lamp differs somewhat from the direct-current lamp, as explained later.

Arc lamps which are operated in series by a constant current, direct or alternating, have a more complicated mechanism than that which is described above. The actuating electromagnet usually has two windings, one of which is in series with the arc and the other is shunted across the arc, and a device called a cutout is arranged for automatically establishing a by-pass for the current when the carbons are burned out or when the lamp gets out of order.

The operation of the series arc lamp is briefly as follows: The first rush of current through the lamp flows almost wholly through the series magnet-winding and the tendency of this current is to separate the carbons more and more; but, as the carbons separate, a larger and larger current flows through the shunt magnet-winding, which, by opposing the series winding, sets a limit to the length of the arc. Then as the carbons waste away the current in the shunt winding still further increases, more and more nearly neutralizing the series winding and allowing the upper carbon to move slowly downwards until finally the clutch which grips the carbon rod is released by a trigger and the carbon falls and is lifted again as at the start, and so on. There is a variety of series arc lamp mechanisms and the above description applies to the arc lamp having what is called a differential actuating magnet. 
137. Volt-ampere characteristics of the electric arc.* - The electric arc, whether it be between rods of pure carbon, or rods of carbon impregnated with metallic salts, or rods of metal, always has this property that the greater the current the less the voltage across the arc, except when the arc is very short. Thus the coördinates of the curves in Figs. I 82 and 183 give some idea of the relation between the current that flows in an arc and the voltage across the arc. The exact relation between current and voltage depends upon the diameter of the carbon rods, upon the degree of purity of the carbon, upon the texture of the car-

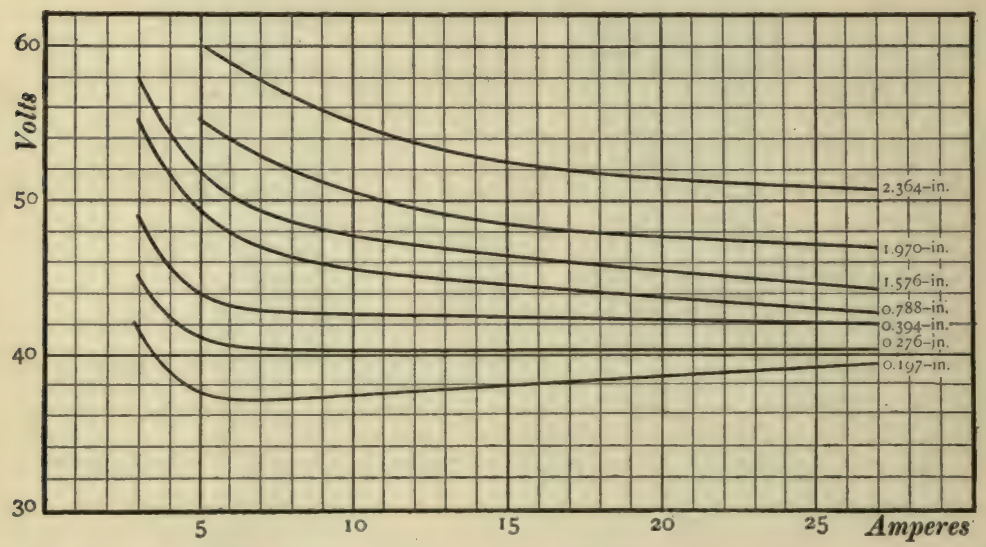

Fig. 182. Volt-ampere characteristics of an open direct-current arc between cored carbons one half inch in diameter.

bon, upon the freedom of circulation of the surrounding air, and upon the nature of the current (direct or alternating), but the relations shown in Figs. 182 and 183 are typical. The numbers attached to the various curves indicate the length of the arc in inches.

When the current flowing in an arc is increased, the arc, that is to say the conducting column of heated vapor, increases in sec-

* For a discussion of the physics of the electric arc, see C. P. Steinmetz, Trans. International Electrical Congress, Vol. II., pp. 710-730, St. Louis, 1904. Also see W. R. Whitney, Trans. American Electrochemical Society, Vol. VII., pp. 29I-299, 1905. 
tional area, and no doubt this increase in sectional area has to do with the decreased voltage required to maintain the arc with increased current.

When the current becomes very large the arc alters greatly in character, emits a loud hissing noise, and constitutes what is called a hissing arc. The transition from the steady arc to the hissing arc does not take place at a sharply defined value of the current; in fact the arc is very unstable in the region of transi-

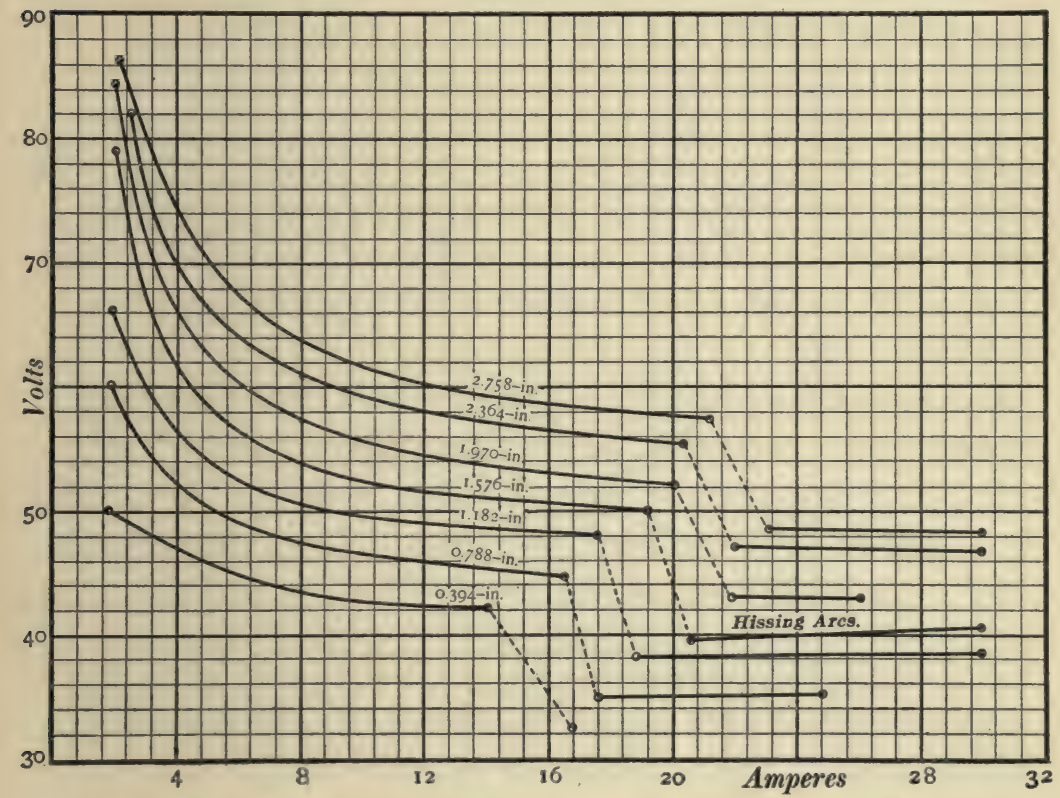

Flg. 183. Volt-ampere characteristics of an open direct-current arc between solid carbons one half inch in diameter.

tion, and when the change to the hissing variety takes place, the current has to be greatly reduced in value to change the arc back to the steady type. This instability of the arc in the region of transition from the steady arc to the hissing arc is indicated in Fig. 183 by the broken portions of the curves, and it must not be confused with the kind of instability that is discussed in the following paragraph. 
The instability of the electric arc when the current is supplied from constant-voltage mains. An arc cannot be maintained between carbon rods connected to constant-voltage mains unless a resistance is connected in series with the arc. This is evident from the volt-ampere characteristic of the arc as follows: If one could imagine an arc burning steadily between constant-voltage mains, then a slight decrease of current would produce a contraction of the arc and an increased voltage would be required to maintain the arc; but this increased voltage would not be forthcoming hence the current would continue to decrease and the arc would go out almost instantly. On the other hand, a slight increase of current would produce an increase in the sectional area of the arc, and the constant voltage would be more than sufficient to maintain the arc, hence the current would continue to increase, rising almost instantly to an excessively large value. These changes are much too rapid to be controlled by the lamp mechanism which moves the carbon.

If a resistance is connected in series with the arc, a decrease of current causes a decrease of the voltage-drop, $R i$, in the resistance, and an increase of current causes an increase of voltagedrop, so that, if the resistance is sufficiently large the change of $R i$ will overbalance the change of voltage across the arc due to any assumed change of current, and the arc will be stable. A resistance must always be connected in series with an arc lamp which is supplied with current from constant-voltage mains. A resistance used in this way is called a ballast. When an arc lamp is operated from constant-voltage alternating-current mains, an inductance, consisting of a coil of wire wound on a laminated iron core, may be and generally is used as a ballast instead of a resistance.

When arc lamps are connected in series and supplied with current from a constant-current generator (or, in the case of alternating current, from a constant-current transformer), no ballast need be used.

The direct-current arc. - The foregoing paragraphs apply in a 
general way to both direct-current arcs and alternating-ourrent arcs. There are, however, differences between direct-current and alternating-current arcs which are of considerable practical importance. In a direct-current arc the positive carbon becomes slightly concave and the negative carbon becomes pointed, as shown in Fig. I 84 . The concave end of the positive carbon is called the crater, it is excessively hot, and it emits a great amount of light. The point of the negative carbon is not nearly so hot as

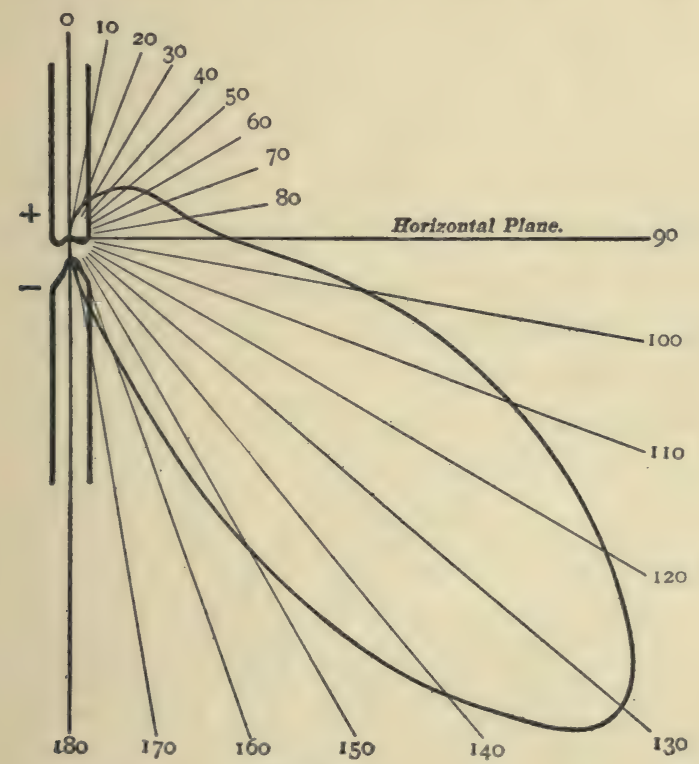

Fig. 184.

the crater so that it emits very much less light, and the column of hot conducting vapor, the arc itself, emits a pale violet light. The result is that the light emitted by a direct-current arc is distributed as indicated by the curved line in Fig. I84. In directcurrent arc lamps the upper carbon is generally the positive carbon so that the greater amount of light may be thrown downwards where it is needed. The direct-current arc lamp which is used in search-lights and projecting lanterns is arranged as shown in Fig. 185 . 
The positive carbon wastes away much faster than the negative carbon, usually about twice as fast. This is due largely to the

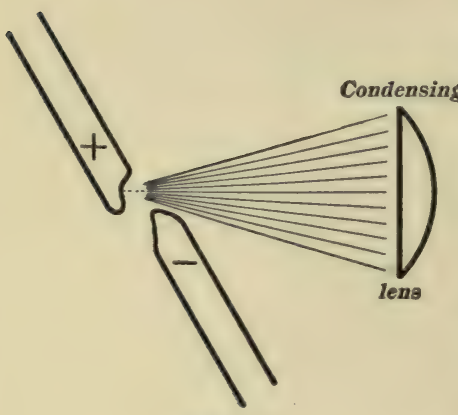

Fig. 185.

higher temperature of the positive carbon which causes it to be burned more rapidly by the oxygen of the air; and it is partly due to something akin to electrolytic action in the arc, for carbon is carried continuously across the arc from the positive carbon to the negative carbon.

The direct-current arc, when it is operating properly, does not make very much noise, although the pulsations of current due to the passage of the successive segments of the commutator under the dynamo brushes always produce an audible tone.

The alternating-current arc. In an alternating-current arc the ends of the carbons are heated nearly to the same extent, the upper carbon being perhaps the hotter of the two, on account of the upward movement of the heated air and vapor. The result is that light is emitted about equally from the ends of both carbons, and the light is distributed as indicated by the curved line in Fig. 186.

On account of the great amount of light that is thrown upwards by an alternating-current arc a

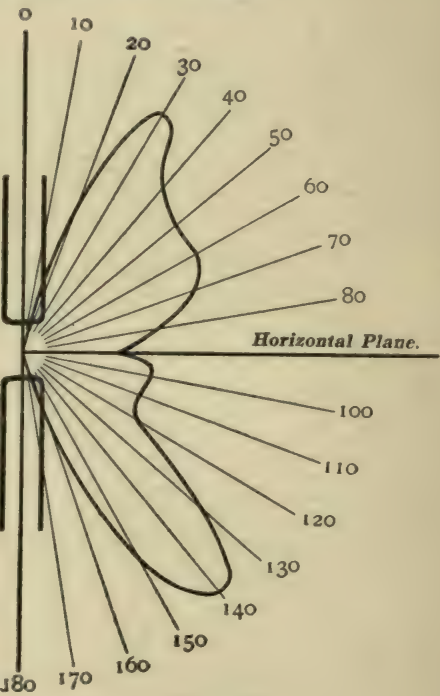

Fig. 186. reflector is usually employed with an alternating-current arc lamp to throw the light downwards.

The light emitted by an alternating-current arc lamp is not 
perfectly continuous, but it pulsates with the rise and fall of the alternating current. Ordinarily these rapid pulsations of the light are not perceptible unless one looks at a moving object which is illuminated by the lamp.

The two carbons of an alternating-current arc lamp waste away at approximately the same rate; the upper carbon, however, is always consumed a little more rapidly than the lower carbon.

The alternating-current arc makes a loud humming noise on account of the repeated heating and cooling and consequent expansion and contraction of the column of conducting vapor.

The alternating-current arc is much more unstable than the direct-current arc, because the alternating current passes repeatedly through zero value and therefore the conducting column of vapor cools slightly and the next pulse of current encounters at first an increased resistance. Cored carbons are therefore generally used in alternating-current arc lamps, that is to say, carbons which have a central core of soft carbon slightly impregnated with metallic salts, the vapors of which are good electrical conductors. The metallic vapors from cored carbons tend, however, to form a coating on the bulb of enclosed arc lamps, and one cored and one solid carbon are therefore generally used in enclosed alternating-current arc lamps.

The instability of the alternating-current arc is very great at low frequencies on account of the longer time that the current is very small before and after each reversal of current; in fact, it is not feasible to operate alternating-current arc lamps at frequencies much lower than about 60 cycles per second.

An important result of the increase of the resistance of the alternating-current arc after each passage of the current through zero is that the power in watts delivered to the arc is less than $E I$, where $E$ is the electromotive force across the arc as measured by an alternating-current voltmeter, and $I$ is the current as measured by an alternating-current ammeter. In fact, the power is equal to $p E I$, where $p$, which is less than unity, is what is called the power factor of the arc. The value of $p$ for the alternating- 
current arc in open air is about 0.85 when the frequency is 60 cycles per second.

Differences between direct-current and alternating-current arc lamps. - The differences which depend upon the peculiarities of the direct-current arc and the alternating-current arc are described above. In the alternating-current lamp the electromagnet that operates the lamp mechanism has a laminated core, and an inductance or reactance coil is used instead of resistance as a ballast when the lamp is connected to constant-voltage mains.

The alternating-current lamp is inherently noisy. The noise of the arc is, however, quite effectually smothered by the bulb of the enclosed arc lamp. The rattling noises due to the vibration of the cores of the electromagnet and of the reactance coil may be greatly reduced by clamping the laminations firmly together, and by suspending the electromagnet on supporting springs to prevent the transmission of its vibrations to the metal case.

The slow consumption of the lower, or negative, carbon in the direct-current enclosed-arc lamp causes only a slight downward displacement of the arc as the lamp continues to burn, the lower carbon being fixed in a rigid support. The approximately equal rates of consumption of the two carbons in an alternating-current enclosed-arc lamp, on the other hand, causes a very considerable downward displacement of the arc as the lamp continues to burn if the lower carbon is held in a rigid support. Therefore the alternating-current arc lamp must have either a mechanism which feeds both carbons, or a long enclosing bulb so that after the lamp is freshly trimmed a considerable downward displacement of the arc is allowable.

138. Enclosed arc lamps versus open arc lamps. - A large item of expense in the maintenance of old style arc lamps, in which the arc burns in the open air, is the cost of the frequent trimming (after about eight hours burning) necessitated by the rapid wasting away of the carbons. This item of expense is greatly reduced by the use of enclosed arc lamps which require trimming after about I 50 hours burning. 
The development of the enclosed arc lamp was due, however, chiefly to the attempt to devise an arc lamp which would operate efficiently across standard I IO-volt mains. Efficient operation in this case depends upon the use of a long arc with a small current so that the greater part of the supply voltage may be expended in the arc, the ballast being barely large enough to make the arc stable. It is impossible however to operate a long low-current arc in the open air, because such an arc is blown out by the least draft of cold air; but when the arc is enclosed in a nearly air-tight bulb this difficulty is avoided. Thus an 80-volt enclosed arc may be satisfactorily operated across I IO-volt mains in series with a ballast which absorbs about 30 volts, and a I 5Ovolt enclosed arc may be operated satisfactorily across 220 -volt mains in series with a ballast which absorbs about 70 volts. These figures are exemplified in commercial enclosed arc lamps for I IOvolt circuits and for 220-volt circuits respectively, I IO-volt lamps usually take from $5 \mathrm{r} / 2$ to $6 \mathrm{x} / 2$ amperes and 220 -volt lamps usually take from $23 / 4$ to $3 \frac{x}{4}$ amperes, according to the adjustment of the spring which opposes the pull of the electromagnet that operates the lamp mechanism.

Another advantage of enclosing the arc of a lamp is that the noise of the arc is thereby muffled. This is especially important when alternating current is used.

The enclosed-arc lamp is now used in all new installations, except those in which the luminous-arc lamp is employed.

Arc lamp rating. - An arc lamp should be rated on the basis of voltage, current, and watts. In fact this mode of rating is generally used with arc lamps of recent design, such as enclosedarc lamps and luminous-arc lamps, and the manufacturer generally specifies a certain consumption of watts per spherical-candle of light emitted. Old style open carbon-arc lamps have however long been rated in candle power. Thus the open carbon-arc lamp which has 47 volts between its terminals with 9.6 amperes flowing through it has an accepted nominal rating of 2,000 candle power, although, as a matter of fact, its spherical-candle power is about 700 . 
139. Arc lamp globes, shades, and diffusers. - The old style open arc lamp is always provided with a globe to shield the arc from drafts of air which are likely to blow the arc out, and the hot enclosing bulb of an enclosed arc lamp is usually shielded from gusts of cold air by an outer globe when it is used out of doors. Thus Fig. I8 I shows an enclosed arc lamp with a clear glass globe for protecting the enclosing bulb.

The intense brilliancy of the electric arc produces a glare that is very unpleasant, even when an arc lamp is suspended at a considerable height overhead out of doors. Therefore the bulb of an enclosed arc lamp is nearly always made of opal glass, and when an enclosed arc lamp is used for inside lighting the enclosing bulb is generally surrounded by an outer opal globe or shade for still further reducing the glare. Thus Fig. I 87 shows an enclosed arc lamp arranged for inside lighting with a shade underneath the enclosing bulb and a large corrugated diffuser above.

Lamp globes are used not only to protect the arc and to eliminate glare, but also to throw the light in a desired direction.*

140. Series grouping of arc lamps across constant-voltage mains. - Where several arc lamps are to be operated continuously, that is, where it is not desired to turn individual lamps off and on, the lamps may be arranged in series groups if the supply voltage is 220 volts or more. Thus it is common practice to operate enclosed arc lamps in series groups of two, across 220-volt mains ; and in series groups of five, across 550 -volt mains. In such cases it is necessary, because of the constant-voltage supply, to insert ballast resistance in circuit to steady the lamps as explained in Art. I37. Failure of one lamp to operate, however, breaks the circuit and puts the entire group of lamps out of service, and if such a result is to be avoided each lamp must be provided with an automatic cut-out arranged to establish a by-pass through an

* An exhaustive series of experimental studies of globes and reflectors is given in a paper entitled "A Photometric Comparison of Illuminating Globes," by R. B. Williamson and J. H. Klinck, Journal of the Franklin Institute, Vol. 149, p. 66, 1900. 
equivalent resistance when the lamp fails to operate. This automatic cut-out, together with the resistance which it substitutes for the lamp, is sometimes mounted in a case entirely separated from the lamp as shown in Fig. I88. When the circuit in the lamp is broken, for example by the failure of the carbons to feed, the cur rent ceases to flow and the cut-out magnet releases its armature which establishes a by-pass through the substitute resistance.

When very many lamps are operated in series the automatic cut-out is a necessity, inasmuch as the very high voltage that is

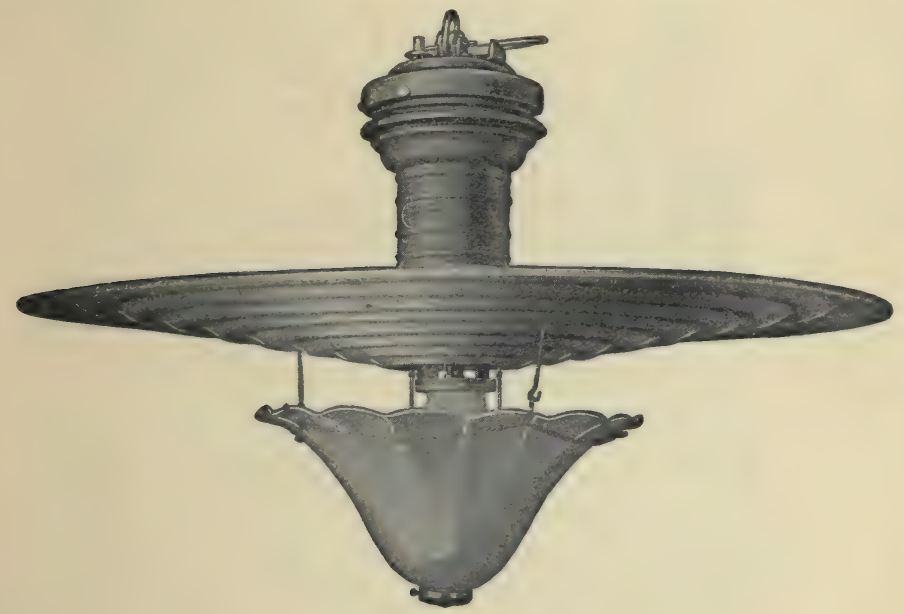

Fir. 187.

used in this case would be likely to force the current through an inoperative lamp, especially if the lamp mechanism includes a shunt winding on its electro-magnet, and the lamp would be destroyed.

141. Constant-current series systems of arc lighting. - Where many arc lamps are to be operated continuously, as in the lighting of city streets, it is best to connect the lamps in series and supply them with constant current. This arrangement is exemplified by numerous old style direct-current open-arc street-lighting plants now in operation, and by the more recently installed series systems using enclosed-arc lamps or luminous-arc lamps. 
The tendency is to favor the use of alternating current for operating street lamps for the reason that a constant current can be delivered to the arc-lamp circuit through a special transformer from the same generator that supplies a constant-voltage distributing system, whereas the use of direct current requires a separate generator. Fig. 189 is a view of the General Electric Company's constant-current transformer for taking a varying current from constant-voltage supply mains and delivering a constant cur-

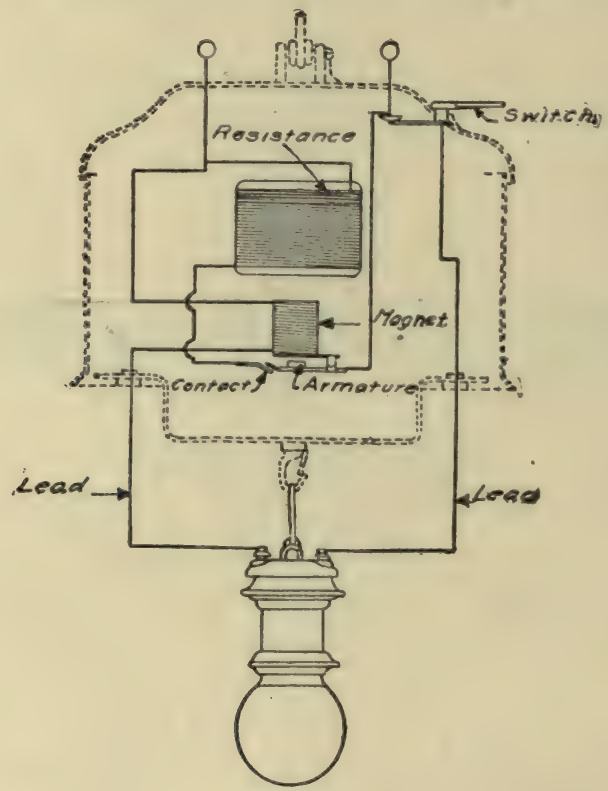

Fig. 188.

rent at varying voltage to a receiving circuit. The action of this transformer is discussed in the second volume of this text.

A general view of the improved* Brush direct-current generator for supplying constant current to arc lamps is shown in Fig. I9O.

When many arc lamps are operated in series it is necessary

* The multi-circuit Brush generator, American Electrician, Vol. XI., pp. 436437, September, 1899 . 
to provide each lamp with an automatic by-pass cut-out, as explained in Art. I40, but no by-pass or ballast resistances are needed. Also when many lamps are operated in series by a constant current the electromagnet which operates the mechanism of each lamp must have a shunt winding connected across the arc as well as a series winding in circuit with the arc.

142. Luminous-arc lamps.* - The light from the carbon-arc lamp comes mostly from the intensely heated tips of the carbons

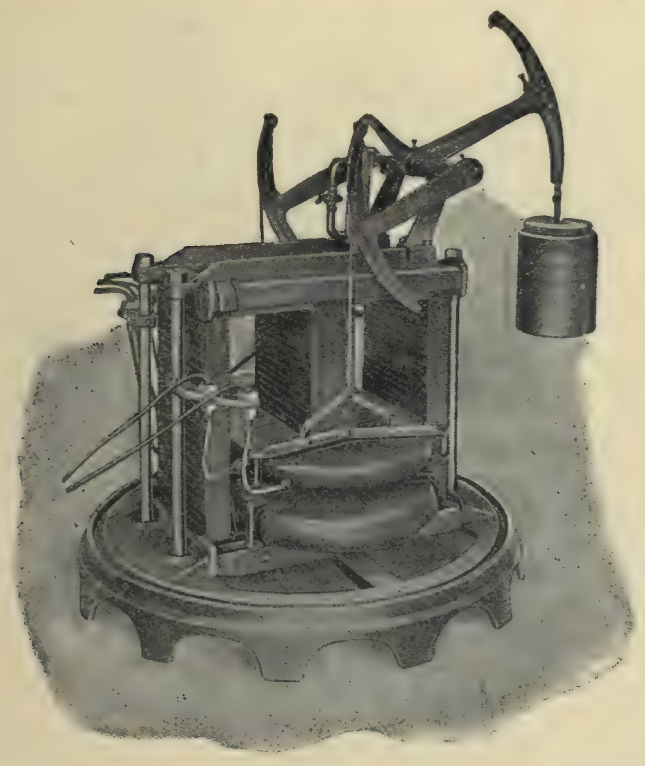

Fig. 189

and the arc itself emits only a pale violet light. The use of carbons strongly impregnated with metallic salts, or the use of rods of metal or of metal oxide instead of carbon, gives an arc which is charged with metal vapor. Such arcs are as a rule brilliantly luminous, and an arc lamp employing such an arc is called a luminous-arc lamp.

* See paper by C. P. Steinmetz, Trans. International Electrical Congress, Vol. II., pp. 7 10-730, St. Louis, 1904 ; and also paper by André Blondel, ibid., pp. $731-767$. 
An objection to the luminous-arc lamp is that a dense smoke is produced by the condensation and oxidation of the metallic vapors, so that the arc cannot be enclosed and the lamp cannot be conveniently used indoors.

The great advantage of the luminous-arc lamp is its high efficiency. Such a lamp consumes from 0.8 to 1.2 watt per spherical-candle of light flux, whereas an ordinary enclosed carbon-arc lamp consumes from 2.0 to 2.2 watts per spherical-candle.

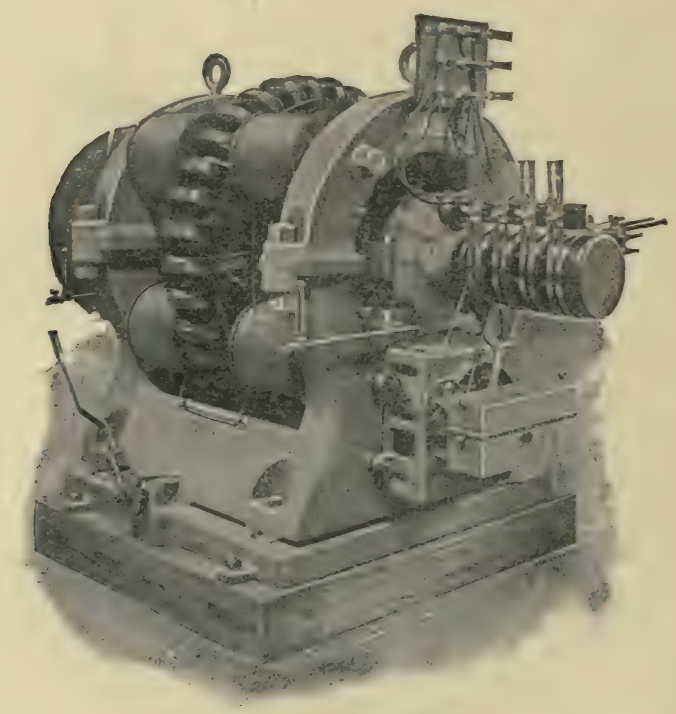

Гg. 190.

In the luminous-arc lamp of Bremer the arc is formed between carbon rods which are highly impregnated, chiefly with salts of calcium. This lamp gives a brilliant yellow light which can be made to approach a pure white by using salts of other metals.

In the luminous-arc lamp of the General Electric Company the arc is formed between a negative terminal (cathode) of iron oxide and a positive terminal (anode) of metal, usually copper. The iron oxide is contained in a thin sheet-iron tube and is fed like the carbon rod of the carbon-arc lamp. The copper anode is a rod which is held in a rigid support. 
143. The carbon-filament glow lamp. - The ordinary incandescent lamp consists of a fine filament of carbon mounted in a highly exhausted glass bulb through the walls of which platinum lead-wires are sealed for connecting the carbon filament to the supply mains. The flow of current through the filament heats it to a high temperature and it emits a steady soft light that is well adapted to inside illumination.

The present process of manufacture is briefly as follows : Clean cotton fiber is dissolved with constant stirring in an aqueous solution of zinc chloride forming a thick syrup-like fluid which is freed from lumps by filtering and from bubbles by heating nearly to the boiling point under reduced pressure. This fluid is then "squirted" through a small hole and the small gelatinous rod is received in alcohol which quickly hardens it so that it can be thoroughly washed in water, wound on a drum and dried. The result is a fairly tough wire or thread of pure cellulose having the appearance of celluloid. This thread is cut into lengths, bent on formers into the desired shape of the lamp filament, packed in charcoal, and carbonized in a furnace. The carbonized filaments are then mounted on the platinum lead-wires and heated electrically to a high temperature one at a time in the vapor of a hydrocarbon such as gasolene. The hot filament decomposes the hydrocarbon vapor and causes a deposit of graphitic carbon on the filament, thus increasing the size of the filament slightly and lowering its resistance to the exact value desired. This process is called "flashing." The finished filament is then placed in the glass bulb which is exhausted and sealed.*

The resistance of the carbon filament of a glow lamp is about half as great when it is at its working temperature as it is at $20^{\circ} \mathrm{C}$., the exact amount of variation depending upon the amount

* See an article on incandescent-lamp manufacture, by Manning K. Eyre, The Electrical World, January 5, 1895.

A modified process of manufacture has recently been developed by the General Electric Company. See a paper by J. W. Howell, "A New Carbon Filament," read at the Ashville Convention of the Am. Inst. of Electrical Engineers, June, 1905, and to be published in volume 24 of the Transactions. 
of graphitic carbon that is deposited upon the filament by the flashing process. Near the working temperature the resistance of the filament does not vary greatly with temperature and the variation, such as it is, may be an increase or a decrease of resistance with rise of temperature according to the amount of carbon deposited on the filament by the flashing process.

144. Relation between voltage, candle-power, efficiency and life of carbon-filament glow lamps. - The voltage of a lamp as specified by the manufacturers is the electromotive force which it is intended should be applied to the terminals of the lamp in service. The effect on the lamp of increasing or decreasing this electromotive force is considered in this article.

Candle-power. - Owing to the difficulty ${ }^{*}$ of measuring the true spherical-candle-power of a lamp it is customary to rate glow lamps on their mean horizontal candle-power, that is, on the candle-power which is indicated by the Bunsen photometer when the lamp is rotated about a vertical axis at a speed of three or four revolutions per second. Thus the curve in Fig. I75 represents the distribution of light around a glow lamp of which the mean horizontal candle-power is 16 and of which the true spherical-candle-power is 13.33 . The spherical-candle-power of a glow lamp is usually from 80 to 85 per cent. of the mean-horizontal candle-power.

Efficiency. - The efficiency of an electric lamp is usually specified by giving the watts consumed per candle-power. $\nmid$ The usual efficiency ratings of carbon-filament glow lamps range from 3 . I to 3.5 watts per mean horizontal candle. High-efficiency lamps (low watts per candle) should be used where the cost of power is high, and low-efficiency lamps should be used where power is cheap.

* It is not difficult to measure true spherical-candle-power with a Matthews integrating photometer; all manufacturers and all large users of glow lamps should use this photometer and base the ratings of their lamps on spherical-candle-power.

t See discussion of lamp efficiency and the thermodynamics of radiation in Art. 135. 
Life. - The carbon filament of a glow lamp deteriorates in service, on account of what seems to be a vaporization of the carbon, and the candle-power and efficiency decrease. The falling off of candle-power is due in part to the fact that as the filament grows smaller its resistance increases, the power delivered to it at the standard voltage is reduced, and the working temperature is lowered so that the light tends to become red instead of a yellowish-white, and it is due in part to the blackening of the bulb by the condensation of the carbon vapor. The falling off in efficiency is due partly to the reduced working temperature of the filament and partly to the absorption of light by the blackened bulb.

A glow lamp frequently becomes very dim and inefficient before the filament actually breaks, and it is usually advisable to discard a lamp and replace it by a new one when the candle-power has fallen to a certain fraction of its initial value. The useful life of a glow lamp is usually reckoned as the number of hours of actual service that the lamp will stand before its candle-power drops to 80 per cent. of its initial value.

The changes of candle-power and efficiency of a glow lamp with the change of voltage between its terminals depend to some extent upon the nature of the filament (ratio of amount of carbon deposited by the flashing process to the amount of carbon in the filament before flashing); and the change of life with the change of voltage depends upon the size of the filament (low candlepower with given voltage, and high voltage with given candlepower, watts per candle being constant, mean a fine filament). However, the following table may be used to estimate the approximate variation of candle-power, efficiency, and life of any ordinary carbon-filament glow lamp. This table is based on the I6-candle-power, Ioo- to I25-volt lamp taking 3.I watts per candle, and giving I 4.7 average candle-power during its useful life of 5 ro hours.

It is to be noted that the basing of this table on the 3.I watt lamp is largely a matter of convenience. In fact the table gives 
the details of behavior of lamps ranging from 4.39 watts per candle to 2.28 watts per candle.

Variation of Candle-Power, Efficiency and Life, * and Costs.

\begin{tabular}{|c|c|c|c|c|c|c|}
\hline 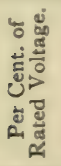 & 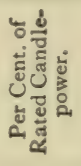 & 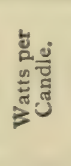 & 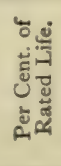 & 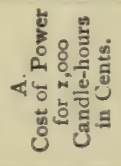 & 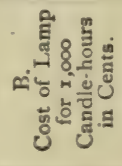 & 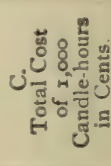 \\
\hline 90 & 56 & 4.39 & 830 & 43.9 & .30 & 44.2 \\
\hline $9 \mathrm{I}$ & 60 & 4. 27 & 650 & 42.7 & .38 & 43. I \\
\hline 92 & 63 & 4.19 & 540 & 41.9 & .45 & 42.3 \\
\hline 93 & 67 & 4.00 & 430 & 40.0 & .57 & 40.6 \\
\hline 94 & 72 & 3.84 & $33^{\circ}$ & 38.4 & .74 & 39.1 \\
\hline 95 & 76 & 3.68 & 270 & 36.8 & $.9 \mathrm{r}$ & 37.7 \\
\hline 96 & 80 & 3.56 & 225 & 35.6 & 1.09 & 36.7 \\
\hline 97 & 85 & 3.43 & 180 & 34.3 & 1.36 & 35.7 \\
\hline 98 & 90 & $3 \cdot 31$ & 147 & $33 . \mathrm{I}$ & 1.66 & 34.8 \\
\hline 99 & 95 & 3.20 & 120 & 32.0 & 2.02 & 34.0 \\
\hline 100 & 100 & 3. 10 & 100 & 31.0 & 2.45 & 33.4 \\
\hline IOI & 105 & 3.01 & $8 I$ & 30. I & 3.03 & 33.1 \\
\hline 102 & I I I & 3.90 & 68 & 29.0 & 3.61 & $3^{2.6}$ \\
\hline 103 & 117 & 2.81 & 57 & 28. I & 4.30 & 32.4 \\
\hline 104 & 123 & 2.73 & 47 & 27.3 & 5.22 & 32.5 \\
\hline 105 & 130 & 2.64 & $3^{8}$ & 26.4 & 6.45 & 32.8 \\
\hline 106 & 136 & 2.57 & 32 & 25.7 & 7.66 & 33.4 \\
\hline 107 & 143 & 2.48 & 27 & 24.8 & 9.07 & 33.9 \\
\hline 108 & I 49 & 2.42 & 23 & 24.2 & 10.64 & 34.8 \\
\hline 109 & I56 & 2.35 & 20 & 23.5 & 12.25 & 35.7 \\
\hline IIO & 164 & 2.28 & 17 & 22.8 & 14.42 & 37.2 \\
\hline
\end{tabular}

Life of 3.1 watt-per-candle lamp is 5 Io hours.

The cost per hour of the light supplied by a glow lamp includes the cost per hour of the electrical power, and the first cost of the lamp divided by the life of the lamp in hours. Column $A$ in the above table is the cost per hour of the power for I,O0O candles; column $B$ gives the lamp cost multiplied by $\mathrm{I}, \mathrm{OOO}$ and divided by the product of candle-power and life in hours; and column $C$ gives the total cost per hour of $\mathrm{I}, 000$ candles. In calculating these costs electrical power is reckoned at Io cents per kilowatt-hour, the cost of a 16 candle-power lamp is taken as 20 cents, the life at

* From General Electric Company, Harrison, N. J., March, 1905. Candle-powers in this table are mean horizontal candle-powers. 
3. I watts per candle is taken as 5 Io hours, and the falling off of the candle-power of a lamp with age is ignored. Under these conditions the total cost of the light is a minimum for an efficiency of about 2.8 watts per candle with a life of 290 hours. The costs given in the above table take no account, however, of the trouble involved in the replacing of old lamps by new ones, which is sometimes very considerable, and therefore the most satisfactory efficiency is somewhat more than 2.8 watts per candle.

The remarkable property of the carbon-filament glow lamp, as shown in the above table, is that great changes of candle-power, efficiency, and life are produced by a slight change of voltage. Thus a one per cent. increase of voltage steadily maintained causes a five per cent. increase of candle-power, about three per cent. decrease in the watts per candle, and about twenty per cent. shortening of life. It is this great sensitiveness of the carbonfilament glow lamp to changes of voltage that makes it necessary to avoid great changes of voltage in the operation of such lamps.

In the purchase of glow lamps the purchaser should specify voltage, candle-power, and efficiency, and of course also the style of base. In meeting these specifications the manufacturer must of course be allowed some lee-way for it is impossible to make large numbers of lamps exactly alike. It is the usual practice for the manufacturer to submit to a purchaser a package of 200 lamps rated at the desired voltage, candle-power and efficiency. The purchaser may then select at random from this package ten lamps to be tested at the rated voltage. If the tests show that any one of the ten lamps gives a candle-power 7.5 per cent. above or below the rated candle-power, or if the average candle-power of the ten lamps is more than 2.5 per cent. above or below the rated candlepower, or if any one of the ten lamps consumes more than 5.5 per cent. above or below the rated watts, or if the average consumption of the ten lamps is more than 2.5 per cent. above or below the rated watts, the whole package of 200 lamps may be rejected by the purchaser. The extent to which the individual lamps in a lot deviate from rated candle-power and watts is shown 
by Fig. I9I in which the coördinates of the dots represent the candle-power and watts of the individual lamps.

145. Metal-filament glow lamps.* - In the first attempt to make a commercial glow lamp fine platinum wire was used for the lamp filament but it was soon found that the filament was very short-lived at the temperature necessary to give a fairly high efficiency. The next step was to use a carbon filament and from

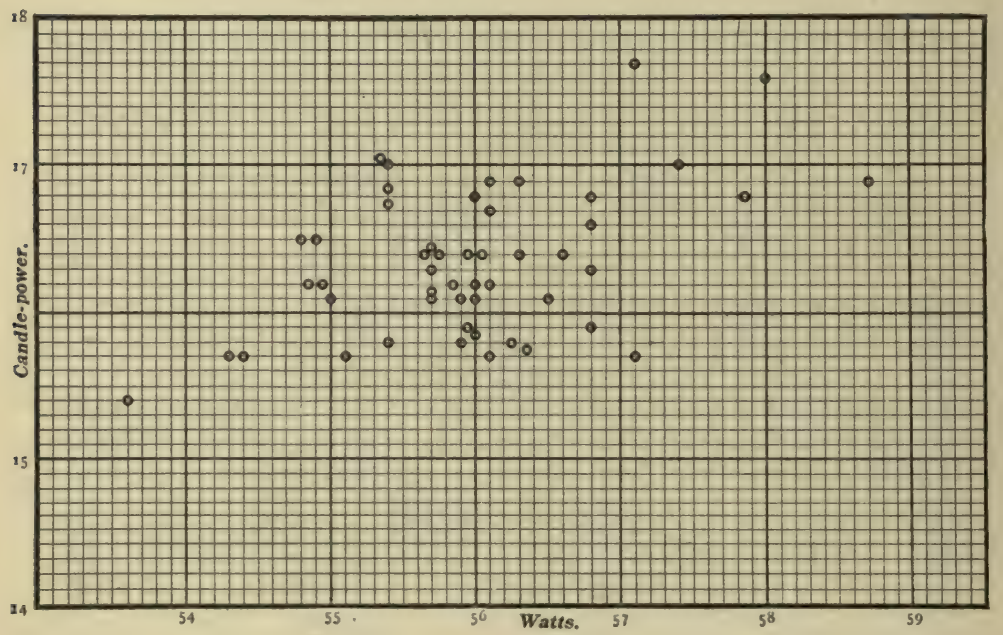

Fig. 191.

about I 88 I to I 900 the carbon-filament lamp was the only rival of the arc lamp.

It has been long known, however, that many metals have higher melting points than platinum, some of them very much higher; but the most refractory metals are extremely brittle at ordinary temperatures and the difficulty of forming these metals into fine wires prevented their being even tried as lamp filaments. Now, however, processes have been developed for the formation

* For a description of the osmium-filament glow lamp see Fritz Blau, Electrotechnische Zeitschrift, Vol. 26, pp. 196-199, February 23, 1905. For a description of the tantalum-filament glow lamp see von Bolton and Feuerlein, Electrotechnische Zeitschrift; Vol. 26, pp. 105-108, January 26, 1905. 
of fine compact filaments of the most refractory and brittle metals, and there are now on the market tantalum-filament glow lamps and osmium-filament glow lamps. These lamps are characterized by the very long and very fine filaments required even for voltages as low as 37 volts, they are not so sensitive to changes of voltage as the carbon-filament glow lamp on account of the fairly rapid increase of resistance of the metal filaments with rise of temperature, and they can be operated satisfactorily at a higher efficiency than the carbon-filament glow lamp. The osmium lamp, for example, can be operated at I.6 watts per sphericalcandle with a useful life of I,500 hours or more, and the tantalum lamp can be operated at I.6 watts per spherical-candle with a useful life of 500 or 600 hours. By useful life is meant the time of continuous operation before the candle-power falls below 80 per cent. of its initial value.

146. The Nernst lamp.* - The luminous element or glower of the Nernst lamp is a small rod of porcelain-like material composed of a mixture of the oxides of the metals of the yttrium group. This rod is as good an insulator as glass or porcelain at ordinary temperatures; like glass or porcelain it becomes a fairly good conductor at a low red heat; and its resistance decreases very rapidly as the temperature rises. Therefore the glower must be heated to a low red heat by external means before current starts to flow through it, and a ballast resistance must be connected in series with the glower to prevent the current from rising indefinitely when once it starts to flow. The initial heating of the glower is accomplished by a fine coil of platinum wire, called the " heater," which is covered with a protecting paste somewhat similar to ordinary whitewash, and when the current starts to flow through the glower it actuates an electromagnet which breaks the heater circuit. The ballast is made of very fine iron wire

* A full discussion of the Nernst lamp is given by A. J. Wurts, Transactions of the American Institute of Electrical Engineers, Vol. XVIII., pp. 545-587, I901.

The process of manufacture of the Nernst lamp is described in The Electrical World and Engineer, Vol. XLIII., pp. 981-985, May 21 , I904. 
mounted in a small glass bulb which is filled with inert gas to protect the iron from oxidation.

The Nernst lamp gives a beautiful white light and its efficiency is claimed to be greater than that of the carbon-filament glow lamp.

147. The mercury-vapor lamp.*-The mercury-vapor lamp consists of a highly exhausted glass tube into the ends of which are sealed two platinum lead-wires. One of these wires carries a large electrode (the anode) of iron or graphite, and the other makes contact with a pool of mercury (the cathode). Either a very high electromotive force or a special starting device is required to start a current flowing through the tube, but when once started a current of several amperes, is maintained by an electromotive force of from 30 volts to IOO volts and the mercury vapor throughout the tube becomes brilliantly luminous. The mercury-vapor lamp of Cooper-Hewitt is started by tilting the glass tube, thus causing the mercury to extend momentarily from end to end, and the current which starts to flow through the fluid mercury continues to flow through the mercury vapor when the thread of mercury breaks. It is necessary to use a ballast resistance in series with the lamp.

The mercury-vapor lamp gives a light which is deficient in the longer wave-lengths (red) and it produces an unpleasant distortion of color values. It is, however, the best source of artificial illumination for photographic purposes and it is used extensively for lighting machine-shops, ware-houses and drafting rooms.

* See von Recklinghausen, Transactions A. I. E. E., Vol. XXII., pp. 7I-90; C. P. Steinmetz, Transactions International Electrical Congress, Vol. II., pp. 7 10730, St. Louis, 1904; E. Weintraub, Transactions American Electrochemical Society, Vol. VII., pp. 273-289, 1905. 


\section{APPENDIX A.}

\section{ELECTROMAGNETS. MAGNETISM OF IRON.}

1. Bunched windings and distributed windings. - The electromagnet, such as is used for the field magnet of a dynamo, consists of a rod of iron surrounded by a winding of insulated wire, through which an electric current flows. The iron rod is called the core of the electromagnet, and the electric current which flows through the winding of wire is called the exciting current. The exciting current tends to produce a magnetic field in the region occupied by the iron core, and the effect of this magnetizing field, as it is called, is to magnetize the core and cause a certain amount of magnetic flux to flow through it.

The iron rod or core usually forms a complete or nearly complete circuit, called a magnetic circuit, through which the magnetic flux flows.

Two distinct cases occur in the arrangement of the windings of wire upon the iron core as follows :

(a) Uniformly distributed winding. - The winding of wire may be distributed uniformly along the entire length of the iron rod or core. In this case the magnetic field which the winding tends to produce in the region occupied by the iron core has sensibly the same value at every point in the rod and is sensibly parallel to the rod at each point. The magnetizing action of such a field upon an iron rod depends simply upon the intensity of the field. Electromagnets having uniformly distributed windings are seldom used in practice except in the magnetic testing of iron, for which purpose the iron to be tested is usually made into a test ring, ${ }^{*}$ which is wound uniformly with wire.

* This applies to the ballistic method of testing iron, which is due to Rowland. In Ewing's method the test piece of iron is in the form of a long slim rcd. 
(b) Bunched winding. - The winding of wire may be bunched at one or more places along the iron core. In this case the magnetic field which the winding tends to produce in the region occupied by the iron core varies greatly in intensity from point to point in the core, and the direction of this field is not parallel to the rod at each point. The magnetizing action of such a field upon an iron rod is discussed in the next article.

Electromagnets usually have bunched windings.

2. The magnetizing action of a bunched winding. Definition of magnetomotive force. - The magnetizing action upon an iron rod of a non-uniform magnetic field, such as the magnetic field produced by a bunched winding, depends upon the average valuc, along the rod, of the component of the magnetizing field parallal to the rod. The product of this average value, into the length of the rod is called the magnetomotive force along the rod. That is:

$$
\mathscr{F}=l A
$$

in which $\mathcal{F}$ is the magnetomotive force along a rod in c. g. s. units, $l$ is the length of the rod in centimeters, and $A$ is the average value along the rod, of the component of the magnetizing field parallel to the rod. The rod may be straight or curved.

A rod passing through a magnetic field determines a certain line or path through the field, and we speak of the magnetomotive force along this path, whether the rod is there or not.

3. Proposition. - The magnetomotive force along a path in a magnetic field is equal to the work done by the magnetic field upon a unit magnet pole while the pole is made to travel along the path, or; the magnetomotive force along the path is equal to $W / m$ where $W$ is the work done by the field upon a pole of strength $m$, while the pole is made to travel along the path. That is:

$$
\mathscr{F}=\frac{W}{m}
$$

in which $\mathcal{F}$ is the magnetomotive force along a path in a magnetic 
field, and $W$ is the work done by the field upon a pole of strength $m$ as the pole travels along the path.

Proof.- The product $m A$ is the average value along the path of the component parallel to the path of the force $\mathscr{F}(=m \mathscr{H})$ with which the field acts on the pole. Therefore $\operatorname{lm} A$ is the work done on the pole by the field as the pole travels from one end of the path to the other. That is :

$$
W=\operatorname{lm} A
$$

Dividing both members of this equation by $m$, and remembering that $l A=\mathscr{F}$ according to equation (I), we have equation (2).

The following discussion of magnetomotive force is based upon the method of calculus. The usual definition of magnetomotive force along a path is that it is the work per unit pole done by the magnetic field upon a magnet pole which is carried along the path. From this definition of magnetomotive force it may be shown that the magnetomotive force along a path is equal to the line integral of the magnetic field along the path, as follows : Consider an element $\Delta l$ of a path $p p^{\prime}$, Fig. I. Let $\mathscr{H}$ represent the intensity of the magnetic field at this element and $\varepsilon$ the angle between $\mathscr{H}$ and $\Delta l$. Then the component of $\mathscr{H}$ parallel to $\Delta l$ is $\mathscr{H} \cos \varepsilon$. Let a magnet test pole of strength $m$ be moved along $\Delta l$. The force with which the magnetic field acts upon this pole is $m \mathscr{H}$, and the component of this force parallel to $\Delta l$

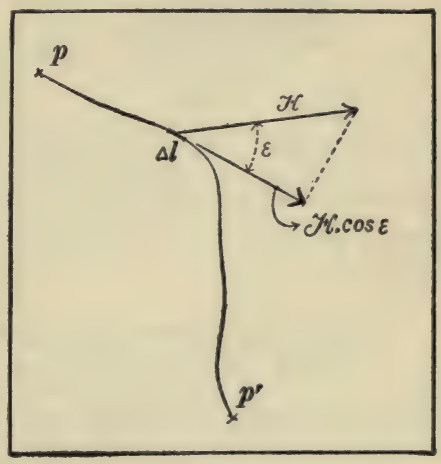

Fig. 1. is $m \mathscr{H} \cos \varepsilon$, so that $m \mathscr{H} \cos \varepsilon \times \Delta l$ is the work $\Delta W$ done by the field on the pole as the pole moves along $\Delta l$. That is :

$$
\Delta W=m \mathscr{H} \cos \varepsilon \cdot \Delta l
$$

and the total work done by the field on the pole while the pole is moved along the path from $p$ to $p$ is :

or

$$
W=m \Sigma \mathscr{H} \cos \varepsilon \cdot \Delta l
$$

$$
\frac{W}{m}=\mathscr{F}=\Sigma \mathscr{H} \cos \varepsilon \cdot \Delta l
$$

The sum $\Sigma \mathscr{H} \cos \varepsilon \cdot \Delta l$ is called the line integral of the magnetic field along the path $p p^{\prime}$. The quotient $\Sigma \mathscr{H} \cos \varepsilon \cdot \Delta l \div l$ is the average value along the path of the component of $\mathscr{H}$ parallel to the path, $l$ being the length of the path. 
4. Magnetomotive force of a coil. - The most important case of magnetization of an iron rod or core by a bunched winding of wire is the case in which the core is in the form of an endless rod which passes through, or links with, the coil as shown in Fig. 2. The magnetomotive force along the rod in this case is entirely

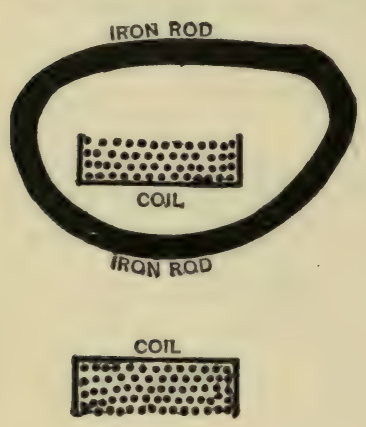

FIg. 2.

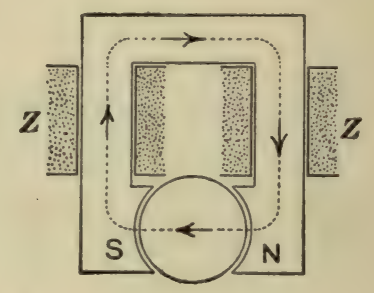

Fig. 3.

independent of the shape and length of the rod and of the shape and size of the coil. It depends only upon the number of turns of wire in the coil and upon the strength of the current in the wire.

This magnetomotive force along an endless rod which links with a coil of wire in which an electric current is flowing, is called the magnetomotive force of the coil.

Examples. - Fig. 3 shows a two-pole dynamo with two field coils $Z Z$. The field magnet iron of this dynamo together with the iron of the armature constitute a nearly endless rod or core of iron which links with both field coils, and which constitutes what is called the magnetic circuit of the dynamo.

Fig. 4 is a sketch of a four-pole dynamo with four field coils $Z Z Z Z$. The iron of the field magnet and armature presents in this case four distinct magnetic circuits, as indicated by the dotted lines. Through each of these magnetic circuits flux is forced by the combined magnetomotive force of the two field coils through which the circuit passes, that is, with which it links. 
The total magnetomotive force acting on any magnetic circuit is equal to the sum of the magnetomotive forces of the coils with which the circuit links.

It is instructive to inspect Figs. 32-39, Chap. II., and note the manner in which the field coils link with the various magnetic circuits. Thus in Fig. 33 each of the four magnetic circuits

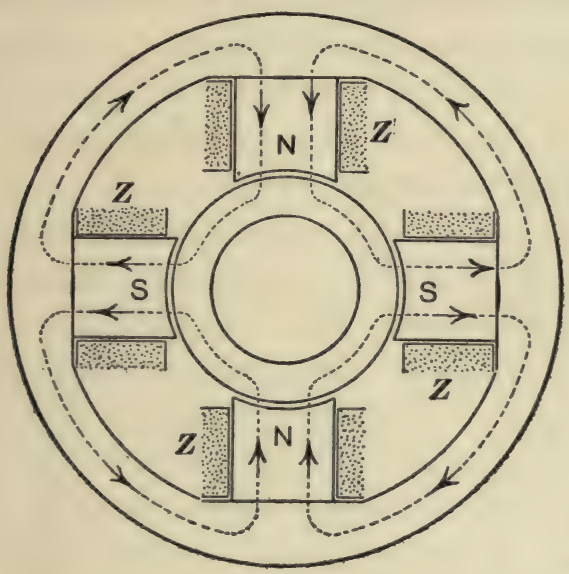

Fig. 4.

links with two field coils, and if, for example, 5,000 ampere-turns are required for each magnetic circuit, then 2,500 ampere-turns must be furnished by each field coil. On the other hand an inspection of Fig. 38 shows that each of the six magnetic circuits links with but one field coil, and if 5,000 ampere-turns are required for each magnetic circuit, then 5,000 ampere-turns must be furnished by each field coil.

5. Proposition. - The magnetomotive force of a coil is given by the equation:

$$
\mathcal{F}=4 \pi Z \imath
$$

in which $Z$ is the number of turns of wire in the coil and $i$ is the

* For proof of this equation see Nichols and Franklin's Elements of Phvsics, Vol. II., p 122. 
strength of the current in the wire in abamperes. When the current is expressed in amperes we have :

$$
\mathscr{F}=\frac{4 \pi}{10} Z i
$$

6. Units of magnetomotive force.-The product, $l \mathscr{H}$, of the length of path in centimeters and intensity of magnetic field in gausses, gives the magnetomotive force along the path (when $l$ and $\mathscr{H}$ are parallel, of course) in c.g.s. units. The name gilbert has been adopted by the American Institute of Electrical Engineers for the c.g.s. unit of magnetomotive force.

The ampere-turn. - The magnetomotive force along a path which links with one turn of wire carrying one ampere of current is called one ampere-turn. The magnetomotive force of any coil, in ampere-turns, is equal to the product of the eurrent flowing in the coil in amperes multiplied by the number of turns of wire in the coil. In magnetic calculations it is usually convenient to reduce ampere-turns of magnetomotive force to c.g.s. units (gilberts), which is done by multiplying ampere-turns by $4 \pi / 10$, according to equation $\left(3^{b}\right)$.

The product of field intensity into length of path gives magnetomotive force, so that the quotient obtained by dividing magnetomotive force by length of path is field intensity. When a magnetomotive force expressed in ampere turns is divided by length of path we have a magnetic field intensity expressed in ampere-turns-per-centimeter, or in ampere-turns-per-inch, as the case may be.

7. Magnetizing force in iron. - When an iron rod is placed in a magnetic field and is magnetized thereby, the actual magnetic field along the rod depends upon the cause of the original field and also upon the newly created magnetic poles of the rod itself. Thus when an iron rod is placed in a coil of wire through which an electric current is flowing, the field along the rod is due to the combined action of the coil and the poles of the rod. 
The magnetizing force $\mathscr{H}$ at a given point in an iron rod is defined as the resultant field intensity at the point due not only to the external cause of the original field, but also to the newly created magnetic poles of the rod. The given point must be assumed to be in air as explained later.

This influence of the poles of a magnetized rod is always such as to reduce the intensity of the original field along the rod, and it is of the nature of a reaction of the magnetized rod upon the original field.

When the iron rod is slim its poles are weak, and if the rod is long its poles are at considerable distances from the middle portions of the rod. In this case the poles of the rod do not produce any appreciable weakening of the original field along the middle portions of the rod.

When the iron rod is in the form of a ring which is magnetized by a winding of wire distributed around the ring, then the rod has no poles and consequently no demagnetizing action on itself. That is to say, the magnetic field produced by the winding of wire is in this case wholly effective in magnetizing the rod.

Example. - An iron rod 20 centimeters long is placed in a magnetic field which, but for the presence of the rod, would be a uniform magnetic field of 60 units intensity, the rod being parallel to the direction of this field. Let us suppose that the rod becomes magnetized to such a degree that the strengths of the poles at the ends of the rod are $+2,700$ units and $-2,700$ units respectively. In this case a point near the middle of the rod is approximately Io centimeters distant from each of the poles, and the actual field, $\mathscr{H}$, near the middle of the rod may be thought of as made up of three parts, namely, $(\alpha)$ the original field of 60 units intensity which is directed towards the $N$ pole of the rod, (b) the field of $2,700 / \mathrm{IO}^{2}$ units intensity due to the $N$ pole of the rod and directed away from the $N$ pole, and $(c)$ the field of $2,700 /$ I $^{2}$ units intensity due to the $S$ pole of the rod and directed towards the $S$ pole. Therefore the net result is a field of which 
the intensity near the middle of the rod is $60-27-27$ units, or 6 units.

A clear idea of the significance of magnetizing force at a point in iron, as above defined, may be obtained as follows :

Figs. 5, 6 and 7 show an iron rod inside of a long coil of wire. The magnetizing field at the point $p$, Fig. 5 , is defined as

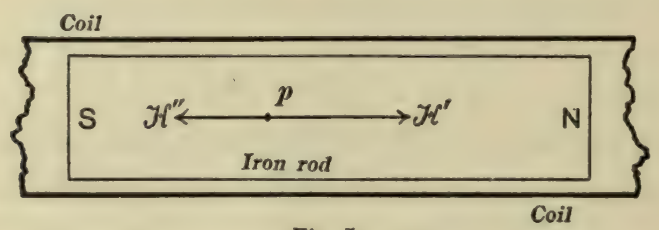

Fig. 5.

the resultant field which would be produced at $p$ by the combined action of the coil and of the poles $N$ and $S$ of the rod. The field which would be produced at $p$ by the coil alone is represented by the arrow $\mathcal{A} f^{\prime}$, the field which would be produced at $p$ by the poles $N$ and $S$ alone, is represented by the arrow $\mathscr{H}^{\prime \prime}$, and the magnetizing field at $p$ is the resultant of $\mathcal{A F}^{\prime}$ and $\mathfrak{d}^{\prime \prime}$.

The fundamental definition of the intensity of a magnetic field at a point as the force in dynes which would act upon a unit test pole placed at the point, has no meaning unless it is possible to place the test pole at the point, that is to say, the point must be in air. But if a cavity is made around the point $p$ as shown by the dotted circle in Fig. 6, the walls of this cavity will be

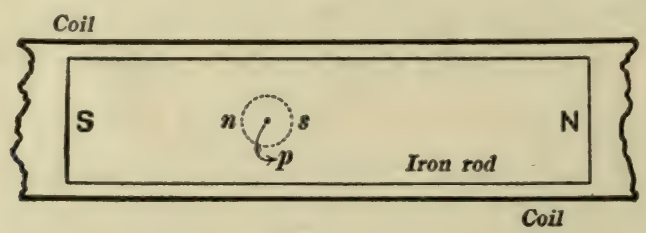

Fig. 6.

magnet poles as indicated by the letters $n, s$, and the field intensity in this cavity will not be that which is due merely to the combined action of the coil and poles $N$ and $S$. If, however, 
the cavity around $p$ is very long and narrow as shown in Fig. 7 , then the poles $n$ and $s$ at the ends of this cavity are weak and far away from $p$, so that these poles $n$ and $s$ do not produce at $p$ any perceptible field, and therefore the field intensity in the nar-

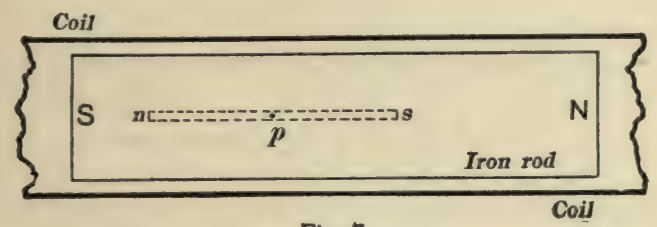

Fig. 7.

row longitudinal cavity shown in Fig. 7 is the resultant of $\mathscr{H}^{\prime}$ and $\mathscr{H}^{\prime \prime}$ of Fig. 5. That is, the field intensity in a narrow longitudinal* cavity is the actual value of the magnetizing field in iron.

8. Intensity of magnetization. - Let $m$ be the strength of the magnetic pole at the end of a magnetized iron rod of which the sectional area is $s$ square centimeters. The pole strength per unit sectional area, namely $m / s$, is called the intensity of magnetization, $\mathfrak{g}$, of the rod. That is :

$$
\mathfrak{g}=\frac{m}{s}
$$

9. Magnetic flux through a magnetized rod. - Consider a long slim iron rod, $s$ square centimeters in sectional area, placed in and parallel to a magnetic field, which, but for the presence of the rod, would be a uniform magnetic field of intensity $\mathscr{H}$. Then the actual intensity of the magnetizing force along the middle portions of the rod is $\mathscr{H}$, as explained in Art. 7. The magnetic field in the neighborhood of each end of the rod is a composite field which is the resultant of two distinct parts, $a$ and $b$; and the magnetic flux which comes up to one end of the rod, flows through the rod, and passes out from the other end of the rod, consists of two distinct parts corresponding to the two parts, $a$ and $b$, of the field near the ends of the rod.

* Parallel to the direction of magnetization of the iron. 
(a) The part $a$ is the original uniform field $\mathscr{H}$. The lines of force of this field are shown by the parallel straight lines in Fig. 8. The amount of magnetic flux which passes out from the end of the rod because of this uniform field $\mathscr{H}$ is $\mathscr{H} \mathcal{H}$ lines.

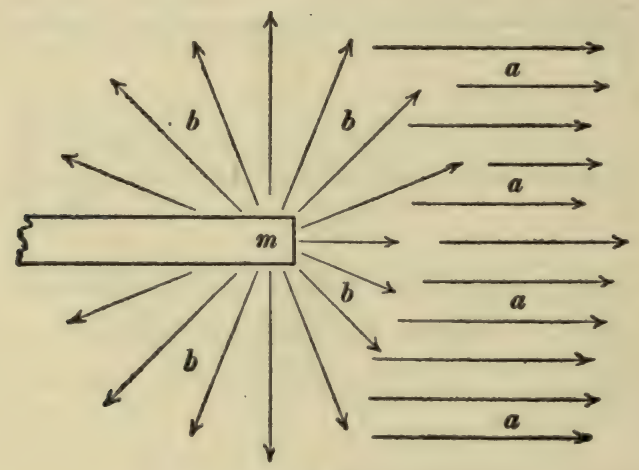

Fig. 8.

(b) The part $b$ is the magnetic field due to the pole $m$ of the rod. The lines of force of this field are shown by the radiating straight lines in Fig. 8. The amount of magnetic flux which passes out from the end of the rod because of this field is $4 \pi m$ lines, as explained in Art. 7, Chapter I.

The total magnetic flux $\Phi$ passing through the rod is equal to the sum of the two parts, $a$ and $b$, so that we have:

$$
\Phi=4 \pi m+\mathscr{I} \mathcal{L}
$$

or, since $m=\mathfrak{s} s$ from equation (4), we have :

$$
\Phi=4 \pi \mathfrak{d} s+\mathscr{H} \mathfrak{H}
$$

The part $4 \pi m$ or $4 \pi \Im s$ of the flux through an iron rod is due to the magnetized condition of the rod, and the part $\mathcal{H} S$ is due to the magnetizing force directly. This part, $\mathscr{H} S$, is the amount of flux which would pass through the region occupied by the rod if the rod were removed. 
10. Flux density in iron.* - The magnetic flux per unit sectional area of an iron rod, namely $\Phi / s$, is called the flux density $\mathscr{B}$ in the rod. That is :

$$
\mathfrak{B}=\frac{\Phi}{s}
$$

Substituting $\mathscr{B} s$ for $\Phi$ in equation (6) we have :

$$
\mathscr{B}=4 \pi \mathfrak{J}+\mathscr{H}
$$

The flux density in an iron rod is equal to the intensity of the magnetic field in a thin crevasse cut across the rod. This is evident when we consider in the first place that flux density in air is identically the same thing as field intensity according to Art. 6, Chap. I, and in the second place that a very thin crevasse does not sensibly disarrange the flux through a rod, so that the flux density in the crevasse is the same as in the rod. If the crevasse is not very narrow, a portion of the flux passes out of the rod, around the crevasse, and back into the rod again. In this case the flux density, or field intensity in the crevasse, is less than the flux density in the part of the rod which is remote from the crevasse.

11. Residual magnetism. Permanent magnets. - An iron rod retains a portion of its magnetism when it is removed from the magnetic field in which it has been magnetized, or, in case of an electromagnet, when the magnetizing current is reduced to zero. The magnetism thus left in a bar of iron, or in an electromagnet, is called residual magnetism. A long slim bar, or a bar which forms a closed or nearly closed magnetic circuit, retains a greater portion of its magnetism than a short thick bar, because of the fact that in case of a short thick bar the poles produce a strong demagnetizing field along the bar. A long slim bar or a closed ring of annealed wrought iron may retain as much as 90 per cent. of its magnetism, but a very weak demagnetizing field or a slight mechanical shock is sufficient to cause soft iron to lose nearly all of its residual magnetism. Cast iron and hard-drawn

* Frequently called magnetic induction or simply induction. 
iron wire retain a smaller portion of their magnetism, but with greater persistence, and hardened steel retains a portion of its magnetism very persistently, even when roughly handled. Magnetized bars of hardened steel are called permanent magnets.

The more persistently a sample of iron or steel retains its residual magnetism, the greater the intensity of the magnetizing force required to magnetize it. Thus hardened bars of steel are magnetized by placing them between the poles of a strong electromagnet or by placing them in a coil of wire through which a strong electric current is caused to flow.

On account of the tendency of iron to retain its magnetism, the quantity $\mathfrak{d}$ in equation (6) is not in general parallel to the magnetizing field $\mathscr{H}$, and the addition of $4 \pi \mathscr{J}$ and $\mathscr{H}$ is strictly a vector addition. However, the tendency of iron to retain its magnetism is usually neglected in practical magnetic calculations relating to electromagnets. To neglect the tendency of iron to retain its magnetism, is equivalent to assuming that $\mathscr{B}, \mathfrak{J}$ and $\mathscr{H}$ are always parallel to each other.

The one case in which the tendency of iron to retain its magnetism is considered in practical calculations of electromagnets is in the calculation of energy losses in the iron. This calculation is discussed in the articles on magnetic hysteresis.

12. Magnetization curves. - When an iron rod is acted upon by a magnetizing force of given intensity, the intensity of magnetization of the rod (and also the flux density in the rod) may have any value whatever, between certain limits, on account of the tendency of the iron to retain its previous magnetic state. Thus the intensity of magnetization of an iron rod is much greater for a given value of $\mathscr{H}$, if this given value of $\mathscr{H}$ is reached by a decrease from a higher value, than if this given value of $\mathscr{H}$ is reached by an increase from a lower value.

If, however, an iron rod is subjected to mechanical shocks, or if the magnetizing field pulsates slightly before it settles to a steady value, then the rod tends to settle to a state which depends only on the value of $\mathscr{H}$, that is, to a state which is independent 
of the previous magnetic state of the rod. Under these conditions, therefore, definite values of $\mathscr{B}$ and $\mathfrak{J}$ are produced by a given value of $\mathscr{H}$. These values are called the normal values $*$ of $\mathfrak{B}$ and $\mathscr{J}$ corresponding to the given value of $\mathscr{H}$. For example, a dynamo having a given exciting current in its field coils, tends to settle to a state in which the magnetization of its field magnet is the normal value corresponding to the given value of its field current, on account of the mechanical vibrations of the machine and on account of slight variations of magnetizing action due to slight changes of field current and of armature current.

The normal $\mathscr{I}$ and $\mathscr{H}$ curve $\dagger$ - The curve of which the abscissas represent values of magnetizing force $\mathscr{H}$ and the ordinates represent the corresponding normal values of $\mathscr{J}$ for a given kind of iron or steel is called the normal $\mathscr{J}$ and $\mathscr{H}$ curve of the iron or steel. Fig. 9 shows the normal $\mathscr{J}$ and $\mathscr{H}$ curves for ordinary annealed wrought iron and for ordinary cast iron. The intensity of magnetization, $\mathscr{J}$, approaches a definite limiting value in a given kind of iron when $\mathscr{H}$ is increased indefinitely. This limiting, or saturation, value of $\mathscr{J}$ is about $I, 730$ units pole per square centimeter of sectional area for annealed wrought iron, and about I,250 units pole per square centimeter sectional area for ordinary cast iron.

The normal $\mathfrak{B}$ and $\mathscr{A}$ curve. - The curve of which the abscissas represent values of magnetizing force $\mathscr{H}$ and the ordinates represent the corresponding normal values of $\mathscr{B}$ for a given kind of iron or steel, is called the normal $\mathscr{B}$ and $\mathscr{H}$ curve of the iron or steel. Fig. Io shows the normal $\mathscr{B}$ and $\mathscr{H}$ curves for annealed wrought iron and for ordinary cast iron. The value of $\mathscr{B}$ increases indefinitely in a given sample of iron when $\mathscr{H}$ is increased indefinitely.

* A normal curve of magnetization of iron. W. S. Franklin and S. S. Clark, Physical Review, Vol. VIII., pp. 304-309.

† Methods for determining these curves experimentally are explained in Nichols and Franklin's Elements of Physics, Vol. II., pages 134-7. 
364 ELEMENTS OF ELECTRICAL ENGINEERING.

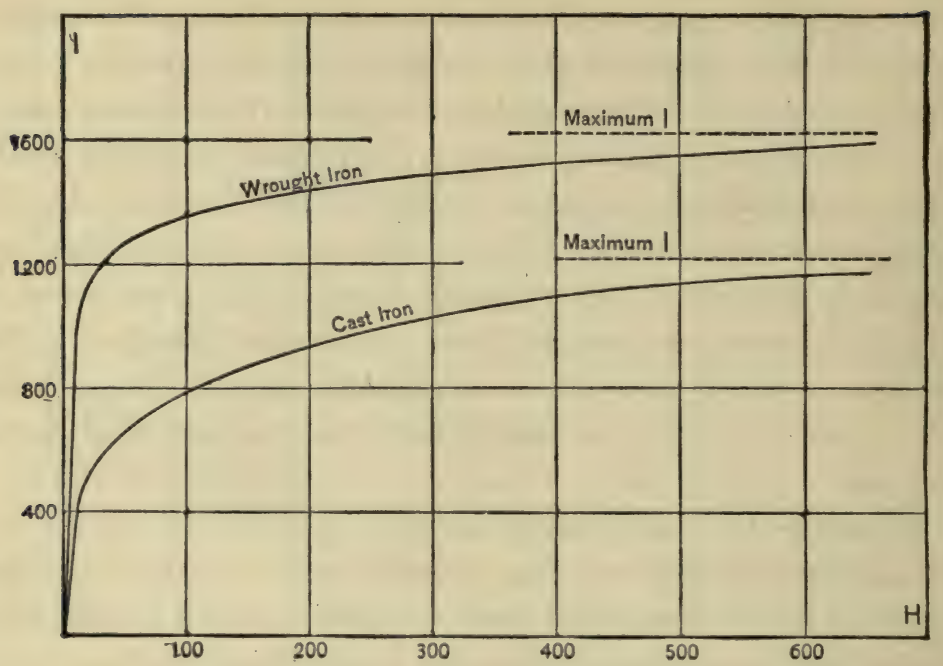

Fig. 9.

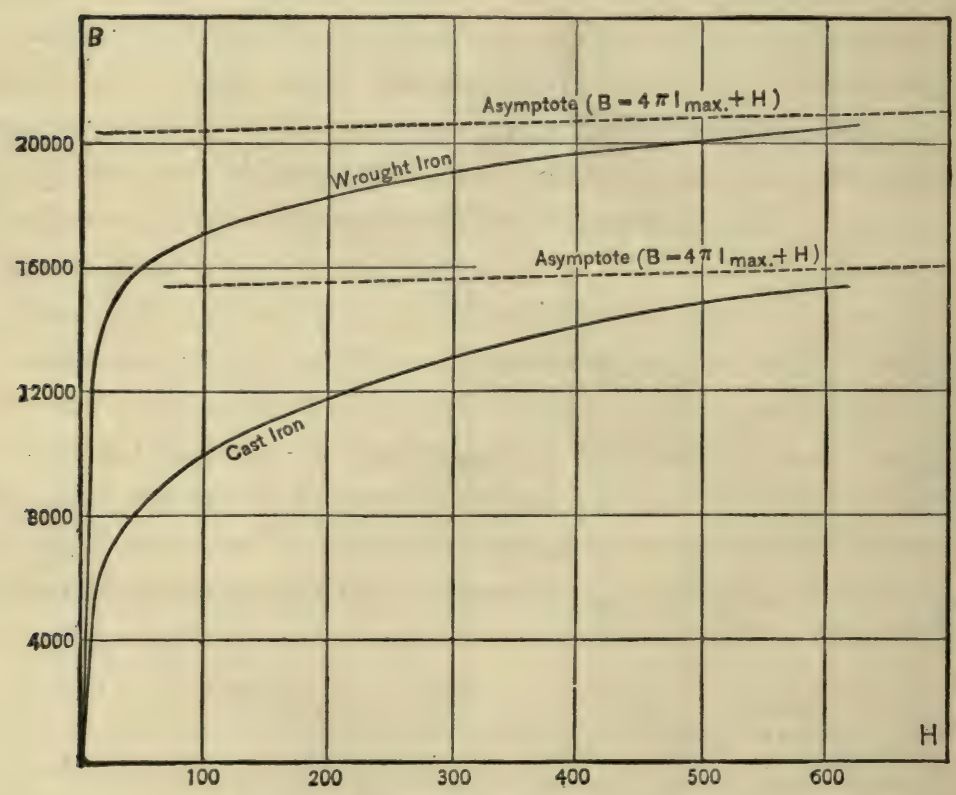

Fig. 10. 
The sharp bend in the $\mathscr{B}$ and $\mathscr{H}$ curve is called the "knee" of the curve. The iron is approximately saturated when $\mathscr{H}$ has been increased beyond the knee of the curve, and any further increase of $\mathscr{H}$ produces only slight increase of $\mathscr{B}$. Thus a magnetizing force of 10 units produces a flux density of 12,400 lines per square centimeter in a certain sample of annealed wrought iron, and a magnetizing force of double this intensity produces only I4,330 lines per square $\mathrm{cm}$., or about ten per cent. increase, in the value of $\mathfrak{B}$. Wrought iron has been subjected to a magnetizing field of about 20,000 units by Ewing who found the corresponding value of $\mathscr{B}$ to be about 40,000 lines per square centimeter, and the corresponding value of $\mathfrak{d}$ to be 1,730 units pole per square centimeter.

The accompanying table gives the corresponding values of $\mathscr{B}$ and $\mathscr{H}$ for wrought iron, for cast iron and for soft cast steel.

\section{TABLE.}

Magnetic Properties of Iron and Steel.

\begin{tabular}{|c|c|c|c|c|c|c|c|c|}
\hline \multicolumn{3}{|c|}{ Wrought Iron (Hopkinson). } & \multicolumn{3}{|c|}{ Cast Iron (M. E. Thompson)* } & \multicolumn{3}{|c|}{ Soft Cast Steel. } \\
\hline $\mathscr{H}$ & $\mathscr{B}$ & $\mu$ & $\mathscr{H}$ & $\mathscr{B}$ & $\mu$ & $\mathscr{H}$ & $\mathscr{B}$ & $\mu$ \\
\hline ro & 12,400 & I, 240.0 & ro & 5,000 & 500.0 & 10 & 9,700 & 970 \\
\hline 20 & 14,330 & 716.5 & 20 & 6,600 & 330.0 & 20 & 13,380 & 669 \\
\hline 30 & 15,100 & 503.3 & 30 & 7,290 & 246.6 & 30 & 14,500 & 483 \\
\hline 40 & $15,55^{\circ}$ & 388.8 & 40 & 7,850 & 195.0 & 40 & 15,250 & $38 r$ \\
\hline 50 & 15,950 & 319.0 & 50 & 8,360 & 169.0 & 50 & 15,840 & 317 \\
\hline 60 & 16,280 & 271.3 & 60 & 8,800 & 146.6 & 60 & 16,300 & 272 \\
\hline 70 & 16,500 & 235.6 & 70 & 9,200 & I3I. 4 & 70 & 16,750 & 239 \\
\hline
\end{tabular}

13. Magnetic permeability.-A long coil of wire produces a magnetic field of intensity $\mathscr{H}$ in its (air-filled) interior, and this field intensity is of course equal to the magnetic flux per unit sectional area, that is, to the flux density, in the air-filled interior of the coil. If the region inside of the coil is filled with iron, the flux density is many times as great as $\mathscr{H}$, as is evident from an inspection of the $\mathscr{B}$ and $\mathscr{H}$ curves of Fig. IO. From this fact

* Thompson, Knight, and Bacon, Transactions American Institute of Electrical Engineers, Vol. IX., 1892. 
there arises the idea of the multiplying effect of an iron core, insofar as the production of magnetic flux is concerned. In conformity with this idea we may write :

$$
\mathscr{B}=\mu \mathfrak{H}
$$

in which $\mu$ is the ratio of a given value of flux density $\mathscr{B}$ to the corresponding value of magnetizing force $\mathscr{H}$. This quantity, $\mu$, is called the permeability of the iron. It is of course not a constant for a given kind of iron (if it were constant the $\mathscr{B}$ and $\mathcal{H}$ curve would be a straight line), but its value varies with the flux density $\mathscr{B}$, as is clearly shown in the tabulated values of $\mathscr{H}, \mathscr{B}$, and $\mu(=\mathscr{B} / \mathscr{H})$ given in Art. I 2 .

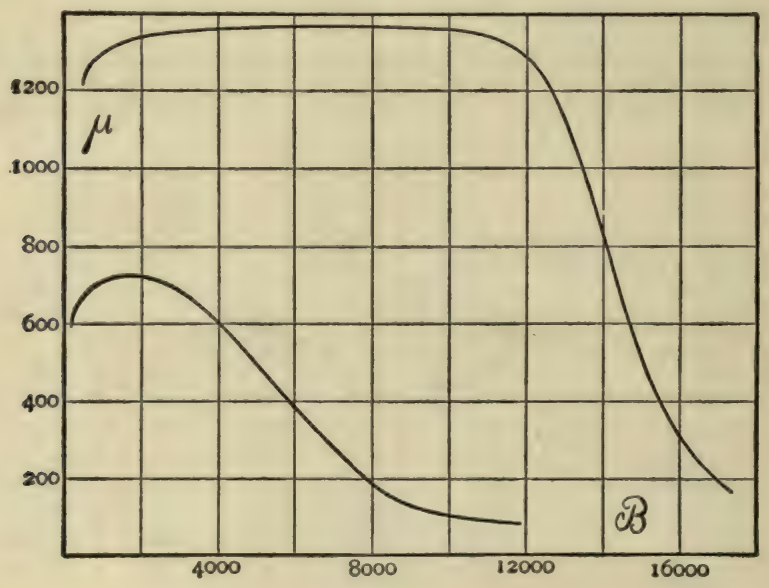

Fig. 11.

$\mathscr{B}$ and $\mu$ curves for wrought iron and cast iron.

The ordinates of the curves in Fig. I I show the values of the permeability $\mu$ of wrought iron and of cast iron for various values of the flux density $\mathfrak{B}$.

The permeability of air and of all non-magnetic materials such as copper, cotton, wood, etc., is unity.

14. Magnetic circuit calculations. - When an iron rod of uniform size and quality, which forms a closed magnetic circuit, is 
subjected to a uniform magnetizing force $\mathscr{H}$ along its entire length, the value of the flux density $\mathscr{B}$ in the rod may be found from the $\mathscr{B}$ and $\mathscr{H}$ curve, and the total flux through the rod may be found by multiplying this flux density by the sectional area of the rod. Or, the uniform value of $\mathscr{H}$ required to give a specified total flux through the rod may be found from the $\mathscr{B}$ and $\mathscr{H}$ curve after the value of $\mathfrak{B}$ has been found by dividing the specified flux by the sectional area of the rod.

When, however, the iron rod varies in size from point to point, or when different parts of the rod are of different kinds of iron, or when there is an air gap in the magnetic circuit, then some kind of an averaging process must be used to calculate the magnetomotive force required to produce a specified flux through the rod, or conversely to calculate the flux produced by a specified magnetomotive force.

This calculation is carried out as follows :

(a) To find the magnetomotive force required to produce a specified flux. - Divide the prescribed flux by the sectional area in square centimeters of each part of the circuit, wrought iron, cast iron, or air, as the case may be. This gives the flux density $\mathscr{B}$ in each part of the magnetic circuit.* Knowing $\mathscr{B}$ for each part of the circuit, take from the $\mathscr{B}$ and $\mathscr{H}$ tables or curves for the different kinds of iron, the value of $\mathscr{H}$ required to produce the known value of $\mathscr{B}$ in each part of the circuit, and multiply the value of $\mathscr{H}$ for each part of the circuit by the length in centimeters of that part. This gives the magnetomotive force required for each part, and the sum of these magnetomotive forces is the total magnetomotive force required. This total magnetomotive force may then be reduced to ampere-turns by multiplying it by $10 / 4 \pi$.

In this calculation it is to be remembered that flux density in air

* It is here assumed that the whole of the magnetic flux passes through each portion of the iron circuit. This is not always the case, for a portion of the magnetic flux may stray through the air. This portion is called the leakage flux. Magnetic leakage is discussed in a subsequent article. 
is $\mathscr{H}$, so that the flux density in the air gap is to be multiplied by the length in centimeters across the gap to give the magnetomotive force required for the gap.

(b) To find the flux produced by a specified magnetomotive force. - In the case of a rod of uniform size and quality this problem $(b)$ is simply the reverse of problem $(a)$. When, however, the parts of a magnetic circuit are of different sizes or of different materials, the flux produced by a specified magnetomotive force is best determined as follows: Calculate, as explained under $(a)$, the magnetomotive force required to produce a series of arbitrarily assumed values of flux. Arrange these results in tabular form, plot them, and trom this plot find the flux corresponding to the prescribed magnetomotive force.

15. Analogy between the magnetic circuit and the electric circuit. Definition of magnetic reluctance. - The statements given in Art. I $4(a)$ and $(b)$ are complete statements of the fundamental principles and methods of calculation of the magnetic circuit. A slight modification of the fundamental methods outlined in Art. I4 (a) and $(b)$ may be used. This modified method is based upon an analogy between the magnetic circuit and the electric circuit. It, however, contains no physical or mathematical principles in addition to those involved in the fundamental method outlined in Art. I4 $(a)$ and $(b)$, and its only advantage is that the fundamental equation (9) is rearranged so as to correspond exactly in form to the equation for Ohm's law.

The fundamental equation

$$
\mathscr{B}=\mu \mathfrak{H}
$$

when applied to an iron rod which forms a magnetic circuit, may be transformed as follows: The magnetic flux $\Phi$ through the rod is equal to $\mathscr{B} s$, whence equation $(i)$ may be written :

$$
\Phi=\mu s \mathscr{H}
$$

Now the magnetomotive force $\mathscr{F}$ along the rod is equal to $\mathscr{H l}$ where $l$ is the length of the rod. Substituting therefore $\mathscr{F} / l$ for $\mathscr{H}$ in equation (ii) we have : 


$$
\Phi=\frac{\mu s \mathscr{F}}{l}
$$

This equation may be rewritten thus:

$$
\Phi=\frac{\mathscr{F}}{\mathscr{R}}
$$

in which $\mathscr{R}$ is written for $\mathrm{I} / \mu \times l / s$. That is,

$$
\mathscr{R}=\frac{\mathrm{I}}{\mu} \cdot \frac{l}{s} .
$$

The quantity $\mathscr{R}$ is called the reluctance of the magnetic circuit, and the reciprocal of the permeability of the iron, $I / \mu$, is called its specific reluctance or reluctivity

A portion of a magnetic circuit one centimeter in length $(l=\mathrm{I})$, and one square centimeter in sectional area $(s=\mathrm{I})$, and made of a material having a permeability of unity $(\mu=\mathrm{I}$, which is the value of $\mu$ for air), has unit reluctance. The name oersted has been adopted for this unit of reluctance by the American Institute of Electrical Engineers.

Equation (IO) is exactly similar in form to the equation expressing Ohm's law, namely, $I=E / R$; and equation (I I) is similar in form to the equation for calculating the resistance of a wire, having given its length and section and the specific resistance or resistivity of its material. This analogy between the magnetic circuit and the electric circuit is, however, physically incomplete, for the magnetic reluctance of an iron circuit and the reluctivity of the iron both increase as the flux increases, whereas the resistance of an electric circuit does not vary with the current, unless changes of temperature occur.

To find the magnetomotive force required to produce a specified magnetic flux, using equations (IO) and (II), proceed as follows: Divide the total flux by the sectional area of each portion of the magnetic circuit thus finding the flux density for each portion. Knowing the flux density $\mathfrak{B}$ for each portion of the circuit take the corresponding values of $\mu$ from the table in 
Art. I 2. Knowing the value of $\mu$, the length $\iota$, and the sectional area $s$ of each portion of the circuit, calculate the magnetic reluctance $\mathscr{R}$ of each portion. Add these separate reluctances together to get the total reluctance of the entire circuit and multiply the total flux $\Phi$ by this total reluctance to get the required magnetomotive force $\mathcal{F}$, according to equation (IO).

16. Two typical examples of magnetic circuit calculations, ignoring magnetic leakage.

(a) Calculation of the amount of field excitation required for a lifting magnet, design of magnet and weight to be lifted being given.

Fig. I 2 is a sketch of a lifting magnet of which the dimensions and material of each part is given. The dotted line represents the middle line of the magnetic circuit and the length of

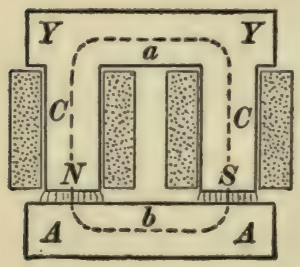

Fig. 12. this line in each part of the circuit may be taken as the mean length $l$ of that part. The flux through the cores $C C$ is so nearly uniform that the sectional area of each core is accurately the sectional area $s$ of the stream of flux in that part of the circuit. The flow of flux through yoke $Y$ and armature $A$ is not uniform, but the actual sectional areas of yoke and armature at $a$ and $b$ represent the mean sectional areas of the flux stream in yoke and armature nearly enough for practical purposes. The length of each air gap (parallel to flux stream of course) is pretty definite, but the flux spreads out greatly where it crosses an air gap and therefore the sectional area of the air gap is indeterminate. When the air gap is short, as in Fig. 12, it is sufficient to take the area of the smaller face (the ends of the cores in Fig. 12) as the sectional area of the air gap.

Let $s$ be the sectional area of each air gap in Fig. I 2 and $\Phi$ the flux crossing each gap. Then the total force in dynes with which the armature is pulled by one field core is 


$$
F=\frac{\mathrm{I}}{8 \pi}\left(\frac{\Phi}{s}\right)^{2} \cdot s \text { or } F=\frac{\Phi^{2}}{8 \pi s}
$$

and the total pull due to both field cores is

$$
F=\frac{\Phi^{2}}{4 \pi s}
$$

When the desired pull $F$ is given in dynes the requisite flux $\Phi$ may be determined from this equation, and the magnetomotive force required to produce this flux may then be calculated as explained in Art. I4 or in Art. I 5 .

(b) Calculation of the amount of field excitation required for a dynamo. - From the prescribed electromotive force, speed, and number of armature conductors $Z$, the necessary amount of magnetic flux $\Phi$ through the armature may be calculated from equation $(2 \mathrm{I} b)$ of Chapter II. The various parts of the magnetic circuit of a dynamo, namely, the yoke, the field cores, the pole pieces, the air gaps, and the armature core, do not as a rule have perfectly definite lengths in the direction of the flux, nor perfectly definite sectional areas at right angles to the flux. Therefore, it is usually desirable to make an outline sketch of the magnetic circuit of the dynamo to scale, as in the above example $(a)$, and estimate the mean length $l$, and the mean sectional area of each part of the magnetic circuit. This done, and the magnetic properties of the iron of each part being given by suitable $\mathscr{B}$ and $\mathscr{H}$ curves, the required number of ampere-turns may be found as explained in Art. 14 or in Art. I 5. Dividing the required field excitation expressed in ampere-turns by the number of turns of wire in the field windings, gives the required field current in amperes.

17. Magnetic leakage. - It has been already pointed out that in most cases magnetic flux is forced through a magnetic circuit by a bunched winding, so that the magnetomotive force is largely concentrated in one part $A$ of the circuit. Therefore the magnetic flux in passing through a portion $B$ of the circuit, which is 
remote from the seat of the magnetomotive force, tends to spread out and pass through the surrounding air as well as through the clearly defined path which is provided for it. This effect is especially prominent when, as in case of the dynamo, the portion $B$ of the magnetic circuit contains an air gap, and also when there is an opposing magnetomotive force in the portion $B$ of the magnetic circuit. Thus in the dynamo the demagnetizing action of the armature current constitutes an opposing magnetomotive force.

Let $\Phi$ be the magnetic flux which enters the armature from a north pole of a field magnet and leaves the armature at a south pole of the field magnet. This is called the useful flux. A certain amount of flux $\phi$ passes through the air from each north pole of the field magnet to the adjacent south pole, or poles, without passing through the armature. This is called the leakage flux. The total flux, $\Phi+\phi$, passes through the field magnet cores.* The ratio $(\Phi+\phi) / \Phi$ is called the coefficient of magnetic leakage of the dynamo. It is always larger than unity and it ranges in value from I.I25 to as much as 2.0. That is, the leakage flux $\phi$ is in some cases as small as 0.125 of the useful flux, and in some cases it is equal to useful flux. The coefficient of magnetic leakage of a dynamo may be determined accurately by experimental tests on the machine, or, if necessary, it may be approximately calculated from the known dimensions of the various parts of the machine.

Experimental determination of magnetic leakage coefficient. The armature of the dynamo to be tested is kept stationary during the test. The terminals of the field winding are connected to supply mains through a rheostat and a reversing switch. The object of the rheostat is to adjust the field excitation to the desired value, and the reversing switch is used for quickly reversing the field excitation. A number of turns of fine wire are wound

* In the field magnet structures shown in Figs. 32, 33, 34, 37 and 39, the whole of $\Phi+\phi$ passes through each magnet core $C$. In the structures shown in Figs. 35, $3^{6}$ and 38 , half of $\Phi+\phi$ passes through each magnet core $C$. 
around the field coils so as to surround the whole (or half) of the total flux $\Phi+\phi$, and another winding of the same number (or half the number) of turns is arranged on the stationary armature so as to surround the useful flux $\Phi$. These windings are connected to a ballistic galvanometer, one at a time, and the throw of the galvanometer is observed when the field current is reversed. The ratio of these two throws is the leakage coefficient.

Calculation of magnetic leakage.- Since magnetic leakage flux passes through the air, it is evident that the calculation of leakage flux must be based upon the calculation of the magnetic reluctances of air gaps. The magnetic reluctance of a short air gap between plane iron faces, parallel to each other or inclined, may be calculated

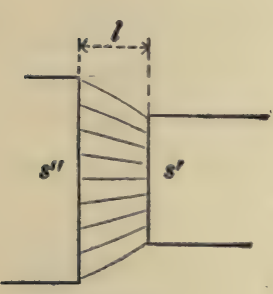

Fig. 13.

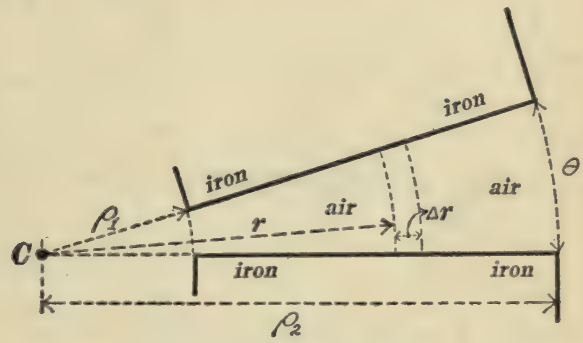

Fig. 14.

with considerable accuracy. The reluctance of a long air gap, that is a gap which is long in comparison with the length and width of the iron faces which bound it, cannot be accurately calculated by any simple formula.

(a) Magnetic reluctance of a short air gap between parallel iron faces. - In this case the sectional area of the gap is equal to, or but very little larger than, the area of the smaller face, and the reluctance of the gap may be accurately calculated by the formula :

$$
\mathscr{R}=\frac{l}{s}
$$

in which $l$ is the length of the gap in centimeters, and $s$ is its sectional area in square centimeters.

(b) Maynetic reluctance of a long air gap between parallel faces. - In this case one may use equation (12) with sufficient accuracy for most purposes, using for $s$ the value $\left(s^{\prime}+s^{\prime \prime}\right) / 2$, where $s^{\prime}$ and $s^{\prime \prime}$ are the areas of the respective faces as shown in Fig. 13.

(c) Magnetic reluctance of a short air gap between similar, rectangular, plane iron faces inclined at an angle $\theta$ as shown in Fig. 14.- - Let $C$ be the line of the intersection of the two faces, and let $\rho_{1}$ and $\rho_{2}$ be the radial distances from $C$ to the edges of the faces as shown in Fig. 14. Then 


$$
\mathscr{R}=\frac{\theta}{L \log _{e} \frac{\rho_{2}}{\rho_{1}}}
$$

in which the angle $\theta$ is expressed in radians, $L$ is the length in centimeters of the two iron faces parallel to the line of intersection $C$, Fig. 14, and $\log \rho_{2} / \rho_{1}$ represents the Naperian logarithm.

Proof of equation (13).- The lines of force in the air gap are arcs of circles with their centers at $C$. Let $\mathcal{F}$ be the magnetomotive force between the iron faces. Consider the magnetic flux $\Delta \Phi$ which crosses from face to face in the region $\Delta r$. The length of air path along which $\Delta \Phi$ crosses from face to face is $r \theta$. Dividing $\mathcal{F}$ by this length of path gives the magnetic field intensity in the region $\Delta r$, according to equation (I). The sectional area of the region $\Delta r$ is $L \times \Delta r$ so that

or

$$
\Delta \Phi=\frac{\nexists}{r \theta} \times L \cdot \Delta r
$$

$$
\Delta \phi=\frac{\mathscr{F} L}{\theta} \cdot \frac{\Delta r}{r}
$$

Integrating this expression from $r=\rho_{1}$ to $r=\rho_{2}$, we have

$$
\Phi=\frac{\mathscr{F} L}{\theta} \cdot \log _{e} \frac{\rho_{2}}{\rho_{1}}
$$

or, since $\phi=\mathscr{F} \mathscr{R}$ from equation (10), we have equation (13) at once.

(d) Magnetic reluctance of a long air gap between inclined faces. - Several modifications of equation (13) have been proposed for the approximate calculation of the reluctance of a long air gap between inclined faces. These modifications are, however, of no value. The best approximate calculations may be made as follows :

If the adjacent edges of the inclined faces are near together, always assume two equal flat rectangular faces which represent the actual faces as nearly as one can judge, and use equation (13).

If the adjacent edges of the inclined faces are not very near together, take the mean sectional area of the two faces for the mean sectional area $s$ of the gap space, estimate the mean length $l$ of the gap as the length of a smoothly curved line starting from the middle of one face at right angles to the face, and leading to the middle of the other face and at right angles to it, and use equation (12).

Note that the angle $\theta$ between the two faces I and I of Fig. 15 is $180^{\circ}$ or $\pi$. The same is true of the faces 2 and 2 , and of the faces 3 and 3 .

18. Two typical examples of magnetic leakage calculations. (a) When magnetic leakage is due to an air gap only, and not to an opposing magnetomotive force. Magnetic leakage around a dynamo armature when the armature current is zero. - In most dynamo calculations the actual useful flux $\Phi$ through the armature 
is given. The flux from a north pole piece to a south pole piece consists of two parts: (I) The useful flux $\Phi$, which passes from a north pole face across an air gap, through the armature core, and across a second air gap into a south pole face, and (2) The leakage flux $\phi$, which passes out from all portions of the surface of a north pole piece, other than the pole face, into the similar portions of the surface of a south pole piece.

To calculate $\phi$ proceed as follows : Knowing $\Phi$ find the flux density in the armature core, then take from tables (or curves) the corresponding value of $\mu$ for the armature core, and calculate the magnetic reluctance of the armature. Next calculate the magnetic reluctance of each gap space between pole faces and armature core. Add these three reluctances together and multiply by $\Phi$ to get the magnetomotive force from pole piece to pole piece. Let this be represented by $\mathcal{F}$.
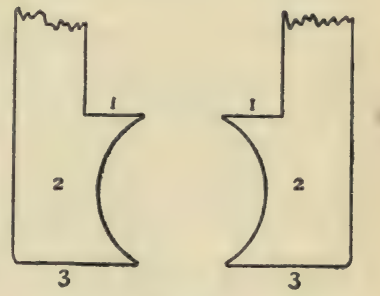

Fig. 15.

The leakage flux $\phi$ is the sum of the fluxes flowing between the surfaces I I, between the surfaces 22 (at both ends of the machine), and between the surfaces 33 , as shown in Fig. I 5 . Calculate the magnetic reluctance of each one of these elementary leakage paths with the help of equations (I2) and (13). Divide $\mathscr{F}$ by the reluctance of each path to find the leakage flux through each path. Finally, add these various leakage fluxes together to get $\phi$.

(b) When magnetic leakage is due to an air gap and an opposing magnetomotive force combined. Magnetic leakage around a dynamo armature when the armature current is not zero. - Let $\Phi$ be the flux through the armature, $\phi$ the leakage flux, $\mathcal{F}$ the magnetomotive force between the pole pieces, and $\mathcal{F}^{\prime}$ the opposing magnetomotive force due to the current in the armature. The flux $\Phi$ is supposed to be given.

To calculate $\phi$ proceed as follows: Find the magnetic reluctance of the armature core and of the gap spaces exactly as 
under ( $a$ ). Add these reluctances and multiply their sum by $\Phi$. This gives the magnetomotive force which is required to overcome the reluctance through which $\Phi$ flows. Add to this the magnetomotive force $\mathscr{F H}^{\prime}$ and we have the total magnetomotive force $\mathcal{F}$ between the pole pieces. Then calculate $\phi$ exactly as under $(a)$.

19. Magnetic reluctances in series and in parallel. - Two portions of a magnetic circuit through which the same flux flows are said to be in series. The combined reluctance of two such portions of a magnetic circuit is equal to the sum of their individual reluctances.

When two or more portions of a magnetic circuit are so related that the total magnetic flux $\Phi$ through the circuit divides and flows in part through each portion, the portions are said to be in parallel. The combined reluctance of a number of branches of a magnetic circuit which terminate in the same branch points, is equal to the reciprocal of the sum of the reciprocals of the individual reluctances.

The total flux $\Phi$ divides up among a number of parallel branches in exactly the same way that an electric current divides up among a number of parallel branches of an electric circuit.

The various leakage paths and the path of the useful flux (across gap spaces and armature core) are all in parallel between the pole pieces of a dynamo.

20. Work required to magnetize iron. - When an iron rod is magnetized by sending an electric current through a coil of wire surrounding the rod, an opposing electromotive force is induced in the coil by the growing magnetism of the rod, and the work done in forcing the current against this opposing electromotive force, is the work expended in magnetizing the rod.

The work $W$, in ergs, which is done in magnetizing $V$ cubic centimeters of iron from a given initial flux density $\mathfrak{B}^{\prime}$ to a given final flux density $\mathfrak{B}^{\prime \prime}$, is given by the equation :

$$
W=\frac{V}{4 \pi} \int_{\mathscr{O}^{\prime}}^{\mathscr{B}^{\prime \prime}} \mathscr{H} \cdot d \mathfrak{B} \text {. }
$$


Proof of equation (14). In order to avoid the complications which arise on account of the perceptible demagnetizing action of the ends of a short iron rod, let us consider a very long slim rod, $l$ centimeters in length, and $s$ square centimeters in sectional area. Suppose this rod to be placed in a long coil having $z$ turns of wire per centimeter of length, or $l z$ total turns. When the coil of wire is first connected to the battery or other source of current, the current in the coil, beginning at zero, rises in value during the time that the rod is being magnetized, and during this time the magnetic flux through the rod is growing in value. Let $d \Phi / d t$ be the rate at which the flux is increasing at a given instant, and let $i$ be the value of the current at this instant. Then $l z \times d \Phi / d t$ is the induced electromotive force in the coil which at the given instant is opposing the current $i$, so that $l_{2} \times d \Phi / d t \times i$ is the rate, $d W / d t$, at which work is being done at the given instant in magnetizing the rod. That is :

so that

$$
\frac{d W}{d t}=l s i \cdot \frac{d \Phi}{d t}
$$

$$
d W=l z i \cdot d \Phi
$$

in which $d W$ is the amount of work done during the time that the flux is increasing by the amount $d \Phi$ and while the current has the mean value $i$.

Now $\Phi=\mathscr{B} s$ or $d \Phi=s \cdot d \mathscr{B}$ from equation (7), and $z i=\mathscr{H} / 4 \pi$ from equation (9), Chapter I., so that the equation $(a)$ becomes:

or, since $l s=V$, we have

$$
d W=\frac{l s}{4 \pi} \mathscr{H} \cdot d \mathfrak{B}
$$

$$
d W=\frac{V}{4 \pi} \mathscr{H} \cdot d \mathfrak{B}
$$

or

$$
W=\frac{V}{4 \pi} \int_{\mathscr{B}^{\prime}}^{\mathfrak{B}^{\prime \prime}} \mathscr{H} \cdot d \mathfrak{B}
$$

In magnetizing a short iron rod, more work is done than is accounted for by equation (I4). The additional work goes to establish the magnetic field in the neighborhood of the magnetic poles of the rod. Equation (14) expresses the work which is spent within the iron.

\section{Graphical representation of work done in magnetizing iron.}

- Let the curve $o p p^{\prime}$, Fig. 16, be drawn so that the coördinates represent corresponding values of $\mathscr{B}$ and $\mathscr{H}$ for a given sample of iron. The branch $o p$ represents the values of $\mathscr{B}$ and $\mathcal{H}$ when the iron is magnetized for the first time, $\mathscr{H}$ beginning at the value zero and increasing until it reaches the value represented by the abscissa $a p$; and the branch $p p^{\prime}$ represents the values of $\mathfrak{B}$ 
and $\mathscr{H}$ when, after the iron has been magnetized up to the point $p$, the value of $\mathscr{A}$ is slowly reduced to zero. The curve of $\mathscr{B}$

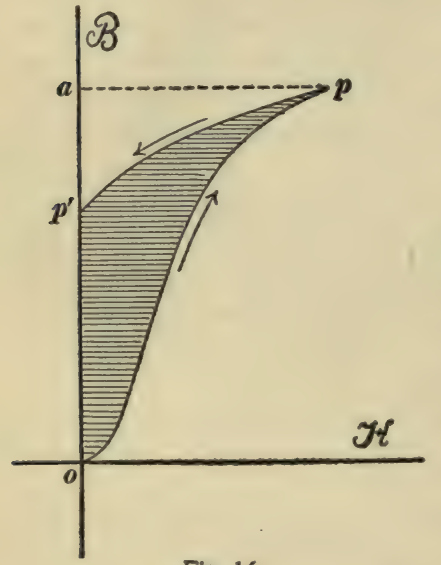

Fig. 16. and $\mathscr{H}$ for decreasing values of $\mathscr{H}$ does not coincide with the curve for increasing values of $\mathscr{H}$. Furthermore, the total area opa represents the work done upon the iron in magnetizing it up to the point $p$, and the area $p p^{\prime} a$ represents the work which is regained from the iron when the magnetizing field drops slowly to zero. The work regained is less than the work required to magnetize the iron. The work which is lost is represented by the shaded area in Fig. 16.

The scale to which area in Fig. 16 represents work, that is, the number of ergs represented by each unit of area, may be determined as follows :

Abscissas represent values of $\mathscr{H}$ to scale, so that we may write

$$
\mathscr{H}=a x
$$

Ordinates represent values of $\mathscr{B}$ to scale, so that we may write

$$
\mathfrak{B}=b y
$$

or

$$
d \mathfrak{B}=b \cdot d y
$$

Substituting these values of $\mathscr{H}$ and $d \mathscr{B}$ in equation (I4) we have

$$
W=\frac{a b V}{4 \pi} \int x \cdot d y
$$

in which $a$ is the number of units of $\mathscr{H}$ represented by one unit of abscissa, and $b$ is the number of units of $\mathscr{B}$ represented by one unit of ordinate in Fig. I6. Now $\int x \cdot d y$ is the area between 
any portion of the $\mathscr{B}$ and $\mathscr{H}$ curve and the $y$-axis. Therefore, $a b V / 4 \pi$ is the number of ergs of work represented by each unit of area between the $\mathscr{B}$ and $\mathcal{H}$ curve and the $y$-axis.

22. Magnetic hysteresis. The magnetic cycle. - The divergence of the $\mathscr{B}$ and $\mathscr{H}$ curve for increasing values of $\mathscr{H}$ from the $\mathscr{B}$ and $\mathscr{A}$ curve for decreasing values of $\mathscr{H}$ is called magnetic hysteresis; or, rather, the tendency of iron to retain a previous magnetic state which is the cause of this divergence is called magnetic hysteresis. One effect of magnetic hysteresis is that the work regained when iron is demagnetized is less than the work which must be spent to magnetize the iron, as pointed out in Art. 2 I.

The magnetization of a given portion of the armature core of a dynamo is repeatedly reversed as the armature rotates, reaching a certain positive value of $\mathscr{B}$ for one position of the armature, an equal negative value of $\mathscr{B}$ for another position of the armature, coming back again to the original positive value of $\mathscr{B}$, and so on. Such a magnetic double-reversal is called a magnetic cycle, inasmuch as the magnetic condition of the iron is precisely the same at the beginning and at the end of the double reversal.

When a mass of iron is magnetized along the $\mathfrak{B}$ and $\mathscr{H}$ curve $o p$ of Fig. I6, and then partially demagnetized along the curve $p p^{\prime}$, a portion of the work done upon the iron during the first stage $o p$ is regained during the stage $p p^{\prime}$, a portion is lost as heat, and a portion remains in the iron as energy of magnetization.

When, however, a mass of iron is carried through a magnetic cycle, the algebraic sum of the work spent upon the iron during the cycle, that is to say, all of the work spent upon the iron during the cycle is lost as heat, inasmuch as the magnetic energy in the iron is exactly the same at the beginning and at the end of the cycle.

Fig. I 7 shows the relation between $\mathscr{B}$ and $\mathscr{H}$ during a complete magnetic cycle. The total work spent on the iron is given by the value of the integral

$$
\frac{a b V}{4 \pi} \cdot \int x \cdot d y
$$


extended over the whole cycle; but the value of $\int x \cdot d y$ extended over the whole cycle is the area enclosed by the $\mathscr{B}$ and $\mathscr{H}$ curve.

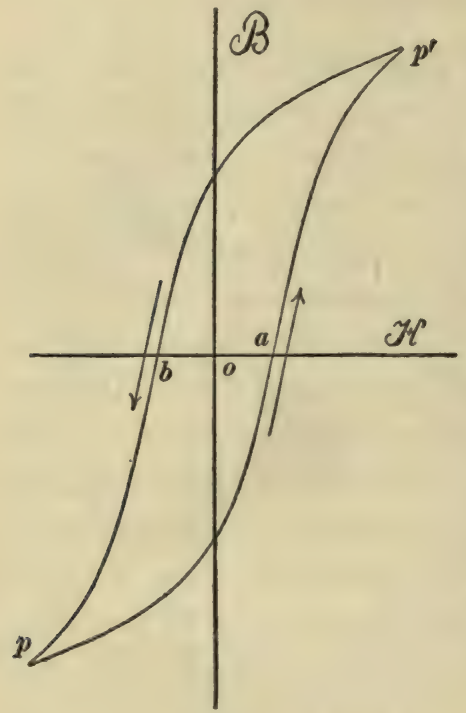

Fig. 17. Therefore, the total energy, in ergs, lost in $V$ cubic centimeters of iron per magnetic cycle is equal to $a b V / 4 \pi \times$ area of $\mathscr{B}$ and $\mathscr{H}$ curve. This energy loss is called the hvsteresis loss and it is all converted into heat.

The hysteresis loss per cycle increases with the range of flux density during the cycle. This energy loss per cycle may be expressed with sufficient accuracy for most practical purposes (from $\mathscr{B}= \pm 2,000$ to $\mathscr{B}= \pm$ 1 2,000) by the empirical equation

$$
W=\eta V \mathfrak{B}^{1.6}
$$

which is due to Steinmetz. In this equation $W$ is the loss of energy in ergs per cycle, $V$ is the volume of the iron in cubic centimeters, $\pm \mathscr{B}$ is the range of flux density during the cycle, and $\eta$ is a constant coefficient, the value of which depends upon the chemical and physical qualities of the iron. The following table gives the values of $\eta$ for different kinds of iron and steel.

\section{TABLE.}

Values of hysteretic coefficient $\eta$.

Best quality of sheet iron for transformer cores, annealed 0.0015

Sheet iron for armature cores, annealed 0.003

Ordinary sheet iron, annealed. 0.004

Soft annealed cast iron. 0.008

Soft machine steel... 0.0095

Cast steel 0.12

Cast iron 0. 16

Hardened stee] 
23. Calculation of loss of power in iron due to hysteresis and eddy currents. - Hysteresis loss. - The product of the energy loss per cycle and the number of cycles per second gives the energy lost per second, or, in other words, the power lost in the iron. Therefore from equation (I6) we have :

$$
P_{h}=\eta f V \mathfrak{B}^{1.6} \times 10^{-7}
$$

in which $P_{h}$ is the loss of power in watts in $V$ cubic centimeters of iron which is subjected to $f$ magnetic cycles per second through the range of the flux density $\pm \mathscr{B}$. Values of $\eta$ are given in the table above.

When a mass of iron is rotated in a magnetic field the direction of magnetization is repeatedly reversed, but in a manner very different from that in which the magnetization is reversed in a stationary mass of iron by reversals of the magnetic field. Baily* found for high flux densities a smaller hysteresis loss in a rotating mass than in a stationary mass of iron for a given maximum flux density, especially when the flux density is very large. Later experiments by Dina $\uparrow$ show a smaller difference than that found by Baily, and both Baily and Dina find the difference to be negligibly small for flux densities up to 16,000 lines per square centimeter.

Eddy current loss. - The loss of energy by eddy currents has no essential connection with the magnetic properties of the iron, but the loss of power by eddy currents is usually considered in conjunction with loss of power by hysteresis. The loss of power by eddy currents is proportional to the volume of the iron, to the square of the number of magnetic cycles per second, to the square of the thickness of the laminations, and to the square of the maximum flux density. Therefore

$$
P_{e}=\epsilon V f^{2} l^{2} \mathfrak{B}^{2}
$$

in which $P_{e}$ is the eddy current loss in watts, $V$ is the volume

* Philosophical Transactions, clxxxvii, p. 715, 1896.

† Elek. Tech. Zeitschrift, I902, p. 4r.

$\ddagger$ For a full discussion of this equation see Steinmetz, Alternating Current Phenomena, third edition, pp. 129 to 149 . 
of the iron in cubic centimeters, $f$ is the number of magnetic cycles per second, $l$ is the thickness of the laminations in centimeters, $\mathfrak{B}$ is the maximum flux density, and $\epsilon$ is a constant depending upon the electrical conductivity of the iron. The value of $\epsilon$ for sheet iron is about $1.6 \times 10^{-11}$. The value 2.5 $\times 10^{-11}$ is more frequently used for $\epsilon$ so as to allow for incomplete electrical insulation between the laminations.

Armature core loss. - The total loss of power in an armature core is due partly to hysteresis and partly to eddy currents. Neither of these parts can be accurately calculated, because the flux density in the core of a toothed armature is far from uniform. This is especially true when the armature flux $\Phi$ is greatly distorted by the armature current. The eddy current loss, as calculated by equation (I8), is all the more uncertain because of the indeterminate character of the insulation between the sheet iron laminations, especially if the edges of the laminations are burred by filing or other machining.

If it is desired to predetermine the armature core loss of a newly designed dynamo, it is less satisfactory to calculate the loss by equations ( I 7 ) and (I 8) than it is to estimate it from experimentally determined values of the armature core loss of other machines. The following table gives the average results of experimentally determined core losses in toothed armatures. These values are tabulated for various values of $f \mathscr{B}_{a}$, the product of the number of magnetic cycles per second and the flux density in the armature core below the slots. The value of $f$ is $p n / 2$ where $p$ is the number of field magnet poles, and $n$ is the speed of the armature in revolutions per second.

\section{TABLE.}

Armature core losses.

\begin{tabular}{c|c||c|c}
\hline$f B_{a} \times 10^{-8}$ & $\begin{array}{c}\text { Watts per Cubic Centimeter } \\
\text { of Laminated Iron. }\end{array}$ & $f B_{a} \times$ ro $^{-5}$ & $\begin{array}{c}\text { Watts per Cubic Centimeter } \\
\text { of Laminated Iron. }\end{array}$ \\
\cline { 1 - 2 } 0.0 & 0.0 & 2.5 & 0.048 \\
0.5 & 0.0094 & 3.0 & 0.060 \\
1.0 & 0.019 & 3.5 & 0.072 \\
1.5 & 0.029 & 4.0 & 0.087 \\
2.0 & $0.03^{8}$ & & \\
\hline
\end{tabular}




\section{APPENDIX B.}

\section{CHARACTERISTIC CURVES.}

24. Definition of the characteristic curve of a generator and of a motor. - (a) Of a generator. - When a dynamo is used as a generator it is usually driven at a definite constant speed, and the current output of the machine may be large or small, according to the resistance of the receiving circuit. When the current output of a generator varies, the electromotive force between its terminals generally changes, and the curve showing the relation between terminal voltage of the generator as ordinates and current output as abscissas, is called its (external) characteristic curve. Sometimes it is desirable to plot the curve showing the relation between current output of generator as ordinates and resistance of receiving circuit as abscissas. This curve is sometimes called the ampere-ohm characteristic of the generator to distinguish it from the volt-ampere characteristic above described.

(b) Of a motor. - When a dynamo is used as a motor it is usually supplied with current from constant voltage mains, and the load on the motor may be large or small according to the amount of power required to drive the machinery to which the motor is connected. When the load on the motor varies, its speed, its current intake, and the torque which it develops, all change in a definite way, and a curve showing the relation between any two of these elements may be called a characteristic curve of the motor. Thus the characteristic curve of a motor may show (I) the relation between speed as ordinates and torque as abscissas, (2) the relation between speed as ordinates and current intake as abscissas, (3) the relation between torque (or, in case of a street car motor, tractive effort) as ordinates and current intake as abscissas. 
A motor may be supplied with constant current in which case the speed and terminal voltage of the motor vary in a definite way with the motor load, and the characteristic curve may in this case show: (I) The relation between speed and torque : (2) The relation between terminal voltage and speed; (3) The relation between terminal voltage and torque. Constant current motor driving, however, seldom or never occurs in practice.

25. The magnetization curve of a dynamo. - A clear understanding of the characteristic curves of a dynamo when used as a generator or as a motor depends upon precise knowledge of the relation between the field current and the flux $\Phi$ which enters the armature from a north pole of the field; or, since the electromotive force, $\Phi Z^{\prime} n$, induced in the armature when the armature is driven at a prescribed constant speed, is proportional to the flux $\Phi$, it is sufficient to know the relation between the induced electromotive $\Phi Z^{\prime} n$ and the field current. The curve which shows this relation is called the magnetization curve of the dynamo.

The magnetization curve of a dynamo may be determined by driving the machine at the prescribed speed, and observing the electromotive force between its brushes $*$ for different observed values of current sent through its field windings from some outside source. Figs. I 8 and 19 are typical magnetization curves of a dynamo which is rated as a 30-kilowatt generator; Fig. I8 shows the magnetization curve of the machine when its field is wound with many turns of fine wire (shunt dynamo), and Fig. I9 shows the magnetization curve of the same machine when its field is wound with few turns of coarse wire (series dynamo).

Dependence of magnetization curve on speed. - When the magnetization curve of a dynamo has been determined for a given speed $n$, the curve for any other speed $n^{\prime}$ may be found by multi-

* When the current in the armature is very small, the electromctive force between the brushes of a dynamo is very nearly equal to the total electromotive force $\Phi Z^{\prime} n$ induced in the armature. 
plying the ordinates of the given curve by $n^{\prime} / n$. This is evident when we consider that the flux $\Phi$ has a definite value for a given value of field current, so that the induced electromotive force, $\Phi Z^{\prime} n$, corresponding to a given value of the field current is proportional to the speed.

Effect of residual magnetism upon the magnetization curve of a dynamo. - When the field current of a dynamo is zero, the armature flux $\Phi$, and the induced electromotive force $\Phi Z^{\prime} n$, are usually not zero, on account of residual magnetism. This effect

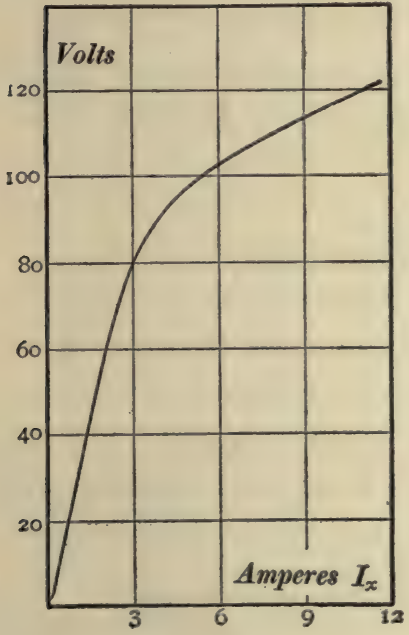

Fig. 18.

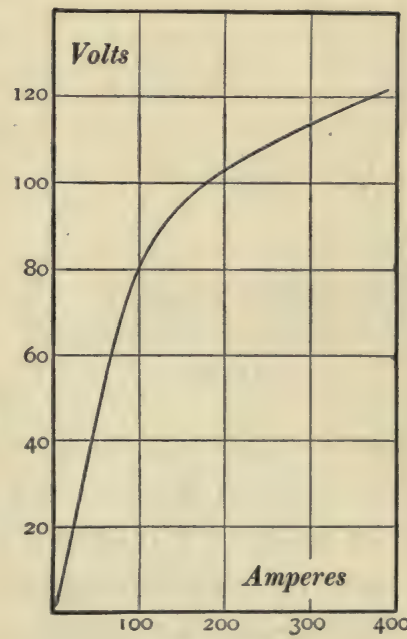

Fig. 19.

is shown in Figs. I 8 and I9. In both of these figures the curves intersect the axis of ordinates at that distance above the origin which represents the value of the induced electromotive force due to the residual magnetism.

Effect of magnetic hysteresis upon the magnetization curve of a dynamo.- On account of magnetic hysteresis, the armature flux $\Phi$ and the induced electromotive force $\Phi Z^{\prime} n$ which correspond to a given value of field current are smaller when the field current is increasing than when the field current is decreasing. Therefore the magnetization curve of a dynamo is slightly different for 
increasing field current and for decreasing field current, as shown in Fig. 20. This effect of magnetic hysteresis upon the magnetization curve of a dynamo is usually ignored in discussing the

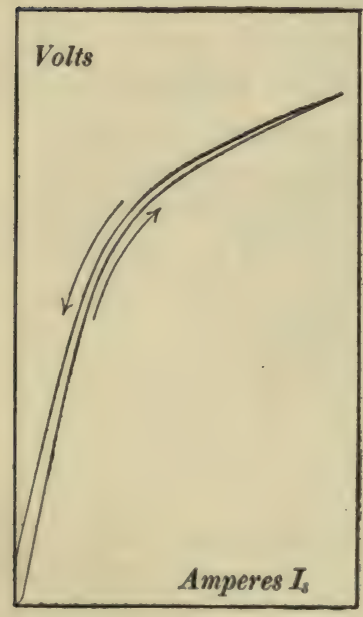

Fig. 20. relation of the magnetization curve to the characteristic curve of the dynamo as generator or as motor. In fact the effect of hysteresis is nearly zero under practical operating conditions, inasmuch as the mechanical vibrations of the dynamo and the slight pulsations of armature and field currents cause the armature flux $\Phi$ to settle to the normal value, as explained in Art. I2, Appen$\operatorname{dix} A$.

\section{CHARACTERISTIC CURVES OF GEN- ERATORS.}

\section{Characteristics of the series gener-} ator.- The characteristic curve of a series generator may be determined experimentally by driving the machine at constant speed and observing corresponding values of current output and terminal voltage for different resistances of the receiving circuit.

External characteristic. Internal characteristic. - The curve $O A$, Fig. $2 \mathrm{I}$, showing the relation between terminal volts $E_{x}$ and current output, is often called the external characteristic to distinguish it from the curve $O C$, showing the relation between total induced electromotive force $E_{a}$ and armature current. (In the series generator the armature current is of course equal to the current output.) This curve, $O C$, is called the internal characteristic, or the total characteristic. When the external characteristic $O A$ is given, the internal or total characteristic $O C$ may be plotted as follows :

The difference between $E_{a}$ and $E_{x}$ is equal to the electromotive force $R I$, which is used to overcome the combined resistance, 
$R$, of the armature and field windings, as explained in Art. 46, Chapter III., so that :

$$
E_{a}=E_{x}+R I
$$

Let the values of $R I$ be plotted for various values of $I$. These plotted points will lie on the straight line $O B$, Fig. 2I, since $R$ is a constant. Plot the curve $O C$, of which the ordinate at each point exceeds the ordinate of the given curve $O A$, by the amount $R I$. The curve $O C$ is the required internal characteristic.

The effect of residual magnetism upon the characteristic curve of a series generator is to cause the curve to intersect the axis of volts above the origin, as shown in Fig. 21 .

Relation betrueen the magnetization curve and the internal or total characteristic of a series generator.-If it were not for the demagnetizing action on the field, of

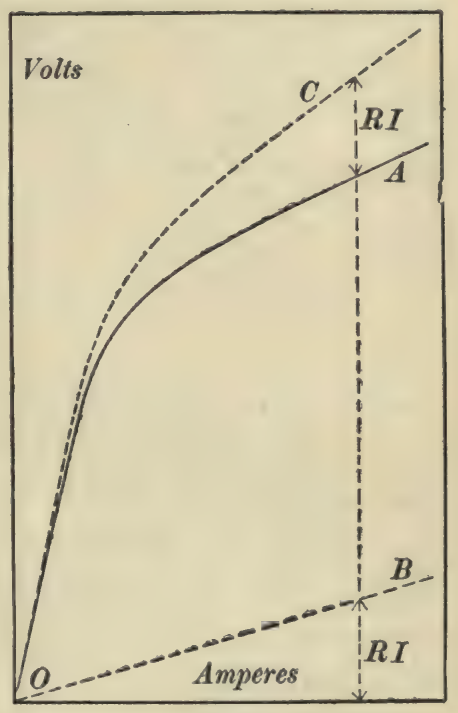

Fig. 21. the current in the armature, the internal or total characteristic of a series dynamo would be identical* with the magnetization curve. However, the effect of the demagnetizing action of the armature current is either to reduce the armature flux and therefore the total induced electromotive force which corresponds to a given field current, or to necessitate an increased field current to give the prescribed total induced electromotive force. Let $d$ be the number of demagnetizing turns $\dagger$ of wire on the armature (per magnetic circuit)

* Except for a slight loss of electromotive force due to the inductance of the elements of the armature winding.

$\dagger$ It is evident from equation (35) that

$$
d=\frac{D}{I_{a}}=\frac{a}{180} \cdot \frac{Z}{p^{\prime}}
$$


through each of which the whole of the armature current may be considered to flow, and let $N$ be the number of turns of wire in the field winding per magnetic circuit. Then the number of ampere-turns, net, acting on each magnetic circuit is $I^{\prime}(N-d)$ when the current output of the generator is $I^{\prime}$ amperes. Now, a current $I$ flowing in the field winding alone gives a total induced electromotive force $E_{a}$, and in order that the same induced electromotive force $E_{a}$ may be obtained when the machine is delivering $I^{\prime}$ amperes as a series generator, the net ampere-turns acting must be equal to $N I$. That is, we must have

$$
\begin{array}{ll} 
& I^{\prime}(N-d)=N I \\
\text { or } \quad & I^{\prime}=\frac{N}{N-d} \cdot I
\end{array}
$$

That is, the current must be increased in the ratio of $(N-d)$ to $N$. Therefore the total characteristic curve of a series generator may be derived from the magnetization curve of the machine by multiplying the abscissas of the magnetization curve by $N /(N-d)$. This result is deduced on the assumption that the demagnetizing action of the armature is strictly proportional to the armature current.

Dependence of the characteristic of a series dynamo on speed. - The flux $\Phi$ has a definite value for a given value of current output of a series generator independently of speed. Consequently the total induced electromotive force $\Phi Z^{\prime} n$ is proportional to the speed of the generator for a given value of current output. Therefore the external characteristic corresponding to speed $n^{\prime}$ may be derived from the given external characteristic corresponding to speed $n$ as follows :

Add $R I$ to each ordinate of the given characteristic, thus finding the internal or total characteristic for the same speed $n$. Multiply the ordinates of this total characteristic by $n^{\prime} / n$, thus findng the total characteristic for speed $n^{\prime}$. Subtract $R I$ from each ordinate of this total characteristic for speed $n^{\prime}$, thus finding the required external characteristic for speed $n^{\prime}$. 
The drooping of the characteristic of the series generator. - It would seem as though the useful flux $\Phi$ through the armature of a series generator should increase more and more with the current output of the machine, but in fact the effect of magnetic leakage around the armature is to cause $\Phi$ to actually decrease in value when the current output is excessive, especially if the field iron becomes saturated before the armature iron. This decrease of $\Phi$ means an actual decrease of $E_{a}$ and of course $E_{x}$ falls off more than $E_{a}$ on account of the armature drop $R_{a} I_{a}$.

Fig. 22 shows the external characteristic (full line curve), and the total characteristic (dotted curve) of a Wood arc lighting series generator, with its governing device disconnected.

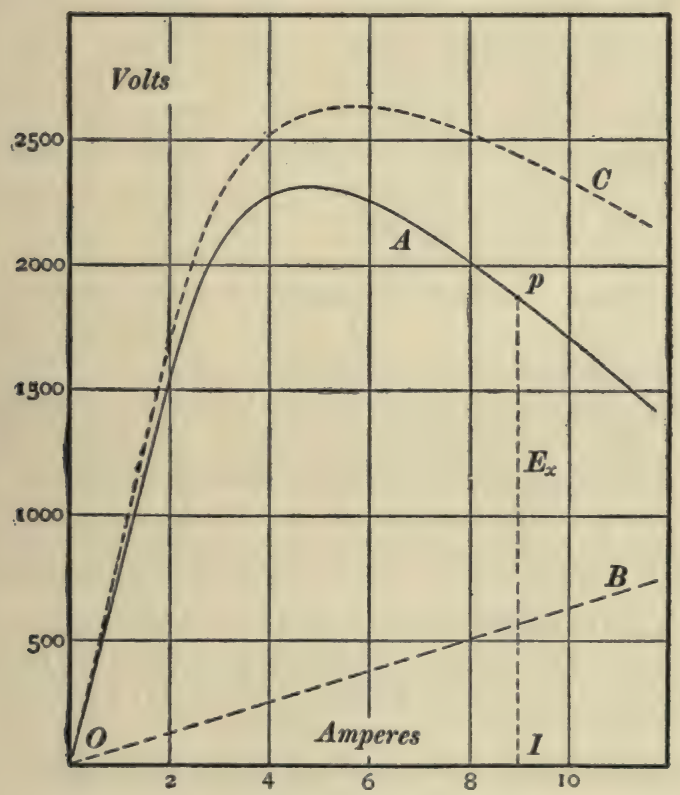

Fig. 22.

Explanation of drooping of series characteristic. - When a series generator has a large coefficient of magnetic leakage, and when the field yoke and cores are nearly saturated magnetically, the armature core being far below saturation, then the total electromotive force induced in the armature decreases with increase of current. This 
effect depends upon the demagnetizing action of the armature current and it may be best explained by a simple numerical example as follows: Given a generator, with considerable positive brush lead, which is delivering a current of 10 amperes with a total induced electromotive force of 2,500 volts. Under these initial conditions suppose that the actual armature flux is $2,500,000$ lines, that the leakage flux is also $\mathbf{2 , 5 0 0 , 0 0 0 ~ l i n e s , ~ s o ~ t h a t ~ t h e ~ f i e l d ~ f l u x ~ i s ~} 5,000,000$ lines (coefficient of magnetic leakage $=2$ ). Let the resistance of the receiving circuit be decreased so that the current output of the generator is increased from ro to II amperes, or by Io per cent. This produces a 10 per cent. increase of ampere-turns on the field and a 10 per cent. increase of the demagnetizing ampere-turns on the armature, the brushes being fixed in position. The field iron being highly saturated, the ro per cent. increase of field ampere-turns produces, say, I per cent. increase of field flux making the field flux equal to 5,050,000 lines. Now, the armature core being far below saturation, a very large part of the opposition to the passage of magnetic flux through the armature is due to the demagnetizing ampere-turns of the armature, and a small part only is due ${ }^{*}$ to the reluctance of the air gaps and the armature core. Let us suppose, for simplicity, that all of the opposition to the passage of the flux through the armature is due to the demagnetizing action of the armature current, or in other words, let us suppose that the magnetic reluctance of air gaps and armature core is zero. Then a Io-per cent. increase of armature current would increase the demagnetizing ampere-turns 10 per cent., the magnetomotive force (ampere-turns), between the pole pieces will be increased ro per cent. and the leakage flux will be increased ro per cent. since the magnetic reluctance of the leakage paths is constant. Therefore the fleld flux becomes $1.01 \times 5,000,000=5,050,000$; and the leakage flux becomes 1.10 $\times$ $2,500,000=2,750,000$; so that the armature flux becomes $(5,050,000-2,750,000)$ which is equal to $2,300,000$. The total induced electromotive force is therefore reduced in the ratio of $2,500,000$ to $2,300,000$ or in the ratio of 1.00 to 0.92 . This shows that under the assumed conditions an actual decrease of 8 per cent. in the total induced electromotive force has resulted from a Io-per cent. increase of current.

Problem: The combined reluctance of the armature core and the air gaps is in fact nearly constant, since the armature core is not highly saturated. Assuming that half of the opposition to the passage of flux through the armature and air gaps is due to this reluctance, and half is due to the demagnetizing ampere-turns on the armature, calculate : $(a)$ The percentage decrease in total induced electromotive force under the above conditions when the current is increased ro per cent., and $(b)$ The coefficient of magnetic leakage with increased current. Answer : (a) 5 per cent.; (b) 2.15.

\section{The ampere-ohm characteristic of the series generator. - For} some purposes it is convenient to represent the current output of a series generator as a function of the resistance of the receiving circuit, by plotting the values of current output as ordinates, and the corresponding values of resistance of the receiving circuit as abscissas. Fig. 23 shows such an ampere-ohm characteristic of 
the Wood arc lighting generator of which the volt-ampere characteristic is shown in Fig. 22.

The ampere-ohm characteristic is derived from the volt-ampere characteristic as follows : Lay off the abscissa of each point $p$ of

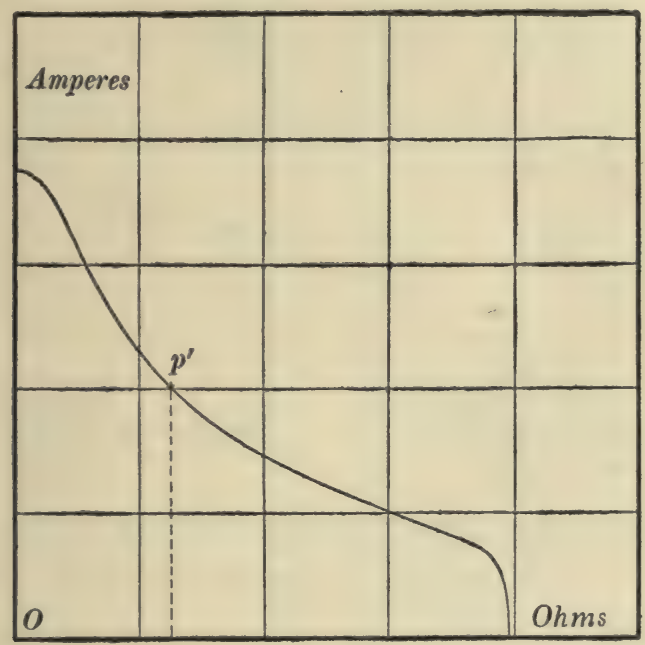

Fig. 23.

the volt-ampere characteristic, Fig. 22, as the ordinate of the corresponding point $p^{\prime}$ of the ampere-ohm characteristic and lay off in each case the ratio $E_{x} / I$, see Fig. 22, as the abscissa of $p^{\prime}$.

28. Examples showing applications of the external characteristic of a series generator. - The examples to be given depend upon the use of what is called the receiving circuit characteristic. This is a curve showing the relation between the electromotive force acting on the receiving circuit and the current produced in the receiving circuit by this electromotive force.

When the receiving circuit consists simply of a resistance $R_{r}$, the electromotive force required to produce a current $I$ is equal to $R_{r} I$, and the receiving circuit characteristic is a straight line, $A B$, as shown in Fig. 24 . 
When the receiving circuit consists, say, of a storage battery which is to be charged by the generator, then the terminal electromotive force $E_{x}$ of the generator is equal to the sum of $R_{r} I$ and the counter electromotive force $e$ of the battery; where

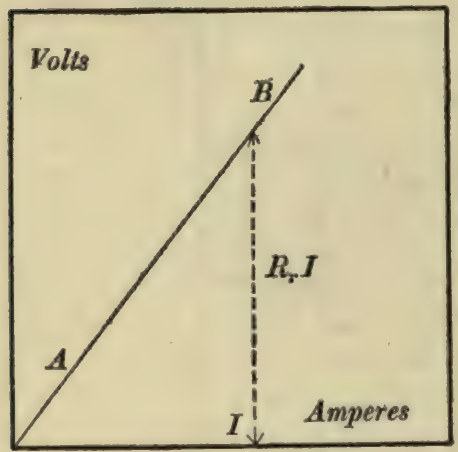

Fig. 24.

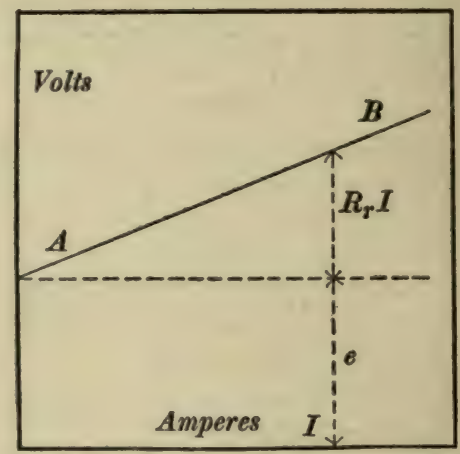

FIg. 25.

$R_{r}$ is the resistance of the battery and connecting wires. In this case the receiving circuit characteristic is the straight line $A B$, shown in Fig. 25.

A receiving circuit containing motors has no definite characteristic in the sense in which the term is here defined, inasmuch as with a given applied voltage the current may have a great variety of values depending upon the motor load.

Now, evidently, the terminal electromotive force of a generator is identical with the electromotive force applied to the receiving circuit, including the line wires, and the current output of a generator is identical with the current flowing through the receiving circuit. Therefore a generator supplying a given receiving circuit operates at that point of its characteristic where the receiving circuit characteristic intersects it.

The point $P$ in Fig. 26 shows the operating conditions of a given series generator running at given speed and supplying current to a receiving circuit of given resistance. With increase of the resistance of the receiving circuit, the line $O P$ becomes steeper, that is, the angle $\theta$, Fig. 26 , increases, inasmuch as the 
tangent of $\theta$ is equal to $R_{r} I / I$, which is equal to $R_{r}$. When the resistance $R_{r}$ has so far increased that the point of intersection is near the knee of the curve at $K$, then either a very slight additional increase of $R_{r}$, or a momentary slowing down of the generator (which reduces the ordinates of the characteristic curve) will lead to a state of affairs in which the generator characteristic and the receiving circuit characteristic do not intersect at all. The resistance $R_{k}$, corresponding to the point $K$, Fig. 26, is called the critical resistance of the series generator for the given speed, inasmuch as the generator when driven at the given speed cannot build up, or if it is already built up, it cannot maintain its

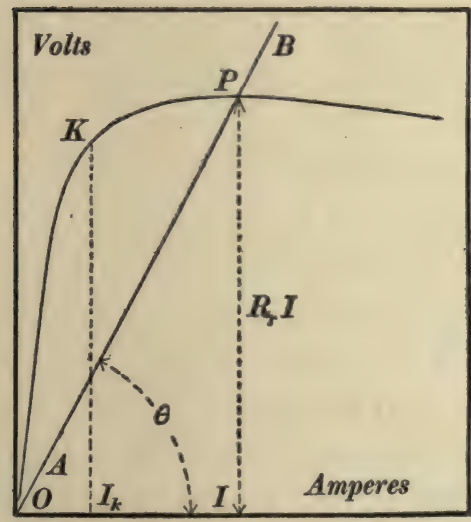

Fig. 26.

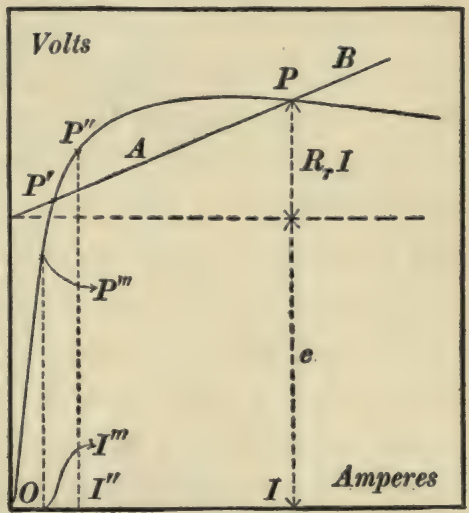

Fig. 27.

field magnetism, if the resistance of the receiving circuit is greater than $R_{k}$. The critical resistance is proportional to the speed at which the generator is driven. The current $I_{k}$, corresponding to the point $K$, is called the critical current of the generator. The critical current does not vary perceptibly with the speed of the generator, but has a definite value for a given generator.

The points $P$ and $P^{\prime}$, Fig. 27 , represent the two presumably possible operating conditions of a given series generator, running at a given speed, and supplying current to charge a storage battery of which the counter-electromotive force is $e$. In fact the 
point $P$ represents a possible running condition, but it is difficult to bring about the state of affairs represented by the point $P$ as explained below, while the point $P^{\prime}$ represents an unstable and therefore impossible condition of running. The instability of the conditions represented by the point $P^{\prime}$ may be shown as follows: Suppose that the generator is in fact operating at the point $P^{\prime}$, and that a slight momentary increase in the speed of the generator takes place. This increases the generator voltage momentarily and also the current and then, even if the generator comes quickly back to its normal speed, it will continue to operate for a very short interval of time at a point $P^{\prime \prime}$ a short distance above $P^{\prime}$; but at the point $P^{\prime \prime}$ the generator voltage is more than sufficient to produce the current $I^{\prime \prime}$ (greater than $e+R_{r} I^{\prime \prime}$ ), and the excess of voltage will cause the current to go on increasing until the generator reaches the point $P$.

On the other hand a momentary drop in the speed of the generator will cause a momentary decrease of current and, even if the generator comes quickly back to its normal speed, it will continue to operate for a very short interval of time at a point $P^{\prime \prime \prime}$ a short distance below $P^{\prime}$; but at the point $P^{\prime \prime \prime}$ the generator voltage is less than $\left\{e+R_{r} I^{\prime \prime \prime}\right\}$, so that the current will continue to fall off more and more. In this latter case (with the storage battery in circuit) the current does not merely drop to zero, but a reversed current is started by the battery. This current causes the generator to build up in the opposite direction, and then both generator and battery work together to produce current, the battery being of course thereby discharged. The point $P^{I V}$, Fig. 28 , shows the operating conditions in this case.

The difficulty in getting a series generator to operate at the point $P$, Fig. 28 , and so charge a storage battery, is due to the fact that when the circuit is first closed (current then equal to zero) the generator begins operating at the point 0 , Fig. 28, and the effect of the battery is to cause the generator always to build up in such a direction as to carry the operating point to $P^{I V}$, thus discharging the battery instead of charging it. Under these con- 
ditions the battery and the generator work together to produce an excessive current, and the power delivered by the battery and by the generator is all used in heating the circuit.

When a street car is coasting down hill it may drive the series dynamos, which are geared to its axles, as generators, if the connections between field and armature are reversed. The difficulty in getting these dynamos to build up in the proper direction to charge the line, as it were, thus returning power to the system, is

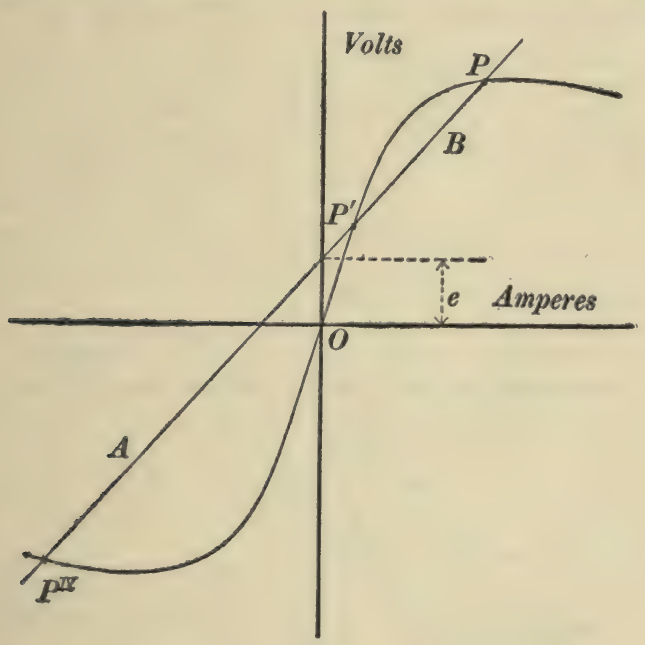

Fig. 28.

precisely the same as that which is described above. The line voltage, between trolley and rail, always causes the series machines to build up in the wrong direction to accomplish the desired result. The action which does take place is that the two series machines build up as generators in the direction such as to help the trolley voltage force an excessive current through the circuit including the machines and the rheostats.

The curious see-saw action of a series generator which supplies current to a motor of which the field magnet is separately excited, is closely related to the above described behavior of a series generator connected to a storage battery. Let a series generator 
driven at constant speed, supply current to the armature of an auxiliary dynamo, of which the field magnet is separately excited. The characteristic of this auxiliary machine at a given speed, considered as a receiving circuit, would be the straight line $P P^{\prime} P^{I V}$, Fig. 28 ; but the counter-electromotive force $e$ of the auxiliary machine increases and decreases with the speed of that machine, so that the line $P P^{\prime} P^{I V}$ moves up and down as the speed of the machine rises and falls. When the circuit is first closed the speed of the auxiliary machine is zero; $e$ is zero, and the series gener-

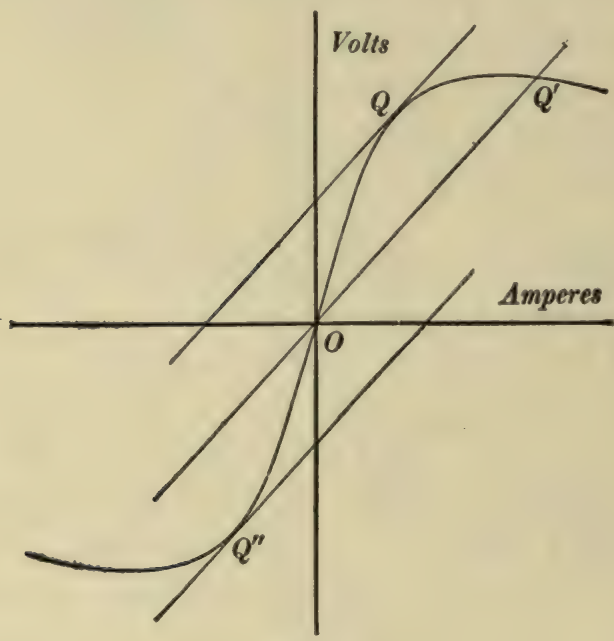

Fig. 29.

ator builds up to the point $Q^{\prime}$, Fig. 29, sending current through the armature of the auxiliary machine. This current causes the auxiliary machine to speed up as a motor, thus increasing $e$. This action continues until the straight line $P P^{\prime} P^{I V}$ rises above the point $Q$, Fig. 29, and does not intersect the positive portion of the characteristic of the series generator. Then the series generator immediately loses its field magnetism, the auxiliary machine continues to run because of the momentum of its armature, and, becoming a generator, its electromotive force $e$ now starts a reversed current through the circuit, thus causing the series gen- 
erator to build up in the reverse direction. During this time the auxiliary machine is acting as a generator, so that it quickly comes to rest as the kinetic energy of its rotating armature is exhausted, and the reversed current, which is now maintained by the reversed series generator, starts the auxiliary machine in the reverse direction as a motor. The auxiliary machine then speeds up until the line $P P^{\prime} P^{I V}$ falls below the point $Q^{\prime \prime}$, Fig. 29, when the series generator again loses its field magnetism, which leads to another reversal as before, and so on.

29. Characteristics of the shunt generator. - The experimental determination of the characteristic curve of the shunt generator is accomplished in essentially the same manner as in the case of the series generator.

The full line curve, Fig. 30, is a typical external characteristic of a shunt generator; the abscissas represent values of current delivered to the receiving circuit, and the ordinates represent the corresponding values of the electromotive force between the generator terminals. The upper portion $c c$ is the part of the characteristic on which the machine usually operates in practice.

The internal or total characteristic is the curve of which the abscissas represent the total current $I_{a}$ in the arma-

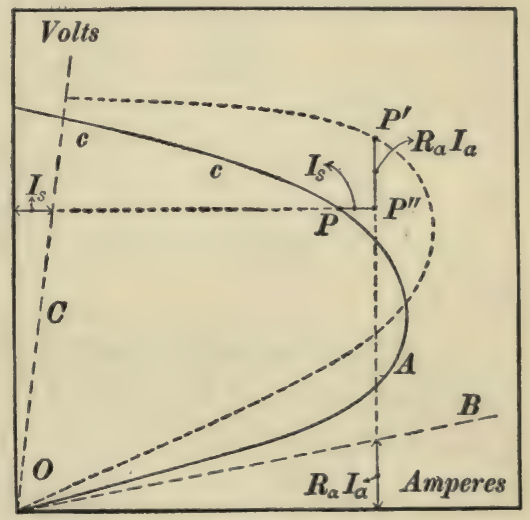

Fig. 30 . ture, and of which the ordinates represent the corresponding values of induced electromotive force $E_{a}\left(=\Phi Z^{\prime} n\right)$.

Consider a point $P$ on the external characteristic. The corresponding point $P^{\prime}$ on the total characteristic is found by increasing the abscissa by $I_{s}$, the value of the shunt field current, and increasing the ordinate by $R_{a} I_{a}$, which is the electromotive force 
lost in overcoming the resistance of the armature. The values of $I_{s}$, corresponding to different values of $E_{x}$, are proportional to $E_{x}\left(=I_{s} R_{s}\right)$, and may be represented by the abscissas of the

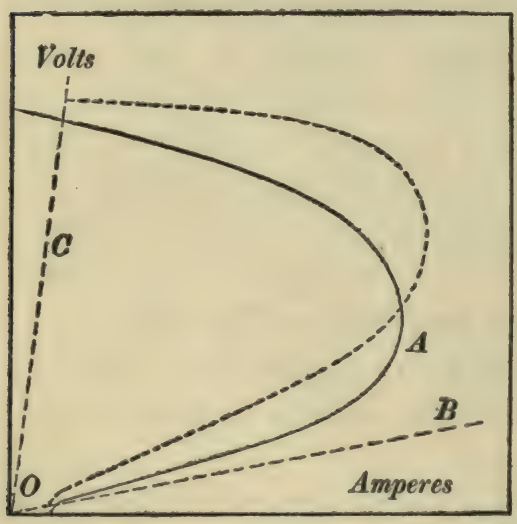

Fig. 31. straight line $O C$; the value of $R_{\alpha} I_{a}$, for various values of $I_{a}$, may be represented by the ordinates of the straight line $O B$; and the total characteristic may be constructed from the external characteristic by first increasing the abscissa of each point $P$, Fig. 30 , by an amount equal to the corresponding abscissa of the line $O C$, and then increasing the ordinate of the point $P^{\prime \prime}$ so reached by an amount equal to the corresponding ordinate of the line $O B$, thus reaching the point $P^{\prime}$ on the total characteristic curve.

The effect of residual magnetism upon the characteristic curve of a shunt generator is to cause the external characteristic to intersect the ampere-axis to the right of the origin, as shown in Fig. 3 I.

30. The ampere-ohm characteristic of the shunt generator.-For some purposes it is convenient to represent the current output of a shunt generator as a function of the resistance of the receiving circuit by plotting the values of current output as ordinates, and the corresponding values of resistance of the receiving circuit as abscissas. Fig. 32 shows a typical ampere-ohm characteristic of a shunt generator. This curve may be derived from the voltampere characteristic, Fig. 30, exactly as in the case of the series generator, as explained in Art. 27.

31. The voltage-speed characteristic of the shunt generator at zero load.- The relation between voltage and speed of a shunt 
generator is more complicated than in the case of the series generator (with given current output), inasmuch as the increase of speed of a shunt generator involves an increase of field excitation. The curve showing the voltage of a shunt generator as a function

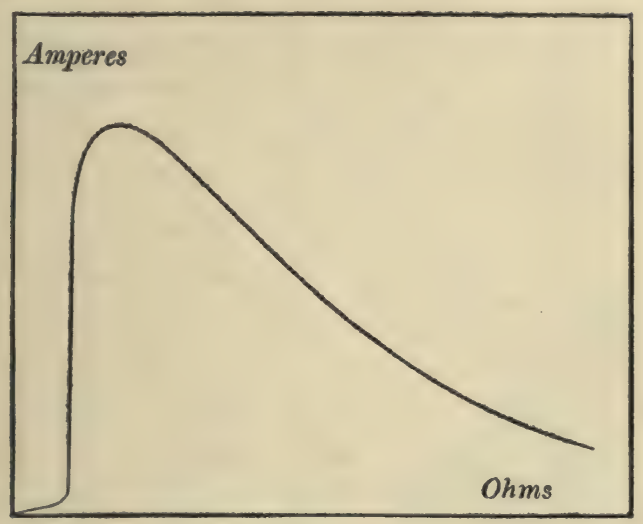

Fig. 32 .

of speed, at zero current output, may be derived from the magnetization curve of the machine as follows :

For the purpose of this derivation it is to be noted that if both the speed $n$ and the shunt field resistance $R$, are imagined to be changed in the same ratio, the electromotive force $E_{a}\left(=E_{x}\right.$ at zero load) will also be changed in that ratio, inasmuch as this change of $E_{a}$ and $R_{s}$ will leave the shunt field current $E_{x} / R_{s}$ unchanged, so that $\Phi$ will be unaltered, and $E_{a}\left(=\Phi Z^{\prime} n\right)$ will vary as $n$.

Let the curve $O A$, Fig. 33 , be the magnetization curve of the shunt machine for a given speed $n$. Draw the straight line $O B$, of which the abscissas represent values of the shunt field current $I_{s}$, and the ordinates represent values of electromotive force, $R_{s} I_{s}$, required to produce the corresponding values of $I_{s}$. The coördinates of the point of intersection of $O A$ and $O B$ will then represent the values of $E_{a}\left(=E_{x}\right.$ at zero current output) and $I$, when the machine is driven at the given speed $n$. 
Lay off a scale of values of $R_{s}$ on the vertical line $C D$, Fig. 33, taking the actual given value of $R_{s}$ as unity.

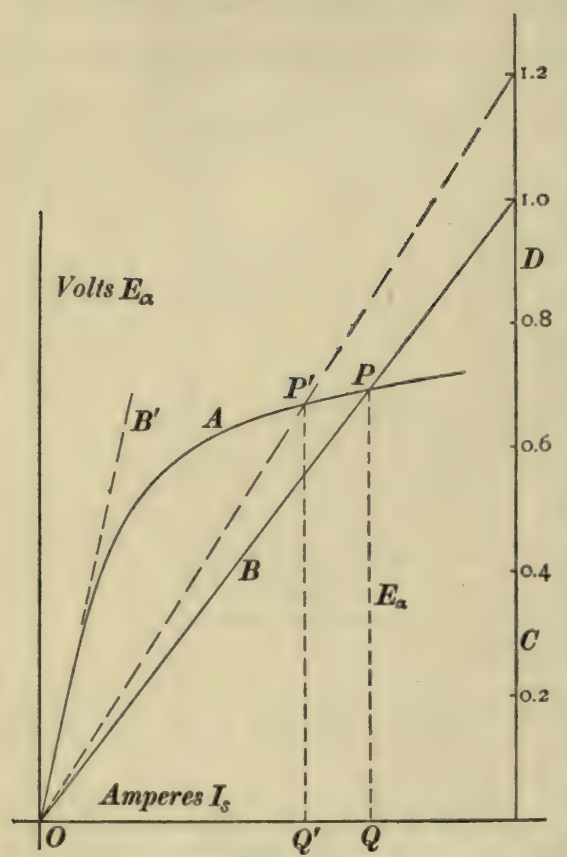

Fig. 33.

The finding of the value of $E_{a}$ for a speed $n^{\prime}$, different from $n$, may be most easily explained by taking a numerical example. Thus, let it be required to find the value of $E_{a}$ for a speed $n^{\prime}$ equal to $n /$ i.2. As a first step imagine the value of $R$ to be increased to 1.2 without change of speed. Under these conditions the ordinate $Q^{\prime} P^{\prime}$, Fig. 33, would be the value of $E_{a}$. As a second step imagine this increased value of $R$, and the original speed $n$ to be both decreased in the ratio $\mathbf{I . 2}$ to I.O. This would bring $R_{s}$ back to its actual value, and it would reduce the value of $E_{a}$, namely $Q^{\prime} P^{\prime}$, in the ratio I.2 to I.O. Therefore the required value of $E_{a}$ at speed $n^{\prime}$ is I/I.2 times $Q^{\prime} P^{\prime}$.

Fig. 34 shows a typical voltage-speed characteristic of a shunt generator at zero current output. If it were not for the effect of the residual magnetism this voltage-speed characteristic would cut the speed axis at S, Fig. 34. The speed corresponding to the abscissa of $S$ is called the critical speed of the given shunt generator. When driven at a speed less than this critical speed the shunt generator cannot build up at all.

The straight line $O B^{\prime}$, which is tangent to the magnetization curve (speed $n$ ) $O A$, Fig. 33 , at the origin, intersects the line 
$C D$ at a number which is equal to $n / n^{\prime \prime}$ where $n^{\prime \prime}$ is the lowest speed (critical speed) at which the given shunt machine can build up as a generator.

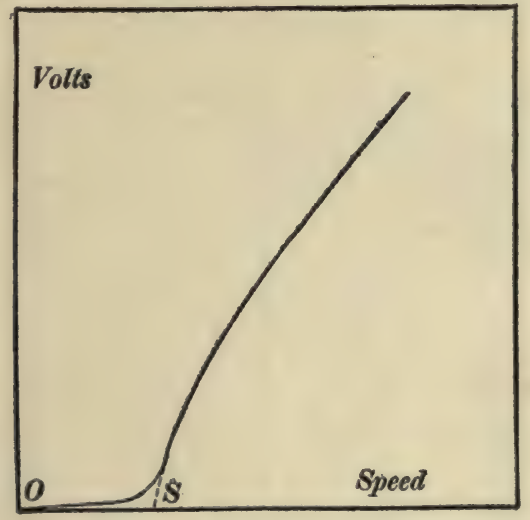

Fig. 34.

32. Relation between the magnetization curve of a shunt dynamo and its total characteristic as a generator.-(a) On the assumption that the demagnetizing action of the armature current is negligible. - In Fig. 35 the magnetization curve $O A^{*}$ of the machine for the given speed is plotted to the left of the origin of coördinates; ordinates of $O A$ of course represent values of $E_{a}$, and abscissas of $O A$, measured to the left from $O$, represent values of shunt field current $I_{s}$. The ordinates of the straight line $O B$ represent values of $R_{a} I_{s}$.

Consider any given point $P$ on the magnetization curve. The ordinate $S P$ is the total induced electromotive force $E_{a}$, so that the point $P^{\prime}$ of the total characteristic which corresponds to $P$, lies on a horizontal line drawn through $P$. Draw the straight line $S C$ of which the abscissas measured to the right from $S$ represent $\left(I_{s}+I_{x}\right)$, or the total armature current $I_{a}$, and of which the ordinates represent the values of $R_{a} I_{a}$. Now, when $P S$ is the total induced electromotive force, $Q S$ is the value of $E_{x}\left(=R_{8} I_{s}\right)$,

* The values of $I_{s}$ are exaggerated in Fig. 35 for the sake of clearness. 
and $P Q$ is the value of $R_{a} I_{a^{*}}$ Therefore $R_{a} I_{a}$ is known, and the abscissa of $P^{\prime}$ is determined by the condition that the corresponding ordinate of $S C$ shall be equal to $R_{a} I_{a}$, as indicated in the figure. The position of $P^{\prime}$ thus determined may be most

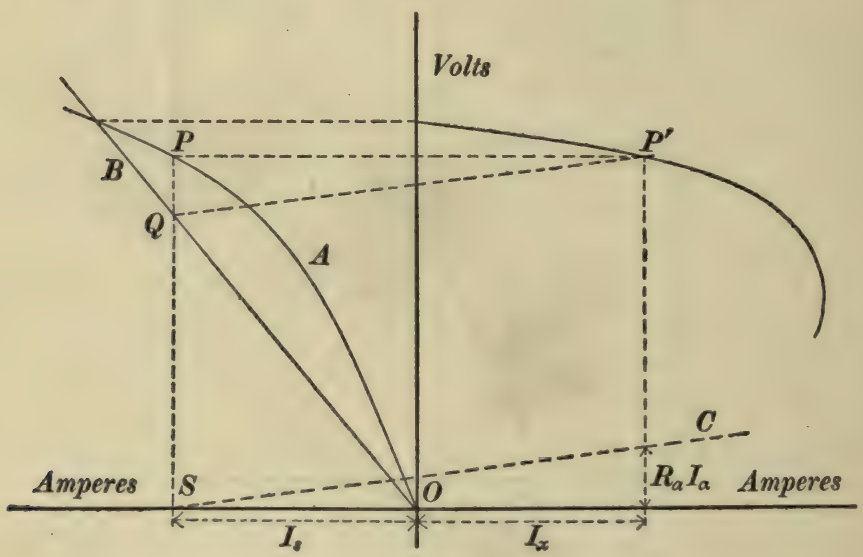

Fig. 35.

easily found by drawing a line through $Q$ parallel to $S C$; this line will intersect the horizontal line through $P$ at the desired point $P^{\prime}$. Additional points on the total characteristic curve may be located in a similar manner.

(b) When the demagnetizing action of the armature current is considered and assumed to be proportional to the armature current. - The curve $O A$, Fig. 36, is the magnetization curve of the machine for the given speed, plotted to the left of the origin $O$, as in Fig. 35. The ordinates of the straight line $O B$, Fig. 36, represent values of $E_{x}\left(=R_{8} I_{s}\right)$ as in Fig. 35 .

Consider any given point $P$ on $O B$, Fig. 36. The abscissa $O S$ is the shunt field current $I_{s}$ and the ordinate $S P$ is $E_{x}$. It is required to find the point $P^{\prime}$ on the total characteristic corresponding to $P$.

Let $N$ be the number of turns of wire in the shunt field winding, and let $d$ be the number of demagnetizing turns on the armature through each of whicb the entire armature current may be considered to flow. Then the net field excitation is $\left(N I_{s}-d I_{a}\right)$ ampere-turns, which may be written $N\left(I_{s}-I_{a} d / N\right)$. Therefore the actual net field excitation and the actual value of $E_{a}$ is that which would be produced by a field current equal to $\left(I_{s}-I_{a} d / N\right)$ acting alone; or, in other words, the distance $Q P, "$ Fig. 36 , is equal to $I_{a} d / N$, $P^{\prime \prime}$ being the unknown point on the magnetization curve corresponding to $P$. Furthermore, the ordinate of $P^{\prime \prime}$ 
is of course $E_{a}$, so that the distance $Q P$ is equal to $E_{a}-E_{x}$ or to $R_{a} I_{a}$. Now $I_{a}$ is as yet unknown, but since $P Q=R_{a} I_{a}$ and $Q P^{\prime \prime}=I_{a} d / N$ it is evident that the direction of $P P^{\prime \prime}$ is independent of $I_{a}$. In fact the direction of $P P^{\prime \prime}$ may be once for all determined by laying off $P M$ equal to $R_{a} I_{a}$ and $M N$ equal to $I_{a} d / N$ for any arbitrary value of $I_{\alpha}$. The line $P N$ then fixes the direction of $P P^{\prime \prime}$, and the point $P^{\prime \prime}$ is found as the intersection of $P N$ and $O A$, as shown in the figure.

Having thus determined the point $P^{\prime \prime}$, the point $P^{\prime}$ on the total characteristic which corresponds to the chosen point $P$, lies in a horizontal line drawn through $P^{\prime \prime}$, and the abscissa of $P^{\prime}$ is determined by the condition that the corresponding ordi-

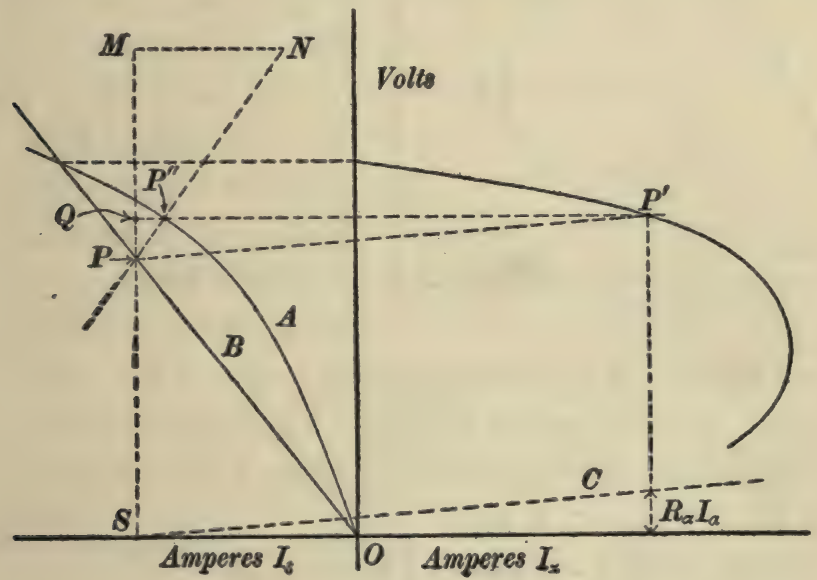

Fig. 36.

nate of $S C$ shall be equal to $P Q\left(=R_{a} I_{a}\right)$. The point $P^{\prime}$ thus determined is most easily located by drawing a line through $P$ parallel to $S C$. Such a line will intersect the horizontal line through $P^{\prime \prime}$ at the desired point $P^{\prime}$.

The value of $d$ is equal to $2 a / 360 \cdot Z / p^{\prime}$, where $a$ is the angle of forward lead of the brushes in degrees, $Z$ is the number of armature inductors and $p^{\prime}$ is the number of paths in parallel between the brushes.

33. Examples showing applications of the external characteristics of a shunt generator. - The point of a characteristic curve at which a shunt generator operates is the point at which the generator characteristic is intersected by the receiving circuit characteristic, exactly as in the case of the series generator.

The curve $O A$, Fig. 37 , is the external characteristic of a given shunt generator, $O B$ is the characteristic of a given receiv- 
ing circuit containing resistance only, and the point $P$ represents the operating conditions of the generator when supplying current to the given receiving circuit. When the resistance $R_{r}$ of the

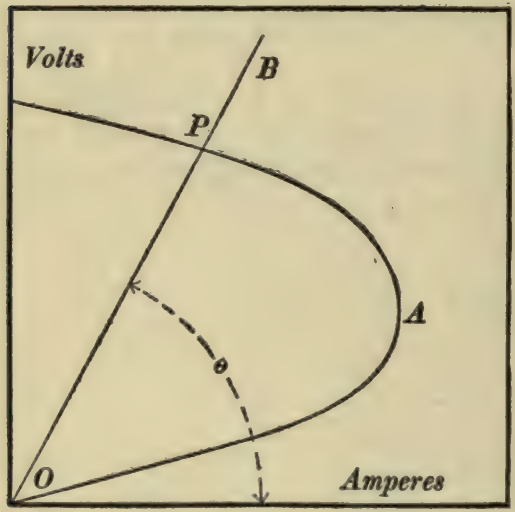

Fig. 37. receiving circuit is increased the angle $\theta$ increases, becoming $90^{\circ}$ for an infinite value of $R_{r}$, that is, when the receiving circuit is open. When $R_{r}$ is decreased more and more the current at first increases to an excessive value and the terminal voltage falls off because of armature drop $R_{a} I_{a}$, because of the decreased field excitation which accompanies the decrease of terminal voltage, and because of the demagnetizing action of the current in the armature. When the field excitation has been reduced considerably, the excessive demagnetizing action of the armature current begins to overpower it and the terminal voltage and current both fall in value.

When $R_{r}$ is zero, that is when the brushes of the shunt generator are connected by a short thick wire, then the voltage between the brushes is zero, the field current is zero, and the only current flowing through the armature and the short circuit between the brushes is

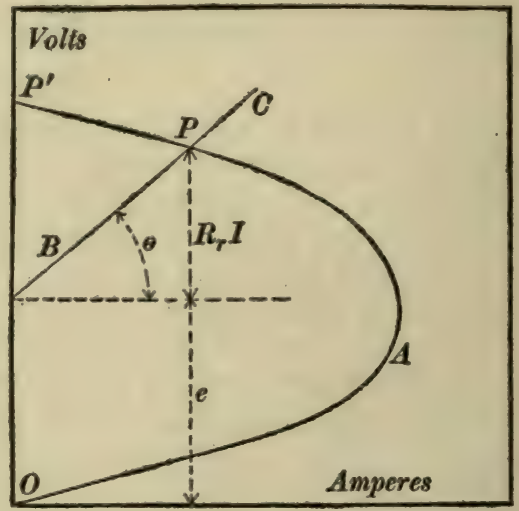

Fig. 38. the current which is due to the residual magnetism of the field magnet of the machine. 
The curve $O A$, Fig. 38 , is the external characteristic of a given shunt generator, the line $B C$ is the characteristic of a receiving circuit consisting of a storage battery of which the counter electromotive force is $e$ volts, and $P$ is the point which represents the conditions of operation of the generator when charging the battery. Before the battery circuit is connected to the generator, the generator is allowed to build up and reach the point $P^{\prime}$ of its characteristic. When the battery is connected the current output quickly increases, the terminal voltage falls off slightly, and the running conditions represented by the point $P$ are quickly established. No difficulty is encountered in charging a storage battery by means of a shunt generator.

Two shunt generators in parallel. - The curves $O A$ and $O B$, Fig. 39, are the external characteristics of two different shunt generators. The curve $O B$ is plotted to the left of the origin with its current output measured to the left for convenience.

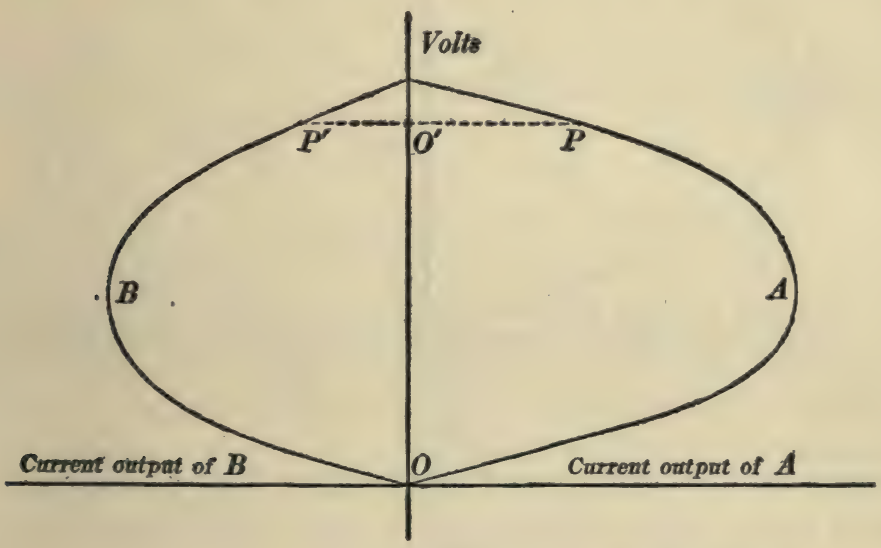

Fig. 39.

These two generators are connected in parallel to a receiving circuit. Under these conditions the terminal voltage must be the same for both generators. Therefore, if the two machines together deliver a specified current, this current is divided between the machines in such a way as to make their terminal voltages 
equal. Suppose, for example, that the combined current output of the two machines is 100 amperes, then the points $P$ and $P^{\prime}$ at which the two machines operate are determined by the two conditions: $(a)$ their ordinates must be the same, and $(b)$ the distance $P^{\prime} P$ must represent 100 amperes. In order that each machine may always deliver half of the total current, $O^{\prime} P$ must always be equal to $O^{\prime} P^{\prime}$, that is the two characteristics must be exactly alike. Fig. 39 shows the characteristics of two shunt generators of which the terminal voltages are equal at no load. Fig. 40 shows the characteristics of two shunt generators of which the

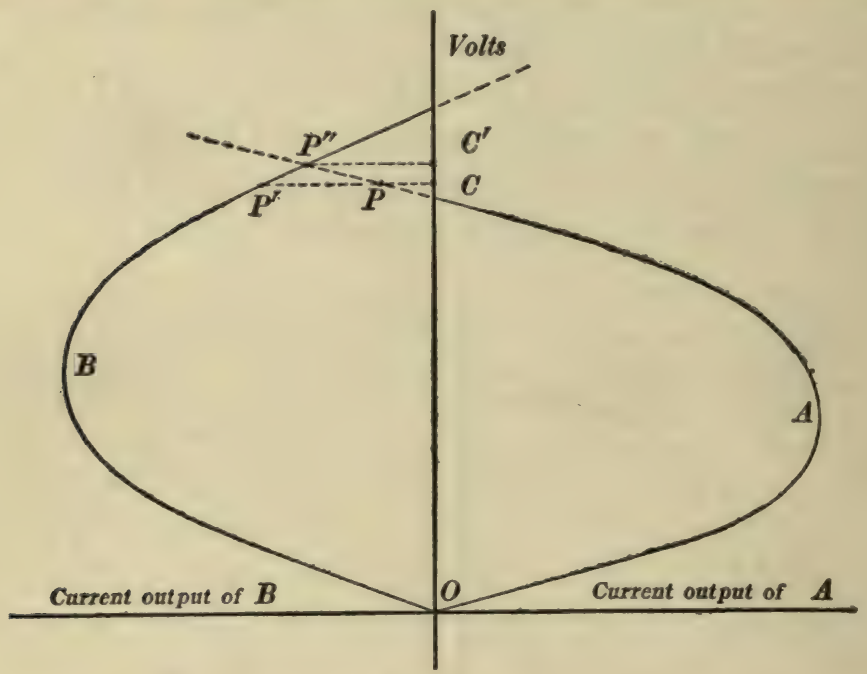

Fig. 40.

terminal voltages are unequal at no load. The dotted extension of each characteristic corresponds to motor action, that is to negative current output, or, in other words, to current flowing through the machine in opposition to its induced electromotive force. In this case the two machines operate at the point $P^{\prime \prime}$ when they are connected in parallel and give a combined current output of zero ; machine $B$ acts as a generator giving out a current equal to $P^{\prime \prime} C^{\prime}$ and this current flows backwards through machine $A$ which of course acts as a motor. 
When the combined current output of $A$ and $B$ is, say, 50 amperes, then the two operating points $P$ and $P^{\prime}$ have equal ordinates and the distance $P P^{\prime}$ represents 50 amperes.

34. Characteristic of the compound generator. - The compound dynamo has been heretofore treated, both as a generator and as a motor, as a modification of the shunt dynamo and there is no need in the present instance to discuss the compound generator minutely. Fig. 4I shows a typical external characteristic of an

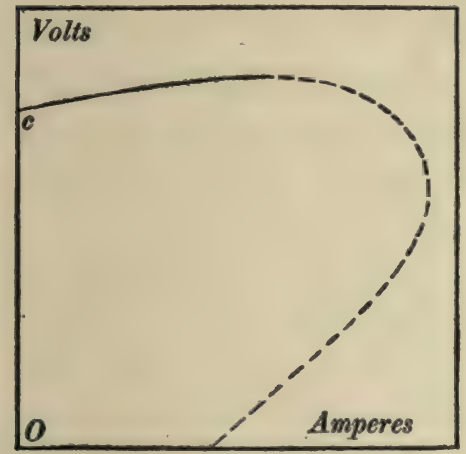

Flg. 41.

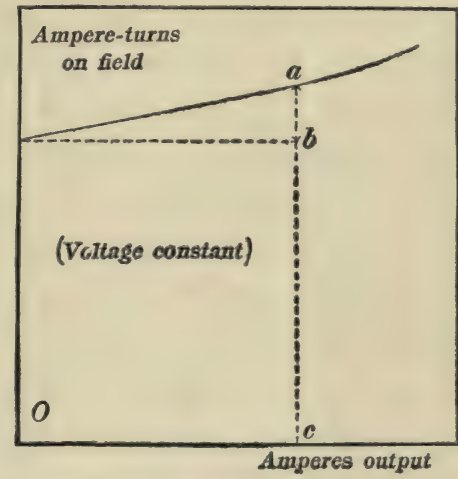

Flg. 42 .

over-compounded generator of which the terminal voltage rises with increase of current up to and beyond full rated load. The rapid fall of terminal voltage in the dotted portion of the curve is due to the great demagnetizing action of the current in the armature, to internal drop $R_{a} I_{a}+R_{c} I_{c}$, and to the decrease of that part of the field excitation which is due to the shunt field winding.

Armature characteristic. Calculation of the number of series field turns for compounding. (a) Flat-compounding. - Consider a given shunt generator driven at constant speed and having a terminal voltage equal to I Io volts when the current output is zero. Under these conditions the field excitation in ampere-turns has a definite value. Let the current output of the generator be increased more and more ; let the field rheostat be manipulated so 
as to keep the terminal voltage of the machine constant; and let corresponding values of armature current $I_{a}$ and of shunt field current $I$, be observed. The field excitation corresponding to any particular value of $I_{s}$ is $N I$ ampere-turns, where $N$ is the number of turns of wire in the shunt field winding. A curve may now be plotted using observed values of $I_{\alpha}$ as abscissas and corresponding values of $N I_{s}$ as ordinates as shown in Fig. 42. This curve is called the armature characteristic of the given generator.

From this armature characteristic the number of turns of wire required in the series field winding for flat-compounding may be determined as follows: Consider the full load armature current represented by $O c$, Fig. 42 , for which a total field excitation $a c$ is required. Of this total field excitation the shunt field winding supplies the amount $b c$, inasmuch as the terminal voltage of the generator is constant. Therefore the series field winding must supply the portion $a b$ of the field excitation, and the required number of series field turns may be found by dividing the ampere-turns represented by $a b$ by the current represented by $O c$ (for the long-shunt compound generator).

(b) Over-compounding.-The following numerical example illustrates sufficiently the calculation of over-compounding. The shunt field winding of a certain I2. I kw. bipolar generator supplies the 4,000 ampere-turns of field excitation required to give a terminal electromotive force of 1 IO volts at zero current output. When the current output is IOO amperes, a field excitation of 5,800 ampere turns is required to give $12 \mathrm{I}$ volts between generator terminals (Io per cent. over-compounding). On account of the increase of terminal voltage from no-load to full load, the shunt field winding supplies $1 \frac{1}{1} \frac{1}{0} \times 4,000=4,400$ ampere turns at full load. Therefore the series field winding must supply I,400 ampere-turns, and the series winding must consist of 14 turns of wire.

\section{CHARACTERISTIC CURVES OF MOTORS.}

35. Experimental determination of speed-torque characteristic of a motor. - The characteristic curve of a generator is definite 
only when certain of the mechanical conditions of driving are constant. Thus we might have a characteristic relation between the current output and electromotive force of a generator driven by an invariable torque, or of a generator driven at invariable speed. The latter condition nearly always occurs in practice; that is to say, electric generators are usually driven at an approximately constant speed. Therefore, the only important type of generator characteristic is that which corresponds to constant speed.

On the other hand, the relation between the speed of a motor and the torque developed by it is definite only when certain of the electrical conditions of driving are constant. Thus we may have a characteristic relation between the speed and torque of a motor which is supplied either with an invariable current, or with current from constant voltage mains. The latter condition, or an approximation to it, usually occurs in practice, and, therefore, the most important type of motor characteristic is that which corresponds to constant impressed electromotive force.

The speed-torque characteristic of a motor may be determined experimentally as follows: Provision is made for supplying the motor current from constant voltage mains. The motor is loaded by means of a Prony brake. The load is varied through a wide range by adjusting the brake, and corresponding values of speed and torque are observed. The observed speeds are then plotted as ordinates, and the correspending values of the observed torques plotted as abscissas.

An interesting characteristic curve of a motor is the torquecurrent curve at stand-still. This curve may be determined experimentally by fixing a lever to the motor pulley and observing, by means of a spring dynamometer, the pull at the end of this lever for various values of armature current. The product of this observed pull into the length of the lever arm gives the value of the torque and these values are to be plotted as ordinates and the corresponding currents as abscissas. This stationary torque exceeds the running torque, for a given value of field excitation and given value of the armature current, by the amount of torque 
required to overcome friction and windage, and to supply the eddy current and hysteresis losses in the armature core.

36. Typical examples of series motor characteristics. - (a) Series motor supplied with current from constant voltage mains. Fig. 43 shows a typical speed-torque characteristic of a series motor supplied with current from constant voltage mains. When the speed is zero the counter electromotive force of the motor

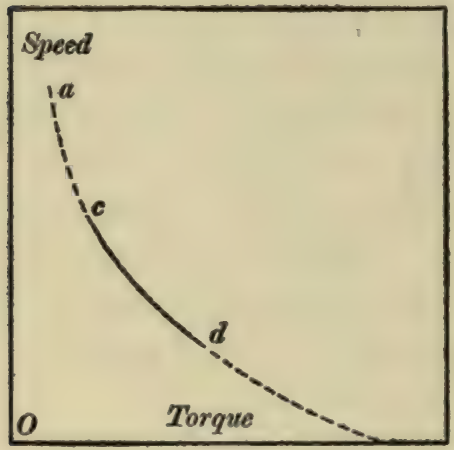

Fig. 43.

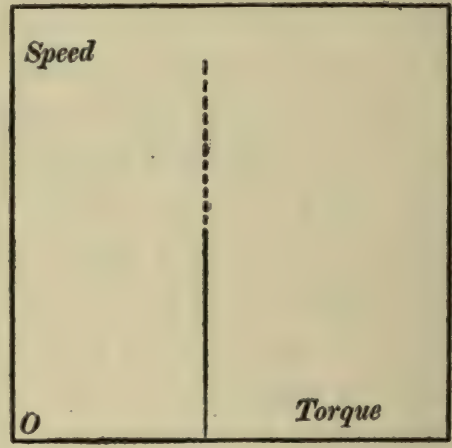

Fig. 44.

is zero; the current through the motor is then very large, and since this current flows through the field winding of the series motor, the field excitation is large. Therefore the flux $\Phi$ is large, and, according to equation (25) Chapter IV., the torque is very large. As the motor speeds up its counter electromotive force increases, the current decreases and the torque decreases. When the motor is unloaded its speed becomes excessive, and the torque drops to the value which is necessary and sufficient to overcome friction, and windage, and, in addition, to supply. to the armature the power lost in the armature core by hysteresis and eddy currents.

The portion $c d$ of the characteristic is the only portion used in practice, inasmuch as the excessive current which flows through the motor at low speeds would produce destructive heating, and the excessive speeds at light loads are dangerous.

(b) Series motor supplied with constant current. - Fig. 44 shows a typical speed-torque characteristic of a series motor 
supplied with constant current. In this case the torque is constant irrespective of the speed and the characteristic is a straight line parallel to the axis of speed. This constancy of torque is evident when we consider that constancy of current implies constancy of field excitation in a series motor, and therefore constancy of flux $\Phi$, so that the torque is constant according to equation (25), Chapter IV.

(c) Series motor supplied with constant current at low speeds and connected to constant voltage mains when the speed reaches a certain value. - These are the conditions which obtain in a railway motor. When the car is started a large resistance is in series with the motor, and as the car speeds up this resistance is slowly cut out so as to keep the current approximately constant at the greatest value that is considered permissible without overheating the motor. When this resistance is all cut out, the cur-

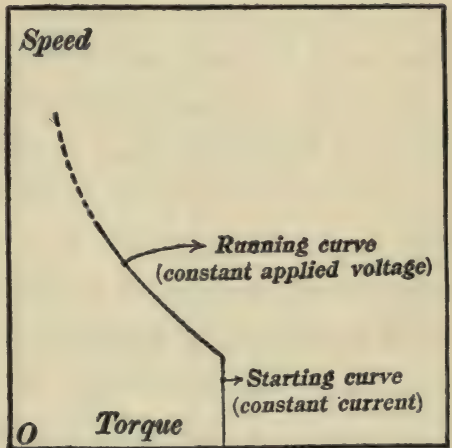

Fig. 45. rent begins to fall off inasmuch as the approximately constant voltage between trolley wire and rail is opposed by an increasing counter electromotive force in the motor. The speed-torque characteristic of a railway motor, under the above conditions, is a combination of the curves shown in Figs. 43 and 44. A typical example of a speed-torque characteristic of a railway motor is shown in Fig. 45.

Two rigidly coupled series motors connected in series. - When two similar series motors are connected in series to supply mains precisely the same current flows through both, and therefore they always develop nearly the same torque whether their speeds are alike or not. If the two motors, so connected, are coupled together mechanically, they run at the same speed, and they share 
a given total load very nearly equally. This state of affairs obtains when the two similar series motors of an electric car are connected in series at starting. If the motorman cuts out the starting resistance too rapidly a large current flows through the two motors, and one of the motors is likely to start the wheels of its truck slipping. The speed of this motor then increases greatly, its counter electromotive force increases, and the current through both motors decreases, thus reducing not only the torque of the slipping motor but the torque of the other motor as well. The result is that the speed of the car falls off unless the motorman quickly throws in more resistance so as to reduce the current and torque sufficiently to enable the slipping wheels to adhere to the rails again. The car will then speed up and the starting resistance may be slowly cut out.

Two rigidly coupled series motors connected in parallel. - When two series motors which are coupled together mechanically are connected in parallel to supply mains, they share the total load with approximate equality unless the machines are very dissimilar. Any slight increase of current through one motor, due, for example to a slight deficiency in the counter electromotive force of that motor, causes an increased field excitation, and an increased counter electromotive force which tends to prevent the increase of current. It is instructive to contrast with this the behavior of mechanically coupled shunt motors connected in parallel, as described in the next article.

37. Typical examples of shunt motor characteristics.-(a) Shunt motor supplied with current from constant voltage mains. - This is the condition under which shunt motors are always used in practice, except that certain modifications are introduced by the use of a rheostat in series with the armature at starting. These modifications are described under $(b)$. It has been pointed out in Chapter IV. that the shunt motor when supplied with current from constant voltage mains, runs at a speed which falls off slightly with increasing load. A typical speed-torque character- 
istic of a shunt motor is shown in Fig. 46. The equation to this curve may be obtained by eliminating $I_{a}$ between equations $(23 c)$ and (25), Chapter IV.; $\Phi$ being assumed constant, the resulting equation is the equation to a straight line. The effect of the demagnetizing action of the armature current is to cause the speed to fall off less with increasing torque than would otherwise be the case, as explained in Art. 6o, Chapter IV.

(b) Shunt motor started by a starting rheostat. - As explained in Art. 59, Chapter IV., the shunt motor (supplied with current from constant voltage mains) is started by first connecting

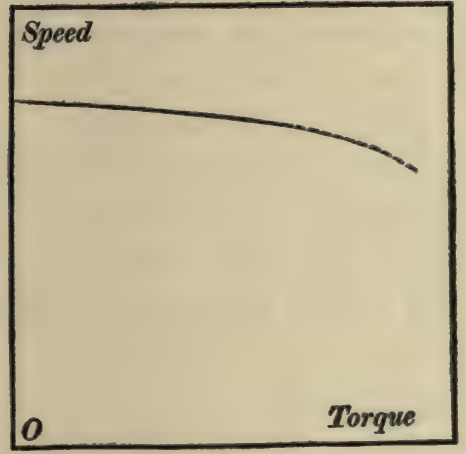

Flg. 46.

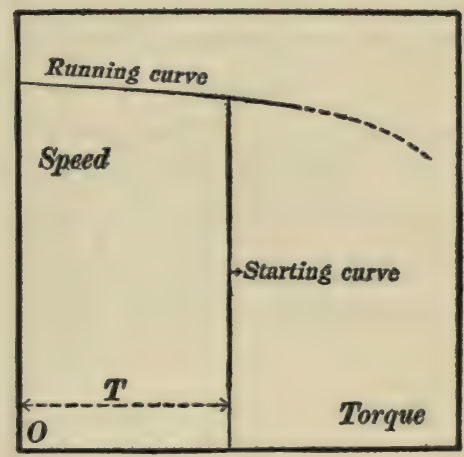

Fig. 47.

its field winding direct to the mains, and then connecting its armature to the mains through an auxiliary resistance which is slowly cut out as the motor speeds up. The ideally correct manipulation of the starting rheostat would be that which keeps the armature current constant until full speed is reached. This would produce a constant torque from the beginning to the end of the starting process, inasmuch as the field excitation of the motor is invariable. The vertical straight line in Fig. 47 represents the ideal starting characteristic of a shunt motor. The starting and accelerating torque $T$ may be made large or small at will.

Two rigidly coupled shunt motors in series. - Two similar shunt motors coupled together mechanically operate satisfactorily and divide a load equally when their armatures are connected in 
series, provided the shunt field winding of each machine is connected directly to the supply mains. If the shunt field windings are designed for the voltage of the supply mains they should of course be connected in parallel to the mains ; if they are designed for half of the supply voltage they should be connected in series. Of course the speed of the motors will be only half of what it would be if the full supply voltage were to be applied to the armature terminals of each machine.

Two rigidly coupled shunt motors in parallel. - When two shunt motors which are coupled together mechanically, are connected in parallel to the supply mains (that is when the armatures are connected in parallel, the field windings being connected directly to the mains), a very slight dissimilarity between the two machines causes a great inequality in the distribution of the total load between them. This is due to the fact that a slight increase of current through one armature causes a decrease of the counter electromotive force in this armature by its demagnetizing action, and this tends to further increase the current through this arma-

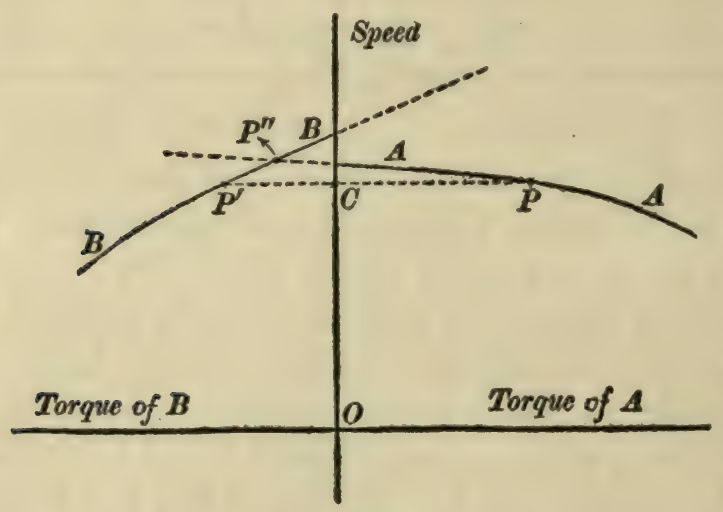

Fig. 48.

ture. At the same time the decrease of current through the other armature causes its counter electromotive force to increase thus tending to reduce the current still more. That is, any 
slight dissimilarity between the two shunt motors is greatly exaggerated in its effect upon the distribution of load between the two machines by these internal electromagnetic actions.

It is instructive to show, by means of the speed-torque curves of two shunt motors, how a given load will be distributed between them. Thus $A A$ and $B B$, Fig. 48 , are the speed-torque curves of two shunt motors, the curve $B B$ being drawn to the left of the origin. The dotted portions of $A A$ and $B B$ correspond to generator action. When the two motors are coupled together mechanically their speed is the same. Under these conditions a given total torque $P^{\prime} P$ is shared unequally by the two motors $\left(P^{\prime} C=\right.$ torque of $B$, and $P C=$ torque of $\left.A\right)$ unless the two motors are exactly alike.

38. Series motor characteristics as used in electric railway engineering. - The characteristics of a motor, supplied, for example, from constant voltage mains, may be expressed not only in terms of speed and torque, but also, if desired, in terms of speed and current, or in terms of torque and current, inasmuch as a definite value of current always corresponds to a given torque or to a given speed.

Fig. 49 shows the characteristic of a single railway motor expressed in terms of speed of car in miles per hour and current taken by the motor. The speed of the motor in revolutions per second bears a definite ratio to the speed of the car in miles per hour; this ratio is known when the gear ratio and size of car wheels are known.

Fig. 50 shows the characteristic of a single railway motor expressed in terms of tractive effort and current taken by the motor. The tractive effort bears a definite relation to the motor torque; this relation is known when the gear ratio, size of car wheels, and motor friction are known.

The speed-current and tractive effort-current characteristics of series motors are of great importance in electric railway engineering, since by their means the details of performance and power 
416 ELEMENTS OF ELECTRICAL ENGINEERING.

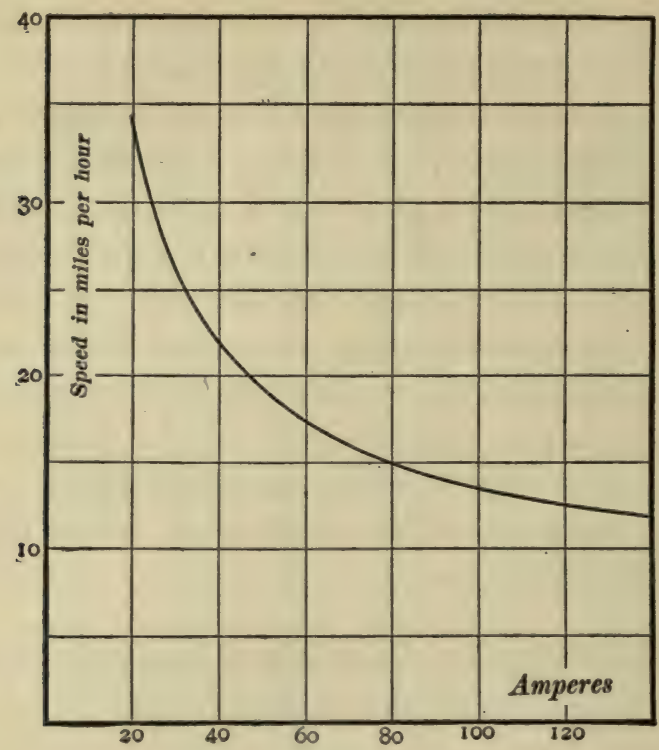

Fig. 49.

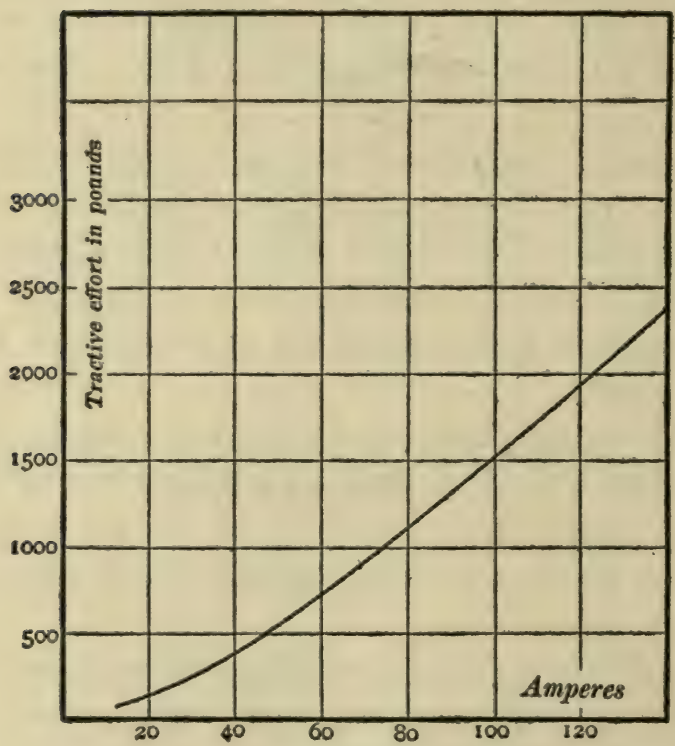

Fig. 50. 
consumption of an electric car or train, with a given equipment of motors may be predetermined; or conversely, with given schedule and road-bed, a proper selection of motors for the given service conditions, may be made.

The following example illustrates the application of series motor characteristics to the calculation of the amount of power delivered to the car wheels by a given motor equipment, and the efficiency obtained under specified conditions.

Example. - A fifteen ton car is equipped with two similar series motors of which the characteristic curves, with 500 volt supply (no starting resistance in circuit), are shown in Figs. 49 and 50. With the motors connected in parallel the car travels at a uniform speed of fifteen miles per hour up a 6.25 per cent. grade. It is required to find the current taken by each motor, the total tractive effort developed by both motors, the total power delivered to both motors, the net power represented by the total tractive effort at the given speed, and the efficiency of the car equipment under the specified conditions.

Solution. - From the curve in Fig. 49 we find the current per motor corresponding to fifteen miles per hour to be 80 amperes, so that the total current delivered to the car is I60 amperes, which at 500 volts represents a power input of 80 kilowatts. From the curve in Fig. 50 we find the corresponding tractive effort per motor to be I, I 25 pounds, so that the total tractive effort is 2,250 pounds. The power in kilowatts represented by this tractive effort at the given speed is the total power delivered by the two motors to the car wheels, and is found to be :

$$
\frac{2,250 \times 15 \times 5,280}{3,600} \times \frac{746}{550} \times \frac{1}{1,000}=67.14 \text { kilowatts }
$$

The efficiency is $67.14 / 80$, which is 0.8393 or 83.93 per cent. 


\section{APPENDIX C.}

\section{ARMATURE WINDINGS.}

39. Classification of armature windings. - Armatures may be classified with respect to the position and arrangement of the windings on the armature core, as (I) ring armatures, (2) drum armatures, and (3) disk armatures. These names were originally applied in view of differences in the external appearance of the armatures, but the distinction here intended refers to essential differences of the windings of wire on the armature core. Thus, in many modern dynamos of large size the armature has a diameter many times as great as its length parallel to the shaft, and the armature core, and to a certain extent the finished armature, has the appearance of a ring; but whether the armature is to be classed as a ring armature or a drum armature is determined solely by the arrangement of the windings.

I. A ring armature is one in which a ring-shaped core of laminated iron has the windings placed upon it in the form of a helix as shown in Fig. I7, Chapter II., and in Fig. 4I, Chapter II. The helical form of the winding in Fig. 4I does not show clearly for the reason that the windings are grouped together in coils, but these coils are connected to each other so as to constitute a continuous helix. A ring armature may be used in connection with a two-pole field magnet as shown in Fig. 17 , or with a multipolar field magnet as shown in Fig. 22. The simple ring winding is sometimes called the helical* winding.

2. A drum armature, as used for a bipolar field magnet, is one in which a cylindrical core of laminated iron has the wire wound lengthwise (parallel to the shaft) on the outside surface of the core, and diametrically (nearly) across the ends. In a drum

* The term "spiral," frequently used to designate this type of winding, is a mis. nomer. 
armature for a multipolar field magnet, the wire is wound lengthwise on the outside surface of the core and across the ends along chords of nearly $90^{\circ}$ for a 4-pole field magnet, along chords of nearly $60^{\circ}$ for a 6 -pole field magnet, along chords of nearly $45^{\circ}$ for an 8-pole field magnet, and so on. The essential difference between the drum armature and the ring armature is that in the drum armature there are no return conductors in the interior of the core; but each conductor on the outside surface of the core is connected to a return conductor which is also on the outside surface and nearly $360^{\circ} / p$ from the first conductor, where $p$ is the number of field poles; whereas, in the ring armature each outside conductor is connected to a return conductor on the inside of the ring.

(3) Disk armatures. - The characteristic feature of the disk armature is that the conductors are arranged in a plane like the spokes of a wheel. These conductors move between two or more pairs of opposite magnet poles which face each other on opposite sides or faces of the disk-like armature. These radial wires are connected at their inner ends in the same manner as are the ends of the conductors of a drum armature at the commutator end of the armature, and the outer ends of the radial wires are connected to each other in the same manner as are the ends of the conductors of a drum armature at the end of the armature opposite to the commutator. Disk arnatures are seldom used nowadays in American practice.

The drum winding is the type which is most used. The great advantage of the drum winding is that, having no conductors which pass through the interior of the armature core, it is possible to wind the armature coils on forming spools, tape, varnish, and bake them, and slip them into place on the armature core as shown in Figs. 48 and 49, Chapter II.

40. Open and closed coil armatures. - In most direct current armatures the winding constitutes a closed circuit of wire as in the simple ring winding shown in Fig. I7, Chapter II. Such armatures are called closed coil armatures. In some direct current armatures, for example, the armatures of the Brush, the Thomson-Houston, and the Westinghouse arc light generators, and in the armatures of most alternators, the windings do not con- 
stitute a closed circuit of wire. Such armatures are called open coil armatures. The simplest form of open coil armature for a direct current dynamo is represented in Fig. 2 I, Chapter II., which shows a single turn of wire with its ends connected to the segments of a two part commutator.

Closed coil armatures always give a much steadier current than open coil armatures, and they have a smaller tendency to cause sparking at the brushes.

41. Inductors. - That portion of the element of an armature winding which actually cuts the lines of force as the armature rotates, and in which electromotive force is induced, is called an inductor. Thus the inductors on a ring armature consist of the wires which lie on the outside surface of the ring. The wires which lie on the interior of the ring cut no lines of force, and are therefore not inductors; they serve only as return paths for the electric current. In the drum armature the inductors are the straight wires lying on the surface of the core parallel to the shaft. The end connections between inductors, including those portions of the straight wires projecting beyond the armature core and pole faces (as in the case of barrel wound drums) are not inductors.

Armature inductors have been called armature conductors in the previous chapters of this text, but in discussing armature windings it is convenient to employ a term which cannot lead to confusion.

42. Armature coil. Element of a winding. - That part of an armature winding which terminates at two commutator bars or segments is called a winding element or a winding unit. A winding element consists usually of a single armature coil, but there may be two or more coils in a winding element. The terms armature coil and winding element are used synonymously hereafter.

In so far as winding connections are concerned, the number of turns of wire in an armature coil is a matter of indifference, and therefore each armature coil may be treated as if it had one turn 
of wire only, and each side of a coil (for a drum winding) may be thought of as a single inductor. The subsequent discussion of lap and wave windings is based upon the assumption that an armature coil consists of two inductors only.

43. Possible number of commutator bars or segments. - It is evident from the discussion of the simple ring winding given in Arts. 28 and 29, Chapter II., that each inductor on a ring winding may be a commutator bar, or be connected to a commu-

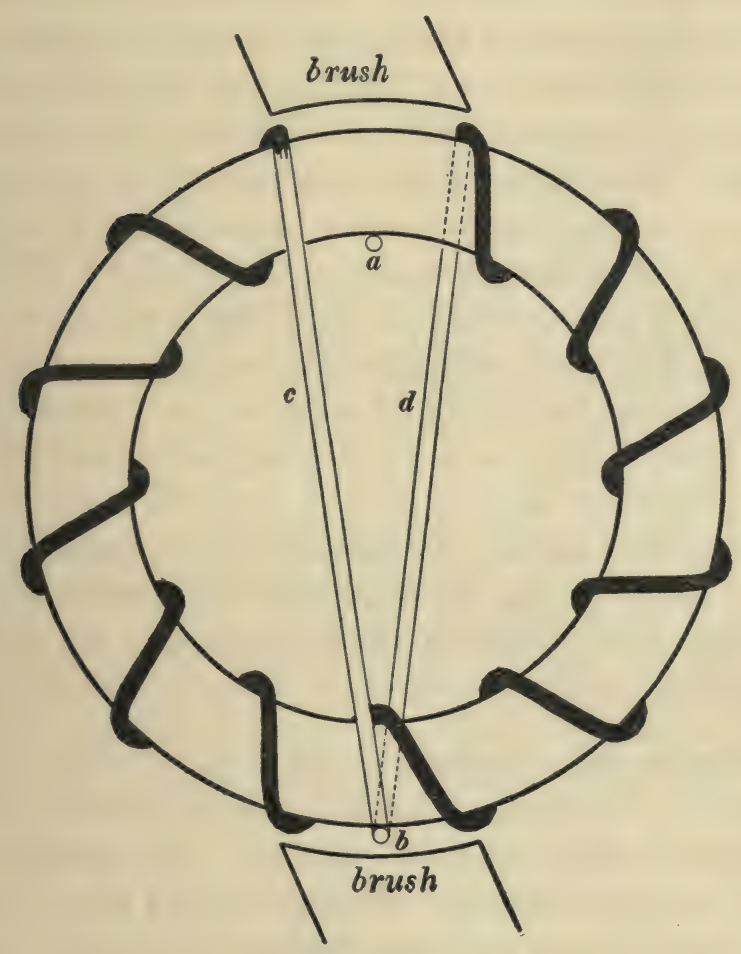

Fig. 51 .

tator bar. Or, as stated in Art. 28, Chapter II., every second inductor, or every third inductor, or every fourth inductor, etc., may be connected to commutator bars. That is to say, the num- 
ber of commutator bars for a simple ring winding may be equal to the total number of inductors $Z$, or the number of bars may be $Z / m$, where $m$ is any integer which is a factor of $Z$.

The necessary relation between the number of inductors and the number of commutator bars in the case of a drum armature may be shown as follows: The drum armature may be looked upon as a ring armature modified by transferring each inside return wire, $a$, Fig. $5 \mathrm{I}$, to the opposite side $*$ of the armature at $b$, the wire $c$ being extended across one end of the armature, and the wire $d$ across the other end. Now, if both $b$ and the adjacent inductor are commutator bars, or are connected to adjacent commutator bars, it will be seen from Fig. 5 I that both wires $c$ and $d$ will connect across directly from the positive brush to the negative brush and the dynamo armature will be short-circuited. Therefore a drum armature cannot have adjacent inductors connected to commutator segments. Every alternate inductor of a drum winding corresponds to an inside return conductor of a ring winding, and, at most, every alternate inductor of a drum winding can be a commutator bar, or be connected to a commutator bar. That is to say, the greatest possible number of commutator bars in case of a drum winding is $Z / 2$, where $Z$ is the number of inductors. The number of commutator bars may of course be less than this. In fact the number of commutator bars in case of a drum winding may be $Z / 2 m$ where $m$ is any integer, and $2 m$ is a factor of $Z$.

44. Lap and wave windings. - There are two different schemes of connecting the ends of the inductors of a drum armature to form a symmetrical closed coil winding. One of these schemes is called the lap winding and the other is called the wave winding. There is no essential difference between these two schemes in case of a bipolar dynamo; but in case of a multipolar dynamo the (simplex) lap winding always gives as many paths between the brushes as there are field poles, while the (simplex) wave

* For a two pole drum armature. 
winding always gives two paths between the brushes whatever the number of field poles may be.

The simple ring winding when used with a multipolar field magnet offers a number of paths between the brushes equal to the number of field poles as explained in Art. 29, Chapter II., and the simple ring winding corresponds in this respect to the lap wound drum.

The windings of a ring armature, when used with a multipolar field magnet, may be grouped in coils, and these coils may be interconnected in such a way as to give a symmetrical closed coil winding which presents two paths between the brushes whatever the number of field poles may be. Such a ring winding corresponds to the wave wound drum.

The following discussion of lap and wave windings is limited to drum armatures.

From the fundamental equation of the dynamo, equation (2I) Chapter II., it is evident that a multipolar dynamo with a wave wound armature $\left(p^{\prime}=2\right)$ gives a greater electromotive force for a given number of armature inductors, than the same dynamo with a lap wound armature $\left(p^{\prime}=p\right)$. High voltage dynamos (especially those of small current output) are usually therefore provided with wave wound armatures, and low voltage dynamos usually have lap wound armatures.

The lap wound armature must have as many brush sets as field poles if every current path in the armature is to be utilized. The wave wound armature needs only two brush sets to bring into service every armature inductor, as pointed out in Art. 50.

45. Lap winding. - Starting at commutator segment No. I, in Figs. 52 and 53 , if we proceed by front end connection to inductor No. I, then along inductor No. I towards the back (or pulley end) of the armature, then across the back (by dotted line in Fig. 52) to inductor No. 6, thence along inductor No. 6 towards the front (or commutator end), then by front end connection to commutator segment No. 2, we shall have completed 
424 ELEMENTS OF ELECTRICAL ENGINEERING.

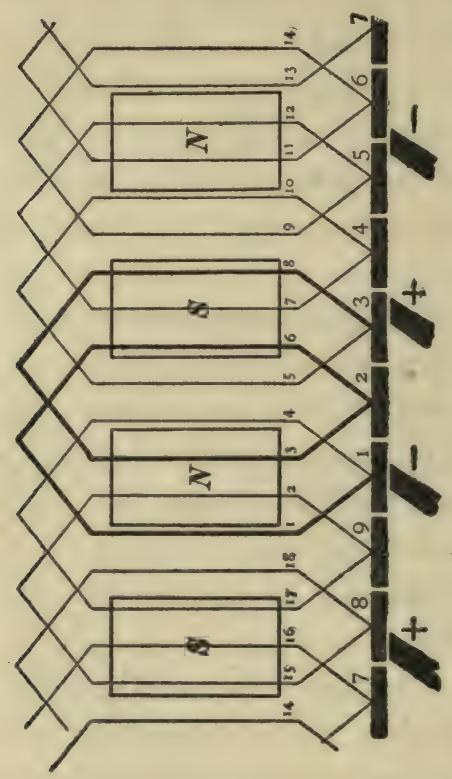

莒

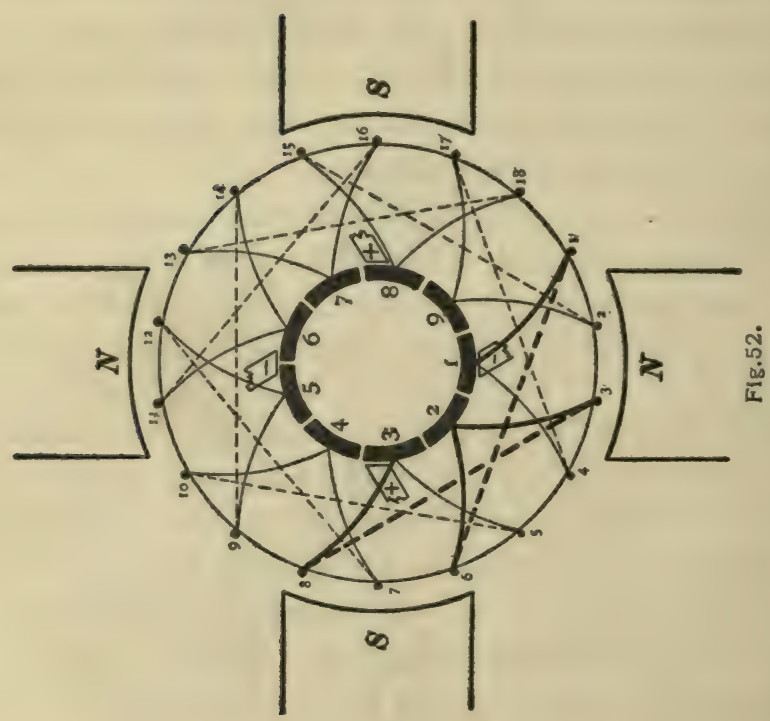


an element of the winding. If we now continue from commutator segment No. 2 along inductor No. 3, across the back to inductor No. 8 , and thence along inductor No. 8 to commutator segment No. 3, we shall have completed the second element of the winding. The rest of the winding is merely a repetition of the steps just described. It is evident from Figs. 52 and 53 , that each winding element consists of an almost closed loop or coil, and that the second element laps over the first, as shown by the heavy lines in Figs. 52 and 53. It is on account of this overlapping of the elements that this winding is called the lap winding.

In Figs. 52 and 53 it can be seen that inductor No. I is connected at the back to inductor No. 6, inductor No. 3 to No. 8 ; inductor No. 5 to No. IO; and so on, that is, each odd numbered inductor is connected to the fifth following inductor. This interval between inductors connected at the back end of the armature is called the back pitch of the winding and it is equal to 5 in Figs. 52 and 53. In this same figure it can be seen that inductor No. I starts from segment No. I, and that inductor No. 4 is also connected to segment No. I, and in a similar manner inductors Nos. 3 and 6 are both connected on the front end to segment No. 2, and so on. This interval between inductors connected together at the front end is called the front pitch of the winding, and it is equal to 3 in Figs. 52 and 53.

In tracing through the winding in Figs. 52 and 53, one progresses in one direction at the back, and in the opposite direction at the front of the armature, and therefore one pitch, say the back pitch, is considered positive and the other pitch is considered negative.

In Figs. 52 and 53 the terminals of an element of the winding are connected to adjacent commutator segments, and the winding is called a simplex or single lap winding.

In what is called a duplex or double lap winding the terminals of an element of the winding are connected, not to adjacent commutator segments, but to every other segment. In what is called a triplex or triple lap winding the terminals of an element of the 
winding are connected to every third commutator segment, and so on. The interval between the terminals of a winding element, expressed in terms of the number of commutator segments, is called the commutator pitch. Thus in Figs. 52 and 53 the commutator pitch is, let us say, $+\mathrm{I}^{*}$. In a duplex winding the commutator pitch is 2 , plus or minus, in a triplex lap winding the commutator pitch is \pm 3 , and so on.

The resultant pitch, $y$, is the algebraic sum of the front pitch and the back pitch. Thus in Figs. 52 and 53 the resultant pitch is

$$
y=y_{b}+y_{f}=5+(-3)=+2
$$

The average pitch is half the arithmetical sum of the front pitch and the back pitch. Thus in Figs. 52 and 53 the average pitch is

$$
y_{a v}=(5+3) / 2=4
$$

46. Wave winding. - Starting at commutator segment No. I in Figs. 54 and 55 , if we proceed by front end connection to inductor No. I, then along inductor No. I towards the back (or pulley end) of the armature, then across the back (by dotted line in Fig. 54) to inductor No. 6, then along inductor No. 6 towards the front (or commutator end), then by front end connection to commutator segment No. 6 , we shall have completed one element of the winding. The next element of the winding is connected similarly from segment No. 6 through inductors No. II and No. I6 to segment No. 2 as shown by the heavy lines in Figs. 54 and 55. The rest of the winding is merely a repetition of the steps just described for the first two elements of the winding.

It is evident from Figs. 54 and 55 that two consecutive winding elements have the appearance of two successive waves or zig-zags. This is shown with especial distinctness in Fig. 55. It

* If front and back pitches are interchanged, the commutator pitch is reversed in sign, that is the first element of the winding (in Figs. 52 and 53) would terminate at segment No. I and segment No. 9. 

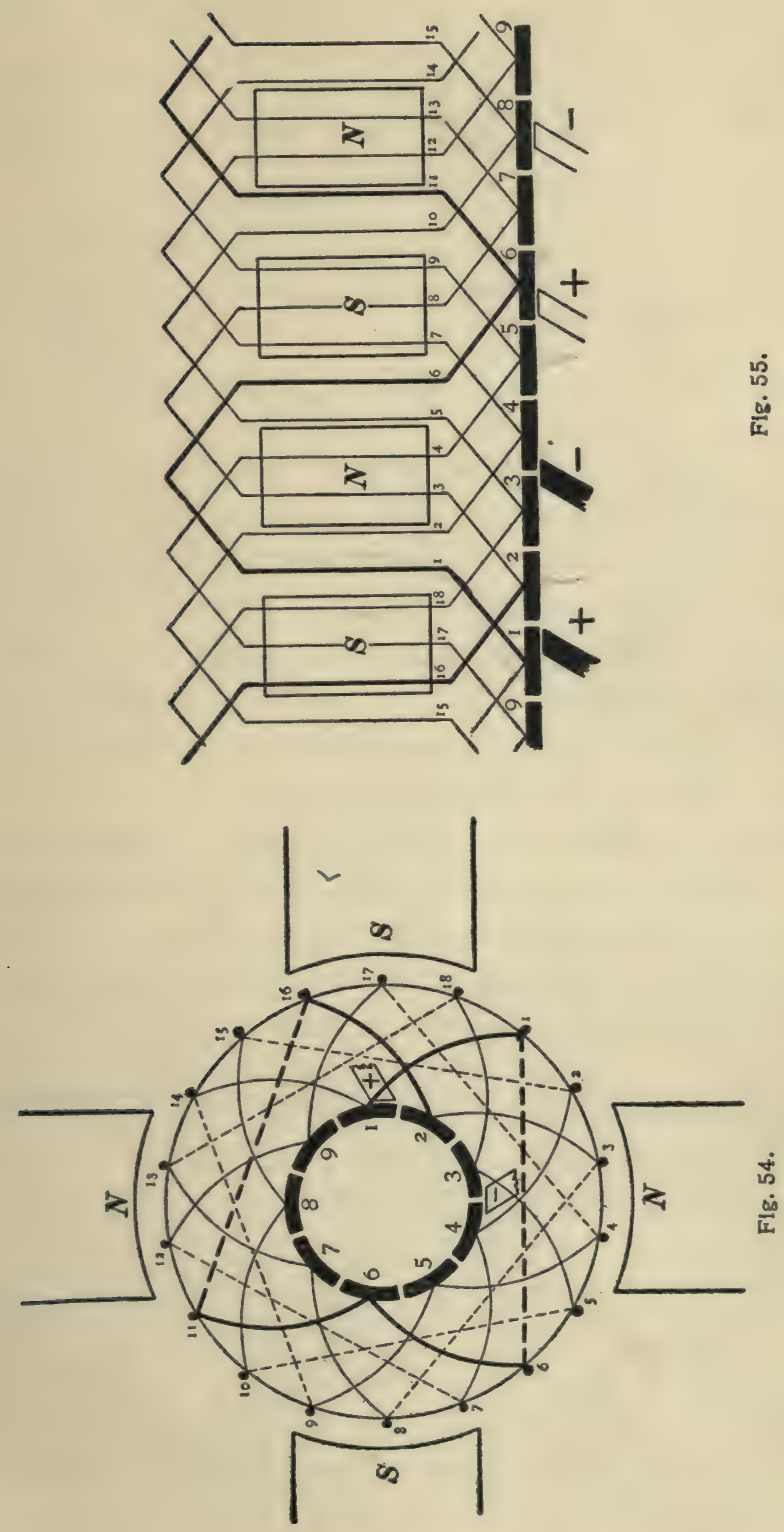
is on account of this wave-like appearance that this winding is called the wave winding.

In Figs. 54 and 55 it may be seen that the back pitch is +5 , that the front pitch is +5 , and that the commutator pitch is also +5 . The resultant pitch is

$$
y=y_{b}+y_{f}=5+5=10
$$

and the average pitch is

$$
y_{a v}=(5+5) / 2=5
$$

Figs. 54 and 55 show a four-pole winding, and it may be seen that starting from commutator segment No. I we come to the next commutator segment No. 2, after passing through two consecutive elements of the winding. Any wave winding which brings one from a given commutator segment to an adjacent segment after passing through $p / 2$ winding elements, where $p$ is the number of field magnet poles for which the winding is made, is called a simplex or single wave winding.

In what is called a duplex or double wave winding, $p / 2$ winding elements lead from a given commutator segment to the next segment but one ; in what is called a triplex or triple wave winding, $p / 2$ winding elements lead from a given commutator segment to the third following segment, and so on.

47. Reëntrancy.-A winding which closes on itself, $i . \ell$., forms a closed (or complete) electrical circuit, is called a closed coil winding; and because such a closed coil winding reënters itself, it is called a reentrant winding.

Armature windings may be singly, doubly, triply, or in general, multiply reëntrant. The number of times a winding reënters, is called the degree of reëntrancy. A singly reëntrant winding is one in which if one starts at inductor No. I and traces through the successive inductors in the order of winding, the whole winding has to be traced through before conductor No. I is reached or reëntered. 
A doubly reëntrant winding is one in which if one starts at inductor No. I and traces through the successive inductors in the order of winding, only one-half of the whole winding has to be traced through before inductor No. I is reached or reëntered; the other half being exactly like this first half.

A triply reëntrant winding, in a similar manner, is a winding which on being traced through from conductor No. I and back to the starting point still leaves two thirds of the total inductors to be traced through.

In tracing through the successive inductors of the winding, one may pass around the armature once, twice, or any number of times before reaching the starting point. The number of times one passes around the armature in tracing through a reentrant set of inductors has no essential relation to the degree of reëntrancy. For example, in tracing through a singly reëntrant drum winding, one may pass around the armature as many as twentyfive or more times.

48. Simplex and multiplex windings. - Consider two similar drum simplex lap windings like Figs. 52 and 53. Let these windings with their commutators be designated as $A$ and $B$. Imagine the winding $B$ to have its inductors sandwiched between the inductors of winding $A$ on the same core, and imagine the commutator segments of winding $B$ to be likewise sandwiched between the commutator segments of winding $A$. We now have an armature core upon which there are 36 inductors and a commutator having 18 segments; odd numbered segments belonging to winding $A$ and even numbered segments to winding $B$. If the original brushes in Figs. 52 and 53 are made thick enough to touch always at least two segments of this doubled commutator, then windings $A$ and $B$ are in parallel between the brushes, and the number of paths in parallel through the armature winding between positive and negative brushes is doubled. The winding here described is called a duplex lap winding and it is doubly reëntrant. 
A triplex winding consists, in the simplest cases, of three similar simplex windings with their commutators, sandwiched together on one core and put in parallel by using sufficiently thick brushes to insure contact with all three windings at each brush.

Any number of simplex wave windings with their commutators may be sandwiched together as above described, giving a multiplex wave winding.

Any winding not a simplex winding is in general called a multiplex winding, although the above description of a multiplex winding as built up of independent and complete simplex windings sandwiched together, is not entirely general.

The advantage of multiplex over simplex windings is that sparking at the brushes is reduced by the use of multiplex windings. In a simplex winding one winding element is shortcircuited when a brush touches two adjacent commutator bars as explained in Art. 78, Chapter VI., and a brush must at certain instants touch two adjacent commutator bars. In a duplex winding no portion of the winding is short-circuited when a brush touches two adjacent commutator bars ; short-circuit only occurs when a brush touches three adjacent bars, and, although a brush must at certain instants touch three adjacent bars in a duplex winding, still the short-circuit includes a greater length of path in the material of the brush than in case of a simplex winding, and the resistance of the brush material is more effective in the prevention of sparking.

49. Number of paths in parallel. Number of inductors in series. - In the simplex lap winding there are $p$ paths in parallel between the positive and the negative brushes, where $p$ is the number of field poles. Therefore there are $Z / p$ inductors in series in each path.

In a simplex wave winding there are two paths in parallel between the positive and the negative brushes irrespective of the number of poles and of the number of brush sets. Therefore 
there are $Z / 2$ inductors in series between the positive and the negative brushes, in the wave winding.

In a multipolar machine, with a given total number of inductors $Z$, the lap winding gives many more paths in parallel between brushes, and fewer inductors in series in each path, than the wave winding Therefore the use of lap winding is sometimes called parallel grouping of the inductors, and the use of wave winding is sometimes called series grouping of the inductors.

Furthermore, on account of the fact that the simplex lap winding has $p$ paths in parallel between brushes, this winding is sometimes called a multiple-circuit winding, and on account of the fact that the simplex wave winding has only two paths in parallel between brushes (independently of the number of poles) this winding is sometimes called a two-circuit winding.

Combining simplex windings to give a multiplex winding always multiplies the number of paths in parallel between brushes. Thus a duplex winding multiplies the number of paths by 2, a triplex winding multiplies the number of paths by 3 and so on. Combining simplex windings to give a multiplex winding does not alter the number of inductors in series between brushes, and hence does not alter the electromotive force of one of the component simplex windings namely the electromotive force between the brushes.

The number of inductors in series between brushes (and hence the electromotive force of the machine) may be multiplied by using a coil of many turns, instead of two inductors, in forming an element of the winding. Thus in Figs. 52 and 53, the element terminating in segments I and 2 may be made into a coil as follows: Let each inductor shown in the figure represent a slot in the armature core. Then starting from segment No. I we may wind a wire up slot No. I and down slot No. 6 any number of times before connecting to segment No. 2. This group of turns constituting one winding element is called a coil or section of the armature winding. 
The use of many turns of wire in a winding element increases the tendency to spark at the brushes.

The conductor of an armature winding is frequently composed of a number of small wires in parallel, instead of one large solid wire, in order that the conductor may be flexible and easily wound. Such a conductor is said to be a stranded conductor. A stranded conductor is electrically a single inductor and is to be counted as such in applying the formula for calculating the electromotive force of a machine.

50. Number of brushes required. - In any closed coil winding whatever, there are $p$ neutral points around the commutator, and $p$ brush sets may be used in any case.

I. Lap Winding. - In the lap winding, simplex or multiplex, $p$ brush sets must be used to permit the delivery of the greatest current output with the least $I^{2} R$ loss in the armature windings.

2. Wave Winding. - In the wave winding, simplex or multiplex, two brush sets, a positive set and a negative set, are necessary and sufficient, but any number of sets up to and including $p$ sets may be used.

The fact that two brush sets are sufficient for the wave winding is shown in Figs. 54 and 55, and is evident from the following considerations: Suppose first that there are four brush sets, one at each neutral point. The commutator segments I and 6, under the two positive brushes are electrically connected through the two inductors Nos. I and 6, and their end connections. The figure shows that these two inductors are situated between the pole pieces and have no electromotive forces induced in them, hence they may be considered simply as cross connections between the two positive neutral points. The negative neutral points are similarly connected by the idle inductors Nos. 5 and IO. In consequence of these cross connections the only function of the second pair of brush sets is to aid the first set in collecting current from and delivering current to the commutator segments by the increased contact surfaces of brushes with commutator; but the distribution of current in the windings 
is in no way altered by the addition or removal of the second pair of brush sets.

When, however, two brushes only are used for a $p$-pole wavewound armature then $p / 2$ winding elements, all in series, are short-circuited under a brush when the brush touches two commutator bars (in a simplex winding). Therefore the wave winding using two brushes has a greater tendency to spark at the brushes than the lap winding with $p$ brushes. This disadvantage of the wave winding is obviated by using $p$ brushes instead of two.*

51. Conditions which must be satisfied by a closed coil drum winding. - Lap and wave windings. - No drum winding can have an odd number of inductors. Both front and back pitches must be odd in a simplex winding. This is due to the fact that odd numbered inductors are to be thought of as returns for the even numbered inductors, and therefore even numbered inductors must be connected at both ends to odd numbered inductors. This requires an odd pitch at both ends.

Both front and back pitches must be approximately equal to $Z / p$ in order that the two inductors which constitute a winding element may move simultaneously under field poles of opposite polarity. Under these conditions electromotive forces induced in the two inductors will be additive. The smallest value of front or back pitch which meets this requirement is a pitch which stretches completely across a pole face, and the largest value of front or back pitch whieh meets this requirement is a pitch which barely stretches across from a given pole tip to the nearest pole tip of like polarity. When front and back pitches differ considerably from $Z / p$ the winding is called a chord winding.

The advantage of the chord winding is that the demagnetizing action of the armature current is to a great extent eliminated thereby, and its disadvantage is that it gives a very narrow neutral zone on the commutator. The elimination of the demagnetizing action is due to the fact that the armature current flows in opposite directions in alternate inductors in each of the two bands of inductors shown in Fig. IOI, Chapter VI.

* See Hobart's Electric Motors, page 48, Whitaker \& Co., London, 1904. 
This may be shown clearly by making a diagram of a chord winding and indicating by arrows the direction of the current in each inductor. The neutral zone on the commutator includes those commutator bars in which terminate all of the winding elements which do not include inductors under the pole faces; between such commutator bars the electromotive force is nearly zero. In a winding of which front and back pitches are very nearly equal to $Z / p$ the neutral zone is nearly as wide as the angular space between adjacent pole tips, because, if the pitch were $Z / p$, every inductor which, for a given position of the armature, lies between pole tips would be connected front and back to other inductors which also lie between pole tips. On the other hand, when the pitch, front or back, differs considerably from $Z / p$, there is only a narrow band of inductors, between a given pair of pole tips, which connect to other inductors not under the pole faces, for a given position of the armature.

For a given number of inductors the front and back pitches must be chosen to comply with the following requirements :

(a) All the winding elements must be similar mechanically and electrically and they must be placed symmetrically on the armature core.*

(b) In tracing through a simplex winding every inductor must be passed over once and once only, and the winding must close on itself or be reëntrant.

(c) In a multiplex winding which consists of a combination of complete and independent simplex windings, each simplex winding must satisfy condition (b).

(d) A multiplex winding which does not consist of complete and independent simplex windings must as a whole satisfy condition $(b)$. Such a multiplex winding is called a singly reëntrant multiplex winding.

(e) In a two layer winding (in slotted armatures, two inductors per slot) the inductors in the lower layer should be considered even numbered, and those in the upper layer odd numbered.

Lap windings. - (a) Front and back pitches are opposite in sign.

(b) Front and back pitches cannot be equal. Equality of

* In some cases armature core stampings already in stock are used for the armature of a newly designed machine, even though a suitable arrangement of the windings necessitates the leaving out of two half-coils in two of the slots. In such a case the two slots which are half filled by the winding, are filled up with the two halves of a "dummy" coil. 
front and back pitches would connect both terminals of a winding element to the same commutator segment.

(c) In simplex lap windings front and back pitches differ by 2 , so that the average pitch is an even number, and the commutator pitch is $\pm \mathrm{I}$.

(d) In a multiplex lap winding front and back pitches differ by $2 m$ where $m$ is the number of component simplex windings, and the commutator pitch is $\pm m$.

(e) The total number of inductors $Z$ may be any even number, except in case of slotted armature cores, in which case $Z$ must be a multiple of the number of slots. (The number of slots may be even or odd.)

Wave windings. - (a) Front and back pitches are alike in sign.

(b) Front and back pitches may be equal or they may differ by any multiple of 2 . When one pitch, the front pitch for example, is made considerably greater than $Z / p$ then the other pitch must be made considerably smaller than $Z / p$. The average pitch of a wave winding is always very nearly equal to $Z / p$ according to $(c)$.

(c) In simplex wave windings $p \times$ average pitch must be equal to $Z \pm 2$. Therefore

$$
Z=p y_{a v} \pm 2
$$

in which $y_{a v}$ is the average pitch.

(d) In multiplex wave windings

$$
Z=p y_{a v} \pm 2 m
$$

in which $m$ is the number of component simplex windings.

In the following table

$$
\begin{aligned}
& p=\text { number of field magnet poles. } \\
& Z=\text { number of armature inductors. } \\
& y=\text { average pitch of winding. } \\
& K=\text { number of commutator segments. }
\end{aligned}
$$

For detailed treatment of armature windings see E. Arnold's Die Gleichstrommaschine, Vol. I., Julius Springer, Berlin, I902; and Parshall and Hobart's Armature Windings, D. Van Nostrand Co., New York, 1895. 
TABLE.

Summary for Drum Windings.

\begin{tabular}{|c|c|c|c|c|c|c|}
\hline & \multicolumn{3}{|c|}{ Lap or Parallel Grouping. } & \multicolumn{3}{|c|}{ Wave or Series Grouping. } \\
\hline & Simplex. & Duplex. & Triplex. & Simplex & Duplex. & Triplex. \\
\hline $\begin{array}{l}\text { No. of Paths in Paral- } \\
\text { lel }(p=\text { number of } \\
\text { poles })\end{array}$ & $p$ & $2 p$ & $3 p$ & 2 & 4 & 6 \\
\hline $\begin{array}{l}\text { No. of Inductors in } \\
\text { Series per Path }\end{array}$ & $Z \mid p$ & $Z / 2 p$ & $Z / 3 p$ & $Z / 2$ & $Z / 4$ & $Z / 6$ \\
\hline No. of Brush Sets. . & $\not p$ & $p$ & $\not p$ & $\begin{array}{l}\text { Min. } 2 \\
\text { Max. } p\end{array}$ & $\begin{array}{l}\text { Min. } 2 \\
\text { Max. } p\end{array}$ & $\begin{array}{l}\text { Min. } 2 \\
\text { Max. } p\end{array}$ \\
\hline $\begin{array}{c}\text { Angular Distance be- } \\
\text { tween Brush Sets. }\end{array}$ & $360 / p$ & $360 / p$ & $360 / p$ & $\begin{array}{l}360 \mid p \\
\text { or an }\end{array}$ & $\begin{array}{l}360 / p \\
\text { odd multiple }\end{array}$ & $\begin{array}{l}360 / p \\
\text { e thereof. }\end{array}$ \\
\hline $\begin{array}{l}\text { No. of Inductors Pos- } \\
\text { sible..... }\end{array}$ & $A_{1}$ & ny even nur & mber. & $p y \pm 2$ & $p y \pm 4$ & $p y \pm 6$ \\
\hline Reëntrancy . . . . & Single. & $\begin{array}{l}\text { Single if } \\
K \text { is odd. } \\
\mathrm{Double} \\
\text { if } K \text { is } \\
\text { even. }\end{array}$ & $\begin{array}{c}\text { Single if } \\
K / 3 \text { is not } \\
\text { an inte- } \\
\text { ger. } \\
\text { Triple if it } \\
\text { is. }\end{array}$ & Single. & $\begin{array}{r}\text { Single if } \\
y \text { is odd. } \\
\text { Double if } \\
y \text { is even. }\end{array}$ & $\begin{array}{l}\text { Single if } \\
y / 3 \text { is not } \\
\text { an inte- } \\
\text { ger. } \\
\text { Triple if it } \\
\text { is. }\end{array}$ \\
\hline
\end{tabular}

52. Typical examples of drum windings. - The following figures, namely, Figs. 56, 57, 58, 59 and 60 , show typical examples of drum windings. In these figures the short radial numbered lines represent the inductors, the crossed lines outside of the circle of inductors represent the connections at the back end of the armature, and the crossed lines between the circle of inductors and the commutator at the center represent the connections at the front end of the armature.

In these figures a small number of armature inductors is chosen for the sake of simplicity, and it is to be noted that for a given number of armature inductors, $Z$, several different combinations of front and back pitches are permissible for a given type of winding, especially when $Z$ is a large number as it usually is in actual machines. Furthermore a change of a few units in the value of $Z$ opens up a greatly increased choice of pitches for a given type of winding. Therefore the examples of windings shown in Figs. 56-60 should be looked upon as special cases chosen from among a great number of possible cases. 
Fig. 56 shows a six pole drum simplex lap winding with 60 inductors, having a back pitch of +9 , a front pitch of - I I, and a commutator pitch of $-\mathrm{I}$. This winding presents six

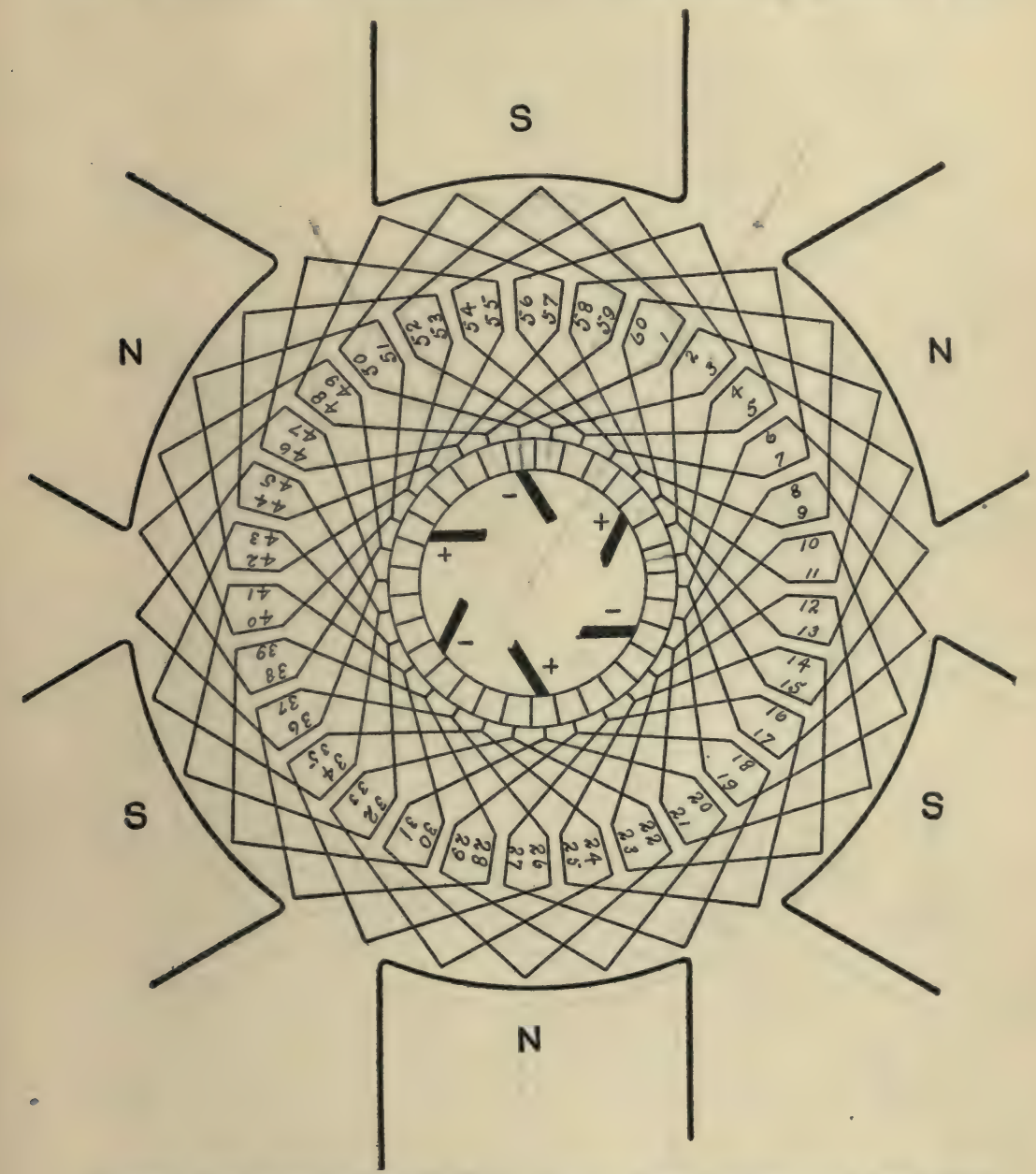

Six-pole drum simplex lap winding. $Z=60$, front pitch $=-\mathrm{II}$, back pitch $=+9$, commutator pitch $=-\mathrm{r}$, number of paths $=6$.

Fig. 56.

paths in parallel between the brushes with one sixth of the inductors in series in each path, and it must have six brush sets as shown in Fig. 56. This winding is singly reëntrant. 
Fig. 57 shows a six pole drum simplex wave winding with 62 inductors, having a back pitch of +9 , a front pitch of + II, an average pitch of 10 , and a commutator pitch of +10 . This

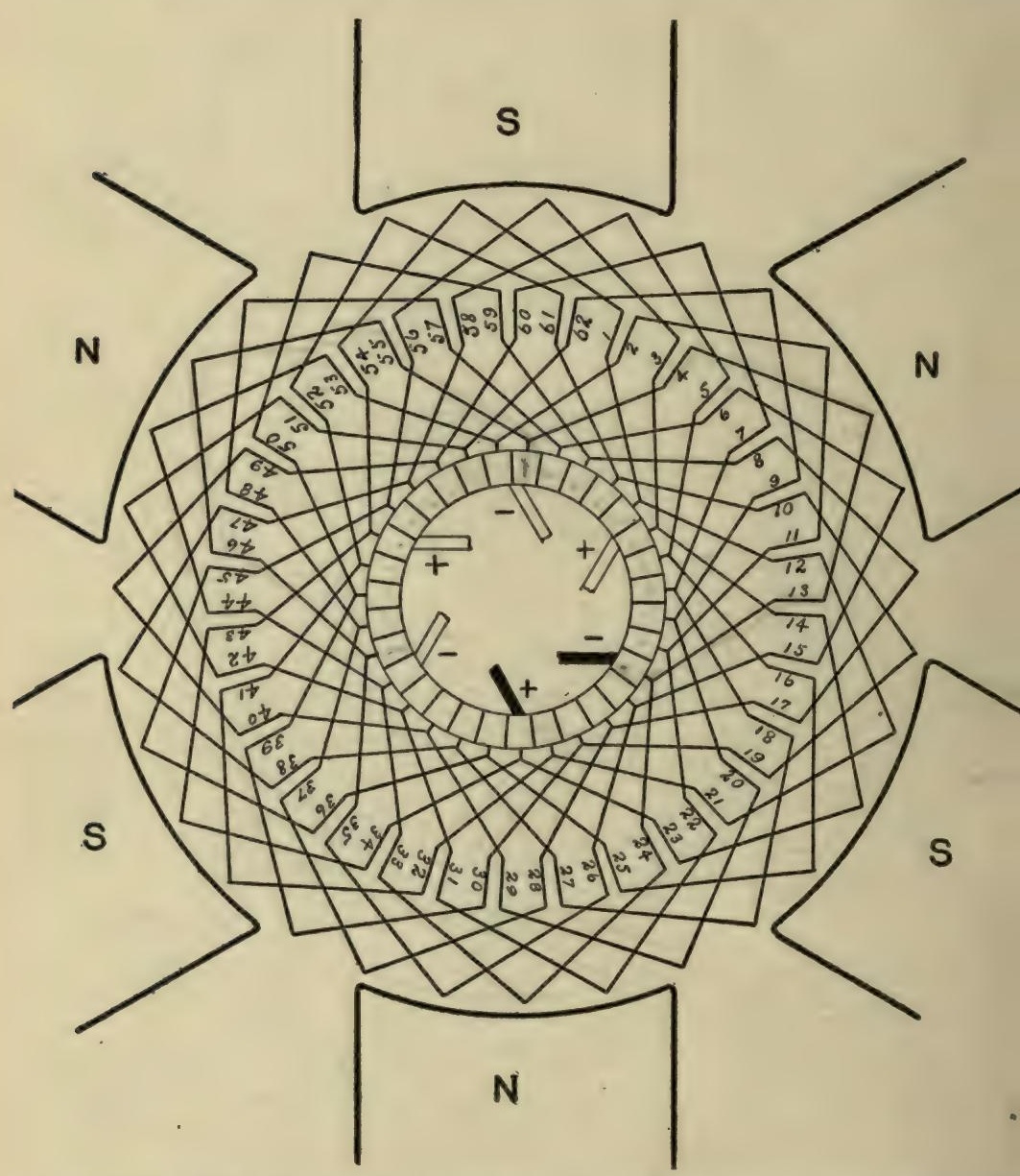

Six-pole drum simplex wave winding. $Z=62$, front pitch $=+1$, back pitch $=+9$, average pitch Io, commutator pitch + Io, number of paths $=\mathbf{2}$.

Fig. 57.

winding presents two paths in parallel between the brushes with one half of the inductors in series in each path, and it needs only 
two brush sets as shown in Fig. 57. This winding is singly reëntrant.

Fig. 58 shows a six pole drum duplex lap winding having 60 inductors. The dotted lines and shaded commutator segments

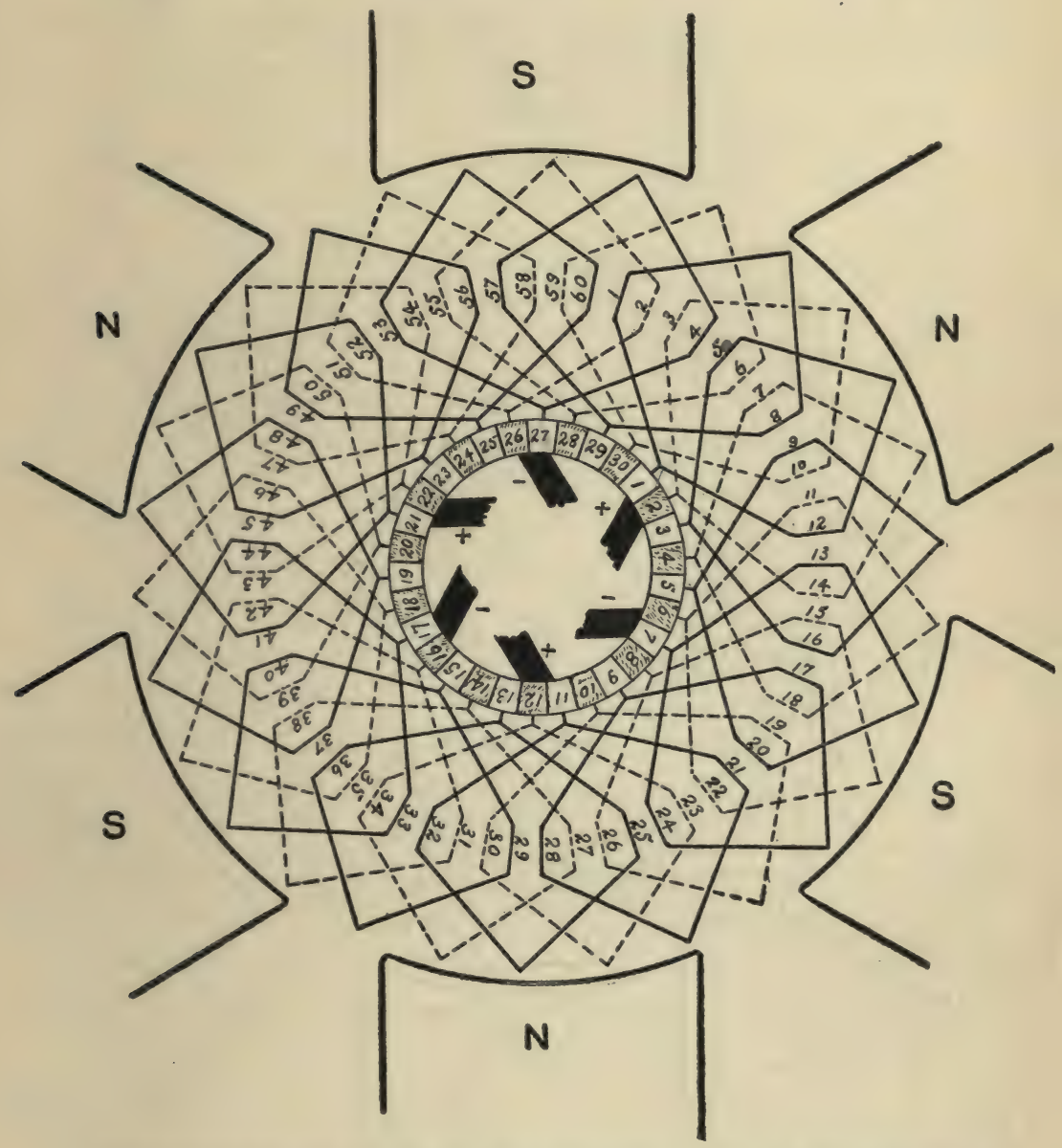

Six-pole drum duplex lap winding, doubly reëntrant. $Z=60$, front pitch $=-\mathbf{I I}$, back pitch $=+7$, commutator pitch $=-2$, number of paths $=12$.

Fig. 58.

represent a complete simplex lap winding having 30 inductors, and the full lines and blank commutator segments represent 
another identically similar simplex winding. These two simplex windings when sandwiched together on one armature core, as shown in Fig. 58, form a duplex lap winding. This duplex winding is of course doubly reëntrant according to Art. 47, inasmuch

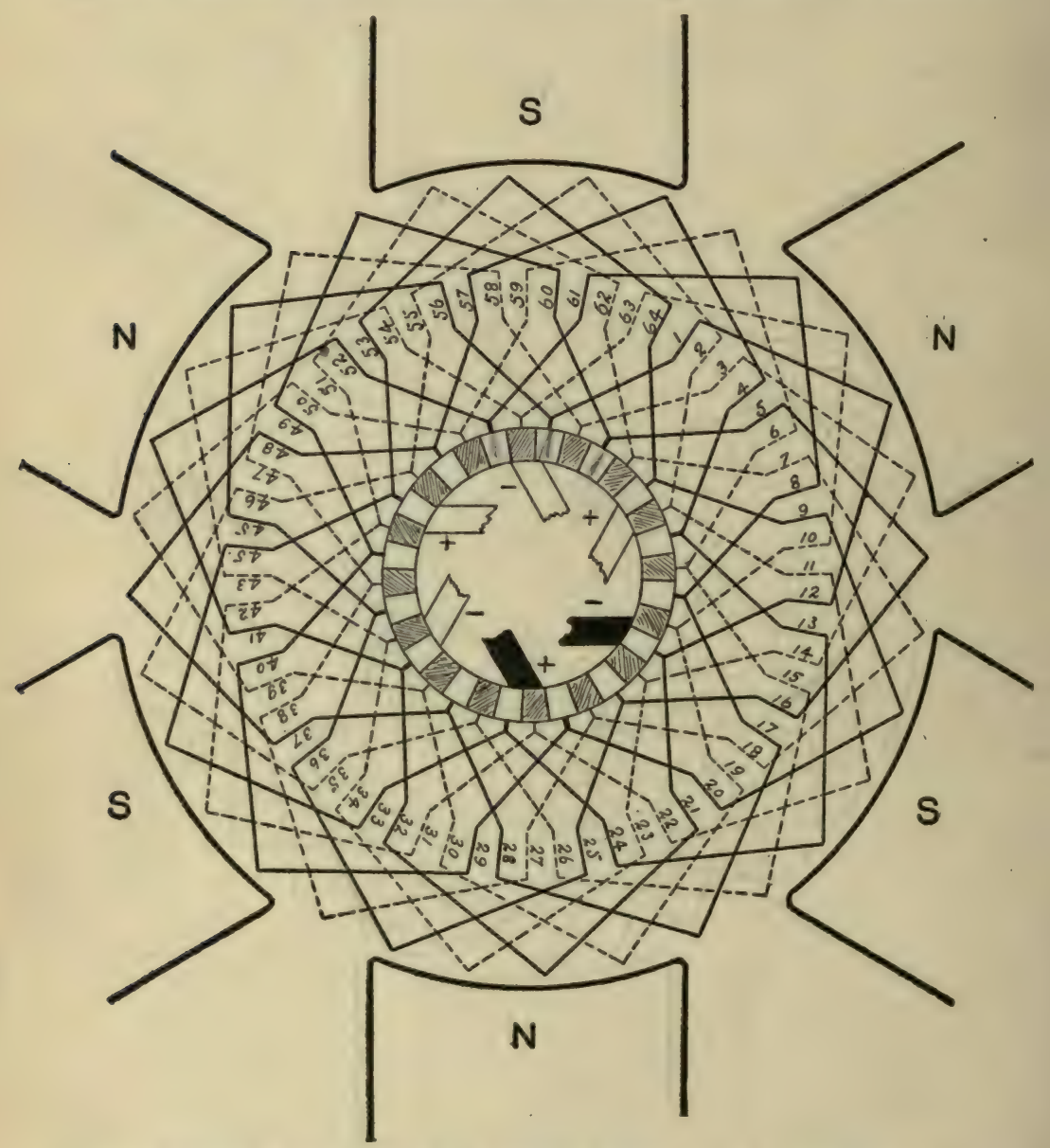

Six-pole drum duplex wave winding, doubly reẽntrant. $Z=64$, front pitch $=+9$, back pitch $=+\mathbf{I}$, average pitch Io, commutator pitch I0, number of paths $=4$.

Fig. 59.

as each of the constituent simplex windings reënters itself. In this winding the back pitch is +7 , the front pitch is $-I I$, and 
the commutator pitch is -2 ; and all of the conditions necessary for duplex lap windings as given in Art. $5 \mathrm{I}$ are satisfied. This winding presents 12 paths in parallel between the brushes, 6 paths through each constituent simplex winding, and it requires 6 brush sets, each brush being thick enough to always touch at least two adjacent commutator segments.

Fig. 59 shows a six pole drum duplex wave winding having 64 inductors. The dotted lines and shaded commutator segments represent a complete simplex wave winding having 32 inductors, and the full lines and blank commutator segments represent another identically similar simplex wave winding. These two simplex windings when sandwiched together, as shown in Fig. 59, form a duplex wave winding. This duplex winding is of course doubly reëntrant according to Art. 47, inasmuch as each of the constituent simplex windings reenters itself. In this winding the back pitch is $+\mathrm{I} I$, the front pitch is +9 , the average pitch is IO, and the commutator pitch is IO; and all the conditions necessary for duplex wave windings as given in Art. $5 \mathrm{I}$ are satisfied. This winding presents four paths in parallel between the brushes, two paths through each constituent simplex winding, and two brush sets are sufficient as shown in Fig. 59.

Fig. 60 shows a six-pole drum duplex wave winding which is singly reëntrant. This duplex winding cannot be looked upon as two simplex windings sandwiched together. In this winding the back pitch is $+\mathrm{II}$, the front pitch is $+\mathrm{II}$, the average pitch is + II and the commutator pitch is + II. This winding presents four paths in parallel between the brushes, two paths each way from each of the two broad brushes, as may be seen by tracing through the windings from brush to brush in Fig. 60. Two brush sets are sufficient for this winding as shown in Fig. 60.

It will be noticed that the inductors of the simplex windings shown in Figs. 56 and 57 are grouped in pairs which are drawn very close together. The same is true of the constituent simplex windings in Figs. 58 and 59. This is intended to show the common arrangement in slotted armatures where the even numbered 
inductors lie in the bottoms of the slots, and the odd numbered inductors lie in the tops of the slots, as shown in Fig. 6I. In

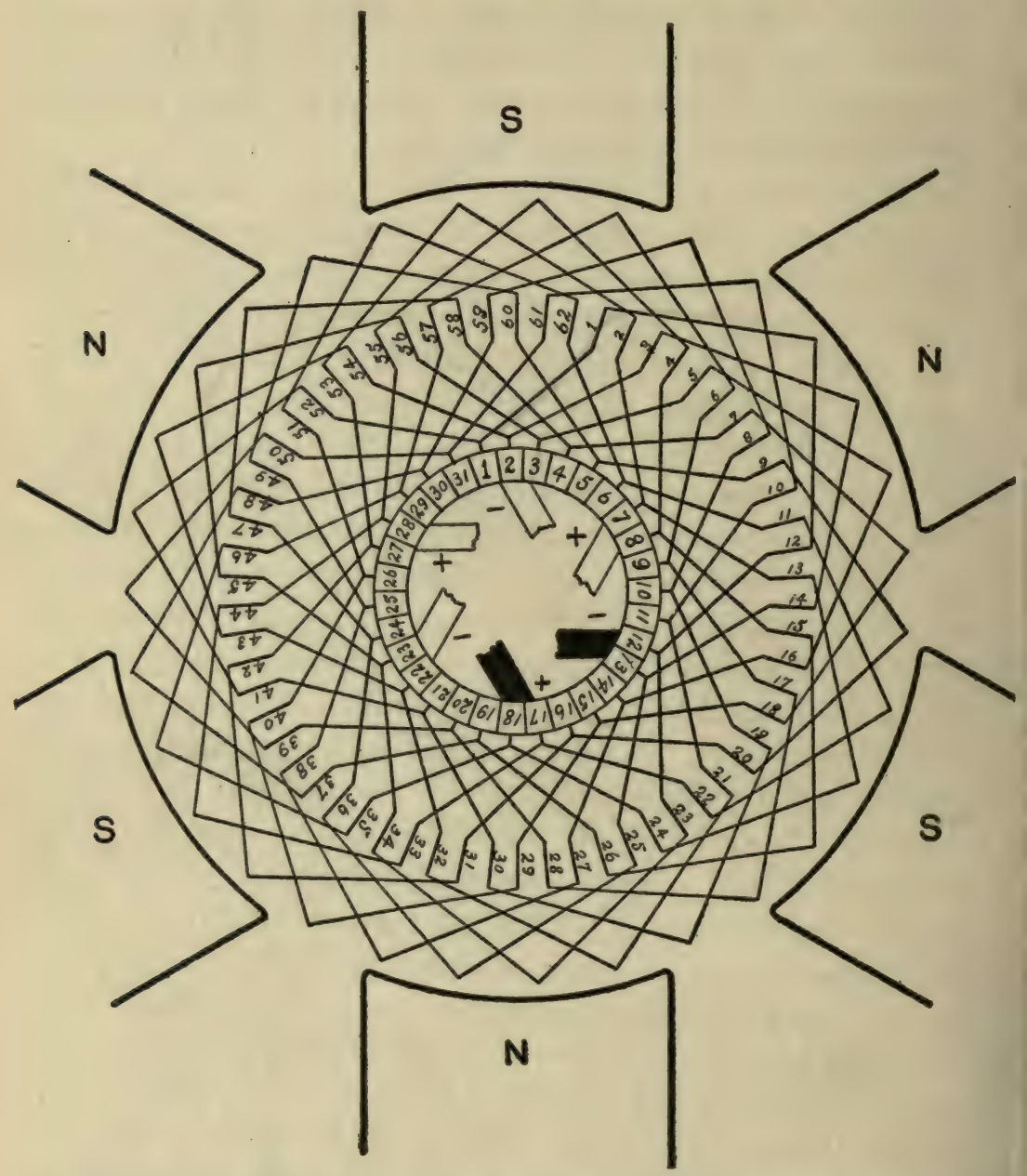

Six-pole drum duplex wave winding, singly reenntrant. $Z=62$, front pitch $=+I I$, back pitch $=+I I$, average pitch $=I I$, commutator pitch $=+I I$, number of paths $=4$.

Fig. 60.

this case the front or back pitch is reckoned by counting consecutive inductors exactly as if they were arranged in one layer. 
If a winding element consists of a coil of $n$ turns of wire instead of two inductors, as explained in Art. 42, then it is convenient to assign numbers to the consecutive bundles of $n$ inductors each, that is, to the consecutive half-coils, instead of assigning numbers to the individual inductors. In this case the pitches, front and back, are reckoned by counting consecutive bundles or half-coils. In the case of slotted armatures wound with formed coils it is

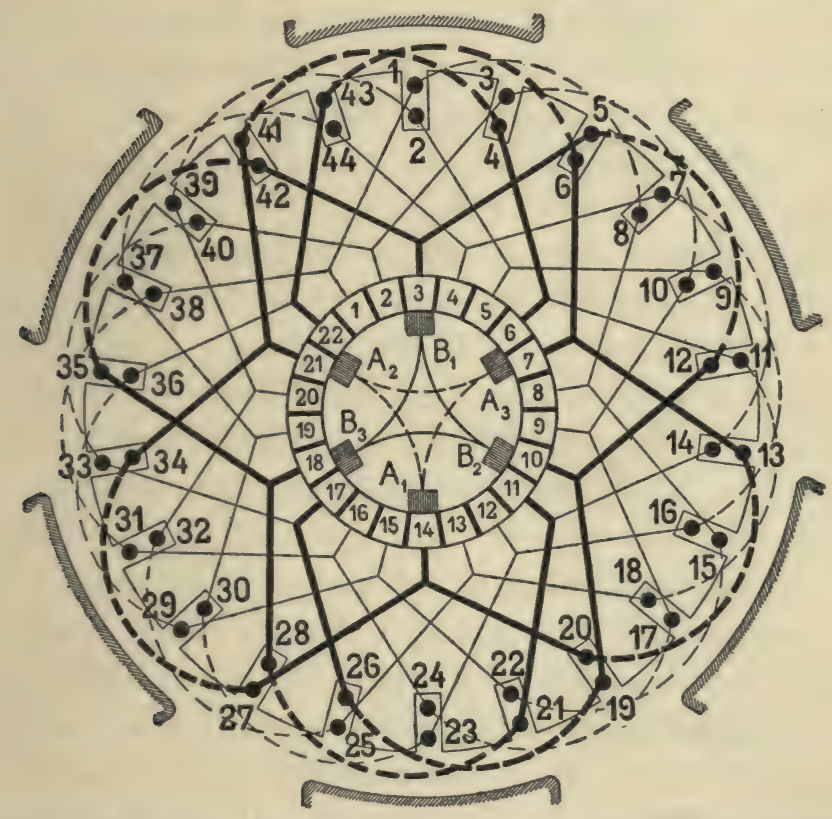

Six-pole drum simplex wave winding. Number of half-coils (which takes the place of $Z$ in the theory of the winding) $=44$, front pitch $=+7$, back pitch $=+7$, commutator pitch $=+7$, throw of coils $=3$, number of paths $=2$.

Fig. 61.

convenient to express the back pitch in terms of the number of slots spanned by the coil, one side of the coil always being in the top of one slot, and the other side being in the bottom of the $n$th following slot, where $n$ is the slot pitch. The number of slots spanned by the coils is called the slot pitch or the throw of the coils. When the throw of the coils is given, the only additional 
information needed for properly connecting the coils (of a simplex winding) is the numbers of the commutator segments to which the terminals of any given coil are to be connected.

The matters above mentioned are illustrated in Fig. 61, which represents a six pole drum simplex wave winding having 22 coils of any given number of turns each, the half-coils being numbered from I to 44 as shown in the figure. The back pitch is $\overline{7}$, inasmuch as half-coil No. 39 is connected across the back, as shown by the dotted line, to half-coil No. 2, and half-coil No. I is connected at the back to No. 8 and so on. The front pitch is also 7 , inasmuch as half-coil No. 2 is connected at the front end to half-coil No. 9, as shown by the full line and so on. The commutator pitch is also 7 , inasmuch as the terminals of the complete coil I-8 are connected to commutator bars No. I and No. 8. The slot pitch or throw of the coils is 3 .

Any one pair of brushes $A, B$ is sufficient for the wave winding shown in Fig. 61. The figure, however, shows three positive brushes $A_{1}, A_{2}$ and $A_{3}$ and three negative brushes $B_{1}, B_{2}$ and $B_{3}$.

53. Further comparison of lap and wave windings. Equipotential connections. - In practice the armature of a dynamo never is exactly centered in the field, but it is usually nearer to one pole or poles than to others, especially after the bearings have become worn. Inaccurate centering of the armature causes more flux to enter or leave the armature at certain poles than at others, so that greater electromotive forces are induced in the inductors under certain poles than in the inductors under other poles. These inequalities of electromotive force exactly balance each other insofar as the closed circuit of the whole winding is concerned, but if there are two or more positive brush sets and two or more negative brush sets these inequalities of electromotive force produce local currents through certain paths of the winding, and through the wires which connect the positive (or negative) brush sets together. These local currents increase the heating of the armature and they tend also to increase the sparking. 
Now the various inductors which constitute a given current path through a wave wound armature are distributed under all of the field magnet poles, so that, in the wave winding, equal electromotive forces are induced in all of the armature paths and there is no perceptible tendency for the production of local currents through certain armature paths, and through the wires which connect the brush sets. On the other hand the various inductors which constitute a current path through a lap wound armature lie under two adjacent field magnet poles and, therefore, an inaccurrate centering of the armature may lead to the production of very considerable local currents through the current paths and brush connections of a multipolar lap-wound armature.

These local currents may be prevented from flowing out through the brushes and across the brush connections, by making permanent low resistance cross-connections in the commutator (or in the armature winding) between each given commutator bar, and every other bar which passes at the same time under a positive brush. These cross-connections are called equipotential connections for the reason that every set of commutator bars so connected will be at the same potential (no electromotive force between them) if the magnetic field is symmetrical. Equipotential connections obviate the increased tendency to sparking due to the local currents, but at the expense of a slightly increased heating of the armature.

54. Modified multiplex wave winding - The above mentioned advantage of the wave winding over the lap winding, namely, that the electromotive forces induced in the various paths of a wave winding are always balanced and do not tend to produce local currents, applies to multiplex wave windings as well as to simplex wave windings. Now, one advantage of the lap winding over the wave winding, for low electromotive force machines, is that a multi-path lap winding does not require an excessive number of commutator bars like the multi-path wave winding. The following modification of the singly reëntrant $m$-plex multipolar wave 
winding gives a winding having $2 m$ balanced paths and having only $\mathrm{I} / m$ th as many commutator bars as the original $m$-plex winding.

Consider a singly reëntrant $m$-plex multipolar wave winding (Fig. 60 shows a singly reëntrant duplex six-pole wave winding). In such a winding a commutator segment is of course inserted at the terminus of each winding element. Suppose, however, that, starting from a given commutator segment, $m$ of these winding elements are wound as one extended winding element before a

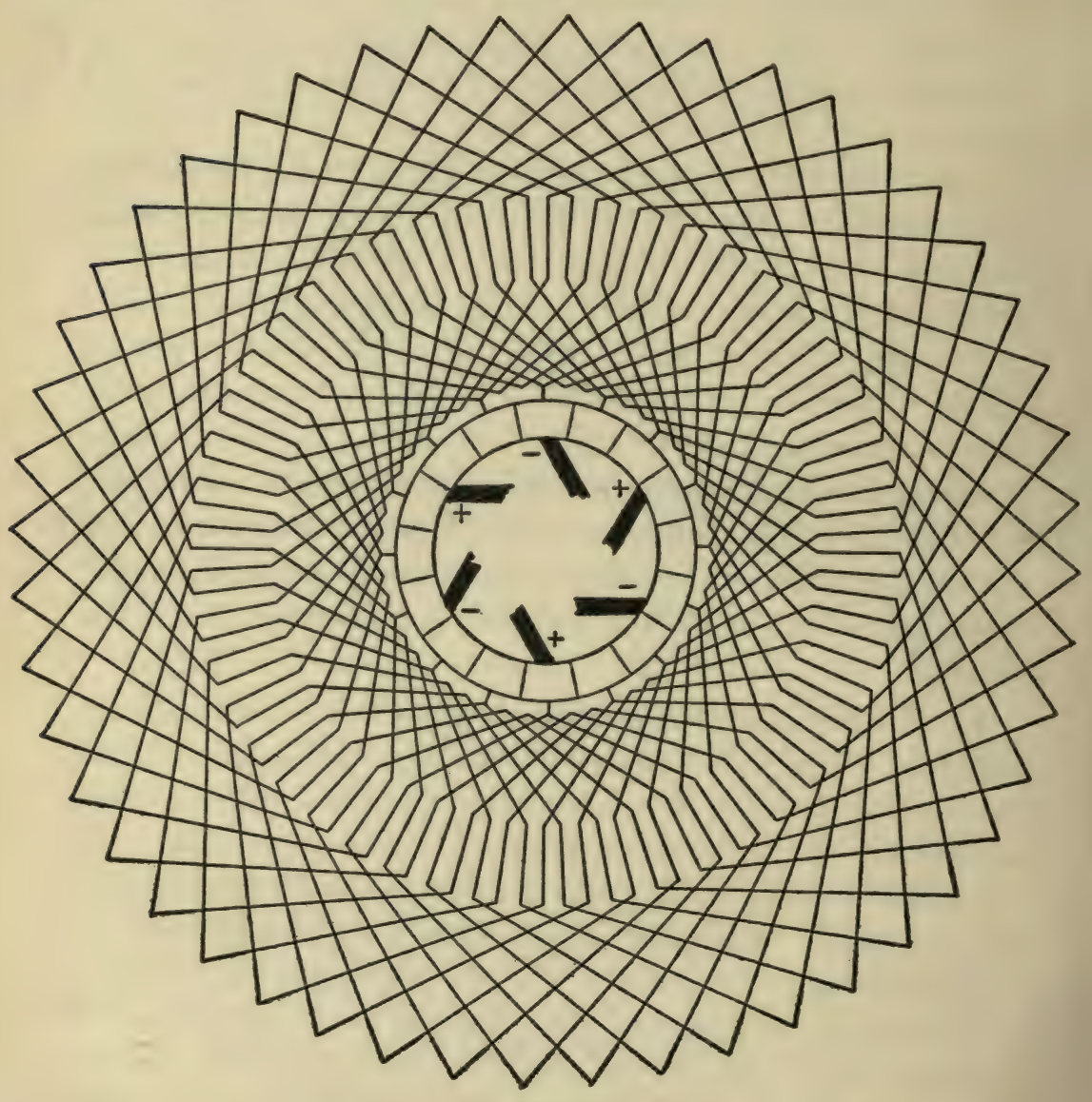

Fig. 62 . 
commutator segment is inserted, then another group of $m$ elements are wound as another extended element, and so on. The result will be a modified type of singly reëntrant wave winding having as many paths as the original $m$-plex winding, namely $2 m$ paths, but having only $\mathrm{I} / m$ th as many commutator segments, each segment being made $m$ times as broad as before.

In the original $m$-plex winding two brushes each broad enough to touch at least $m$ commutator segments are sufficient. In the modified winding the brushes touch one segment (or at most two) at a time, and two brushes are not sufficient.

Fig. 62 shows one of these modified six-pole wave windings with 16 commutator bars. This winding may be thought of as derived from a singly reëntrant triplex wave winding with a front pitch of 17 , and a back pitch of 17 . In this case it is necessary to use all six of the narrow brushes in order to utilize the six paths in the armature. 


\section{APPENDIX D.}

\section{PROBLEMS.}

Chapter I. Elementary Electricity and Magnetism.

1. Define the following terms and state the unit in terms of which each is expressed in the c.g.s. system : $(a)$ force, $(b)$ work, $(c)$ potential energy, $(d)$ kinetic energy, and $(e)$ power.

2. Define the watt. Define the horse-power. Find the number of watts in one horse-power using the following data: one foot $=30.48$ centimeters, one pound $=453.6$ grams, acceleration of gravity $=980$ centimeters per second per second.

3. (a) Find the number of kilogram-meters per second in one horse-power. (b) Find the number of ergs per second and the number of watts in one kilogram-meter per second.

4. A pulley Io inches in diameter runs at a speed of $\mathrm{I}, 000$ revolutions per minute and delivers Io horse-power. Find (a) the torque acting on the pulley in pound-inches, and $(b)$ the tangential pull at the rim of the pulley in pounds. Ans. (a) 63I.I pound-inches; (b) 126.22 pounds.

5. (a) Electrical energy for lighting costs 15 cents per kilowatt-hour, what is the cost per month (30 days) of operating a 16 candle-power lamp which takes 3 . I watts per candle, the lamp being in service three hours each day? (b) Electrical energy is supplied at 5 cents per kilowatt-hour for driving a Io horse-power motor. The efficiency of the motor at full load is 85 per cent. Find the cost per horse-power-hour of the mechanical output of the motor. Ans. (a) 67 cents; (b) 4.38 cents.

6. Two steel bars each $50 \mathrm{~cm}$. long, $2 \mathrm{~cm}$. wide and $2 \mathrm{~cm}$. thick are magnetized to an intensity of 800 units pole per square centimeter of sectional area. (a) Calculate the strength of each pole. (b) Calculate the force in dynes, and in pounds, with which 
the north pole of one rod attracts the south pole of the other rod when the poles are at an approximate distance of 50 centimeters from each other. Ans. (a) 3,200 units pole; (b) 4,096 dynes, 0.00924 pounds.

Note. - A slim rod of the best quality of hardened magnet steel retains a permanent magnetization of about 800 units pole per square centimeter sectional area.

7. One of the magnets of problem 6 is supported horizontally at its center of gravity. Find how far from the center a tengram weight must be placed to balance the tilting action of the vertical component of the earth's field, the value of which is 0.52 gauss; the acceleration of gravity is 980 centimeters per second per second. Ans. 8.49 centimeters.

8. Calculate the direction and intensity of the magnetic field due to both poles of one of the magnets specified in problem 6 at a point which is 40 centimeters from one pole and 30 centimeters from the other pole. Ans. Intensity 4.079 gausses; makes an angle of $23^{\circ} 27^{\prime}$ with axis of magnet.

9. The earth's magnetic field at a certain place dips $63^{\circ}$ below the horizon and its intensity is 0.58 gauss. A room $5 \times 6 \times 3$ meters high stands with its two $3 \times 6$ meter walls in the magnetic meridian. (a) Find the magnetic flux into the room across its ceiling and out of the room across its floor. (b) Find the magnetic flux into the room across its south wall and out of the room across its north wall. Ans. (a) I 55,040 maxwells, (b) 39,495 maxwells.

10. One of the pole faces of a dynamo has an area of 20 $\mathrm{cm}$. $\times 30 \mathrm{~cm}$. and 1,800,000 lines (maxwells) of flux pass from the pole face into the smooth core of the armature. What is the intensity of the magnetic field in the air gap between the pole face and the armature core? Ans. 3,000 gausses.

11. Calculate the magnetic flux which passes out from a north pole of one of the magnets specified in problem 6. Ans. 40,210 maxwells.

12. The armature of a dynamo has a length, under the pole face, of 30 centimeters. The magnetic field intensity in the gap 
space is 3,000 gausses. The armature is covered with straight wires parallel to its axis. Each of these wires carries 75 amperes. Calculate the side push on each wire in dynes and in pounds. Ans. 675,000 dynes or 1.518 pounds.

13. A horizontal wire ro meters long, stretched due magnetic east and west, and carrying a current, is pushed upwards by the horizontal component of the earth's magnetic field with a force of 3,000 dynes. What is the direction and strength of the current in the wire, the horizontal component of the earth's field being 0.2 gauss? Ans. I 50 amperes flowing towards the east.

14. A horizontal electric light wire, stretched due magnetic north and south, carries a current of $\mathrm{I}, 000$ amperes flowing towards the north. The wire is 250 meters long, the intensity of the earth's magnetic field is 0.58 gauss, and the magnetic dip is $63^{\circ}$. Find the value of the force with which the earth's magnetic field pushes on the wire and specify its direction. Ans. 2.906 pounds, pushing towards the west.

15. A circular coil of wire of $20 \mathrm{~cm}$. radius has 15 turns of wire. How much current is required in the coil to give a magnetic field intensity of one gauss at the center of the coil ? Ans.

\subsection{2 amperes.}

16. A circular coil of wire of $20 \mathrm{~cm}$. radius has $\mathrm{I}_{5}$ turns of wire and it carries 20 amperes. Calculate the magnetic field intensity: $(a)$ at the center of the coil, $(b)$ at a point in the axis of the coil and distant $100 \mathrm{~cm}$. from the plane of the coil, and $(c)$ at a point in the axis of the coil and distant $200 \mathrm{~cm}$. from the plane of the coil. Ans. (a) 9.42; (b) 0.07109; (c) 0.00929 gauss.

17. A solenoid five feet long has 1,600 turns of wire, calculate the magnetic field intensity inside of the solenoid due to a current of 10 amperes in the wire. Ans. 131.8 gausses.

18. A long iron rod two inches in diameter has one of its ends projecting into the solenoid specified in problem I7. Assuming that the rod is magnetized to an intensity of 1,500 units pole per square $\mathrm{cm}$. section, calculate the force tending to draw the rod 
into the solenoid when a current of 20 amperes flows in the wire. Ans. 8.01 $4 \times 10^{6}$ dynes.

NotE. - One pole of the rod is in the intense field inside of the solenoid and is pulled with a force of $m \mathscr{H}$ dynes. The other pole of the rod is remote from the solenoid and the force acting upon it may be neglected.

19. A current of 0.5 ampere flowing through a glow lamp generates 150 calories of heat in 10 seconds. (a) Required the resistance of the lamp in ohms. (b) What power is expended in the lamp? Express in watts and in horse-power. Ans. (a) $252 \mathrm{ohms}$; (b) 63 watts or 0.0844 horse-power.

20. A wire having a resistance of $250 \mathrm{ohms}$ is coiled in a vessel containing 2,000 grams of oil of which the specific heat is 0.60 . The vessel itself weighs 200 grams and its specific heat is 0.95 . A current of 1.5 amperes is passed through the coil of wire. How long will it take to raise the temperature of the oil and the vessel one centigrade degree? Ans. 9. I I seconds.

21. The field coil of a dynamo contains 25 pounds of copper (specific heat 0.094), weight of cotton insulation negligible. The resistance of the coil is Ioo ohms. (a) At what rate does the temperature of the coil begin to rise when a current of 0.5 ampere is started in the coil ? (b) How long would it take for the temperature of the coil to rise $20^{\circ} \mathrm{C}$. if no heat were given off from the coil by radiation? Ans. (a) 0.0056 centigrade degrees per second; (b) 59 minutes, 3 I seconds.

22. What is the resistance at $25^{\circ} \mathrm{C}$. of one mile of commercial copper wire 200 mils in diameter? Ans. 1.426 ohms.

Note. - One mil is a thousandth of an inch. One circular mil is the area of a circle one mil in diameter. The area of a circle $d$ mils in diameter is $d^{2}$ circular mils. When length of wire is expressed in feet and sectional area in circular mils the numerical value of $k$ in equation (12), Chapter I., for commercial copper at $25^{\circ} \mathrm{C}$. is about I0.8 ohms.

23. A given spool wound full of copper wire IO2 mils in diameter has a resistance of $\mathrm{I} .5$ ohms. An exactly similar spool is wound full of copper wire 204 mils in diameter, what is its resistance? Ans. $0.0938 \mathrm{ohm}$. 
24. Find the resistance at $25^{\circ} \mathrm{C}$. of a rectangular copper bus bar 50 feet long, 4 inches wide, and $1 / 2$ inch thick. Ans. 0.000212 ohm.

NOTE. - Square inches $\times 1,000,000 \times 4 / \pi$ gives circular mils.

25. What is the resistance at $25^{\circ} \mathrm{C}$. of a steel rail 30 feet long weighing $600 \mathrm{lbs}$ ? One cubic inch of steel weighs $0.28 \mathrm{lb}$. and the specific resistance of the steel is 8 times that of copper. Ans. $0.000342 \mathrm{ohm}$.

26. What is the resistance at $25^{\circ} \mathrm{C}$. of a 20 foot length of wrought iron pipe, I inch inside diameter and I $3 / 8$ inches outside diameter? Specific resistance of wrought iron is 7 times that of copper. Ans. $0.001695 \mathrm{ohm}$.

27. A transmission line 2,000 feet long (each wire) is to have a total resistance of $0.40 \mathrm{ohm} \mathrm{:} \mathrm{(a)} \mathrm{Find} \mathrm{diameter} \mathrm{of} \mathrm{wire} \mathrm{in} \mathrm{mils.}$ One cubic inch of copper weighs $0.32 \mathrm{lb}$; $(b)$ find the weight of the above copper wire (bare); and $(c)$ find its total cost at I 5 cents per lb. Ans. (a) 328.6 mils ; (b) I,303 lbs.; (c) \$I 95.45.

28. A given weight of copper is manufactured into $I, 000$ feet of wire 128 mils in diameter having a resistance of $0.629 \mathrm{ohm}$. The same amount of copper is made into 32 mil wire; find: (a) its length; and (b) its resistance. Ans. (a) I6,000 feet; (b) 161.02 ohms.

29. The weight of a cubic inch of copper is $0.32 \mathrm{lb}$. and the resistance of an inch cube of copper between opposite faces at $20^{\circ} \mathrm{C}$. is $0.00000068 \mathrm{ohm}$. Derive a formula giving the resistance at $20^{\circ} \mathrm{C}$. of copper wire in terms of its length in feet and its weight in lbs. Ans. $R=0.00003133 L^{2} / W$.

30. A sample of commercial copper wire three feet long and I 20 mils in diameter is found by test to have the same resistance as 26.2 inches of pure copper wire 100 mils in diameter at the same temperature. Find the conductivity (reciprocal of specific resistance) of the sample expressed in per cent. of the conductivity of pure copper. Ans. 95.42 per cent.

31. A copper transmission line has a resistance of $5 \mathrm{ohms}$ at $-20^{\circ} \mathrm{F}$. What is its resistance at $90^{\circ} \mathrm{F}$ ? Ans. $6.236 \mathrm{ohms}$. 
32. The field coil of a dynamo (copper wire) has a resistance of $30 \mathrm{ohms}$ after it has been standing for a long time in a room at a temperature of $68^{\circ} \mathrm{F}$. After the machine has been running fully loaded for several hours the resistance of the field coil is $35 \mathrm{ohms}$, what is the temperature of the coil ? Ans. $149^{\circ} \mathrm{F}$.

33. An ordinary 16-candle-power i Io-volt glow lamp has a resistance of $400 \mathrm{ohms}$ at $0^{\circ} \mathrm{C}$. and $220 \mathrm{ohms}$ at a white heat, say, $1500^{\circ} \mathrm{C}$. What is the average temperature coefficient of its resistance between $0^{\circ} \mathrm{C}$. and $1500^{\circ} \mathrm{C}$.? Ans. -0.0003 per centigrade degree.

34. The specific resistance of commercial copper is 1,600 c.g.s. units at $\mathrm{O}^{\circ} \mathrm{C}$., find from this: $(a)$ the resistance at $\mathrm{O}^{\circ} \mathrm{C}$. of a copper wire I mil in diameter and one foot long; and $(b)$ the resistance of a copper bar one inch long and one square inch in sectional area. Ans. (a) $9.63 \mathrm{ohms} \mathrm{;} \mathrm{(b)} 0.00000063 \mathrm{ohm}$.

35. When a certain electric generator is giving out no current it takes 1.75 horse-power to drive it. When the generator delivers a current of I 50 amperes it takes 25 ho e-power to drive it. Assuming that the increased power is all used in the maintenance of the 150 amperes of current, find the electromotive force of the generator. Ans. I I 5.7 volts.

36. An incandescent lamp takes 0.6 ampere when the electromotive force between its terminals is I I volts. Find the power delivered to the lamp in watts and in horse-power. Ans. 66 watts or 0.0884 horse-power.

37. A so-called 2,000 candle-power arc lamp has a current of 9.6 amperes flowing through it, and an electromotive force of 47 volts between its terminals. (a) Find how many kilowatt-hours and how many horse-power-hours of work are delivered to the lamp during a run of eleven hours. (b) If the charge for a run of eleven hours is 27 cents, find the price of power per kilowatthour. Ans. (a) 4.96 kilowatt-hours or 6.66 horse-power-hours ; (b) 5.43 cents.

38. A storage battery consisting of 54 cells connected in series has a resistance of $0.0002 \mathrm{ohm}$ per cell, and an electromotive 
force per cell which ranges from 2 volts at the begin ning to 1.85 volts at the end of the discharge. The battery supplies current to I 00 glow lamps (each having $220 \mathrm{ohms}$ resistance) connected in parallel between copper wires 0.325 inch in diameter at a distance of 200 feet from the battery. Find the electromotive force between the terminals of the group of lamps at the beginning and at the end of the discharge of the storage battery. Ans. I05.6 volts and 97.5 volts.

39. The electromotive force of a battery is 15 volts (measured of course on open circuit). The battery terminals are connected by a wire, when it is observed that a current of 1.5 amperes is produced and the electromotive force between the battery terminals is 9 volts. Find the resistance of the wire and the apparent resistance of the battery. Ans. 6 ohms and 4 ohms.

Note. - When a voltaic cell is called upon to give current, the terminal voltage of the cell falls off, not only on account of the ri drop in the cell, but also on account of what is called polarization. This problem is to be solved on the assumption that the whole of the decrease in terminal voltage is due to $r i$ drop, and the value of the resistance as calculated on this assumption is greater than the true resistance of the battery.

40. Find the total electromotive force that must be induced in a dynamo armature to send a charging current of 100 amperes through a storage battery consisting of 54 cells connected in series. Each cell has an average counter electromotive force of 2.3 volts, the resistance of each cell is $0.0004 \mathrm{ohm}$, the resistance of the dynamo armature is $0.02 \mathrm{ohm}$, and the resistance of the leads is $0.03 \mathrm{ohm}$. Ans. 131.36 volts.

41. A dynamo having an electromotive force of I I 5 volts between its terminals delivers 200 amperes to a group of glow lamps I, Ooo feet distant from the generator. Find: $(a)$ the size of copper wire for the mains in order that 95 per cent. of the power output of the generator may be delivered to the lamps ; (b) the electromotive force between the mains at the lamps. Ans. (a) 752,000 circular mils; (b) 109.25 volts.

42. What size of copper wire is required to deliver current at I Io volts to a IO-horse-power motor of 85 per cent. efficiency, 
the motor being 2,000 feet from the generator, and the electromotive force between the generator terminals being 125 volts. Ans. 230,000 circular mils.

43. A motor receiving 100 kilowatts of power is at a distance of I 5 miles from the generator. Line wires 200 mils in diameter are to be used. The line loss is to be ro per cent. of the generator output. Find: $(a)$ the current; $(b)$ the voltage at the generator; and $(c)$ the voltage at the motor. Ans. (a) I6.12 amperes; (b) 6,894 volts; (c) 6,204 volts.

Note. - High voltage direct current power transmission is not used in American practice.

44. Six cells of battery each having an electromotive force (on open circuit) of 2 . I volts and a resistance of $5 \mathrm{ohms}$ are connected as shown in Fig. $44 P$. The resistance of $a b$ is 150 ohms. (a) Locate the point $P$ so that no current will be indicated by the galvanometer $G$.

(b) The contact $P$ being at the middle point of $a b$ and the total resistance of the path $M G P$ being $25 \mathrm{ohms}$ find: (I) the current in each path $a, b$ and $G$, and (2) the electromotive force between the terminals of the left-hand cell

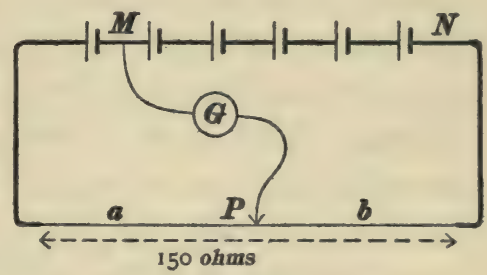

Fig. $44 P$. and the voltage between $M$ and $N$. Ans. (a) $a / b=0.2 ;(b)$ 0.042 ampere in $a, 0.0924$ ampere in $b, 0.0504$ ampere in $G$; I.89 volts between terminals of left-hand cell, and 8.19 volts between $M$ and $N$.

45. A one-ohm coil is tested and found to have a resistance of I.004 ohm. (a) What length of German silver wire having a resistance of I 5 ohms per meter must be connected in parallel between the terminals of the coil to give a combined resistance of I ohm? (b) Suppose that an error of 5 per cent. is made in measuring off the required length of the German silver; what will be the percentage error of the corrected one-ohm coil ? Ans. (a) I6.73 meters; (b) about $\frac{2}{10}$ of one per cent. 
46. The resistances in the various branches of a Wheatstone network are indicated in Fig. 46P. The battery has an electromotive force of 6 volts in the direction of the arrow. Find

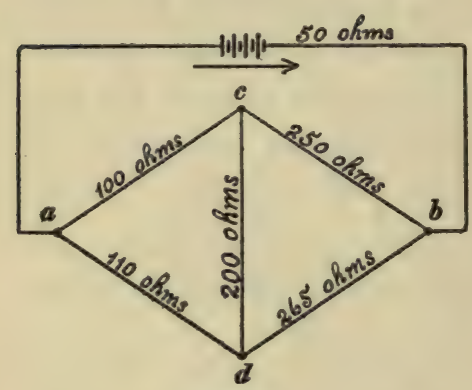

Fig. 46P. the current in the galvanometer branch $c d$ and specify its direction. Ans. 0.0001024 ampere from $d$ towards $c$.

47. The network shown in Fig. $46 P$ has a one-volt voltaic cell of negligible resistance connected in the branch $d b$ tending to force current from $d$ towards $b$. Find the current in the branch $c d$ and specify its direction. Ans. 0.000735 ampere from $c$ towards $d$.

48. A direct-reading ammeter has a resistance of $0.05 \mathrm{ohm}$. The instrument is provided with a shunt so that the total current passing through the instrument and shunt is Io times the ammeter reading. What is the resistance of the shunt? Would it be practicable to construct such a shunt, measure its resistance by a Wheatstone's bridge, and connect it to the ammeter terminals ? If not, how could such a shunt be accurately adjusted? Ans. $0.00556 \mathrm{ohm}$.

49. The scale of a direct-reading millivoltmeter has Ioo divisions, each division corresponding to one millivolt between the terminals of the instrument. This instrument is connected to the terminals of a low resistance shunt and each division of the scale corresponds to 0.25 ampere in the shunt. What is the resistance of the shunt? Ans. $0.004 \mathrm{ohm}$.

50. A millivoltmeter has a resistance of $\mathrm{I} 5.4 \mathrm{ohms}$. What resistance must be connected in series with the instrument so that the scale reading may give volts instead of millivolts? Ans. I $5,384.6$ ohms.

51. A direct-reading voltmeter $V$, Fig. p. $5 \mathrm{I}$, having 16,000 ohms resistance, is connected from main $A$ to earth. The voltmeter 
gives a reading of 2.6 volts and the electromotive force between the mains is I I volts. Find the insulation resistance between main $B$ and the earth on the assumption that the insulation resistance of main $A$ is: (a) infinite; $(b)$ the same as that of main $B ;(c)$ one-tenth of that of main $B$. Ans. (a) 660,900 ohms; (b) 644,900 ohms ; (c) 500,900 ohms.

52. The armature $D$, Fig. 52 $P$, of a dynamo is connected to

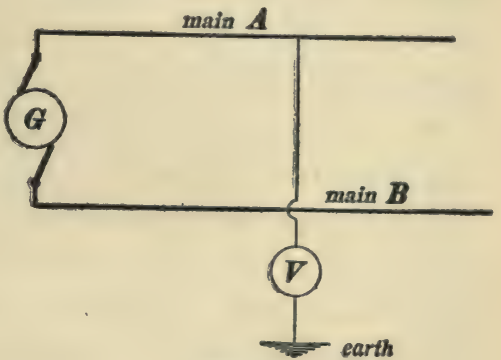

Fig. $51 P$. supply mains in series with an ammeter $A$ and a suitable rheostat $R$, and the ammeter reads 50 amperes. The terminal leads $\|$ of a low reading voltmeter $V$ are connected to the two commu-

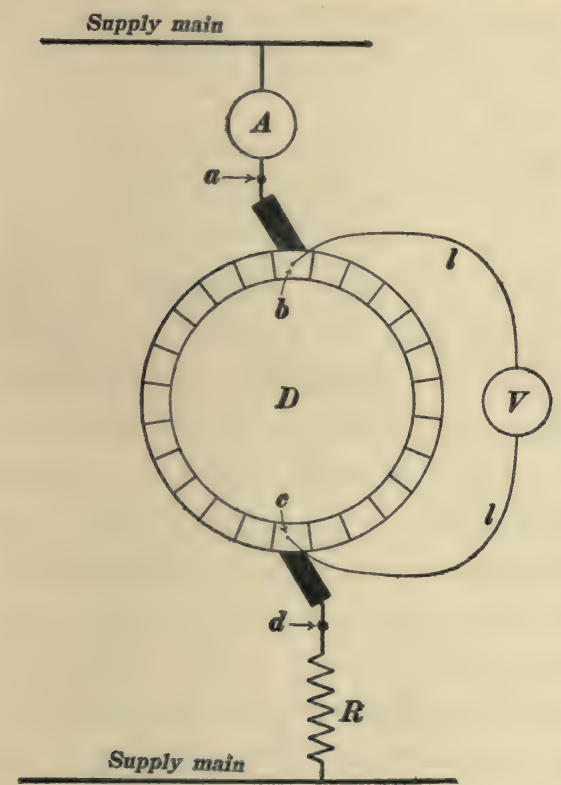

Fig. 52P. tator segments $b$ and $c$ and the voltmeter reads 4.2 volts. The leads $l l$ are then connected to brush lead $a$ and segment $b$ and the voltmeter reads I.I volts; the same reading is obtained when the leads are connected to segment $c$ and brush lead $d$. Find: ( $I$ ) the resistance of the armature between commutator segments $b$ and $c$; (2) the resistance of each brush, including contact resistance between brush and commutator; and (3) the total resistance between $a$ and $d$. Ans. (I) 0.084 ohm; (2) $0.022 \mathrm{ohm;} \mathrm{(3)} 0.128 \mathrm{ohm}$.

53. A straight horizontal metal bar 3 meters long falls parallel 
to itself at a uniform velocity of I,O0O centimeters per second. The bar points magnetic east and west and the horizontal component of the earth's magnetic field is 0.2 gauss. How much electromotive force is induced in the bar? Express the result in c.g.s. units and in volts. Ans. 60,000 abvolts or 0.0006 volt.

54. The straight metal spoke of a wheel is 40 centimeters long. The plane of the wheel is vertical and magnetic east and west. The horizontal component of the earth's magnetic field is 0.2 gauss. (a) How many lines of force does the spoke cut in one revolution? (b) How much electromotive force is induced in the spoke when the wheel makes 25 revolutions per second? Ans. (a) I,005.3 maxwells; (b) 0.00025 I volt.

55. The wheel of problem 54 is replaced by

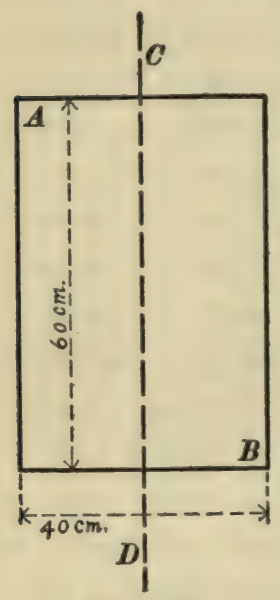

Fig. $56 P$. a metal disk 80 centimeters in diameter. What is the electromotive force between the center and the circumference of the disk when its speed is 25 revolutions per second? Ans 0.00025 I volt.

56. A wire is bent to form a rectangle $A B$, Fig. $56 P$, which is rotated about the vertical axis $C D$ at a speed of 25 revolutions per second. The horizontal component of the earth's magnetic field is 0.2 gauss. (a) How much magnetic flux passes through $A B$ when its plane is magnetic east and west? (b) How much flux passes through $A B$ one quarter of a revolution later? (c) What is the average value of the electromotive force which is induced in the wire $A B$ during this quarter of a revolution? Ans. (a) 480 maxwells; (b) zero; (c) 0.00048 volt.

57. A conductor $A$, Fig. $57 P$, perpendicular to the plane of the paper, is caused to move in the direction of one of the arrows. Specify the direction of the induced electromotive force in each of the four cases.

58. The pole face of a dynamo is 30 centimeters long, parallel 
to the axis of the armature, and the field intensity in the air gap between the pole face and the armature core is 6,000 gausses. The wires on the armature are 12 centimeters from the axis of the armature, and the speed of the armature is $\mathrm{I}, 800$ revolutions per minute. Find the electromotive force in volts induced in each

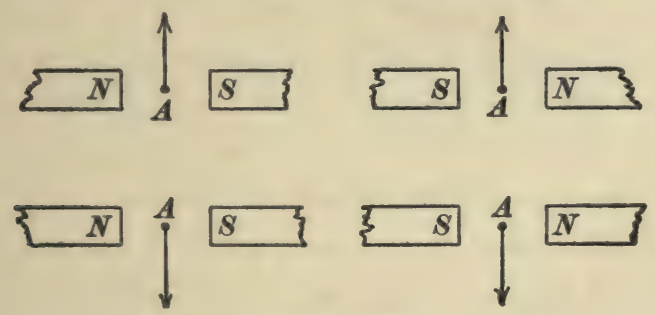

Fig, $57 P$.

armature wire ( $30 \mathrm{~cm}$. long) as it sweeps across the pole face. Ans. 4.072 volts.

59. The core of an induction coil carries 100,000 lines of flux when current is flowing through the primary coil. When the primary circuit is broken the flux through the core drops to 10,000 lines in 0.002 second. How many turns of wire are required in the secondary coil in order that an average electromotive force of 5,000 volts may be induced in it during the 0.002 second? Ans. I I, I Io turns.

\section{Chapter II. The Dynamo.}

60. A coil of wire wound on one tooth of an alternator armature like that shown in Fig. I6, Chapter II., has 80 turns of wire. When the tooth is squarely under a north pole of the field magnet the magnetic flux through the coil is 2,500,000 maxwells. The field magnet has ten poles and the armature makes 25 revolutions per second. Find the average value of the electromotive force induced in the armature coil while the tooth on which it is wound moves from a position squarely under a north pole to a position squarely under a south pole of the field magnet. Ans. r,ooo volts. 
61. The ring armature of a bipolar direct-current dynamo has 260 turns of wire upon it, the armature is driven at a speed of I,200 revolutions per minute, and the magnetic flux from one pole face into the armature core is $3,500,000$ lines. Calculate the electromotive force of the dynamo in volts. Ans. I 82 volts.

62. The armature specified in problem 61 has 400 feet of 320 mil copper wire wound upon it. What is the resistance of the armature from brush to brush? Ans. 0.01055 ohm at $25^{\circ} \mathrm{C}$.

63. The armature specified in problems 61 and 62 is used with a six-pole field magnet with six sets of brushes. (a) What is the resistance of the armature between positive and negative brush sets? (b) The flux per pole of this six-pole field magnet is 600,000 lines. What is the electromotive force of this six-pole dynamo? Ans. (a) 0.00II 7 ohm at $25^{\circ} \mathrm{C}$.; (b) 31.2 volts.

64. The field winding of a shunt generator has a resistance of $40 \mathrm{ohms}$ and the electromotive force between its brushes is I IO volts. (a) What is the value of the field current? (b) What amount of power is expended in field excitation? (c) The fullload current output of the generator being 50 amperes (at I IO volts), express the field current in per cent. of the full-load current output, and express the power consumed in field excitation in per cent. of the full-load power output of the generator. Ans. (a) 2.75 amperes; (b) 302.5 watts; (c) 5.5 per cent., 5.5 per cent.

65. The field winding of a series generator has a resistance of O. I $2 \mathrm{I} \mathrm{ohm}$. The full-load current output of the generator is 50 amperes at IIO volts between the generator terminals. What electromotive force is lost in the field winding at full-load? (b) How much power is consumed in field excitation at full-load ? Express the volts lost in the field winding in per cent. of the fullload terminal voltage of the machine, and express the power consumed in field excitation in terms of the full-load power output of the generator. Ans. (a) 6.05 volts or 5.5 per cent. ; (b) 302.5 watts or 5.5 per cent.

66. The motor of a motor-generator set takes 100 amperes at 
I I volts from supply mains and delivers 85 per cent. of its power intake to the generator which it drives. This generator in its turn delivers 84 per cent. of its power intake to a receiving circuit at 20 volts. Find the current delivered by the generator. Ans. 392.7 amperes.

67. A homopolar dynamo like Fig. 62, Chapter II., is driven at a speed of 3,000 revolutions per minute. The flux passing through the cylindrical armature $A A$ is 5 million lines. What is the electromotive force between positive and negative brushes? Ans. 2.5 volts.

\section{Chapter III. The Dynamo as a Generator.}

68. A given shunt generator gives an induced electromotive force of I IO volts when run at a speed of I,200 revolutions per minute with a total resistance of $56 \mathrm{ohms}$ in its shunt field circuit. If this machine is driven at a speed of 1,500 revolutions per minute what must be the total resistance in the shunt field circuit to cause the machine to generate an electromotive force of 137.5 volts? Ans. 70 ohms.

69. The electromotive force of a shunt generator decreases from I I volts to 93 volts when the speed is reduced from I,OOO to 900 revolutions per minute. The armature flux at the higher speed is $1,000,000$ lines. (a) What is the armature flux at the lower speed? (b) What would the electromotive force of the generator be at the lower speed if its armature flux were kept constant at a value of $1,000,000$ lines? (c) What causes the flux to decrease with the speed? Ans. (a) 939,390 lines ; (b) 99 volts.

70. The resistance of a generator armature, including brushes and brush contacts, is $0.14 \mathrm{ohm}$. The total electromotive force induced in the armature is 120 volts. Find the value of the electromotive force between the brushes when the current in the armature is 70 amperes. Ans. I 10.2 volts.

71. A battery consisting of ten Daniell cells has an electromotive force of 10.8 volts (measured on open circuit) and an internal 
resistance of 4 ohms. Find: $(a)$ the terminal voltage of the battery when it is delivering a current of $\mathrm{I} .2$ amperes; $(b)$ the resistance of the external circuit. Ans. (a) 6 volts; (b) 5 ohms.

72. The electromotive force of a shunt generator rises from I IO volts at full-load to I 30 volts when the receiving circuit is disconnected. What is the precentage regulation of the machine? Ans. I 8.2 per cent.

73. The electromotive force of a shunt generator driven at constant speed decreases from II 5 volts when the brushes are in the neutral axis to 90 volts when the brushes have $20^{\circ}$ forward lead. When the field of this generator is separately excited the electromotive force decreases from I I 5 volts when the brushes are in the neutral axis to 100 volts when the brushes have $20^{\circ}$ forward lead. One million lines of armature flux are required to give II 5 volts when the brushes are in the neutral axis. What is the value of the armature flux when the machine is giving, self-excited, an electromotive force of 90 volts with $20^{\circ}$ forward brush lead? The armature current in all the above is assumed to be negligibly small. Ans. 900,000 maxwells.

74. A separately excited generator with brushes in the neutral axis gives an electromotive force of I I 5 volts. The electromotive force betwen one of the main brushes of this generator and an auxiliary brush $15^{\circ}$ from it in the direction of rotation is 8 volts. What would the electromotive force between the main brushes be if they were given a forward lead of $15^{\circ}$ ? The armature current is assumed to be negligible. Ans. 99 volts.

75. A certain bipolar shunt generator like Fig. 35 , page 52 , is rated to give its full-load current of 50 amperes at a terminal voltage of 100 volts. To give 100 volts at zero-load a field excitation of 8,100 ampere-turns is required. At full-load a field excitation of 10,500 ampere-turns is required. Find the number of turns required in the series field coil to give flat compounding. Ans. 48 turns.

NoTE. - The ampere-turn is a unit of magnetomotive force and it is fully explained in Appendix A. 
76. Given a two-pole dynamo having a ring armature 20 centimeters long and 34 centimeters outside diameter with 250 turns of wire $(=Z)$, and 50 commutator bars which are connected to the armature windings by radial connectors. The resistance of the armature between brushes is $0.4 \mathrm{ohm}$. A double pilot brush is made by tying two light copper strips to the sides of a stick of which the thickness is equal to the width of a commutator bar, and these two copper strips are connected to a voltmeter. The dynamo is driven as a generator at a speed of $\mathrm{I}, 200$ revolutions per minute at zero-load, and the voltmeter reads 8 . I volts when the double pilot brush is held against the commutator anywhere within the limits of a pole face. Find: $(a)$ the mean intensity of the magnetic field in the air gap between the pole face and the armature core.

The dynamo, driven as a generator at the same speed as before, now delivers a current of 40 amperes from its armature, and the voltmeter is observed to read 2.47 volts and II . 56 volts when the double pilot brush is held against the commutator under the leading tip and the trailing tip respectively of a pole piece. Find : $(b)$ the field intensity in the air gap, under a leading pole tip, and (c) ditto under a trailing pole tip. Ans. (a) 3,79I gausses; (b) I,456 gausses; (c) 5,7 I I gausses.

NOTE. - The voltmeter reading $(b)$ or $(c)$ is the induced electromotive force minus the $r i$ drop in a section of the armature winding. This problem illustrates a point which is discussed in Art. 77.

77. A series generator has a total internal resistance of 0.2 ohm. When the generator is driven at a speed of $\mathrm{I}, 000$ revolutions per minute it gives a terminal voltage of 99 volts with a current output of 5 amperes. Increasing the current output to Io amperes increases the useful flux, $\Phi$, by 50 per cent. Find the terminal voltage of the generator when, driven at a speed of I, 200 revolutions per minute, it delivers a current of 10 amperes. Ans. I 78 volts. 
Chapter IV. The Operation of the Dynamo as a Motor.

78. A shunt motor, connected to I I O-volt supply mains, takes 2.6 amperes through its shunt field winding, and, when the motor is unloaded, it takes 3.0 amperes through its armature and runs at a speed of 997 revolutions per minute. The rated fullload armature current of this motor is 50 amperes, and the resistance of its armature including brushes and brush contacts is O. I I ohm. At what speed would this motor run if the friction, eddy current, and hysteresis losses in the armature were zero? Ans. I,OOO revolutions per minute.

Note. - This speed is called the ideal zero-load speed of the motor. It is the speed at which the motor would run if no torque were required to supply the losses due to friction, eddy currents, and hysteresis. If these losses were zero, the armature current at zero-load would be zero, the $r i$ drop in the armature would be zero, and the induced electromotive force would be equal to $E_{x}$.

79. From the data given in problem 78 find: (a) the counter electromotive force of the motor at full-load, and $(b)$ the full-load speed of the motor (assuming $\Phi$ to be constant). Ans. (a) 104.5 volts ; (b) 945.5 revolutions per minute.

80. The actual full-load speed of the motor of problem 78 is observed to be 980 revolutions per minute instead of the speed as calculated in problem 79. Find the value of the ratio $\Phi / \Phi^{\prime}$ where $\Phi$ is the armature flux due to the field winding alone, and $\Phi^{\prime}$ is the actual value of the armature flux at full-load due to the combined action of field and armature currents. Ans. I.036.

81. (a) Calculate the resistance that must be connected in series with the armature of the motor of problem 78 to give a speed of . 500 revolutions per minute under full-load (50 amperes in the armature); (b) find the speed of the motor when, with this resistance in the armature circuit, the load is reduced to one half ( 25 amperes in the armature) and to one quarter (1 2.5 amperes in the armature) respectively. In this problem assume that $\Phi$ remains the same as at zero-load. Ans. (a) $0.99 \mathrm{ohm;} \mathrm{(b)} 750$ and 875 revolutions per minute.

82. (a) Find the value of the induction factor, $\Phi Z^{\prime}$, of the 
motor specified in problem 78 ; and $(b)$ calculate the torque in pound-inches developed by the motor when its armature current is 50 amperes, assuming $\Phi$ to have the same value at zero-load and at full-load. Ans. (a) 6.6 ; (b) 465.3 pound-inches.

83. A four-pole motor has a four-path armature $30 \mathrm{~cm}$. in diameter and $35 \mathrm{~cm}$. long, that is to say, the armature core and pole faces are $35 \mathrm{~cm}$. long in a direction parallel to the shaft; 0.7 of the periphery of the armature is under the four pole faces, and there are 250 conductors on the surface of the armature. The mean intensity of the magnetic field in the air gap is 4,500 gausses, and the total current flowing through the armature is IOO amperes. (a) Calculate the side push in dynes acting on each wire under the pole faces, from this calculate the total torque in dyne-centimeters and reduce the result to pound-inches. (b) Calculate the value of $\Phi$ and the value of $Z^{\prime}$ from the above data, and then calculate the torque in pound-inches by equation (25). Ans. (a) 393,750 dynes and 9I 5 pound-inches; (b) 9I5 pound-inches.

84. Find the current which would flow through the armature of the motor specified in problem 78 if the armature were to be connected directly to the I I O-volt supply mains at starting. Ans. I,000 amperes.

85. The resistance of the field circuit of the shunt motor specified in problem 78 is doubled by adjusting a field rheostat, the actual zero-load speed of the motor is observed to rise to 1,500 revolutions per minute, and the armature current to 3.3 amperes. In what ratio has the armature flux $\Phi$ been changed by the doubling of the resistance of the field circuit? Ans. I : 0.664 .

86. One terminal of a voltmeter is connected by a lead to the positive brush (which is in the neutral axis) of the motor specified in problem 78 , the other voltmeter lead is touched to the commutator at an angular distance of $15^{\circ}$ ahead of the positive brush, and the voltmeter indicates 9.2 volts. What would be the ideal zero-load speed of the motor if its main brushes were given a forward lead of $15^{\circ}$ ? Ans. I, 200.9 revolutions per minute. 
NOTE. - Imagine the machine to be running at its ideal zero-load speed with $\mathrm{I}$ IO volts applied to its brushes which are in the neutral axis. Under these conditions the induced electromotive force (counter electromotive force) between the brushes is 110 volts, and the induced electromotive force between two auxiliary brushes $15^{\circ}$ ahead of the neutral axis is $(110-2 e)$. If the main brushes are now shifted $15^{\circ}$ forwards, taking the place of the two auxiliary brushes, then the speed must increase sufficiently to make the induced etectromotive force equal to the applied voltage. In this problem and the one that follows the armature current is assumed to be negligibly small. It is to be noted that the speed equation $\left(23^{b}\right)$, page 98 , is true only when the brushes are in the neutral axis.

87. The ideal zero-load speed of the motor specified in problem 78 increases to I, 700 revolutions per minute when the brushes are shifted $25^{\circ}$ from the neutral axis. Find the electromotive force between: $(a)$ an auxiliary pair of brushes in the neutral axis ; $(b)$ one of the main brushes and an auxiliary brush in the neutral axis. Ans. (a) 187 volts; (b) 38.5 volts or 148.5 volts.

88. Ignoring the demagnetizing action of the current in the armature, calculate: $(a)$ the resistance that must be connected in series with the armature of the motor specified in problem 78 to reduce its speed to 800 revolutions per minute when its load is such as to make the armature intake 50 amperes; $(b)$ the watts lost in this resistance expressed in per cent. of the total power intake of the motor. Ans. (a) $0.33 \mathrm{ohm}$; (b) 833 watts or 14.4 per cent.

89. The field current of the motor described in problem 78 is increased to 4.8 amperes by cutting out a portion of the field rheostat. This reduces the full-load speed of the motor to 800 revolutions per minute. Calculate the increase of power delivered to the field circuit due to the increase of field current from 2.6 to 4.8 amperes, and express it in per cent. of the power lost in the armature rheostat in problem 88. Ans. 242 watts or 29. I per cent.

90. The motor of problem 78 has its field permanently connected to I I O-volt mains and its speed is reduced to 200 revolutions per minute at full-load ( 50 amperes in the armature) by having its armature connected to low voltage supply mains. Calculate: (a) the electromotive force of the low voltage mains, 
the full-load speed on the I I O-volt mains being 980 revolutions per minute; $(b)$ the horse-power developed by the armature at this reduced speed expressed as a fraction of the horse-power developed by the armature when running under full-load on I I Ovolt mains at a speed of 980 revolutions per minute. Ans. (a) 26.8 volts ; (b) 20.4 per cent.

91. The actual full-load speed of the motor of problem 78 is 980 revolutions per minute (armature current 50 amperes). This machine is driven as a generator at a speed of 980 revolutions per minute, and the field rheostat is adjusted to give the same field current (2.6 amperes). Find : $(a)$ the terminal voltage of the generator when the armature current is 50 amperes; $(b)$ the total resistance of the field circuit (field coils plus field rheostat) when the machine is operated as a motor and as a generator respectively; (c) the resistance which has been cut out of the field rheostat. Ans. (a) 99 volts; (b) 42.3 and 38 . I ohms; (c) 4.2 ohms.

NOTE. - It is assumed in this problem that the armature flux $\Phi$ is the same in value whether the machine operates as motor or generator. This is true if the backward lead of the brushes for the motor is equal to the forward lead of the brushes for the generator, armature current being the same in each case.

92. A given shunt generator is rated at io kilowatts at Ioo volts between its terminals when driven at a speed of $\mathrm{I}, 200$ revolutions per minute. The resistance of the shunt field winding is $40 \mathrm{ohms}$ (hot) and the resistance of the armature is $0.08 \mathrm{ohm}$ (hot). Find: (a) the armature current of the generator at full load; and $(b)$ the speed of the machine when driven as a motor from Ioo-volt mains with a load such that the armature current is equal to that found under $(a)$. Ans. (a) I02.5 amperes; $(b)$ I, oI 8 revolutions per minute.

93. Find the speed of a railway motor in revolutions per minute corresponding to a car speed of fifteen miles per hour, the gear ratio between the motor and the axle being $14: 68$, and the diameter of the car wheels being 33 inches. Ans. 742 revolutions per minute. 
94. Find the armature torque in pound-inches corresponding to a tractive effort of 2,000 pounds developed by a street railway motor, the gear ratio between the motor armature and the axle being $14: 68$, and the diameter of the car wheels being 33 inches. Ignore motor, gear, and axle friction in this calculation. Ans. 6,794 pound-inches.

\section{Chapter V. Power Losses and Efficiency.}

95. (a) Find field loss and the stray power loss of the motor specified in problem 78 . (b) Find the efficiency of this machine when operated as a generator with an armature current of 50 amperes and an electromotive force of I IO volts between its brushes, assuming the stray power loss to be that found under $(a)$. (c) Find the stray power loss and efficiency of the generator under the conditions specified in $(b)$ making a correction for the difference of induced voltage in the armature. Ans. (a) 286 watts, and 329 watts; (b) 85.4 per cent.; (c) 347 watts, 85.42 per cent.

NOTE. - The difference between the uncorrected and corrected values of the stray power loss, amounting to only 18 watts, is negligible in its effect upon the calculated efficiency of the machine. In the following problems the uncorrected value of the stray power loss is used.

96. Find the efficiency of conversion and the electrical efficiency of the generator specified in problem 95, when its armature current is 50 amperes and its terminal voltage is I IO volts. Ans. 94.6 and 90.3 per cent.

97. Find the true efficiency, the efficiency of conversion, and the electrical efficiency of the generator specified in problem 95, when the armature current is 10 amperes and the terminal voltage is I Io volts. Ans. 56.5, 77.2, 73. I per cent.

NOTE. - In this problem neglect the change of field loss due to the adjustment of the field rheostat which must be made to keep the terminal voltage constant.

The stray power loss of a generator at light loads is in fact less than at full-load; this difference depends not only upon the change of induced electromotive force, but also upon the change of distribution of flux in the armature core due to armature reaction. The change of stray-power loss due to armature reaction cannot be calculated and is generally ignored in efficiency calculations. 
98. (a) Calculate the efficiency of the motor specified in problem 78 , driven as stated from I IO-volt supply mains, when the current intake of its armature has each of the following values : 5 , I O, 15, 25, 35 and 50 amperes. (b) For what armature current is the efficiency a maximum ? (c) Calculate the maximum value. Ans. (a) 26. I, $548,669,77.5,82.0$ and 846 per cent ; (b) 74.8 amperes, (c) 85.6 per cent.

99. The motor referred to in problem 98 is run ten hours each day as follows: I hour with 5 amperes armature intake, 2 hours with I 5 amperes armature intake, 2 hours with 25 amperes armature intake, 4 hours with 35 amperes armature intake, and I hour with 50 amperes armature intake. What is the mean allday efficiency of the motor under these conditions of service? Ans. 78.4 per cent.

NotE. - The all day efficiency of a dynamo is the total output in watt-hours divided by the total input in watt-hours during the day. Thus in this problem find the total input in watt-hours and the total output in watt-hours during the ro-hour run and divide the output by the input.

Evidently a high all-day efficiency, when a motor is run for a large portion of the time at light load, depends upon high efficiency at light loads, and this in turn depends upon small constant losses (field loss plus stray power loss).

100. What is the efficiency of conversion and the mechanical efficiency of the above motor . (a) when its armature current intake is 15 amperes, and $(b)$ when its armature current intake is 50 amperes. Ans. (a) 83.9 and 79.8 per cent; $(b) 90.3$ and 93.7 per cent.

101. From the results of problem 98 calculate the output of the motor in horse-power corresponding to each value of current intake of the armature, and plot a curve showing efficiencies as ordinates and outputs of power in horse-power as abscissas.

102 A certain shunt motor is used as a dynamometer to measure the power absorbed by a printing press. The stray power loss corresponding to $E_{a}=525$ volts is found by previous test to be 450 watts, and the armature resistance is $0.53 \mathrm{ohm}$ including resistance of brushes and of brush contacts. During the test the current delivered to the armature of the motor is 20.2 
amperes, and the voltage across the brushes is 512 volts. Find power delivered to the press. Ans. Stray power during test 430 watts; power to drive press 9,696 watts.

103. A certain shunt motor when connected to Ioo-volt supply mains takes an armature current of 4 amperes and runs at a speed of $\mathrm{x}, 063$ revolutions per minute at zero load. The armature resistance is $\mathrm{O} . \mathrm{I} \mathrm{ohm}$. Find the stray power loss in watts and the corresponding torque in pound-inches. What opposition to rotation is overcome by this torque? Ans. 398.4 watts; 31.7 pound-inches.

104. An efficiency test of a shunt motor (rated at 5 horsepower, 220 volts, I,200 revolutions per minute) by the stray power method gave the following results: Volts between brushes 220 , and current delivered to armature 2.0 amperes. The machine was then shut down, a current of Io amperes was passed through the armature, and a voltmeter across brushes read 5.0 volts. Find: (a) stray power loss in watts ; $(b)$ full-load current in the armature; (c) full-load power intake; $(d)$ true efficiency at full load; and $(e)$ true efficiency at half load. The resistance (hot) of the shunt field winding is 733 ohms. Ans. (a) 438 watts; (b) I 9.85 amperes; (c) 4,433 watts ; (d) 84. I per cent.; (e) 76.7 per cent.

Note. - For a given power output, $P$, in watts, the armature current of the motor is determined by the quadratic equation :

$$
P=E_{x}\left(I_{a}+I_{s}\right)-F-S-R_{a} I_{a}^{2}
$$

in which $F\left(=E_{x} I_{s}\right)$ is the shunt field loss and $S$ is the stray power loss, both of which are assumed to be independent of load.

105. A shunt dynamo when run as a generator at a speed of 800 revolutions per minute delivers I I kilowatts at a terminal voltage of I 10 volts, with a field current of 2 amperes. The resistance of the armature (hot) is $0.05 \mathrm{ohm}$. When this machine is run as a motor taking 102 amperes from I IO-volt mains, it delivers 12.8 horse-power at its pulley. Find: $(a)$ counter electromotive force of the motor; $(b)$ speed of the motor; $(c)$ induction factor, $\Phi Z^{\prime}$ of the motor; (d) stray power loss in the motor; $(e)$ 
full-load torque developed in the generator; and $(f)$ full-load torque developed in the motor. Ans. (a) 105 volts; $(b) 729$ revolutions per minute; (c) 8.63; (d) 950 watts; (e) I,240 pound-inches ; I, 2 I 8 pound-inches.

NoTE. - The armature current is so nearly the same for motor and generator in the specifications of this problem that the armature flux may be assumed to be the same. See note to problem 91.

106. A certain 6.5-kilowatt shunt generator, giving I I 5 volts between its terminals, has an armature resistance of $0.29 \mathrm{ohm}$, a field resistance of $57.5 \mathrm{ohms}$, and a stray power loss of 495 watts. Find the armature current for which the efficiency is a maximum, and find the value of this maximum efficiency. Ans. 50 amperes, 79.2 per cent.

NotE. - This problem and the next are based on the condition that maximum efficiency occurs when the variable loss is equal to the constant loss.

107. A certain 5 horse-power I IO-volt shunt motor with a speed of I,200 revolutions per minute has a field loss of I IO watts and a stray power loss of 250 watts, both assumed to be independent of load. The armature resistance is $0.4 \mathrm{ohm}$. Find the value of the armature current intake for which the efficiency of the motor is a maximum, and find the value of this maximum efficiency. Ans. 30 amperes ; 78.9 per cent.

108. A Io-kilowatt Ioo-volt long-shunt flat-compound generator has a stray power loss of 600 watts, the resistance of its armature including brushes and brush contacts is $0.04 \mathrm{ohm}$, the resistance of its series field winding is $0.0 \mathrm{I} \mathrm{ohm}$, and the resistance of its shunt field winding is 25 ohms. (a) Calculate the watts lost in armature winding and series field coil for various armature currents up to IO4 amperes and plot a curve showing these losses as ordinates and the corresponding external currents as abscissas. What is the equation to this curve? What is the name of this curve? (b) Plot a curve showing total losses as ordinates and values of external current $I_{x}$ as abscissas; and $(c)$ plot a curve showing efficiencies as ordinates and external currents as abscissas. 
109. A certain short-shunt compound generator delivers io kilowatts of power at a terminal voltage of 100 volts at full-load. The resistance of armature, series field coils and shunt field coils are $0.04 \mathrm{ohm}, 0.03 \mathrm{ohm}$ and $20 \mathrm{ohms}$ respectively; and the stray power loss is found by test to be 430 watts. Find at full-load : (a) voltage between brushes; (b) armature current; (c) total electromotive power induced in the armature windings; $(d)$ total electrical power developed in the armature; $(e)$ electrical efficiency; $(f)$ commercial efficiency. Ans. (a) IO3 volts; $(b)$ I05. I 5 amperes; (c) I07.2 I volts; (d) II,300 watts; (e) 88.5 per cent. ; $(f) 85.4$ per cent.

110. A certain shunt motor is tested for efficiency at full-load by the Prony brake method and the following observations are obtained : speed, I,200 revolutions per minute ; reading of spring balance, I 8.75 pounds; perpendicular distance from center of pulley to the center line of the spring balance, 28 inches; electromotive force applied, I Io volts ; total current intake, 83.6 amperes. Find: (a) output in horse-power and in watts; $(b)$ torque in pound-feet; $(c)$ efficiency (true) of the motor; and $(d)$ tangential pull in pounds at the rim of the motor pulley which is 10 inches in diameter. Ans. (a) 9.98 horse-power or 7,455 watts; (b) 43.75 pound-feet; (c) 8 I.I per cent.; (d) I05 pounds.

111. A certain 6-pole, 250 -kilowatt, 550-volt generator runs at a speed of 320 revolutions per minute. The commutator is 37.4 inches in diameter. The machine has six sets of carbon brushes, each set has four brushes, each brush has r.09 square inches of contact surface with the commutator, the brush pressure is 1.25 pounds per square inch, and the coefficient of friction of the carbon brushes upon the copper commutator is 0.3 . Find : $(a)$ the watts lost in brush friction; (b) the watts lost in contact resistance between the brushes and the commutator at full load, neglecting the shunt current. Ans. (a) 695 watts; (b) 9I I watts.

NoTE. - Under ordinary conditions as to peripheral velocity and brush pressure the contact resistance of carbon brushes in ohms per square inch of contact surface is 
approximately equal to the reciprocal of the current density in amperes per square inch of contact surface. An elaborate discussion of brush contact resistance in its dependence upon peripheral velocity of commutator, brush pressure, quality of carbon, and current density is to be found in E. Arnold's Die Gleichstrommaschine, Vol. I., pp. $478-484$.

112. The generator specified in problem 106 has its field separately excited so as to give its full rated voltage at zero-load. Assuming that this constant field excitation leads to the production of a constant total induced electromotive force, $E_{a}$, at all loads, find: $(a)$ the value of the resistance of the external circuit for which the power output of this separately excited generator is the greatest possible; $(b)$ the corresponding value of the current ; (c) the value of this maximum power output ; $(d)$ the corresponding terminal voltage of the generator; and $(e)$ the corresponding electrical efficiency of the generator. Ans. $(a) 0.29 \mathrm{ohm} ;(b)$ I 98.3 amperes; $(c)$ I I,400 watts; $(d) 57.5$ volts; $(e) 50$ per cent.

NoTE. - The armature current corresponding to maximum possible output of a generator (or motor) is greatly in excess of the greatest permissible armature current and it would cause excessive sparking at the brushes and excessive overheating of the armature windings. The assumption of constant armature flux $\Phi$ is made to simplify the problem. In fact the armature flux falls off greatly with such excessive armature currents.

113. Assuming that the armature flux, $\Phi$, of the motor specified in problem 78 is constant at all loads, find: $(a)$ the value of the counter electromotive force and speed of the motor for which its power output has the greatest possible value, (b) the value of this greatest possible output; and (c) the efficiency of conversion of the motor at its greatest possible power output, ignoring the power delivered to its field winding. Ans. (a) 55 volts and 500 revolutions per minute; (b) 27, I I I watts; $(c) 50$ per cent.

Note. - See note to problem II 2.

114. A certain belted compound-wound machine, purchased second-hand, is rated as a 250 -kilowatt 550 -volt generator, at a speed of 320 revolutions per minute. The diameter of the armature is 46 inches and the gross length of the armature core is I 2.3 inches. Calculate the rating of this machine according to 
Art. 73 , page 148 , in order to check the above rating as claimed by the dealer. Ans. 265 kilowatts.

115. A certain enclosed motor is rated by the manufacturers as a 220-volt, 27.5-horse-power motor at a speed of 600 revolutions per minute. The diameter of the armature is 15 inches and the gross length of the armature core is 13 inches. Calculate its rating according to Art. 73, page 148. Ans. 34 horse-power.

116. A certain four-pole dynamo is rated as a 5-horse-power I IO-volt motor to run at a speed of 600 revolutions per minute. This motor is connected to 220 -volt mains and the armature flux $\Phi$ is increased 25 per cent. by the 100 per cent. increase of field current. (a) At what speed would this motor run under these conditions? (b) The resistance of the shunt field winding is 100 ohms, how much resistance must be connected in series with the field winding to increase the motor speed to $\mathrm{I}, 200$ revolutions per minute on 220-volt mains? (c) What would the power rating of this motor be at this speed of $\mathrm{I}, 200$ revolutions per minute? $(d)$ Would it be permissible to drive this motor at a speed of I,200 revolutions per minute from 220-volt mains, the diameter of the armature being Io inches and the number of commutator segments being 60? Ans. (a) 960 revolutions per minute; (b) 100 ohms; $(c)$ Io horse-power; $(d)$ the machine would in all probability operate satisfactorily.

NoTE. - In dynamos of medium current rating an average electromotive force of 20 volts per commutator segment is the greatest that can be used without danger of sparking. By average electromotive force per segment is meant $E / n$ where $E$ is the electromotive force between the brushes and $n$ is equal to $K\rceil p$, where $K$ is the total number of commutator bars and $p$ is the number of field magnet poles.

A well constructed armature can be expected to withstand a speed which corresponds to a peripheral velocity of from 3,000 to 4,000 feet per minute.

117. A certain bipolar drum-wound armature has 384 conductors; its pole faces have an angular breadth of $120^{\circ}$; and its full-load armature current is 200 amperes. Find : $(a)$ per cent. of armature periphery covered by poles; (b) the number of demagnetizing ampere-turns on the armature if the angle of lead of the brushes is $30^{\circ} ;(c)$ the cross-magnetizing ampere-turns. 
Ans. (a) 67 per cent ; (b) 6,400 ampere-turns per magnetic circuit ; (c) I 2,800 ampere-turns per pole.

118. A certain 6-pole, 250-kilowatt, 550-volt railway generator has a six-path armature winding with I,200 conductors. The commutator has 600 segments, and at full load the brushes are shifted forwards through an angle represented by 8 commutator segments. Find : $(a)$ the angle of lead of the brushes in degrees ; and $(b)$ the armature demagnetizing ampere-turns per magnetic circuit at full-load, neglecting the shunt current. Ans. (a) $4.8^{\circ}$; (b) 2,424 ampere-turns.

119. The pole faces of the generator specified in problem i i 8 cover 70 per cent. of the periphery of the armature, and the radial length of the air gap between the pole faces and the armature core is 0.527 inch. Find: $(a)$ the cross-magnetizing ampereturns per pole on the armature at full-load; $(b)$ the intensity of the magnetic field under the pole tips due to the armature current alone; $(c)$ the intensity of the magnetic field under the leading and trailing pole tips due to the combined action of field coils and armature, the field intensity in the air gap due to field coils alone being 6,500 gausses at full-load. Ans. (a) 10,640 ampere-turns per pole ; $(b) 5,000$ gausses ; (c) I, 500 gausses and I I, 500 gausses.

NoTE. - The radial length of the air gap in this dynamo is in fact 0.313 inch, but the effect of the high degree of magnetic saturation of the teeth of this armature core is equivalent to a greatly increased radial length of air gap.

120. A bipolar generator has a drum armature with 420 conductors and each pole face subtends an angle of $120^{\circ}$. The distance across the gap space is 0.6 centimeter and the field intensity in the gap space due to the field winding alone is 8,000 gausses. What is the greatest permissible current in the armature of the generator if it is assumed that sparkless running requires the resultant field intensity under the leading pole tips to be at least 25 per cent. of the field intensity due to the field winding alone? Ans. $8 \mathrm{r} .8$ amperes.

121. The drum armature specified in problem $\mathrm{I} 20$ is arranged so as to be suitable for a four-pole field magnet, the pole faces cover- 
ing the same percentage of the armature periphery as before, the length of the air gap being the same, and the field intensity in the gap space due to the field winding alone being 8,000 gausses. What is the greatest permissible current in the armature of the machine if it is assumed that sparkless running requires the resultant field intensity under the leading pole tips to be at least 25 per cent. of the field intensity due to the field winding alone? This four-pole drum armature winding has two paths between positive and negative brushes. Ans. 163.6 amperes.

122. The pole faces of the generator specified in problem I $2 \mathrm{I}$ are widened so as to cover 75 per cent. of the armature periphery and the gap space is increased to 0.7 centimeter. Find the greatest permissible current output of the machine if it is assumed that sparkless running requires the field intensity under the leading pole tips to be at least 25 per cent. of the field intensity of 8,000 gausses which is due to the field winding alone. Ans. I78 amperes.

123. Find the armature demagnetizing ampere-turns per magnetic circuit and the number of turns required in each series field coil to balance the same, in a four-pole roo-kilowatt, 500-volt generator, having a four-path armature winding with 500 conductors; forward lead of the brushes is $10^{\circ}$, and 0.868 of the armature current flows through the series field coils. Ans. I,389 ampere-turns and 4 turns.

124. The field coil of a dynamo rises 46 centigrade degrees above a room temperature of $35^{\circ} \mathrm{C}$., what would be its rise of temperature above a room temperature of $25^{\circ}$ C.? Ans. 44.39 centigrade degrees.

Note. - Let $t^{\prime}$ be the temperature rise above $25^{\circ} \mathrm{C}$. and $t^{\prime \prime}$ the temperature rise above $35^{\circ} \mathrm{C}$. In the first case the actual temperature of the winding is $25+t^{\prime}$ and its resistance, $R^{\prime}$, is $R_{0}\left[\mathbf{I}+\beta\left(25+t^{\prime}\right)\right]$. In the second case the actual temperature of the winding is $35+t^{\prime \prime}$ and its resistance, $R^{\prime \prime}$, is $R_{0}\left[\mathbf{I}+\beta\left(35+t^{\prime \prime}\right)\right]$. The rates at which heat is generated by the given current in the two cases are proportional to $R^{\prime}$ and $R^{\prime \prime}$ respectively, and, since the rise of temperature is proportional (very nearly) to the rates of generation of heat, therefore

$$
t^{\prime}: t^{\prime \prime}:: R^{\prime}: R^{\prime \prime} \text {. }
$$


The A.I.E.E., rule, see page 165, Art. 28 , applied to this problem would give 43.7 instead of the correct value 44.39. The rule is, however, nearly enough exact for all practical purposes. Indeed tenths of degrees are wholly meaningless in connection with such uncertain quantities as rises of temperature of dynamo machines, so that to the nearest degree the A.I.E.E. rule and the rigorous formula give the same result.

125. The armature of a dynamo has a resistance between commutator bars $b$ and $c$ (see problem 52) of $0.0678 \mathrm{ohm}$ at room temperature of $10^{\circ} \mathrm{C}$. After the dynamo has been run for a long time at full load it is shut down and the resistance of the armature between commutator bars is found to be $0.0806 \mathrm{ohm}$. Find the difference between room temperature and the running temperature of the armature; $(b)$ what would this temperature difference be if the room temperature were $25^{\circ}$ C.? Ans. (a) 49 centigrade degrees; (b) 53 centigrade degrees.

126. The armature and all field windings of a given 500-kilowatt 500-volt generator are connected together (machine not running) and a circuit is made from one main of a 500-volt supply, through a direct reading voltmeter to one brush of the given generator, whence a very small current passes through the armature and field insulation to the iron frame of the machine which is connected to the other main of the 500-volt supply. The voltmeter indicates 6.2 volts and its resistance is $55,000 \mathrm{ohms}$. Find the insulation resistance of the given generator as connected. Ans. 4.38 megohms.

Note. - The voltmeter, arranged as specified in this problem, is used as an am. meter, and the current flowing through it is equal to its reading in volts divided by its resistance in ohms.

\section{Chapter ViI. The Practical Operation of Dynamos.}

127. The voltage of an over-compounded generator rises from I 5 volts at zero-load to I 25 volts at full-load. When the series field coil is not used the machine becomes a shunt generator and its voltage falls from I I 5 volts at zero-load to 90 volts at full-load. What would the voltage of this generator be if it were operated alone at full-load, with its series field coil in par- 
allel with the series field coil of another similar generator which is not in operation? Ans. 107.5 volts.

Note. - The effect, direct and indirect, of the full-load current in the series field winding of this generator is to raise its voltage at full-load from 90 volts to 125 volts and it may be roughly assumed that one half of the full-load current in the series field winding will raise the full-load voltage half as much.

128. A current of 200 amperes is to be delivered at 450 volts to an extension of an electric railway at a "feeding point" which is five miles from the power station. Two plans are considered for accomplishing this result, namely: (I) To install a copper feeder circuit (the resistance of the ground and rail return being very uncertain, a return feeder wire is here assumed for the sake of definiteness) which will deliver the prescribed current with a drop of 100 volts, and (2) to install a feeder circuit of smaller wire which will deliver the current with a drop of 300 volts, and a 40 -kilowatt booster for raising the station voltage of 550 volts up to 750 volts, which is required by this small feeder.

The feeder required by plan (I) costs more than the feeder required by plan (2) at the rate of $171 / 2$ cents per pound for the additional copper ; the cost of electrical energy at the switchboard is 1.75 cents per kilowatt-hour; the cost of the booster and its accessories including the motor to drive it is $\$ 2,200$; the efficiency of the booster set, motor and generator combined, is 75 per cent .; the depreciation of the booster is 8 per cent. per annum; the depreciation of the feeders is 5 per cent. per annum; and the interest and taxes on invested capital is 6 per cent. per annum.

Find the maximum number of hours per day that the full-load current of 200 amperes can be transmitted by plan (2) at a lower actual cost than by plan (I). Ans. 5.97 hours.

Note. - The weight of bare copper wire in pounds is equal to 0.00000303 $X$ length in feet $X$ section in circular mils.

The cost of large sizes of weather-proof insulated wire may be based on the weight of the bare copper. Thus the high price of $171 / 2$ cents per pound for the bare copper is intended to include the cost of the insulation.

129. Given a direct reading ammeter (or voltmeter) of the direct-current type of which the scale divisions are of equal length 
so that an observer can read, say, to $\frac{1}{10}$ of an ampere anywhere on the scale. (a) Find the percentage error due to the error of reading when the reading is I ampere; $(b)$ find ditto when the reading is Io amperes. Ans. (a) Io per cent.; (b) I per cent.

130. An electrodynamometer-ammeter (a Siemens instrument) has a scale of equal parts. The position of the pointer can be read to, say, $1 / 2$ of a division anywhere on the scale. (a) Find how many times as great the error in amperes, due to the given error in the reading, is when the reading of the pointer is 25 divisions than when the reading of the pointer is 250 divisions; (b) find the percentage error in each case and the ratio of these percentage errors. Ans. (a) 3.I6; (b) I per cent., $\frac{1}{10}$ per cent. ; ratio $\mathrm{IO}: \mathrm{I}$.

131. Given a direct-reading ammeter (or voltmeter) of the alternating current type of which the actual length of any scale division under the pointer is proportional to the reading of the pointer; a scale division being supposed to correspond to, say, O. I ampere over the entire scale. The position of the pointer can be read to a certain fraction of a degree of angle, so that the error in amperes due to an error of reading is inversely proportional to the length of the scale division under the pointer, that is, inversely proportional to the reading of the pointer. (a) Find how many times as great the error in amperes due to an error in reading is when the instrument reads I ampere than when the instrument indicates ro amperes. (b) Find how many times as great the percentage error is in the first case than in the second case. Ans. (a) Io times as great; (b) Ioo times as great.

Note. - Ammeters and voltmeters which are suitable for direct-current circuits only, always give a deflection which is approximately proportional to the current or voltage. Therefore the scales of direct-reading instruments of this type are scales of approximately equal parts.

Ammeters and voltmeters which are suitable for both alternating and direct-current circuits always give a deflection which is nearly proportional to the square of the current or voltage. Let $y$ be the deflection of such an instrument read on a scale of equal parts or, say in degrees, let $d y$ be one of the divisions of this scale, and let $x$ be the current or voltage corresponding to the deflection $y$. Then

$$
y=k x^{2} \text { and } d y=2 k x d x
$$


where $k$ is a constant. Now, in a direct-reading instrument each scale division corresponds to a certain constant fractional part of an ampere or volt which may be represented by $d x$. Then the length of a scale division of such a direct-reading instrument expressed in degrees is given by the value of $d y$, which according to the above equation is proportional to $x$, which is the reading in amperes or volts of the directreading instrument.

Problems 129, 130, and 131 touch upon a matter of very great practical importance and two simple examples covering the entire ground may be worth while. A standardized direct current ammeter (or voltmeter) which indicates 100 amperes (or volts) with a certain degree of precision will indicate 50 amperes (or volts) with the same actual error or with twice the percentage error. A standardized alternating current ammeter (or voltmeter) which indicates 100 amperes (or volts) with a certain degree of precision will indicate 50 amperes (or volts) with twice as large an actual error or with four times as large a percentage error.

132. A wattmeter is connected as shown in Fig. $12 \mathrm{I}$, Chapter VII. The resistance of the coil $B$ is 5 ohms. The wattmeter indicates 55 watts when used to measure the power delivered to a lamp which takes one ampere of current. What is the true power delivered to the lamp? Ans. 50 watts.

133. A wattmeter is connected as shown in Fig. I22, Chapter VII. The resistance of the circuit $A R$ is $\mathrm{I}, 200$ ohms. The wattmeter indicates 60 watts when used to measure the power delivered to a I IO-volt glow lamp. What is the true power delivered to the lamp? Ans. 49.92 watts.

134. A Thomson watt-hour meter without a starting coil starts on a 75-watt load. The meter is adjusted to give a true watthour record when run on a 500-watt load. What will the instrument indicate after running for 4 hours on a constant load of 200 watts, running friction being assumed to be equal to half of starting friction. See note to problem I 36 . Ans. 702.8 watt-hours.

135. The watt-hour meter specified in problem 134 is provided with a starting coil so as to start, on I IO-volt mains, when the power delivered to the receiving circuit is 40 watts. At what load will the meter start on 55 -volt mains? See note to problem I36. Ans. 66.25 watts.

136. The watt-hour meter of problem I 35 is adjusted to record a 500-watt load correctly on I ro-volt mains. At what load will it record correctly on 55-volt main? Ans. 5,750 watts. 
NoTE. - The driving torque, not counting that due to the starting coil, is propor tional to the watts delivered to the receiving circuit, and it may be conveniently expressed in "watts." The driving torque due to the starting coil (with given voltage between the supply mains) may be expressed as the difference between the starting watts with and without the starting coil. The running friction (a torque) may be expressed as one half the starting load in watts without the starting coil. The speed of the meter may be conveniently expressed in "watt-hours recorded per hour."

Subtracting from the total driving torque (including the torque due to the starting coil) the running friction, gives the net torque used to overcome the retarding action of the damping magnets, and the speed of the meter is proportional to this net torque. The torque produced by the starting coil is proportional to the square of the voltage between the mains.

137. The operation costs of a 405-kilowatt lighting plant including the distributing system are: an invariable charge of $\$ 22,000$ per year, which includes interest, depreciation, repairs, insurance, taxes, one half of the cost of wages, and one quarter of the cost of fuel and supplies; and a variable charge of 2.05 cents per kilowatt-hour of station output. Find the proper net prices to be charged per kilowatt-hour for: $(\alpha)$ domestic lighting which comes wholly within the daily period of two hours of full station-load; $(b)$ store lighting of which one third comes during the daily period of full station-load, and $(c)$ motive power no portion of which comes during the period of full station-load. Allow for a profit equal to I 5 per cent. of the gross income. Ans. (a) II.I 6 cents, $(b) 5.33$ cents, and (c) 2.4 I cents.

Note. - The separating of the operation costs of a station into an invariable part $A$ and a variable part $b P$, which is proportional to the station-load $P$, is only approximately correct. The total actual cost of operating the station at various loads may be determined from actual station tests. From these data a curve may be plotted showing the costs as ordinates and the station-loads as abscissas. A straight line may now be drawn so as to represent this curve as nearly as may be. Then $A$ is the intercept of this straight line on the $y$-axis, and $b$ is the tangent of the angle between this straight line and the $x$-axis.

The cost price per kilowatt-hour during the two hours of full load is $\$ 22,000$ divided by $405 \times 2 \times 365$, to which must be added 2.05 cents; whereas the cost price per kilowatt-hour during the remainder of the day is 2.05 cents.

138. Find the proper prices to be charged per kilowatt-hour for the three classes of service specified in problem 137 , the daily period of full station-load being three hours. Ans. (a) 8.25 cents ; (b) 4.36 cents ; (c) 2.4 I cents. 


\section{Chapter ViII. Storage Batteries.}

139. A primary battery which is free from local action gives a current of 1.5 amperes for 50 hours. Calculate the number of grams of zinc consumed. Ans. 91 grams.

Note. - The electrochemical equivalent of zinc is 0.000337 gram per ampere per second.

140. A Grenet cell consumes 12.5 grams of zinc during the time that the current from the cell is depositing 8 grams of silver in an electrolytic cell containing a solution of silver nitrate. What portion of the zinc is consumed by local action? Ans. 80.7 per cent.

Note. - The electrochemical equivalent of silver is 0.001118 gram per ampere per second. See Note to problem 139.

141. The charge and discharge curves, shown in Fig. I43, Chapter VIII., apply to the case in which an 80 ampere-hour cell is charged for 8 hours and 5 minutes by a steady current of IO amperes, and discharged for 8 hours by a steady current of IO amperes. Calculate : $(a)$ the energy in watt-hours delivered to the cell in charging, $(b)$ the energy in watt-hours delivered by the cell in discharging; and $(c)$ find the efficiency of the cell. Ans. (a) I 85.7 watt-hours; (b) I 59.4 watt-hours; (c) 85.3 per cent.

142. The total weight of all the grids of a I 20 ampere-hour storage cell (discharged) is 40 pounds, and one-third of this weight is active material. (a) Calculate from electrochemical data the weight of $\mathrm{PbSO}_{4}$ that is converted into $\mathrm{PbO}_{2}$ on the positive grids, and the weight of $\mathrm{PbSO}_{4}$ that is converted into $\mathrm{Pb}$ on the negative grids when the cell is charged. (b) What part of the socalled active material is really active? Ans. (a) I.49 pounds of $\mathrm{PbSO}_{4}$ on the positive grids ; I.49 pounds of $\mathrm{PbSO}_{4}$ on the negative grids ; (b) 22.4 per cent.

Note. - One ampere in one hour liberates 37.4 milligrams of hydrogen from $\mathrm{H}_{2} \mathrm{SO}_{4}$. The weight of $\mathrm{PbSO}_{4}$ may be calculated from the weight of the hydrogen by the ordinary methods of chemical arithmetic. The atomic weights are as follows : $\mathrm{H}=\mathrm{r}, \mathrm{O}=16, \mathrm{~S}=32$, and $\mathrm{Pb}=207$. 
143. How many grams of $\mathrm{SO}_{3}$ are removed from the electrolyte during the discharge of a 120 ampere-hour storage cell ? Ans. 359 grams, half of which is absorbed by each electrode.

144. The electromotive force of a storage cell is observed to be 2.05 volts on open-circuit and the electromotive force is observed to fall suddenly to 1.95 volts when a current of 50 amperes is taken from the cell. What is the resistance of the cell? Ans. $0.002 \mathrm{ohm}$.

145. A storage battery consisting of $6 \mathrm{I}$ cells has an internal resistance of $0.03 \mathrm{ohm}$. The battery delivers a current of 100 amperes at I IO volts to lighting mains when the resistance of the rheostat, $R$, shown in Fig. I46, Chapter VIII., is $0.12 \mathrm{ohm}$. $(a)$ What is the voltage between the mains when half of the lamps are suddenly turned off? (b) Calculate the resistance required in the rheostat, $R$, to bring the voltage across the lighting mains back to I IO volts. Ans. (a) II 7.5 volts; (b) $0.27 \mathrm{ohm}$.

146. The lamps in Fig. I 50, Chapter VIII., require a constant electromotive force of IIO volts and the current demanded by the elevator motor varies from zero to 150 amperes with an average value of 25 amperes. The booster, $M$, is provided with the two opposing field windings, $S$ and $S^{\prime}$. (The winding, $S^{\prime}$, may be assumed to be connected to the constant-voltage mains in parallel with the lamps for the sake of simplicity.) The constant field excitation due to $S^{\prime}$ is equal to $3^{n}$, and the opposing field excitation due to $S$ is $3^{n}$ when the current flowing through $S$ is 25 amperes; where $n$ is the ampere-turns on the field of $M$ required to produce 10 volts between the brushes of $M$. The storage battery consists of $5 \mathrm{I}$ cells with a minimum voltage of 96.9 volts (I.9 volts per cell) and a maximum voltage of I 22.4 volts (2.4 volts per cell). The minimum occurs, of course, when the battery is nearly discharged and the elevator motor is taking its maximum current of 150 amperes, and the maximum occurs when the battery is nearly charged and the elevator motor is taking no current at all. Find the current delivered through $M$ and $S$ to the elevator motor and battery : $(\alpha)$ when the battery is nearly discharged and 
the elevator motor is taking its maximum current of 150 amperes, and $(b)$ when the battery is nearly charged and the elevator motor is taking no current at all. Ans. (a) 35.9 amperes; (b)

\section{I4.7 amperes.}

Note. - Assume that the voltage of the booster is proportional to its net field excitation, ro volts for each $n$ ampere-turns.

The student may calculate the values of the current corresponding to $(a)$ and $(b)$ on the supposition that the winding, $S^{\prime}$, is connected as shown in Fig. 150, $3^{n}$ being the field excitation due to $S^{\prime}$ when the voltage between the terminals of $S^{\prime}$ is IIO volts. The answers in this case are : $(a) 32.9$ amperes; and $(b) 17.5$ amperes.

\section{Chapter IX. Electric Distribution and Wiring.}

147. Five hundred glow lamps each taking one-half an ampere at I IO volts are supplied with current from a II 5.5-volt generator at a distance of $\mathrm{I}, \mathrm{OOO}$ feet from the lamps. Find: $(a)$ The size of copper wire required, $(b)$ the total weight of the wire, and $(c)$ the total cost of the wire at I 6 cents per pound. Ans. (a) 982,000 circular mils ; (b) 5,950 pounds ; (c) 953 dollars.

148. Five hundred glow lamps each taking one-half an ampere at I IO volts are supplied with current from a 23 I-volt generator at a distance of $\mathrm{I}, 000$ feet from the lamps. The Edison three-wire system is used and the system is balanced. Find: (a) The size of copper wire required for the outside mains; (b) the total weight of all three mains, the middle main having onehalf the sectional area of either outside main; and $(c)$ the total cost of the three mains at 16 cents per pound. Ans. (a) 245,500 circular mils; (b) 1,860 pounds; (c) 298 dollars.

149. The three-wire system of problem 148 supplies 300 lamps (I 50 amperes) on one side and 200 lamps (I00 amperes) on the other side, all at a distance of I,000 feet from a $23 \mathrm{I}$-volt generator. A balancer is used in the station to take care of the current in the middle main and to keep the voltage between the middle main and each outside main equal to I 5.5 volts. Find : (a) The voltage across the set of 300 lamps; and $(b)$ the voltage across the set of 200 lamps. Ans. (a) 104.5 volts; (b) I I 5.5 volts. 
Note. - If the lamps above specified are all exactly alike it is evident that the current in each lamp of the 300 set cannot be the same as the current in each lamp of the 200 set. It is usual, however, in wiring calculations to consider that lamps of a given size and type take a definite amount of current irrespective of the slight variations of voltage.

150. The three-wire system of problem 148 supplies 300 lamps (I 50 amperes) on one side and 100 lamps (50 amperes) on the other side, and the total voltage of $23 \mathrm{I}$ volts at the generator is equally divided by the balancer as explained in problem I49. Find: $(a)$ The voltage across the set of 300 lamps; and $(b)$ the voltage across the set of IOO lamps. Ans. (a) IOO. I volts; (b) I 22. I volts.

151. A span, 150 feet long, of hard-drawn copper wire, No. 8 Brown and Sharpe gauge, is to be strung at a temperature of $75^{\circ} \mathrm{F}$. at a place where the winter temperature sinks to $-20^{\circ} \mathrm{F}$. The maximum tension of the wire is to be 164 pounds. Find : (a) The sag at $-20^{\circ} \mathrm{F}$; (b) the sag at $75^{\circ} \mathrm{F}$, , and $(c)$ the tension at $75^{\circ} \mathrm{F}$. Ans. (a) 0.86 foot; (b) 2.86 feet ; (c) 49 pounds.

152. A group of ten lamps, each taking one-half an ampere, is ten feet distant from I I 5-volt mains. (a) Find the size of wire required in order to give a drop of 5 volts. (b) What size of wire (rubber insulation) would be required according to the table of safe carrying capacity given in Art. I 16, Chap. IX. ? Ans. (a) 14.7 mils diameter; (b) No. 16 Brown and Sharpe gauge (5 I mils diameter).

Note. - It is evident that a wire $\mathbf{1 4 . 7}$ mils in diameter would be excessively heated by a current of 5 amperes. Usually the size of wire for supplying lamps near to the generator or center of distribution is determined by the table of safe carrying capacity.

The insurance rules of the National Electrical Code forbid the use of wire smaller than No. 14 Brown and Sharpe gauge for house wiring.

153. A pair of street mains leading out from a central station delivers 50 amperes of current to a consumer at a distance of 200 feet from the station, 75 amperes to a second consumer at a distance of 350 feet from the station, and 40 amperes to a third consumer at a distance of 600 feet from the station. The station voltage is I 5 volts and the voltage at the distant end of mains is I IO volts. Find: $(a)$ The size, weight, and cost of mains of uniform size; and $(b)$ the size of each section of the mains, and their total weight 
and cost, when the size is reduced in steps so as to give the specified voltage-drop with a minimum amount of copper. The cost of copper is to be taken at 16 cents per pound. Ans. (a) 260,300 circular mils, 946.5 poinds, I 51.2 dollars, (b) first section 319,700 circular mils, second section 267,000 circular mils, third section I 57,400 circular mils, 868 pounds, I 39 dollars.

Note. - In estimating the distance, $L$, of the "center of gravity" of the consumers in the above problem, one may use one ampere as the unit instead of one lamp.

154. An electric railway 33,300 feet in length is divided into three sections of which the lengths, 9,000 feet, 10,800 feet and I 3,500 feet, are proportional to the schedule speeds of cars on the respective sections so that a car running from end to end of the line traverses each section in Io minutes. Four cars are always on the first section five minutes apart going each way, two cars are always on the second section ten minutes apart going each way, and a single car is always on the third section making the round trip in 20 minutes. Owing to frequent stops on the first section the cars take an average current of 125 amperes each, on the second section the stops are less frequent and each car takes an average of 105 amperes, and on the third section the stops are least frequent and the car that is always on this section takes an average of 95 amperes.

The "center of gravity" of the four cars that are always on the first section is at the middle of the section, the "center of gravity" of the two cars that are always on the second section is at the middle of that section, and the most unfavorable position of the single car that is always on the third section is when it is at the extreme end of the line. Assume, therefore, that 500 amperes are delivered continuously at the middle of the first section ( 4,500 feet from the city end), that 2 IO amperes are delivered continuously at the middle of the second section (14,400 feet from the city end), and that 95 amperes are delivered continuously at the extreme end of the line. If the power house is located at the city end of the line, find: $(a)$ The size of each section of the feeder to give a total drop of 75 volts at the ex- 
treme end of the line with a minimum amount of copper; and (b) the total cost of feeder copper at 16 cents per pound. Ans. (a) First 4,500 feet of feeder I,980,000 circular mils, next 9,900 feet of feeder I, 2 I 9,000 circular mils, and remaining 18,900 feet of feeder 680,000 circular mils; (b) 16,400 dollars.

Note. - The resistance of the bonded track, which is used as a return feeder, is very uncertain and it is here to be assumed equal to zero for the sake of simplicity.

155. (a) Find the position in which the power house should be placed on the railway specified in problem I 54 in order that the feeder copper may be reduced to a minimum; (b) find the size of each section of the feeder on the assumption that the two cars on the middle section are in the most unfavorable positions, namely, at the two ends of the section, and on the assumption that the car on the third section is at the extreme end of the section; and $(c)$ find the total cost of the feeder copper at 16 cents per pound. Total drop to each end of the line to be 75 volts. Ans. (a) I0,480 feet from the city end of the railway; $(b)$ first section $44 \mathrm{I}, 000$ circular mils, city end of second section ( $\mathrm{I}, 480$ feet) 485,200 circular mils, suburban end of second section $(9,320$ feet) 536,700 circular mils, and third section 369,900 circular mils ; (c) 7, I I9 dollars.

Note. - The power house should be placed at the "center of gravity" of a sys" tem in the sense in which this term is defined in Art. I19, Chap. IX., in order that the amount of feeder copper may be a minimum. Similarly, a center of distribution, from which electric lamps are to be supplied by street mains, should be located at the "center of gravity" of the consumers, each consumer being "weighted" in proportion to the current delivered to him.

The feeder on city section of 9,000 feet is assumed to be of uniform section of $44 \mathbf{I}$, ooo circular mils throughout, but it would be advisable in fact to make the extreme city end of this section of the feeder much smaller than 44I,000 circular mils.

156. A nearly reëntrant row of Ioo lamps, each taking onehalf an ampere, is to be wired in accordance with the return loop scheme, using wire of uniform size. The row is 200 feet long, one end of the row is 50 feet from the service point, and the other end of the row is 60 feet from the service point. The voltage at the service point is I I 5 volts. Find the size of wire to 
give 105 volts at the middle lamp of the row. Ans. I4,040 circular mils sectional area.

Note. - Such problems as this and problem 157 are most easily solved on the assumption that the given group of lamps is equivalent to a 50-ampere load distributed with ideal uniformity over the whole length of 200 feet.

157. Find the voltage at each end lamp in the row specified in problem I 56 . Ans. 108.85 volts at one end and 109.23 volts at the other end.

Note. - The drop along $a b$ (or $c d$ ), Fig. 164b, is equal to $\frac{\rho I}{X} \int_{x=0}^{x=x}(X-x) d x$, which is equal to $\pi / 2 \rho X I$, where $\rho X$ is the resistance of $a b$ (or $c d$ ) and $I$ is the total current delivered to the group of lamps. Compare Art. I 19, Chap. IX. Therefore the voltage at one end of the row is 115 volts minus the drop in $\left(60+-\frac{0}{2} 0\right)$ feet of the service wire, and the voltage at the other end of the row is 115 volts minus the drop in $\left(50+\frac{20}{2}\right)$ feet of the service wire; with full current of 50 amperes in each case.

158. All the lamps except the middle lamp in the row specified in problem 156 are turned off. Find the rise of voltage at the middle lamp. Ans. From I05 volts to I I 4.88 volts.

159. Find the size of wire required to supply the row of 100 lamps specified in problem 156 by the simple parallel scheme, both service wires being led from the service point to the nearer end of the row: $(a)$ when the drop between the service point and the most remote lamp is Io volts ; $(b)$ when the drop to the most remote lamp exceeds the drop to the nearest lamp by the amount (I09.23 - I05) volts; and (c) when the drop to the most remote lamp is 5 volts. Ans. (a) 16,200 circular mils sectional area ; (b) 25,600 circular mils; (c) 32,400 circular mils.

Note. - In case $(a)$ we have the same total drop as in problem 156 , but the voltage at the lamps ranges from 105 volts at the remote end to IIr.67 volts at the near end of the row, that is a range of 6.67 volts; whereas with the return loop scheme as specified in problem 156 the voltage at the lamps ranges from 105 at the middle lamp to a maximum of 109.23 volts, that is, a range of only 4.23 volts, and the wire in the return loop scheme is the smaller.

In case $(b)$ the voltage at the lamps has the same range as in problem 156 and the lamps therefore would operate equally well as in the return loop scheme as specified in problem 156, provided the lamps are all in use or all out of use, but the wire in case $(b)$ is nearly twice as heavy as in problem 156 . This shows in a striking way the saving of copper by the return loop scheme, for the same range of voltage among 
the lamps, when the group of lamps forms a nearly reëntrant row and when the lamps are always either all on or all off.

On the other hand, the result of problem 158 shows that the return loop as specified in problem 156 is not at all suited to the case in which part of the lamps in the group are turned off, the effect being to cause a very considerable rise of voltage at the remaining lamp (or lamps).

160. A group of 50 lamps each taking 1.0 ampere is to be installed in a church at a distance of one mile from a lighting station. It is understood that whenever any of the lamps are in use all are in use, so that the drop in the feeders which supply the lamps may have any value that economy demands. The lamps are to be operated for 300 hours each year. The cost of power at the station is 3.5 cents per kilowatt-hour, the cost of copper is 16 cents per pound, the annual charge on the cost of the wire is IO per cent. (interest 6 per cent., depreciation 3 per cent., and taxes I per cent.), and the station voltage is 125 volts. Find: (a) The size of the feeders to give a balance between loss of power and cost of copper, and $(b)$ the voltage at the lamps. Ans. (a) 76,370 circular mils; (b) 50.45 volts.

Note 1 . - The only objection to the application of the economic principle of the balance between loss of power and cost of copper to a case like the one here considered is that the voltage at the lamps may be very different from the voltage which prevails in the other parts of the lighting system, so that the station management would have to be careful to supply special lamps suited to the special voltage. It is evident that it is expensive at best to supply the fifty lamps at a distance of a mile, for, under the conditions of problem 160 , it requires $\$ 391.40$ worth of copper with a loss of \$39.14 worth of power each year, and to transmit the required power, 2.522 kilowatts (or $\mathbf{2 2 . 9 3}$ amperes with IIO volts at the lamps), with 15 volts drop would take $\$ 892$ worth of copper with a loss of $\$ 3.61$ worth of power each year.

Note 2. - It is instructive to solve problem 160 graphically as follows : Assume, say, 50,000,60,000, 70,000, 80,000, 90,000, and 100,000 circular mils. Use these sectional areas as abscissas of two curves, $A$ and $B$; the ordinates of curve $A$ representing the values in dollars of the power lost each year, and the ordinates of curve $B$, representing the annual charge in dollars on the total cost of the copper. Then plot a third curve, $C$, of which each ordinate is the sum of the corresponding ordinates of curves $A$ and $B$, and the abscissa corresponding to the minimum ordinate of this curve, $C$, is the required sectional area.

161. A consumer pays Io cents per kilowatt-hour not only for the energy he uses in his lamps but also for the energy that is lost in the wires that lead from the watt-hour meter to his lamps. 
If the customer uses his lamps 2 hours per day the year round, find the size of wires he should use in his house, in circular mils per ampere, for greatest economy, the cost of copper being I 6 cents per pound, and the interest and depreciation being 8 per cent. Ans. 4,507 circular mils per ampere.

162. The cost of power at the switch-board in an arc-lighting station is 2 cents per kilowatt-hour. The plant supplies a current of 6.6 amperes to a circuit of 50 arc lamps which are operated on a moonlight schedule for 2, 160 hours each year. The cost of copper is $2 \mathrm{I}$ cents per pound, and the annual charge on the cost of the wire is I I per cent. (interest 6 per cent., depreciation 3.5 per cent., and taxes I. 5 per cent.). The cost of the wire is high and its depreciation is large because the wire is insulated. The price of $2 \mathrm{I}$ cents per pound is on the net weight of copper in the wire and this price is intended to cover the cost of the insulation. Find the size of wire to give a balance between loss of power and cost of copper. Ans. I 7,040 circular mils.

Note. - The falling of an arc light wire into the street would be very dangerous on account of the high voltage. Therefore, it is important that an arc-lamp circuit be very substantial. It is usually not considered allowable for this reason, to use wire smaller than No. 6 Brown and Sharpe gauge $(26,000$ circular mils) for an arc-lamp circuit.

163. Find the cost to the station owners of 16.5 kilowatts of power delivered for 1,200 hours each year at 220 volts over a special line to a single customer at a distance of one mile from the station, the wire being of such size as to give a balance between loss of power and cost of copper. Determine the size of wire on the basis of a 6 per cent. annual charge on the cost of the copper at I 6 cents per pound, the cost of power at the switch-board being 2.5 cents per kilowatt-hour. Reckon the total cost of the line at 2.25 times the cost of the copper, and reckon the total annual cost of interest, depreciation, taxes, and maintenance of line at I 5 per cent. of the total cost of the line. Ans. $\$ 1,176.80$ per year, or about 6 cents per kilowatt-hour delivered.

164. Given ten groups of lamps, each group taking Io amperes, 
the groups being Io feet apart. The lamps are supplied with current from II 5-volt mains according to the wiring scheme shown in Fig. 168, Chap. IX. The end group, $b b$ (see figure), is $I 0$ feet from the mains, the point, $p p$, is 70 feet from the mains, and No. 2 Brown and Sharpe gauge copper wire is used throughout. Make a drawing like Fig. I 57b, Chap. IX., showing the voltage at every group of lamps. Sample answer: I.964 volts drop to the group of lamps which is 50 feet from the service point.

Note. - It would be permissible for practical purposes to calculate drops to the various lamps in this problem on the assumption that the lamps constitute a load which is distributed with ideal uniformity. It is here intended, however, that the drops be calculated as they actually are and so represented in the drawing.

165. The core of an insulated cable is a cylindrical rod of copper $\frac{3}{8}$ inch in diameter, the lead sheath is $1 \frac{1}{2}$ inches inside diameter, and the space between the core and the sheath is filled with rubber of which the electrical strength is, say, 120,000 volts per centimeter. Find the greatest voltage between sheath and core that the rubber insulation can sustain. Ans. 79, 130 volts.

I66. An electromotive force of 104,800 volts is applied very suddenly to two parallel wires 6 mils in diameter and 6 inches apart center to center. What is the diameter of the cylindrical region about each wire throughout which the electrical stress exceeds the electrical strength of the air, namely, 26,000 volts per centimeter? Ans. One-half an inch in diameter.

Note. - After the voltage has been applied and the air inside of the region onehalf an inch in diameter has broken down electrically, a thin cylindrical layer of air at a distance of $\frac{1}{4}$ inch from the wire is electrically charged, and the lines of force which pass outwards from this thin layer of air being in tension pull outwards on the layer and cause convection currents which very quickly alter the distribution of electric field between the wires.

167. The accompanying figure, Fig. I $67 P$, shows two motors, $M$ and $M^{\prime}$, two groups of glow lamps, $L$ and $L^{\prime}$, and a group of arc lamps, $A$, all supplied from the I 15 -volt service point, $P$. The distances are all so smail that the sizes of all wires are to be determined from the table of safe carrying capacities. The motor, $M$, takes 35 amperes, the motor, $M^{\prime}$, takes I 8 amperes, the group, $L$, contains 7 half-ampere lamps, the group, $L^{\prime}$, contains 3 halfampere lamps, and each arc lamp takes 5 amperes.

(a) Make a sketch of Fig. $167 P$ and indicate the values of the current at the points $a, b, c, d, e$ and $f ;(b)$ indicate the size of 
each wire assuming rubber insulated wire to be used; $(c)$ show the location and mark the current rating of every fusible cut-out and branch block required by the National Electrical Code.

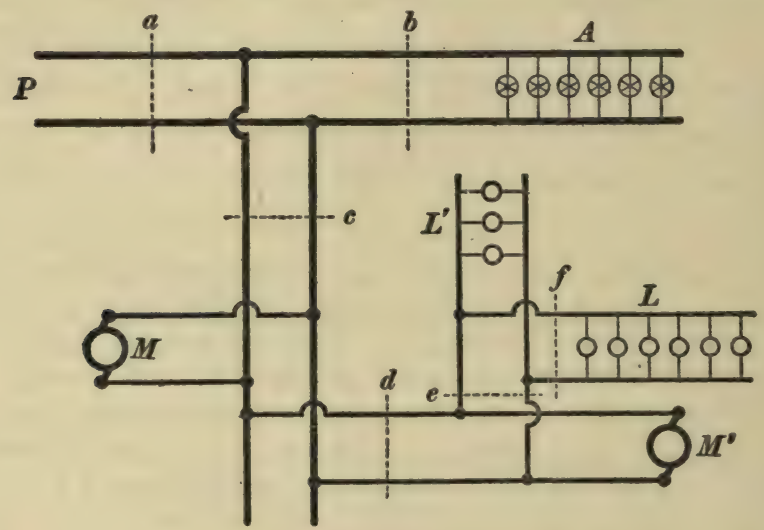

Fig. $167 P$.

Chapter X. Photometry and Electric Lighting.

168. The intensity of illumination at a distance of four feet from a I6-candle lamp is sufficient for easy reading of ordinary book type. (a) Find the distance from a 20-candle lamp at which the lamp gives the same intensity of illumination; (b) express this intensity of illumination in spherical-candles of light falling on each square foot of illuminated surface; and $(c)$ express this intensity of illumination in luxes. Ans. (a) 4.47 feet; (b) 0.0796 sphericalcandles per square foot; (c) I 2.2 I luxes.

Note. - One lux is the intensity of illumination produced by a Hefner lamp at a distance of one meter.

169. The glow lamp which is used as a standard in a Bunsen photometer has a candle power of 16.8 candles in the direction towards the photometer screen. Another lamp, $B$, is placed at the other end of the photometer bar; and when the screen is adjusted - to equality of illumination on both sides, it is 2.6I meters from the lamp, $B$, and I.80 meters from the standard lamp. What is 
the candle power of $B$ in the direction towards the screen? Ans. 35.3 candle power.

170. The lamp, $B$, of problem $\mathrm{I} 69$ is placed at a distance of 0.70 meter from the center of a large mirror which reflects the light from $B$ along the photometer bar towards the photometer screen ; and when the screen is again adjusted to equality of illumination on both sides, it is 1.85 meters from the standard lamp and 1.82 meters from the center of the mirror. The lamp, $B$, presents towards the mirror in this case the same face that was presented towards the screen in problem 169 . Find the factor by which the apparent candle power of any lamp, when measured by the light reflected from the above mirror, must be multiplied in order to correct for the loss of light at the mirror. Ans. I. I 3 .

171. A beam of light consisting of parallel rays has a sectional intensity of 300 luxes. Find the conical intensity of the beam after it passes through a lens of which the focal length is 50 centimeters. Ans. 75 hefners.

Note. - The sectional intensity of the conical beam of light at the lens is the same as the sectional intensity of the given beam, namely, 300 luxes, that is 300 times as great as the sectional intensity of the light at a distance of roo centimeters from a Hefner lamp, or 75 times as great as the sectional intensity at a distance of 50 centimeters from a Hefner lamp. Therefore the conical intensity of the convergent beam is 75 hefners.

It is perhaps more intelligible to state the solution as follows : Let us take as the unit of light flux the amoun. of light in one square centimeter of a beam of which the sectional intensity is one lux. This unit of light is one lux-cm. ${ }^{2}$ Consider one square centimeter of the surface of the lens. The cone defined by lines drawn from the periphery of this portion of the lens to the focus has a solid angle of $\mathrm{I} \mathrm{cm} .^{2}$ divided by $(50 \mathrm{~cm} \text {. })^{2}$ which is equal to $\frac{1}{2500}$. This cone contains 300 lux $-\mathrm{cm} . .^{2}$ of light so that the conical intensity of the conical beam is 300 lux $-\mathrm{cm} .{ }^{2}$ divided by $\frac{1}{2} 5_{00}$ which is equal to 750,000 lux $-\mathrm{cm} .{ }^{2}$, and it only remains to reduce this to hefners To this end consider one square centimeter of area at a distance of 100 centimeters from a Hefner lamp. The amount of light passing through this area is one lux $-\mathrm{cm} .^{\mathrm{s}}$ and the solid angle subtended by the area is $\frac{1}{10} \sigma 00$, so that one hefner is equal to Io, 000 lux $-\mathrm{cm}^{2}$.

Solid angle is measured by a pure ratio, area divided by radius squared; and therefore light flux and conical intensity of light have the same physical dimensions, and both may be expressed in lux-cm. ${ }^{2}$ 
172. An open-arc lamp is placed at a distance of five feet from a converging lens and an image of the arc is formed at a distance of one foot beyond the lens. The light from the lamp has a conical intensity of 2,500 candles. Assuming that the luminous surface of the lamp is negligibly small, and ignoring loss of light at the lens by reflection and absorption, find the conical intensity of the beam beyond the image. Ans. I00 candles.

Note. - Consider the cone $A$ formed by lines drawn from the arc to the periphery of the lens, the cone $B$ formed by lines drawn from the image to the periphery of the lens, and the cone $a$ formed by lines drawn from the center of the lens to the periphery of the luminous surface of the lamp. The size of the luminous area of the lamp is negligibly small when the solid angle of the cone $a$ is negligible in comparison with the solid angle of the cone $B$.

All of the light in cone $A$ is in cone $B$ after it passes through the lens, so that the conical intensities of the light in cones $A$ and $B$ are inversely as the solid angles of these cones, and these solid angles are inversely proportional to the squares of the distances of the lens from the lamp and from the image, respectively.

173. Calculate the spherical-candle-power of a bare glow lamp from the data given in Fig. 175, Chap. X. Ans. I 3.33 sphericalcandles.

174. Calculate from the data given in Fig. I76, Chap. X., the spherical-candle-power of the glow lamp of problem 173 when it is provided with an aluminum cone shade, and express the amount of light absorbed by the shade as a percentage of the spherical-candle power of the bare lamp. Ans. 8.26 spherical candles; 38 per cent. of the light is lost.

175. (a) How many I 6 candle-power lamps are required to illuminate a lecture hall $50 \times 75$ feet with a moderately high ceiling? (b) How many I6 candle-power lamps would be required to give exactly the same average intensity of illumination in a room twice as long, twice as wide and twice as high with the same kind of walls and ceiling and the same kind of furniture? Why? (c) The mean coefficient of absorption of walls, ceiling and furniture in the room specified under $a$ is 40 per cent. How many I6-candle-power lamps would be required to give the same illumination in a room of the same size in which the mean coefficient of absorption of walls, ceiling and furniture is 55 per cent? 
Ans. (a) 47 lamps; (b) four times as many, because the area of the light-absorbing surfaces in the larger room is four times as great as in the smaller room; (c) 65 lamps.

Note. - To specify the number of lamps required for illuminating a room as so many per square foot of floor area, is to assume that the wall area is proportional to the floor area, absorption coefficient being fixed in value.

176. A direct-current arc lamp gives the following distribution of light:

Angle from vertical, $10^{\circ} 20^{\circ} 30^{\circ} 40^{\circ} \quad 50^{\circ} \quad 60^{\circ} \quad 70^{\circ} \quad 80^{\circ}$ Candle-power, $\quad 290 \quad 440670108012201080795 \quad 580$

Calculate the intensities of illumination at points along a level open street distant $h \tan 10^{\circ}, h \tan 20^{\circ}, h \tan 30^{\circ}, h \tan 40^{\circ}$, etc., horizontally from the lamp : $(a)$ when the height, $h$, of the lamp above the street is 15 feet, and $(b)$ when the height, $h$, of the lamp above the street is 50 feet.

Express the intensities of illumination in "candle-feet," that is in terms of the intensity of illumination produced by the beam from a standard candle falling perpendicularly upon a screen at a distance of one foot from the candle.

Plot two curves showing horizontal distances from the lamp as abscissas and intensities of illumination as ordinates. Sample answer. Intensity of illumination of the surface of the street at a distance of 26 feet horizontally from the lamp is 0.600 "candlefeet" when the lamp is I 5 feet above the ground.

Note. - The distance, $d$, in feet of one of the points on the street from the lamp is equal to $\sqrt{h^{2}+h^{2} \tan ^{2} \theta}$ where $\theta$ is one of the angles given in the problem. The sectional intensity of any one of the beams above specified, at distance $d$ feet from the lamp is equal to the candle-power of the beam divided by $d^{2}$, this result being expressed in "candle-feet." Furthermore, one unit sectional area of this beam at the given point on the street is spread over $\mathbf{I} / \cos \theta$ units of area of ground, so that the intensity of illumination of the surface of the street at the given point is $\cos \theta$ times the sectional intensity (in "candle-feet") of the given beam at distance $d$ feet from the lamp.

177. Draw a tangent to one of the volt-ampere arc characteristics in Fig. 182, Chap. X., and let $e$ volts be the intercept on the volt-axis and $i$ amperes the intercept on the ampere-axis, of this tangent. Let $E$ volts and $I$ amperes be the coördinates of the 
point of tangency. Show that $e / i$ is the least resistance which will cause the arc to be stable with a current of $I$ amperes and an electromotive force of $E$ volts across the arc.

Note. - Plot the combined volt-ampere characteristic of the arc and the resistance $e / i$ in series, by adding to each ordinate of the given volt-ampere characteristic $e / i$ times the corresponding abscissa. This combined volt-ampere characteristic will show a decreasing total voltage with increasing current (unstable) when the current is less than $I$, it will be horizontal at the point corresponding to $I$, and it will show an increasing total voltage with increasing current (stable) when the current is greater than $I$.

178. When the cost of electric power is Io cents per kilowatthour, what is the total cost of 1,000 candle-hours delivered by a carbon-filament glow lamp costing I 8 cents, giving an average of 15 candles during its life of 600 hours, and consuming an average of 54 watts? Ans. 38 cents.

179. At what price could an osmium-filament glow lamp be sold to make the total cost of light io per cent. less than by the use of the carbon-filament lamp specified in problem 178 , the osmium lamp giving an average of $15 \mathrm{I} / 2$ candles during a life of I, 500 hours, and consuming an average of 24 watts ? Ans. $\$ 4.35$.

180. An enclosed arc lamp costing I 5 dollars is used 3,500 hours each year for street lighting. The depreciation of the lamp is, say, $3 \frac{1}{2}$ dollars per year, including interest on its cost. The lamp is trimmed and cleaned thirty-five times per year at a cost of $\mathrm{I} 3 / 4$ dollars. The lamp consumes 500 watts and delivers 225 spherical-candles of light. Find the total cost of I,000 sphericalcandle-hours, the cost of power being ro cents per kilowatt-hour. Ans. 22.9 cents.

Note. - Power for street arc lighting should be reckoned at a cost very much less than ro cents per kilowatt-hour on account of the fact that the lights are operated steadily for a long time each day. The cost of ro cents per kilowatt-hour is here specified in order that the result of problem 180 may be compared intelligently with the result of problem 178 . It is to be noted furthermore that 1,000 candle-hours from a glow lamp means at present I, 000 mean-horizontal candle-hours, not 1,000 spherical-candle-hours. The latter is about 15 per cent. less than the former, so that the result of problem 178 should be divided by 0.85 to make it strictly comparable with the result of problem 180 .

Even though the cost per spherical-candle-hour be considerably less by the use of an enclosed arc lamp than by the use of a glow lamp, the latter may be really cheaper 
on account of the fact that a portion of a street, for example, may be much better lighted by a number of distributed glow lamps than by one arc lamp even though the glow lamps aggregate much less spherical-candle power than the arc lamp.

181. A street is to be lighted for 3,600 hours each year. The street has trees along it so that satisfactory illumination requires, either, $(a)$ one 225-candle-power 364-watt enclosed arc lamp every 250 feet, costing $\$ 5.25$ per year for interest, depreciation, cleaning and trimming, or $(b)$ three 32 -candle-power I I 2-watt glow lamps every 250 feet, each lasting 600 hours, and costing 25 cents, including the cost of replacing when burned out. The price paid for power is 3 cents per kilowatt-hour. What is the total annual cost of lighting 250 feet of the street: $(a)$ By enclosed arc lamps, and $(b)$ by glow lamps. Ans. $(a) \$ 44.56$; $(b)$ $\$ 40.79$.

Note 1 . - The arc lamp consumes less power here than is specified in problem 180 inasmuch as street arcs are usually connected in series and operated by a constantcurrent generator (or transformer) so that there is no ballast resistance in the lamps.

Note 2. - It is very important to consider that a special generator (or transformer) must be used to supply series-connected arc lamps, whereas glow lamps can be operated from the same generator that supplies current for glow lamps for house lighting. Therefore, the cost of operating series-connected arcs is rather more than $\$ 44.56$ each per year, as compared with $\$ 40.79$ for three 32 -candle-power glow lamps, on account of the interest on the cost and the depreciation of the special generator (or transformer) required in the former case.

\section{Appendix A. Magnetism of Iron.}

182. The intensity of the magnetic field in the air gap between the pole face and the armature core of a dynamo is 3,500 gausses and the field is at right angles to pole face and armature surface. The distance across the air gap is $3 / 8$ inch. Find the magnetomotive force across the air gap in gilberts and in ampere-turns. Ans. 3,333.5 gilberts, or 2,650 ampere-turns.

183. Find the magnetomotive force in gilberts and in ampereturns along a vertical line io meters long at a place where the intensity of the earth's magnetic field is 0.56 gauss and its dip is $72^{\circ}$. Ans. 533 gilberts or 424 ampere-turns. 
184. A slim rod 25 centimeters long is made into a link which passes through a coil of 50 turns of wire in which a current of I 5 amperes is flowing. Find the average value along the rod of the component parallel to the rod of the magnetic field due to the coil. Express the result in gausses. Ans. 37.7 gausses.

185. Reduce a field intensity of 25 ampere-turns per inch to gausses. Ans. 12.37 gausses.

186. Show by dotted lines the various magnetic circuits in Figs. 32 to 39 of Chap. II. Let $\mathcal{F}$ represent the magnetomotive force of a single field coil in each figure. What is the magnetomotive force acting on each magnetic circuit? Ans. $2 \mathscr{F}$ in Fig. $32 ; 2 \mathscr{F}$ in Fig. 33 ; $\mathcal{F}$ in Fig. $34 ; \mathscr{F}$ in Fig. $35 ; 2 \mathscr{F}$ in Fig. $36 ; 2 \mathscr{F}$ in Fig. $37 ; \mathcal{F}$ in Fig. 38 ; and $2 \mathscr{F}$ in Fig. 39.

187. An iron rod $2 \times 2$ centimeters square and 20 centimeters long is magnetized to an intensity of $\mathrm{I}, 000$ units pole per square centimeter section when it is placed in a region which, but for the action of the poles of the rod, would be a uniform field parallel to the rod and of an intensity of 102 gausses. Assuming the poles of the rod to be concentrated at its ends calculate the net magnetizing field at the center of the rod. Ans. 22 gausses.

188. Find the total magnetic flux through the middle part of the iron rod specified in problem 187. Ans. 50,353.6 maxwells or lines.

Note. - One part of the flux is $4 \pi m$ and the other part is $\mathscr{H} s$, where $\mathscr{H}$ is the net magnetizing field at the middle of the rod.

189. A bar magnet of hard steel is $2 \times 2$ centimeters square and 20 centimeters long, and the strength of each pole of the magnet is 2,000 units. The magnet is placed in a region which, but for the presence of the magnet, would not be a magnetic field. Find the total magnetic flux through the middle part of the magnet. Ans. 24,972.8 maxwells.

Note. - In this case the net magnetizing force, $\mathscr{H}$, at the middle of the bar is a demagnetizing force, and the flux due to $\mathscr{H}$ is subtracted from $4 \pi \mathrm{m}$.

190. A transformer has a sheet iron core of which the uniform sectional area is 120 square centimeters. The mean length of 
the magnetic circuit formed by the core is Ioo centimeters. How much current must be passed through a winding of 500 turns of wire to produce a magnetic flux of $1,767,000$ lines through the core. Ans. 3.88 amperes.

Note. - The student should plot carefully the $\mathscr{B}$ and $\mathscr{H} \mathcal{H}$ curves for wrought iron, cast iron, and soft steel from the tables given in Art. 12, Appendix A, and use these curves in finding corresponding values of $\mathscr{B}$ and $\mathscr{H}$ in this and the following problems.

191. How much flux will be produced through the wrought iron core specified in problem 190 by a current of 8.2 amperes through the 500 turns of wire? Ans. 1,920,000 lines.

192. The magnetic circuit of a dynamo consists of wrought iron (in armature core and in field cores), cast iron (in field yoke and pole pieces), and air gap. The wrought iron portion is 50 centimeters long and $\mathrm{I} 2 \mathrm{O}$ square centimeters in sectional area, the cast iron portion is 40 centimeters long and 220 square centimeters in sectional area, and the air portion is 2.5 centimeters long and 300 square centimeters in sectional area. How many ampere-turns are required to force $1,600,000$ lines of flux through this circuit, ignoring magnetic leakage? Ans. I 2, 100 ampereturns.

Note. - On account of magnetic leakage the magnetic flux through field yoke, field cores and pole pieces is larger, sometimes very much larger, than the flux which flows across the air gaps and through the armature core. Therefore a slightly greater magnetomotive force is required to overcome the magnetic reluctance of field yoke, field cores and pole pieces than would be required if the magnetic leakage were zero. Magnetic leakage is never really negligible, but it can be satisfactorily determined only by tests on a finished machine.

193. How much magnetic flux would be forced through the magnetic circuit specified in problem 192 by a magnetomotive force of 13,500 ampere-turns. Ans. 1,723,000 lines.

Note. - This problem is to be solved by calculating a series of values of ampereturns for various assigned values of flux. These results are to be plotted. The flux corresponding to 13,500 ampere-turns may then be taken from this curve.

194. A straight wrought iron rod 2 inches in diameter and 24 inches long is wound with 1,000 turns of wire. Calculate approximately the current required to force 203 , 100 lines of flux through the rod and back through the air. Ans. IO.I amperes. 
Note - The magnetic reluctance of the air between two similar iron spheres, $r$ centimeters.in radius and $d$ centimeters apart, center to center, is equal to $(d-r) / 2 \pi d r$, when $d$ is large compared with $r$. In this case $r$ may be dropped from the numerator as negligible and we have $I / 2 \pi r$ as the magnetic reluctance of the air between two iron spheres, each $r$ centimeters in radius.

In the above problem the poles of the iron rod may be taken to be two iron spheres 2 inches in diameter at a distance apart which is large compared with 2 inches. The magnetic reluctance of the air return path is therefore approximately equal to 0.0627 oersted $(=\mathbf{I} / 2 \pi r)$. The magnetic reluctance of the iron part of the magnetic circuit in this problem is about 0.002 oersted and it is entirely negligible, inasmuch as the larger reluctance of the air part of the circuit is calculated by an approximate formula.

195. The magnetic reluctance of the two air gaps of a large bipolar generator is 0.0006 oersted, the reluctance of the armature core is 0.00005 oersted, the reluctance of the leakage paths through the air from pole-piece to pole-piece is 0.0022 oersted, and the useful flux through the armature is $6,000,000$ maxwells. Find: (a) the magnetomotive force between the pole-pieces in ampere-turns; (b) the magnetomotive force in ampere-turns across the two air gaps; $(c)$ the magnetomotive force in ampereturns across the armature core; $(d)$ the leakage flux; $(e)$ the total field flux; $(f)$ the magnetic leakage coefficient of the machine; and $(g)$ the total magnetic reluctance from pole-piece to pole-piece. Ans. (a) 3, I IO ampere-turns; (b) 2,870.8 ampere-turns; (c) 239.2 ampere-turns; $(d)$ I,773,000 maxwells; (e) 7,773,000 maxwells; $(f)$ 1.295; $(g) 0.000502$ oersted.

196. A 6-pole generator with a full load rating of 275 kilowatts at 550 volts has a simplex wave armature winding ( 2 paths through the armature); the armature has I,200 conductors and the commutator has 300 segments. At full load the brushes have a forward lead of four commutator segments. Find the number of series turns required on each field core $C$ (when the field windings are arranged like Fig. 33, Chap. II.) to counteract the demagnetizing action of the armature at full load. Ans. 8 turns.

Note. - The number of ampere-turns required on the field per magnetic circuit to balance $D$ demagnetizing ampere-turns on the armature per magnetic circuit is equal to

$$
D\left(\mathbf{I}+\frac{\text { field reluctance }}{\text { leakage reluctance }}\right)
$$


where field reluctance means the total magnetic reluctance of yoke, magnet cores, and pole-pieces and leakage reluctance means the total magnetic reluctance of the air paths between two adjacent pole-pieces. The field reluctance is generally a small fraction of the leakage reluctance so that the ratio of the two may be neglected in the above expression.

197. (a) Find the work in ergs and in foot-pounds spent in magnetizing a wrought iron bar 3 inches $\times 3$ inches $\times 20$ inches long from a neutral condition to $\mathscr{B}=16,000$ lines per square centimeter, using the tabular values of $\mathscr{B}$ and $\mathscr{H}$ given in Art. I2, Appendix A. (b) Find the work in ergs and in foot-pounds required to magnetize a cast-iron rod of the same size from a neutral condition to $\mathfrak{B}=9,000$ lines per square centimeter. Ans. (a) $32.6 \times 10^{6} \mathrm{ergs}$ or 2.406 foot-pounds; (b) $33.9 \times 10^{6} \mathrm{ergs}$ or 2.50 foot-pounds.

Note. - Plot the $\mathscr{B}$ and $\mathscr{H}$ curves from the tabular values given in Art. 12, Appendix A. Divide the areas between the curves and the $\mathscr{B}$ axis into a number of parallelograms. Calculate the area of each parallelogram and thus find the total area.

198. A transformer core contains 96 cubic inches of the best quality of transformer iron and the laminations, which are thoroughly insulated from each other, are o.or4 inch thick. The core, is carried through 133 magnetic cycles per second between the limits $\mathscr{B}= \pm 3,500$ gausses, by means of an alternating current. Find the hysteresis loss and the eddy current loss in the core both in watts. Ans. Hysteresis loss 14.7 watts; Eddy current loss 6.9 watts.

199. The hysteresis loop (Fig. I7, Appendix A), of a sample of iron for a magnetic cycle having the limits $\mathfrak{B}= \pm$ I 2,500 lines per square centimeter, has an area of 9.52 square inches. In plotting the loop each inch of ordinate represents 2,000 units of $\mathscr{B}$ and each inch of abscissa represents 5 units of $\mathscr{H}$. Find the energy lost in 25 cubic centimeters of the iron during the magnetic cycle. Ans. 189,400 ergs.

200. The electromagnet shown in Fig. I 2, Appendix A, has the following dimensions. The cylindrical cores, $C C$, are of annealed wrought iron 2 inches in diameter, 8 inches long, and 7 inches apart from center to center. The yoke, $Y Y$, and the 
armature, $A A$, are also of wrought iton, $2 \times 2$ inches square, and the air gap between the armature and the ends of the cores is $\frac{1}{16}$ inch. Find the total number of ampere-turns required to give a pull of 9345 pounds on the armature. Ans. 6, I75 ampereturns.

Note. - Neglect magnetic leakage and reluctance of joints between cores and yoke. Assume length of magnetic path in yoke and in armature each equal to 9 inches.

\section{Appendix B. Characteristic Curves.}

201. Given the following data, specifying the external characteristic of a series generator :

$\begin{array}{ccccccccc}E_{x} & 65 . \mathrm{I} & \text { I21 O } & \text { I38.0 } & \text { I 50.0 } & \text { I 58.3 } & \text { I63.5 } & \text { I64.3 } & \text { volts } \\ I & 12 & 24 & 30 & 36 & 42 & 48 & 54 & \text { amp }\end{array}$

The combined resistance of armature and field winding is 055 ohm. Plot the external characteristic from the above data, and derive and plot the total characteristic, ignoring the demagnetizing action of the current in the armature. Ans. Coördinates of one point $E_{a}=\mathrm{I} 54.5$ volts, $\mathrm{I}_{a}=30.0$ amperes.

202. The speed of the above generator is doubled; derive and plot the altered external characteristic, and the altered total characteristic. Ans. Coördinates of one point, $E_{\alpha}=292.5$ volts, $I_{x}=30.0$ amperes.

203. The field of the series generator of problem $20 \mathrm{I}$ is wound with 0.8 as many turns of wire as in problem 201 , the wire being smaller in size so as to have the same resistance. Derive the data for the external characteristic of the new machine from the data of problem 20I, ignoring the demagnetizing action of the current in the armature, and plot the new external characteristic. Ans. Coördinates of one point, $E_{x}=\mathrm{I} 33.87$ volts, $I_{x}=37.5$ amperes.

204. Derive the data for the external ampere-ohm characteristic of the series generator of problem $20 \mathrm{I}$, and plot the external ampere-ohm characteristic of the machine. Ans. Coördinates of one point, $I_{x}=30$ amperes, $R_{x}=4.6 \mathrm{ohms}$. 
205. Given the following data specifying the external characteristic of a shunt generator :

$\begin{array}{ccccccccr}E_{x} & 216 & 207 & 197 & \text { I } 87 & \text { 175 } & \text { 161 } & \text { 146 } & \text { volts. } \\ I_{x} & 0 & 4 & 8 & \text { 12 } & \text { 16 } & 20 & 24 & \text { amp. }\end{array}$

The resistance of the armature is $0.4 \mathrm{ohm}$ and the resistance of the shunt field winding is I 6.7 ohms. Plot the external characteristic from the above data, and derive and plot the total characteristic, ignoring the demagnetizing action of the current in the armature. Ans. Coördinates of one point, $E_{o}=182$ volts, $I_{a}=17.5$ amperes.

206. Derive the data for the external ampere-ohm characteristic of the shunt generator of problem 205 and plot the external ampere-ohm characteristic of the machine. Ans. Coördinates of one point, $I_{x}=16$ amperes, $R_{x}=10.94 \mathrm{ohms}$.

207. Given the following data for the magnetization curve of a shunt generator at a speed of $\mathrm{I}, 200$ revolutions per minute:

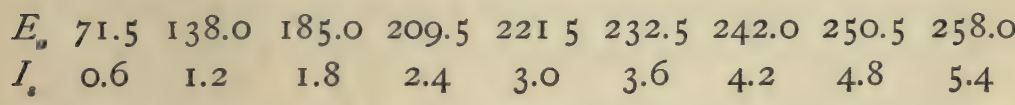

Plot this magnetization curve and derive from it the voltage-speed characteristic of the machine at zero load, the resistance of the shunt field winding being $80 \mathrm{ohms.} \mathrm{Armature} \mathrm{resistance} \mathrm{ignored.}$ Ans. Coördinates of one point, 430 volts at 2,000 revolutions per minute.

208. Derive the total characteristic of the shunt generator of problem 207 from its magnetization curve, the resistance of the armature being $0.15 \mathrm{ohm}$ Ignore the demagnetizing action of the current in the armature. Ans. Coördinates of one point, $E_{a}=209.2$ volts, $I_{a}=\mathrm{I} 14.7$ amperes.

209. (a) A short-shunt compound generator, rated as a IIOkilowatt machine, is Io per cent. overcompounded. That is, it gives between its terminals 500 volts at zero load and 550 volts at full load. Plot the external characteristic of the machine, assuming it to be a straight line between zero load and full load. 
(b) Derive and plot the total characteristic of the machine, the resistances of armature, shunt field winding and series field winding being $0.09 \mathrm{ohm}, \mathrm{I} 00 \mathrm{ohms}$, and $0.04 \mathrm{ohm}$, respectively. Ans. Coördinate of one point, $E_{a}=576.5$ volts, $I_{a}=205.58$ amperes.

210. Given the following data specifying the armature characteristic of a shunt generator, the voltage between the brushes being kept at 220 volts.

$\begin{array}{cccccccc}I_{a} & 2.7 & 20 & 40 & 60 & 80 & \text { 100 } & \text { 1 20 amp. } \\ I . & 2.7 & 2.84 & 3.05 & 3.28 & 3.57 & 3.9 & 4.26\end{array}$

(a) Plot this curve and also plot the curve between $I_{x}$ and $I_{8^{\circ}}$. The shunt field winding of the machine has 2,000 turns. (b) Find the number of turns of wire required in a series field winding (shortshunt connection) to bring the voltage between the brushes to 220 volts when $I_{x}$ is 100 amperes. (c) What fraction of the 100 amperes would have to be shunted round the series winding to give exactly 220 volts between the brushes when $I_{x}$ is 100 amperes? (d) What fractional part of $I_{x}$ would have to be shunted round the series winding to give exactly 220 volts between the brushes when $I_{x}$ is 50 amperes? Ans. (b) 26 turns; (c) 2.3 per cent. ; (d) 22.9 per cent.

211. The generator specified in problem 209 requires a total of 6,500 ampere-turns to give 500 volts at zero load, and a total of 9,750 ampere-turns to give 550 volts at full load $\left(I_{x}=200\right.$ amperes). How many turns of wire are there in the series field winding? Ans. 13 turns.

212. The curve in Fig. 49, Appendix B, gives the relation between speed-of-car and amperes-per-motor when the two motors on the car are connected in parallel between the 500-volt trolley wire and the track with rheostat resistance all cut out. Derive from this curve the speed-ampere curve of the two motors when they are connected in series to the 500-volt supply, the resistance of each motor being $0.50 \mathrm{ohm}$. Ans. Coördinates of one point, speed 6.85 miles per hour, current 80 amperes.

Note. - For any given current $i$ in a motor the $R i$ drop in the motor is $0.5 \times i$, and the $R i$ drop in the two motors connected in series is $2 \times 0.5 \times i$. Therefore 
the counter electromotive force of one motor in Fig. 49, Appendix B, is 500 volts minus $0.5 \times i$ and the counter electromotive force of the two motors when they are connected in series is 500 minus $2 \times 0.5 \times i$. Hence for given current $i$ in each motor the counter electromotive force per motor is 500 volts $-0.5 \times i$ when the motors are in parallel and $1 / 2(500$ volts $-2 \times 0.5 \times i)$ when the motors are in series; and the speeds corresponding to the given current $i$ are to each other as these counter electromotive forces.

213. The curve in Fig. 50, Appendix B, gives the relation between the tractive effort developed by a single motor and the current in the motor. Derive the curve showing the relation between the total tractive effort and the total current taken from the trolley wire for two motors : $(a)$ connected in parallel, and $(b)$ connected in series. Ans. Coördinates of one point : $(a)$ tractive effort 2,250 pounds, current 160 amperes; $(b)$ tractive effort 2,250 pounds, current 80 amperes.

Note. - To make these transformations it is only necessary to remember that a certain current in a motor means so much tractive effort.

214. The curves derived in problems 212 and 213 for two motors connected in series apply to the two motors on a fifteenton car, with 500 volts between trolley and rail, and with no resistance in series with the motors. Under these conditions the car travels at a speed of 6.85 miles per hour up a 6.25 per cent. grade. Find $(a)$ The current taken from the trolley; $(b)$ the total tractive effort developed; $(c)$ the total power delivered to the car ; $(d)$ the net power represented by the total tractive effort at the given speed; and $(e)$ the efficiency of the car equipment under the specified conditions. Ans. (a) 80 amperes; (b) 2,250 pounds; $(c) 40$ kilowatts; $(d) 30.65$ kilowatts; $(e) 76.6$ per cent.

Note. - See explanation of example in Art. $3^{8}$ of Appendix B.

215. The magnet core of a 4-pole shunt dynamo has a diameter of 8 inches, including thickness of brass bobbin plus inside insulation. The bobbin is wound with wire to a depth of $I \frac{x}{2}$ inches. The terminal voltage of the machine is I IO volts, of which 20 per cent. is to be absorbed in the field rheostat. Two thousand ampere-turns are to be provided by each of the four 
spools, which are to be connected in series as usual. The temperature of the winding in service is $60^{\circ} \mathrm{C}$. Find the size of wire required. Ans. 2,7 10 circular mils.

Note. - The ampere-turns in a shunt field winding are equal to the quotient of the voltage between the terminals of the winding divided by the resistance of a mean turn of the wire. Of course, a commercial size of wire would have to be used in winding the above coils and the portion of the voltage absorbed in the field rheostat would be slightly different from 20 per cent.

216. The length of winding space on each of the bobbins of problem $2 \mathrm{I} 5$ is 3 inches. Find: (a) Resistance at $60^{\circ} \mathrm{C}$. of the four bobbins in series when wound with No. 16 Brown and Sharp gauge double cotton-covered copper wire (outside diameter of wire 0.063 inch; assume no bedding of the layers); (b) find watts dissipated in each bobbin using resistance at $60^{\circ} \mathrm{C}$. ; and $(c)$ calculate the approximate rise of temperature of the bobbins above the air. Ans. (a) $51.6 \mathrm{ohms}$; (b) 40.6 watts ; (c) $31.4{ }^{\circ} \mathrm{C}$. rise of temperature.

Note. - Dynamo designers estimate the rise in temperature of a field winding by the formula

$$
t=\frac{8 \mathrm{o} P}{A}
$$

in which $t$ is the estimated rise of temperature of the winding above the air in centigrade degrees, $P$ is the power in watts dissipated in the winding, and $A$ is the area in square inches of the cylindrical surface of the winding. This formula is only roughly approximate, and it is to be applied only to medium-sized field spools with a depth of winding of from 0.75 inch to 2 inches.

217. A certain 200-volt, 50-kilowatt, 4-pole shunt generator has its four field spools connected in series and it is designed to run at speed of 500 revolutions per minute. This machine is to be driven at a speed of 250 revolutions per minute and to develop Ioo volts. (a) What change is necessary in the arrangement of the field winding? (b) What is the current capacity of the machine at the reduced speed? (c) What is the power rating of the machine at the reduced speed? Ans. (a) The four field spools are arranged two in series by two in parallel ; $(b) 250$ amperes; (c) 25 kilowatts. 


\section{Appendix C. Armature Windings.}

218. Draw a 2-pole drum armature winding having 18 inductors; front pitch 7 , back pitch 9, showing connections to commutator bars. Make the armature circle 4 inches in diameter. Indicate back connections by dotted lines and front connections by full lines, as shown in Fig. 54, Appendix C.

219. Draw a simplex lap winding for a 4-pole drum armature having I 8 inductors; front pitch 5 , back pitch 3 . Show connections to commutator bars and indicate positions of poles and brushes. Make the drawing same size and style as Fig. 56, Appendix C.

220. Draw a simplex wave winding for a 4-pole drum armature having 18 inductors; front pitch 5, back pitch 3. Show connections to commutator bars and indicate positions of poles and brushes. Make the drawing same size and style as Fig. 57, Appendix C.

221. Draw a doubly reëntrant duplex lap winding for a 4-pole drum armature having 36 inductors ; front pitch 10, back pitch 6 . Show connections to commutator bars and indicate positions of poles and brushes. Make the drawing same size and style as Fig. 58, Appendix C.

Note. - A duplex lap or wave winding must have both front and back pitches even, when all even numbered inductors belong to the one and all odd numbered inductors belong to the other of the two constituent simplex windings.

222. Draw a doubly reëntrant duplex wave winding for a 4-pole drum armature having 36 inductors; front pitch Io and back pitch 6. Show connections to commutator bars and indicate positions of poles and brushes. Make the drawing same size and style as Fig. 59, Appendix C.

223. Draw a singly reëntrant duplex lap winding for a 4-pole drum armature having 34 inductors; front pitch 7 and back pitch I I. Show connections to commutator bars and indicate positions of poles and brushes. Make drawing same size and style as Fig. 6o, Appendix C. 
Note. - In a singly reêntrant duplex winding, lap or wave, both front and back pitches must be odd.

224. Draw a singly reëntrant duplex wave winding for a 4-pole drum armature having 32 inductors; front pitch 9 and back pitch 9. Show connections to commutator bars and indicate positions of poles and brushes. Make the drawing same size and style as Fig. 60, Appendix C.

225. Given a 4-pole drum armature which is to be wound with 20 inductors. (a) Can it be wound with a lap winding? Can it be wound with a wave winding? (c) State what windings are possible when the number of inductors is taken as 22 and 24 respectively, and give front and back pitches for each case.

226. Given a 6-pole drum armature having 40 inductors arranged as a duplex wave winding. What are the front and back pitches and what is the degree of reëntrancy?

227. Given a 6-pole drum armature having 52 inductors. State the possible varieties of winding, and give front, back and commutator pitches, and degree of reëntrancy in each case.

228. Given a 6-pole drum armature having 50 inductors. State the possible varieties of winding, and give front, back, and commutator pitches, and degree of reëntrancy in each case.

229 A 4-pole drum armature is to have from 46 to 50 inductors, the exact number being chosen so that they may be arranged as a singly reëntrant duplex wave winding having onefourth as many commutator bars as inductors. What number of inductors is necessary? Make a drawing somewhat similar to Fig. 62, Appendix C, showing the winding and showing the positions of the poles and brushes.

230. A drum armature is to have 500 inductors and it is to be driven at a speed of 600 revolutions per minute.

(I) The number of poles is 6 and the useful flux per pole is $4.8 \times 10^{6}$ lines. Find electromotive force generated: $(a)$ when the inductors are arranged as a simplex lap winding; $(b)$ when the inductors are arranged as a duplex wave winding. Ans. (a) 240 volts ; (b) 360 volts. 
(2) The number of poles is 8 and the useful flux per pole is $3.6 \times 10^{6}$. Find the electromotive force generated: $(a)$ when the inductors are arranged as a simplex lap winding; $(b)$ when the inductors are arranged as a duplex lap winding. Ans. (a) I 80 volts ; (b) 90 volts.

231. In order to generate the desired electromotive force in a 6-pole drum armature it is decided to use about 670 inductors and arrange them as a singly reëntrant triplex wave winding. (a) What is the exact number of inductors required; and (b) what are the front and back pitches?

232. A 4-pole simplex lap drum winding has 576 inductors. The diameter of the bare wire is $0.08 \mathrm{I}$ inch, and the mean length of one "armature turn" (consisting of two inductors and two end connectors) is 16 inches. Find the resistance (cold) of the winding from positive to negative brushes. Ans. $0.0395 \mathrm{ohm}$.

233. An 8-pole drum armature has a duplex lap winding with r,258 inductors. Find: (a) front, back, and commutator pitches ; (b) degree of reëntrancy; and $(c)$ number of paths between the brushes. Is the winding symmetrical? Why?

234. A certain Siemens and Halske I4-pole I,O0o kilowatt generator has a quintuple wave wound drum armature with I, I 44 inductors. Find : (a) front, back, and commutator pitches ; and (b) degree of reëntrancy.

235. An 8-pole duplex wave-wound drum armature is to be driven at a speed of 100 revolutions per minute, and the useful flux per pole is $8.3 \times 10^{6}$ lines. How many inductors are needed to generate 150 volts? Ans. 542 inductors. 



\section{INDEX.}

Ab-amperre, definition of the, 16

Ab-ohm, definition of the, 20

Absorption of light, influence of, on illumination, 324

Ab.volt, definition of the, 24

All-day efficiency, definition of, 469

Alternating current arc, the, 334

Alternator, the, 36

Aluminum wire, weights and resistances of, 307

Ammeters, 200 errors of, 479

Ampère, definition of the, 16

Ampere-turn, the, 356

Angle of lead of brushes, 94

Anti-parallel wiring scheme (see return loop).

Arc, the electric, characteristics of, 330 alternating-current, 334

direct-current, 332

lamp, the, 327

$$
\text { rating, } 337
$$

Arc lamps on constant-voltage mains, 336 series grouping of, $33^{8}$

lighting, constant-current series system, 339

Armature, closed-coil and open-coil, 40 coil or section, 420 core loss, calculation of, 382 cross-magnetizing action of, $\mathbf{I} 5 \mathbf{I}$ definition of, $36,3^{8}$ demagnetizing action of, $15 \mathrm{I}$ faults, testing for, 182 inductors, 420

loss in generators and motors, 129 neutral axis of, 93

reaction, $\mathbf{1 5} \mathbf{I}$ compensation of, $\mathbf{I} 60$

winding, dependence of brushes upon, 432

the disk, 419
Armature winding, the drum, 418 element of, 420 the helical, 418

lap, 423 modified multiplex, 445 multi-circuit, 43r open-coil and closed-coil, 4I9 parallel grouping of, see lap. reêntrancy of, 428 the ring, 418 table of conditions, 436 two-circuit, 43 I simplex and multiplex, 429 series grouping of, see wave. wave, 426

Armature windings, 418 examples of, 436

Armatures, smooth and slotted, 67

Axis of commutation, 93 neutral, of armature, 93

Balancer, the motor-generator, 274 the three-wire, 273

Battery, the storage (see storage battery).

the voltaic or primary, 239

Bipolar versus multipolar dynamos, 65

Booster, the compound, $26 \mathrm{I}$

the differential, 26r

the negative, 259

the shunt, 257

with carbon rheostat control, 26r

Boosters, automatic, 258

Brush arc-lighting generator, the, 342

contact resistance, $472-473$

holders, 63

lead, $9 \mathrm{r}$

Brushes, 63

number of on a dynamo, 432

Building-up of a generator, 80

Bunsen photometer, the, 313 
Candle, the spherical-, $3 \mathbf{I I}$

the standard, 3 ro

Candle-foot, the, definition of, 3 I 2

Candle-power of carbon-filament glow lamps, 344

Carrying capacity, safe, of wires, 284

Cast iron, magnetic properties of, 365

Characteristic curves of a compound generator, 407

of dynamos, 383

of generators, 75

of motors, 408-417

of series generators, $386-397$

of shunt generators, 397-407

of motors, 123

of the electric arc, 330

of railway motors, 415

Circuit breakers and fuses, 205

Circular mil, definition of, $45 \mathrm{I}$

Collecting rings, definition of, 37

Commutator bars, number of, 421

definition of, 39

details of, 63

Commutation, axis of, 93

the phenomena, I6I

Compass, the, I

Compensating field winding, 160

Compound generator, the, 47,86 adjustment of, 90 connections of, 79

motor, the, I 16

Constant-current distribution, 74

transformer, the, $34 \mathrm{I}$

-voltage distribution, 74

Control of voltage of generators, 88

of speed of motors, 109

Controller, the series-parallel, I 21

Cooper-Hewitt lamp, see mercury vapor lamp.

Cost of electrical power, 223

of steam power, $22 \mathrm{I}$

Costs, installation and operation of stations, 220, 230

Coulomb's law, 3

Counter-electromotive-force cells, use of, 256

definition of, 97
Cumulative compound motor, the, II 7

Current, electric, strength of, 15

Density of wires, 283

Dettmar's three-wire generator, 272

Differential booster, the, 26 I compound motor, the, 116

Diffusers, lamp, 338

Direct-current arc, 332 dynamo, $3^{8}$

Distribution and wiring, 266

constant-current, 74

constant-voltage, 74

Edison three-wire system of, 268

electric, size of wires for, 275

parallel system of, 266

series system of, 266

Dobrowolsky's three-wire generator, 273

Drum armature, the, 418

Dynamo, fundamental equation of, 43

ratings, 169

speeds, 145

the, 35

the alternating-current, 36

the direct-current, 38

the homopolar, $7 \mathrm{I}$

the multipolar direct-current, $4 \mathrm{I}$

Dynamometer, use of motor as a, 143

Dynamos, diseases of, 175

heating of, 148

insulation of, 167

management of, 172

operation of, 172

sparking of, I 5 I

sparking of, conditions of, r6 $\mathbf{I}$

special designs of, 69

Dynamotor, the, 70

Eddy current loss in iron, 381

currents, 49

Edison three-wire system of distribution, 268

Efficiencies of generators and motors, 127

Efficiency, all-day, of a dynamo, 469

of carbon-filament glow lamps, 344

of conversion of a generator, 135

of a motor, $14 \mathrm{I}$ 
Efficiency, electrical, of a generator, 135 of a generator, calculation of, 132 of generator, variation of with output, 136

of lamps, 326

mechanical, of a motor, $14 \mathrm{I}$

of a motor, calculation of, 138

Siemens' law, 99

variation of, with output, $14 \mathrm{x}$

Electric arc, the, characteristics of, 330

current, see current.

distribution, size of wires for, 275

lamp, the, 325

lighting, 308

strength of air between parallel wires, 302

of cable insulation, 3 or

of insulation, dependence of size of wires upon, 299

Electrical efficiency of a generator, 135 power, cost of, 223

systems of selling, 232

resistance, see resistance.

Electromotive force, definition of, 22 induced, 30

Electrolysis, 238

Electrolyte, definition of, 238

Electromagnets, 35 I

Enclosed-arc lamp, the, 336

End-cell control, 256

Equalizing arrangement, the, 184

Expansion, coefficients of linear, 283

Exciter dynamo, the, 38

External characteristic of series generator, 386

of shunt generator, 397

Faults in armatures, testing for, 182

Feeder control, 195

Field excitation of generators, 44, 87

of motors, $4^{8}$

separate, 44

loss in generators and motors, 127

magnet, definition of, $36,3^{8}$

magnetic, 4

rheostat, the, 88

switch, non-sparking, 188
Fire risks, 219

Flat-compounding of a generator, 86

" Floating battery,"' the, 258

Flux density in iron, $36 \mathrm{r}$

magnetic, definition of, 8

of light required for illumination, 324

Foucault currents (see eddy currents).

Fuses and circuit breakers, 205

Galvanized wire, weight of, 283

Generator, the, 35

the compound, 47,86 adjustment of, 90 connections of, 79

the double current, 273

efficiency, calculation of, 132

electromotive force of, 82

the flat-compound, 86

and motor efficiencies, 127

losses, 127

output of, $8 \mathbf{I}$

the over-compound, 86

separately excited, the, 85

the series, 46

series, connections of, 78

the shunt, 45

shunt, connections of, 79

the split-pole, 272

Generators, characteristic curves of, 75

characteristic curves of, 386-407

for Edison three-wire system, 27 I

field excitation of, 44,87

and motors, diseases of, 175

management of, $\mathbf{r}_{\mathbf{7}} \mathbf{2}$

starting of, 174

operation of, in parallel, I 84

rating of, 82

voltage control of, 88

voltage regulation of, 88

Gilbert, the definition of, 356

Glare of a lamp, definition of, 322

Globes, lamp, $33^{8}$

Glow lamp, the carbon-filament, 343 metal-filament, 348

Grounds and ground detectors, 209

Guarantees and ratings, 145

standard, 164 
Heating of dynamos, 148

Heat, radiant, definition of, 308 run, the, 149

Hefner, the, 3 I I

the spherical-, $3 \mathbf{I} \mathbf{I}$

lamp, the, 3 ro

Homopolar dynamo, the, $7 \mathbf{I}$

Hysteretic coefficients, table of, 380

Hysteresis loss in iron, 380

magnetic, 379

Illumination, the problem of, 32 I intensity of, 312

Impressed electromotive force, definition of, 97

Incandescent lamp, see glow lamp.

Induced electromotive force, $3^{\circ}$

Inductors, armature, 420

Insulation of dynamos, 167

of pole lines, 304

strength of, 299

of underground, station, and house wires, 305

Internal characteristic of series generator, 386

of shunt generator, 398

Iron and steel wire, weights and resistances of, 307

Joule's law, 20

Kelvin's law of economy, 295

limitations of, 299

Lamination of dynamo iron, 49

Lamp, the arc, 327

efficiency, 326

the electric, 325

the Hefner, 3 ro

globes, shades and diffusers, 338

the mercury-vapor, $35^{\circ}$

metal-filament glow, $34^{8}$

the Nernst, 349

the osmium, $34^{8}$

the tantalum, 348

Lamps, $\operatorname{dim}$ versus bright, 323

standard, 3 ro
Lap windings, 423

Lead, angle of, 94

of brushes, $9 \mathrm{r}$

backward, 94

forward, 94

Leakage, magnetic, $37 \mathrm{I}$

Lenz's law, 30

Life of carbon-filament glow lamps, 344

Light, amount of required for illumination, 324

conical intensity of, $3 \mathrm{II}$

definition, 308

distribution of, around a lamp, 314

flux measurement of, 316

luminous intensity of, 309

physical intensity of, 308

sectional intensity of, 3 I I

Lighting, electric, 308

of streets, 325

Lightning and lightning arresters, 2 I I

Linear expansion, coefficients of, $28_{3}$

Lines, mechanical stresses in, 276

Load-factor of a station, definition of, 226

Local action and voltaic action, 240

Loss, armature, in generators and motors, 129

field, in generators and motors, 127

stray power, in generators and motors, I 29, 130

Losses in generators and motors, 127

Lumen, definition of the, 312

Lux, the, definition of, 3 II

Magnetic circuit, the, 366

field, direction of, 5

due to a wire, 13

in a long solenoid, 19

homogeneous and non-homogeneous, 5

intensity of, 4

figures, 7

flux, definition of, 8

in iron, 359

hysteresis, 379

leakage, $37 \mathrm{I}$

coefficient of a dynamo, 372

po-meability, 365 
Magnetic properties of iron and steel, table, 365

reluctance, 368

Magnetism of iron, $35 \mathrm{I}$

residual, $36 \mathbf{r}$

Magnetization curve of a dynamo, 384 curves, 362

intensity of, 359

of iron, 12

Magnetizing force in iron, 356

Magnetomotive force of a coil, 354 definition of, $35^{2}$

units of, 356

Magnet, the, I

$$
\text { pole, sign of, } 3
$$

windings, bunched and distributed, $35 \mathrm{I}$

Magnets, mutual action of, $\mathbf{I}$ permanent, 36 I

Matthews photometer, the, 318

Maximum demand meter, the, 235

Mechanical efficiency of a motor, I4I

Mercury-vapor lamp, 350

Meter, the maximum demand, 235 the two-rate, 235

Mil, definition of the, $45 \mathrm{I}$

Motor and generator efficiencies, 127

$$
\text { losses, } 127
$$

direction of running of, 95

efficiency, calculation of, 138

Siemens' law of, 99

fundamental equation of, 97

shunt, starting of, 112

the, 35

the compound, 116

the " multi speed," 106

the series, 118

the shunt, ror

speed control of, 105

regulation of, 104

torque, 98

Motor generator balancer, the, 274

the, 70

Motors and generators, characteristic curves of, 123, 408-4I 7

diseases of, 175

field excitation of, 48
Motors and generators, management of, 172

starting of, I 74

speed control of, comparison of methods, 109

Multiplex armature winding, 429

Multipolar versus bipolar dynamos, 65

Multivoltage speed control of motors, 108

National Electrical Code, the, 220

Nernst lamp, the, 349

Network of conductors, solution of, $\mathbf{2 7}$

Neutral axis of armature, 93

Oersted, the, 369

Ohm, definition of the, 20

Ohm's law, 23

Open-arc lamp, the, 336

Operation test of a station, 199

Osmium lamp, the, 267,348

Output of a generator, $8 \mathbf{r}$

Over-compounding of a generator, 86

Parallel connections, 24 grouping of glow lamps on arc cir. cuits, 267 operation of generators, 184 system of distribution, 266

Performance test of a station, 199

Permanent magnets, $36 \mathbf{I}$

Permeability magnetic, 365

Photometer, the Bunsen, $3 \mathbf{I} 3$ the integrating, 318 the Matthews, 318

Photometry, 308, 309 simple, 309

Pole-line construction (reference), 305 lines, insulation of, 304

mechanical stresses in, 276 magnet, sign of, 3 strength of $\mathrm{a}, 2$

Poles, distributed and concentrated, 2 of a magnet, $\mathbf{I}$

Power, electrical, systems of selling, 232 cost of, 22 I, 223 rating of generators, 82

Radiant heat, definition of, 308 
Railway motors, characteristics of, $4 \mathrm{I} 5$

Rating of arc lamps, 337

of generators, 82

Ratings and guarantees, I45 standard, $x_{4}$

estimation of, from speed and dimensions, $\mathbf{1 4 8 ,} \mathbf{1 6 5}$

of dynamos, 169

Reaction, armature, $15 \mathrm{r}$

Reëntrancy of an armature winding, 428

Regulation, voltage, as a factor in wiring, 285

of generators, 88

Reluctance, magnetic, 368

Reluctivity, 369

Residual magnetism, definition of, 36 r

Resistance, definition of, 20

of brush contacts, $472-473$

temperature coefficient of, 21

Resistances and weights of copper wire, 306

Resistivity, see specific resistance.

Return-loop wiring scheme, 292

Rheostat, starting, the, 112

Ring armature, the, 418

Safety, personal, rules for, I33

Series connections, 24

generator and motor compared, 96 connections of, 78

the, 46

grouping of glow lamps, 266

motor, the, 118

parallel controller, 12 I

system of distribution, 266

Shades, lamp, $33^{8}$

Shunt booster, the, 257

generator and motor compared, 95 connections of, 79

the, 45

motor, Ior

speed control of, ro5 regulation of, 104

starting, I 12

Siemens' law of efficiency of a motor, 99

Sign, algebraic, of magnet pole, 3

Simplex armature winding, 429
Size of wires for electric distribution, 275

Solenoid, definition of, $\mathbf{1} 9$

Sparking of dynamos, $\mathbf{I}_{5} \mathbf{I}$

conditions of, $\mathbf{1} 6 \mathbf{I}$

Specific gravity (see density).

resistance, 21

Spectro-photometry, 309

Speed control of motors, comparison of methods, 109

of shunt motor, 105

regulation of shunt motor, 104

Speeds of dynamos, 145

Standard lamps, 3 I0

ratings and guarantees, 164

Starting a generator or motor, method of, 174

of shunt motor, 112

rheostat, the, II 2

Steam power, cost of, 22 I

Steel and iron wire, weights and resistances of, 307

magnetic properties of, 365

Storage batteries, management and care of, 250

use of, 254

weights and costs, 250

battery, action of, 242-247

grids, 247

the, 242

cell, the, 242

Stray power loss in generators and motors, 129, 130

Street lighting, 325

Table of armature-winding conditions, 436

candle-power and efficiency of glow lamps, 344

carrying capacities, 284

costs of installation, $23^{\circ}$

of operation, $23 \mathrm{I}$

density of wires, 283

hysteretic coefficients, $3^{80}$

magnetic properties of iron and steel, 365

resistances and weights of copper wires, 306 
Table of tensile strengths of wood, 278 of wires, 283

\section{units, 34}

Tantalum lamp, the; 348

Tensile strength of wood, table, 278 strengths of wires, table, 283

Three-wire balancers, 273 generators, $27 \mathrm{I}$ system, the Edison, 268

Torque of a motor, 98

Total characteristics of generators (see internal characteristics).

Transformer, the constant-current, 34I

Two-rate meter, the, 235

Underwriter's rules, 219

Unipolar dynamo, the, $7 x$

Units, table of, 34

Voltaic action and local action, 240 battery, the, 239 cell, the, 239

Voltage control of generators, 88 regulation of generators, 88

Volt, definition of the, 24

Voltmeter multiplying coils, 202

Voltmeters, 20x errors of, 479

Volts-per-commutator-segment of a dynamo, 474
Ward Leonard method of speed control of motors, 108

Watt-hour meter, the, 204

Wattmeter, the, 202

Wattmeters, errors of, 480

Wave windings, 426

Weight of galvanized wire, 283

Weights and resistances of copper wire, 306

of wires, 283

Windings, armature, see armature.

Wire, weights of, 283

weights and resistances of, 306

Wires for electric distribution, size of, $\mathbf{2 7 5}$ heating of, 284

information concerning (reference), 305

mechanical stresses in, 276

testile strengths of, $\mathbf{2 8 3}$

Wiring calculations for concentrated loads, 286

based on power loss, 296

distributed loads, 288

in constant-voltage systems, 286

for return-loop scheme, 294

charts, 287

outside and inside (reference), 305

Wood, tensile strength of, table, 278

Wrought iron, magnetic properties of, 365 



$s 1^{-219}$ 
PLEASE DO NOT REMOVE CARDS OR SLIPS FROM THIS POCKET

\section{UNIVERSITY OF TORONTO LIBRARY}

S\&M

A

219 
